\title{
Marc Schaefer
}

\section{Recherches sur la famille et l'oeuvre des Silbermann en Alsace}

Walcker-Stiftung für orgelwissenschaftliche Forschung 


\section{Veröffentlichungen der}

Walcker-Stiftung für orgelwissenschaftliche Forschung

Band 23

publiziert auf http://www.walcker-stiftung.de/

(C) Walckerstiftung für orgelwissenschaftliche Forschung

c/o PD Dr. Roland Eberlein

Franz-Raveaux-Str. 16

50827 Köln 


\section{Avertissement}

La présente version de ma thèse de doctorat de $3^{\text {ème }}$ cycle est une reproduction quasi inchangée du texte dactylographié d'origine datant de 1984, pour ce qui est du texte et de la mise en page. En particulier il m'a paru important de maintenir inchangée la répartition du texte par rapport à la pagination, afin de permettre au lecteur de retrouver des renvois occasionnels à mon texte faits dans des publications subséquentes. Pour cela il a été nécessaire de maintenir la présentation ainsi que la police et la taille des caractères. Il en va de même de quelques irrégularités au niveau des interlignes et de la pagination. J'ai renoncé délibérément à retravailler et à actualiser le texte. Toutefois quelques erreurs ont été corrigées. Signalons que dans la transcription des pièces justificatives le $ß$ allemand a été remplacé par ss, étant donné que les machines à écrire françaises de l'époque ne comportaient pas de $B$.

\section{Vorbemerkung}

Bei der vorliegenden Fassung meiner Dissertation handelt es sich um eine in Text und Layout weitgehend unveränderte Nachschrift des ursprünglichen Typoskripts aus dem Jahr 1984. Insbesondere schien es mir wichtig, daß die Zuordnung des Textes zu den Seitenzahlen weitgehend unverändert bleibt, damit der Leser Verweisen auf meine Arbeit nachgehen kann, die sich in der bisher erschienenen Literatur gelegentlich finden. Das erforderte eine Beibehaltung von Layout, Schrifttyp und Schriftgröße sowie von gewissen Unregelmäßigkeiten im Zeilenabstand und in der Seitenzählung. Auf eine Überarbeitung und Aktualisierung des Textes wurde bewußt verzichtet, allerdings habe ich einige Korrekturen angebracht, wo mir dies geboten erschien. Bei der Wiedergabe historischer Dokumente in den »Pièces justificatives « ist zu berücksichtigen, $§$ durch ss ersetzt wurde, da es damals auf französischen Schreibmaschinen kein ß gab.

Straßburg, im November 2012

Marc Schaefer 
UNIVERSITE DES SCIENCES HUMAINES DE STRASBOURG
FACULTE DES SCIENCES HISTORIQUES

Thèse de Musicologie présentée pour l'obtention du Doctorat de 3ème Cycle:

RECHERCHES SUR LA FAMILLE ET L'OEUVRE DES

SILBERMANN EN ALSACE.

INTRODUCTION AUX "ARCHIVES SILBERMANN DE PARIS"

Préparée sous la Direction de

M. Ie Doyen Georges LIVET

par MarC SCHAEFER 


\section{Introduction}

a) Aperçu général sur l'orgue en France à la fin du $17^{\circ}$ siècle.

Vers la fin du $17^{\circ}$ siècle l'orgue classique français, tel qu'il sera décrit et codifié un siècle plus tard par Dom Bedos (1), est déjà formé. La diffusion de ce type d'orgue unifié, qui n'a guère varié pendant plus d'un siècle, a été favorisée par des conditions politiques et économiques. Paris est la capitale d'un Etat relativement centralisé. Il n'y a plus en France de capitales de province dont l'importance et le rayonnement artistique puissent être comparés à ceux des grandes villes d'Allemagne ou d'Italie. La richesse n'est plus détenue par les princes, mais par les institutions de l'Eglise, chapitres de cathédrales et monastères. L'on sait que les échanges étaient très nombreux entre ces derniers. L'orgue classique français sera "parisien"; les "facteurs du Roy" donneront le ton. Ils travaillent dans toute la France, uniformisant le style. De ce fait les particularités provinciales sont en France beaucoup moins marquées que dans d'autres pays, par exemple l'Allemagne.

Alexandre Thierry (1646-1699), facteur d'orgues du Roi, fut le meilleur organier de sa génération. Il fut l'auteur des orgues de St. Séverin (1675), de Saint-Cyr (1685), des Invalides (1683), de St. Eustache (1681). A la fin de sa vie il s'associa à Julien Tribuot, puis à Robert Clicquot (v. 1645 - v. 1719). Citons encore Jean de Joyeuse (v. 1638 - 1698) (3), Pierre François Deslandes, Jacques Carouge, Robert Ingout.

Quelles furent les caractéristiques de cette facture? Les usages sont établis dès le milieu du siècle. Les principaux et les anches ont les corps en étain et les pieds en plomb. Les basses de Pédale et les basses bouchées des Manuels sont en bois. Les jeux de la synthèse flûtée sont en plomb. Les sommiers sont à gravures. La mécanique, du type "suspendu", est d'une grande simplicité et d'une remarquable souplesse.

Les plans sonores des claviers de Grand Orgue et de Positif sont presque toujours complets, en ce sens qu'ils comportent, d'une 
part, tous les rangs pour un Plenum directement proportionné aux buffets, d'autre part, les jeux formant le cornet décomposé, et s'il y a Trompette et Clairon - le cornet composé, de cinq rangs. Il commence en général au $c^{\prime}$ et se trouve posté en hauteur, derrière les tuyaux de façade. Les anches utilisées sont toujours les mêmes: Trompette 8, Clairon 4, Cromorne 8, Voix humaine 8. Le $3^{\circ}$ clavier est en général un demi-clavier. S'il commande un Récit, sa tuyauterie est placée au-dessus du Grand Orgue; s'il s'agit d'un Echo, la tuyauterie est placée dans le soubassement. Ce clavier d'Echo possède souvent un petit plein-jeu d'Echo, qui disparấtra vers le milieu du $18^{\circ}$ siècle.

La tessiture des orgues est encore variable. L'octave courte est encore pratiquée. Souvent les claviers comptent 48 notes, C D c''' sans le premier do dièse. Les claviers de pédale avaient souvent un ravalement, notamment pour les jeux d'anches, soit au contre-La, soit même au contre-Fa. Le diapason était celui de l'Opéra de Paris c'est-à-dire environ deux demi-tons plus bas. Le tempérament était inégal. (4)

L'orgue français classique forme un ensemble cohérent, typisé et rationalisé à l'extrême. Les contemporains ne parlaient guère des compositions des orgues: Il suffisait de caractériser l'instrument par la hauteur du plus grand tuyau de façade et le nombre de claviers. On distinguait aussi un "petit 8" d'un "grand 8" qui possédait un Bourdon 16.

La fin du siècle voit les travaux du mathématicien et acousticien Joseph Sauveur (1653-1716), qui ne seront pas sans influence sur l'évolution du Plein-Jeu classique français. (5) Le plein-jeu de type normand-flamand, formule qui sera retenue par Dom Bedos un siècle plus tard, et dont il reste un témoin au Petit-Andely (R. Ingout 1674), sera supplanté par le plein-jeu nouvelle manière, au début du règne de Louis XIV: plein-jeu cymbalisé pratiqué par Robert Clicquot, Deslandes, Jean de Joyeuse (Auch 1688, Béziers 1679) .

A cette époque les grands principes de la registration française sont arrêtés. (6) Les "mélanges" les plus usités sont au nombre de trois: Le Plein-jeu réunit les Principaux du 16 pieds jusqu'au 2 pieds, les Fournitures et Cymbales, avec les Bourdons de 16 et de 8, tant au Grand Orgue qu'au Positif. Les Fournitures et Cymbales contiennent des Octaves et des Quintes.

Le Jeu de Tierce, que Nivers appelle aussi "le gros jeu de dimi- 
nutions" comprend les rangs du Cornet décomposé, tant au Grand Orgue qu'au Positif. Depuis le dernier tiers du $17^{\circ}$ siècle, la série s'est enrichie de la Grosse Tierce, qui fait appel à la fondamentale de 16 pieds du grand clavier. De façon générale la Tierce occupe une place de choix en France.

Le Grand Jeu se compose du Jeu de Tierce, auquel vient s'ajouter l'ensemble des anches: Trompettes, Clairons, Cromorne ainsi que le Cornet composé.

Les autres mélanges sont destinés à faire chanter une voix en solo.

Une nouvelle musique voit le jour. Le style polyphonique d'un Titelouze est peu à peu abandonné au profit d'un style mélodique, inauguré par Nivers en 1665, par ses récits de solos. Cette musique est destinée au culte catholique et s'inscrit dans le déroulement de l'office. La fin du siècle voit apparâttre toute une série de "Livres d'Orgue", émanation caractéristique de l'orgue classique français.

Nivers, 1665, 1667, 1675.

Lebègue, 1676, 1678-79, 1685.

Gigault, 1685 .

Raison, 1688.

D'Anglebert, 1689 .

Boyvin, 1689 .

F.Couperin, 1690 .

Jullien, 1690.

Chaumont, 1695.

De Grigny, 1699.

Quelques compositions d'orgues de la fin du $17^{\circ}$ siècle serviront à illustrer l'orgue classique français parvenu à sa maturité.

Le Petit Andely. Robert Ingout 1674. (7)

Cet instrument reste l'unique témoin de la facture d'orgues française du $17^{\circ}$ siècle.

$\begin{array}{lll}\text { Positif } & \text { Grand Orgue } & \text { Echo } \\ \text { Bourdon 8 } & \text { Bourdon 16 } & \text { Bourdon 8 } \\ \text { Prestant 4 } & \text { Montre 8 } & \text { Prestant 4 } \\ \text { Flûte 4 } & \text { Bourdon 8 } & \text { Nazard 3 } \\ \text { Nazard 3 } & \text { Prestant 4 } & \text { Doublette 2 } \\ \text { Doublette 2 } & \text { Flûte 4 } & \text { Tierce 1 3/5 } \\ \text { Tierce 1 3/5 } & \text { Grosse Tierce 3 1/5 } & \text { Fourniture II } \\ \text { Fourniture III } & \text { Nazard 3 } & \text { Régale 8 }\end{array}$


Cymbale II

Doublette 2

Cromorne 8

Tierce $13 / 5$

Larigot 1 1/3

Cornet V

Fourniture IV

Cymbale III

Trompette 8

Clairon 4

Voix humaine 8

L'étendue des claviers est de 48 notes (C D - C'''). Celle de l'Echo est de 37 notes (c - C'''). La Pédale semble avoir disparu lors de la Révolution. Tremblant fort, Tremblant doux, Rossignol. Accord un demi-ton au-dessous de notre diapason habituel. Voici le relevé complet des pleins-jeux:

Fourniture Grand Orgue

\begin{tabular}{|c|c|c|}
\hline C & $f$ & $f^{\prime}$ \\
\hline $11 / 3$ & $22 / 3$ & $51 / 3$ \\
\hline 1 & 2 & 4 \\
\hline $2 / 3$ & $11 / 3$ & $22 / 3$ \\
\hline $1 / 2$ & 1 & 2 \\
\hline
\end{tabular}

Cymbale Grand Orgue

$\begin{array}{llllllll}C & C & f & C^{\prime} & f^{\prime} & C^{\prime \prime} & f^{\prime \prime} \\ 1 / 2 & 2 / 3 & 1 & 11 / 3 & 2 & 22 / 3 & 4 & 22 / 3 \\ 1 / 3 & 1 / 2 & 2 / 3 & 1 & 11 / 3 & 2 & 2\end{array}$

\section{Fourniture Positif}

C

1

$2 / 3$

$1 / 2$

$\underline{\text { Cymbale Positif }}$

$\mathrm{C}$

$1 / 3$

C

$1 / 2$

$1 / 4$

$1 / 3$

Fourniture Echo

C

$2 / 3$

$1 / 2$

f

2

$11 / 3$

1

$\mathrm{f}$

$2 / 3$

$1 / 2$
$C^{\prime}$

1

$2 / 3$ f

4

$22 / 3$

2

f

$C^{\prime}$ '

f' '

$11 / 3$

$22 / 3$

1

2

$11 / 3$

2 
Saint-Quentin, Robert Clicquot et Alexandre Thierry, 1697. (8)

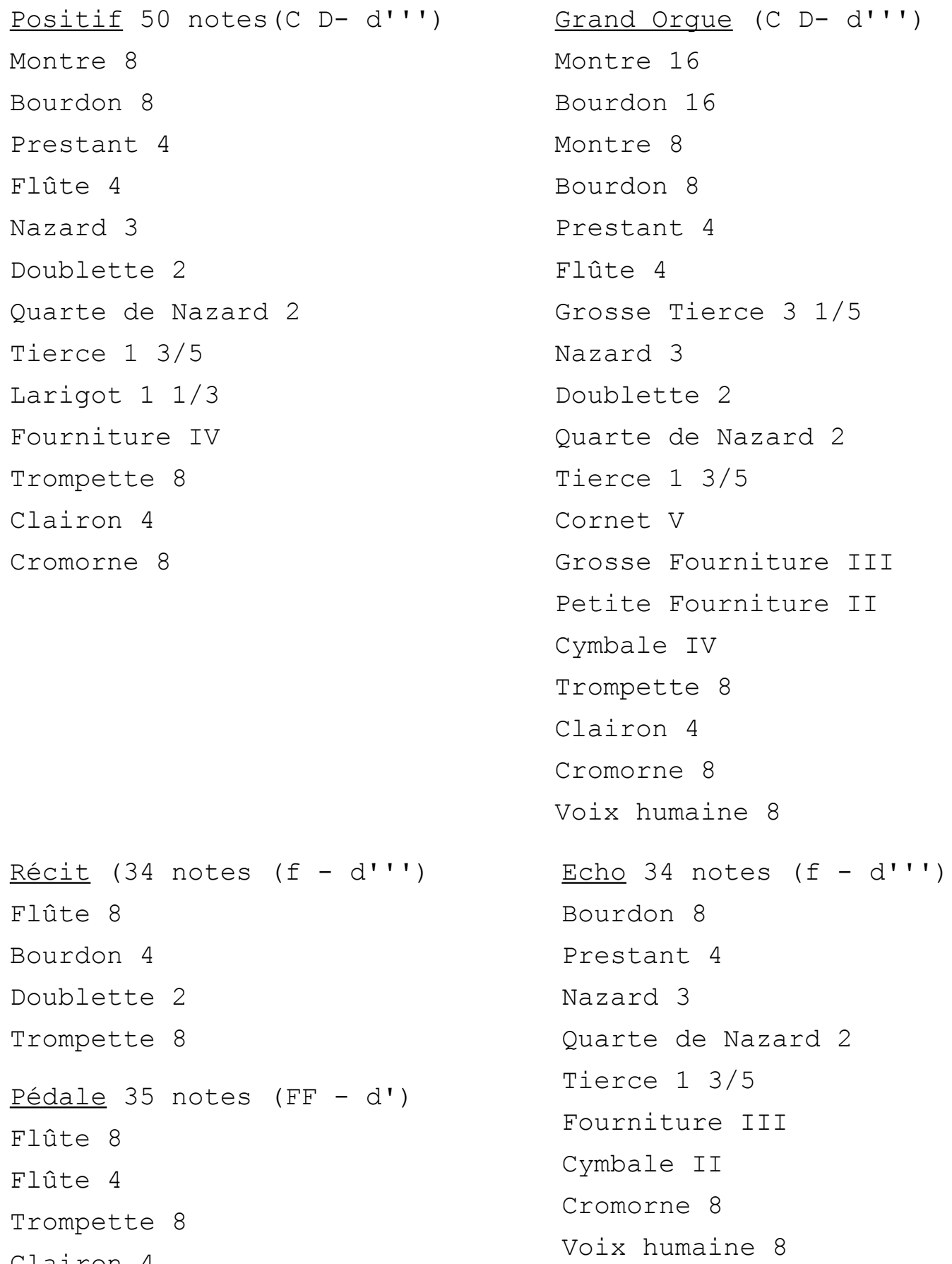


Auch. Jean de Joyeuse, 1688-1693. (3)

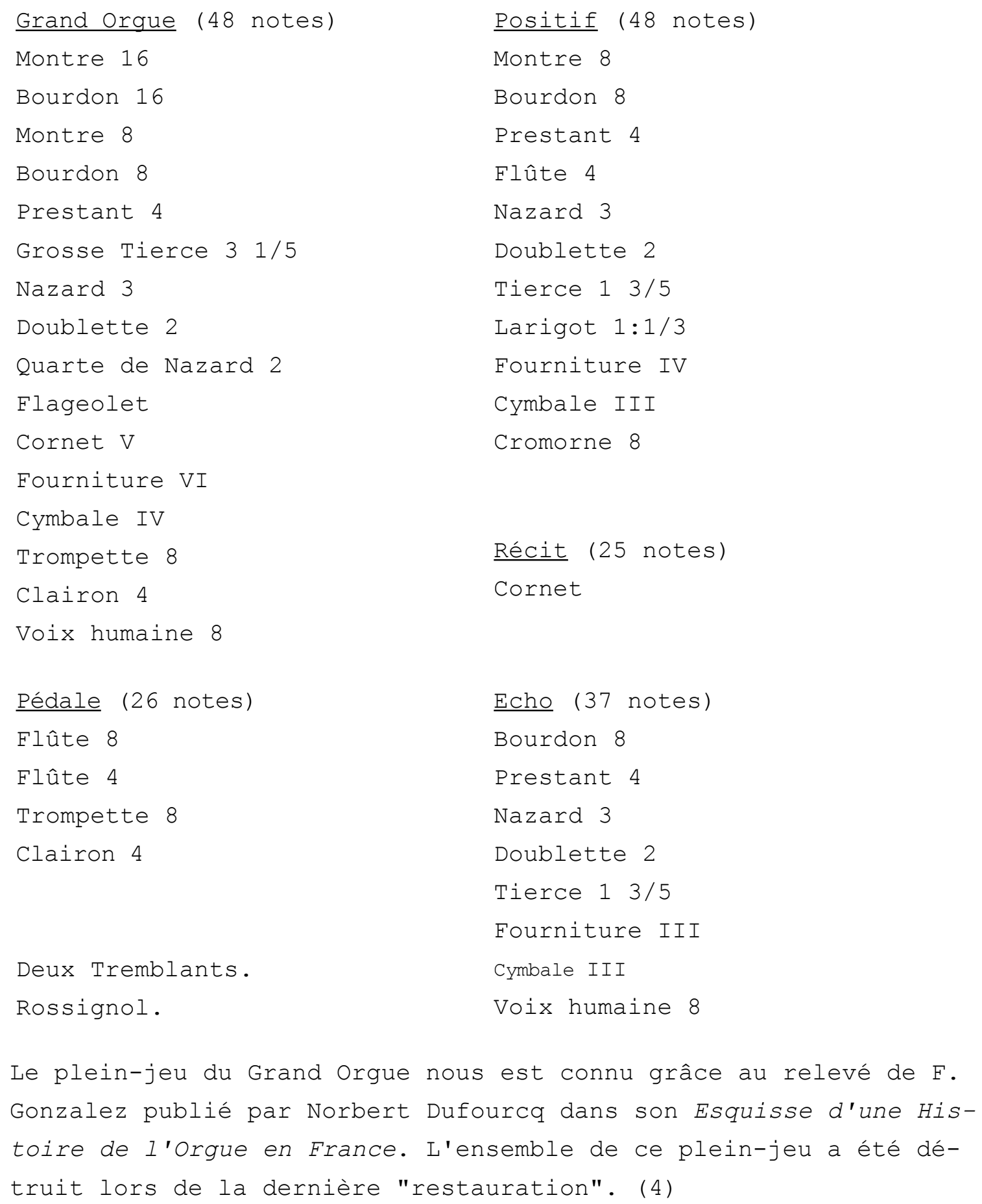


Paris. St.Germain-des-Prés. Alexandre Thierry 1667. (9)

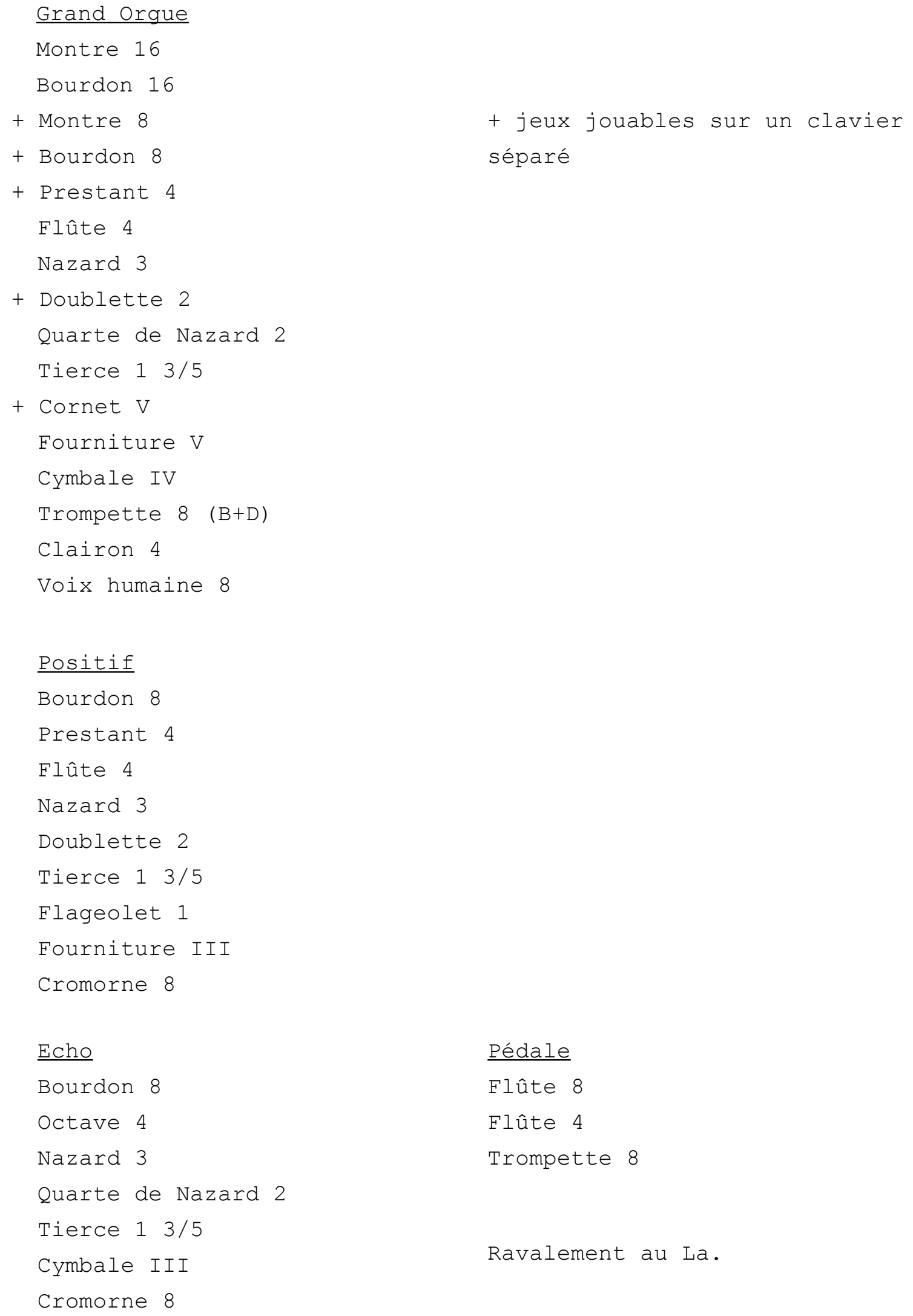


(1) Dom François Bedos de Celles, L'Art du facteur d'orgues, 3 vol. in-folio, 1766-1778.

(2) Georges Lhôte, Remarques sur l'orgue français, ISO-Information $\mathrm{N}^{\circ} 1,1969, \mathrm{p} .57 \mathrm{sS}$.

(3) Voir Norbert Dufourcq, Jean de Joyeuse et la pénétration de la facture d'orgues parisienne dans le Midi de la France au XVIIme siècle, Paris 1958.

(4) Jean Fellot, L'orgue classique français, Musique de tous les temps, Saint-Michel-de-Provence, 1962.

(5) Léon Souberbielle, La Théorie Louisquatorzienne du Plain Jeu de l'orgue français, une source de son histoire, à Paris, en 1702, Paris 1971.

(6) Fenner Douglass, The Language of the Classical French Organ, Yale 1969.

(7) Le Petit Andely, Musique de tous les temps, Saint-Michel-deProvence, 1963. - L'Orgue, illustration sonore à l'orgue du Petit Andely, Musique de tous les temps, 1962.

(8) Félix Raugel, Les grandes Orgues et les Organistes de la Basilique de Saint-Quentin, extrait de la Revue L'Orgue, $\mathrm{N}^{\circ}{ }^{\circ} 122-$ 123 (1967).

(9) Paris, Bibliothèque Nationale, Ms. fr. 18818, p. 212-214. 
b) La facture d'orgues en Allemagne à la fin du $17^{\circ}$ siècle.

Alors qu'en France la facture d'orgues est uniformisée à l'extrême, la facture allemande présente, à la même époque, une grande variété de styles et de traditions régionales. Le commerce florissant des villes hanséatiques, l'autorité des princes-évèques sont autant de facteurs favorisant le développement et le maintien de styles régionaux. C'est précisément dans les villes d'Allemagne du Nord que s'épanouit, dans les dernières décennies du siècle, l'activité prodigieuse d'Arp Schnitger. (1) Alors qu'en Allemagne la plupart des chefs-d'oeuvre de Schnitger ont été victimes soit des destructions des guerres, soit de l'outrage des restaurations abusives, les orgues de Groningue (Aa-Kerk) et d'Uithuizen aux PaysBas rendent encore aujourd'hui témoignage de l'art accompli de cet organier. La construction de l'orgue de St. Nicolas de Hambourg en 1682-87 établit solidement la réputation d'Arp Schnitger. Avec ses 67 jeux, cet instrument sera le plus grand orgue d'Allemagne et même d'Europe pendant un certain temps. Les différents plans sonores, y compris la Pédale, forment un tout bien homogène, offrant de nombreuses possibilités de registrations solistes (Sesquialtera, anches douces dans des tessitures variées). L'échelle des harmoniques est exploitée depuis le 32 pieds jusqu'au 1 pied. Les pleins-jeux présentent une plus grande acuité, vers le haut du clavier, qu'en France. Il convient de noter que, comme en France, le plafond du plein-jeu au milieu du clavier, c'est-à-dire en c', demeure le 2/3 de pied. Les mixtures de schnitger comportent parfois des Tierces, et même des quartes et sixtes. La composition de la Pédale est tellement complète (Principaux, Flütes, Mixtures, Anches) qu'elle rend superflue la Tirasse. Les jeux de Pédale sont la plupart du temps placés dans des tourelles de Pédale, séparées du buffet principal de part et d'autre (ou une seule tourelle comme à Norden). Les plans sont très nettement séparés et comportent très souvent un Brustwerk ou un Oberwerk. Les grands instruments ont un Positif, mais celui-ci tend à disparâ̂tre, alors qu'en France il sera pratiqué jusqu'au $19^{\circ}$ siècle. 
Peu à peu Schnitger abandonne le sommier à ressorts au profit du sommier à gravures. La mécanique, un peu moins souple qu'en France, utilise souvent des équerres. L'octave courte, condamnée par Werckmeister, est pratiquée assez longtemps en Allemagne du Nord. Le tempérament est inégal (mésotonique modifié) et le diapason est de façon générale plus haut qu'en France (jusqu'à une Tierce).

En Allemagne du Sud les jeux d'anches sont moins nombreux. Les Pédales sont moins fournies. On cultive avec soin une grande quantité de jeux de fonds colorés et caractéristiques. L'Allemagne connait une grande variété de Flûtes (Hohlflöte, Spitzflöte, Gemshorn, Waldflöte, Nachthorn, Blockflöte) et des jeux étroits, notamment en Franconie (Quintatön, Viola di Gamba, Salicional).

Souvent la tuyauterie est en plomb, à l'exception des tuyaux de façade. Parfois ceux-ci sont également en plomb, recouverts d'une feuille d'étain. Les basses des Bourdons sont également en métal.

Le théoricien de la facture d'orgues allemande est à cette époque Andreas Werckmeister (1645-1706). En 1698 il publie à Quedlinburg son Erweiterte und verbesserte Orgel=Probe.

La littérature d'orgue qui voit le jour est essentiellement d'inspiration protestante. Elle est liée au choral luthérien. Citons les principaux compositeurs de la fin du siècle:

D. Buxtehude (1637-1707), organiste à Ste. Marie de Lübeck;

V. Lübeck (1654 ou 1656-1740), organiste à St. Cosmae à Stade et plus tard à St. Nicolas de Hambourg;

J. Kuhnau (1660-1722), organiste à St. Thomas de Leipzig à partir de 1684;

N. Bruhns (1665-1697), organiste à Husum dès 1689;

G. Böhm (1661-1733), organiste à St. Jean de Lüneburg;

J.A.Reincken (1623-1722), organiste à Ste. Catherine de Hambourg;

J. Christoph Bach (1642-1703), organiste à Eisenach en 1665;

J. Caspar Kerll (1627-1693), organiste à la Chapelle impériale à Vienne à partir de 1677;

J. P. Alberti (1642-1710), organiste de la cathédrale de Merseburg;

J. H. Buttstedt (1666-1727), élève de Pachelbel, et organiste à la Predigerkirche d'Erfurt à partir de 1691;

J. C. F. Fischer (v. 1670-1746), mâ̂tre de chapelle au service du margrave Ludwig von Baden d'abord à Schlackenwerth (Bohême), puis à Rastatt; 
J. Krieger (1652-1735), organiste à Zittau en 1681;

N. Vetter (1666-1710), organiste à Erfurt;

Matthias Weckmann (1621-1674), organiste de St.Jacobi à Hambourg;

F. W. Zachow (1663-1712), organiste de Ste.Marie à Halle;

G. F. Kauffmann (1679-1735), qui succède à J. P. Alberti en 1698

comme organiste à Merseburg;

Franz Xaver Murschhauser (né en 1663 à Saverne, mort en 1738 à Mu-

nich) publie son Octo-Tonium novum Organicum, Augsbourg 1696;

Johann Pachelbel (1653-1706), organiste à Nuremberg, publie en 1699

son Hexacordum Apollinis, dédié à Buxtehude; ses Choräle zum

Präambulieren avaient paru en 1693; ses Musicalische Sterbens

Gedancken aus 4 variirten Chorälen bestehend sont imprimées à

Erfurt en 1683; ses oeuvres représentent une synthèse d'éléments d'Allemagne du sud et d'Allemagne centrale.

Sebastian Anton Scherer (1631-1712), organiste de la cathédrale d'Ulm; après avoir été organiste de St. Thomas à Strasbourg de 1684 à 1685; sa Partitura in cymbalo et organo voit le jour à Ulm en 1664 .

N'oublions pas Georg Muffat (né à Megève en 1653, mort à Passau en 1703). Elève de rhétorique au Collège des Jésuites à Molsheim, il fut organiste du Grand Choeur, replié à Molsheim, et quitta l'Alsace en 1674. (2) En 1690 il publia son Apparatus musicoorganisticus à Salzbourg.

Quelques compositions d'orgues donneront un aperçu sur la diversité des styles régionaux. L'orgue de Passau, construit par L. Freundt, de Passau, en 1688, fut joué par Muffat de 1690 à 1695. (3)

I.

Prinzipal 8

Copula 8

Flöte 8

Oktav 4

Oktavcopula 4

Quint 2 2/3

Superoktav 2

Mixtur VI
II.

Copula 8

Spitzviolin 8

Prinzipal 4

Spitzflöte 4

Superoctav 2

Quintadecima 1

Tremblant.

Accouplement des claviers.
Péd.

Portun 16

Octav 8

Puner 8

Quint $51 / 3$

Superoktav 4

Mixtur IV

Posaune 8

Notons l'absence de jeux d'anches aux claviers manuels. 
Au Musée de Munich se trouve un petit orgue provenant de Thalkirchen et datant de 1630 . Il reflète les mêmes tendances:

Clavier manuel:

Flaut 8

Prinzipal 4

Copel 4

Octav 2

Quint $11 / 3$

Mixtur III l'

Cimpel I $1 / 4^{\prime}$
Pédale:

Subbass 16

Octavbass 8

Quintbass $51 / 3$

La composition de l'orgue de St.Pierre-et-Paul de Weimar, qui sera joué par J.G.Walther, était la suivante (Christoph Junge 1685):

Grand Orgue:

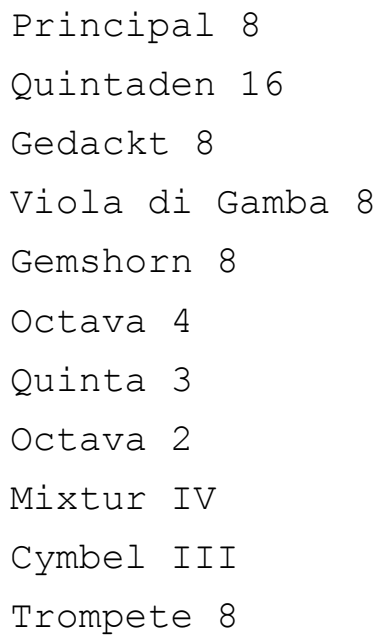

Positif: étain

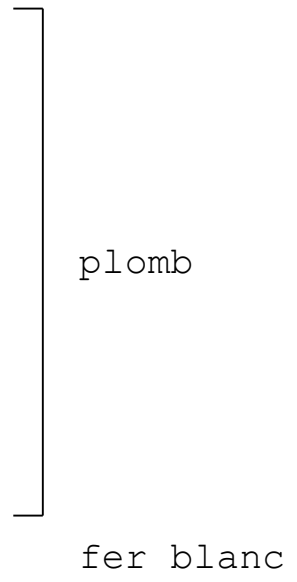

étain

plomb 
Pédale:

$\begin{array}{ll}\text { Subbass } 16 & \text { bois } \\ \text { Posaune } 16 & \text { bois } \\ \text { Trompete } 8 & \text { fer blanc } \\ \text { Cornet } 2 & \text { fer blanc }\end{array}$

2 tirasses, accouplement des claviers.

2 tremblants.

6 soufflets.

Zimbelstern RP.

Le style d'Allemagne centrale est représenté par l'orgue de Johann Jost Schleich, de Lohr, à Amorbach, 1685-1693:

Oberwerk, 42 notes:

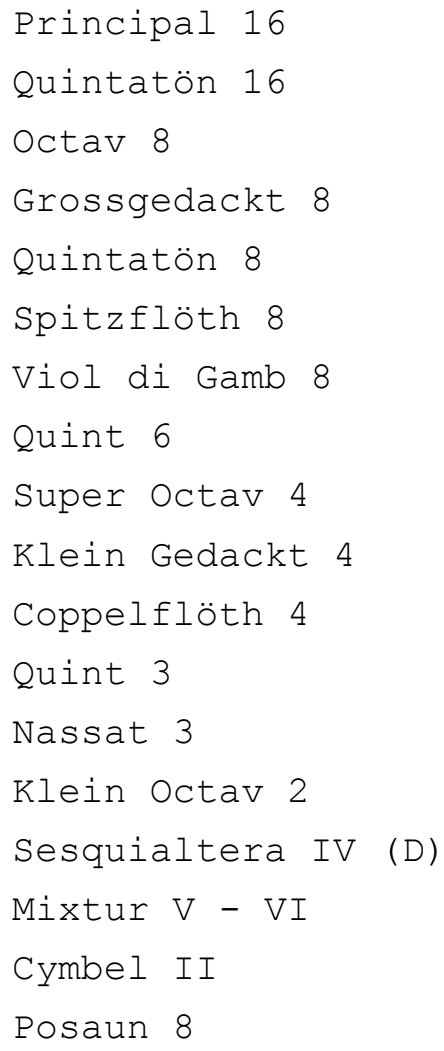


Pédale: 18 notes:

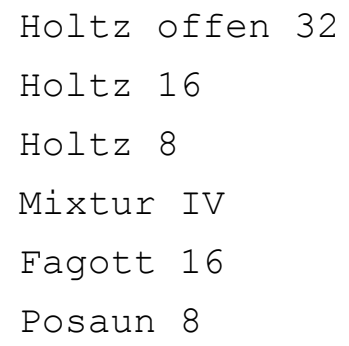

9 soufflets $(4)$

Voici une composition typique d'Arp Schnitger: l'orgue du couvent St. Jean à Hambourg (1680), transféré en 1816 à Cappel.

Hauptwerk, C D E - C'''

Quintadena 16

Prinzipal 8

Hollfloit 8

Oktave 4

Spitzfloit 4

Nasat 3

Gemshorn 2

Rauschpfeife II

Mixtur V - VI

Zimbel III

Trompete 8

Rückpositiv, C D E - C'''

Gedackt 8

Quintadena 8

Prinzipal 4

Floit 4

Oktave 2

Siffloit 1 1/3

Sesquialtera II

Terzian II

Scharff IV - VI

Dulzian 16

Pédale, C D - d'

Untersatz 16

Oktave 8

Oktave 4

Nachthorn 2

Rauschpfeife II 


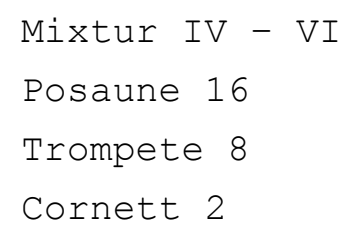

Tremblant.

Zimbelstern.

Accouplement des claviers, pas de tirasse.

accord $3 / 5$ de ton plus haut.

Composition de la Mixture V - VI du Grand Orque:

$\begin{array}{lllll}C & C & 9 & C^{\prime} \\ 11 / 3 & 2 & 2 & 4 / 3 & 4 \\ 1 & 11 / 3 & 2 & 2 & 2 / 3 \\ 2 / 3 & 1 & 1 & 2 & \\ 1 / 2 & 2 / 3 & 1 / 3 & 2 & \\ 1 / 2 & 2 / 3 & 1 & 1 / 3 & 1 / 3\end{array}$

Composition du Scharff IV - VI du Positif:

\begin{tabular}{|c|c|c|c|c|c|}
\hline C & C & $g$ & $C^{\prime}$ & $g^{\prime}$ & $C^{\prime}{ }^{\prime}$ \\
\hline 1 & $11 / 3$ & 2 & $22 / 3$ & $22 / 3$ & $22 / 3$ \\
\hline $2 / 3$ & 1 & $11 / 3$ & 2 & 2 & $22 / 3$ \\
\hline $1 / 2$ & $2 / 3$ & 1 & $11 / 3$ & $11 / 3$ & 2 \\
\hline \multirow[t]{3}{*}{$1 / 3$} & $1 / 2$ & $2 / 3$ & 1 & $11 / 3$ & 2 \\
\hline & & $1 / 2$ & $2 / 3$ & 1 & $11 / 3$ \\
\hline & & & & $2 / 3$ & $11 / 3$ \\
\hline
\end{tabular}


(1) Gustav Fock, Arp Schnitger und seine Schule, Kassel 1974.

(2) Marc Schaefer, L'orgue Silbermann de l'église paroissiale StGeorges de Molsheim, ancienne église des Jésuites, in Société d'Histoire et d'Archéologie de Molsheim, Annuaire 1971, p. 47 .

(3) J. Kappel, Der Dom des hl. Stephans zu Passau, Regensburg 1912 .

(4) E.F.Schmid - F. Bösken, Die Orgeln von Amorbach, Mayence 1963. 
c) La facture d'orgues en Alsace à la fin du $17^{\circ}$ siècle.

Quels furent les facteurs d'orgues, autochtones ou non, qui travaillaient en Alsace avant l'arrivée d'André Silbermann en 1699? Quelles étaient les compositions et les caractères généraux des instruments construits par ces facteurs? Ces questions méritent d'être posées, car elles nous aideront à comprendre la nature des premiers travaux d'André Silbermann. Nous pourrons ainsi distinguer ce qui dans sa production est un tribut payé aux traditions locales, et d'autre part ce qui appartient au "goût nouveau".

Dans la seconde moitié du $17^{\circ}$ siècle nous trouvons les noms de $\mathrm{F}$. Freundt, J.C. Spiess, J.C. Besthorn, C. et J.J. Eby, J.S. Füchslein, A. Geiger, C. Schaff, G.C. Lautensack, Wing, Gäusler, M. Tretzscher, J.J.Baldner et son gendre H. Hochwaldt-Reichert, T. Dressel. Vers 1700 S'y ajouteront F. Ring et C. Legros, qui seront les prédécesseurs immédiats d'André Silbermann à Strasbourg.

Frantz Freundt, qui signe tantôt Frantz tantôt Frantziscus, était établi à "Rothenburg am Neckar", c'est-à-dire Rottenburg près de Tübingen. Nous ignorons s'il était en parenté avec Johann Georg Freundt, de Passau, l'auteur de l'orgue de Klosterneuburg, 1642. Le 29 octobre 1683 F. Freundt signe un contrat avec la paroisse du Temple-Neuf (Predigerkirohe) à Strasbourg pour la réparation et l'amélioration de l'orgue. Malheureusement ce contrat n'indique pas la composition de l'instrument. Quelques conclusions peuvent cependant être tirées: L'orgue avait deux claviers de 46 notes, et le pédalier en comptait 25. La Pédale avait deux jeux, dépourvus de tirage de jeux: Soubasee 16 et Octave 8. Freundt se proposait d'y ajouter une Mixture de 6 rangs basée sur le 4 pieds, et dont 3 range devaient servir également au clavier manuel. Le 8 pieds de Pédale devait être jouable au clavier du bas, afin d' avoir deux jeux de 8 pieds à chacun des claviers manuels. Notons que Freundt devait pourvoir les jeux de Pédale de tirants, pour le cas d'un éventuel cornement ("wann vielleicht eine Pfeiff heulen sollte"). La réparation était évaluée à 170 florins, 2 rézaux de blé et autant de seigle, 6 mesures de vin, une corde de bois, et la fourniture de tous les matériaux nécessaires.

En 1687 Freundt répare l'orgue de St.Nicolas à Strasbourg pour 3 fl. (1) 
Johan Carol Spiess, dont nous ignorons l'origine, se signale par trois réparations d'orgues à Strasbourg: St. Guillaume en 1684 (2), St. Nicolas en 1690 (3), et St. Thomas en 1692. (4)

Joh. Sebastian Fichslein (Fixlein, Füchssle) répare l'orgue de St. Nicolas de Strasbourg en 1693, 1694, 1695 et 1696. (5)

Un dénommé Gäusler, de Lucerne, répare et accorde l'orgue de Ribeauvillé en 1657 pour $50 \mathrm{fl}$. et un demi foudre de vin. (6)

En 1698 l'orgue de l'église paroissiale de Kaysersberg est réparé par Caesarius Schaff, de Horb (Wurtemberg). (7)

Le bénédictin Anton Geiger, né vers 1640 à Lure, décédé au couvent de Luxeuil en 1711, répare et déplace l'orgue de l'abbaye bénédictine de Murbach en 1691. (8)

Selon Lobstein (9) le facteur d'orgues Wing répara l'orgue de l'église St. Thomas à Strasbourg, et y plaça une Voix humaine le 16 octobre 1700. A mon avis il s'agit là du facteur Ring, dont nous parlerons au prochain chapitre.

Nous connaissons deux facteurs du nom d'Eby. Christophe Eby, de Saverne, accorde l'orgue Baldner de Bouxwiller en 1671, et par abonnement jusqu'en 1675 (10). Est-il identique à "Christophorus Aeby von Solothurren" qui fait marché avec la paroisse d'Ernen (Valais) pour la construction d'un orgue de dix jeux, le 13 juillet 1679 ? (11)

L'autre facteur d'orgues de ce nom, Hans Jakob Eby, était installé à Ensisheim. (12) Il fit les orgues neufs d'Erstein en 1660 et de Munster en 1661. (13)

Johann Conrad Besthorn était un tonnelier, qui s'occupait sporadiquement de facture d'orgues. Les protocolles de la corporation des tonneliers nous donnent quelques renseignements, pas toujours favorables, à son sujet (14). On y signale des irrégularités et des querelles avec ses apprentis. Originaire de Zerbst-an-der-Nuthe il apprend le métier chez la veuve de Daniel Böhringer à Strasbourg en 1681. L'année suivante, son chef-d'oeuvre étant approuvé par les XV et par les inspecteurs ("Schawer") de la corporation, il passe mâ̂tre tonnelier. Malgré les démèlés qu'il a avec sa corporation, il est élu maître des redevances ("Gölten Meister") en 1694, membre 
du tribunal de la corporation en 1695 et "Weinkiesser" en 1699. Quel fut son activité dans le domaine de la facture d'orgues? Selon Jean André Silbermann, il répara l'orgue de Bouxwiller en 1698.(15) En 1702 il répara celui de St.Guillaume à Strasbourg, (16) puis nous le retrouvons à Bouxwiller en 1704 et 1710 (17). Après cette date sa trace se perd. Toujours selon Silbermann, il habitait au quai des Bâteliers ("am Holtz Staden neben dem Friedischen Haus mit dem Ercker, dem Vogelsgesang zu"). Citons encore Silbermann: "Er soll ein Atheist gewesen seyn, und hat ein schreckliches Ende genommen."

Matthias Tretzscher, facteur d'orgues à Kulmbach en Franconie orientale, vint à Strasbourg en 1658 pour voir l'orgue de la cathédrale. Le 4 novembre de la même année il est chargé de réparer et de transformer l'orgue, qui avait été restauré en 1608 par Antonius Neuknecht.

Né le 23 mars 1626 à Lichtenstadt (Bohême), Matthias Tretzscher (18) apprit la facture d'orgues chez son beau-frère Andreas Schedlich et chez son beau-père Jacob Schedlich. Il fit un stage chez David Schedlich à Nuremberg. De confession luthérienne, il décéda en 1686.

Avant de venir à Strasbourg, Tretzscher avait construit des orgues à Bayreuth (1653, 2 claviers, Pédale, 20 jeux), Heilsbronn (1654, 2 claviers, 20 jeux), Weissenstadt (1654), Wunsiedel (1655, 2 claviers, Pédale, 15 jeux), Kulmbach (1657-1661, 1 cl., Péd., 11 jeux), Cronach (1657). A Strasbourg il travailla de 1658 à 1650, en collaboration avec son élève Tobias Dressel (19). Il réalisa la composition suivante, qui est attestée d'une part par le manuscrit de Heckheler et par le devis de restauration d'André Silbermann (P.J.p. 310-315):

Composition de l'orque de la cathédrale de Strasbourg en 1660.

Oberwerk: 1) Principal 16

2) Octav 8

3) Quintadehn 8

4) Spitzflöthen 8

5) Quint 6

6) Superoctav 4

7) Gembshorn 4 ou Spitzflöth 4

8) Mixtur 5 à 6 rangs

9) Cimbale 3 à 4 rangs 
Rückwerk :
1) Principal 8
2) Coppel 8
3) Viol de Gamba 8
4) Octave 4
5) Flöth 4
6) Quinta 3
7) Nazard 3
8) Octave 2 ou Cymbale 2 rangs
9) Sesquialtera 2 rangs
10) Mixtur 4 ou 5 rangs

Brustpositif: 1) Gedeckt 8 en bois

2) Principal 4

3) Octave 2

4) Quinte 1 1/2

5) Superoctav 1

6) Nasatt

7) Cimbale

Pédale: $\quad$ 1) Gross Principal Subbass 32 en façade

2) Octav 16

3) Posaune 16

3 claviers de 48 notes (C D- C''')

pédalier de 24 notes ( C D- C')

2 accouplements, tirasse Oberwerk;

tremblant pour le Brustpositif;

appel du Positif de dos;

5 sommiers;

12 soufflets (datant de 1608) à plis multiples;

total des tuyaux: 1900, dont 768 à l'oberwerk, 720 au Positif, 336 au Brustpositif, 76 à la Pédale.

accord au ton de cornet.

F 32 en façade. Les notes C D Ds et E en Bourdon 32 en bois et 16 pieds ouvert.

Toute la tuyauterie était en métal, à l'exception de ces 8 tuyaux de pédale, et du Bourdon 8 du Brustwerk.

A la même époque (1658-1660) Tretzscher construisit un petit orgue sur le jubé de la cathédrale, instrument que les protestants transférèrent au Temple-Neuf lorsque la cathédrale fut rendue au culte catholique en 1681. 
Il nous reste à étudier le facteur strasbourgeois le plus productif de la seconde moitié du $17^{\circ}$ siècle: Johann Jacob Baldner (Hanss Jacob Baltner, Baltdner). Nous le rencontrons à Bouxwiller, Obernai, Haguenau et surtout Strasbourg, où il travaille à la cathédrale, à St. Thomas, à St. Nicolas, à St. Pierre-le-Jeune protestant, à St. Pierre-le-Vieux protestant et à St. Guillaume. Sa période d'activité s'étend de 1642 à 1680 .

Johann Jacob Baldner fait partie d'une grande famille strasbourgeoise dont l'histoire reste à écrire. Il est né à Strasbourg le 17. 11.1606 de Hans Baldner, (20) instrumentiste et organiste aveugle et de Johanna Betz, mariés le 18. 3. 1601 à St. Thomas.(21) Hans Baldner, né à Strasbourg en 1574 (baptisé le onzième dimanche après la Trinité), (22) de Jacob Baldner, pêcheur et membre du Magistrat, acheta une maison à Strasbourg en 1603 ("bey Sanct Barbaren Gassen in der Hellen genant"). (23) Neuf enfants sont issus de son mariage avec Johanna, fille d'Elias Betz, orfèvre. En voici les dates de baptême:

28. 1. 1602: Catharina (24)

16. 1. 1605: Maria (25)

17. 11. 1606: Hans Jacob

15. 7. 1609: Johannes (26)

22. 7. 1612: Agnes (27)

26. 4. 1615: Anna Catharina (28)

$17^{\circ}$ dim. P. Trin. 1616: Anna Margaretha (29)

14. 12. 1619: Anna (30).

Hans Baldner est décédé entre 1619 et 1622. En effet le 15. 9. 1622 est célébré le mariage de Johanna, fille de feu Hans Baldner fille dont nous n'avons pas retrouvé la date de naissance. (31) Johann Jacob Baldner semble s'être marié trois fois. D'une union avec Elisabeth $\mathrm{N}$. sont nés cinq enfants, dont voici les dates de baptême:

3. 7. 1639: Johanna (32)

6. 2. 1642: Johannes (33)

21. 10. 1643: Salome (34)

3. 5. 1646: Hans Jacob (II) (35)

30. 3. 1648: Anna Margaretha (36), qui en 1668 épousa le menuisier Heinrich Hochwaldt Reichert, originaire de Meseritz en Pologne. Le 9 décembre de la même année celui-ci acquit le droit de bourgeoisie à Strasbourg (37).

Le 8. 3. 1652 Johann Jacob Baldner conclut un deuxième mariage avec Aurelia, fille d'Andreas Peter, bourgeois et pêcheur à Wibels- 
heim (38). Elle mourut le 10.2. 1671 à l'âge de 55 ans (39). Jean André Silbermann, qui consacre quelques lignes à Baldner (40) mentionne cette date, qu'il avait trouvée aux Archives de la Ville. Deux ans plus tard, Johann Jakob Baldner se remarie, le 10. 4. 1673, avec Elisabeth, fille de Diebold Otter, bourgeois et tisserand ("Leineweber") à Gerstheim (41).

Baldner mourut le 3.2. 1683, âgé de 76 ans, 2 mois et une semaine(42). Le 5. 3. 1687 sa veuve se remarie avec Nicolaus Nonnenmacher, bourgeois et arquebusier, ainsi que huissier de justice auprès de la corporation des maçons (43).

Voici, groupées par instruments, les différentes interventions de Baldner:

Bouxwiller.

En 1648 il répare l'orgue, qui date de 1614, (44) pour 15 1b., ainsi que $12 \mathrm{~s}$. et $4 \mathrm{~d}$. pour son logement. Dix ans plus tard, nouvelle réparation pour 10 lb., $12 \mathrm{s.,}$ ainsi que 3 lb., $11 \mathrm{~s}$. et $10 \mathrm{~d}$. pour la nourriture pendant 14 jours (45). En 1668 il construisit un orgue neuf pour $400 \mathrm{fl}$., et 70 rézaux moitié blé, moitié seigle, ainsi que l'ancien orgue qui fut démonté les 25 et 26 mai. Le peintre Thomas Franciscus Monori, qui séjourna à Bouxwiller de 1669 à 1673, fut chargé de peindre le buffet d'orgue ainsi que la tribune pour $120 \mathrm{fl} ., 20$ rézaux de grains et 12 mesures de vin. On versa $4 \mathrm{fl} .8 \mathrm{s.}$, une mésure de vin et un rézal de grains au serrurier Griessen pour avoir aidé pendant onze jours lors de l'accord du nouvel instrument. Les frais de nourriture de Baldner à l'Hôpital de Bouxwiller s'élevèrent à une livre et $10 \mathrm{~d}$. Baldner et Monori consommèrent ensemble 14 mesures de vin. Le nouvel orgue fut inauguré le 25 juin 1668, avec la participation de musiciens strasbourgeois. On dépensa 10 deniers pour la fourniture de cordes pour les instruments. (46)

La construction de cet orgue revêt une grande importance, car l'instrument est en partie conservé. Transféré en 1777 en l'église catholique de Bouxwiller, il est aujourd'hui le plus vieil orgue d'Alsace, le seul témoin de la facture d'orgues en Alsace au dixseptième siècle. Son buffet est le deuxième du point de vue ancienneté, venant immédiatement après celui de la cathédrale de Strasbourg (1489). Ses tuyaux sont les seuls qui soient antérieurs à 1700 .

La composition de l'orgue n'est pas connue. Il semble que l'instrument n'ait eu à l'origine qu'un seul clavier, car en 1737 le me- 
nuisier Schild ajouta le Positif, pour $400 \mathrm{fl}$, selon un document d'archives noté par J.A. Silbermann (47). Actuellement l'instrument compte 19 jeux.

\section{Description du matériel, selon l'ordre des chapes:}

\section{Grand Orgue}

1) Montre 8, jeu ancien. C et D en cuivre, de Kern 1960.

2) Cornet $\mathrm{V}$, jeu ancien, commençant en $\mathrm{C}^{\prime}$. Métal d'aspect jaunâtre. Bourdon 8 à calottes soudées, sans cheminées. Les 5 notes complémentaires cs''' - f''' ne comportent pas de Tierce. Faux-sommier en chêne.

3) Bourdon 8, jeu ancien. Métal d'aspect jaunâtre. 15 tuyaux en bois, C, D - ds, ayant deux côtés en chêne. Calottes soudées, sans cheminées. Plusieurs pieds refaite en 1960.

4) Cymbale III, de Kern 1960, à la place d'un Salicional 8.

\begin{tabular}{|c|c|c|c|c|c|}
\hline C & A & e & a & $e^{\prime}$ & $a^{\prime} '$ \\
\hline $1 / 2$ & $2 / 3$ & 1 & $11 / 3$ & 2 & $22 / 3$ \\
\hline $1 / 3$ & $1 / 2$ & $2 / 3$ & 1 & $11 / 3$ & 2 \\
\hline $1 / 4$ & $1 / 3$ & $1 / 2$ & $2 / 3$ & 1 & $11 / 3$ \\
\hline
\end{tabular}

5) Flûte 4, en bois, jeu ancien. 7 tuyaux de métal de provenance diverse, ainsi que les 5 tuyaux du sommier complémentaire.

6) Prestant 4, jeu ancien, métal d'apparence jaunâtre. C D Ds E en spotted. Plusieurs pieds refaits en 1960.

7) Doublette 2, jeu ancien, en métal d'aspect jaunâtre.

8) Eourniture III, jeu ancien, qui avait autrefois 4 rangs.

\begin{tabular}{|c|c|c|c|}
\hline C & $\mathrm{f}$ & $f^{\prime}$ & $f^{\prime} '$ \\
\hline $11 / 3$ & 2 & $22 / 3$ & 4 \\
\hline 1 & $11 / 3$ & 2 & $22 / 3$ \\
\hline $2 / 3$ & 1 & $11 / 3$ & 2 \\
\hline
\end{tabular}

9) Trompette 8, jeu de Stiehr. C D Ds pavillons en cuivre, 1960. Noyaux en bois de Cs à H. C noyau neuf. Compléments de Stiehr également, mais dépareillés. Ratelier supérieur en contreplaqué.

\section{Positif}

1) Prestant 4, jeu ancien. Métal d'aspect jaunâtre.

2) Bourdon 8, en bois, jeu ancien, 2 côtés en chêne.

3) Sesquialtera II, de Kern 1960, à la place d'une Flûte conique 4 C $\quad \mathrm{C} \quad \mathrm{g}$

$11 / 3 \quad 13 / 5 \quad 22 / 3$

$1 \quad 11 / 3 \quad 13 / 5$

Le rang de Tierce, cylindrique, est moderne. Le rang de Quinte, conique, est ancien, d'aspect jaunâtre. 
4) Cymbale III, de Kern 1960, en métal martelé, à la place d'une Voix céleste 4.

\begin{tabular}{|c|c|c|c|c|c|}
\hline C & G & $g$ & $C^{\prime}$ & $g^{\prime}$ & $C^{\prime} '$ \\
\hline $2 / 3$ & 1 & $11 / 3$ & 2 & $22 / 3$ & 4 \\
\hline $1 / 2$ & $2 / 3$ & 1 & $11 / 3$ & 2 & $22 / 3$ \\
\hline $1 / 3$ & $1 / 2$ & $2 / 3$ & 1 & $11 / 3$ & 2 \\
\hline
\end{tabular}

Les reprises sont une réédition du Positif de Marmoutier!

5) Doublette 2, jeu en partie ancien, en métal jaunâtre.

6) Cromhorne 8, de Kern 1960, en spotted, à la place d'un Hautbois 8 .

\section{Pédale}

1) Soubasse 16, jeu ancien, bouché, en bois.

2) Flûte 8, jeu ancien, en bois, ouvert. Pieds octogonaux.

3) Prestant 4, Violoncelle 8 coupé par Kern en 1960.

4) Dulciane 16, jeu de Kern 1960, en cuivre, à la place d'un Fagott 8 . Les pavillons ont 8' de longueur.

\section{Les sommiers}

\section{Grand Orgue}

2 sommiers diatoniques anciens, (C D - e''', 48 notes sans Cs)

$$
\begin{aligned}
& \text { - C Ds - h'' } \\
& \text { - DE - C''' }
\end{aligned}
$$

1 sommier complémentaire de 5 notes, chromatique, datant d'avant la restauration de 1960 .

Les chapes sont vissées.

Division:

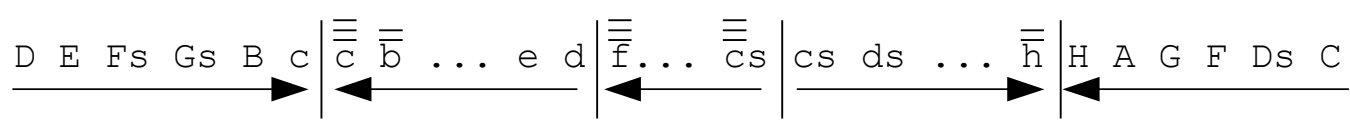

\section{Positif}

1 sommier de 53 notes (C D - f''' sans Cs)

Chapes vissées.

Division:

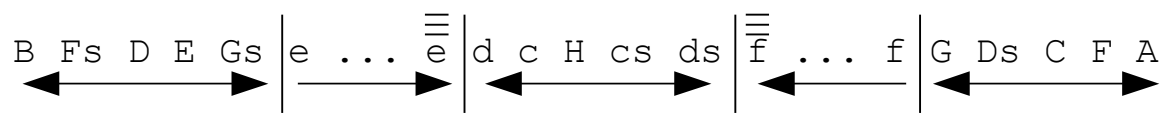

\section{Pédale}

2 sommiers diatoniques, de 24 notes (C D - C' sans Cs) à l'origine. Actuellement 27 notes, Cs CS' et d'ayant été ajoutés en 1960 de la manière suivante: le premier sommier va de $\mathrm{C}$ à b, le second de $\mathrm{Cs}$ à h. Dans ce dernier sont intercalées les notes c' cs' et d', entre 
les gravures Cs Ds P et G.

Un tremblant, aujourd'hui hors service, est situé sur le portevent alimentant le Positif, sous le plancher.

La mécanique

Au Grand Orgue, mécanique suspendue, bras d'abrégés en fer.

Au Positif, pilotes, équerres, vergettes.

A la Pédale, rouleaux d'abrégés en fer, modernes.

Tirage de jeux entièrement refait en 1960 .

Claviers anciens, plaqués d'ébène.

Répartition des tirants de jeux à la console:

+ Cornet V G.O.

+ Flûte 4 G.O.

+ Cymbale III G.O.

+ Prestant 4 G.O.

+ Doublette 2 G.O.

+ Fourniture III G.O.

+ Trompette 8 G.O.

+ Flûte 8 Péd.

+ Bourdon 8 G.O.

+ Soubasse 16 Péd.

+ Montre 8 G.O.

+ Dulciane 16 Péd.

+ Bourdon 8 Pos.

+ Prestant 4 Péd. + Prest. 4 Pos.

+ Cymbale III Pos. + Tacet

+ Cromorne 8

$\begin{aligned}+ \text { Tirasse G. O. } & + \text { Sesqui. Pos. } \\ & + \text { Doubl. } 2 \text { Pos. }\end{aligned}$

\section{Le buffet}

Le buffet est en pin, actuellement peint en blanc comme l'ensemble de la balustrade. Le soubassement a été considérablement diminué en hauteur. Les entablements des deux grandes tourelles plates ont été amincis. Les ornements des consoles latérales comportent des motifs de cornes d'abondance et des éléments du monde végétal. Au Positif on trouve déjà une conception Louis XVI, bien que la structure des cinq plate-faces soit assez bien adaptée à celle du grand buffet. Il est regrettable que le manque de hauteur n'ait pas permis le remontage intégral du buffet, qui donne une impression d'écrasement.

Les tuyaux de façade, tant du Grand Orgue que du Positif, ont heureusement échappé à la réquisition par les autorités allemandes en 1917. (48)

Revenons à Baldner. Il accorde l'orgue de Bouxwiller en 1670, (49) mais de 1671 à 1675 c'est le facteur Christoph Eby, de Saverne, qui se charge des accords (voir ci-dessus), et nous ignorons le motif de ce revirement. 
Strasbourg, st. Guillaume.

Une réparation importante de l'instrument datant de 1611 est signalée par Lobstein pour l'année 1643. (50) Selon Vogeleis (51) elle fut exécutée vraisemblablement par J.J. Baldner. D'après J.A. Silbermann (52), l'instrument démonté par son père en 1727 était l'oeuvre de Baldner. Le sommier du Grand Orgue avait des séparations dans les gravures. Strasbourg, St. Pierre-le-Jeune protestant.

En 1642 J.J. Baldner restaura l'orgue de la nef de l'église St. Pierre-le-Jeune. Les archives de la paroisse ont consigné la composition de l'instrument au lendemain de cette restauration (P.J. p.417) Clavier manuel: Pédale:
1) Principal
1) Soubasse
2) Copel
2) Principal
3) Octav
3) Octav
4) Superoctav
4) Mixtur
5) Quint
6) Flöten
7) Mixtur
8) Schweiglein
9) Zincken
5) Posaunen
10) Zimbel

Les jeux du clavier manuel se trouvaient sur un sommier placé au centre. Ils étaient tous en étain.

Les jeux de Pédale, Principal, Octave, Mixture et Posaune, également en étain, sauf trois en bois, étaient placés sur deux sommiers, de part et d'autre (53).

Cet instrument était accroché en nid d'hirondelle au mur nord de la nef. Les traces des portes d'accès sont visibles dans les combles du bas-coté.

Strasbourg, st. Pierre le-Vieux protestant.

Des réparations sont signalées par Lobstein pour 1645 (54). En 1666 Baldner exécuta une autre réparation, ignorée de Lobstein et de Vogeleis. Le facteur s'engage à étancher les fuites aux sommiers, soufflets et porte-vents, au moyen de peau, à l'exclusion de parchemin. Il nettoie et réharmonise la tuyauterie et polit les tuyaux de façade. Les tuyaux mal fixés sont pourvus d'attaches, et l'orgue est accordé dans son ensemble. La paroisse paye $40 \mathrm{fl}$. à Baldner, le salaire du souffleur et du serrurier restant à la charge de la paroisse. Les matériaux sont fournis par le facteur. 
Strasbourg, St. Thomas.

Baldner répara cet instrument à plusieurs reprises. En 1672 celui-

ci avait la composition suivante, résultat des remaniements de 1613

et 1626: (55)

Grand Orgue

Principal

Quintatön

Octav

Gross Guint

Superoctav

Klein Quint

Zimbel

Mixtur 6 rangs

Zincken

\section{Positif}

7 registres.

Pédale

Principal

Octav (8)

Superoctav (4)

Mixtur

Posaune

Tremblant.

Soufflets à plis multiples, situés dans une chambre.

Buffet peint.

Buffet de Grand Orgue en plate-faces, les deux plus grandes étant à l'extérieur.

Buffet de Positif en forme d'hexagone (56)

Claviers commençant au Fa.

J.J.Baldner est chargé d'une réparation en 1672. Il répare la

tuyauterie, les soufflets, les sommiers, les porte-vents; il

nettoie, réharmonise et réaccorde l'instrument, pour $150 \mathrm{fl}$.

L'adjonction d'un sommier pour les notes C. D. et E. coûterait 250 fl. (55).

Selon Lobstein (57) ces réparations donnèrent lieu à un procès en 1673. Baldner aurait réparé ses torts en 1677.

Toujours est-il qu'en 1674 Baldner demande $12 \mathrm{fl}$. pour accorder l'orgue, ainsi que 4 rézaux de blé ou $12 \mathrm{fl}$. en argent, pour l'entretien annuel de l'orgue à compter de 1674 . Georges Christophe Lauttensakh, organiste à la cathédrale, 
remplissait les fonctions d'expert.

Strasbourg, St. Nicolas.

En 1595 la paroisse St. Nicolas avait acheté l'orgue de choeur du Temple-Neuf. Jean Klein, de Donauwörth, avait assuré le transfert, sous la surveillance de Bernard Schmidt, expert. Cet orgue, qui comportait un positif de dos, fut rénové par Israel Gellinger en 1610. (58)

Baldner accorda cet orgue régulièrement. Des quittances des années 1649 à 1655 sont conservées (59).

En 1668 Baldner fut chargé de construire un orgue neuf, travail qui fut mené à bien en 1669-1670 pour la somme de 350 thaler, les matériaux étant fournis par la paroisse (60). Les soufflets furent placés en décembre 1669 (40). Aux archives de la paroisse se trouve la liste des collectes faites en 1668-1669 pour la construction de l'orgue, ainsi qu'un répertoire des dépenses occasionnées par la fourniture des matériaux à Baldner, depuis le 12 mars jusqu'au 24 décembre 1669. Le gendre de Baldner, le menuisier Henri Hochwaldt, y est cité comme étant son collaborateur.

En 1707 André et Gottfried Silbermann furent chargés de construire un nouvel orgue à St. Nicolas. Ils proposèrent de réutiliser le buffet de Baldner, tout en le transformant. Un projet, écrit de la main d'André Silbermann, mais ni daté ni signé, donne la composition de l'orgue de Baldner vers 1707 (61). (P.J.p.284-286)

\section{Grand-Orgue}

$\begin{array}{lr}\text { 1) Quintaton } & 16 \\ \text { 2) Principal } & 8 \\ \text { 3) Viole de gambe } & 8 \\ \text { 4) Nazard bouché } & 6 \\ \text { 5) Octave } & 4 \\ \text { 6) Holflöt } & 4 \\ \text { 7) Quinte } & 3 \\ \text { 8) Superoctave } & 2 \\ \text { 9) Mixture 4 rangs } & 1\end{array}$

Positif

$\begin{array}{ll}\text { 1) Quintaton } & 8 \\ \text { 2) Coppel } & 8 \\ \text { 3) Principal } & 4 \\ \text { 4) Octave } & 2 \\ \text { 5) Flûte } & 2 \\ \text { 6) Superoctave } & 1\end{array}$


Pédale

1) Soubasse 16

2) Octavebasse 8

8 soufflets, à plis multiples, actionnés au pied.

En 1676 et 1677 Baldner accorda et révisa l'instrument. (59)

\section{Strasbourg, cathédrale.}

Des interventions de Baldner sont attestées pour la période allant de 1650 à 1680, soit pour le grand orgue de la nef, soit pour le petit orgue du jubé, soit enfin pour l'horloge astronomique.

a) Le-grand orgue.

1650-1651: accord du posaune, pour 15 sols. (62)

1651-1652: accord du posaune pendant l'année, pour 2 livres. (63)

1652-1653: accord et réparation de l'orgue pendant l'année, pour 4 livres, 18 sols et 4 deniers. (64) Deux souffleurs étaient engagés pour ces travaux. Notons qu'en 1652 Georges Christophe Lautensack (père) succède à Philippe Frédéric Böddecker comme organiste de la cathédrale. Il quittera ce poste en 1681 pour devenir organiste au Temple-Neuf

1653-1654: entretien annuel, pour 1 livre, 12 sols.(65)

1656-1657: après deux années passées sous silence, accord du posaune et du régale, pour 1 livre. (66)

1657-1658: interventions de Baldner, pour 1 livre. La même année l'Oeuvre Notre-Dame dépense 2 livres et 19 sols pour frais d' hébergement du facteur d'orgues Pierre Holtz, et 1 livre et 10 sols pour ses frais de voyage.(67) Rappelons que de 1658 à 1660 l'orgue de la cathédrale fut reconstruit par Tretzscher et Dressel. Le nom de Baldner ne réapparaît dans les comptes de l'Oeuvre Notre-Dame qu'en 1668. D'ailleurs entre 1658 et 1666 aucune activité de Baldner n'est signalée en Alsace.

1669-1670: réparation du grand orgue, pour 20 livres. Trois souffleurs prêtèrent main forte pendant neuf jours et demi. (68)

1672-1673: réparations et accords, pour 2 livres (69)

1673-1674: réparation des soufflets, déplacement des poids en pierre, pour 15 sols. (70)

1674-1675: accord de l'orgue, pour Pâcques, pour 15 sols. (71)

1679-1680: dernière mention de l'activité de Baldner. Il accorde le Rückpositif et le Brustpositif, il encolle le sommier avec du parchemin et les tampons de laye avec de la peau, il répare les soufflets, pour 10 livres, alors que trois souffleurs travaillent pendant 17 jours. (72) 
b) Le petit orgue.

1668-1669: Le petit orgue du jubé, achevé par Tretzscher le 12 avril 1660, fut réparé par Baldner pour 22 livres et 10 sols. Le souffleur Nicolas Geörger fut mis à contribution pendant 24 jours. (73)

1675-1676: Baldner et son gendre, Heinrich Hochwaldt Reichert, "tous deux facteurs d'orgues", sont chargés d'une réparation. Le 3 août 1676 ils touchent 67 livres et 10 sols, ainsi que 2 mesures de vin. Les souffleurs, qui ont travaillé pendant onze jours, recoivent 3 sols et 4 deniers par jour, soit 1 livre 16 sols et 8 deniers. (74)

C) L'horloge astronomique.

L'horloge astronomique de la cathédrale fut réparée en 1669-1670. Selon la chronique, les travaux, commencés à la Saint-Jean 1669, furent achevés le 21 mars de l'année suivante, à l'exception du mouvement des éclipses. En 1670 Baldner fit un tuyau neuf pour le coq, au prix de 15 sols. (75)

\section{Obernai.}

En 1655 Baldner répara l'orgue de l'église paroissiale d'obernai, construit en 1616 par Dietrich Wagner. (76)

\section{Haguenau.}

L'orgue de l'église paroissiale St. Georges de Haguenau fut réparé par Baldner en 1666. (77)

\section{Strasbourg, Temple-Neuf.}

Le marché du 29 octobre 1683 pour la réparation de l'orgue du Temple-Neuf par F. Freundt fait allusion à une réparation faite précédemment par "un facteur d'orgues et son gendre, le menuisier." Il s'agit vraissemblablement de Baldner et de son gendre Heinrich Hochwaldt Reichert.

Voilà donc le bilan de l'activité de Baldner, pour autant que nous en trouvions trace dans les archives. Deux constructions neuves (Bouxwiller et Strasbourg, St. Nicolas), et pour le reste une série de réparations, qui ne furent pas toujours jugées satisfaisantes. Néanmoins Baldner s'inscrit dans une tradition locale, qui ne manquera pas d'influencer le jeune André Silbermann et son frère Gottfried. Ce qui reste de Baldner dans 1'orgue de Bouxwiller en porte témoignage. 
Essayons de tirer quelques conclusions de ce survol de la facture d'orgues en Alsace vers la fin du $17^{\circ}$ siècle. Les rares compositions d'orgues dont le relevé nous soit parvenu permettent de définir quelques caractéristiques.

Fréquemment les jeux de Pédale, en particulier le 16 pieds, n'avaient pas de tirants de jeux, et parlaient en permanence. (16' de l'orgue de choeur du Temple-Neuf à Strasbourg 1635, 16' et 8' à l'orgue de Brumath vers 1675; 16' et 8' de l'orgue du Temple-Neuf avant 1683; 16' et 8' de l'orgue de St. Nicolas à Strasbourg construit par Baldner en 1669). Dans les exemples cités, la Pédale ne comptait pas d'autres jeux. Nous trouvons encore des exemples de ce type au début du $18^{\circ}$ siècle, par exemple à Kaysersberg (16' et 8' de l'orgue de Waltrin 1719), à Munster (16' et 8' de l'orgue de C. Legros 1706). Il est à remarquer que les premiers instruments d'André Silbermann auront également des Pédales dépourvues de tirants pour ces jeux-là: St. Nicolas à Strasbourg 1707 (16' et 8'), St.Pierre-le-Vieux protestant à Strasbourg,170Q (16' et 8'), Temple-Neuf à Strasbourg, Pédale d'André et Gottfried Silbermann 1708 (16' et 8'), Marmoutier (16' en 1710, 16' et 8' à partir de $1746)$.

Certaines compositions de Pédale sont plus riches et comportent notamment une Mixture: St. Thomas à Strasbourg 1613, St-Pierre-leJeune protestant à Strasbourg 1642, Temple-Neuf avant 1683. Il semble que ce soit là un type plutôt archäqque, mais que nous retrouverons également chez André Silbermann dans ses premiers instruments: Mixture de 6 rangs à la Pédale du Temple-Neuf en 1708, Fourniture de 4 rangs à St-Pierre-le-Vieux protestant en 1709, et à la cathédrale de Bâle en 1711. Les deux grands instruments avec Oberwerk de Jean André Silbermann auront également une Mixture de 6 rangs, de même les quatre orgues à trois claviers de Gottfried.

Les compositions connues contiennent relativement peu de jeux d'anches: aucun au Temple-Neuf en 1635, sur 17 jeux; aucun à st. Nicolas en 1669, sur 17 jeux; un seul à la cathédrale de Strasbourg en 1659, sur 29 jeux; deux à St. Pierre-le-Jeune prot. en 1642, sur 15 jeux. La plupart des orgues contiennent certains jeux de fonds qui semblent faire partie de la tradition locale et que l'on ne rencontrera presque plus au $18^{\circ}$ siècle: Viole de Gambe 8, Quintaton 16 et 8, Flûtes coniques 8 et 4 (Spitzflöte, Gemshorn), Hohlflöte 4 et Schweiglein 2. Chez André Silbermann cette variété de jeux de 
fonds sera progressivement délaissée au profit du schéma de l'orgue français. 


\section{Conclusion}

Si nous jetons un coup d'oeil sur la facture d'orgues française et allemande vers 1700, nous constatons que dans les deux pays elle est à son apogée. C'est l'époque où Arp Schnitger construit ses grands chefs-d'oeuvres en Allemagne du Nord et aux Pays-Bas, d'une qualité technique et sonore dont les orgues de Groningue (Aa-Kerk) ou d'Uithuizen aux Pays-Bas rendent encore aujourd'hui un éclatant témoignage. (78)

En France la facture d'orgues prend une forme très caractéristique et rationalisée à l'extrême. C'est l'ère des Alexandre Thierry, Robert Clicquot, Jean de Joyeuse, Robert Ingout, Carouge, Tribuot, Deslandes. (79)

Vers 1700 la facture d'orgues en Alsace est le fait d'une tradition locale qui tend à se perdre. Le dix-huitième siècle sera fortement marqué par la pénétration du goût français dans les milieux alsaciens (80). Si dans l'architecture religieuse on constate une influence allemande - les mâttres du Vorarlberg par exemple - aussi bien que française - Massol, Blondel, d'Ixnard -, dans la facture d'orgues le goût du jour, la manière nouvelle, seront avant tout français. André Silbermann, suivi par ses fils, en sera le propagateur. Il ne sera d'ailleurs pas le seul. Pour s'en convaincre il suffit d'examiner les compositions d'un Legros, d'un Boulay, d'un Rohrer, d'un Dupont, tous influencés par les mâttres parisiens. Toutefois l'influence française n'a rien d'exclusif. D'autres influences continuent à s'exercer - tout comme en architecture - dans une cohabitation pacifique. 
(1) Arch. de la paroisse St. Nicolas à Strasbourg. P.J. des comptes 1687.

(2) J.P.Lobstein, Beiträge ..., Strasbourg 1840, p. 87.

(3) Arch. de la paroisse St. Nicolas à Strasbourg. P.J. des comptes 1690. Spiess répara également un régale.

(4) J.P.Lobstein, ouvr. cité, p. 57.

(5) Arch. de la paroisse St. Nicolas à Strasbourg. P.J. des comptes 1694-1696.

(6) Médard Barth, Elsass "das Land der Orqeln" ..., Haguenau 1966, p. 304 .

(7) Martin Vogeleis, Quellen und Bausteine ..., Strasbourg 1911, P. .542.

(8) M. Barth, ouvr. cité, p. 234.

(9) J.F.Lobstein, ouvr. cité, p. 57.

(10) Carl Klein, Pfarrerbuch und Kirchenchronik..., Strasbourg 1914, p. 73-77.

(11) Staatsarchiv Sitten. Fonds J.-M. Jost-Arnold. K 118. Communication de M. Hans Füglister, Grimisuat.

(12) M. Vogeleis, ouvr. cité, p. 527.

(13) R. Friedel, Geschichte des Fleckens Erstein, Erstein 1927, p. 518. - F. Hecker, Die Stadt und das Tal zu Mûnster, Munster 1890, p. 180 .

(14) AMS. Corporation des tonneliers, $\mathrm{N}^{\circ} 10-12$.

(15) J.A.Silbermann, ms. "Von Orgelmachern", p. 318.

(16) J.F.Lobstein, ouvr. cité, p. 87.

(17) C. Klein, ouvr. cité, p. 101 et 103.

(18) Hans Hofner, Matthias Tretzscher, ein Kulmbacher Orgelbauer der Barockzeit, Ars Organi 23 (1964), p. 655-678.

(19) H. Hofner, Die Tretzscherschüler aus der Kulmbacher Orgelbauwerkstatt, Ars Organi 28 (1866), p. 949-962.

(20) AMS. N 248, baptêmes St. Thomas, fol. 241 b, N 1647.

(21) AMS. N 248, mariages St. Thomas, fol. $148 \mathrm{~b}, \mathrm{~N}^{\circ} 386$.

(22) AMS. N 27 III, baptêmes St. Guillaume, fol. 50 b.

(23) AMS. Chambre des Contrats $\mathrm{N}^{\circ} 344$, 1603, fol. 124 b.

(24) AMS. N 217, baptêmes Temple-Neuf, fol. 125 a.

(25) AMS. N 248, baptêmes St. Thomas, fol. 219 b, N 1460 .

(26) AMS. N 248, baptêmes St. Thomas, fol. 286 b, $\mathrm{N}^{\circ} 1943$.

(27) AMS. N 248, baptêmes St. Thomas, fol. 340 a, $N^{\circ} 2269$.

(28) AMS. N 248, baptêmes St. Thomas, fol. 376 b, N² 2550.

(29) AMS. N 248, baptêmes St. Thomas, fol. 391 a.

(30) AMS. N 248, baptêmes St. Thomas, fol. 428 a, N² 2989. 
(31) AMS. N 248, baptêmes St. Thomas, fol. 452 b, N 1452.

(32) AMS. N 220, baptêmes Temple-Neuf, fol. 116 a.

(33) AMS. N 220, baptêmes Temple-Neuf, fol. $177 \mathrm{a}$.

(34) AMS. N 220, baptêmes Temple-Neuf, fol. 220 a.

(35) AMS. N 220, baptêmes Temple-Neuf, fol. 278 a.

(36) AMS. N 220, baptêmes Temple-Neuf, fol. 326 a.

(37) AMS. Livre de Bourgeoisie IV, col. 367.

(38) AMS. M 109, mariages Temple-Neuf, fol. 542 a.

(39) AMS. D 170, décès Temple-Neuf, fol. 48 b.

(40) J.A.Silbermann, ms. Von Orgelmachern, p. 183.

(41) AMS. M 111, mariages Temple-Neuf, fol. 163 a.

(42) AMS. D 170, décès Temple-Neuf, fol. 107 a.

(43) AMS. N 251, mariages St.Thomas, p. 126, $\mathrm{N}^{\circ}$ 739, et M 112, mariages Temple-Neuf, fol. 147 b.

(44) C. Klein, ouvr. cité, p. 62.

(45) C. Klein, ouvr. cité, p. 67.

(46) C. Klein, ouvr. cité, p. 70.

(47) J.A.Silbermann, ms. Els. Orgeln, p. 419-421.

(48) Pour l'histoire des orgues de Bouxwiller, voir SHAS 1961/111.

(49) C. Klein, ouvr. cité, p. 71.

(50) J.F.Lobstein, ourr. cité, p.87.

(51) M. Vogeleis, ouvr. cité, p. 506.

(52) J.A.Silbermann, ms. Els. Orgeln, p. 439-440.

(53) Le nombre de sommiers indiqué par M. Vogeleis, ouvr. cité, p. 505, est à rectifier.

(54) J.F.Lobstein, ouvr. cité, p. 74.

(55) AMS. Arch. du Chapitre de St. Thomas, 30.

(56) J.A.Silbermann, ms. Els. Orgeln, p. 431-435.

(57) J.F.Lobstein, ourr. Cité, p. 57.

(58) Arch. de la paroisse St. Nicolas, Strasbourg, PI, Alte Protocol der Fabric zu S. Nicol, fol. 63 b, 76 b.

(59) Arch. de la paroisse St. Nicolas, P.J. des comptes.

(60) J.F.Lobstein, ouvr. cité, p. 83, qui indique la date de 1668.

(61) Arch. de la paroisse SV. Nicolas, $N^{\circ}$ 64. - P.Meyer-Siat, Die Orgeln zu St-Nicolas in Strassburg, Acta Organologica, V (1971) p.140-159.

(62) AMS. V.F.W. 43, 1650-51, fol. 50 b.

(63) AMS. V.F.W. 43, 1651-52, fol. 49 a.

(64) AMS. V.F.W. 43, 1652-53, fol. 55 a.

(65) AMS. V.F.W. 43, 1653-54, fol. 52 a.

(66) AMS, V.F.W. 43, 1656-57, fol. 52 a. 
(67) AMS. V.F.W. 43, 1657-58, fol. 54 a, 72 a, 72 b.

(68) AMS. V.F.W. 43, 1669-70, fol. 67 b.

(69) AMS. V.F.W. 43, 1672-73, fol. 62 a.

(70) AMS. V.F.W. 43, 1673-74, fol. 60 a.

(71) AMS. V.F.W. 43, 1674-75, fol. 62 b.

(72) AMS. V.F.W. 43, 1679-80, fol. 52 a-b.

(73) AMS. V.F.W. 43, 1668-69, fol. 66 a.

(74) AMS. V.F.W. 43, 1665-76, , fol. 59 a.

(75) AMS. V.F.W. 43, 1670-71, fol. 66 b. - BNU, MS 1373, fol.8 b.

(76) X. Ohresser, L'ancienne église d'obernai, Archives de I'Eglise d'Alsace, 8 (1957), p. 82.

(77) AMH, GG 293, 38.

(78) Voir Gustav Fock, Arp Schnitger und seine Schule, Kassel 1974.

(79) Voir bibliographie sur l'orgue en France.

(80) H. Haug, Le style Louis XIV à Strasbourg. Essai sur la transition entre la "manière allemande" et le "goût français" (1681-1730), in Archives Alsaciennes d'Histoire de I'Art, 3/1924, p. 65-111. 
Introduction aux "Archives Silbermann de Paris."

A côté de son activité prodigieuse de facteur d'orgues, Jean André Silbermann avait une âme de collectionneur et d'historien. On connaît les deux ouvrages parus de son vivant: Local-Geschichte der Stadt Strassburg, avec vignettes et 16 planches gravées par Weis. Strasbourg 1775, 232 p. in-folio, et Beschreibung von Hohenburg oder dem St. Odilienberg, samt umliegender Gegend, avec 20 gravures de Weiss. Strasbourg 1781, 134 p. in-12. Jean André Silbermann avait réuni un important cabinet numismatique, dont l'inventaire est conservé. Il possédait également un cabinet de curiosités. Il avait laissé de nombreux manuscrits d'histoire locale ainsi que des dessins et croquis de monuments. Cette collection fut vendue à la Ville de Strasbourg en 1784 et périt - du moins en très grande partie - dans l'incendie de la Bibliothèque en 1870 .

Un certain nombre de manuscrits d'organologie, qui étaient restés dans la famille de Jean Henri Silbermann (1727-1799), ont échappé à ce sort et sont aujourd'hui conservés dans la descendance, en propriété privée, à Paris. D'où le nom d'"Archives Silbermann de Paris." Un microfilm, incomplet, de ces documents est conservé à la Bibliothèque Nationale à Paris.

Longtemps inaccessible aux chercheurs, cette documentation a été consultée par le chanoine F.X.Mathias vers 1927. Son importance fut mise en lumière à l'occasion du Congrès d'Orgue de Strasbourg de 1932 . 
Les notes prises par Mathias ont ensuite permis à J. Wörsching, puis à P. Smets, de faire des publications plus importantes sur les Silbermann.

Les manuscrits conservés à Paris se présentent sous forme de 5 volumes in-8, auquels s'ajoute un volume in-12 ainsi qu'une liasse de feuilles volantes. Le tout est rédigé par Jean André Silbermann en langue allemande.

\section{$\underline{\text { Contenu des manuscrits d'organologie de Jean André Silbermann }}$}

Volume 1.

Titre au dos de la reliure:"ORGELN VON ANDREAS SILBERMANN.T.V" Titre à la page de titre: "Bericht / derer / Von meinem lieben Vater / Andreas Silbermann / Neben seinen vielen Instrumenten=Arbeiten / verfertigten Orglen."

Description et histoire des 35 orgues construits par André Silbermann, le père de l'auteur. Période: 1699-1784. Aire géographique: Alsace, Suisse, Pays-de-Bade. Notices complémentaires par Josias Silbermann, fils de l'auteur. 1 vol. in-4, $209 \mathrm{p}$. Abréviation utilisée par Mathias-Wörsching-Smets: SA I. Cote de la Bibliothèque Nationale à Paris: Vm. Micr. 405 (5).

\section{Volume 2.}

Titre au dos de la reliure: "EINRICHTUNG DER ORC-EL111. T.IV." Il n'y a pas de page de titre générale. Chaque chapitre est intitulé "Einrichtung, Intonnir= und Stimmung der Orge1..." 
Compte-rendu, noté au jour le jour et souvent en style télégraphique, de la construction, du montage, de l'harmonisation et de l'accordage de 31 orgues de Jean André Silbermann.

Période: 1746-1780.

Aire géographique: Alsace, Lorraine, Pays-de-Bade, Suisse.

1 vol. in-4, $261 \mathrm{p}$.

Abréviation utilisée par Mathias-Wörsching-Smets: SA II.

Cote de la Bibliothèque Nationale à Paris: Vm. Micr. 405 (4).

\section{Volume 3.}

Titre au dos de la reliure: ELSASSISCHE ORGELN. T. I."

Titre à la page de titre: "Von / Elsassischen Orgeln / wovon ich die meisten bey Gelegenheit / selbsten gesehen, oder Nachricht davon / erhalten habe."

Description de 126 orgues d'Alsace ne provenant pas des ateliers des Silbermann.

Croquis à l'encre ou au crayon, par l'auteur.

Période: $17^{\circ}$ et $18^{\circ}$ siècles.

1 vol. in-4, $487 \mathrm{p}$.

Abréviation utilisée par Mathias-Wörsching-Smets: SB I.

Cote de la Bibliothèque Nationale à Paris: Vm. Micr. 405 (1).

\section{Volume 4.}

Titre au dos de la reliure: AUSWAERTIGE ORGELN. T. II."

Titre à la page de titre: "Anmerkungen / Einiger ausser dem Elsass stehenden / Orgeln. / Davon ich viele gesehen, oder aus erhaltenen / Nachrichten hier verzeichnet habe. / Darunter sind keine Silbermännische befindlich."

Notices consacrées à 123 orgues d'Europe, en dehors de l'Alsace, ne provenant pas des ateliers des Silbermann. 
Croquis à l'encre ou au crayon, par l'auteur.

Pièces jointes: gravures d'orgues, lettres de facteurs d'orgues et d'organistes.

Période: $17^{\circ}$ et $18^{\circ}$ siècles.

Aire géographique: Allemagne, France, Italie, Autriche, Pays-Bas, Suisse, Angleterre, Tchecoslovaquie.

1-vol. in-4, $450 \mathrm{p}$.

Abréviation utilisée par Mathias-Wörsching-Smets: SB II.

Cote de la Bibliothèque Nationale à Paris: Vm. Micr. 405 (2).

\section{Volume 5.}

Titre au dos de la reliure: "VON ORGELMACHERN. T. III."

Titre à la page de titre: "Bericht / von Orgelmachern / auch Organisten welche sich auf Orgeln / verstanden, oder vielmehr haben ver- / stehen wollen."

Notices biographiques sur 182 facteurs d'orgues du $18^{\circ}$ siècle de France, d'Allemagne, d'Italie, d'Autriche, de Suisse et des Pays-Bas .

Pièces jointes: lettres de facteurs d'orgues et d'organistes. 1 vol. in-4, $318 \mathrm{p}$.

Abréviation utilisée par Mathias-Wörsching-Smets: SB III. Cote de la Bibliothèque Nationale à Paris: Vm. Micr. 405 (3).

\section{Volume 6.}

Ce volume ne porte pas de titre général. Mathias l'appelle le "Livre de la spécification des prix" (abréviation: Sp.).

Il contient des renseignements techniques et commerciaux destinés à l'usage personnel et secret de l'auteur. Il est rédigé en caractères extrêmement petits et quelquefois en cryptographie. Période: 1734-1753. 
Vu son caractère ésotérique, ce volume n'a guère été exploité par Mathias. Nous en donnons les titres des chapitres successifs:

- Specification / Aller / Zum Orgelwesen / Gehoerigen Holtz Sorten.

- Orgel Kästen / und / Zierathen.

- Zinn und Bley. / Der Preiss und die / Legierung desselben.

- Gewicht / Unterschiedlichen Scheinen / von 16, 8, und 4 Schühigen

/ Werckern / Und kleinen Cabinet=Örgelein / wie auch / Ein

Überschlag eines 32 Füssigen / Scheins / Auch das Gewicht Aller

Gewöhn- / lichen Manual= und Pedal= Register.

- Zeit= Arbeit / An Schein=Pfeiffen / und dem Innern Pfeiffenwerck, Wie auch Mund=Stücken / Und / denen Höltzern Pfeiffen.

- Windladen.

- Zeit Arbeit und / Matherialien / an Blassbälgen.

- Zeit Arbeit / bey / Aufsetzung Intonnir= und Stimmung derer Orgeln.

- Haupt Specification / derer Scheinen.

- Unterschiedliche / Orgeln / die /Herr Gottfried Silbermann / Ihro Königl.- Majestät In Pohlen / und Churf.- Sächsischer Hoff=/ und Land= Orgelbauer / verfertiget.

- Unterschiedliche / Frembte / Orgel= Wercke.

- Schreiner + Arbeit / und Holtz / An Orgel Kästen / wie auch / Anmerckung einiger Zeit Arbeit / Ferner / Vom Fürnissen.

- Bildhauer = Arbeit / vor Orgeln.

- Schlosser = Arbeit / vor / Orgel Kästen.

- Speculationen / Und Zeit Arbeit / An Orgel = Rissen.

1 vol. in-12, $128 \mathrm{p}$. 
Volume 7.

Liasse de feuilles volantes, pour lesquelles Mathias utilise l'abréviation: Fv. Elles sont glissées dans une reliure semblable à celle des 5 premiers volumes, et qui porte le titre au dos: ORGELN VON ... SILBERMANN.

La liasse contient des projets de devis de Jean André Silbermann, des brouillons de lettres, des plans et des croquis. 36 p.

\section{Volume 8.}

Ces archives parisiennes sont complétées par un volume conservé à la Bibliothèque Nationale et Universitaire de Strasbourg sous la cote: Ms. 1541. (Als. 862). Le titre noté au fichier est le suivant: "Notices des orgues construites à Strasbourg de 1703 à 1783, par Silbermann père et fils." Le volume lui-même ne porte aucun titre. Il contient des notices sur les orgues d'André et de Jean André Silbermann, rédigées par Jean André Silbermann, avec des adjonctions de la main de son fils Josias.

Ce volume a fait partie de la bibliothèque de F.C.Heitz et servit de source à J.F.Lobstein. Pour un certain nombre d'orgues Silbermann il constitue l'unique source de renseignements. 1 vol. in-4, $84 \mathrm{p}$.

L'ensemble de ces manuscrits d'organologie totalise 1973 pages. Il va sans dire que leur importance est capitale, non seulement pour la connaissance de l'oeuvre des Silbermann, mais également pour l'histoire de l'orgue en général. 


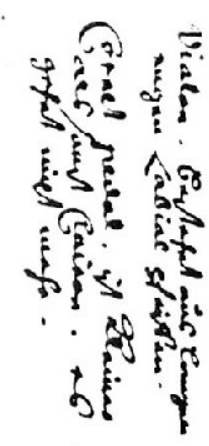

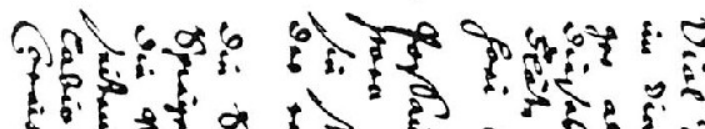
की की की

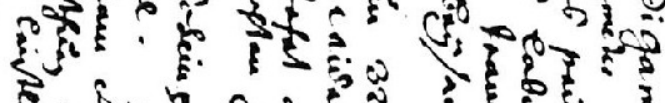

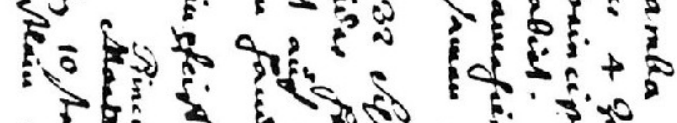

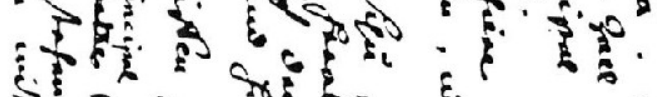

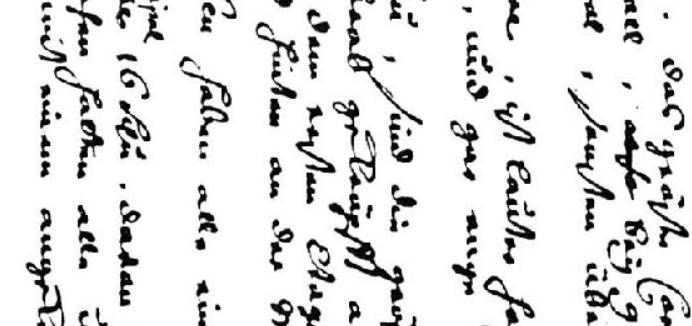

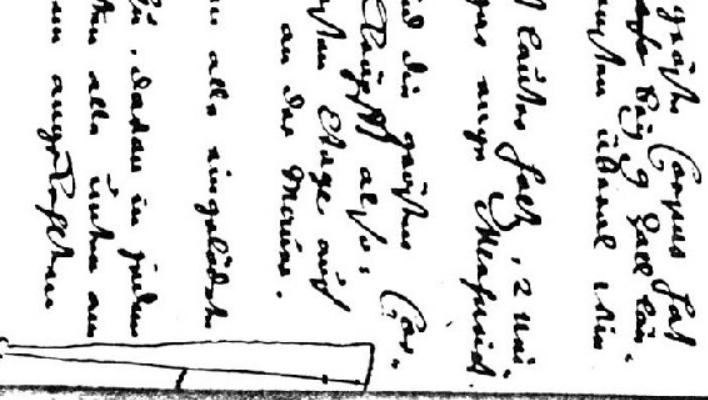

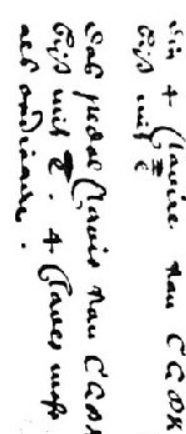

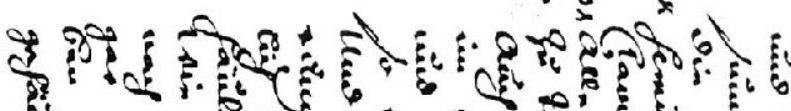
- H.

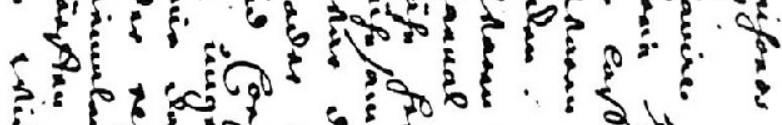
की की

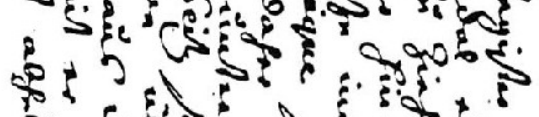

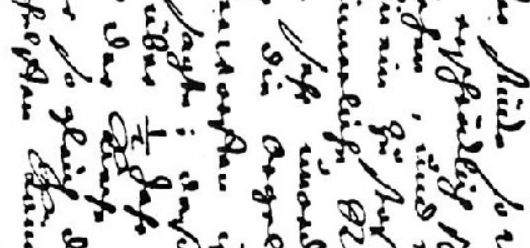
6.5.

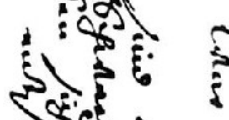

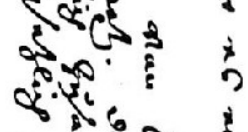
की 5ै

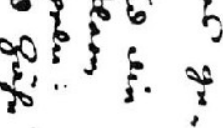

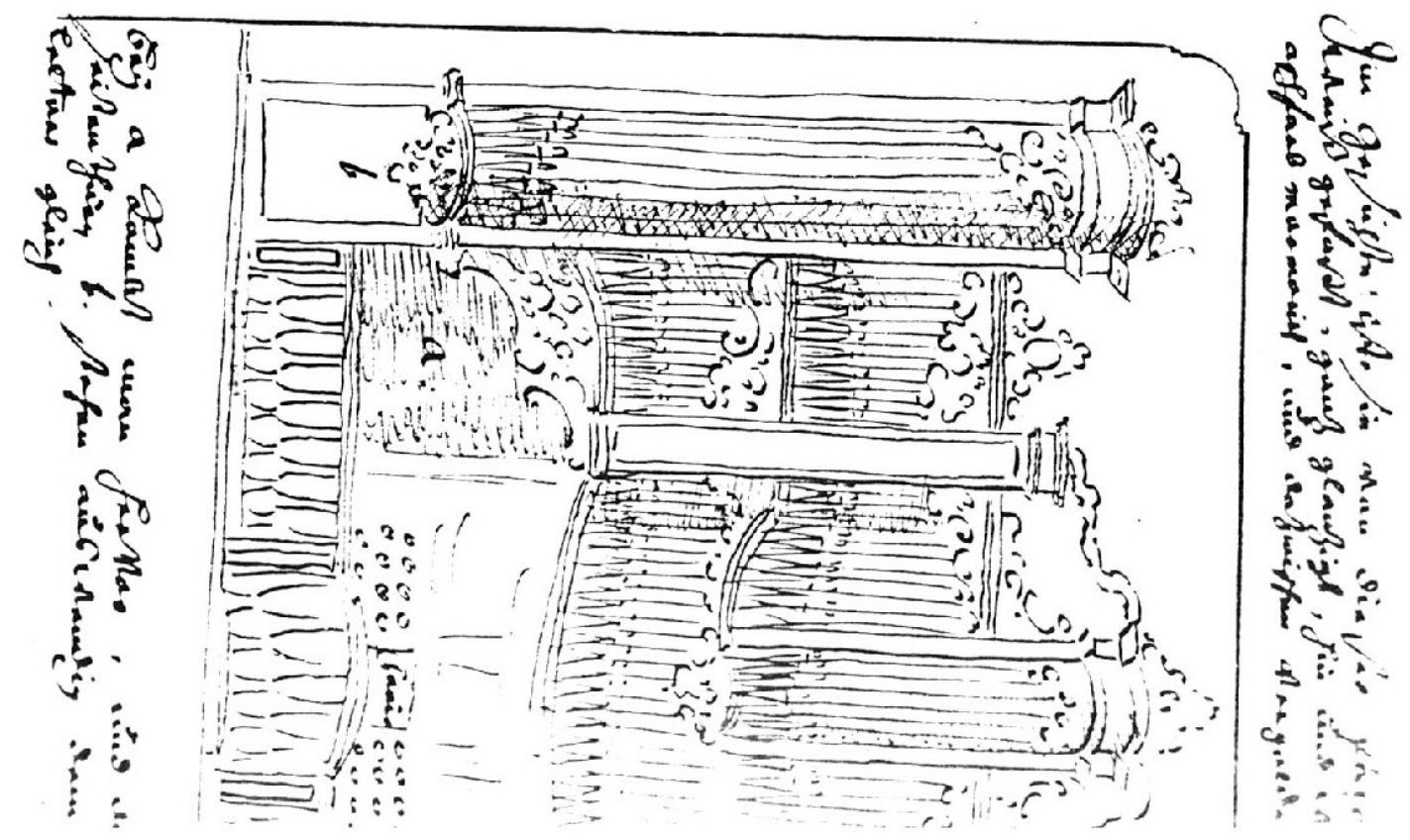


Le prétendu apprentissage d'André Silbermann chez Casparini.

Dans les ouvrages sur la famille Silbermann il est coutume de trouver un chapitre consacré à Casparini. Aussi bien Flade que Rupp, Mathias, Wörsching, Dähnert, pour n'en citer que les principaux, admettent qu'André Silbermann, avant de se rendre en Alsace, a appris le métier de facteur d'orgues chez E. Casparini à Görlitz. Il aurait fait un stage, dont la durée va jusqu'à six ans pour certains auteurs, et aurait participé à la construction de l'orgue de l'église Saint-Pierre-et-Saint-Paul de Görlitz. (1)

A qui remonte cette tradition? Aucun des auteurs susdits n'indique sa source, à l'exception de Flade(2) qui renvoie à J.F.Lobstein(3). Or Lobstein passe Casparini sous silence.

Il faut attendre 1861 pour voir apparaitre une relation écrite de l'apprentissage d'André Silbermann chez Casparini, dans l'ouvrage de Ludwig Mooser, Das Brüderpaar die Orgelbaumeister Andreas und Gottfried Silbermann. Historische Skizze nach kirchlichen und amtlichen Urkunden entworfen von Ludwig Mooser, Strasbourg, Gustave Silbermann, 1861. "Ludwig Mooser" n'est qu'un nom de plume pour Joseph Krömer (1815 - 1880), qui fut instituteur, cantor et organiste à Brand près de Freiberg en Saxe. (4) En 1857 il avait fait paraître Gottfried Silbermann der Orgelbauer. Ein historisches Lebensbild, Langensalza 1857. Il s'agit là de biographies romancées à l'extrême et sans aucune prétention scientifique. Elles sont d'ailleurs remplies de contradictions. (5) Mooser n'a pas eu de scrupules à inventer de toutes pièces des légendes, dont les plus exploitées ont été sans conteste, d'une part l'enlèvement d'une nonne du Couvent de Saint-Marguerite à Strasbourg par Gottfried Silbermann, et d'autre part l'apprentissage d'André Silbermann chez Casparini. Ce dernier point fut repris en 1911 par Vogeleis, qui indique comme source "Mooser, l.i.c.". Les auteurs subséquents lui font confiance, sûrs de transmettre une donnée scientifique.(6) La tradition de l'apprentissage d'André Silbermann chez Casparini repose donc sur un fondement scripturaire dénué de toute solidité. Nous ajouterons les remarques suivantes:

1) Le contrat pour la construction de l'orgue de Görlitz fut signé par Eugenius Casparini et son fils Adamus Oratius le 12 octobre 1697. Les travaux ne furent achevés qu'en 1703 (expertise le 5 juillet, inauguration le 19 août). (7) Ils durèrent 
donc six ans. André Silbermann est-il resté à Görlitz pendant tout ce temps? Non, car en 1699 sa présence est attestée à Bouxwiller en Alsace (8). D'autre part, si l'on admet que les travaux aient commencé en 1698, André Silbermann n'aurait pu faire qu'un séjour très bref à Görlitz; il n'aurait participé qu'au début des travaux.

2) Jean André Silbermann, qui dans ses notices utilise des indications laissées par son père, ne mentionne pas Casparini comme ayant été le mâttre de celui-ci. Dans le volume qu'il consacre aux facteurs d'orgues, il ne donne qu'une indication très sommaire:

"Casparini, Eugenius. Ein Niderlausitzer.

Casparini, Adam. Sohn des vorigen, lebte am End des vorigen biss ins gegenwärtige Jahrhundert. Casparini, Johann Gottlob. Sohn des vorigen." (9)

3) Lors de son voyage en Saxe en 1741, Jean André Silbermann eut l'occasion de voir et d'entendre l'orgue de Görlitz. Le jugement qu'il porta sur cet instrument est extrêmement dur et négatif.(10) Dans un autre récit, il va jusqu'à écrire: "Es ist diese Orgel eine von denen schlechtesten die ich auf meiner Reyss gesehen und gehöret."(11) Notons également les remarques désobligeantes de Jean-Sébastien Bach sur cet orgue(12) .

4) L'on s'est évertué à trouver dans l'oeuvre d'André Silbermann des influences de l'école de Casparini, sans succès. On a voulu voir dans le jeu de Viole de Gambe qu'André construira en 1703 au Couvent de Sainte-Marguerite à Strasbourg une réminiscence de Görlitz. Or ce jeu étant couramment construit en Alsace, bien avant l'arrivée des Silbermann. Les Baldner, Ring, Tretzscher le pratiquaient, dans la deuxième moitié du $17^{\circ}$ siècle, à Strasbourg. (13) Bien au contraire il est très difficile de trouver une relation quelconque entre les factures d'André Silbermann et de Casparini.(14)

5) Dans la lettre qu'il adressa le 7 février 1747 à son neveu Jean André, Gottfried Silbermann relate que son frère André avait travaillé chez Frédéric Ring à Strasbourg.(15) Cet apprentissage a eu lieu avant le printemps 1701, car Ring est décédé le 16 mars 1701. J'ai eu l'occasion d'attirer l'attention sur l'importance de Ring pour la formation d'André, et indirectement de Gottfried Silbermann. (16) 
(1) E. Flade, Der Orgelbauer Gottfried Silbermann, Leipzig 1926, p. 1 - 27; 20 édition, Gottfried Silbermann, Leipzig 1953, p. $1-54$.

E. Rupp, Die Entwicklungsgeschichte der Orgelbaukunst, Einsiedeln 1929, p. 34.

F.X.Mathias, articles dans la Revue Catholique d'Alsace, 1928 et 1929 .

J. Wörsching, Die Orgelbauer=Familie Silbermann in Strassburg i.E., Mayence s.d. (1941) p. 8 - 14; réédition par P. Smets, Mayence s.d. (1960) p. $14-24$.

U. Dähnert, Die Orgeln Gottfried Silbermanns in Mitteldeutschland, Leipzig 1953, p. 12.

(2) E. Flade, ouvr. Cité, éd. 1953, p. 57, note 149. A la page suivante, note 150, Flade renvoie même aux Archives Silbermann!

(3) J.P.Lobstein, Beiträge zur Geschichte der Musik im Elsass und besonders in Strassburg, Strasbourg 1840. Lobstein, qui utilise des notes manuscrites de Silbermann, consacre tout un chapitre à la famille Silbermann (p. 106 - 114).

(4) W. Müller, Auf den Spuren von Gottfried Silbermann, Berlin 1968, p. 28 , note 4.

(5) Par exemple, André est tantôt le frère, tantôt l'oncle de Gottfried Silbermann. W. Müller, dans l'ouvrage cité, p. 5051, note 11, doute qu'André Silbermann ait travaillé chez Casparini.

(6) M.Vogeleis, Quellen und Bausteine zu einer Geschichte der Musik und des Theaters im Elsass 500-1800, Strasbourg 1911, p. 587. Mooser est encore cité p. 593. L'essentiel du texte de L. Mooser concernant Casparini se trouve en note chez Wörsching-Smets, ouvrage cité, p. 35.

(7) L. Burgemeister, Der Orgelbau in Schlesien, $2^{\circ}$ éd. Frankfortsur-le-Main 1973, p. 135.

(8) J.A.Silbermann, ms. Elsässische Orgeln, p. 421.- C. Klein, Pfarrerbuch und Kirchenchronik der älteren evangelischen Gemeinde zu Buchsweiler, Strasbourg 1914, p. 96-97.

(9) J.A.Silbermann, ms. Von Orgelmachern, p.144.

(10) J.A.Silbermann, ms. Auswärtige Orgeln, p.76-81.

(11) J.A.Silbermann, ms. Unterschiedliche frembte Orgel=Wercke, Orgel in der Kirche St. Petri et Pauli in Görlitz.

(12) J.A.Silbermann, ms. Ausw. Org., p. 77, reproduit dans BachDokumente, vol. II, 1969, p.389. 
(13) J.J.Baldner, orgue de Saint-Nicolas à Strasbourg, 1668, Viole de Gambe 8 au Grand-Orgue. F.Ring, orgue du Temple-Neuf à Strasbourg, 1700, Viole de Gambe 8 au Grand-Orgue. M.Tretzscher, orgue de la cathédrale de Strasbourg, 1658-1659, Viole de Gambe 8 au Grand-Orgue.

(14) U.Dähnert, Die Orgellandschaften Sachsen und Thüringen, dans Acta Organologica, vol.I, 1967, p. 54.

(15) J.A.Silbermann, ms. Els. Org., p. 351-354. Le texte est reproduit dans Wörsching-Smets, p. 27-28.

(16) M.Schaefer, Les anciennes orgues Silbermann du Temple-Neuf à Strasbourg, dans La musique en Alsace hier et aujourd'hui, Strasbourg 1970, p. 95-122. 
Le travail d'André Silbermann auprès de Frédéric Ring à Strasbourg.

Une lettre de Gottfried Silbermann à son neveu Jean André, en date du 7. 2. 1749, nous renseigne sur les origines de l'orgue du Temple Neuf à Strasbourg et sur le rôle joué par Frédéric Ring au début de la carrière d'André Silbermann à Strasbourg. (1) Elle nous apprend qu'André travailla un certain temps auprès de Frédéric Ring dans la facture d'instruments à clavier ("in Instrumenten-Arbeiten"). Ensuite, avant de s'établir définitivement à Strasbourg, il répara des orgues dans différents couvents en Alsace. Ayant appris la mort de Frédéric Ring, survenue le 16. 3. 1701 avant que celui-ci n'ait pu terminer la construction de l'orgue du Temple-Neuf, André se mit en route pour Strasbourg dans l'espoir d'être chargé de mener à bien cet ouvrage. On lui préféra le facteur d'orgues Claude Legros, de Metz. Cette affaire fut néanmoins l'occasion pour André de s' installer à Strasbourg. Il logea chez le menuisier Michael Tenninger, rue Sainte-Barbe, et s'occupa de facture d'instruments à clavier (par quoi il faut entendre épinettes, clavecins, clavicordes).

Qui était Frédéric Ring? Faute de renseignements précis, F. X. Mathias (2) et d'autres après lui (3) l'ont considéré comme un obscur facteur d'orgues du Palatinat, dépourvu de talents et n'ayant laissé aucune trace remarquable. Ceci afin de rehausser le blason des Silbermann. Nous verrons dans un instant que ces affirmations péjoratives étaient erronées et bien inutiles. Ring était facteur d'orgues et de clavecins à Strasbourg. Le buffet d'orgues de l'église catholique de Ribeauvillé, classé "monument historique" (4) ainsi qu'un clavecin à deux claviers, sont là pour témoigner non seulement des talents de ce facteur, mais aussi de l'influence profonde qu'il exerça sur André Silbermann et indirectement sur Gottfried. (5)

Frédéric (Friderich) Ring est né le 5 mai 1666 à Berstett (BasRhin), fils aîné de Dominicus Ring, pasteur à Berstett et de son épouse Dorothea Heupel. Il fut baptisé à Berstett le 8 mai 1666 par le pasteur Andreas Barth, de Lampertheim. Ses parrains et marraine furent: Georg Conrad Schaualitzky von Muckothal, représenté par Johann Jacob von Berstett, Gottfried Rederer, de Strasbourg, et Maria Charitas von Berstett, née von Rathsamhausen. (6) 
Sans vouloir établir la généalogie complète de la famille Ring, disons quelques mots du père de Frédéric. Dominique Ring est né à Strasbourg le 25. 10. 1637, fils de Dominique Ring et d'Anne Marie Heck. A Pentecôte 1665 il épouse en premières noces Dorothée Heupel, fille du pasteur Jean Frédéric Heupel. Celle-ci meurt le 3. 12. 1686. Dominique Ring épouse en secondes noces Catherine Schmid, le 29. 10. 1692. Celle-ci était la veuve de Jean Louis Eggers, commerçant. Après le décès de Dominique Ring, elle épousa en troisièmes noces Emmanuel Fischer, commerçant, le 5. 5. 1700. Dominique Ring fit ses études de théologie à Strasbourg. Le 30. 4. 1665 il reçut l'ordination à la Cathédrale. Il occupa les postes suivants:

1662-1664: aumônier à l'Hôpital de Strasbourg, 1664-1665: pasteur adjoint à Berstett, 1665-1682: pasteur à Berstett, 1682-1691: vicaire à Ste-Aurélie à Strasbourg, 1691-1698: pasteur de Ste-Aurélie, chanoine de St. Thomas. Il mourut le 3. 11. 1698. (7) Notons que dans sa notice nécrologique on vante les mérites de son fils Frédéric, facteur d'orgues et d'instruments. (8)

Revenons à Frédéric Ring. Le 20 juin 1689, âgé de vingt-trois ans, il entre dans la corporation des charpentiers et achète le droit de bourgeoisie pour la somme de 3 livres, 2 sols et 6 deniers. (9) Le 12 octobre de la même année il épouse Anne Catherine Artopoeus, fille de feu Jean Georges Artopoeus, pasteur à St. Guillaume à Strasbourg. Le mariage est célébré en l'église St. Thomas. (10) Cinq enfants sont issus de cette union:

1) Frédéric, né le 20.10. 1690 (11)

2) Marie Dorothée, née le 7. 3. 1693 (12)

3) Anne Catherine, née le 3.1.1695 (13), décédée le 5.8.1697(14)

4) Jean, né le 14.12. 1697 (15)

5) Jean Daniel, né le 5. 4. 1700 (16), décédé le 25.9. 1703 (17)

Dans les documents ci-dessus, Ring porte le titre de menuisier et facteur d'orgues à partir de 1697, alors qu'il est âgé de trenteet-un ans. L'on ignore auprès de quel mâ̂tre il apprit le métier de facteur d'orgues. A Strasbourg il n'y avait à cette date aucun facteur d'orgues important.

Que connaissons-nous de l'activité de Frédéric Ring? Par la lettre de Gottfried Silbermann nous savons qu'il était facteur de clave- 
cins. Par une chance extraordinaire il se trouve qu'il subsiste un clavecin de Ring construit en 1700. Fait d'autant plus remarquable que tous les clavecins d'André Silbermann sont réputés disparus. En 1955 Josef Hirt (18) écrivit qu'un clavecin de Frédéric Ring était attesté pour l'année 1700, mais sans donner de précisions. En 1959 Raymond Russell (19) mentionne qu'un clavecin de Ring, datant d'environ 1700, était exposé à l'International Inventions Exhibition de Londres en 1885. Il avait deux claviers, et comportait deux jeux de 8' et deux jeux de 4', l'un de ces derniers étant uniquement un jeu de résonance. La trace de cet instrument, dit Russell, est perdue. Or nous l'avons retrouvé en 1974 chez le facteur de clavecins Martin Scholz (Maison Hug) à Bâle, qui doit le restaurer. Il appartient à Madame Eleonore Klinckerfuss, de Stuttgart-Sillenbuch, dont le grand-père, Appollo Klinckerfuss (Stuttgart 10.12. 1840 - 29.9.1923), acheta l'instrument en 1870 à Wissembourg. Luimême facteur de pianos, élève de Pape et de Kriegelstein à Paris, (20) Klinckerfuss restaura le clavecin de Ring, l'exposa à Londres en 1885 et obtint une médaille d'argent. Selon une lettre adressée par le pianiste Hans von Bülow (1830-1894) au mâttre de chapelle Schletterer à Augsbourg, ce clavecin aurait appartenu à Georg Muffat (1653-1704). Cette tradition nous semble peu vraisemblable, car Muffat avait quitté l'Alsace dès 1674 et dirigea la Chapelle du prince-évêque à Passau de 1690 jusqu'à sa mort. Toujours est-il que Hans von Bülow qualifia le clavecin de Ring de magnifique.(21)

Quelle fut l'activité de Ring en facture d'orgues? Selon Lobstein (22) il répara l'orgue de St-Thomas à Strasbourg en 1696 et y plaça une Voix humaine le 16 octobre 1700. Nous n'avons trouvé aucun document d'archives concernant ce travail.

Le seul travail attesté par des documents d'archives, et il est d'importance, est la construction de l'orgue du Temple-Neuf à Strasbourg en 1700-1701 (23). Le devis, daté d'octobre 1700, prévoyait la composition suivante:

Clavier du haut: 1) Bourdon 8

2) Quinte $22 / 3$

3) Octave 2

4) Cymbale

5) Cornet V

Clavier du milieu: 1) Principal 8

2) Viole de Gambe 8

3) Bourdon 8

4) Prestant 4

5) Quinte 2 2/3 


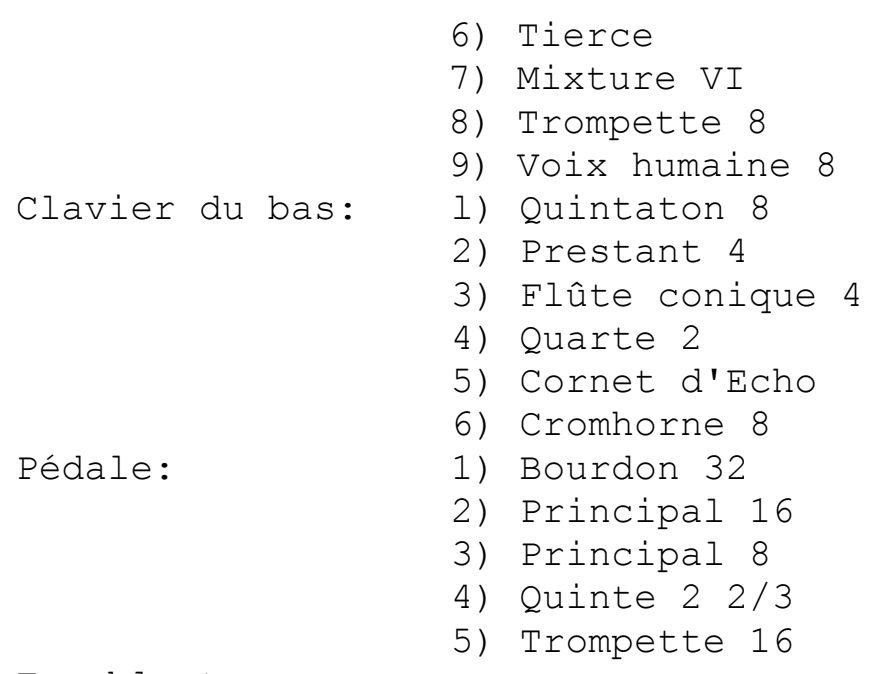

Tremblant.

Ring pouvait utiliser à son gré le le matériel de l'ancien orgue, qui après la réparation par F. Freundt en 1683 comptait quatorze jeux. Le buffet devait être neuf, selon le plan dessiné par Ring lui-même. Les claviers devaient avoir 50 notes chacun. Ils devaient permettre à l'organiste de transposer d'un ton et d'un demi-ton. Le nouvel orgue devait être achevé pour Pâques 1701.

Dans cette composition nous sommes frappés par l'absence de 4 pieds au clavier du haut, de 2 pieds au Grand Orgue, de 4 pieds à la Pédale. Par ailleurs on y relève des éléments de tradition locale: Viole de Gambe 8, Quintaton 8, Flûte conique 4 et Quarte 2(Schweiglen). Alors que la Pédale, basée sur le 32 pieds, a très nettement une fonction de clavier de basse, on note déjà des influences françaises: Cornet V au clavier du haut, Cornet d'Echo à celui du bas, jeu de Tierce - quoiqu'incomplet - au Grand Orgue.

Le buffet, conservé à l'église catholique de Ribeauvillé où il fut placé en 1749 par Rohrer, est de toute beauté. Le travail de sculpture est très élaboré. L'architecture groupe trois tourelles séparées par deux plate-faces assez étroites et non centrées. Le plan des tourelles n'est pas en demi-cercle comme le feront les silbermann de Strasbourg à partir de 1708, mais plus aplati: Ce sera le parti des premiers instruments d'André ainsi que de la plupart de ceux de Gottfried.

La répartition des différents plans sonores est assez particulière. Alors que le Grand Orgue occupe sa place traditionnelle, le clavier du haut est un véritable "Oberwerk", disposition qui se traduit dans le buffet par trois petits buffets placés sur les tourelles. 
Le clavier du bas était sans doute destiné au soubassement. Celuici comporte des panneaux ajourés. Notons le riche emploi du motif des feuilles d'acanthe, notamment sur la frise séparant le soubassement et le grand corps. Cette particularité sera reprise par André Silbermann au couvent de Ste-Marguerite (1704) et à StPierre-le-Vieux protestant (1708) à Strasbourg, ainsi qu'à l'Abbaye de Marmoutier (1710).

Les tourelles, plus larges que les plate-faces, comportent 9 tuyaux pour la tourelle centrale, et 7 pour les tourelles latérales. Les plate-faces ont chacune 7 tuyaux.

La structure des petits buffets d'Oberwerk sera utilisée par Jean André Silbermann dans ses tourelles tripartites constituées par une tourelle centrale imbriquée dans deux demi-plates-faces, à partir de 1737 (Colmar Unterlinden). De plus, le petit buffet central préfigure le type de buffet de Saint-Quirin (1746), avec ses platesfaces latérales.

L'influence de Frédéric Ring sur la facture des Silbermann, aussi bien en Alsace qu'en Saxe, est indéniable. A quelle date se situe le travail d'André Silbermann auprès de Ring? Frédéric Ring meurt subitement le 16 mars 1701 d'une embolie, à l'âge de trente-quatre ans (24). Il n'a pu terminer l'orgue du Temple-Neuf. Le buffet est en place, mais les sommiers ne sont pas achevés. André Silbermann travaille à Haguenau à cette époque et ne se rend à Strasbourg que lorsqu'il apprend la mort de Ring. Si l'on admet que la venue en Alsace d'André Silbermann se situe en 1699, il est permis de penser qu'il travailla chez Ring en 1699-1700. 
(1) Jean André Silbermann a recopié cette lettre dans son volume consacré aux orgues d'Alsace, Elsässische Orgeln, p. 351-354, à propos des anciens orgues du Temple-Neuf à Strasbourg. F.X. Mathias en a donné une traduction dans son article "André Silbermann s'établit à Strasbourg" dans les Echos des Sanctuaires de Sainte-Odile, 2, 1930, p. 58-60. Le texte original a été reproduit par smets dans la réédition de l'ouvrage de Wörsching, Die Orgelbauer-Familie Silbermann in Strassburg i.E., Mayence s.d. (1960), p. 27.

(2) F.X. Mathias, Compte-Rendu du Congrès d'Orgue tenu à I'Université de Strasbourg du 5 au 8 mai 1932, Strasbourg 1934, p.245.

(3) Jean Ringue, Les facteurs d'orgues Silbermann en Alsace, in Caecilia, 1952, p. 60-61.

(4) Voir les photos du buffet de Ribeauvillé dans Médard Barth, Elsass "das Land der Orgeln" im 19. Jahrhundert, Haguenau 1966, pl. 19, et Norbert Dufourcq, Le Livre de l'Orgue Français, t.2, Le Buffet, Paris 1969, fig. 505.

(5) Marc Schaefer, Les anciennes orgues Silbermann du Temple-Neuf à Strasbourg, in La Musique en Alsace, hier et aujourd'hui, Strasbourg 1970, p. 95 ss.

(6) Archives paroissiales de Berstett, Kirchenbuch 1664-1685,p.28 (P.J. p.213).

(7) M.-J. Bopp, Die evangelischen Geistlichen und Theologen in Elsass und Lothringen, Neustadt a.d.Aisch, 1959, p.442, $\mathrm{N}^{\circ}$ 4261.

(8) AMS, Arch. du Chapitre de St-Thomas, $\mathrm{N}^{\circ} 449$, Progr. funebr. $\mathrm{N}^{\circ}$ 102. En voici un extrait: "...quatuor liberos peperit, inter quos unus superest filius, tribus caeteris, duabus scil. puellis \& altero filio ante patrem praemissis. A superstite vero filio, artifice singularis ingenii, Organorum pnevmaticorum \& aliorum instrumentorum Musicorum confectore, Scriniatioque DN. Friderico Ring, conjugem habente Annam Catharinam, plur. Rev. DN. Joh. Georg. Artopoei, Pastoris quondam Wilhelmitani filiam, nepotes vidit duos, duasque neptes, quarum altera avum praecessit; supersunt nepotes, Fridericus \& Johannes, neptis Dorothea, quos Deus servet."

(9) AMS, Charpentiers 6.

Sontag, den 20t. Juny 1689.

Newzünfftige:

Friederich Ring, Schreiner producirt seinen Stallschein de 
dato den 18. Juny 1689. undt erkaufft das Burgerrecht umb 3. 1b. 2. s. 6. d. die er gleich erlegt undt bezalt, juravit eod.

(10) AMS, M 122, fol. 13, $\mathrm{N}^{\circ} 63$ (P.J. p.214)

(11) AMS, N 252, fol. 70 b, $\mathrm{N}^{\circ} 287$ (P.J. p.215)

(12) AMS, N 252, fol. 119 a, $\mathrm{N}^{\circ} 497$ (P.J. p.216)

(13) AMS, N 252, fol. 158 a, $\mathrm{N}^{\circ} 662$ (P.J. p.217)

(14) AMS, D 201, fol. 25 a, $\mathrm{N}^{\circ} 10$ (P.J. p.218)

(15) AMS, N 252, fol. 214 a, $\mathrm{N}^{\circ} 931$ (P.J. p.219)

(16) AMS, N 252, fol. 251 a, $\mathrm{N}^{\circ} 1104$ (P.J.p.220)

(17) AMS, D 200, fol. 106 b, $N^{\circ} 708$ (P.J. p.222)

(18) Franz Josef Hirt, Meisterwerke des Klavierbaus, Olten 1955, p. 494; 2/1981, p. 223.

(19) Raymond Russell, The harpsichord and clavichord, Londres 1959, 2/1973, P. 99.

(20) F. J. Hirt, ouvr. cité, p. 447.

(21) Jörg-Dieter Hummel, Friderich Ring, der vergessene Instrumentenbauer aus dem Elsass und sein Clavicymbal von 1700, mit Beiträgen von Marc Schaefer, Strassburg, Martin Scholz, Basel und einem Geleitwort von Wilhelm Kempff, Augsburg 1976.

(22) J. P. Lobstein, ouvr. cité, p. 57. "Wing" est probablement une erreur d'impression. Par ailleurs le nom de Ring est souvent orthographié "Rink" ou "Rinck". Lui-même signe toujours "Ring".

(23) Voir M. Schaefer, art. Cité, qui reproduit le devis de Ring. (24) AMS, D 200, fol. $91 \mathrm{a}-\mathrm{b}, \mathrm{N}^{\circ} 602$ (P.J. p.221) 
56

y.riknorify
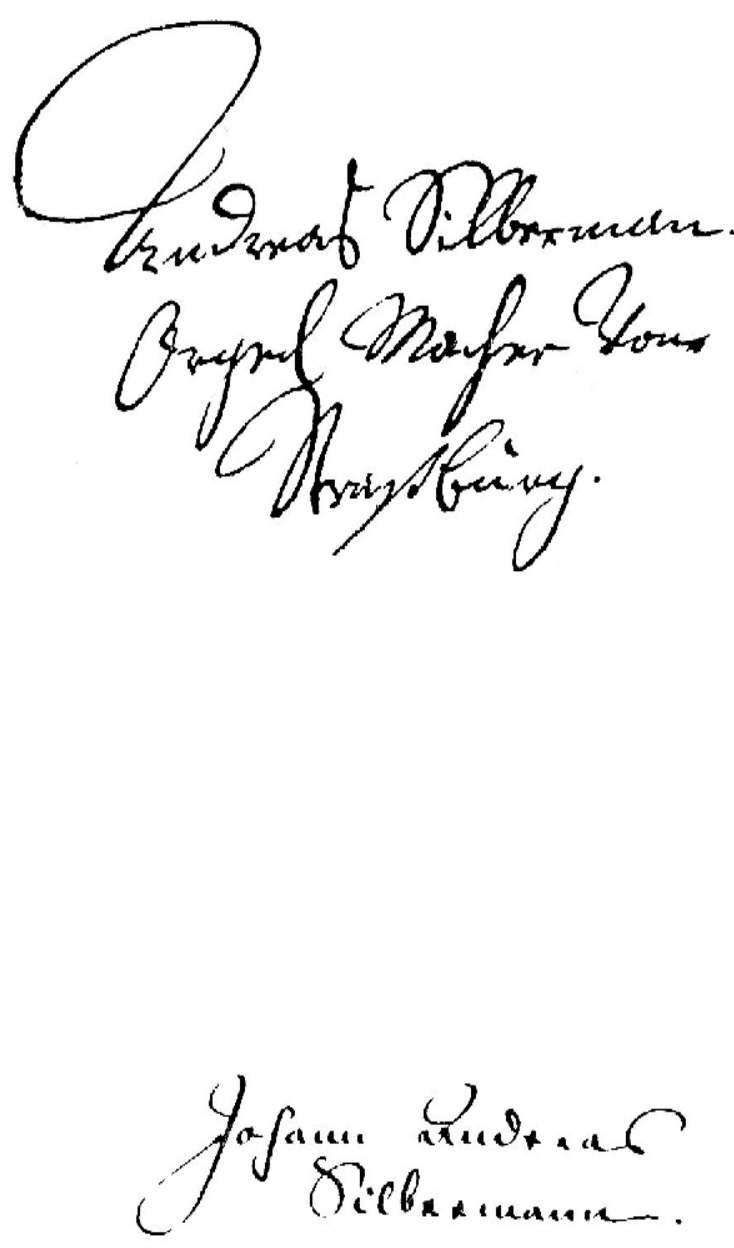


\section{La généalogie de la famille Silbermann.}

Il n'existe actuellement aucune généalogie complète de la famille Silbermann. Plusieurs auteurs ont publié des éléments épars, et les généalogies présentées comme telles sont incomplètes et contiennent des erreurs.

En 1840, l'avocat J. F. Lobstein, dans ses Beiträge zur Geschichte der Musik im Elsass, consacre un chapitre à la famille silbermann. Sa généalogie est très sommaire et n'englobe pas la branche saxonne.

La même remarque vaut pour Vogeleis, qui dans ses Quellen und Bausteine zu einer Geschichte der Musik und des Theaters im Elsass, Strasbourg 1911, consacre quatre pages à la famille Silbermann. Elle ne comprend qu'une douzaine d'individus.

Ernst Flade, Der Orgelbauer Gottfried Silbermann, Leipzig 1926, donne un arbre généalogique plus développé, mais qui reste sélectif et qui s'arrête à Gustave Silbermann, l'imprimeur. Les renseignements donnés par Flade dans cette première édition de son livre ont le mérite d'être exacts. Disons d'emblée que ce n'est pas le cas de la seconde édition.

Dans le Compte-rendu du Congrès d'Orgue de 1932, F. X. Mathias tente de compléter les indications de Vogeleis et de Flade grâce aux renseignements donnés par une prétendue "feuille volante" des Archives Silbermann de Paris. L'élément nouveau est un capitaine de cavalerie lourde de Saxe, Georg Silbermann, qui serait arrivé en Alsace à la Guerre de Trente Ans. Nous aurons à revenir à ce Georg Silbermann. Disons pour le moment que la feuille volante en question ne se trouve pas dans les Archives Silbermann de Paris, et que si ce Georg Silbermann a existé, il n'est pas prouvé qu'il ait un lien avec la famille des facteurs d'orgues.

J. Wörsching, Die Orgelbauer=Familie Silbermann in Strassburg i. E., Mayence, s.d., reprend le tableau de Flade, tout en y ajoutant le capitaine de Mathias.

Dans la deuxième édition de Flade, 1953, la situation devient critique. Flade cite deux filles de Jean André Silbermann, l'éminent facteur d'orgues strasbourgeois, qui auraient épousé l'une le facteur d'orgues Conrad Sauer, l'autre le facteur d'orgues Alffter- 
mann, de Strasbourg. Or nous connaissons les trois femmes de Conrad Sauer: aucune n'est née Silbermann. Quant à Alfftermann, qui s'appelait Alffermann et qui était facteur d'orgues à Landau, il n'était non plus un gendre de Silbermann. Par ailleurs, plusieurs dates de cette généalogie se révèlent erronées.

Dans sa réédition de l'ouvrage de Wörsching, P. Smets augmente la confusion en inventant les prénoms des deux filles de Silbermann et en donnant leur année de naissance. Or, pour ce qui est de Sauer, aucune des deux filles de Jean André Silbermann ne s'appelait Friederike. Quant à Alffermann, il est décédé quelques années après la prétendue date de naissance de Katharine Silbermann, d'ailleurs née en 1754 et non en 1753.

Pour terminer cet aperçu critique, citons l'ouvrage de Werner Müller, Auf den Spuren Gottfried Silbermanns, Berlin et Kassel 1968, 6/1982. Il ne contient pas d'arbre généalogique, mais donne de nombreuses précisions en ce qui concerne les antécédants saxons.

Nous avons essayé de dresser une nomenclature complète de la famille Silbermann, en tout cas pour la branche strasbourgeoise. Tous les noms et toutes les dates sont étayés et corroborés par des documents d'archives, registres paroissiaux, état-civil. Nos recherches sont allées dans trois directions:

1) La famille des facteurs d'orgues strasbourgeois, dont les origines sont à chercher en saxe. Deux familles parisiennes descendent de cette lignée. L'une d'elles est propriétaire des Archives Silbermann.

2) Les ancêtres de M. Xavier Silbermann, actuellement facteur d' orgues à Thonon. Nos recherches se sont arrêtées en 1623 à Haguenau, sans que nous n'ayons pu établir de lien avec la famille précitée.

3) Une lignée haut-rhinoise, dont les origines remontent à 1646, et dont des descendants habitent Spechbach-le-Haut, Burnhauptle-Haut et Sentheim.

$$
\begin{aligned}
& \text { Abréviations: } \\
& \text { ○ = né } \\
& \text { ○० = marié } \\
& +\quad=\text { décédé } \\
& \text { b. = baptisé } \\
& \text { bg. = bourgeois }
\end{aligned}
$$




\section{Généalogie $N^{\circ} 1$.}

Les ancêtres des facteurs d'orgues saxons et strasbourgeois.

Après ce que nous venons de dire au sujet des généalogies traditionnelles, on ne sera pas étonné de ne plus voir mentionnés certains noms. C'est le cas notamment du premier ancêtre, sculpteur né vers 1530 à Graupen, selon Flade. Rien ne permet d'affirmer que ce personnage a existé, et encore moins d'y voir l'ancêtre de la famille.

Le nom Silbermann était très répandu en Saxe, et en particulier dans l'Erzgebirge. Werner Müller cite plusieurs Silbermann ayant vécu à la fin du $16^{\circ}$ siècle, mais dont il faut se garder, en l'absence de preuves, de faire des membres de la famille qui nous occupe. Il s'agit par exemple de Magdalena Silbermann, fille de Benedix Silbermann, de Grund, qui le 7. 5. 1593 épouse Nicol Braun, d'Oberbobritzsch; ou encore Matz et Franz Silbermann, cités dans les registres d'impôts de Freiberg en 1546. (1)

$$
\mathrm{N}^{\circ} 1
$$

Georg Silbermann

+ avant 1619 à Kleinbobritzsch

Enfants: 1 . Veit $\left(\mathrm{N}^{\circ} 2\right)$

2. Christoph o 1595

3. Georg $\circ 1597$

4. Abraham o 1601

5. fille

6. fille

7. fille

$$
\mathrm{N}^{\circ} 2
$$

Veit Silbermann

( du $N^{\circ} 1$ )

o entre 1584 et 1591

$+1.3 .1666$

oo 1) Anna Klemm 1619

oo 2) Barbara Arnold 1623

Enfants: trois enfants 
oo 3) Justina Seifert $1634(+22.2 \cdot 1666)$

Enfants: 1. enfant mort à 3 ans

2. Abraham $01637+1684$

3. Michael $\left(\mathrm{N}^{\circ} 3\right)$

4. Andreas o 1646

oo 4) Maria Richter, de Birkersdorf

$\mathrm{N}^{\circ} 3$

Michael Silbermann

$\left(\mathrm{du} \mathrm{N} \mathrm{N}^{\circ}\right.$ 2) $\quad$ (28. 8. 1640 à Kleinbobritzsch. Charpentier.

oo 1) Christina Tröger 9. 7. 1660 (+ 6.5.1676)

Enfants: 1. Sabina + après quelques semaines

2. Michael $\left(\mathrm{N}^{\circ} 4\right)$

3. Georg $\left(\mathrm{N}^{\circ} 5\right)$

4. Christian $\left(\mathrm{N}^{\circ} 6\right)$

oo 2) Anna Maria Preussler 17. 9. 1676

Enfants: 1. Andreas $\left(\mathrm{N}^{\circ} 7\right)$

2. Gottfried o 14. 1. 1683 à Kleinbobritzsch

facteur d'orgues à Freiberg

+ 4. 8. 1753 à Dresden, célibataire

3. Abraham o 1696

$$
\mathrm{N}^{\circ} 4 \text {. }
$$

Michael Silbermann

$\left(\mathrm{du} \mathrm{N}^{\circ} 3\right)$

- 23. 9. 1666 à Kleinbobritzsch

oo 1) Rosina Köhler 23. 11. 1685

à Kleinbobritzsch

oo 2) Christina Richter 1692 (+ 1. 12. 1694)

Enfant: fils mort-né 29.11. 1694

oo 3) Christina Kirchner 13.1. 1696

Enfants: 1. Michael o 16.10.1696

oo Agneta Sophia Stöber 1727 à Frauenstein

2. Johann George o 19. 5. 1698 à Frauenstein

facteur d'orgues

$+1.9 .1749$

3. fils o 1710 


$$
\mathrm{N}^{\circ} 5 \text {. }
$$

Georg Silbermann
$\left(\mathrm{du} \mathrm{N}^{\circ} 3\right)$
- $11.11 .1670+1735$
chirurgien à Glashütte
oo Sophia Herrmann 1696

Enfant: Georg Friedrich $\circ$ 1699, chirurgien à Glashutte ( ${ }^{\circ}$ 9)

$$
\mathrm{N}^{\circ} 6 \text {. }
$$

Christian Silbermann

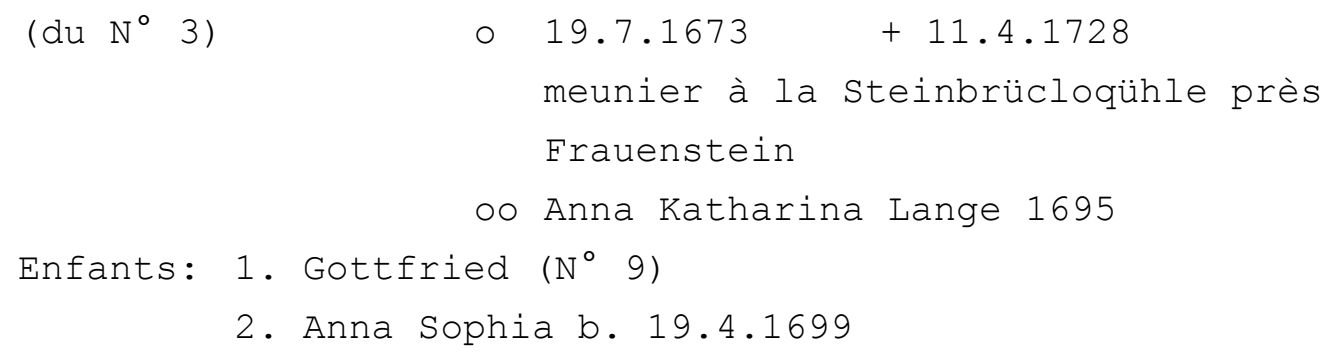




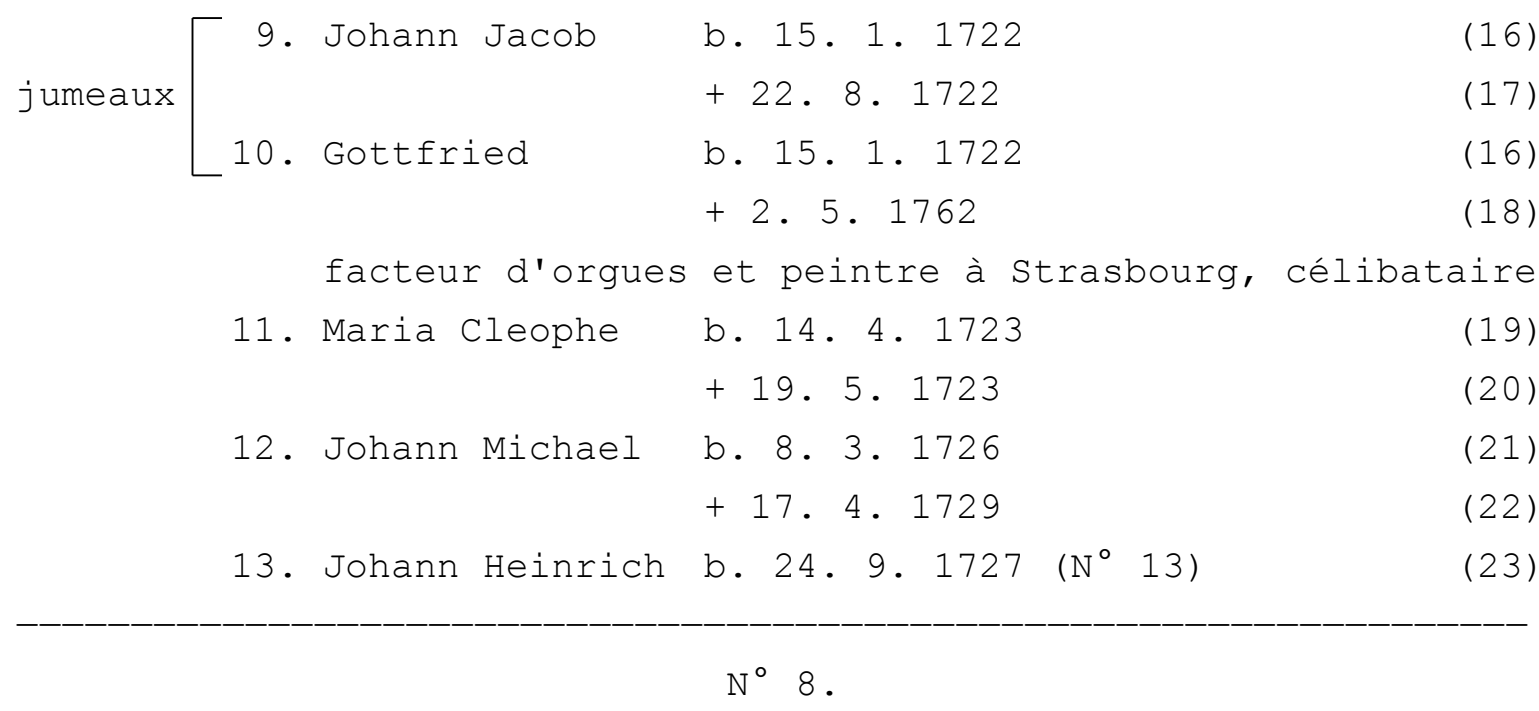

Georg Friedrich Silbermann

$\begin{array}{ll}\left(\mathrm{du} \mathrm{N} \mathrm{N}^{\circ} \text { 5) }\right. & \\ & \\ \text { Enfant: Georg Friedrich } & \text { chirurgien à Glashütte } \\ & \circ 1735 \\ & \text { chef d'équipe à Glashütte } \\ & +1787\end{array}$

N 9

Gottfried Silbermann

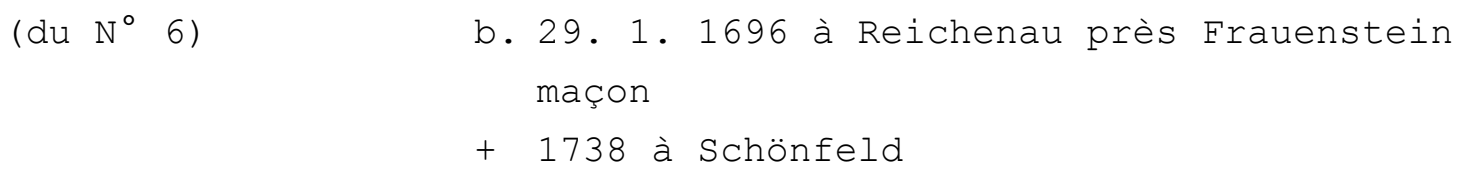

Enfants: 3 filles

4 fils, dont le dernier:

Gottfried $\quad$ o 1734

maçon

oo sans enfants

+ 25. 2. 1808 à Lichtenberg

$N^{\circ} 10$.

Anna Maria Silbermann
(du $\mathrm{N}^{\circ} 7$ )
b. 18. 3. 1709 à Strasbourg
+ 19.9. 1760 à Strasbourg
oo 1) Philipp Jacob Nacken,
négociant à Strasbourg,
fils de Jonas Nacken, négociant à Francfort/M.
veuf de Margaretha Salome Huth. + 25. 3. 1748 


$$
\begin{aligned}
& \text { à Strasbourg } \\
& \text { ०० 1. 8. } 1731 \text { à Strasbourg }
\end{aligned}
$$

oo 2) 16. 1. 1752 Johannes Saum, veuf,

marchand de vins à Strasbourg,

Fabric-Pfleger de St. Nicolas

+ 1. 5. 1761 à Strasbourg

$$
\mathrm{N}^{\circ} 11 \text {. }
$$

\begin{tabular}{|c|c|c|c|}
\hline \multirow{2}{*}{$\left(\begin{array}{lll}d u & N^{\circ} & \end{array}\right)$} & \multicolumn{3}{|c|}{ - 24. 6. 1712 à Strasbourg } \\
\hline & \multicolumn{3}{|c|}{$\begin{array}{l}\text { facteur d'orgues à Strasbourg, membre } \\
\text { du Grand Conseil de la ville de Strasbou } \\
\text { oo 1) } 22.8 \text {. } 1742 \text { Anna Salome Mannberger, f } \\
\text { de Johann Nicolaus Mannberger, coutelier } \\
\text { Strasbourg, } 01725\end{array}$} \\
\hline \multirow[t]{10}{*}{ Enfants: } & 1. Johann & Andreas & $\begin{array}{l}\text { b. } 17 \cdot 6 \cdot 1743 \\
+14 \cdot 6 \cdot 1744\end{array}$ \\
\hline & 2. Johann & Daniel & $\begin{array}{l}\circ 2.10 .1745 \\
\text { facteur d'orgues à Strasbourg } \\
+2.8 .1770 \text { célibataire }\end{array}$ \\
\hline & \multicolumn{2}{|c|}{ 3. Margaretha Salome } & $\begin{array}{l}\circ 17 \cdot 7 \cdot 1749 \\
+6 \cdot 5 \cdot 1750\end{array}$ \\
\hline & \multicolumn{2}{|c|}{ 4. Margaretha Salome } & $\begin{array}{l}\circ 21 \cdot 12 \cdot 1750 \\
+23 \cdot 1 \cdot 1760\end{array}$ \\
\hline & 5. Johann & Andreas & ○ 14.12. $1752\left(\mathrm{~N}^{\circ} 14\right)$ \\
\hline & 6. Cathar & ina Dorothea & $\begin{array}{l}\circ 1 \cdot 11 \cdot 1754 \\
+22 \cdot 12 \cdot 1763\end{array}$ \\
\hline & 7. Johann & Gottfried & $\begin{array}{l}\circ 21 \cdot 1 \cdot 1758 \\
+2 \cdot 4 \cdot 1758\end{array}$ \\
\hline & \multirow{2}{*}{\multicolumn{2}{|c|}{ 8. Gottfried }} & ० 4.1. 1760 \\
\hline & & & $+6 \cdot 7 \cdot 1762$ \\
\hline & 9. Johann & Josias & $\begin{array}{l}\text { o } 17.2 .1765 \\
\text { facteur d'orgues à Strasbourg } \\
+3.6 .1786 \text { célibataire }\end{array}$ \\
\hline
\end{tabular}

Johann Andreas Silbermann

\section{rg}

ille 
fille de Johann Jacob Schatz, directeur du Gymnase protestant et bibliothécaire de l'Université de Strasbourg, et de Anna Margaretha Arnold, o 1724

+10.3 .1808 à Strasbourg

Enfants: sans enfants.

$+11 \cdot 2 \cdot 1783$ à Strasbourg

$$
\mathrm{N}^{\circ} 12 \text {. }
$$

Johann Daniel Silbermann

$\left(\mathrm{du} \mathrm{N}^{\circ} 7\right)$

b. 31. 5. 1717 à Strasbourg

+ 9. 5. 1766 à Leipzig

facteur d'orgues à Strasbourg puis à Dresden

oo 1) 6. 3. 1748 Maria Elisabetha Baer

à Strasbourg

+ 13. 9. 1750 à Soultz

fille de Johann Philipp Baer, négociant à Strasbourg

Enfant: Ludwig o 18. 8. 1749 à Strasbourg,

+ 12. 11. 1750 à Strasbourg

oo 2) janvier 1766 Susanna Christiana

Rockenthien, veuve Theile, à Leipzig

b. 11. 12. 1745 à Leipzig

fille de Johann Friedrich Rockenthien,

négociant à Leipzig

○o en premières noces, à Leipzig,

18. 10. 1763, Christian Gottlob Theile,

négociant à Dresden.

Enfants: sans enfants.

$$
\mathrm{N}^{\circ} 13 .
$$

Johann Heinrich Silbermann

$\left(\mathrm{du} \mathrm{N}^{\circ} 7\right.$ )

b. 24.9. 1727 à Strasbourg

+ 15. 1. 1799 à Strasbourg

facteur d'orgues et d'instruments à strasbourg

o 17. 8. 1757 à Strasbourg, Catharina

Margaretha Mosseder

- 1736 à Strasbourg

+26.3 .1796 à Strasbourg 
fille de Johann Jacob Mosseder, marchand de vins et de Catharina Margaretha Schuch, à Strasbourg

Enfants: 1. Catharina Henrietta o 9. 1. $1759\left(\mathrm{~N}^{\circ} 15\right)$

2. Johann Heinrich o 3.6.1760

$+15.4 .1762$

3. Johann Friedrich

- 21.6 .1762

+ 9.3. 1805 célibataire

organiste et facteur de clavecins

4. Friederica Magdalena O 19. 12. $1768\left(\mathrm{~N}^{\circ} 16\right)$

5. Johann Heinrich 0 16. 7. $1771\left(\mathrm{~N}^{\circ} 17\right)$

$$
\mathrm{N}^{\circ} 14 \text {. }
$$

Johann Andreas Silbermann

$\left(\mathrm{du} \mathrm{N}^{\circ} 11\right)$

- 14. 12. 1752 à Strasbourg

+28.3 .1794 à Strasbourg

négociant

○o 14. 5. 1783 à Strasbourg, Anna Elisabetha

Karth, fille de Johann Niclaus Karth,

négociant à Strasbourg, et de Maria

Elisabetha Mylius

Enfants: 1. Johann Heinrich o 17. 11. 1784 à Strasbourg

2. Johann Andreas o 22. 3. 1786 à Strasbourg $\left(N^{\circ} 18\right)$

3. Carl Daniel o 4. 6. 1788 à Strasbourg

4. Theodor Friedrich o 14. 7. 1790 à Strasbourg membre du Conservatoire de Musique à Paris + 5. 6. 1816 à Strasbourg, célibataire

\section{$\mathrm{N}^{\circ} 15$.}

Catharina Henrietta Silbermann

$$
\begin{aligned}
& \text { (du } \mathrm{N}^{\circ} \text { 13) } \quad \text { 9. 1. } 1759 \text { à Strasbourg } \\
& +\quad \text { 16. } 4.1833 \text { à Strasbourg } \\
& \text { ○o } 25.8 .1783 \text { à Strasbourg, Johann Müller, } \\
& \text { pasteur à Gundershoffen, fils de Johann } \\
& \text { Georg Müller, instituteur à Gertwiller, } \\
& \text { et de Maria Magdalena Wilhelm, } \\
& \quad 16.8 .1753 \\
& +30.1 .1847 \text { à Strasbourg }
\end{aligned}
$$

Enfant: Johann Heinrich $\quad 1784$ à Gundershoffen 


$$
\mathrm{N}^{\circ} 16 .
$$

Friederica Magdalena Silbermann

$$
\begin{aligned}
& \text { (du } \mathrm{N}^{\circ} \text { 13) } \quad \text { o 19.12. } 1768 \text { à Strasbourg } \\
& \text { + 8. 12. } 1829 \text { à Strasbourg } \\
& \text { oo 17. 9. } 1794 \text { à Strasbourg, Philippe } \\
& \text { Jacques Strohl, marchand-fourreur } \\
& \text { o 13. 4. } 1767 \text { à Strasbourg }
\end{aligned}
$$

Enfants: 1. Philippe Théodore

$$
+8.9 .1861
$$

2. Sophie Adèle

$$
+2.3 .1835
$$

3. Frédérique Emilie

$$
+12 \cdot 6 \cdot 1851
$$

oo 1818 Jean-Louis Koehler, fondé de pouvoirs à Strasbourg

$$
\mathrm{N}^{\circ} 17 .
$$

Jean Henri Silbermann

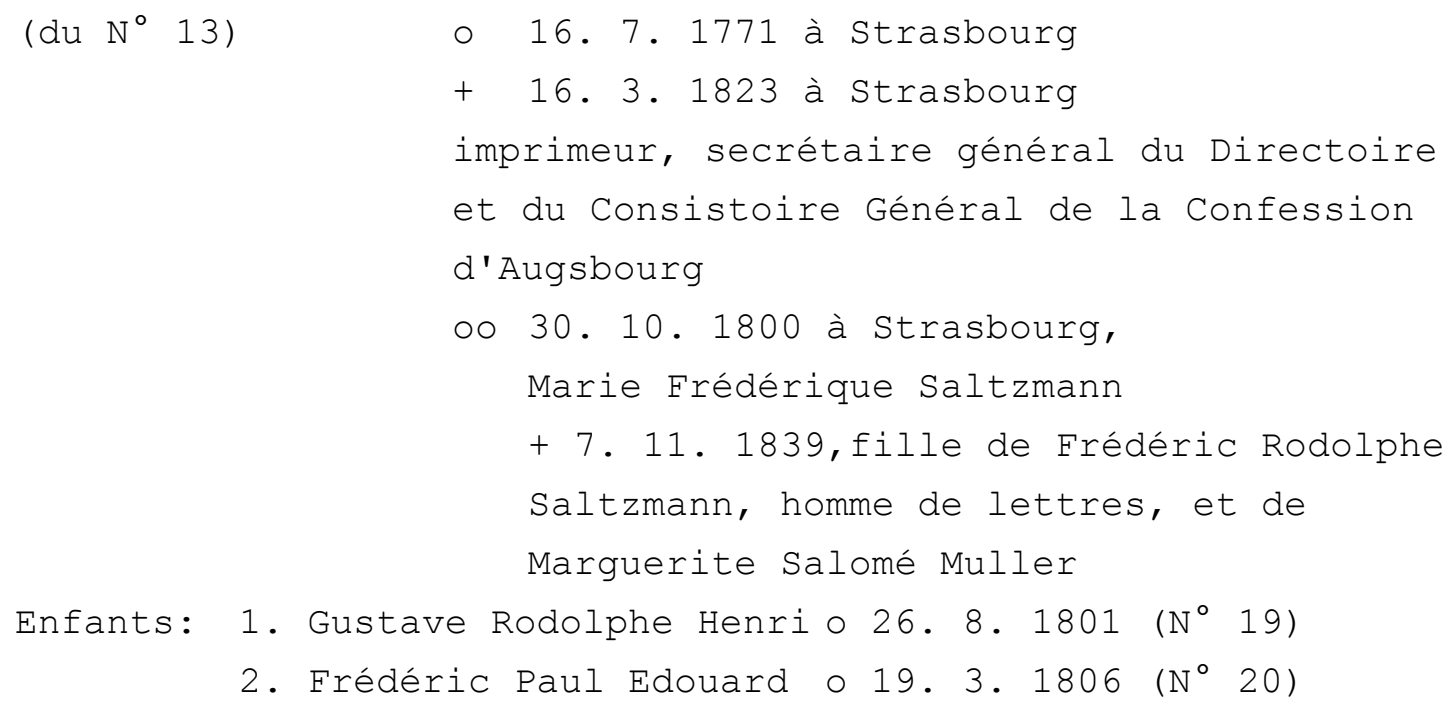

$$
\mathrm{N}^{\circ} 18 \text {. }
$$

Jean André Silbermann

$$
\begin{aligned}
\left(\mathrm{du} \mathrm{N}^{\circ}\right. \text { 14) } & 22.3 .1786 \text { à Strasbourg } \\
& \text { négociant à Paris, marchand de rubans } \\
& \text { ○o } 10.7 .1813 \text { à Strasbourg, Sophie Frédérique } \\
& \text { Graffenauer } 013.4 .1792, \\
& +12.9 .1865 \text { à Strasbourg }
\end{aligned}
$$


fille de Jean Daniel Graffenauer,

négociant, + 21.10.1805

et de Marguerite Elisabeth Redslob

Enfants: 1. 23. 2. 1815, enfant mort-né, du sexe féminin

2. Jean André Emile o 31.4-1816

3. Sophie Emilie

- 6.3.1818 à Strasbourg

+8.12 .1819 à Schiltigheim

4. 30.11.1819, enfant mort-né, du sexe féminin

$$
\mathrm{N}^{\circ} 19 .
$$

Gustave Rodolphe Henri Silbermann

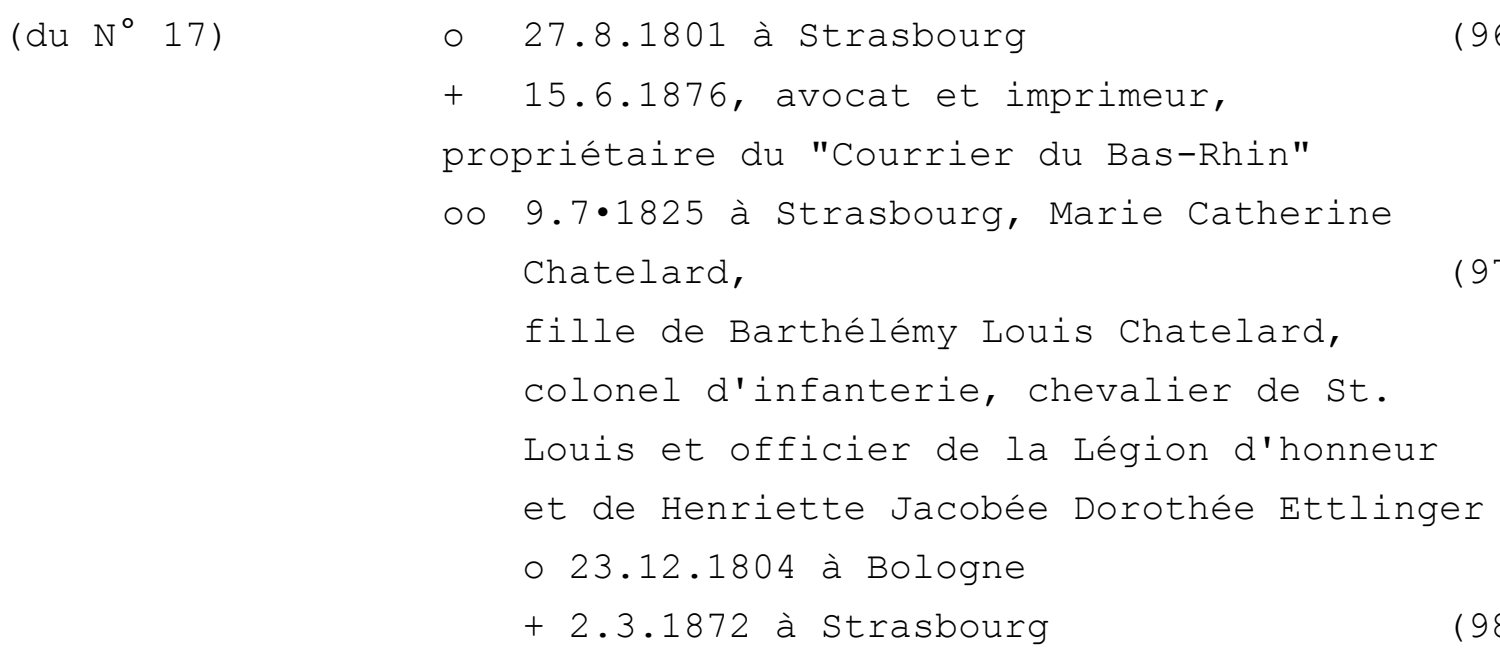

Enfants: 1. Frédérique Henriette Pauline

- 23.12.1826 à Strasbourg

+10.8 .1839 à Strasbourg

2. Marie Mathilde $\quad 13.2 .1834\left(\mathrm{~N}^{\circ} 21\right)$

3. Jenny Constance

$$
\begin{aligned}
& \text { - 1.3.1838 à Strasbourg } \\
& +25.2 .1840 \text { à Strasbourg }
\end{aligned}
$$

$$
\mathrm{N}^{\circ} 20 \text {. }
$$

Frédéric Paul Edouard Silbermann

$$
\begin{aligned}
\left(\mathrm{du} \mathrm{N} \mathrm{N}^{\circ}\right. \text { 17) } & \bigcirc 9.3 .1806 \text { à Strasbourg } \\
& +1878 \\
& \text { sous-préfet à St-Omer } \\
& \text { oo Camille Feray }
\end{aligned}
$$

Enfant: Camille Henriette Valentine 
Marie Mathilde Silbermann

(du $\mathrm{N}^{\circ}$ 19)

- 13.2.1834 à Strasbourg

+1921 à Paris

oo 2.7.1857 à Strasbourg, Edmond Henri David Hartung,

"chef d'escadron au corps d'état-major, employé au dépôt de la guerre, officier de la Légion d'hônneur, décoré de l'ordre du Médjidié et de la médaille de la reine d'Angleterre" o 19.8.1819 à Strasbourg fils de Chrétien Aloyse Henri Adam François Hartung, Dr. en médecine à Strasbourg, chevalier de la Légion d'honneur et de Marguerite Salomé Kusian à Strasbourg

Enfant: Marie oo Mallet dont la petite-fille, Madame Rosetti-Balanesco, est propriétaire des Archives Silbermann à Paris. 


\section{Généalogie $\mathrm{N}^{\circ} 1$.}

\section{$\underline{\text { Notes }}$}

(1) Werner Müller, Auf den Spuren Gottfried Silbermanns, Kassel et Bâle, 1968, p. 7 ss. En ce qui concerne la branche saxonne de la famille, on peut s'appuyer sur les recherches de $\mathrm{W}$. Müller.

(2) Pfarramt Frauenstein, Taufbeurkundung $N^{\circ} 17 / 1678$ (P.J. p. 223).

(3) AMS. M 53 fol. 113 a (P.J. p.224).

(4) AMS. D 93 fol. 187 a (P.J. p.244).

(5) AMS. N 111 fol. 217 b (P.J. p.225).

(6) AMS. D 94 fol. 5 b (P.J. p.226).

(7) AMS. N 111 fol. 297 a (P.J. p.228).

(8) AMS. D 93 fol. 60 b (P.J. p.229).

(9) AMS. N 111 fol. 332 b (P.J. p.230).

(10) AMS. D 93 fol. 74 a (P.J. p.232).

(11) AMS. N 112 fol. 22 b (P.J. p.231).

(12) AMS. N 112 fol. 55 b (P.J. p.233).

(13) AMS. D 93 fol. 90 a (P.J. p.234).

(14) AMS. N 112 fol. 99 a (P.J. p.235).

(15) AMS. N 94 fol. 19 b (P.J. p.236).

(16) AMS. N 112 fol. 129 b (P.J. p.237).

(17) AMS. D 94 fol. 23 a (P.J. p.238).

(18) AMS. D 96 fol. 115 b (P.J. p.251).

(19) AMS. N 112 fol. 161 b (P.J. p.239).

(20) AMS. D 93 fol. 110 b (P.J. p.240).

(21) AMS. N 112 fol. 236 a (P.J. p.241).

(22) AMS. D 93 fol. 153 a (P.J. p.243).

(23) AMS. N 112 fol. 276 b (P.J. p.242).

(24) AMS. N 111 fol. 176 a.

(25) AMS. D 96 fol. 106 a.

(26) AMS. D 204 fol. 62 a.

(27) AMS. M 122 fol. 221 b.

(28) AMS. N 254 fol. 102 b.

(29) AMS. N 254 fol. 124 b.

(30) AMS. N 254 fol. 301 a.

(31) AMS. M 54 fol. 115 a. et M 123 fol. 79 a.

(32) AMS. D 96 fol. 110 a.

(33) AMS. N 111 fol. 252 a (P.J. p.227). La date de naissance est indiquée dans l'acte de décès. Elle figure en outre, de la 
main de Jean André Silbermann, au revers du portrait dessiné par F.B.Frey en 1742. La date du baptême, 26 juin, figure à tort comme date de naissance au bas de la gravure de Guérin, publiée par Friese en 1791. Cette erreur a été reprise par tous les auteurs.

(34) AMS. M 114 fol. 211 a (P.J. p.245). Le voyage qu'effectua Jean André Silbermann en Saxe au printemps 1741 ne fut donc pas un voyage de noces, comme le prétendent Mathias et Wörsching! Voir M. Schaefer, Jean André Silbermann, in SaintQuirin, Harmonia Mundi 1965, p. 24.

(35) AMS. D 99 fol. 12 b (P.J. p.254).

(36) AMS. N 114 p. 24

(37) AMS. D 95 fol. 68 a.

(38) AMS. N 114 fol. 109 a (P.J. p.246).

(39) AMS. D 99 fol. 33 a.

(40) AMS. N 114 fol. 287 a.

(41) AMS. D 96 fol. 38 b.

(42) AMS. N 114 fol. 360 b.

(43) AMS, D 96 fol. 99 b.

(44) AMS. N 114 fol. 445 a (P.J. p.249).

(45) AMS. N 114 p. 527

(46) AMS. D 98 fol. 39 b.

(47) AMS. N 115 p.106

(48) AMS. D 96 fol. 86 a.

(49) AMS. N $115 \mathrm{p} \cdot 178$

(50) AMS. D 96 fol. 116 a.

(51) AMS. N 115 p. 463 (P.J. p.252).

(52) AMS. D 207 fol. 203 a (P.J. p.257).

(53) AMS. M 55 fol. 124 b. et M 123 fol. 222 b (P.J. p.255).

(54) AMS. Décès 1808

(55) AMS. D 207 fol. 158 a (P.J. p.256).

(56) AMS. N 112 fol. 22 b (P.J. p.231).

(57) Leipzig, Ratsleichenbuch der Stadt Leipzig, vol. 30 fol. 308 b (P.J. p.253).

(58) AMS. M 54 fol. 90 a (P.J. p.247).

(59) AMS. D 96 fol. 41 a (P.J. p.248).

(60) AMS. N 114 fol. 293 a.

(61) AMS. D 95 fol. 103 a.

(62) Wöchentliche Nachrichten und Anmerkungen die Musik betreffend, Leipzig 1766.

(63) Leipzig, Taufregister der St.-Thomaskirchgemeinde Leipzig, $1745, \mathrm{p} \cdot 52$ b. $\mathrm{N}^{\circ} 444$. 
(64) AMS. N 112 fol. 276 b (P.J. p.242).

(65) AMS. Décès an VII, $N^{\circ} 366$ (P.J. p.258).

(66) AMS. M 54 fol. 143 b.

(67) AMS. Décès an IV, $N^{\circ} 652$

(68) AMS. N 230 fol. 531 a.

(69) AMS. D 206 p. 53

(70) AMS. N 255 p. 137

(71) AMS. Décès an XIII, fol. 181

(72) AMS. N 114 fol. 445 a (P.J. p.249).

(73) AMS. Décès an II, $N^{\circ} 2689$

(74) AMS. M 124 fol. 107

(75) AMS. N 256 p. 681

(76) AMS. N 258 fol. 1 b.

(77) AMS. N 258 fol. 20 a.

(78) AMS. Décès 1816, $\mathrm{N}^{\circ} 867$

(79) AMS. N 230 fol. 421 a (P.J. p.250).

(80) AMS. Décès 1833, $\mathrm{N}^{\circ} 809$

(81) AMS. M 124 fol. 112 b.

(82) Bopp, Die evangelischen Geistlichen, $\mathrm{N}^{\circ} 3653$

(83) AMS. N 255, p. 497

(84) AMS. Décès 1829, $\mathrm{N}^{\circ} 2139$

(85) AMS. Mariages an II, $\mathrm{n}^{\circ} 1215$

(86) Généalogie de la famille Koehler à Paris.

(87) AMS. N 255 P. 671

(88) AMS. Décès 1823, $\mathrm{N}^{\circ} 426$

(89) AMS. Mariages an IX, $\mathrm{N}^{\circ} 83$

(90) AMS. N 256 fol. 381 b.

(91) AMS. Mariages 1813, $\mathrm{N}^{\circ} 233$

(92) AMS. Décès 1865, N 1856

(93) AMS. Décès 1815, $\mathrm{N}^{\circ} 326$

(94) AMS. Naissances 1816, $\mathrm{N}^{\circ} 834$

(95) AMS. Décès 1819, $\mathrm{N}^{\circ} 1695$

(96) AMS. Naissances an IX, N ${ }^{\circ} 2020$

(97) AMS. Mariages 1825, $\mathrm{N}^{\circ} 256$

(98) AMS. Décès 1872, $\mathrm{N}^{\circ} 478$

(99) AIS. Naissances 1826, $\mathrm{N}^{\circ} 1960$

(100) AMS. Décès 1839, $\mathrm{N}^{\circ} 1584$

(101) AMS. Naissances 1838, $N^{\circ} 356$

(102) AMS. Décès 1840, $\mathrm{N}^{\circ} 4 \mathrm{ft}+1$

(103) AMS. Naissances 1806, $\mathrm{N}^{\circ} 972$

(104) AMS. Naissances 1833, $\mathrm{N}^{\circ} 1482$

(105) AMS. Naissances 1834, $\mathrm{N}^{\circ} 263$

(106) AMS. Mariages 1857, $\mathrm{N}^{\circ} 301$. 
Généalogie $N^{\circ} 2$.

Les ancêtres du facteur d'orques Xavier Silbermann à Thonon.

Lorsqu'en 1699 André Silbermann arrive en Alsace, il existe sur place au moins deux familles qui portent le nom de Silbermann, l'une dans la région de Haguenau, l'autre en Haute-Alsace. Le hasard veut qu'en suivant la lignée haguenovienne jusqu'à nos jours, l'on aboutisse à un facteur d'orgues, M. Xavier Silbermann, établi à Thonon. L'état actuel de nos recherches ne permet pas de conclure à une parenté avec la famille issue de Saxe.

Par souci de clarté nous avons choisi la présentation descendante, sélective et agnatique, pour cette généalogie.

$$
\mathrm{N}^{\circ} 1
$$

Georges Silbermann

boulanger à Haguenau

oo Anne

(1)

$$
\mathrm{N}^{\circ} 2
$$

Jean Adam Silbermann

boulanger et meunier à Haguenau

b. 9. 10. 1623 à Haguenau

oo 1) Dorothée ca. 1656

oo 2) Anne Marguerite ca. 1669

$$
\mathrm{N}^{\circ} 3
$$

Jean Adam Silbermann

chirurgien à Oberbronn

b. 18.10.1675 à Haguenau

+ 8.8.1730 à Oberbronn

oo 1) Marie Salomé Hoffmann

+ 26.2.1727 à Oberbronn

oo 2) 16.8.1728 Marie Anne Hön de Landau

oo 3) 9.10.1729 Marguerite Latour de Reichshoffen 


$$
\mathrm{N}^{\circ} 4
$$

François Joseph Silbermann

chirurgien à Oberbronn, procureur fiscal

bailliage d'oberbronn et Niederbronn

○ 5.4. 1706 à Oberbronn

+ 12. 5. 1770 à Oberbronn

oo Marie Eve Weis 9. 4. 1731 à Dauendorf

$$
\mathrm{N}^{\circ} 5 \text {. }
$$

Philippe Joseph Silbermann

"scriniarius" à Oberbronn

b. 10. 3. 1737 à Oberbronn

oo 1) 20. 5. 1765 Marie Madeleine Schmitt

à Oberbronn

oo 2) 15. 1. 1770 Anne Elisabeth Schad

à Oberbronn

$$
N^{\circ} 6 \text {. }
$$

François Antoine Silbermann

tailleur à Heiligenberg

- 27. 5. 1772 à Reichshoffen

+ 6. 4. 1849 à Heiligenberg

oo 14. 4. 1798 Catherine Munsch à Heiligenberg

$$
\mathrm{N}^{\circ} 7 \text {. }
$$

François Joseph Silbermann

tailleur à Heiligenberg

- 19. 4. 1804 à Heiligenberg

oo Marie Anne Diebinger

$$
\mathrm{N}^{\circ} 8
$$

Didier Silbermann

$$
\begin{aligned}
& \text { ○ 27. 3. } 1834 \text { à Heiligenberg } \\
& \text { oo Marie Anne Brandner }
\end{aligned}
$$




$$
\mathrm{N}^{\circ} 9 .
$$

François Joseph Silbermann

$$
\begin{aligned}
& \text { tailleur à Heiligenberg } \\
& \text { ○ 17. 7. } 1871 \text { à Dinsheim } \\
& \text { oo Marie Elchinger }
\end{aligned}
$$

$$
\mathrm{N}^{\circ} 10 \text {. }
$$

Joseph Simon Silbermann

$$
\begin{aligned}
& \text { - 20. 10. } 1907 \text { à Dinsheim } \\
& +\quad 26 \cdot 5 \cdot 1970 \text { à Hoerdt }
\end{aligned}
$$

$$
\mathrm{N}^{\circ} 11 \text {. }
$$

Xavier Silbermann

facteur d'orgues à Thonon

- 23. 5. 1934 à Mutzig

oo 23. 4. 1964 Marie-Rose Fastenrath

à Obernai

$$
\mathrm{N}^{\circ} 12 \text {. }
$$

Christian Silbermann

- 2. 6. 1971

à Thonon 


\section{Généalogie $\mathrm{N}^{\circ} 2$.}

\section{$\underline{\text { Notes }}$}

(1) AMH. GG 79, $\mathrm{N}^{\circ} 1031$.

(2) AMH. GG 109, $\mathrm{N}^{\circ} 541$.

(3) ABR. 3 E 340/13, fol. 29 b.

(4) ABR. 3 E 340/13, fol. 24 a.

(5) ABR. 3 E 340/13, p. 34.

(6) ABR. 3 E 340/13, fol. 28 .

(7) ABR. 3 E 340/13, fol. 40 a.

(8) ABR. 3 E 340/13, fol. 6 b.

(9) ABR. 3 E 340/20, p. 115.

(10) ABR. 3 E 86/7, p. 129.

(11) ABR. 3 E 340/14, p. 53.

(12) ABR. 3 E 340/19, p. 2

(13) ABR. 3 E 340/19, p. 61.

(14) Mairie de Heiligenberg, Actes de décès 1849.

(15) Mairie de Heiligenberg, Actes de mariages An VI.

(16) Mairie de Heiligenberg, Actes de naissances An XII.

(17) Mairie de Heiligenberg, Actes de naissances 1834.

(18) Mairie de Dinsheim, Actes de naissances 1871.

(19) Mairie de Strasbourg, Décès 1970 (transcription). 
Généalogie $\mathrm{N}^{\circ} 3$.

La lignée haut-rhinoise originaire de Burnhaupt-le-Bas.

Selon Mathias (1) cette famille, établie depuis le milieu du $17^{\circ}$ siècle dans le Haut-Rhin, serait issue de Saxe, tout comme nos facteurs d'orgues. "Dès la guerre de 30 ans - écrit-il - Georges Silbermann, sans doute grand-oncle d'André arriva en Alsace comme capitaine de cavalerie lourde de Saxe. En 1648 il passa à la religion catholique, épousa une Alsacienne d'origine suisse et s'établit à Burnhaupt-le-Bas (Haut-Rhin)."

Cette affirmation repose-t-elle sur des données sûres? Les registres paroissiaux de Burnhaupt-le-Bas (2) ne comportent aucun mariage pour la période 1630 à 1673. Par contre en 1646 on note le baptême de Georges, fils de Georges Silbermann et d'Anne Bauchler, sans aucune autre indication. C'est là le premier acte concernant la famille Silbermann inscrit dans les registres de Burnhaupt-le-Bas et il faut continuer jusqu'en 1672 pour trouver le deuxième. Il s'agit du baptême d'une fille de Georges Silbermann et de Marguerite Peff.

De ces indications il résulte que Georges Silbermann était catholique dès avant 1648. Il n'est pas établi que sa femme ait été une Alsacienne d'origine suisse. Son mariage ne se situe pas en 1648, mais avant 1646 .

Qu'en est-il des fonctions militaires de Georges Silbermann? La fonction d'officier de cavalerie (Rittmeister), était attribuée, à l'époque qui nous intéresse, en priorité à des officiers d'origine noble. Les listes des officiers de l'armée saxonne sont conservées à partir de 1616 au Staatsarchiv de Dresde. Elles ne contiennent aucun officier du nom de Silbermann. Il est donc possible d'affirmer, les documents d'archives étant dignes de foi, que Georges Silbermann n'a pas été officier ou capitaine de cavalerie de l'armée de Saxe.

Il faut donc mettre en doute le lien de parenté qui unirait la famille Silbermann du Haut-Rhin à celle issue de Saxe et comprenant les facteurs d'orgues. Cette lignée haut-rhinoise a d'ailleurs été florissante et ne comporte pas moins de onze générations.

Les membres de cette famille sont à chercher à Burnhaupt-le-Bas, Spechbach-le-Haut, Soultz, Dannemarie, Mulhouse, Delle, Wettolsheim, Sentheim. 


\section{Généalogie $N^{\circ} 3$.}

\section{$\underline{\text { Notes }}$}

(1) F.X. Mathias, Compte-Rendu du Congrès d'orgue ..., Strasbourg 1934, p. 245

(2) AHR, Registres paroissiaux de Burnhaupt-le-Bas. Les baptêmes manquent entre 1631 et 1639, et les décès entre 1631 et 1673 . Communication de M. Chr. Wilsdorf en date du 8.11.1974. 
Documents iconographiques concernant la famille Silbermann.

Si aucun document ne nous transmet les traits d'André ou de Gottfried Silbermann, nous avons la chance de posséder des portraits de plusieurs autres membres de la famille, notamment de Jean André et de son frère Jean Henri.

1.) Jean André Silbermann. Pastel de François Bernardin Frey, peintre originaire de Guebwiller (1716-1806). Buste de trois quarts à gauche, porte habit et gilet noirs, et tient un tricorne noir galonné d'or sous le bras gauche. Fond bleu, à gauche une colonne. Hauteur $59 \mathrm{~cm}$, largeur $45 \mathrm{~cm}$.

Au revers, inscription de la main du modèle:

Johann Andreas Silbermann nat. 1712. den 24 Juny

Aetatis 30 anos.

Le portrait fut donc réalisé en 1742, année de mariage de Jean André Silbermann. Il se trouve, de même que le suivant, au Musée des Beaux-Arts de la Ville de Strasbourg. (1)

2.) Anne Salomé Mannberger (1725-1769), première femme de Jean André Silbermann. Pastel de F.B.Frey. Buste de trois quarts à droite. Elle porte un corsage de soie bleu ciel, broché de grosses fleurs polychromes. Un fichu de dentelle fermé devant par trois roses. Au cou, collier d'or à six rangs et ruban noir avec croix. Fond bleu, à gauche un buisson. Hauteur $59 \mathrm{~cm}$, largeur $45 \mathrm{~cm}$. Au revers, inscription de la main de Jean André Silbermann:

Anna Salomea Silbermännin. Johann Andreas Silbermanns Ehegeliebste, war gebohren hier in Strassburg den 14. November 1725. Verheurathet den 22. August 1742. Starb zu meinem grössten Leidwesen den 3. May 1769.

3.) Jean André Silbermann. Gravure de Christophe Guérin, graveur strasbourgeois, d'après Jean-Georges Tanisch, peintre allemand établi à Strasbourg en 1751, et qui francisa son nom en Daniche (1705-1775) (2). Buste de trois quarts, portant habit et gilet. La gravure fut publiée par J. Friese, Neue Vaterländische Geschichte der Stadt Strassburg, Strasbourg, chez Lorenz et Schuler, 1791. Inscriptions:

Daniche filius pinxit. C. Guerin sculpsit. 
JOH. ANDREAS SILBERMANN.

Orgelbauer und Alterthums Forscher

geb. zu Strasburg den 26 Jun. 1712.

gestorben den 11 Febr. 1783.

Dimensions de la plaque: Hauteur 17,1 cm. Largeur: 9,9 cm.

Dimensions du médaillon: $9,8 \mathrm{~cm} \times 8 \mathrm{~cm}$.

Le dessin a dû être fait au plus tard en 1775, année de décès de Daniche.

4.) Jean Henri Silbermann (1727-1799). Portrait par Daniche, 1775. Buste de trois quarts à droite.

Au revers, inscription de la main du modèle:

Dieses Portrait ist 1775 im Monat Sept.

von Herrn Tanisch älterer Sohn, im 48.ten

Jahr meines Alters gemahlt worden.

Propriété privée.

5.) Catherine Marguerite Mosseder (1736-1796), femme de Jean Henri

Silbermann. Portrait par Mademoiselle Daniche, 1775. Buste de trois quarts à gauche.

Au revers, inscription de la main de Jean Henri Silbermann:

Dieses Porträt meiner Frau ist 1775 im Monat Oktober von Mademoiselle Tanisch im 39.ten Jahr ihres Alters gemahlt worden.

Propriété privée.

6.) Jean Jacques Strohl (1767-1833), époux de Frédérique Madeleine Silbermann. Portrait anonyme. Buste de trois quarts à gauche. Propriété privée.

7.) Le même. Portrait de profil, en uniforme. Propriété privée.

8.) Frédérique Madeleine Silbermann (1768-1829), épouse de Jean Jacques Strohl. Portrait anonyme. Buste de trois quarts à gauche. Propriété privée. 
Le texte suivant, tiré des Archives de la Fondation St-Thomas à Strasbourg, nous a fait entrevoir un instant la possibilité de découvrir un masque mortuaire de Jean André Silbermann. En 1837 Madame veuve Silbermann fait don au Séminaire Protestant du "masque de feu M. Jean André Silbermann auteur de la topographie historique de la ville de Strasbourg". (3) Or la veuve de Jean André Silbermann, Anne Elisabeth Schatz, est décédée en 1808. Il s'agit certainement de la veuve de Jean Henri Silbermann, Secrétaire Général du Directoire de l'Eglise de la Confession d'Augsbourg, décédé en 1823. Ce qui n'empêche que le masque en question ait bien été celui de Jean André. Quoi qu'il en soit, sa trace est aujourd'hui perdue. 
(1) Musée des Beaux-Arts de la Ville de Strasbourg. Catalogue des peintures anciennes, p. 204. $\mathrm{N}^{\circ} 395$ et 396. Ancien fonds, provenance inconnue, Inv. $n^{\circ} 6$ et 5 . Des copies de ces deux portraits se trouvent en propriété privée, à Paris.

(2) Hans Haug, L'Art en Alsace, Paris 1962, p. 197.

(3) Arch. de la Fondation St-Thomas. Procès-verbaux du Séminaire Protestant. 1836-1843. Séance du 14 décembre 1837, p. 960. 
Répertoire chronologique des orgues construits par André

\section{Silbermann.}

1. Strasbourg, Couvent Sainte-Marguerite

1703

2. Strasbourg, Collegium Wilhelmitanum, Positif $\mathrm{N}^{\circ}$ I 1706

3. Strasbourg, église protestante Saint-Nicolas

1707

4. Strasbourg, église protestante Saint-Pierre-le-Vieux

1709

5. Marmoutier, église abbatiale

6. Bâle, cathédrale

1711

7. Bâle, église protestante Saint-Pierre

1712

8. Obernai, église catholique Saints-Pierre-et-Paul

9. Geudertheim, église simultanée

10. Strasbourg, cathédrale

11. Strasbourg, abbaye Saint-Etienne

12. Strasbourg, Madame d'Andlau, Positif $N^{\circ}$ II

13. Strasbourg, Monsieur Keck, Positif $\mathrm{N}^{\circ} \mathrm{V}$

14. Strasbourg, église protestante Sainte-Aurélie

15. Strasbourg, couvent Sainte-Madeleine

16. Bâle, église protestante Saint-Léonard

17. Strasbourg, Monsieur Vigera, Positif $\mathrm{N}^{\circ}$ VI

18. Strasbourg, église protestante Saint-Pierre-le-Jeune, Positif $\mathrm{N}^{\circ}$ IV

19. Marmoutier, église abbatiale, Positif $\mathrm{N}^{\circ}$ IX

20. Wissembourg, église simultanée Saint-Jean

21. Saint-Léonard, collégiale

22. Kolbsheim, Madame Linck, Positif $\mathrm{N}^{\circ}$ III

23. Altenheim, église protestante

24. Bischwiller, église protestante

25. Strasbourg, couvent de la Toussaint, Positif $\mathrm{N}^{\circ}$ VII V. 1725 
26. Colmar, église des Dominicains

1726

27. Strasbourg, église protestante Saint-Guillaume

1728

28. Vieux-Thann, couvent des Dominicaines

1729

29. Altorf, église abbatiale

1730

30. Ebersmunster, église abbatiale

31. Colmar, église protestante Saint-Matthieu

1732

32. Koenigsbruck, couvent des Cisterciennes

1732

33. Saverne, Château des Rohan, Positif $N^{\circ}$ VIII

V. 1733

34. Rosheim, église catholique Saints-Pierre-et-Paul

1733 
Répertoire chronologique des orgues construits par Jean André Silbermann.

1. Muhlbach, église simultanée

2. Strasbourg, église protestante Saint-Thomas, Positif

3. Barr, église simultanée, Positif

4. Colmar, couvent des Dominicaines d'Unterlinden

5. Barr, église simultanée

6. Marbach, couvent des Augustins

7. Strasbourg, église protestante Saint-Thomas

8. Ensisheim, église catholique

9. Strasbourg, couvent de la Toussaint

10. Guebwiller, église des Dominicains

11. Saint-Quirin, église du Prieuré

12. Bischoffsheim, église catholique

13. Guémar, église catholique

14. Saint-Jean-Saverne, église abbatiale

15. Balbronn, église simultanée

16. Strasbourg, église protestante du Temple-Neuf

17. Soultz, église catholique

18. Sélestat, couvent des Dominicaines de Sylo

19. Sélestat, couvent des Franciscains

20. Muttersholtz, église simultanée

21. Alspach, couvent des Clarisses

22. Villingen, église abbatiale Saint-Georges

23. Baden-Baden, collégiale

24. Baden-Baden, collégiale, orgue de choeur

25. Woerth, église simultanée

26. Colmar, collégiale Saint-Martin 
28. Offendorf, église catholique

29. Kehl, église du village

1758

30. Amtenhausen, église abbatiale

31. Scherwiller, église catholique

32. Rosheim, église catholique Saint-Etienne

33. Hipsheim, chapelle du pèlerinage Saint-Ludan

34. Arlesheim, collégiale

35. Strasbourg, église catholique Saint-Pierre-le-Jeune

36. Strasbourg, église catholique Saint-Jean

37. Châtenois, église catholique

38. Wissembourg, église protestante Saint-Michel

39. Mulhouse, église protestante Saint-Etienne

40. Bettenhoffen, église catholique

41. Bâle, église française Saint-Jean (Predigerkirche)

42. Sélestat, église catholique Saint-Georges

43. Ettenheimmunster, abbaye bénédictine

44. Bâle, église protestante Saint-Théodore

45. Riegel, église catholique

46. Guebwiller, couvent des Dominicaines d'Engelporthen

47. Colmar, couvent des Catherinettes

48. Sankt-Blasien, abbaye bénédictine

49. Meissenheim, église protestante

50. Sankt-Märgen, collégiale

51. Bouxwiller, église protestante

52. Offenburg, couvent des Franciscains

53. Blodelsheim, église catholique

54. Strasbourg, église protestante Saint-Pierre-le-Jeune

55. Molsheim, église des Jésuites

56. Gries, église simultanée

57. Lahr, collégiale 
Répartition confessionnelle des orques construits par André et Jean André Silbermann.

Les Silbermann ont construit des orgues aussi bien pour des églises luthériennes que pour des abbayes bénédictines. Les deux instruments les plus importants et les plus célèbres construits par Jean André Silbermann furent ceux du Temple-Neuf à Strasbourg et de l'abbaye bénédictine de Sankt-Blasien en Forêt-Noire. En ce qui concerne la structure et la composition des orgues des Silbermann, nous pouvons affirmer qu'elles sont indépendantes de leur destination confessionnelle. Elles varient en fonction d'autres critères. Les considérations économiques paraissent déterminantes; elles sont ensuite nuancées par des aspects proprement musicaux.

Ces deux critères sont d'ailleurs très liés, car un édifice important du point de vue acoustique et musical est déjà le fait d'une situation économique donnée, selon l'importance de telle paroisse ou de telle abbaye.

L'importance de la décoration d'un buffet d'orgue peut aussi être le résultat d'un souhait particulier, comme ce fut le cas à l'église Saint-Thomas à Strasbourg.

La présence de tel ou tel registre dans une composition peut être liée à une certaine pratique musicale, soit l'accompagnement du chant d'assemblée, soit l'accompagnement de cantates. A Mulhouse Jean André Silbermann indique que le jeu d'anches de 16 pieds à la Pédale sera soit une Bombarde soit une Posaune, en fonction de l'intensité du chant de l'assemblée. Au Temple-Neuf à Strasbourg, le jeu de Basson 8 placé en 1756 à l'Oberwerk permettra à la paroise de licencier le bassoniste de l'orchestre ...

Les Silbermann ont été appelés à construire des orgues aussi bien "catholiques" que "protestants" en fonction de leur réputation de qualité. Bien entendu, l'argument confessionnel était parfois invoqué dans le jeu de la concurrence. Notamment Rohrer et Waltrin semblent avoir fait valoir le fait qu'ils étaient catholiques pour obtenir des commandes dans les abbayes. Jean André Silbermann rapporte le fait à propos d'Ebersmunster:

"... indeme dasige Herren weder einen Chatolicken noch einen Lutheraner begehrten, sondern einen guten Orgelmacher." (1) 
Numériquement, nous pouvons faire les constatations suivantes: André Silbermann a construit 16 orgues "catholiques" (y compris le Positif destiné au Cardinal de Rohan à Saverne). Deux projets non exécutés concernent des églises catholiques: Weingarten et Frauenalb. D'autre part il a construit 13 orgues "protestants" (compte non tenu de son intervention en 1708 au Temple-Neuf). Un orgue fut placé dans une église régie par le simultaneum (Wissembourg). Dans cette évaluation ne sont pas compris les quatre orgues d'appartement construits par André Silbermann.

Chez Jean André Silbermann le pourcentage d'orgues "catholiques" est plus élevé: 38 orgues "catholiques" contre 12 orgues "protestants." Sept instruments furent placés dans des églises où le simultaneum était en vigueur: Muhlbach, Barr (2 orgues), Balbronn, Muttersholtz, Woerth et Gries. Parmi les projets non exécutés, cinq concernent des églises catholiques, et deux des églises protestantes.

Les relations humaines entre les Silbermann, luthériens, et les autorités religieuses catholiques furent toujours excellentes, courtoises, voire amicales. A propos d'un séjour de Jean Henri et de Jean André Silbermann chez les Prémontrés du Mont-Sainte-Odile en 1750, ce dernier rapporte l'anecdote suivante:

"Es war diese zeit über so schön und angenehm Wetter wie am Sommer, derowegen hatte ich viele Freude mich in dieser Gegend recht umzusehen, und weilen ich unterschiedliche Zeichnungen machte, so wäre ich länger geblieben, wan nicht mein Bruder Heinrich villeicht von denen beständigen Fastenspeissen das Fieber bekommen hätte." (2)

En 1730 Jean André aide son père à installer chez les Soeurs Grises de Haguenau un Positif tout d'abord destiné à l'abbaye de Marmoutier. Il fait le commentaire que voici, au sujet de leur séjour à Haguenau:

"Die Zeit über als wir da arbeiteten speissten wir gemeiniglich im Convent an der Frau Mutter Priorin Tisch. Weilen sich die Closterfrauen flattirten unter denen vielen Orgeln die in Hagenau meist neu gemacht worden die beste zu haben, so waren sie recht bemühet uns auf das Beste aufzuwarten. Sie bedauerter nichts mehres als dass sie uns wegen der Fastenzeit mit nichts anders als Fastenspeisse aufwarten können. Es konten sich aber unsere lutherische Mägen gar wohl darein schücken." 
(1) Jean André Silbermann, ms. "Von Orgelmachern", p. 18. Notons que J. Wörsching, op. cit., p. 64, rapporte cette remarque à propos de la cathédrale de Strasbourg, au lieu de l'abbaye d'Ebersmunster. De plus, l'énoncé de la citation est erroné.

(2) Jean André Silbermann, ms. "Orgeln von Andreas Silbermann", p. 60 .

(3) Jean André Silbermann, ms. "Orgeln von Andreas Silbermann", p. 74 . 
Les orgues d'André Silbermann

Compositions, selon l'ordre chronologique 
Strasbourg, couvent Ste. Marguerite, 1703

Manual

Echo

Pédale

Tremblant doux

Tremblant fort

4 soufflets

ton de cornet
1. Bourdon 8

2. Prestant 4

3. Flûte 4

4. Gambe 4

5. Nazard $22 / 3$ basse/dessus

6. Doublette 2

7. Tierce $13 / 5$ basse/dessus

8. Cornet à partir de es'

9. Fourniture 3 rangs

10. Cromhorne 8 basse/dessus

Cornet d'Echo 5 rangs

Pédale d'une octave 8 ? 
Strasbourg. Positif $\mathrm{N}^{\circ}$ I (Collegium Wilhelmitanum), 1706

Manual

Tremblant doux

2 soufflets
1. Bourdon

2. Prestant

3. Nazard

4. Doublette

5. Tierce

6. Cornet 5 rangs

7. Fourniture 3 rangs

8. Trompette de récit
8

4

$22 / 3$

2

$13 / 5$

$f^{\prime}-C^{\prime}{ }^{\prime}$

$8 \mathrm{f}^{\prime}-\mathrm{C}^{\prime} \mathrm{I}^{\prime}$ 
Strasbourg, St-Nicolas, 1707

\begin{tabular}{|c|c|c|c|c|}
\hline Manual & 1. Montre & & 8 & \\
\hline & 2. Bourdon & & 8 & \\
\hline & 3. Viol di gamba & & 8 & \\
\hline & 4. Prestant & & 4 & \\
\hline & 5. Nazard & 2 & $2 / 3$ & \\
\hline & 6. Doublette & & 2 & \\
\hline & 7. Tierce & 1 & $3 / 5$ & \\
\hline & 8. Cornet 5 rangs & & & \\
\hline & 9. Fourniture 4 rangs & & & \\
\hline & 10. Cymbale 3 rangs & & & \\
\hline Positif & 1. Bourdon & & 8 & \\
\hline & 2. Prestant & & 4 & \\
\hline & 3. Nazard & 2 & $2 / 3$ & \\
\hline & 4. Doublette & & 2 & \\
\hline & 5. Tierce & 1 & $3 / 5$ & \\
\hline & 6. Fourniture 3 rangs & & & \\
\hline Pédale & 1. Soubasse ouverte & & 16 & sans tirant \\
\hline & 2. Octavebasse & & 8 & sans tirant \\
\hline
\end{tabular}

Tremblant doux

Tremblant fort

6 soufflets

ton de cornet 
Strasbourg, Temple-Neuf, nouvelle Pédale, 1708

$\begin{array}{llr}\text { Pédale } & \text { 1. Soubasse ouverte } & 16 \\ \text { C - C' } & \text { 2. Octavebasse } & 8 \\ \text { 3. Quinte } & 51 / 3 \\ \text { 4. Prestant } & 4 \\ \text { 5. Fourniture } 6 \text { rangs } & 22 / 3 \text { sans reprises } \\ \text { 6. Trompette } & 8 \text { en fer-blanc } \\ \text { 7. Clairon } & 4 \text { en fer-blanc }\end{array}$


Strasbourg, église protestante Saint-Pierre-le-Vieux, 1709

Manual

Positif

Pédale
1. Bourdon 16

2. Montre

3. Bourdon

4. Prestant

5. Nazard

6. Doublette

7. Tierce

8. Cornet 5 rangs

9. Fourniture 3 rangs

10. Cymbale 3 rangs

11. Trompette

1. Bourdon

2. Prestant

3. Nazard

4. Doublette

5. Tierce

6. Fourniture 3 rangs

1. Soubasse bouchée

2. Octavebasse

3. Prestant

4. Fourniture 4 rangs

5. Trompette

6. Clairon
16
8

8

4

$22 / 3$

2

$13 / 5$

$11 / 3$

8 placée en 1738 par J.A. Silbermann

Tremblant doux

Tremblant fort

4 soufflets

ton de cornet 
Marmoutier, église abbatiale, 1710

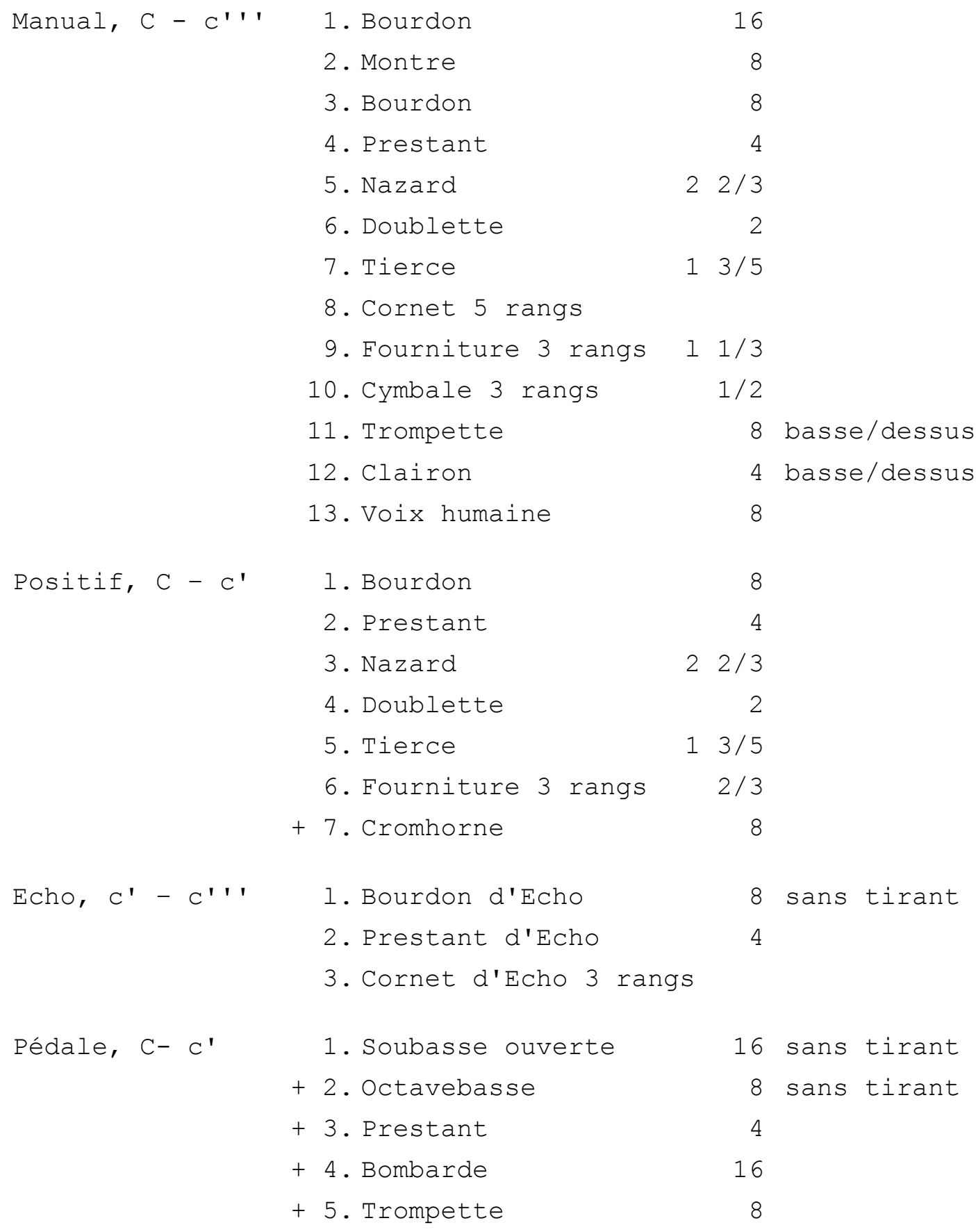

Tremblant doux

Tremblant fort

4 soufflets

ton d'opéra

+ jeux placés par J.A.Silbarmann en 1746 
Bâle, cathédrale, 1711

\begin{tabular}{|c|c|c|}
\hline Manual & 1. Montre & 8 \\
\hline \multirow[t]{9}{*}{$C D-C^{\prime}{ }^{\prime}$} & 2. Bourdon & 8 \\
\hline & 3. Prestant & 4 \\
\hline & 4. Nazard & $22 / 3$ \\
\hline & 5. Doublette & 2 \\
\hline & 6. Tierce & $13 / 5$ \\
\hline & 7. Fourniture 4 rangs & \\
\hline & 8. Cymbale 3 rangs & \\
\hline & 9. Trompette & 8 \\
\hline & 10. Voix humaine & 8 \\
\hline Positif & 1. Bourdon & 8 \\
\hline \multirow[t]{5}{*}{ C D- C''' } & 2. Prestant & 4 \\
\hline & 3. Nazard & $22 / 3$ \\
\hline & 4. Doublette & 2 \\
\hline & 5. Tierce & $13 / 5$ \\
\hline & 6. Fourniture 3 rangs & \\
\hline \multirow[t]{5}{*}{ Pédale } & 1. Montre & 16 à partir de $F$ \\
\hline & 2. Octavebasse & 8 \\
\hline & 3. Prestant & 4 \\
\hline & 4. Fourniture 4 rangs & \\
\hline & 5. Bombarde & 16 en bois \\
\hline
\end{tabular}


Bâle, église Saint-Pierre, 1712

\begin{tabular}{|c|c|c|c|}
\hline Manual & 1. Bourdon & & 16 \\
\hline \multirow[t]{11}{*}{$C D-C^{\prime}{ }^{\prime}$} & 2. Montre & & \\
\hline & 3. Bourdon & & \\
\hline & 4. Prestant & & \\
\hline & 5. Nazard & 2 & $2 / 3$ \\
\hline & 6. Doublette & & \\
\hline & 7. Tierce & 1 & $3 /$ \\
\hline & 8. Cornet 5 rangs & & \\
\hline & 9. Fourniture 3 rangs & & \\
\hline & 10. Cymbale 3 rangs & & \\
\hline & 11. Trompette & & \\
\hline & 12. Voix humaine & & \\
\hline Positif & 1. Bourdon 8 & & \\
\hline \multirow[t]{6}{*}{$C D-C^{\prime}{ }^{\prime}$} & 2. Prestant & & \\
\hline & 3. Nazard & 2 & $2 / 3$ \\
\hline & 4. Doublette & & \\
\hline & 5. Tierce & 1 & $3 / 5$ \\
\hline & 6. Fourniture 3 rangs & & \\
\hline & 7. Cromhorne & & \\
\hline
\end{tabular}

Echo, C' - C''' Cornet d'Echo 5 rangs

Pédale

1. Soubasse 16

2. Octavebasse 8

3. Prestant 4

4. Bombarde 16 en bois

5. Trompette 8

6. Clairon 4

Tremblant doux

Tremblant fort

3 soufflets

ton de choeur 
Obernai, église Saints-Pierre-et-Paul, 1713

Manual

1. Montre 8

2. Bourdon 8

3. Prestant 4

4. Flûte 4

5. Nazard $22 / 3$

6. Doublette 2

7. Tierce $13 / 5$

8. Cornet 5 rangs

9. Fourniture 3 rangs

10. Cymbale 3 rangs

11. Trompette 8

12. Voix humaine 8 prévue

Positif

1. Bourdon 8

2. Prestant 4

3. Nazard $22 / 3$

4. Doublette 2

5. Tierce $13 / 5$

6. Fourniture 3 rangs

7. Cromhorne

8 placé en 1784 par Josias Silbermann

Echo, C' - c''' Cornet d'Echo 5 rangs placé en 1784 par Josias Silbermann

Pédale

1. Soubasse

16

2. Octavebasse

8

3. Trompette 8

4. Clairon

4 prévu

Tremblant doux

Tremblant fort

ton de choeur 
Geudertheim, église simultanée, 1715

Manual

1. Bourdon 8

2. Prestant 4

3. Flûte 4

4. Nazard $22 / 3$

5. Doublette 2

6. Fourniture 3 rangs

7. Cymbale 3 rangs

Pédale, $\mathrm{C}-\mathrm{g}$

Soubasse

20 notes 
Strasbourg, cathédrale, 1714 - 1716

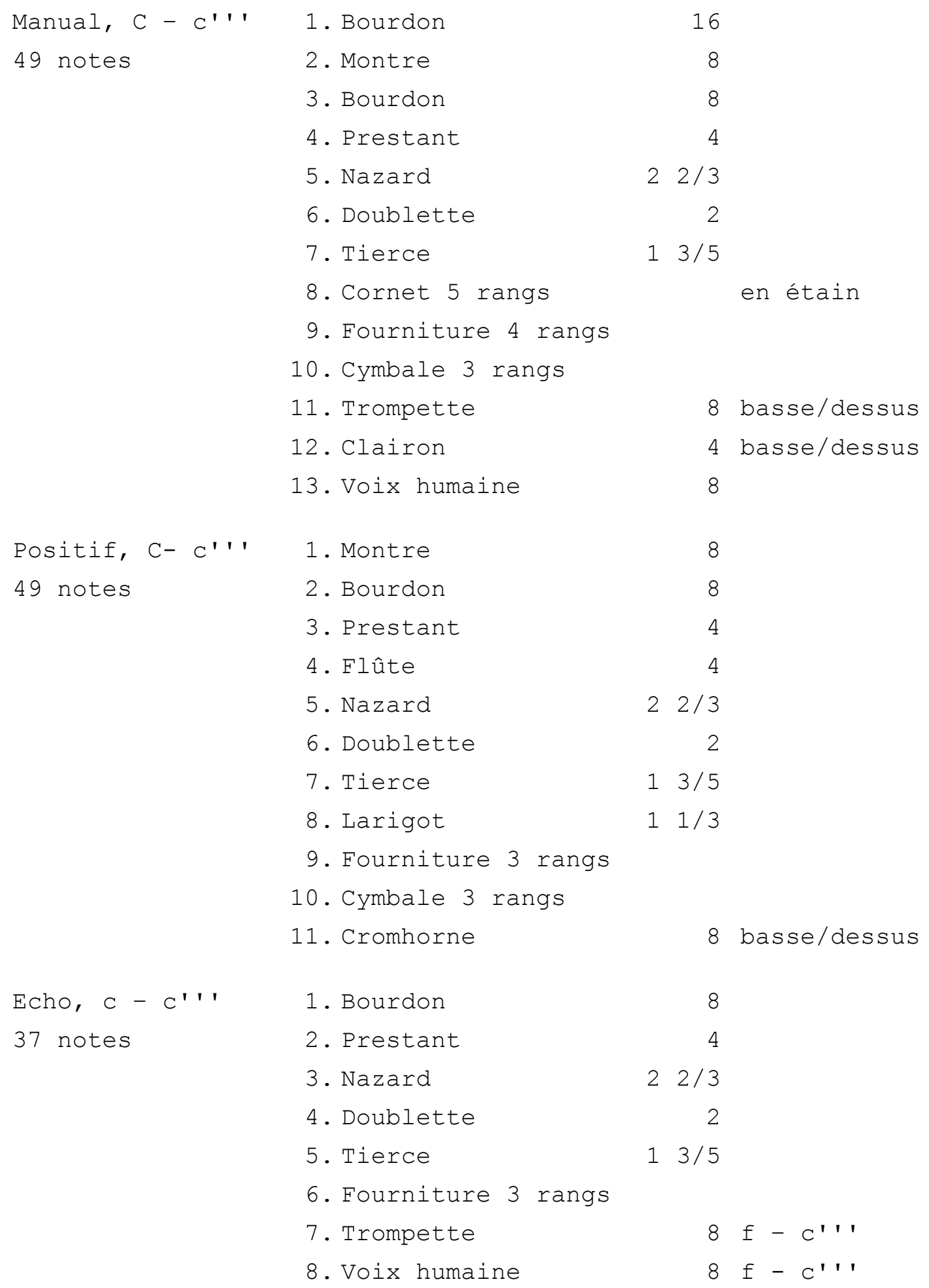




\begin{tabular}{|c|c|c|}
\hline \multirow{7}{*}{$\begin{array}{l}\text { Pédale, } C-C^{\prime} \\
25 \text { notes }\end{array}$} & 1. Montre & 16 en façade \\
\hline & 2. Soubasse & 16 bouchée \\
\hline & 3. Octavebasse & 8 en métal \\
\hline & 4. Prestant & 4 en métal \\
\hline & 5. Bombarde & 16 en étain \\
\hline & 6. Trompette & 8 \\
\hline & 7. Clairon & 4 \\
\hline
\end{tabular}

Tremblant doux

Tremblant fort

6 soufflets à un pli

ton d'opéra 
Strasbourg, Saint-Etienne, 1716

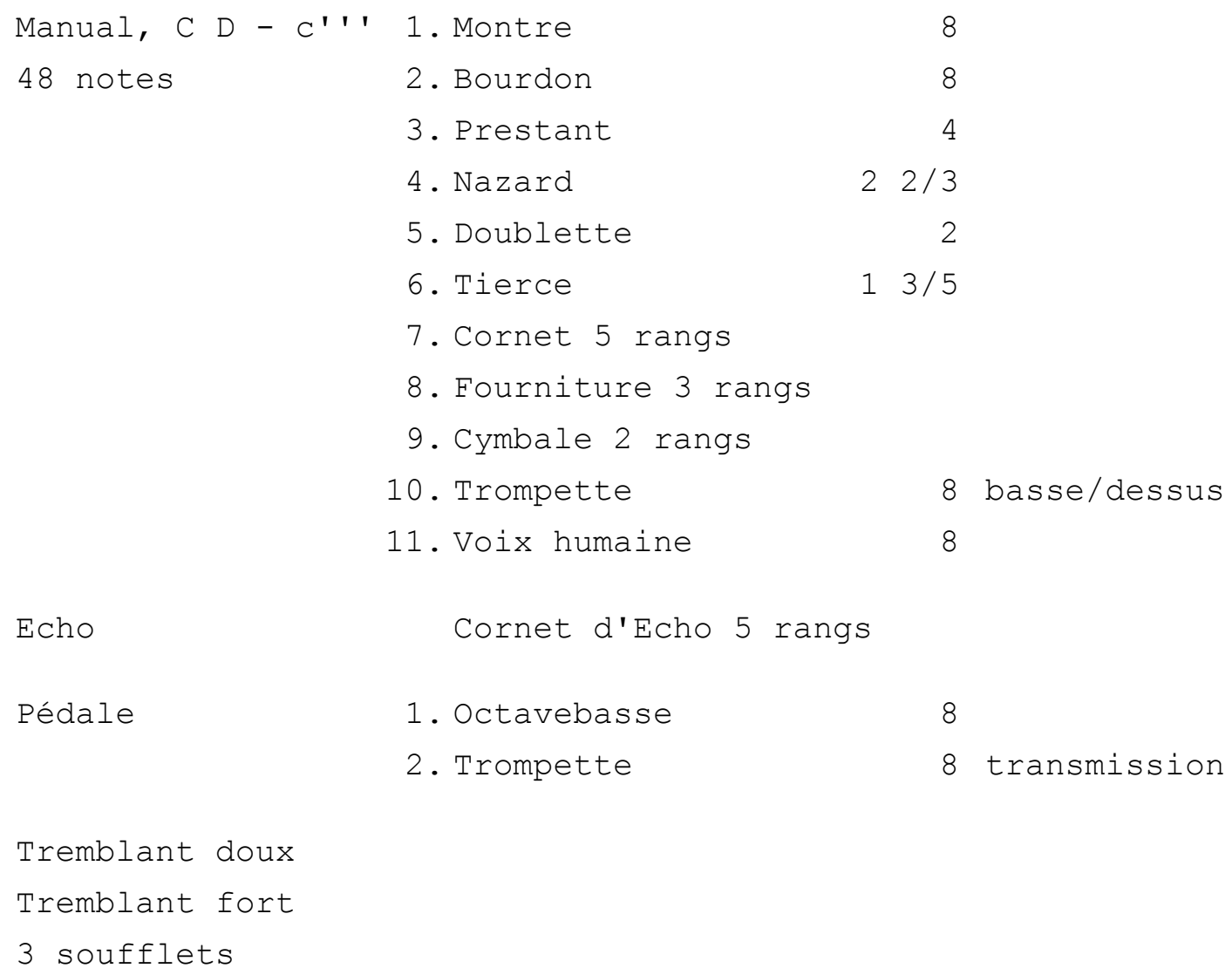


Strasbourg, Positif $\mathrm{N}^{\circ}$ II (Madame d'Andlau), 1717

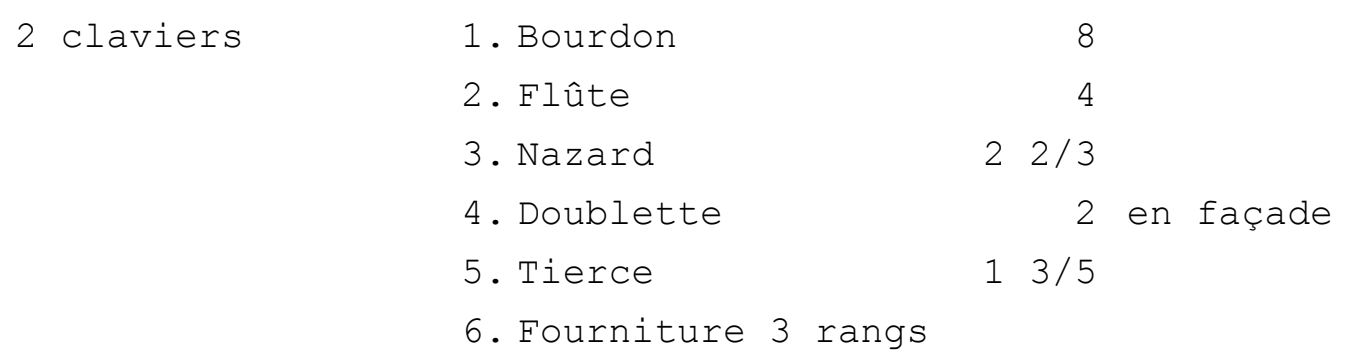


Strasbourg, Positif $\mathrm{N}^{\circ} \mathrm{V}$ (Brasseur Keck), V. 1718

Manual

1. Bourdon

8

2. Prestant

4

3. Doublette

2

4. Fourniture 3 rangs

Tremblant doux

2 soufflets

ton de Cornet 
Strasbourg, Sainte-Aurélie, 1718

\begin{tabular}{|c|c|c|c|}
\hline Manual, C - C''' & 1. Montre & 8 & \\
\hline 49 notes & 2. Bourdon & 8 & \\
\hline & 3. Prestant & 4 & \\
\hline & 4. F1ûte & 4 & \\
\hline & 5. Quinte & $22 / 3$ & \\
\hline & 6. Doublette & 2 & \\
\hline & 7. Tierce & $13 / 5$ & prévue \\
\hline & 8. Cornet 5 rangs & & \\
\hline & 9. Fourniture 3 rangs & & \\
\hline & 10. Cymbale 3 rangs & & \\
\hline & 11. Voix humaine & 8 & \\
\hline Positif, C - C''' & 1. Bourdon & 8 & \\
\hline 49 notes & 2. Prestant & 4 & \\
\hline & 3. Nazard & $22 / 3$ & prévue \\
\hline & 4. Doublette & 2 & \\
\hline & 5. Tierce & $13 / 5$ & prévue \\
\hline & 6. Fourniture & & \\
\hline Pédale & 1. Soubasse & 16 & \\
\hline & 2. Octavebasse & 8 & \\
\hline & 3. Trompette & 8 & \\
\hline Tremblant doux & & & \\
\hline 3 soufflets & & & \\
\hline tirasse fixe & & & \\
\hline on de Cornet & & & \\
\hline
\end{tabular}


Strasbourg, Sainte-Madeleine, 1718

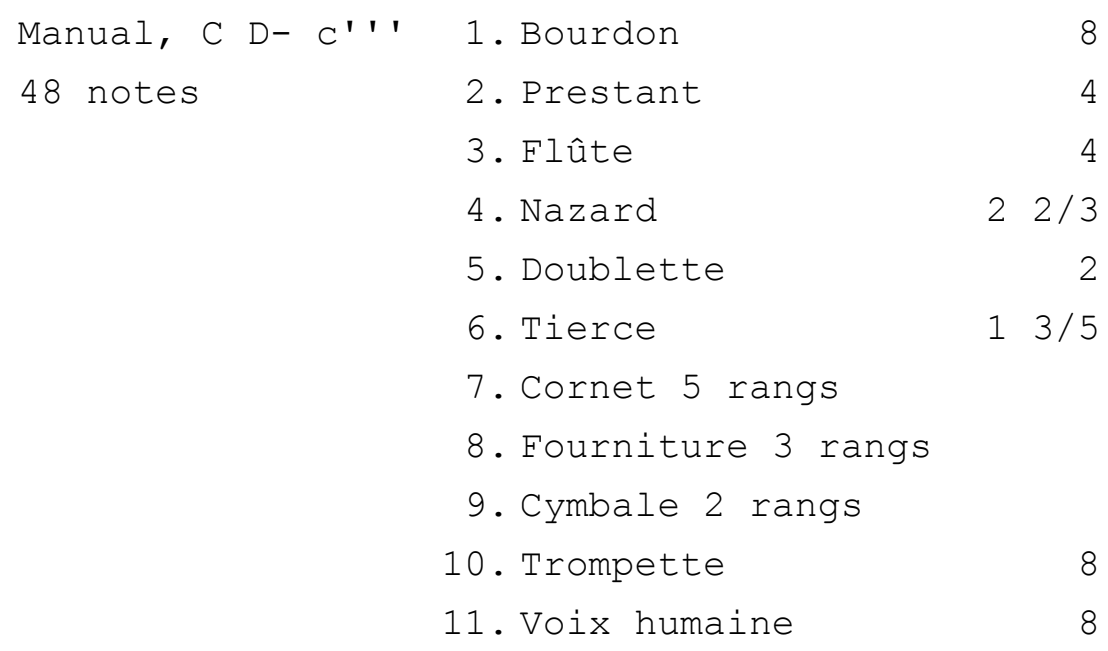

Echo Cornet d'Echo 5 rangs

Pédale, C D - 9

19 notes

2 soufflets
4

4

2

8

8 transm. 
Bâle, Saint-Léonard, 1718

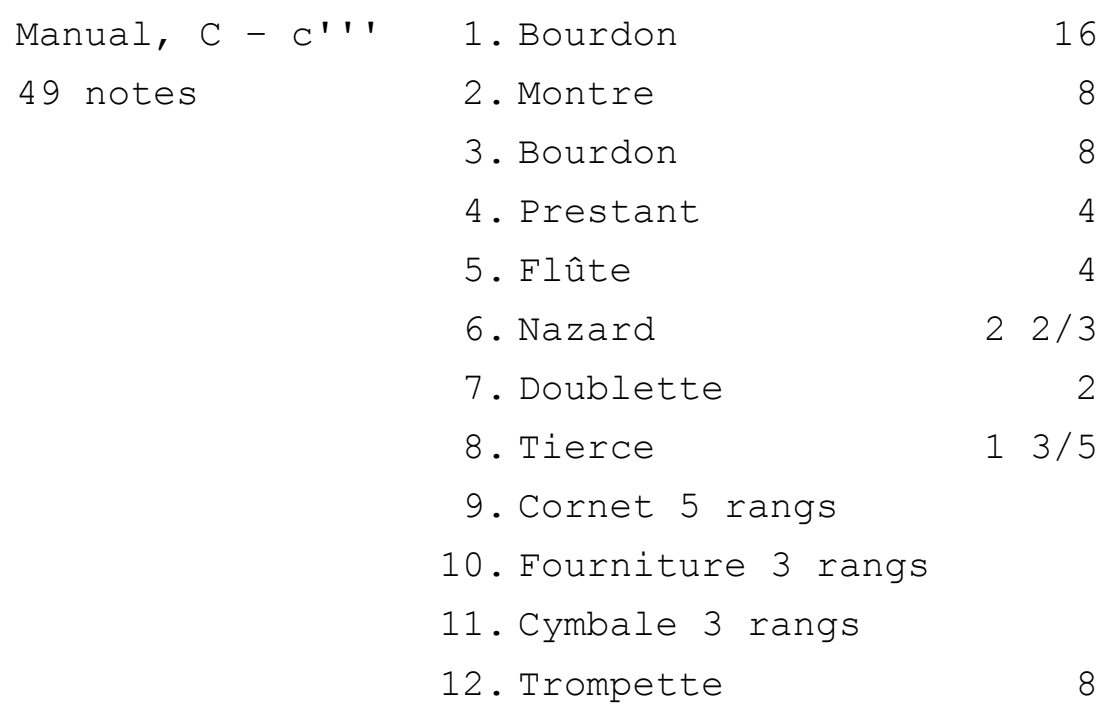

Positif ajouté en 1771 par J.A. Silbermann

C - C''', 49 notes 1. Bourdon 8

2. Prestant 4

3. Nazard $22 / 3$

4. Doublette 2

5. Tierce $13 / 5$

6 . Fourniture 3 rangs

7. Cromhorne 8

Pédale, C - C' 1. Soubasse ouverte 16

25 notes 2. Octavebasse 8

3. Trompette 8

2 Tremblants

3 soufflets

ton de choeur 
Strasbourg, Positif $\mathrm{N}^{\circ}$ VI (Monsieur Vigera), après 1718

Manual

1. Bourdon

8

2. Prestant

4

3. Doublette

2

4. Fourniture 3 rangs 
Marmoutier, Positif $\mathrm{N}^{\circ}$ IX, 1719

$1^{\circ}$ clavier, C-C''' 1. Bourdon 8

49 notes 2. Prestant 4

3. Nazard $22 / 3$

4. Doublette 2

5. Tierce $13 / 5$

6. Fourniture 3 rangs

7. Cromhorne 8 basse/dessus

$2^{\circ}$ clavier, $C^{\prime}-C^{\prime}{ }^{\prime}$ Cornet 5 rangs

25 notes

Tremblant doux

2 soufflets 
Strasbourg, Positif $\mathrm{N}^{\circ}$ IV (Saint-Pierre-le-Jeune), 1719

Manual

1. Bourdon

8

2. Flûte 4

3. Doublette 2

4. Fourniture 3 rangs 
Weingarten, projet non exécuté, 1720

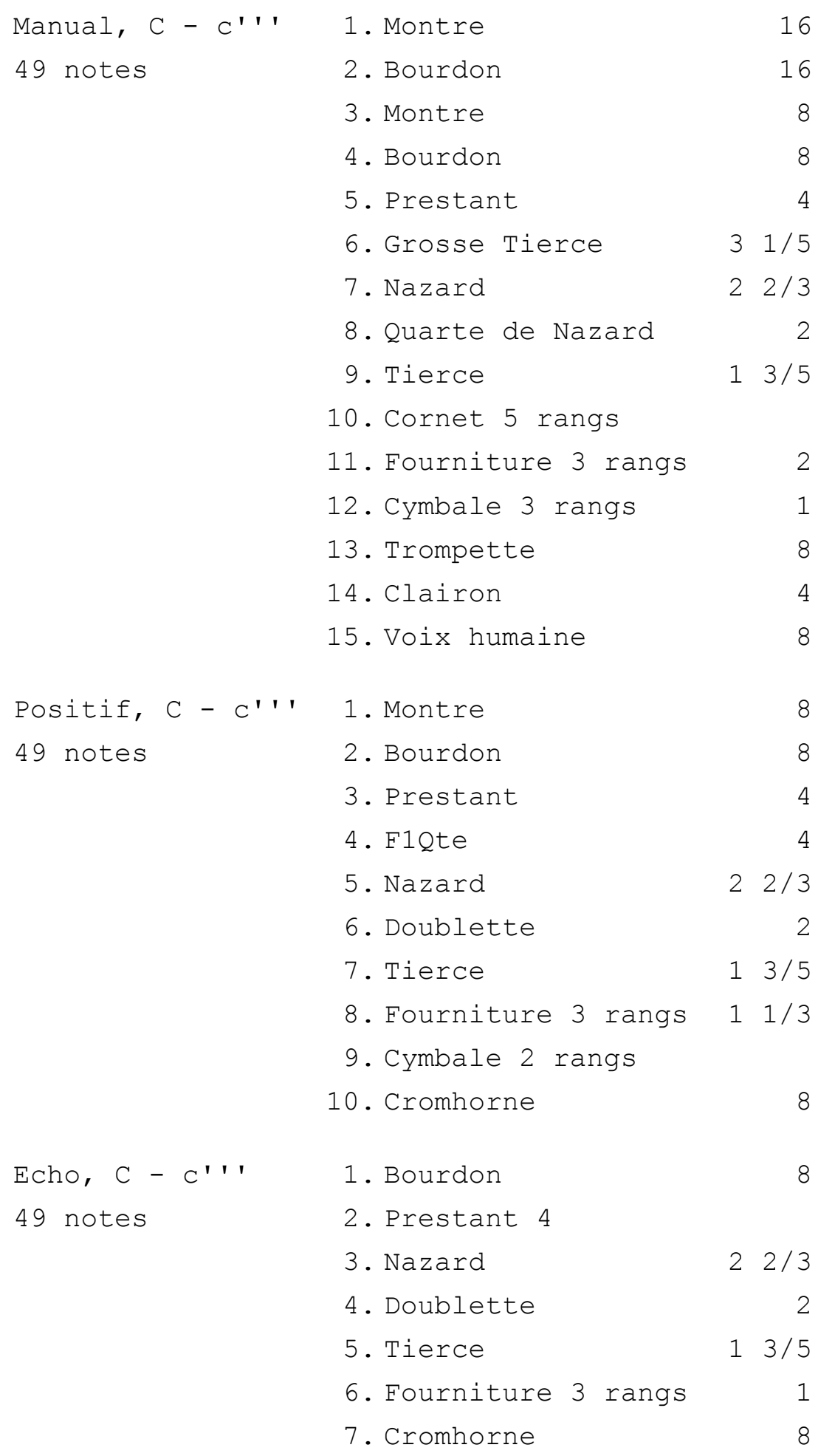


Pédale, C - C'

25 notes

\section{Tremblants}

1. Soubasse

16

2. Octavebasse

3. Prestant

4. Bombarde

5. Trompette

6. Clairon
8

4

16 en étain

8

4 
Wissembourg, Saint-Jean, 1720

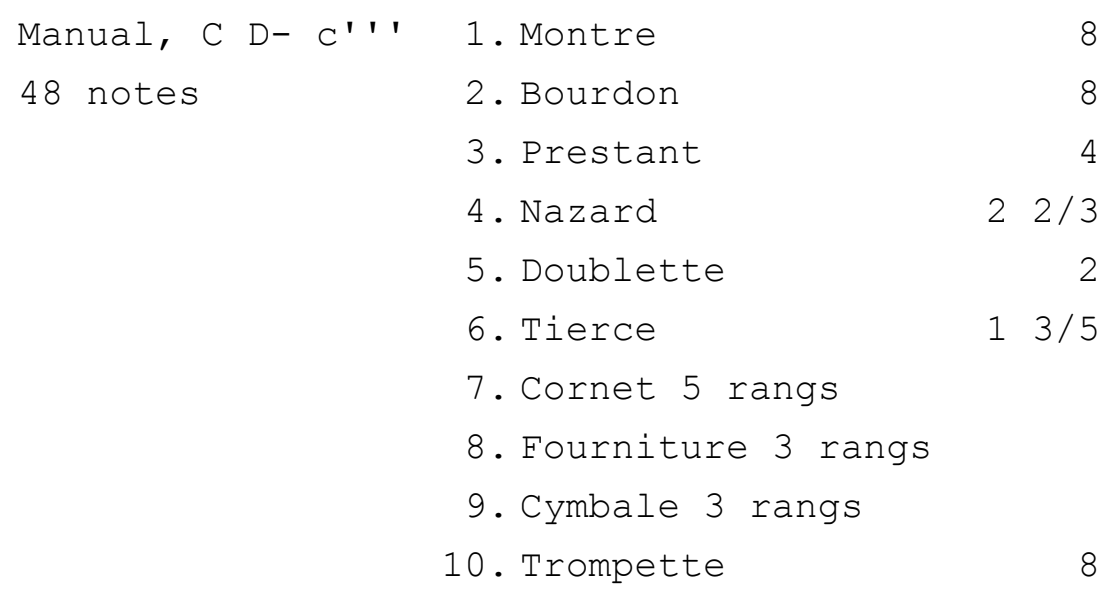

Echo Cornet d'Echo 5 rangs

Pédale

1. Soubasse

16

2. Octavebasse

3. Trompette

8

3 soufflets 
Saint-Léonard, église collégiale, 1721

Manual

1. Bourdon

8

2. Prestant

4

3. Nazard

$22 / 3$

4. Doublette

2

5. Tierce

$13 / 5$

6. Cornet

prévu

7. Fourniture 3 rangs

8. Cymbale 2 rangs

9. Trompette

8 prévue

Echo

Cornet d'Echo

prévu

2 soufflets 
Kolbsheim, Positif $\mathrm{N}^{\circ}$ III (Mme. Linck), 1722

$\begin{array}{ll}\text { 1. Bourdon } & 8 \\ \text { 2. Flûte } & 4 \\ \text { 3. Doublette } & 2 \\ \text { 4. Fourniture 2 rangs } & \end{array}$


Altenheim, église protestante, 1722

$\begin{array}{rlr}\text { Manual, C D- C''' } & \text { 1. Bourdon } \\ 48 \text { notes } & \text { 2. Prestant } \\ & \text { 3. Flute } \\ & \text { 4. Nazard } \\ & \text { 5. Doublette } 2 / 3 \\ & \text { 6. Fourniture 3 rangs } \\ & \text { 7. Cymbale 2 rangs }\end{array}$

Pédale, C D - c(?) Soubasse 16

12 notes (?)

Tremblant doux 
Bischwiller, église protestante, 1724

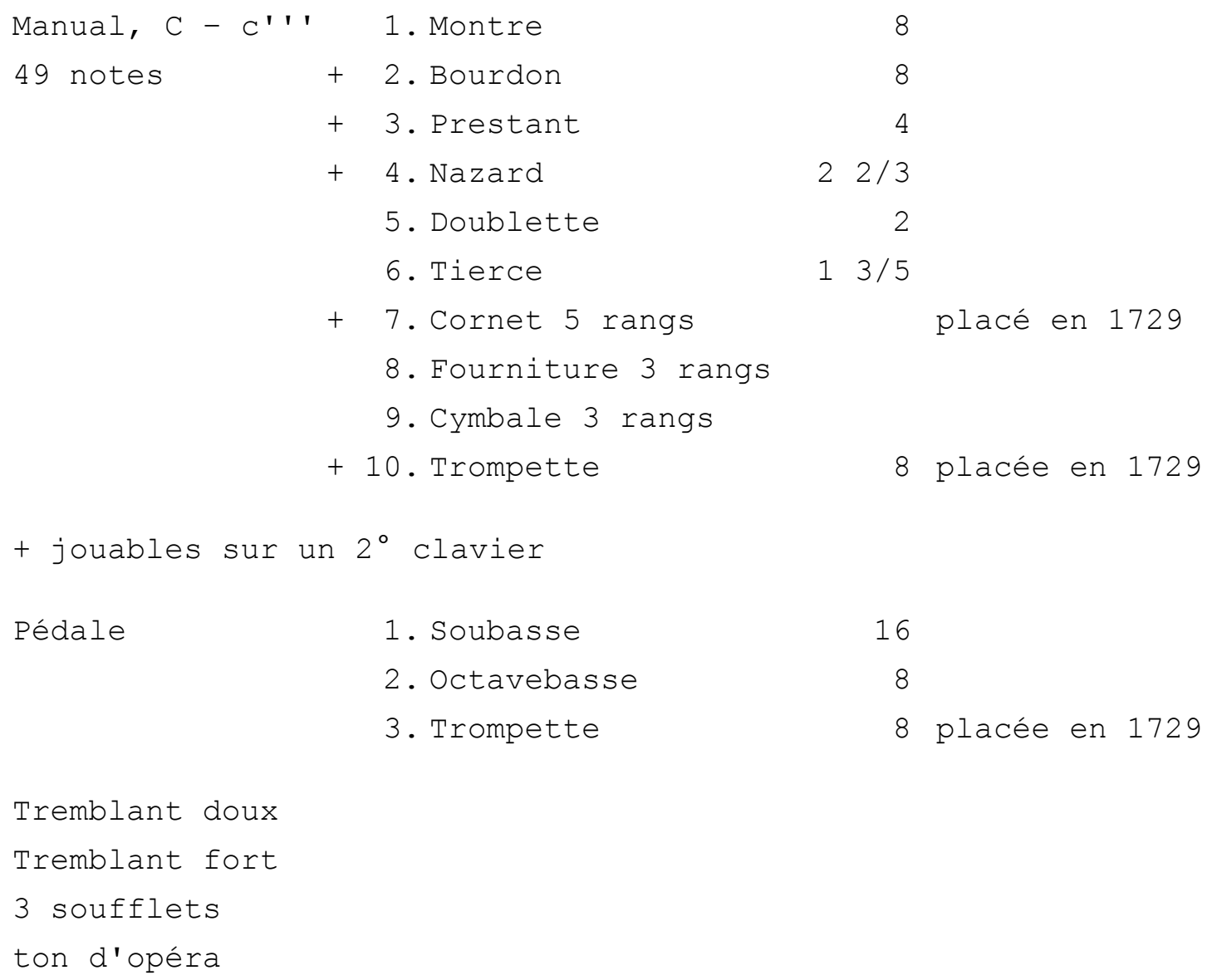


Strasbourg, Eglise de la Toussaint, Positif $N^{\circ}$ VII, V. 1725

\begin{tabular}{|c|c|c|}
\hline \multirow{6}{*}{$\begin{array}{l}\text { Manual, C D - d''' } \\
50 \text { notes }\end{array}$} & 1. Bourdon & 8 \\
\hline & 2. Flûte & 4 \\
\hline & 3. Doublette & 2 \\
\hline & 4. Cornet 4 rangs & \\
\hline & 5. Fourniture 2 rangs & \\
\hline & 6. Cromhorne & 8 basse/dessus \\
\hline
\end{tabular}

2 soufflets 
Colmar, Dominicains, 1726

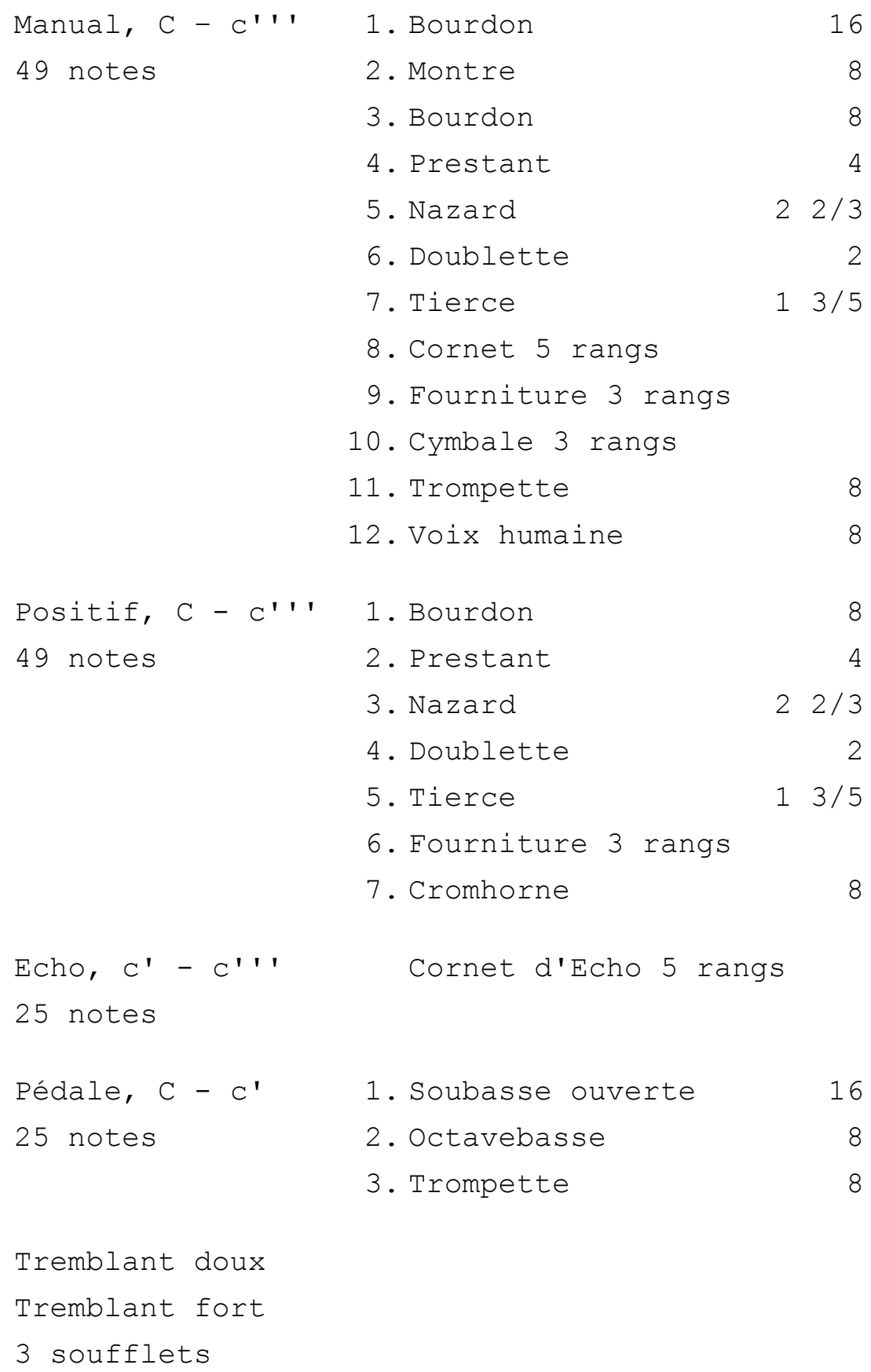


Strasbourg, église Saint-Guillaume, 1728

\begin{tabular}{|c|c|c|c|c|}
\hline \multirow{2}{*}{$\begin{array}{l}\text { Manual, C - } \\
49 \text { notes }\end{array}$} & 1. Bourdon & \multicolumn{2}{|r|}{16} & \\
\hline & 2. Montre & & 8 & \\
\hline & 3. Bourdon & & 8 & \\
\hline & 4. Nazard & 2 & $2 / 3$ & \\
\hline & 5. Doublette & & 2 & \\
\hline & 6. Tierce & 1 & $3 / 5$ & \\
\hline & 7. Cornet 5 rangs & & & \\
\hline & 8. Fourniture 3 rangs & & & \\
\hline & 9. Cymbale 3 rangs & & & \\
\hline & 10. Trompette & & 8 & placée par J.A. \\
\hline & & & & Silbermann en 1734 \\
\hline & 11. Voix humaine & & 8 & placée par J.A. \\
\hline & & & & Silbermann en 1734 \\
\hline Positif, C - C''' & 1. Bourdon & & 8 & \\
\hline 49 notes & 2. Prestant & & 4 & \\
\hline & 3. Nazard & 2 & $2 / 3$ & \\
\hline & 4. Doublette & & 2 & \\
\hline & 5. Tierce & 1 & $3 / 5$ & \\
\hline & 6. Fourniture 3 rangs & & & \\
\hline Pédale, $\mathrm{C}-\mathrm{C}^{\prime}$ & 1. Soubasse ouverte & & 16 & \\
\hline 25 notes & 2. Bombarde & & 16 & \\
\hline & 3. Trompette & & 8 & \\
\hline Tremblants & & & & \\
\hline soufflets & & & & \\
\hline
\end{tabular}


Vieux-Thann, Dominicaines, 1729

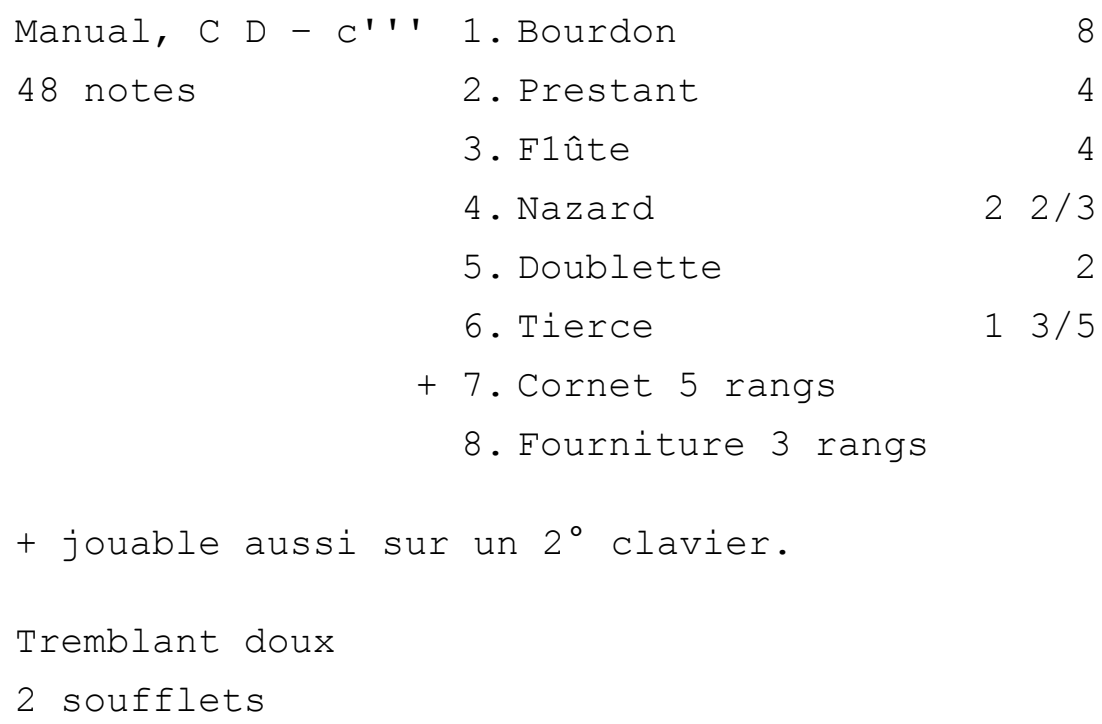


Altorf, église abbatiale, 1730

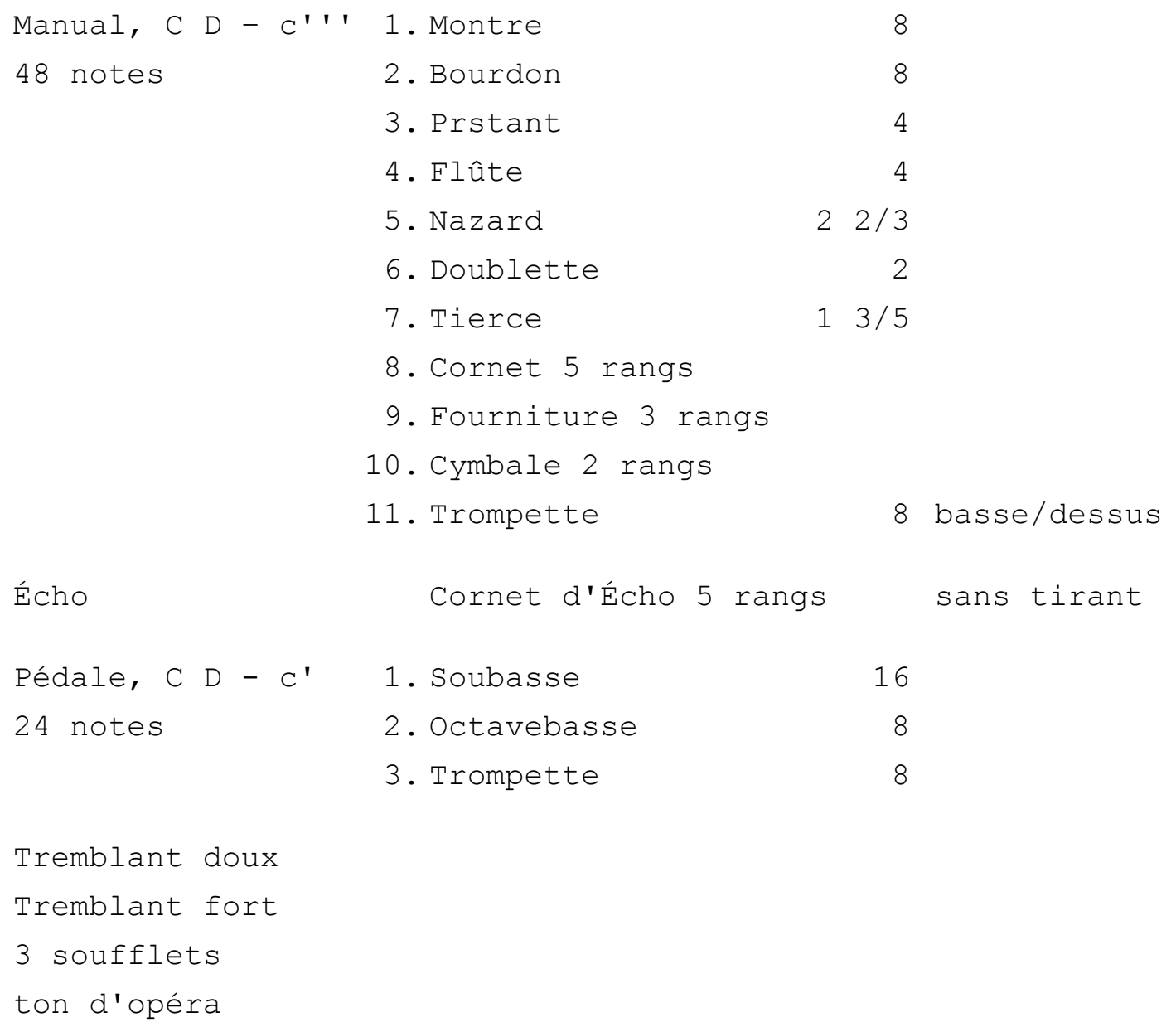


Frauenalb, église abbatiale (projet non exécuté), 1730

\begin{tabular}{|c|c|c|c|}
\hline Manual & 1. Montre & & 8 \\
\hline & 2. Bourdon & & 8 \\
\hline & 3. Prestant & & 4 \\
\hline & 4. Flûte & & 4 \\
\hline & 5. Nazard & 2 & $2 / 3$ \\
\hline & 6. Doublette & & 2 \\
\hline & 7. Tierce & 1 & $3 / 5$ \\
\hline & 8. Fourniture 3 rangs & 1 & $1 / 3$ \\
\hline & 9. Cymbale 2 rangs & & $1 / 2$ \\
\hline & 10. Trompette & & 8 \\
\hline & 11. Voix humaine & & 8 \\
\hline Positif & 1. Bourdon & & 8 \\
\hline & 2. Prestant & & 4 \\
\hline & 3. Nazard & 2 & $2 / 3$ \\
\hline & 4. Doublette & & 2 \\
\hline & 5. Tierce & 1 & $3 / 5$ \\
\hline & 6. Fourniture 3 rangs & & I \\
\hline Pédale & 1. Soubasse & & 16 \\
\hline & 2. Trompette & & 8 \\
\hline
\end{tabular}


Ebersmunster, église abbatiale, 1731

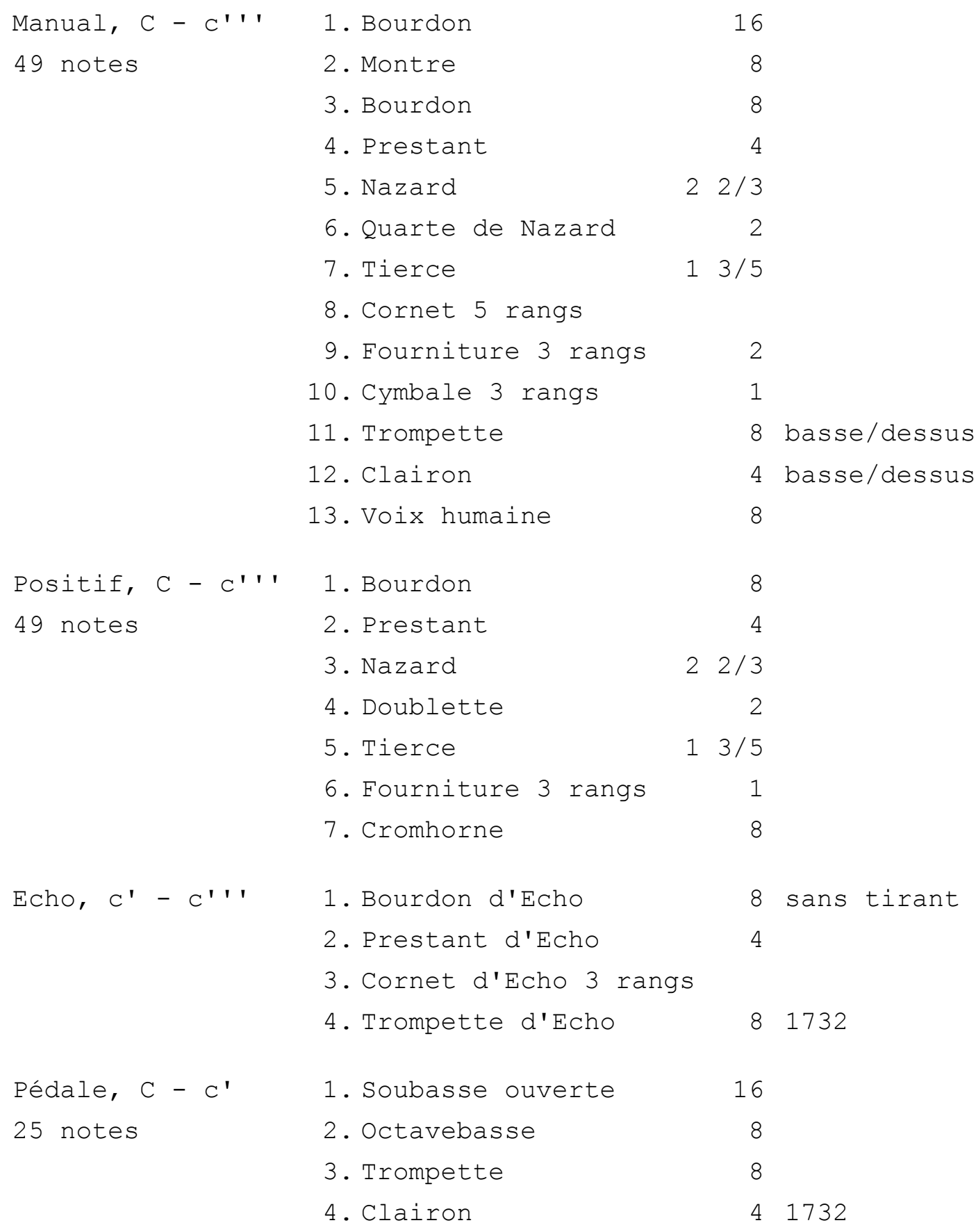

Tremblant doux

Tremblant fort

4 soufflets

ton d'opéra 
Colmar, église protestante, 1732

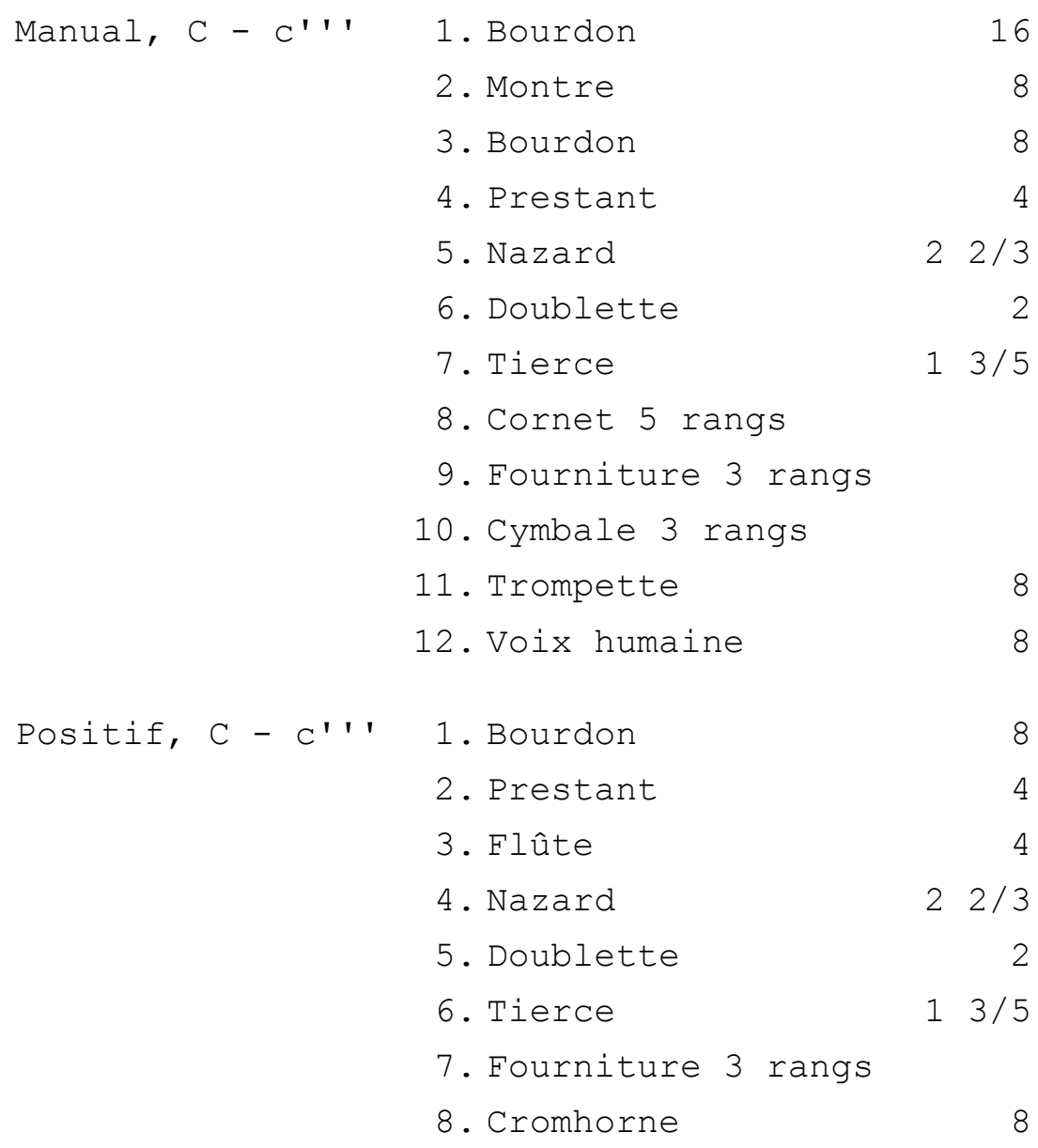

Echo

Cornet d'Echo 5 rangs

Pédale

1. Soubasse ouverte 16

2. Octavebasse 8

3. Trompette 8

Tremblant doux

Tremblant fort

4 soufflets 
Koenigsbruck, couvent de Cisterciennes, 1732

$\begin{array}{rr}\text { Manual, } 48 \text { notes } & \text { 1. Bourdon } \\ & \text { 2. Prestant } \\ & \text { 3. Flûte } \\ & \text { 4. Nazard } \\ & \text { 5. Doublette } \\ & \text { 6. Tierce } 2 / 3 \\ & \text { 7. Cornet 5 rangs } \\ & \text { 8. Fourniture 3 rangs }\end{array}$

2 soufflets 
Saverne, Positif ${ }^{\circ}$ VIII (Château des Rohan), 1733

Manual

1. Bourdon

8

2. Flûte 4

3. Doublette 2

4. Fourniture 
Rosheim, église Saints-Pierre-et-Paul, 1733

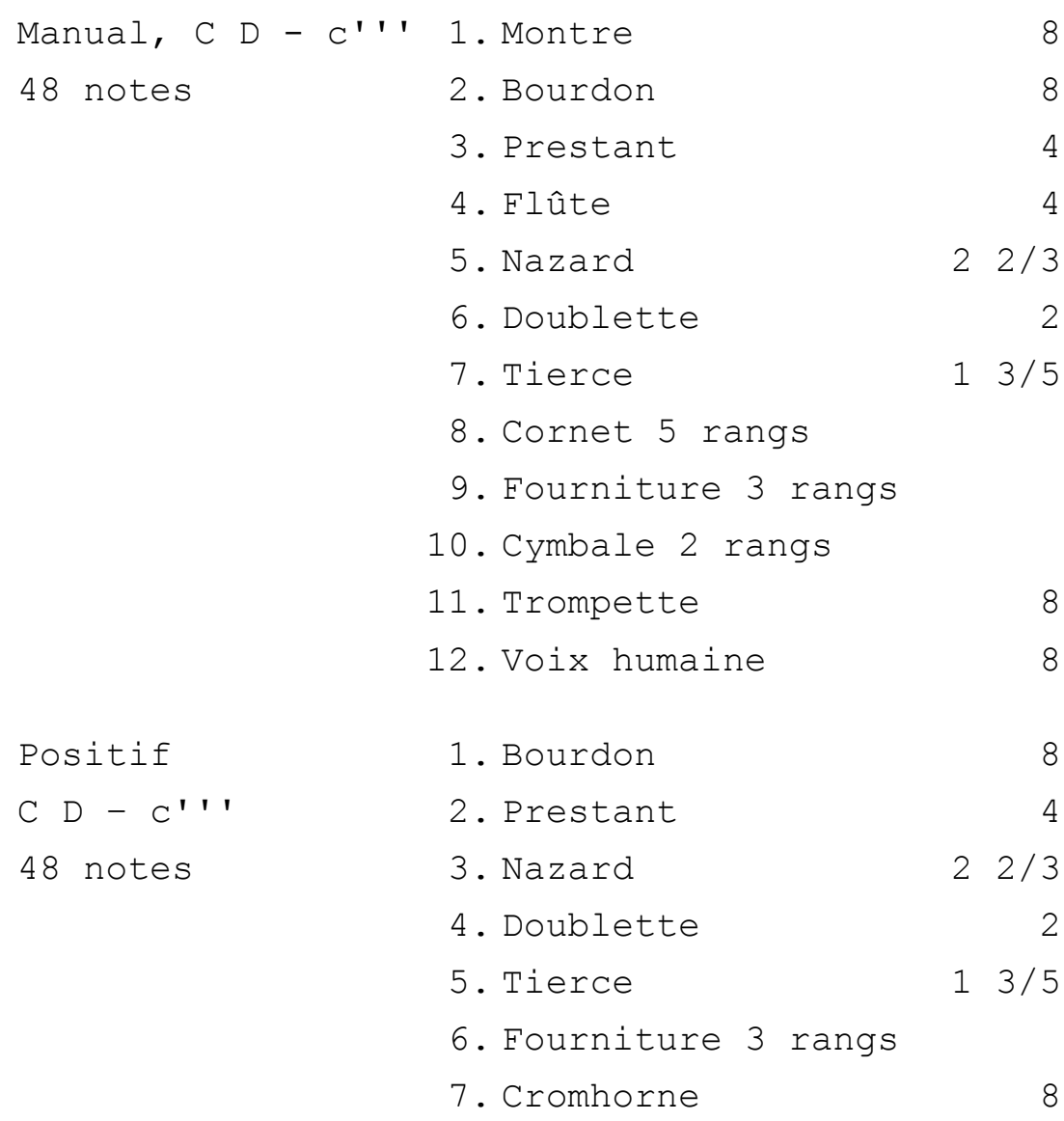

Echo Cornet d'Echo 5 rangs

Pédale, C D - C'

1. Soubasse

16

24 notes

2. Octavebasse

3. Trompette

8

3 soufflets

ton d'opéra 
Les orques de Jean-André Silbermann

Compositions, selon l'ordre chronologique 
Muhlbach, église mixte, 1736

$\begin{array}{rlr}\text { Manual, C D - C''' } & \text { 1. Bourdon } \\ & \text { 2. Prestant } & 8 \\ & \text { 3. Flûte } & 4 \\ & \text { 4. Nazard 2 2/3 } \\ & \text { 5. Doublette } \\ & \text { 6. Tierce } \\ & \text { 7. Fourniture 3 rangs } \\ & \text { 8. Cymbale 3 rangs } \\ & \text { 1. Bourdon } 3 / 5 \\ \text { Pédale, C D- C } & \\ \text { 12 notes } & \end{array}$


Neuf-Brisach, église catholique Saint-Louis, 1736

- projet non exécuté -

$\begin{aligned} \text { Manual, C - C''' } & \text { 1. Bourdon } \\ 49 \text { notes } & \text { 2. Prestant } \\ & \text { 3. Flûte } \\ & \text { 4. Nazard } \\ & \text { 5. Doublette } \\ & \text { 6. Tierce } 2 / 3 \\ & \text { 7. Cornet 5 rangs } \\ & \text { 8. Fourniture 3 rangs } \\ & \text { 9. Cromhorne } \\ & \end{aligned}$

basse/dessus

Echo Cornet d'Echo 5 rangs

Pédale, C - c'

1. Bourdon

8

25 notes

2. Trompette

8

Tremblant doux

Tremblant fort

2 ou 3 soufflets

ton d'opéra 
Strasbourg, Saint-Thomas, Positif, 1737

Manual

1. Bourdon

8

2. Flûte

4

3. Nazard

$22 / 3$

4. Doublette

2

5. Tierce

$13 / 5$

6. Fourniture 3 rangs 
Barr, Positif, 1737

$\begin{array}{llr}\text { Manual, C D - C''' } & \text { 1. Bourdon } \\ 48 \text { notes } & \text { 2. Flûte } & 8 \\ & \text { 3. Nazard } & 4 \\ & \text { 4. Doublette } & 2 / 3 \\ & \end{array}$

2 soufflets 
Colmar, couvent des Dominicaines d'Unterlinden, 1737-1738

\begin{tabular}{|c|c|c|c|}
\hline \multirow[t]{9}{*}{ Manual } & 1. Bourdon & 8 & \\
\hline & 2. Prestant & 4 & \\
\hline & 3. Flûte & 4 & \\
\hline & 4. Nazard & $22 / 3$ & \\
\hline & 5. Doublette & 2 & \\
\hline & 6. Tierce & $13 / 5$ & \\
\hline & 7. Cornet 5 rangs & & \\
\hline & 8. Fourniture 3 rangs & & \\
\hline & 9. Cromhorne & 8 & basse/dessus \\
\hline \multirow[t]{3}{*}{ Echo } & 1. Bourdon d'Echo & 8 & \\
\hline & 2. Prestant d'Echo & 4 & \\
\hline & 3. Cornet d'Echo 3 rang & & \\
\hline Positif & 1. Bourdon & 8 & \\
\hline \multirow[t]{5}{*}{ ajouté en 1743} & 2. Flûte & 4 & \\
\hline & 3. Quinte & $22 / 3$ & \\
\hline & 4. Doublette & 2 & \\
\hline & 5. Tierce & $13 / 5$ & \\
\hline & 6. Trompette de Récit & 8 & \\
\hline Pédale, C - C' & 1. Octavebasse & 8 & \\
\hline 25 notes & 2. Trompette & 8 & \\
\hline
\end{tabular}


Barr, église mixte, 1738-1739

\begin{tabular}{|c|c|c|c|}
\hline \multirow{2}{*}{$\begin{array}{l}\text { Manual, C - C''' } \\
49 \text { notes }\end{array}$} & 1. Bourdon & 8 & 1738 \\
\hline & 2. Prestant & 4 & 1738 \\
\hline & 3. Flûte & 4 & 1738 \\
\hline & 4. Nazard & $22 / 3$ & 1739 \\
\hline & 5. Doublette & 2 & 1738 \\
\hline & 6. Tierce & $13 / 5$ & 1739 \\
\hline & 7. Cornet 5 rangs & & 1739 \\
\hline & 8. Fourniture 3 rangs & & 1738 \\
\hline & 9. Cymbale 3 rangs & & 1738 \\
\hline & 10. Cromhorne & 8 & basse/dessus 1739 \\
\hline Pédale, C - g & 1. Soubasse & 16 & 1738 \\
\hline 20 notes & 2. Octavebasse & 8 & 1738 \\
\hline & 3. Trompette & 8 & 1739 \\
\hline
\end{tabular}


Marbach, Couvent des Augustins, 1738-1739

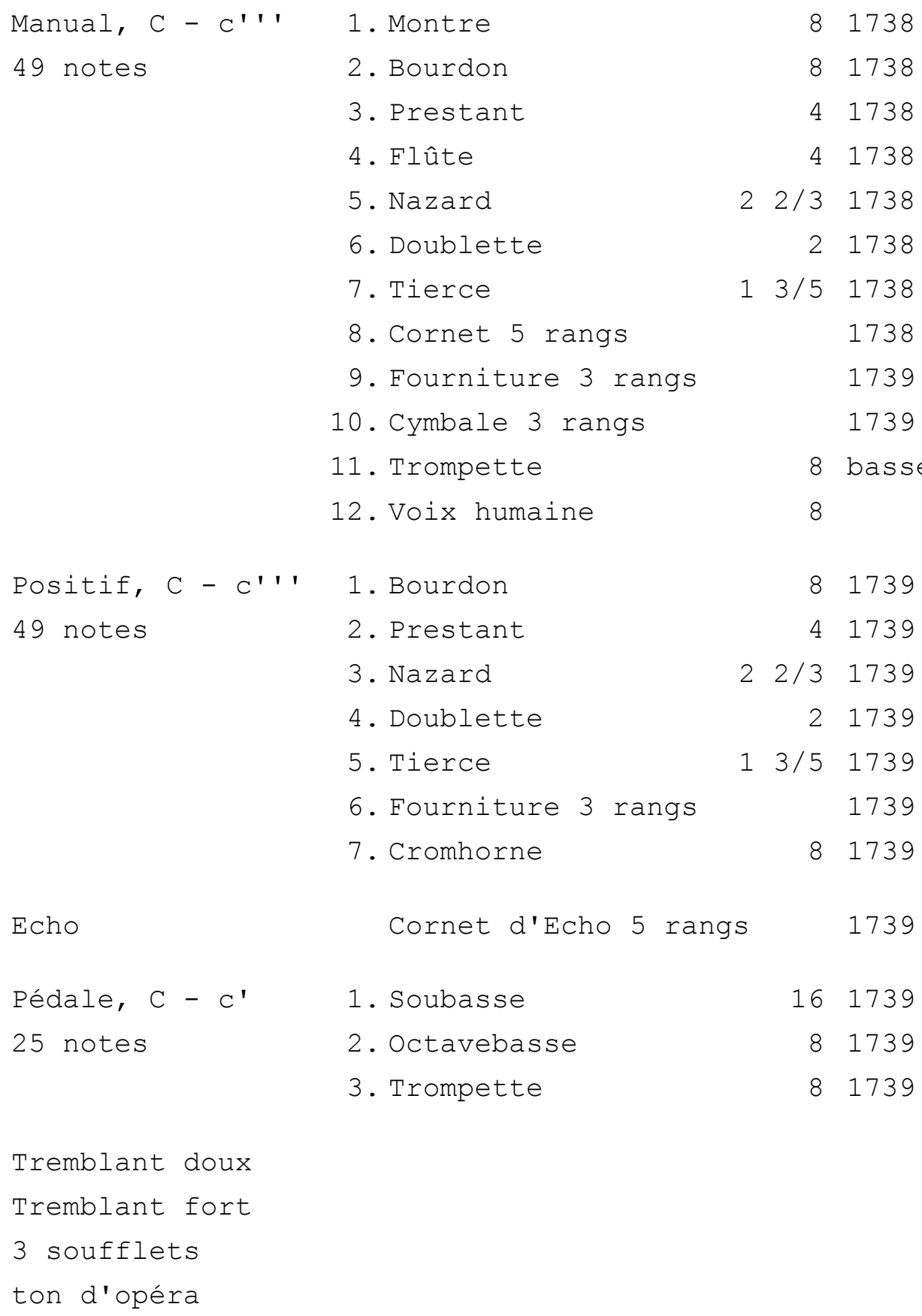

Echo Cornet d'Echo 5 rangs 1739 
Strasbourg, église catholique Saint-Pierre-le-Vieux, 1739

- projet non exécuté

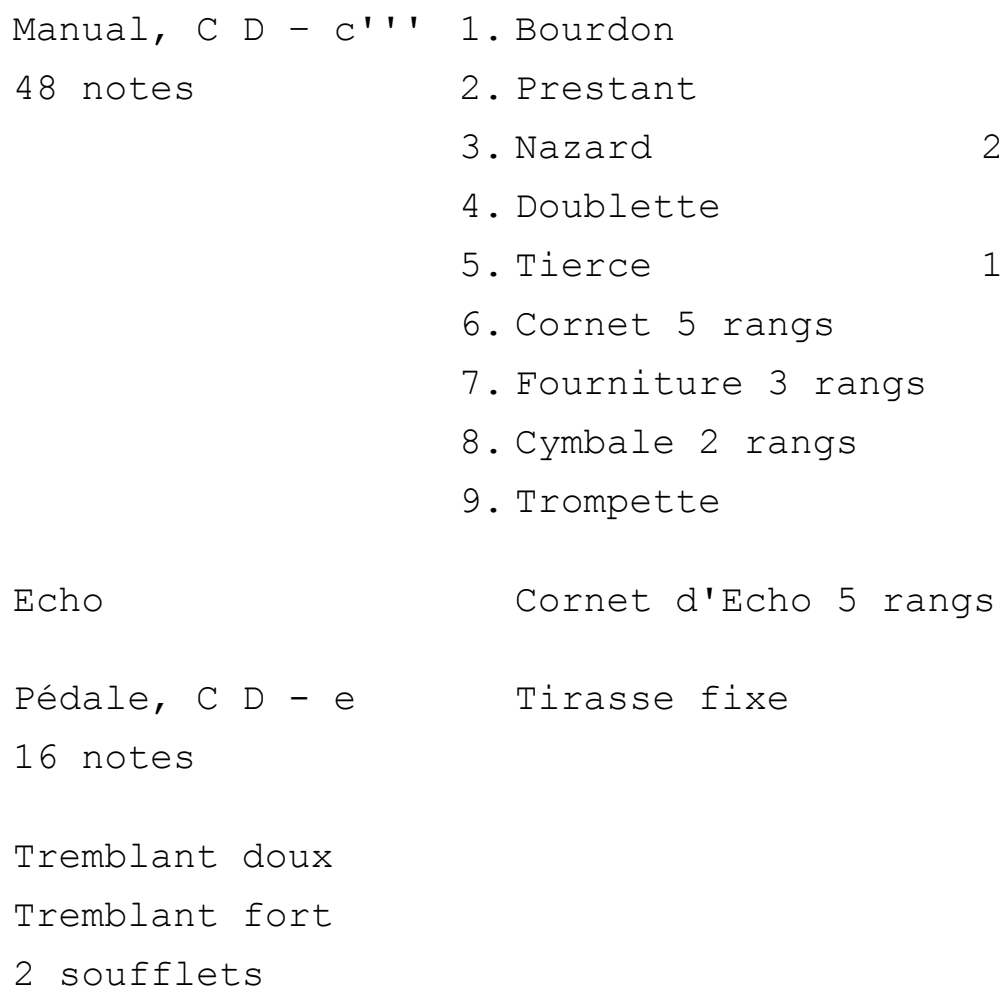

8 basse/dessus

$22 / 3$

2

$13 / 5$

8

4

2


Strasbourg, Saint-Thomas, 1740-1741

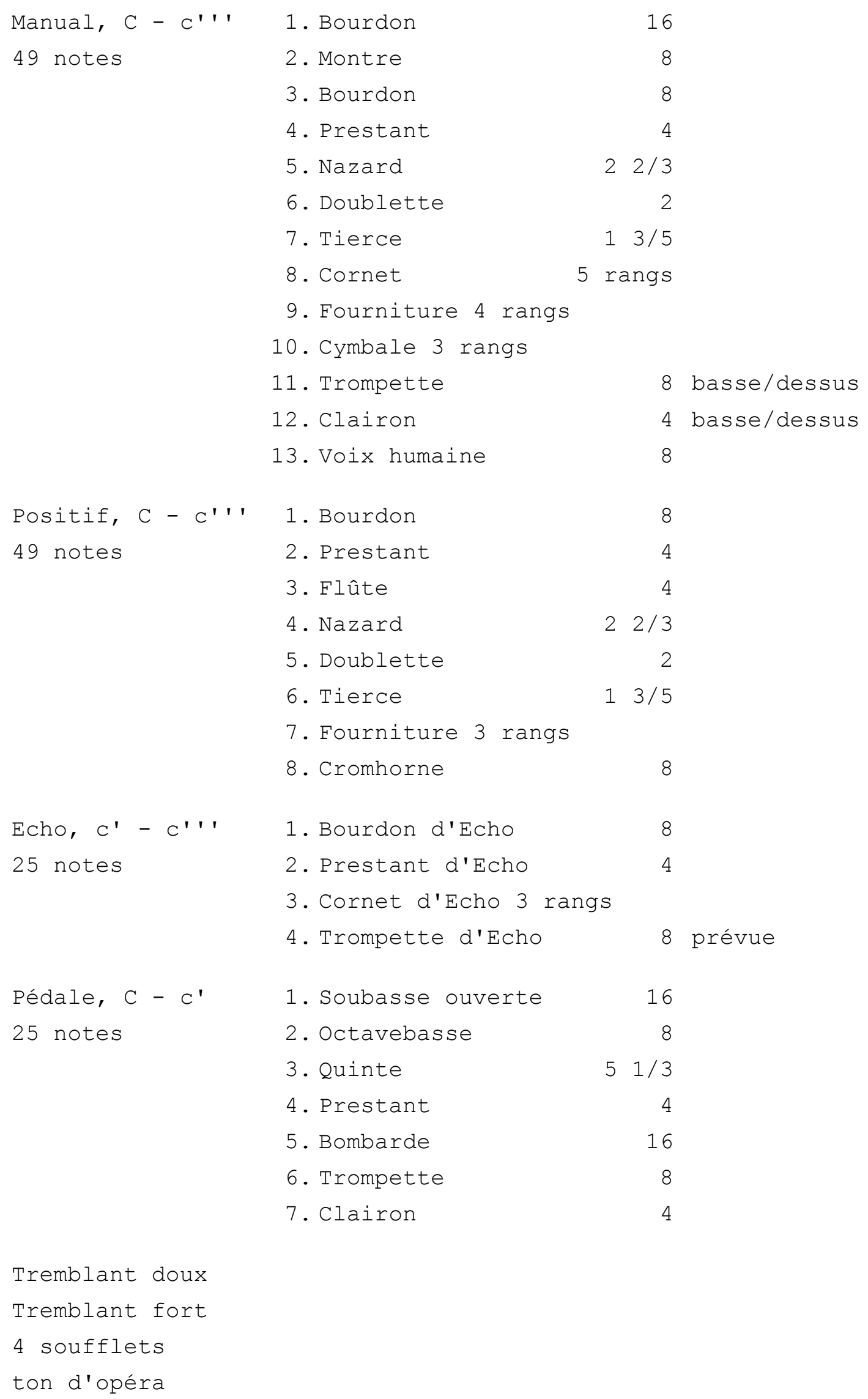


Ensisheim, église catholique, 1742

\begin{tabular}{|c|c|c|c|}
\hline Manual, C - C''' & 1. Bourdon & & 16 \\
\hline 49 notes & 2. Montre & & 8 \\
\hline & 3. Bourdon & & 8 \\
\hline & 4. Prestant & & 4 \\
\hline & 5. Flûte & & 4 \\
\hline & 6. Nazard & 2 & $2 / 3$ \\
\hline & 7. Doublette & & 2 \\
\hline & 8. Tierce $13 / 5$ & & \\
\hline & 9. Cornet 5 rangs & & \\
\hline & 10. Fourniture 3 rangs & & \\
\hline & 11. Cymbale 3 rangs & & \\
\hline & 12. Trompette & & 8 \\
\hline & 13. Voix humaine & & 8 \\
\hline Positif, C - C''' & 1. Bourdon & & 8 \\
\hline 49 notes & 2. Prestant & & 4 \\
\hline & 3. Flûte & & 4 \\
\hline & 4. Nazard & 2 & $2 / 3$ \\
\hline & 5. Doublette & & 2 \\
\hline & 6. Tierce & 1 & $3 / 5$ \\
\hline & 7. Fourniture 3 rangs & & \\
\hline & 8. Carillon 2 rangs & & \\
\hline & 9. Cromhorne & & 8 \\
\hline Echo, $c^{\prime}-c^{\prime}{ }^{\prime}$ & 1. Cornet d'Echo 5 rang & & \\
\hline 25 notes & 2. Voix humaine & & 8 \\
\hline Pédale, C - C' & 1. Soubasse ouverte & & 16 \\
\hline 25 notes & 2. Octavebasse & & 8 \\
\hline & 3. Trompette & & 8 \\
\hline & 4. Clairon & & 4 \\
\hline
\end{tabular}


Strasbourg, Couvent de la Toussaint, 1743

\begin{tabular}{|c|c|c|c|}
\hline \multirow{2}{*}{$\begin{array}{l}\text { Manual, C - C''' } \\
49 \text { notes }\end{array}$} & 1. Bourdon & 8 & \\
\hline & 2. Prestant & 4 & \\
\hline & 3. Flûte & 4 & \\
\hline & 4. Nazard & $22 / 3$ & \\
\hline & 5. Doublette & 2 & \\
\hline & 6. Tierce & $3 / 5$ & \\
\hline & 7. Cornet 4 rangs & & \\
\hline & 8. Fourniture 2 rangs & $1 / 2$ & \\
\hline & 9. Cromhorne & 8 & \\
\hline $\begin{array}{l}\text { Echo, C' }-C^{\prime}{ }^{\prime} \\
25 \text { notes }\end{array}$ & Cornet d'Echo 5 rangs & & \\
\hline Pédale, C - c & 1. Octavebasse & 8 & \\
\hline 13 notes & 2. Trompette & 8 & placée en 1745 \\
\hline
\end{tabular}


Strasbourg, Saint-Pierre-le-Vieux catholique, 1744

$$
\text { - projet non exécuté - }
$$

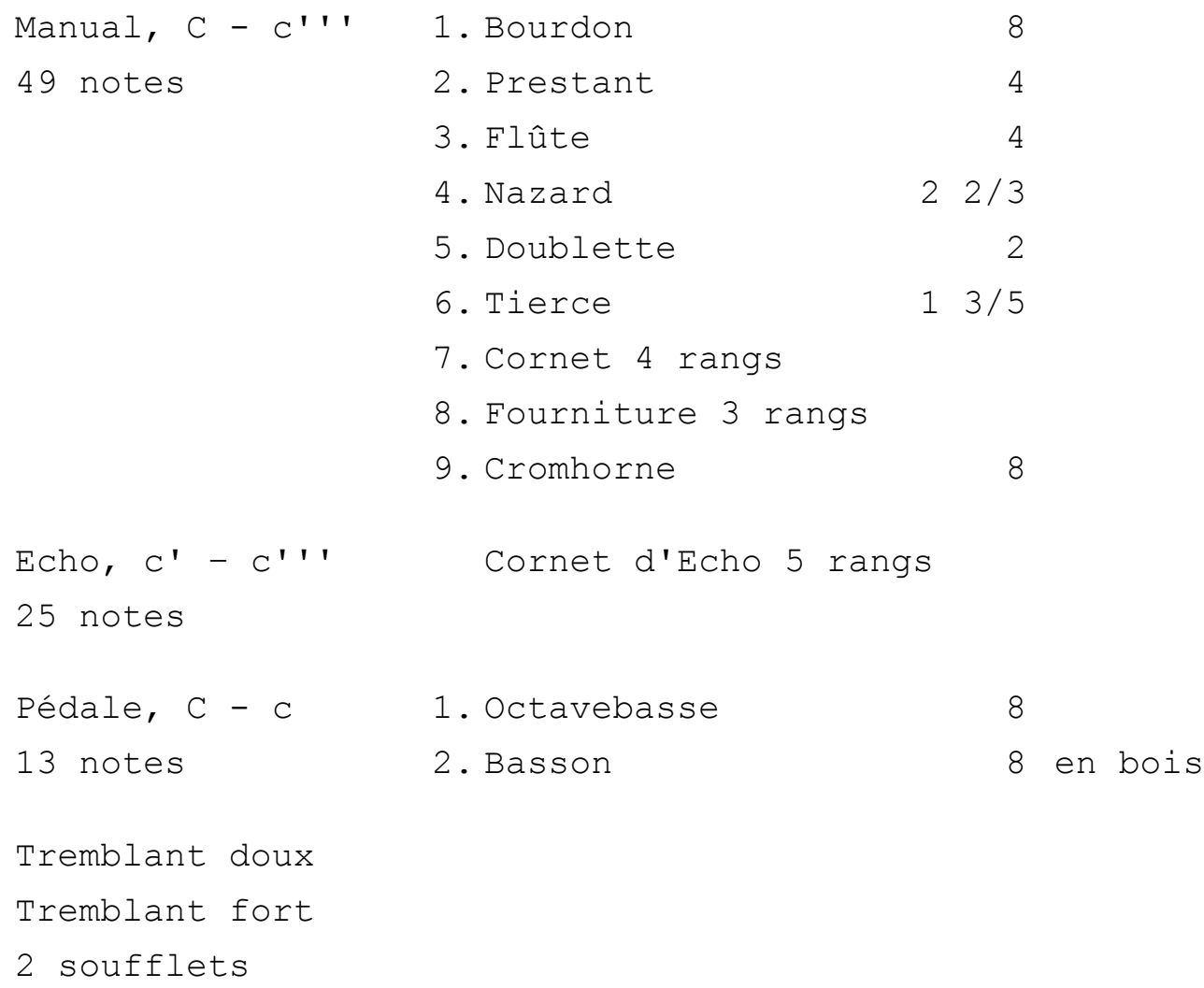


Guebwiller, Dominicains, 1745

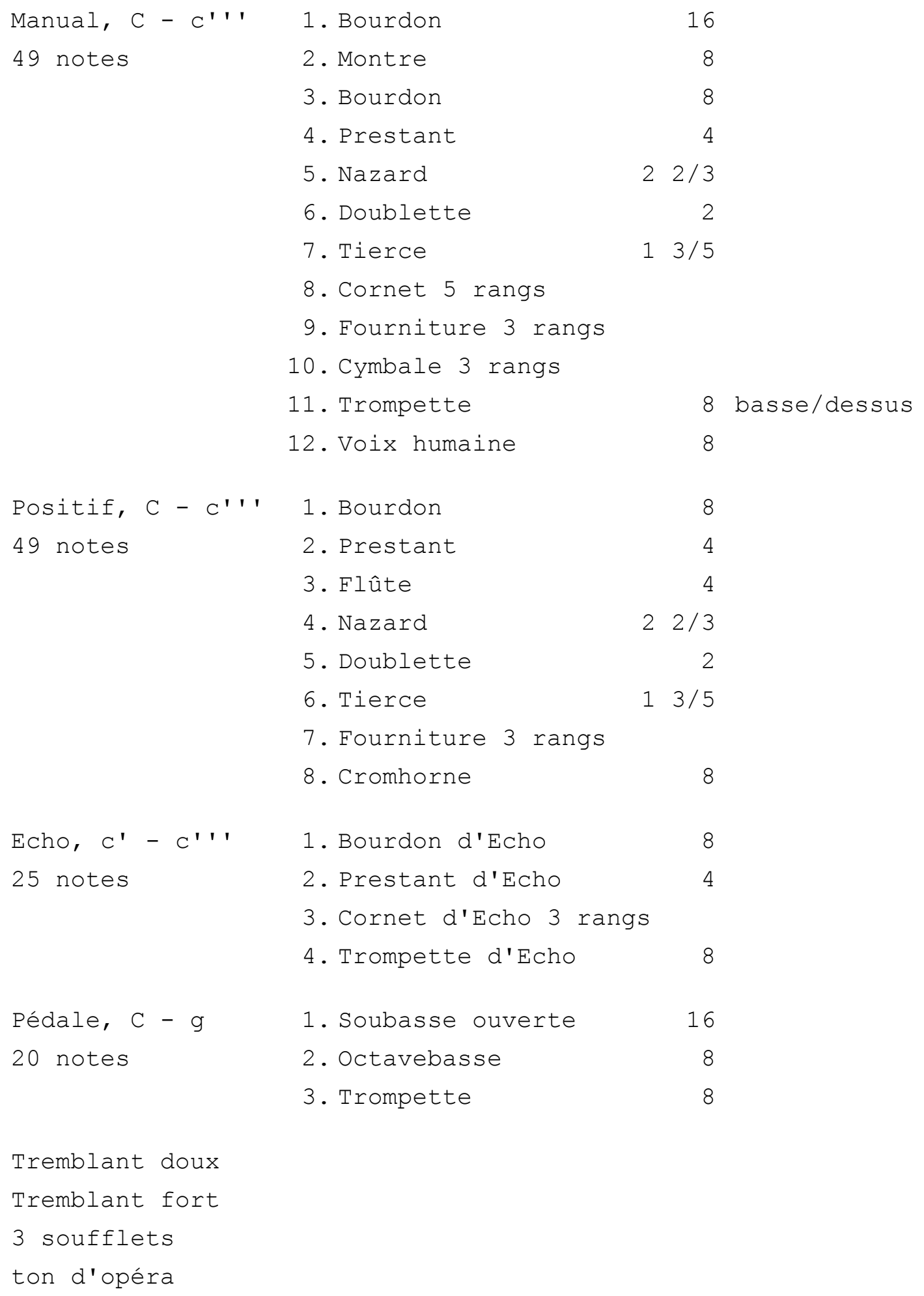


Saint-Quirin, Prieuré, 1746

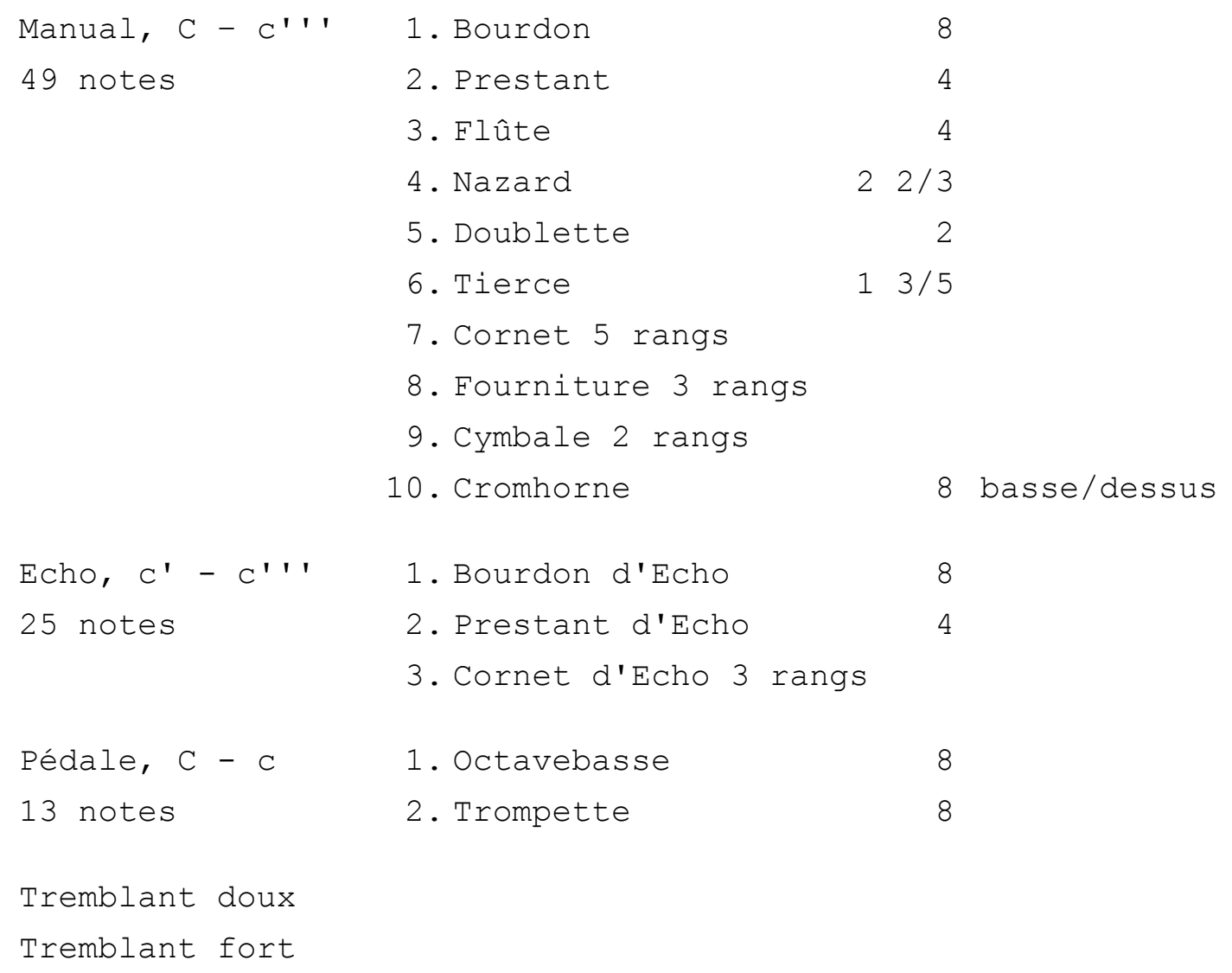


Bischoffsheim, église catholique, 1746

Manual

1. Bourdon

2. Prestant

3. Nazard

4. Doublette

5. Tierce

6. Cornet

7. Fourniture

8. Cymbale 2 rangs
8

4

$22 / 3$

2

$13 / 5$

Tremblant doux 
Guémar, église catholique, 1746

$\begin{array}{lr}\text { Manual } & \text { 1. Bourdon } \\ \text { 2. Prestant } & 4 \\ \text { 3. Flûte } & 4 \\ & \text { 4. Nazard } \\ & \text { 5. Doublette } \\ & \text { 6. Tierce } \\ & \text { 7. Cornet 5 rangs } \\ & \text { 8. Fourniture 3 rangs } \\ & \text { 9. Cymbale 3 rangs } \\ \text { 10. Cromhorne } & \end{array}$

8

4

4

2

8 basse/dessus

Echo

Cornet d'Echo

Pédale

1. Octavebasse

8

2. Trompette

8 
Saint-Jean-Saverne, église abbatiale, 1747

$\begin{array}{lr}\text { Manual } & 8 \\ \text { 1. Bourdon } & 4 \\ \text { 2. Prestant } & 4 \\ \text { 3. Flûte } & 22 / 3 \\ & \text { 4. Nazard } \\ & \text { 5. Doublette } \\ & \text { 6. Tierce } \\ & \text { 7. Cornet 5 rangs } \\ \text { 8. Fourniture 3 rangs } & \\ \text { 9. Cymbale 2 rangs } & \\ \text { 10. Cromhorne } & 8\end{array}$

8

4

4

- Flúte

13

2

5. Doublette

$3 / 5$

7. Cornet 5 rangs

8. Fourniture 3 rangs

10. Cromhorne

8 basse/dessus

Echo

1. Bourdon d'Echo 8

2. Prestant d'Echo 4

3. Cornet d'Echo 3 rangs

Pédale

1. Octavebasse 8

2. Trompette 8

Tremblant doux

Tremblant fort 
Balbronn, église simultanée, 1747

$\begin{array}{ll}\text { Manual, C }- \text { C''' } & \text { 1. Bourdon } \\ 49 \text { notes } & \text { 2. Prestant } \\ & \text { 3. Quinte } \\ & \text { 4. Doublette } 2 / 3 \\ & \text { 5. Tierce } \\ & \text { 6. Cornet 5 rangs } \\ & \text { 7. Fourniture 3 rangs } \\ & \text { 8. Cymbale 3 rangs }\end{array}$

Tremblant doux

2 soufflets

ton d'opéra 
Strasbourg, Temple-Neuf, 1749

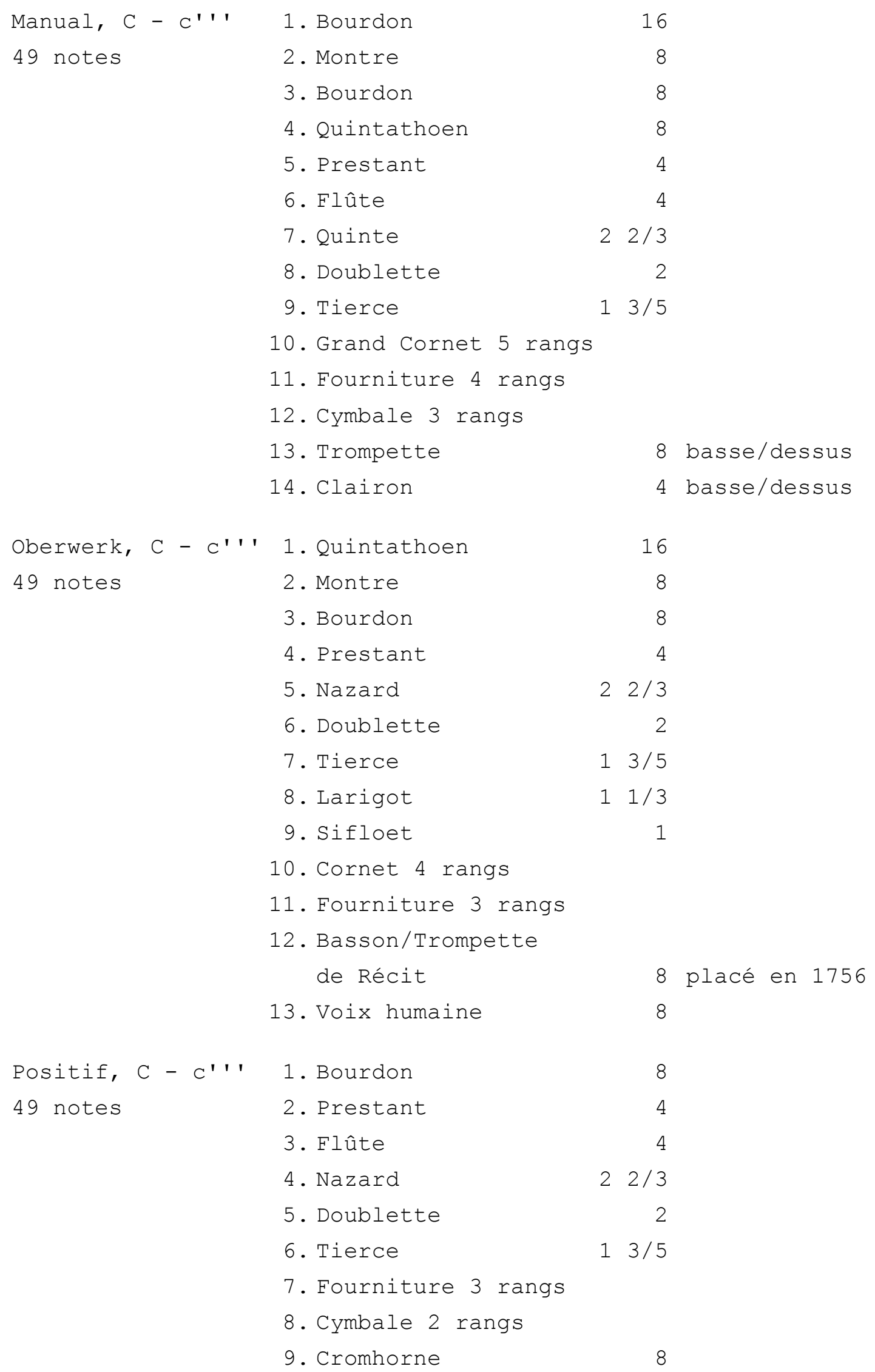




\begin{tabular}{|c|c|c|}
\hline \multirow{9}{*}{$\begin{array}{l}\text { Pédale, } C-C^{\prime} \\
25 \text { notes }\end{array}$} & 1. Montre & 16 en bois \\
\hline & 2. Soubasse & 16 \\
\hline & 3. Octavebasse & 8 \\
\hline & 4. Prestant & 4 \\
\hline & 5. Fourniture 6 rangs & \\
\hline & 6. Bombarde & 16 en étain \\
\hline & 7. Trompette & 8 \\
\hline & 8. Clairon & 4 \\
\hline & 9. Cornetto & 2 \\
\hline
\end{tabular}

Tremblant doux Manual

Tremblant fort Manual

Tremblant doux Oberwerk

6 soufflets

ton d'opéra 
Soultz, église catholique, 1750

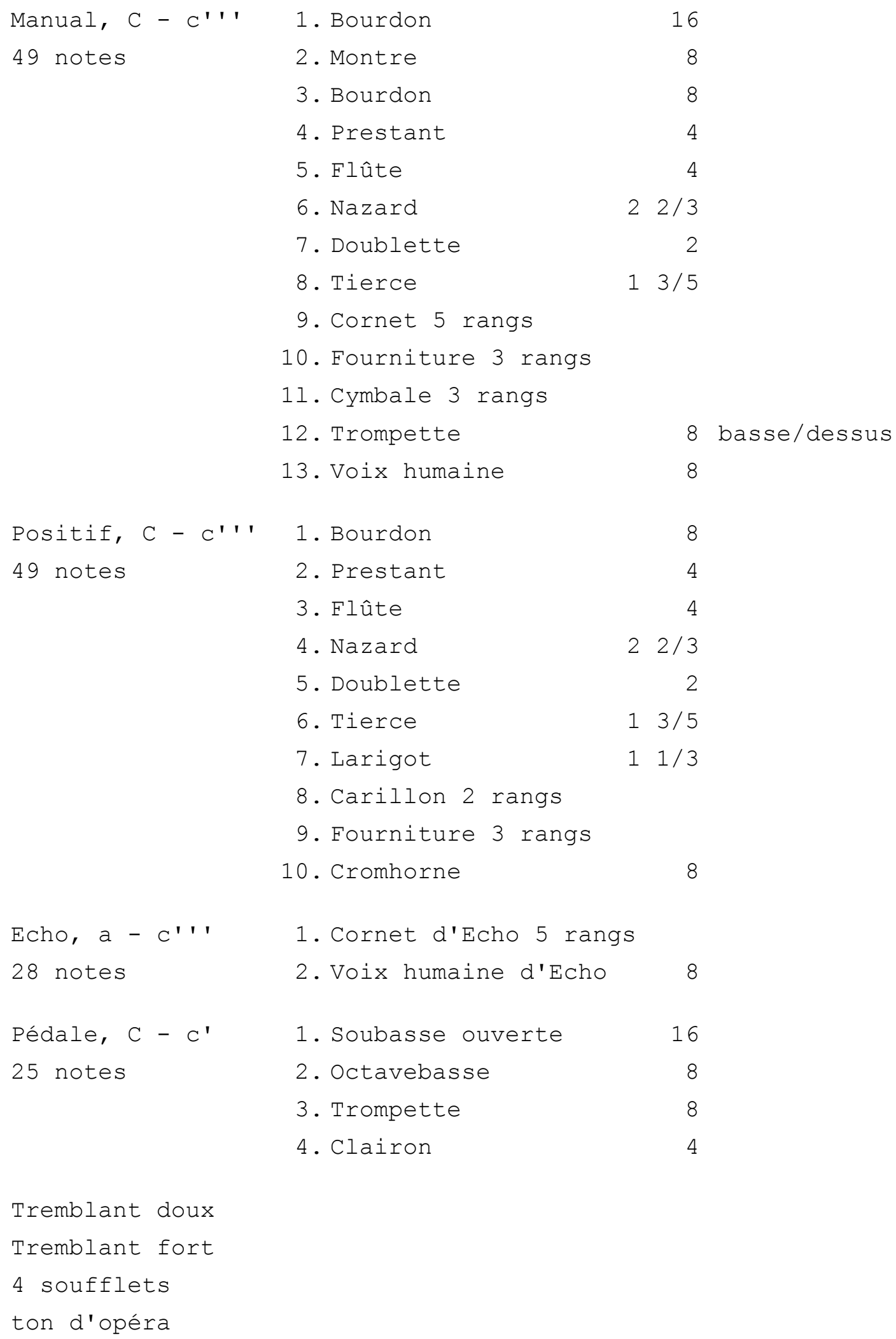

Tremblant doux Tremblant fort 
Sélestat, Couvent des Dominicaines de Sylo, 1750-1751

\begin{tabular}{|c|c|c|c|}
\hline Manual, C - C''' & 1. Bourdon & 8 & \\
\hline \multirow{8}{*}{49 notes } & 2. Prestant & 4 & \\
\hline & 3. Flûte & 4 & \\
\hline & 4. Nazard & $22 / 3$ & \\
\hline & 5. Doublette & 2 & \\
\hline & 6. Tierce & $13 / 5$ & \\
\hline & 7. Cornet 5 rangs & & \\
\hline & 8. Fourniture 3 rangs & & \\
\hline & 9. Cromhorne & 8 & basse/dessus \\
\hline \multirow[t]{3}{*}{ Echo } & 1. Bourdon d'Echo & 8 & \\
\hline & 2. Prestant d'Echo & 4 & \\
\hline & 3. Cornet d'Echo 3 rang & & \\
\hline Positif, $C-C^{\prime \prime}$ & 1. Bourdon & 8 & \\
\hline 49 notes & 2. Flûte & 4 & \\
\hline \multirow[t]{4}{*}{ ajouté en 1751} & 3. Quinte & $22 / 3$ & \\
\hline & 4. Doublette & 2 & \\
\hline & 5. Tierce & $13 / 5$ & \\
\hline & 6. Trompette de Récit & 8 & \\
\hline Pédale, C $-\mathrm{C}^{\prime}$ & 1. Octavebasse & 8 & \\
\hline 25 notes & 2. Trompette & 8 & \\
\hline
\end{tabular}


Sélestat, Couvent des Franciscains, 1751

Manual

1. Montre 8

2. Bourdon 8

3. Prestant 4

4. Nazard $22 / 3$

5. Doublette 2

6. Tierce $13 / 5$

7. Cornet 5 rangs

8. Fourniture

9. Cymbale

10. Trompette 8

Echo

1. Bourdon d'Echo 8

2. Prestant d'Echo 4

3. Cornet d'Echo 3 rangs

Pédale

1. Soubasse 16

2. Octavebasse

3. Trompette

Positif prévu

Tremblant doux

Tremblant fort

3 soufflets

ton d'opéra 
Muttersholtz, église simultanée, 1751

$\begin{array}{ll}\text { Manual, C - C''' } & \text { 1. Bourdon } \\ 49 \text { notes } & \text { 2. Prestant }\end{array}$

Pédale

? 
Alspach, Couvent des Clarisses, 1751

Manual

1. Bourdon

8

2. Prestant

4

3. Flûte 4

4. Nazard

$22 / 3$

5. Doublette 2

6. Tierce

$13 / 5$

7. Cornet 5 rangs

8. Fourniture

9. Cromhorne

Echo Cornet d'Echo 5 rangs

Pédale

1. Octavebasse 8

2. Trompette 8

Tremblant doux

Tremblant fort 
Villingen, église Saint-Georges, 1752

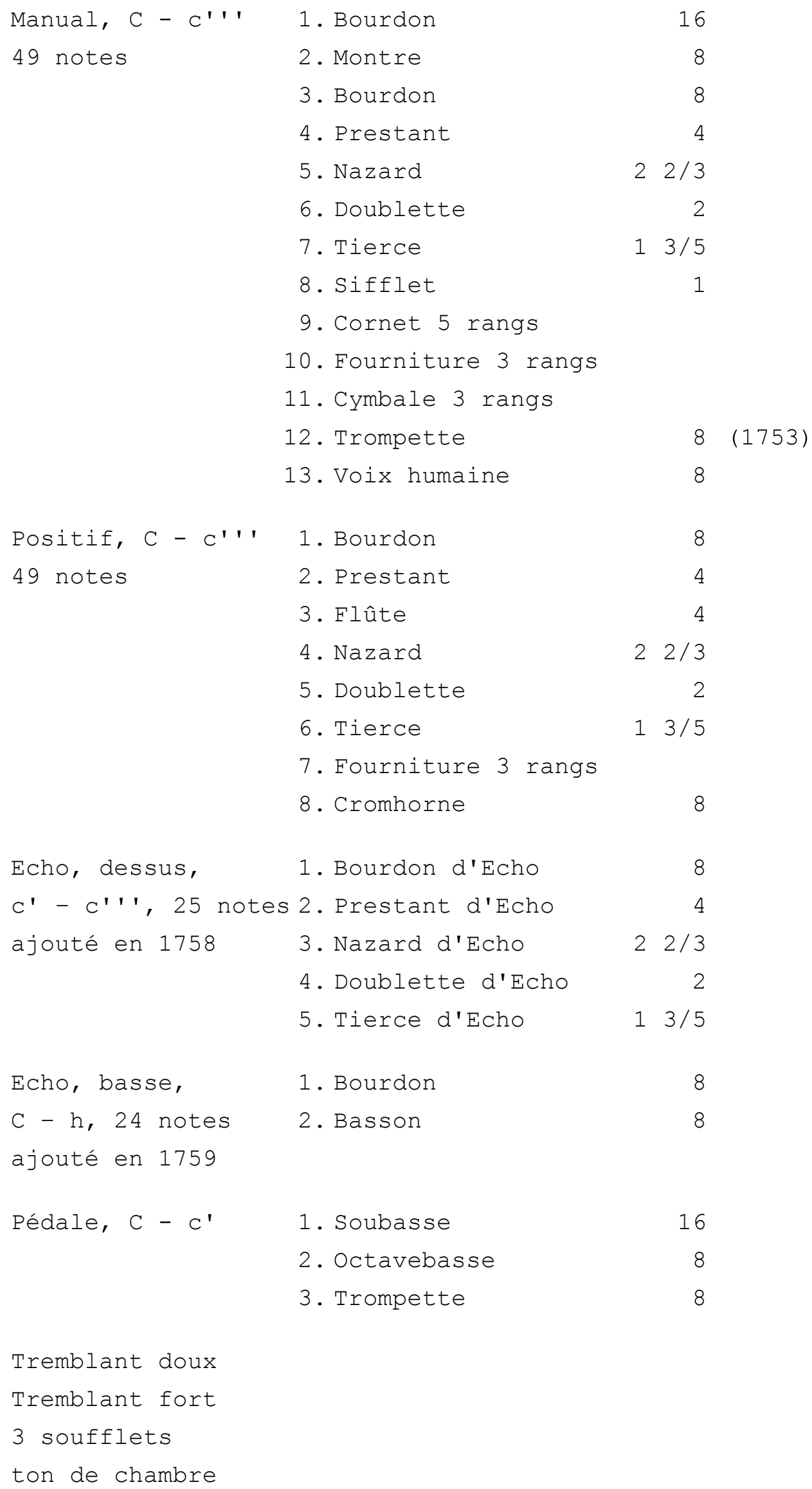


Baden-Baden, collégiale, 1753

\begin{tabular}{|c|c|c|c|}
\hline \multirow{2}{*}{$\begin{array}{l}\text { Manual, C - C''' } \\
49 \text { notes }\end{array}$} & 1. Montre & 8 & \\
\hline & 2. Bourdon & 8 & \\
\hline & 3. Prestant & 4 & \\
\hline & 4. Nazard & $22 / 3$ & \\
\hline & 5. Doublette & 2 & \\
\hline & 6. Tierce & $13 / 5$ & \\
\hline & 7. Sifflet & 1 & \\
\hline & 8. Cornet 5 rangs & & \\
\hline & 9. Fourniture & & \\
\hline & 10. Cymbale & & \\
\hline & 11. Trompette & 8 & place prévue (B/D) \\
\hline & 12. Voix humaine & 8 & place prévue \\
\hline Positif, C - C''' & 1. Bourdon & 8 & \\
\hline 49 notes & 2. Prestant & 4 & \\
\hline & 3. Flûte & 4 & \\
\hline & 4. Nazard & $22 / 3$ & \\
\hline & 5. Doublette & 2 & \\
\hline & 6. Tierce & $13 / 5$ & \\
\hline & 7. Fourniture & & \\
\hline & 8. Cromhorne & 8 & place prévue \\
\hline Pédale & 1. Soubasse & 16 & \\
\hline & 2. Octavebasse & 8 & \\
\hline & 3. Trompette & 8 & place prévue \\
\hline
\end{tabular}

Tremblant doux

Tremblant fort 
Baden-Baden, collégiale, orgue de choeur, 1754

$$
5 \text { jeux }
$$


Woerth, église mixte, 1754

$\begin{array}{lr}\text { Manual } & \text { 1. Bourdon } \\ \text { 2. Prestant } & 8 \\ \text { 3. Flûte } & 4 \\ \text { 4. Nazard } & 2 / 3 \\ \text { 5. Doublette } & 12 / 5 \\ \text { 6. Tierce } & \\ \text { 7. Cornet 5 rangs } & \\ \text { 8. Fourniture 3 rangs } & \\ \text { 9. Cymbale 3 rangs } & \\ \text { 1. Soubasse } & 8 \\ \text { 2. Octavebasse } & 8 \\ \text { 3. Trompette } & \end{array}$

Tremblant doux 
Colmar, Collégiale Saint-Martin, 1755

\begin{tabular}{|c|c|c|c|c|}
\hline Manual , & $C-C^{\prime} \cdot 1$ & 1. Bourdon & 16 & \\
\hline \multirow[t]{13}{*}{49 notes } & & 2. Montre & 8 & \\
\hline & & 3. Bourdon & 8 & \\
\hline & & 4. Prestant & 4 & \\
\hline & & 5. Nazard & $22 / 3$ & \\
\hline & & 6. Doublette & 2 & \\
\hline & & 7. Tierce & $13 / 5$ & \\
\hline & & 8. Sifflet & 1 & \\
\hline & & 9. Cornet 5 rangs & & \\
\hline & & 10. Fourniture 4 rangs & & \\
\hline & & 11. Cymbale 3 rangs & & \\
\hline & & 12. Trompette & 8 & basse/dessus \\
\hline & & 13. Clairon & 4 & basse/dessus \\
\hline & & 14. Voix humaine & 8 & \\
\hline \multicolumn{2}{|c|}{ Positif, $C-C^{\prime \prime \prime}$} & 1. Montre & 8 & \\
\hline \multirow[t]{10}{*}{49 notes } & & 2. Bourdon & 8 & \\
\hline & & 3. Prestant & 4 & \\
\hline & & 4. Flûte & 4 & \\
\hline & & 5. Nazard & $22 / 3$ & \\
\hline & & 6. Doublette & 2 & \\
\hline & & 7. Tierce & $13 / 5$ & \\
\hline & & 8. Larigot & $11 / 3$ & \\
\hline & & 9. Fourniture 3 & rangs & \\
\hline & & 10. Carillon 2 rangs & & \\
\hline & & 11. Cromhorne & 8 & \\
\hline \multirow[t]{7}{*}{ Echo } & & 1. Bourdon & 8 & \\
\hline & & 2. Prestant & 4 & \\
\hline & & 3. Nazard & $22 / 3$ & \\
\hline & & 4. Doublette & 2 & \\
\hline & & 5. Tierce & $13 / 5$ & \\
\hline & & 6. Trompette & 8 & \\
\hline & & 7. Voix humaine & 8 & \\
\hline
\end{tabular}




$\begin{array}{llr}\text { Pédale, C }- \text { C' }^{\prime} & \text { 1. Soubasse ouverte } & 16 \\ 25 \text { notes } & \text { 2. Octavebasse } & 8 \\ & \text { 3. Prestant } & 4 \\ \text { 4. Bombarde } & 16 \text { en étain } \\ \text { 5. Trompette } & 8 \\ \text { 6. Clairon } & 4\end{array}$

2 Tremblants doux

Tremblant fort

4 soufflets

ton d'opéra 
Pairis, abbaye cistercienne, 1755

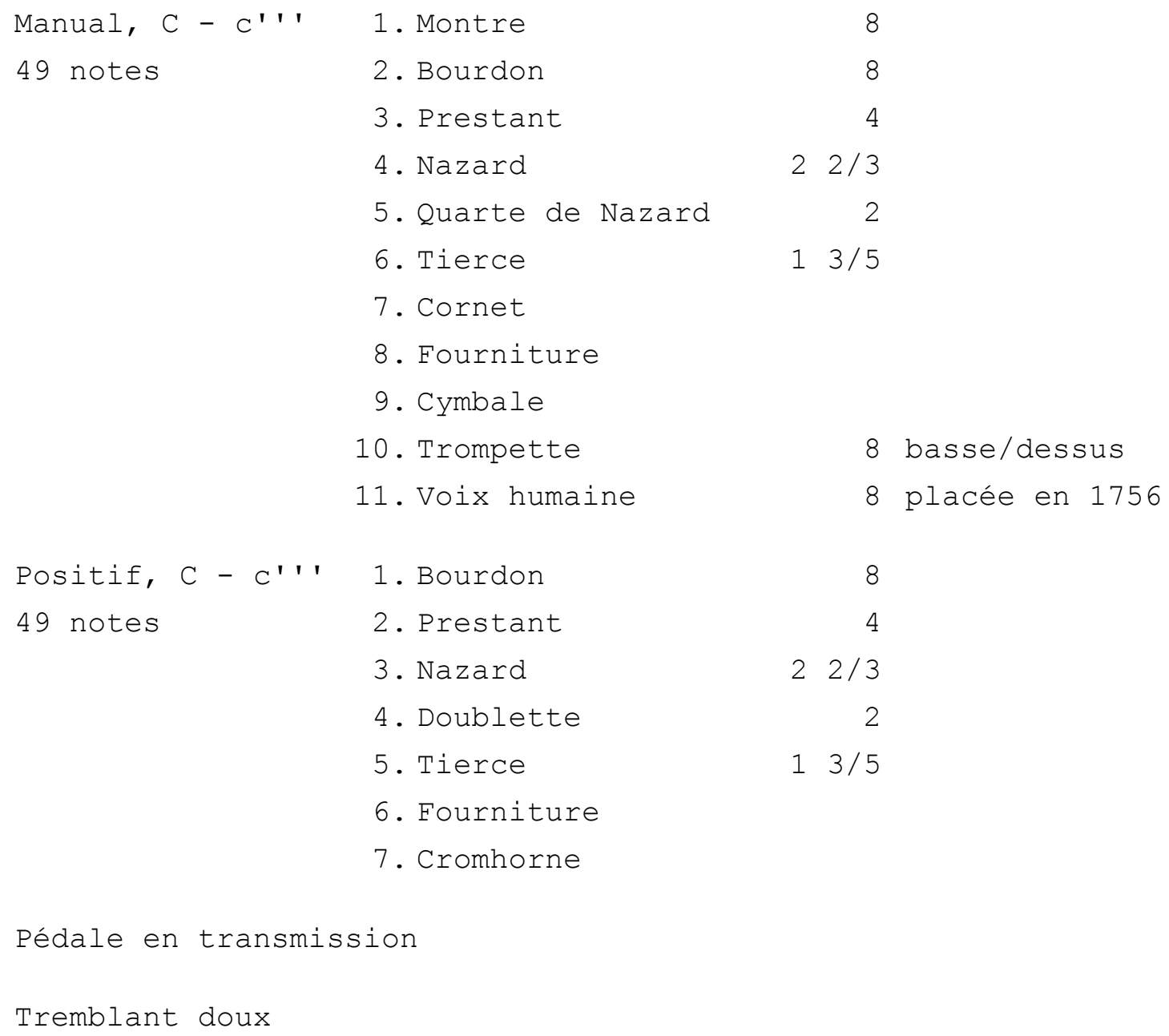


Offendorf, église catholique, 1756

Manual

1. Bourdon 8

2. Prestant 4

3. Flûte 4

4. Nazard $22 / 3$

5. Doublette 2

6. Tierce $13 / 5$

7. Cornet

8. Fourniture 3 rangs

9. Chalumeau 8

Pédale 1. Octavebasse 8

2. Prestant 4

Tremblant 
Kehl, église du village, 1758

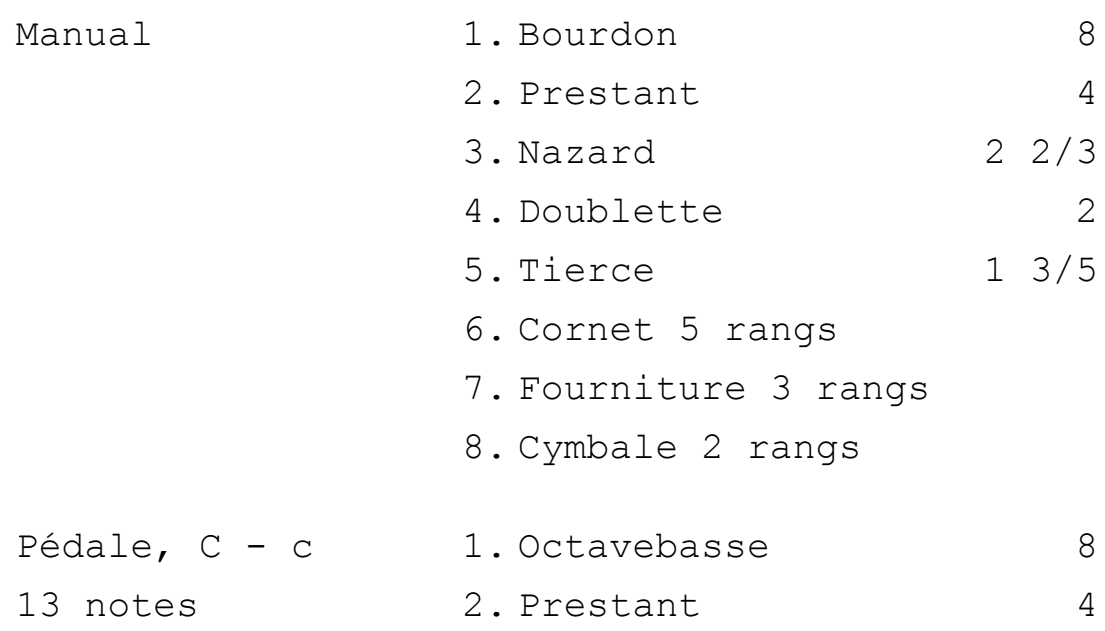


Amtenhausen, église abbatiale, 1758

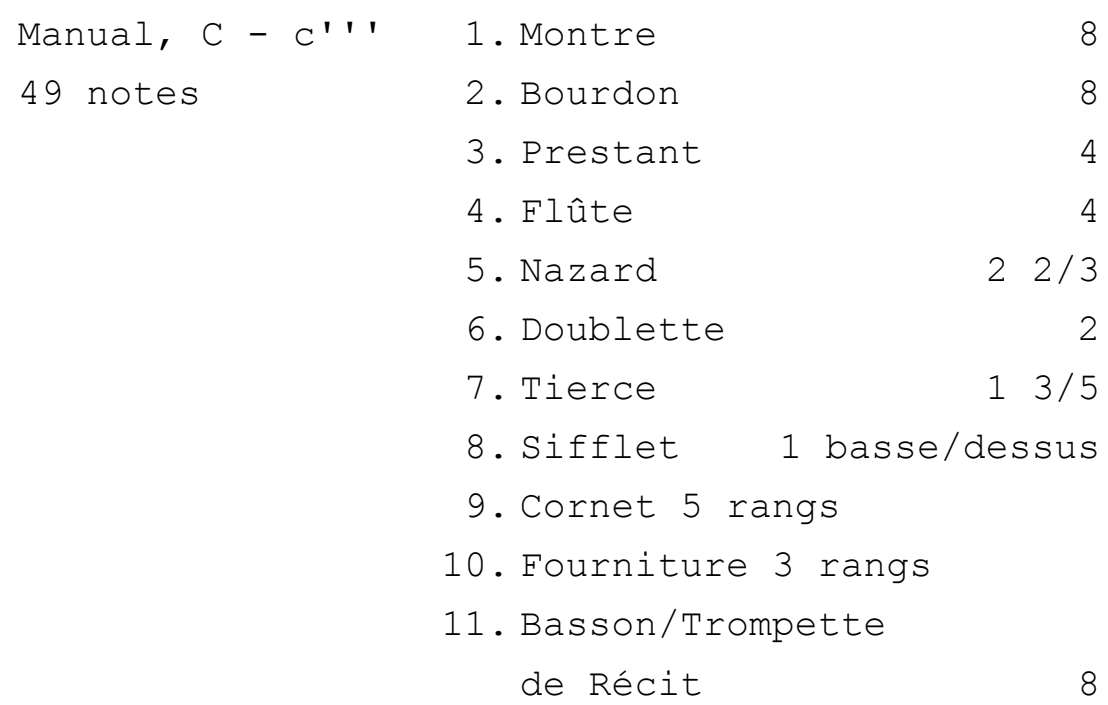
8 8 4

3

8 basse/dessus

Pédale, C - c

en transmission

13 notes

Tremblants 
Scherwiller, église catholique, 1759

Manual

1. Bourdon

8

2. Prestant

4

3. Flûte 4

4. Nazard

$22 / 3$

5. Doublette 2

6. Tierce

$13 / 5$

7. Cornet

8. Fourniture

9. Cromhorne

Pédale en transmission 
Rosheim, église Saint-Etienne, 1760

\begin{tabular}{|c|c|c|c|}
\hline \multirow{11}{*}{$\begin{array}{l}\text { Manual, } C-C^{\prime \prime} \\
49 \text { notes }\end{array}$} & 1. Montre & 8 & \\
\hline & 2. Bourdon & 8 & \\
\hline & 3. Prestant & 4 & \\
\hline & 4. Flûte & 4 & \\
\hline & 5. Nazard & $22 / 3$ & \\
\hline & 6. Doublette & 2 & \\
\hline & 7. Tierce & $13 / 5$ & \\
\hline & 8. Sifflet & 1 & basse/dessus \\
\hline & 9. Cornet 5 rangs & & \\
\hline & 10. Fourniture 3 rangs & & \\
\hline & 11. Trompette & 8 & basse/dessus \\
\hline
\end{tabular}

Tremblant doux

Tremblant fort 
Hipsheim, chapelle Saint-Ludan, 1760

$\begin{array}{lr}\text { Manual } & \text { Bourdon } \\ \text { 2. Prestant } & 4 \\ \text { 3. Nazard } & 2 \text { 2/3 } \\ \text { 4. Doublette } & 2 \\ \text { 5. Tierce } & 13 / 5 \\ \text { 6. Cornet } & \\ \text { 7. Fourniture } & \\ \text { 8. Cymbale (remplacée par Cromhorne 8 en 1777) }\end{array}$


Arlesheim, collégiale, 1761

\begin{tabular}{|c|c|c|c|c|}
\hline \multirow{2}{*}{$\begin{array}{l}\text { Manual, C - C''' } \\
49 \text { notes }\end{array}$} & 1. Bourdon & \multicolumn{2}{|r|}{16} & \\
\hline & 2. Montre & & 8 & \\
\hline & 3. Bourdon & & 8 & \\
\hline & 4. Prestant & & 4 & \\
\hline & 5. Nazard & 2 & $2 / 3$ & \\
\hline & 6. Doublette & & 2 & \\
\hline & 7. Tierce & 1 & $3 / 5$ & \\
\hline & 8.Sifflet & & 1 & \\
\hline & 9. Cornet 5 rangs & & & \\
\hline & 10. Fourniture 3 rangs & & & \\
\hline & 11. Cymbale 2 rangs & & & \\
\hline & 12. Trompette & & 8 & basse/dessus \\
\hline & 13. Voix humaine & & 8 & \\
\hline Positif, C- C''' & 1. Bourdon & & 8 & \\
\hline 49 notes & 2. Prestant & & 4 & \\
\hline & 3. Flûte & & 4 & \\
\hline & 4. Nazard & 2 & $2 / 3$ & \\
\hline & 5. Doublette & & 2 & \\
\hline & 6. Tierce & 1 & $3 / 5$ & \\
\hline & 7. Larigot & 1 & $1 / 3$ & \\
\hline & 8. Fourniture 3 rangs & & & \\
\hline & 9. Cromhorne & & 8 & \\
\hline Echo basse, $\mathrm{C}-\mathrm{h}$ & 1. Bourdon & & 8 & \\
\hline 24 notes & 2. Prestant & & 4 & \\
\hline & 3. Nazard & 2 & $2 / 3$ & (?) \\
\hline & 4. Doublette & & 2 & (?) \\
\hline & 5. Basson & & 8 & \\
\hline Echo dessus, $\mathrm{C}^{\prime}$ - & $C^{\prime} \prime^{\prime}$ & & & \\
\hline 25 notes & 1. Bourdon & & 8 & \\
\hline & 2. Prestant & & 4 & \\
\hline & 3. Nazard & 2 & $2 / 3$ & \\
\hline & 4. Doublette & & 2 & \\
\hline & 5. Tierce & 1 & $3 / 5$ & \\
\hline & 6. Flageolet & & 1 & \\
\hline
\end{tabular}


Pédale, c - c'

25 notes

Tremblant doux

Tremblant fort

ton de chambre
16

$\begin{array}{ll}\text { 2. Octavebasse } & 8 \\ \text { 3. Trompette } & 8\end{array}$

$\begin{array}{ll}\text { 2. Octavebasse } & 8 \\ \text { 3. Trompette } & 8\end{array}$

3. rompette 
Sarrebruck, église luthérienne (Ludwigskirche), 1762

- projet non exécuté -

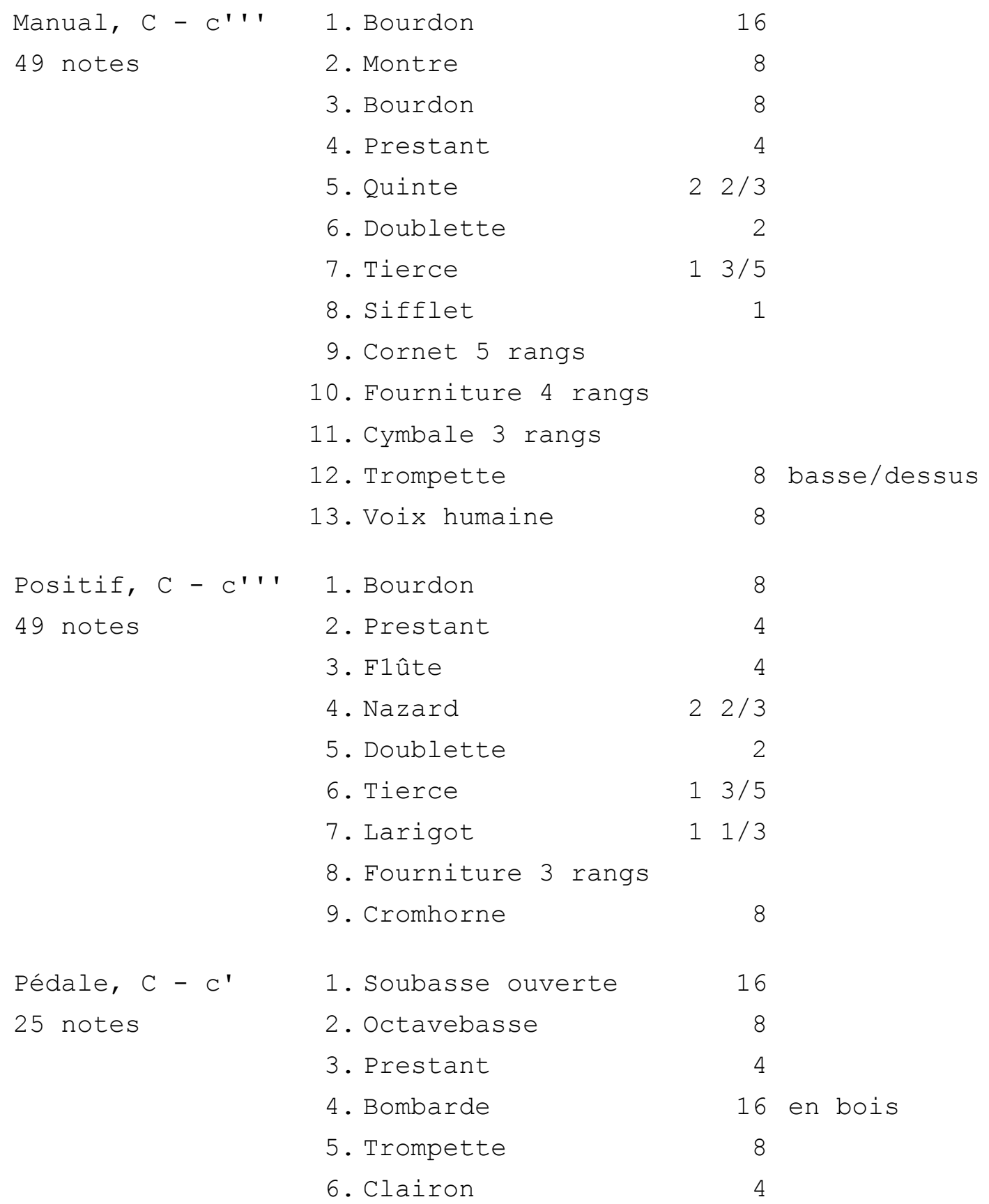

Tremblant doux

Tremblant fort

4 soufflets 
Strasbourg, église catholique Saint-Pierre-le-Jeune, 1762

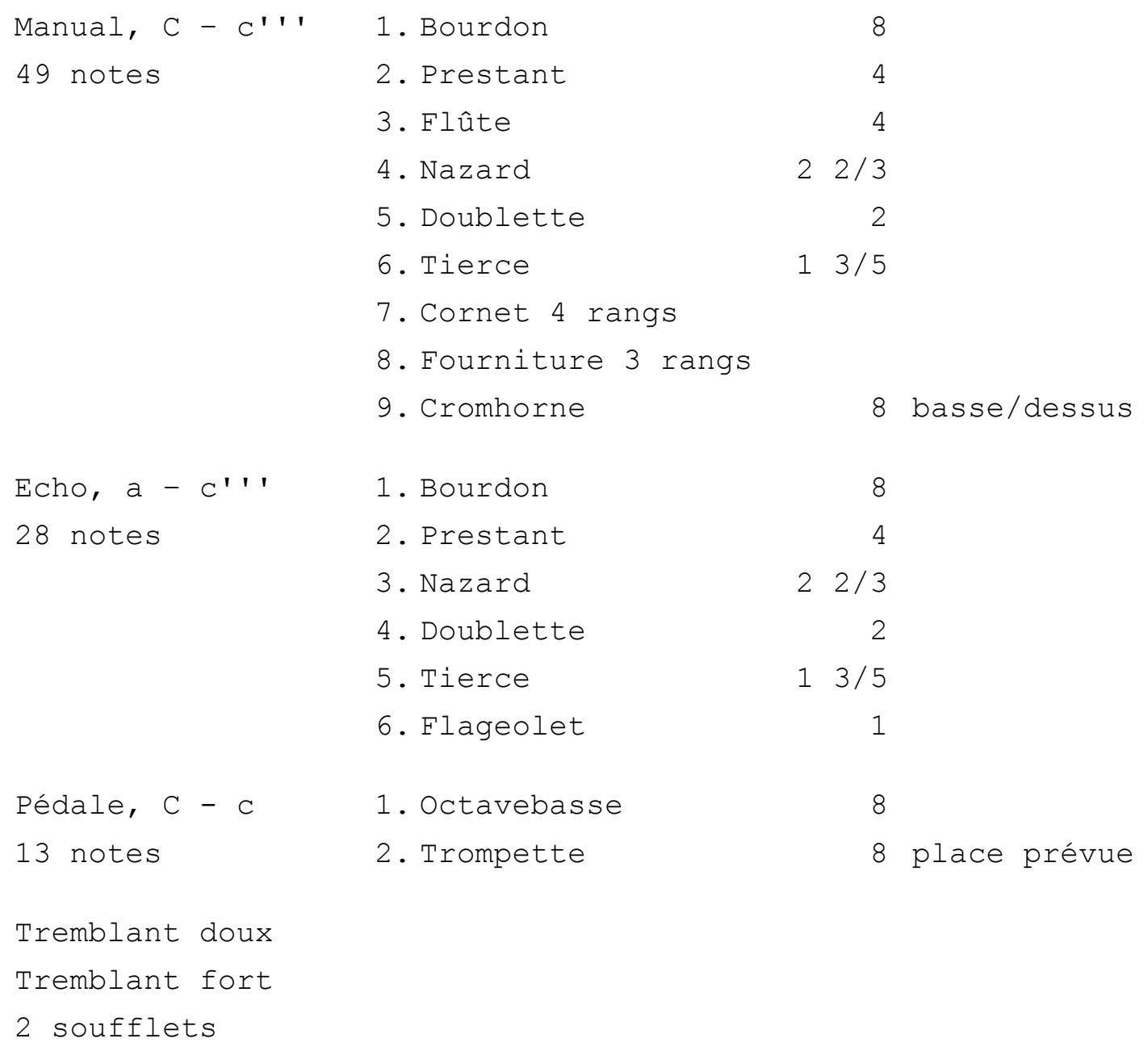


Strasbourg, église catholique Saint-Jean, 1763

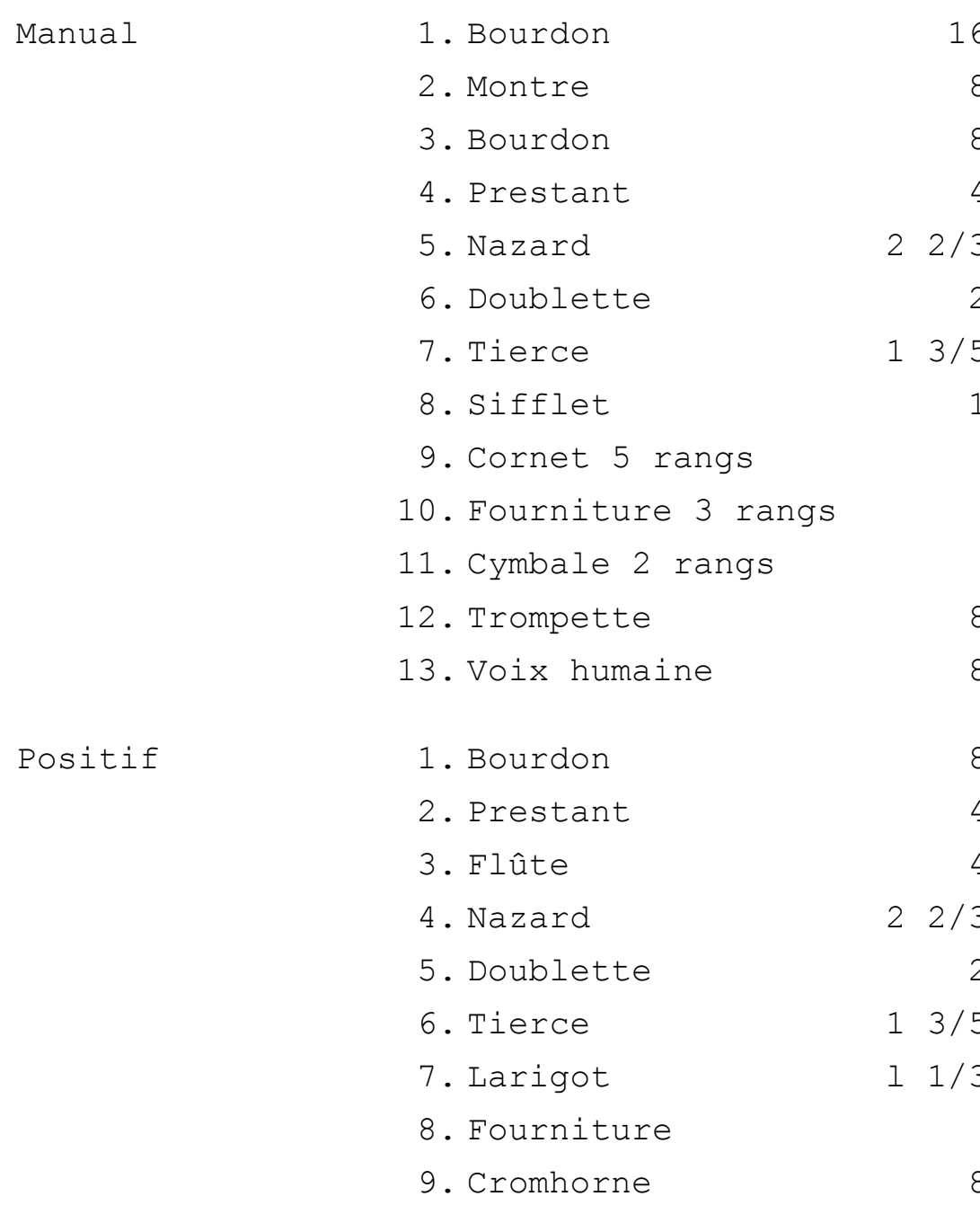

$\begin{array}{llr}\text { Echo basse } & \text { 1. Bourdon } \\ \text { 2. Prestant } & 8 \\ \text { 3. Basson } & 4 \\ \text { Echo dessus } & \text { 1. Bourdon } \\ \text { 2. Prestant } & 8 \\ \text { 3. Nazard } & 4 \\ \text { 4. Doublette } & 2 / 3 \\ \text { 5. Tierce } & 13 / 5\end{array}$


Pédale, c - c'

25 notes

Tremblant doux

Tremblant fort
16

2. Octavebasse 8

3. Trompette 8 
Wissembourg, église luthérienne Saint-Michel, 1765

Manual

Pédale

Tremblant fort
1. Bourdon 8

2. Prestant

3. Nazard

$22 / 3$

4. Doublette

2

5. Tierce

$13 / 5$

6. Larigot

$11 / 3$

7. Sifflet

8. Cornet 5 rangs

9. Fourniture 3 rangs

10. Cymbale 2 rangs

1. Soubasse

16

2. Octavebasse 4 1

8 
Châtenois, église catholique, 1765

\begin{tabular}{|c|c|c|c|}
\hline \multirow{2}{*}{$\begin{array}{l}\text { Manual, } C-C^{\prime \prime \prime} \\
49 \text { notes }\end{array}$} & 1. Montre & 8 & \\
\hline & 2. Bourdon & 8 & \\
\hline & 3. Prestant & 4 & \\
\hline & 4. Nazard & $22 / 3$ & \\
\hline & 5. Doublette & 2 & \\
\hline & 6. Tierce & $13 / 5$ & \\
\hline & 7. Sifflet & 1 & basse/dessus \\
\hline & 8. Cornet 5 rangs & & \\
\hline & 9. Fourniture 3 rangs & & \\
\hline & 10. Trompette & 8 & basse/dessus \\
\hline Echo, $C^{\prime}-C^{\prime}{ }^{\prime}$ & 1. Bourdon & 8 & \\
\hline 25 notes & 2. Prestant & 4 & \\
\hline & 3. Nazard & $22 / 3$ & \\
\hline & 4. Doublette & 2 & \\
\hline & 5. Tierce & $13 / 5$ & \\
\hline & 6. Flageolet & 1 & \\
\hline Pédale, C - c & 1. Soubasse & 16 & \\
\hline 13 notes & 2. Octavebasse & 8 & \\
\hline & 3. Trompette & 8 & \\
\hline Tremblant doux & & & \\
\hline Tremblant fort & & & \\
\hline 3 soufflets & & & \\
\hline on de chambre & & & \\
\hline
\end{tabular}


Mulhouse, église protestante Saint-Etienne, 1765-1766

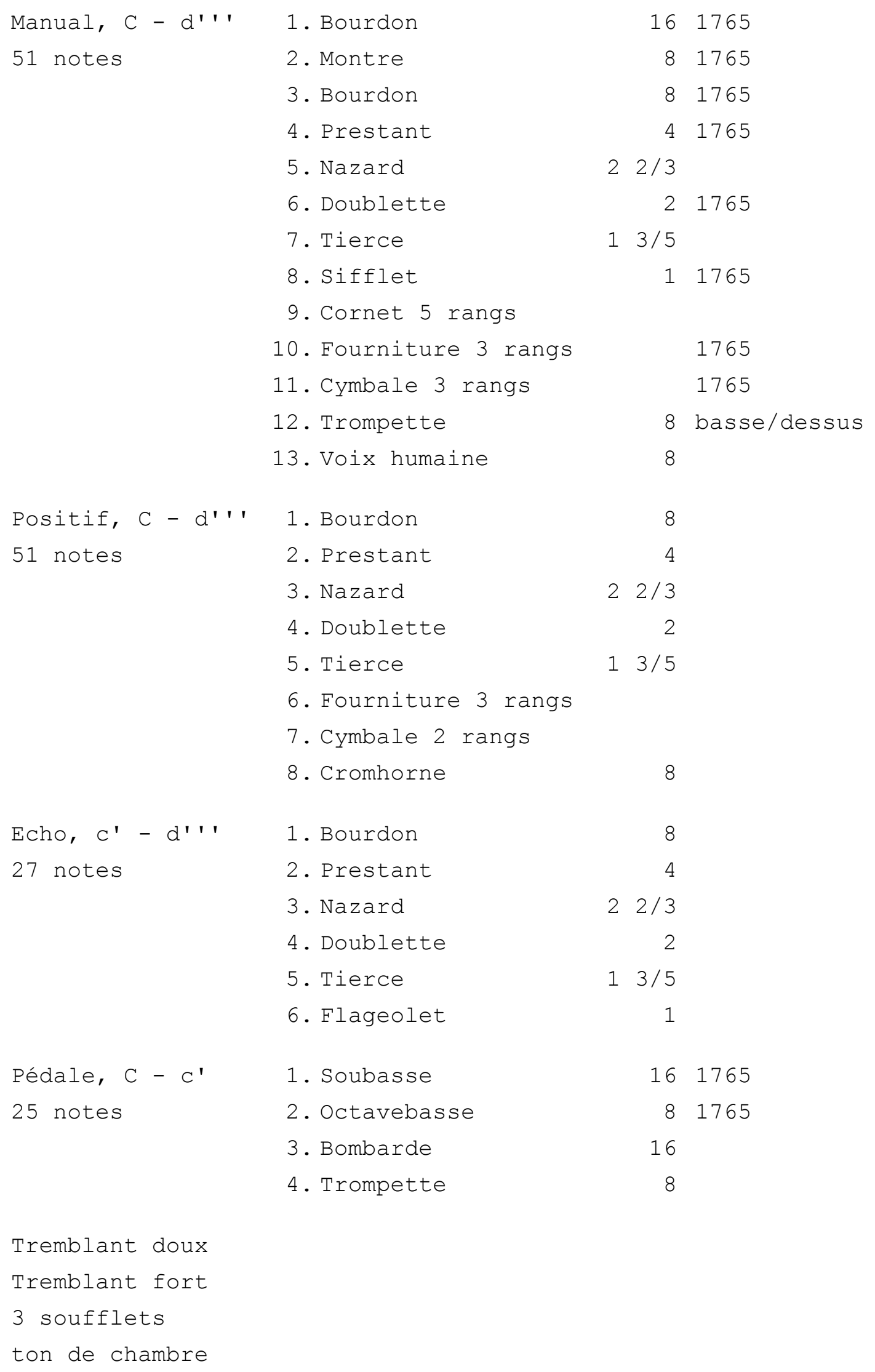


Bettenhoffen, église catholique, 1767

\begin{tabular}{|c|c|c|c|}
\hline \multirow{9}{*}{ Manual } & 1. Bourdon & 8 & \\
\hline & 2. Prestant & 4 & \\
\hline & 3. Flûte & 4 & \\
\hline & 4. Nazard & $22 / 3$ & \\
\hline & 5. Doublette & 2 & \\
\hline & 6. Tierce & $13 / 5$ & \\
\hline & 7. Cornet & & \\
\hline & 8. Fourniture & & \\
\hline & 9. Cromhorne & 8 & basse/dessus \\
\hline \multirow[t]{6}{*}{ Echo } & 1. Bourdon & 8 & \\
\hline & 2. Prestant & 4 & \\
\hline & 3. Nazard & $22 / 3$ & \\
\hline & 4. Doublette & 2 & \\
\hline & 5. Tierce & $13 / 5$ & \\
\hline & 6. Flageolet & 1 & \\
\hline \multirow[t]{3}{*}{ Pédale } & 1. Octavebasse & 8 & \\
\hline & 2. Prestant & 4 & \\
\hline & 3. Trompette & 8 & \\
\hline
\end{tabular}

Tremblant doux

Tremblant fort 
Bâle, église française Saint-Jean (Predigerkirche), 1767-1769

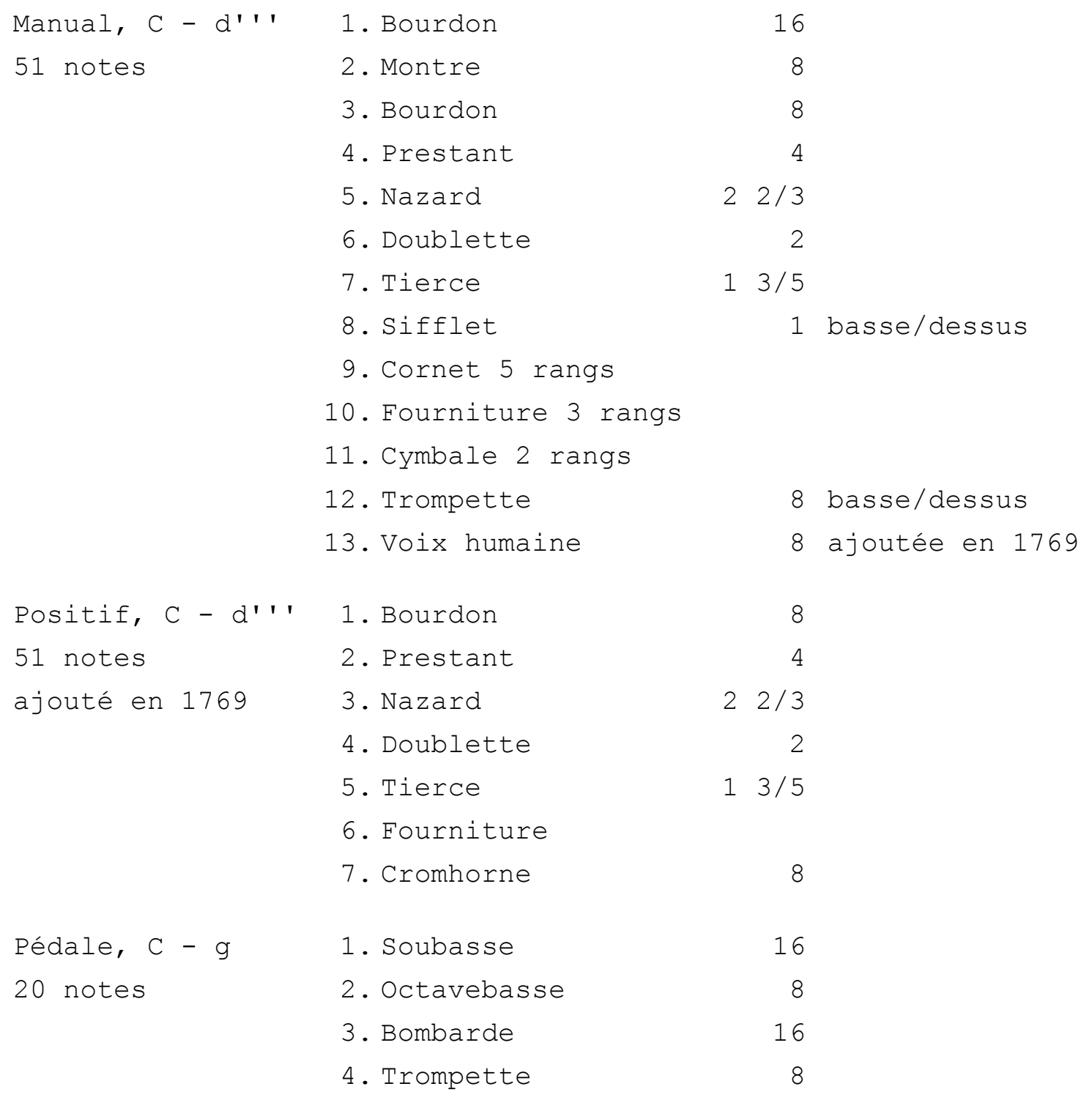

Tremblant doux

Tremblant fort

3 soufflets

ton de chambre 
Sélestat, église catholique Saint-Georges, 1768

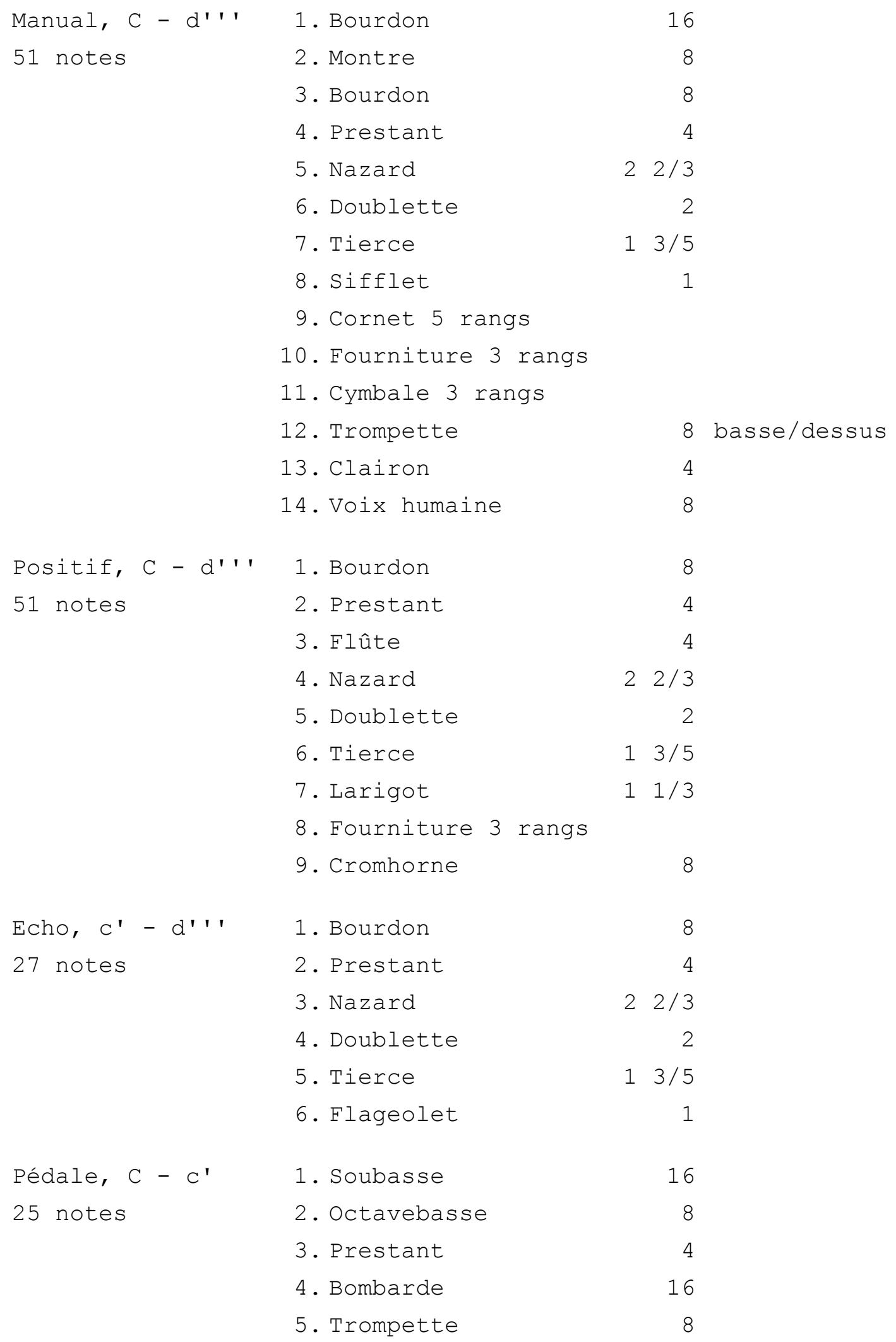

Tremblant doux

Tremblant fort 
Neuwiller-les-Saverne, église Saints-Pierre-et-Paul, 1769 - projet non exécuté -

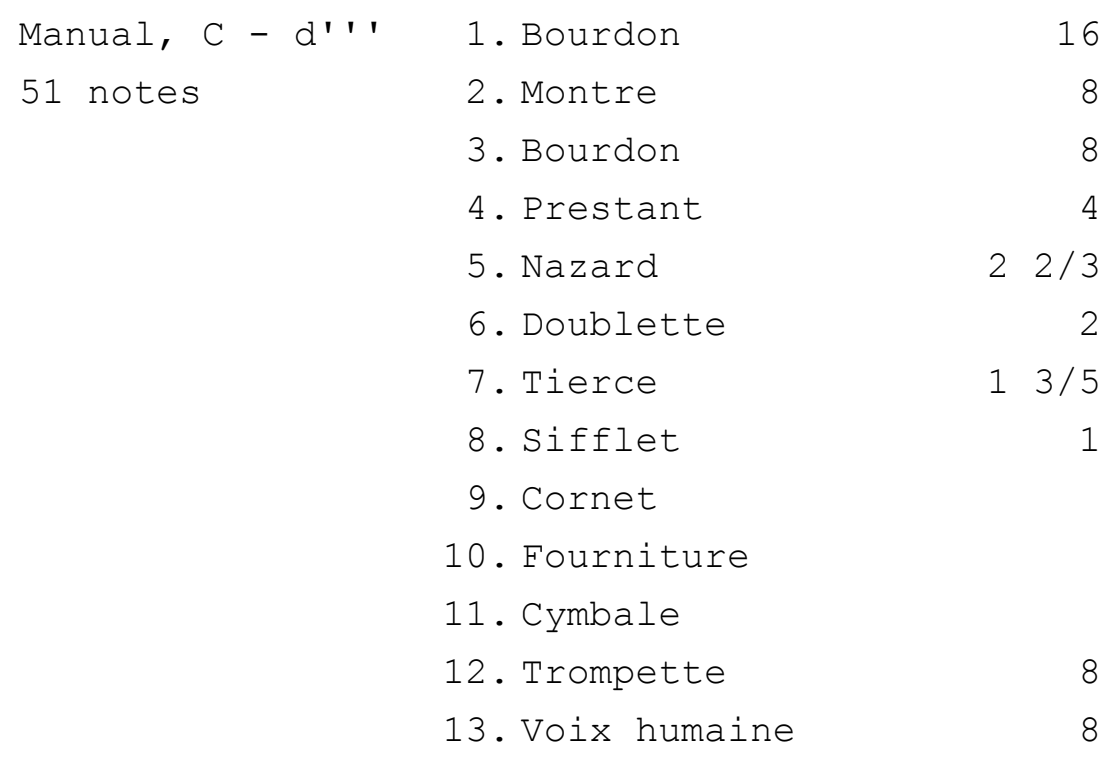

Positif, C - d''' 1. Bourdon 8

51 notes 2. Prestant 4

3. Flûte 4

4. Nazard $22 / 3$

5. Doublette 2

6. Tierce $13 / 5$

7. Larigot $11 / 3$

8. Fourniture

9. Cromhorne 8

Echo

1. Bourdon 8

2. Prestant 4

3. Nazard $22 / 3$

4. Doublette 2

5. Tierce $13 / 5$

6. Flageolet 1

Pédale, $\mathrm{C}-\mathrm{g}$ 1. Soubasse 16

20 notes 2. Octavebasse 8

3. Prestant 4

4. Trompette 8

5. Clairon 4

3 Tremblants

3 soufflets

ton de chambre 
Bâle, église Saint-Théodore, 1770

\begin{tabular}{|c|c|c|c|}
\hline \multirow{13}{*}{$\begin{array}{l}\text { Manual, } C-d \text { '' } \\
51 \text { notes }\end{array}$} & 1. Bourdon & 16 & \\
\hline & 2. Montre & 8 & \\
\hline & 3. Bourdon & 8 & \\
\hline & 4. Prestant & 4 & \\
\hline & 5. Nazard & $22 / 3$ & \\
\hline & 6. Doublette & 2 & \\
\hline & 7. Tierce & $13 / 5$ & \\
\hline & 8. Sifflet & 1 & \\
\hline & 9. Cornet 5 rangs & & \\
\hline & 10. Fourniture 3 rangs & & \\
\hline & 11. Cymbale 2 rangs & & \\
\hline & 12. Trompette & 8 & basse/dessus \\
\hline & 13. Voix humaine & 8 & \\
\hline Positif, C - d''' & 1. Bourdon & 8 & \\
\hline 51 notes & 2. Prestant & 4 & \\
\hline & 3. Nazard & $22 / 3$ & \\
\hline & 4. Doublette & 2 & \\
\hline & 5. Tierce & $13 / 5$ & \\
\hline & 6. Fourniture 3 rangs & & \\
\hline & 7. Cromhorne & 8 & \\
\hline Echo & 1. Bourdon & 8 & \\
\hline & 2. Prestant & 4 & \\
\hline & 3. Nazard & $22 / 3$ & \\
\hline & 4. Doublette & 2 & \\
\hline & 5. Tierce & $13 / 5$ & \\
\hline & 6. Flageolet & 1 & \\
\hline Pédale, C - g & 1. Soubasse & 16 & \\
\hline 20 notes & 2. Octavebasse & 8 & \\
\hline & 3. Bombarde & 16 & \\
\hline & 4. Trompette & 8 & \\
\hline
\end{tabular}


Riegel, église catholique, 1770

\begin{tabular}{|c|c|c|c|}
\hline Manual, c - d''' & 1. Montre & 8 & \\
\hline \multirow[t]{10}{*}{51 notes } & 2. Bourdon & 8 & \\
\hline & 3. Prestant & 4 & \\
\hline & 4. Flûte & 4 & \\
\hline & 5. Nazard & $22 / 3$ & \\
\hline & 6. Doublette & 2 & \\
\hline & 7. Tierce & $13 / 5$ & \\
\hline & 8. Sifflet & 1 & basse/dessus \\
\hline & 9. Cornet 5 rangs & & \\
\hline & 10. Fourniture 3 rangs & & \\
\hline & 11. Basson/Trompette & 8 & basse/dessus \\
\hline Pédale, $\mathrm{C}-\mathrm{d}$ & 1. Soubasse & 16 & \\
\hline \multirow[t]{2}{*}{15 notes } & 2. Octavebasse & 8 & \\
\hline & 3. Trompette & 8 & \\
\hline 2 Tremblants & & & \\
\hline 2 soufflets & & & \\
\hline
\end{tabular}


Guebwiller, couvent des Dominicaines, 1771

\begin{tabular}{|c|c|c|c|}
\hline \multirow{2}{*}{$\begin{array}{l}\text { Manual, } C-d ' \cdot ' \\
51 \text { notes }\end{array}$} & 1. Bourdon & 8 & $C-c$ ouvert \\
\hline & 2. Prestant & 4 & \\
\hline & 3. Flûte & 4 & \\
\hline & 4. Nazard & $22 / 3$ & \\
\hline & 5. Doublette & 2 & \\
\hline & 6. Tierce & $13 / 5$ & \\
\hline & 7. Sifflet & 1 & basse/dessus \\
\hline & 8. Cornet 5 rangs & & \\
\hline & 9. Fourniture 3 rangs & & \\
\hline & 10. Basson/Trompette & 8 & basse/dessus \\
\hline Pédale, C - c & jeux empruntés au M & anual: & \\
\hline 13 notes & 1. Bourdon & 8 & \\
\hline & 2. Prestant & 4 & \\
\hline & 3. Basson & 8 & \\
\hline
\end{tabular}

Tremblant doux

Tremblant fort

2 soufflets

ton de chambre 
Colmar, couvent des Catherinettes, 1772

\begin{tabular}{|c|c|c|c|}
\hline \multirow{11}{*}{$\begin{array}{l}\text { Manual, C } \\
51 \text { notes }\end{array}$} & 1. Montre & 8 & \\
\hline & 2. Bourdon & 8 & \\
\hline & 3. Prestant & 4 & \\
\hline & 4. Nazard & $22 / 3$ & \\
\hline & 5. Doublette & 2 & \\
\hline & 6. Tierce & $13 / 5$ & \\
\hline & 7. Larigot & $11 / 3$ & \\
\hline & 8. Sifflet & 1 & basse/dessus \\
\hline & 9. Cornet 5 rangs & & \\
\hline & 10. Fourniture 3 rangs & & \\
\hline & 11. Basson/Trompette & 8 & basse/dessus \\
\hline \multirow{6}{*}{$\begin{array}{l}\text { Positif, } C-d ' \text { ' } \\
51 \text { notes }\end{array}$} & 1. Bourdon & 8 & \\
\hline & 2. Flûte & 4 & \\
\hline & 3. Quinte & $22 / 3$ & \\
\hline & 4. Doublette & 2 & \\
\hline & 5. Tierce & $13 / 5$ & \\
\hline & 6. Fourniture 2 rangs & & \\
\hline \multirow{6}{*}{$\begin{array}{l}\text { Echo, c' - d''' } \\
27 \text { notes }\end{array}$} & 1. Bourdon & 8 & \\
\hline & 2. Prestant & 4 & \\
\hline & 3. Nazard & $22 / 3$ & \\
\hline & 4. Doublette & 2 & \\
\hline & 5. Tierce & $13 / 5$ & \\
\hline & 6. Flageolet & 1 & \\
\hline \multirow{5}{*}{$\begin{array}{l}\text { Pédale, } C-c \\
13 \text { notes }\end{array}$} & jeux empruntés au M & anual: & \\
\hline & 1. Montre & 8 & \\
\hline & 2. Bourdon & 8 & \\
\hline & 3. Prestant & 4 & \\
\hline & 4. Basson & 8 & \\
\hline
\end{tabular}

Tremblant doux

Tremblant fort

2 soufflets

ton d'opéra 
Sankt-Blasien, abbaye bénédictine, 1774

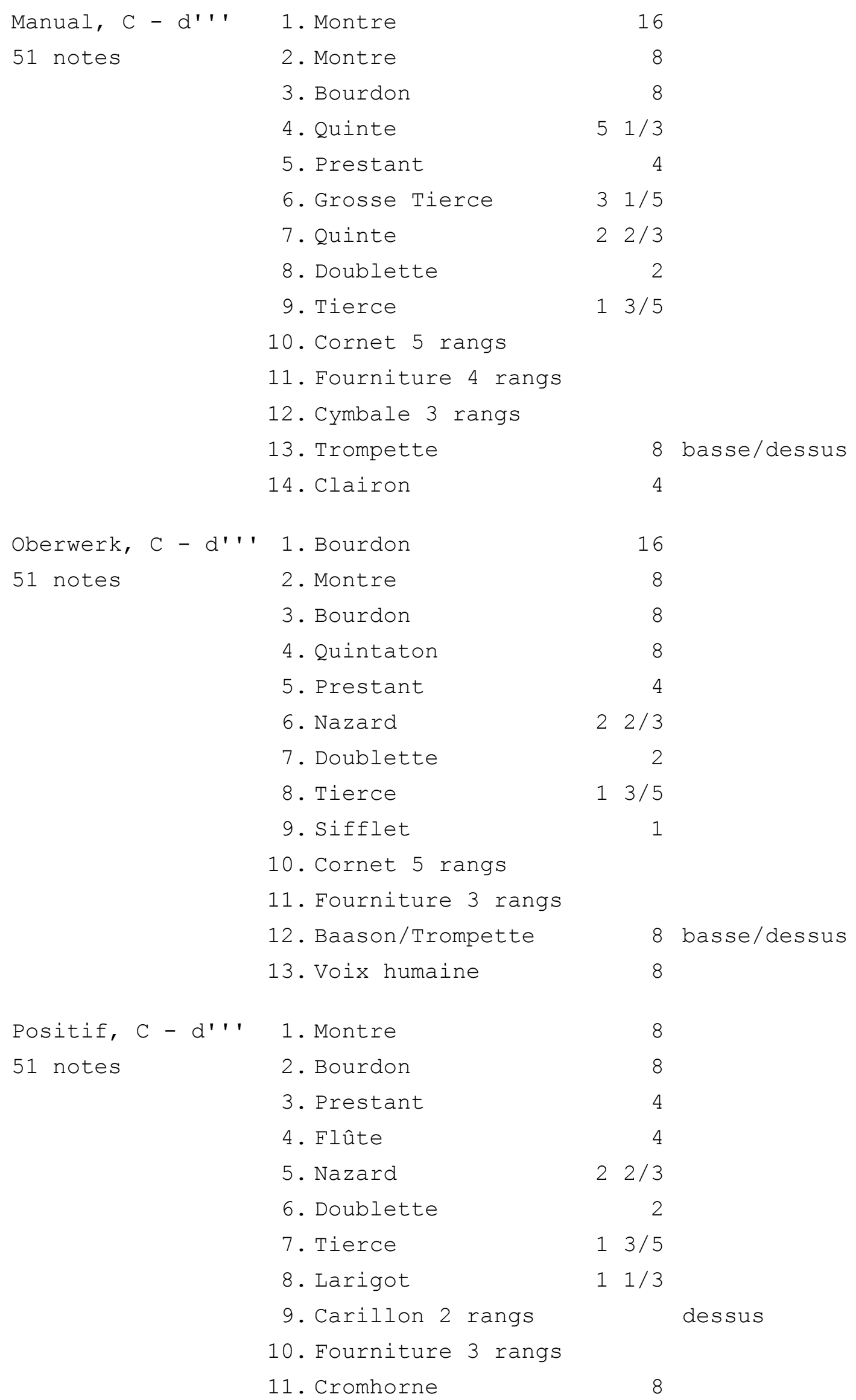


Pédale, c - c'

1. Principal

16

25 notes

2. Soubasse

16

3. Octavebasse

8

4. Prestant

4

5. Bombarde

16

6. Trompette

8

7. Clairon

4

8. Cornetto

2

Tremblant doux

Tremblant fort

Tremblant doux ("Schwebung") à l'Oberwerk

Accouplement Oberwerk/Manual

Accouplement Positif/Manual

6 soufflets

ton de chambre 
Schuttern, abbaye bénédictine, 1775

- projet non exécuté -

\begin{tabular}{|c|c|c|c|c|}
\hline Manual, C - d''' & 1. Bourdon & & 16 & \\
\hline 51 notes & 2. Montre & & 8 & \\
\hline & 3. Bourdon & & 8 & \\
\hline & 4. Quintaton & & 8 & \\
\hline & 5. Prestant & & 4 & \\
\hline & 6. Quinte & 2 & $2 / 3$ & \\
\hline & 7. Doublette & & 2 & \\
\hline & 8. Tierce & 1 & $3 / 5$ & \\
\hline & 9. Sifflet & & 1 & \\
\hline & 10. Cornet 5 rangs & & & \\
\hline & 11. Fourniture 3 rangs & & & \\
\hline & 12. Cymbale 2 rangs & & & \\
\hline & 13. Trompette & & 8 & basse/dessus \\
\hline Oberwerk, C - d''' & 1. Montre & & 8 & \\
\hline 51 notes & 2. Bourdon & & 8 & \\
\hline & 3. Prestant & & 4 & \\
\hline & 4. Flûte & & 4 & \\
\hline & 5. Nazard & 2 & $2 / 3$ & \\
\hline & 6. Doublette & & 2 & \\
\hline & 7. Tierce & 1 & $3 / 5$ & \\
\hline & 8. Larigot & 1 & $1 / 3$ & \\
\hline & 9. Fourniture 3 rangs & & & \\
\hline & 10. Basson/Chalumeau & & 8 & basse/dessus \\
\hline & 11. Voix humaine & & 8 & \\
\hline Pédale, C $-C^{\prime}$ & 1. Soubasse & & 16 & \\
\hline 25 notes & 2. Octavebasse & & 8 & \\
\hline & 3. Trompette & & 8 & \\
\hline
\end{tabular}

Tremblant

Accouplement Oberwerk/Manual

5 soufflets

ton de chambre 
Mittelhausen, église protestante, 1776

- projet non exécuté -

$\begin{array}{lr}\text { Manual } & 8 \\ \text { 1. Bourdon } & 4 \\ \text { 3. Prestant } & 22 / 3 \\ \text { 4. Doublette } & 2 \\ \text { 5. Tierce } & 13 / 5 \\ \text { 6. Cornet 4 rangs } & \\ \text { 7. Fourniture } & \end{array}$

Pédale

1. Octavebasse 8

2. Prestant 4 
Meissenheim, église protestante, 1776

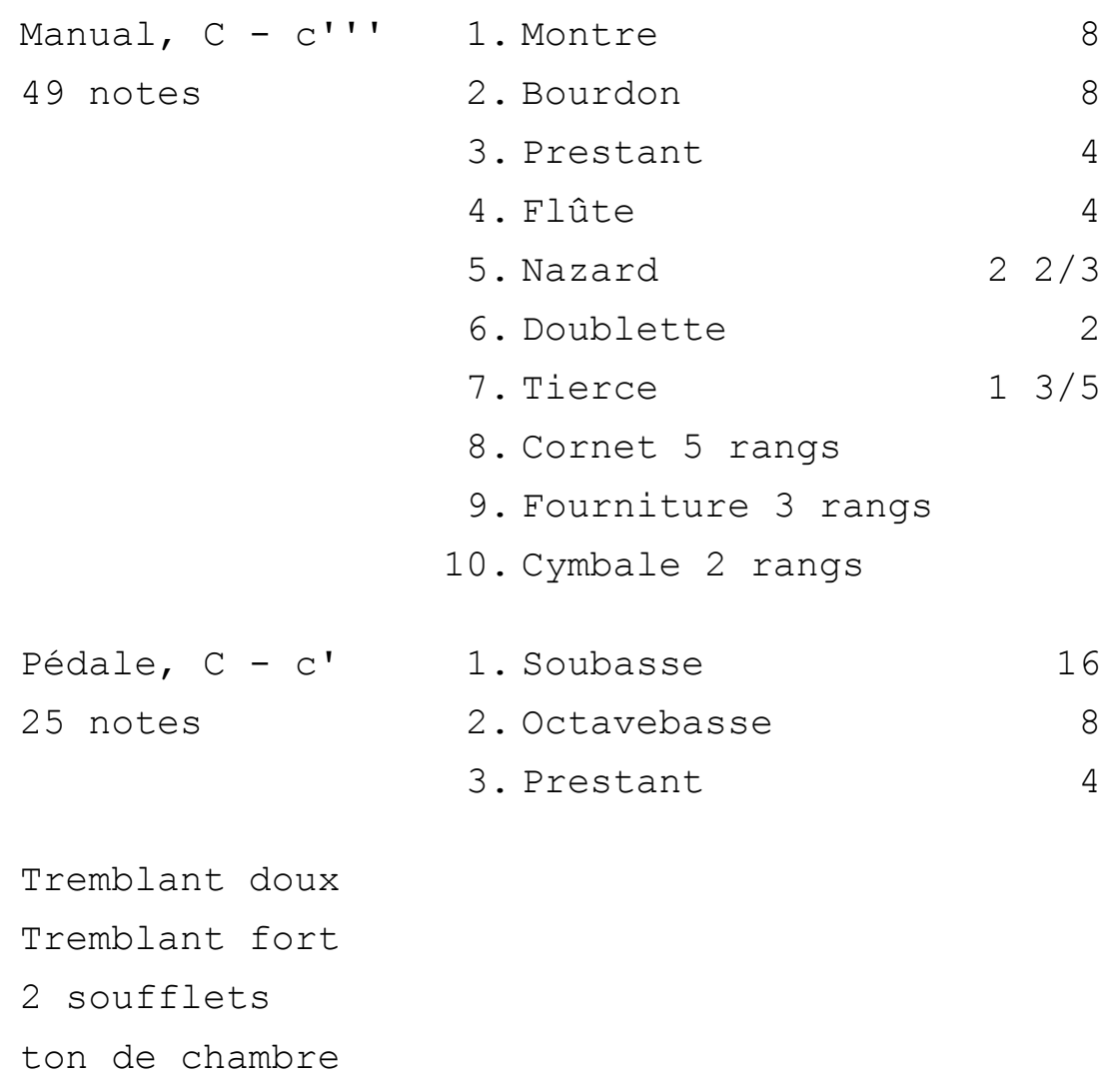


Sankt-Märgen, collégiale, 1777

\begin{tabular}{|c|c|c|}
\hline \multirow{11}{*}{$\begin{array}{l}\text { Manual, } c-d \text { '' } \\
51 \text { notes }\end{array}$} & 1. Montre & 8 \\
\hline & 2. Bourdon & 8 \\
\hline & 3. Prestant & 4 \\
\hline & 4. Flûte & 4 \\
\hline & 5. Nazard & $22 / 3$ \\
\hline & 6. Doublette & 2 \\
\hline & 7. Tierce & $13 / 5$ \\
\hline & 8. Larigot & $11 / 3$ \\
\hline & 9. Cornet 5 rangs & \\
\hline & 10. Fourniture 3 rangs & \\
\hline & 11. Basson/Trompette & 8 \\
\hline
\end{tabular}

Pédale, C - c jeux empruntés au Manual:

13 notes

$\begin{array}{ll}\text { 1. Montre } & 8 \\ \text { 2. Bourdon } & 8 \\ \text { 3. Prestant } & 4 \\ \text { 4. Basson } & 8\end{array}$

Tremblant

2 soufflets

ton de chambre 
Bouxwiller, église protestante, 1778

\begin{tabular}{|c|c|c|c|}
\hline \multirow{13}{*}{$\begin{array}{l}\text { Manual, } C-d \text { '' } \\
51 \text { notes }\end{array}$} & 1. Bourdon & 16 & \\
\hline & 2. Montre & 8 & \\
\hline & 3. Bourdon & 8 & \\
\hline & 4. Prestant & 4 & \\
\hline & 5. Quinte & $22 / 3$ & \\
\hline & 6. Doublette & 2 & \\
\hline & 7. Tierce & $13 / 5$ & \\
\hline & 8. Sifflet & 1 & \\
\hline & 9. Cornet 5 rangs & & \\
\hline & 10. Fourniture 3 rangs & & \\
\hline & 11. Cymbale 2 rangs & & \\
\hline & 12. Trompette & 8 & basse/dessus \\
\hline & 13. Voix humaine & 8 & \\
\hline Positif, $c$ - d''' & 1. Bourdon & 8 & \\
\hline \multirow[t]{7}{*}{51 notes } & 2. Prestant & 4 & \\
\hline & 3. Flûte & 4 & \\
\hline & 4. Nazard & $22 / 3$ & \\
\hline & 5. Doublette & 2 & \\
\hline & 6. Tierce & $13 / 5$ & \\
\hline & 7. Fourniture 3 rangs & & \\
\hline & 8. Cromhorne & 8 & \\
\hline Pédale, C - g & 1. Soubasse & 16 & \\
\hline \multirow[t]{3}{*}{20 notes } & 2. Octavebasse & 8 & \\
\hline & 3. Bombarde & 16 & \\
\hline & 4. Trompette & 8 & \\
\hline
\end{tabular}

Tremblant doux

Tremblant fort

3 soufflets

ton de chambre 
Offenburg, couvent des Franciscains, 1779

\begin{tabular}{|c|c|c|c|}
\hline Manual, c - d''' & 1. Montre & 8 & \\
\hline \multirow[t]{9}{*}{51 notes } & 2. Bourdon & 8 & \\
\hline & 3. Prestant & 4 & \\
\hline & 4. Nazard & $22 / 3$ & \\
\hline & 5. Doublette & 2 & \\
\hline & 6. Tierce & $13 / 5$ & \\
\hline & 7. Larigot & $11 / 3$ & \\
\hline & 8. Cornet 5 rangs & & \\
\hline & 9. Fourniture 3 rangs & & \\
\hline & 10. Basson/Trompette & 8 & basse/dessus \\
\hline \multicolumn{4}{|c|}{ Positif intérieur, C - d''' } \\
\hline \multirow[t]{4}{*}{51 notes } & 1. Bourdon & 8 & \\
\hline & 2. Flûte & 4 & \\
\hline & 3. Doublette & 2 & \\
\hline & 4. Sifflet & 1 & \\
\hline Pédale, c - c & 1. Soubasse & 16 & \\
\hline \multirow[t]{2}{*}{13 notes } & 2. Octavebasse & 8 & \\
\hline & 3. Trompette & 8 & \\
\hline
\end{tabular}


Blodelsheim, église catholique, 1779

\begin{tabular}{|c|c|c|c|}
\hline \multirow{2}{*}{$\begin{array}{l}\text { Manual, } C-d \text { '' } \\
51 \text { notes }\end{array}$} & 1. Bourdon & 8 & \\
\hline & 2. Prestant & 4 & \\
\hline & 3. Flûte & 4 & \\
\hline & 4. Nazard & $22 / 3$ & \\
\hline & 5. Doublette & 2 & \\
\hline & 6. Tierce & $13 / 5$ & \\
\hline & 7. Sifflet & & basse/dessus \\
\hline & 8. Cornet 5 rangs & & \\
\hline & 9. Fourniture 3 rangs & & \\
\hline & 10. Cromhorne & 8 & basse/dessus \\
\hline
\end{tabular}

Pédale, C - c jeux empruntés au Manual:

13 notes

$\begin{array}{ll}\text { 1. Bourdon } & 8 \\ \text { 2. Prestant } & 4 \\ \text { 3. Cromhorne } & 8\end{array}$


Strasbourg, église protestante Saint-Pierre-le-jeune, 1780

\begin{tabular}{|c|c|c|c|}
\hline Manual, $c-d '$ '' & 1. Bourdon & 16 & \\
\hline \multirow[t]{11}{*}{51 notes } & 2. Montre & 8 & \\
\hline & 3. Bourdon & 8 & \\
\hline & 4. Prestant & 4 & \\
\hline & 5. Quinte & $22 / 3$ & \\
\hline & 6. Doublette & 2 & \\
\hline & 7. Tierce & $13 / 5$ & \\
\hline & 8. Larigot & $11 / 3$ & \\
\hline & 9. Cornet 5 rangs & & \\
\hline & 10. Fourniture 3 rangs & & \\
\hline & 11. Cymbale 3 rangs & & \\
\hline & 12. Trompette & 8 & basse/dessus \\
\hline Pédale, C- g & 1. Soubasse & 16 & \\
\hline \multirow[t]{3}{*}{20 notes } & 2. Octavebasse & 8 & \\
\hline & 3. Bombarde & 16 & \\
\hline & 4. Trompette & 8 & \\
\hline
\end{tabular}

Tremblant doux

Tremblant fort

3 soufflets

ton de chambre 
Molsheim, église des Jésuites, 1781

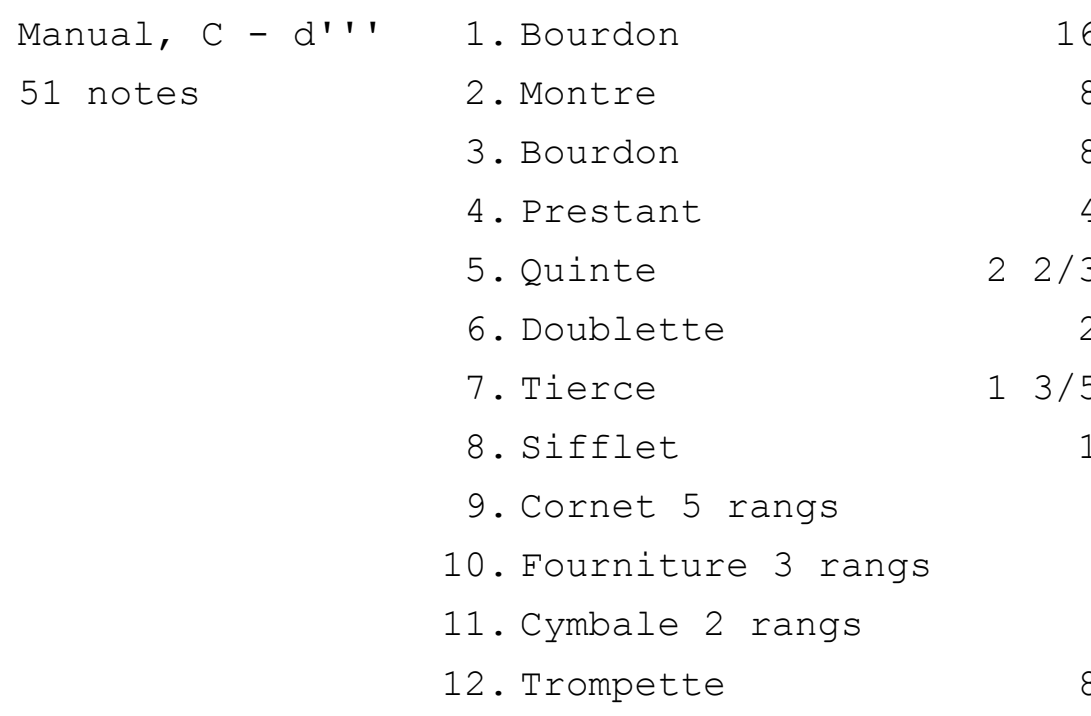

16

8

8

4

13

2

1 basse/dessus

8 basse/dessus

Echo basse, $\mathrm{C}-\mathrm{h}$ 1. Bourdon 8

24 notes 2. Prestant 4

3. Flûte 4

4. Doublette 2

5. Basson 8

Echo dessus, $C^{\prime}-d^{\prime}$ ''

27 notes
1. Bourdon
8
2. Prestant
4
3. Flûte
4
4. Nazard
$22 / 3$
5. Doublette
2
6. Tierce
$13 / 5$

Pédale, $c-c^{\prime}$

1. Soubasse

16

25 notes

2. Octavebasse

8

3. Trompette

8

Tremblant fort

ton de chambre 
Gries, église simultanée, 1781

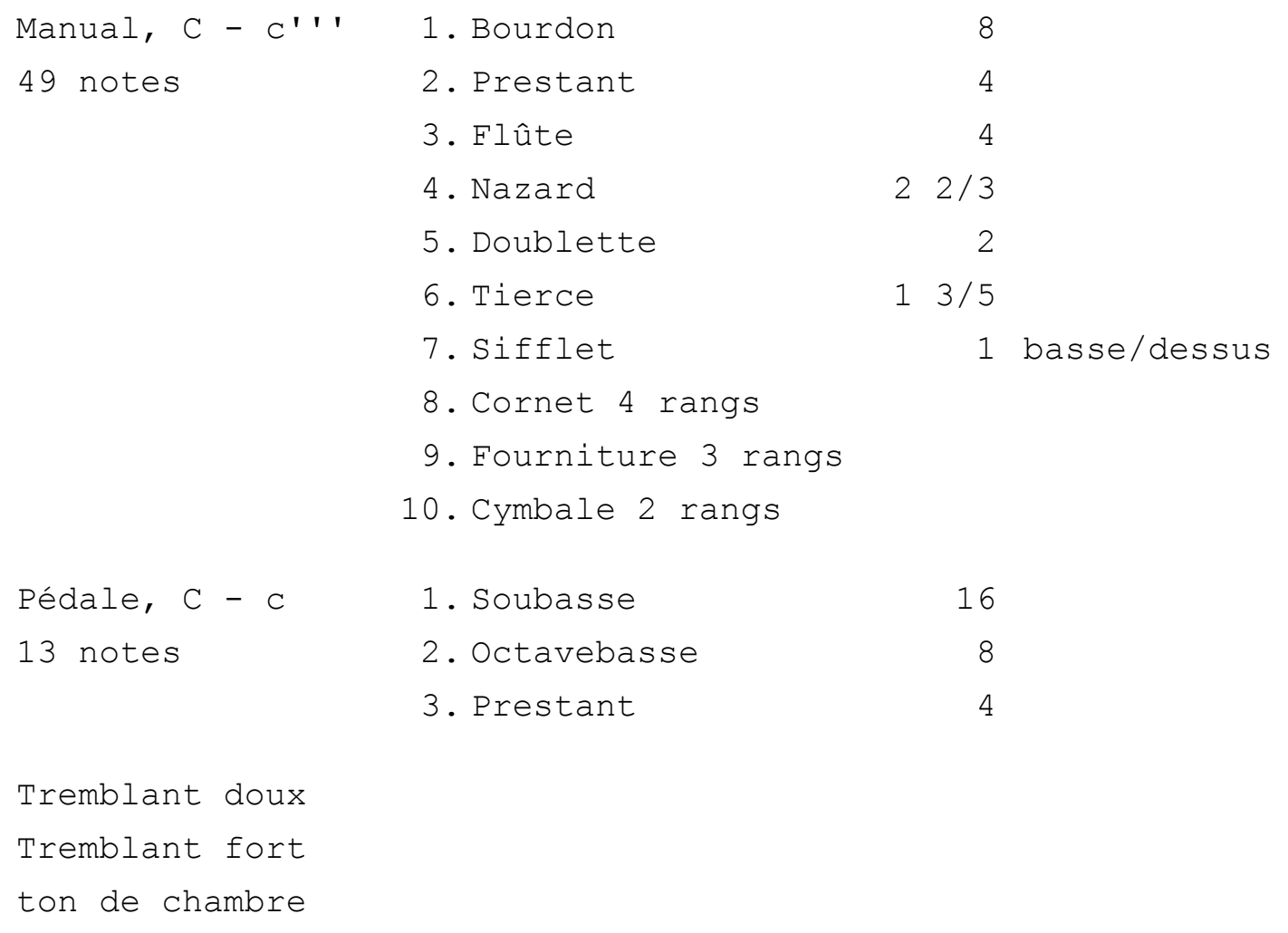


Lahr, collégiale, 1783

\begin{tabular}{|c|c|c|c|}
\hline \multirow{13}{*}{$\begin{array}{l}\text { Manual, c } \\
51 \text { notes }\end{array}$} & 1. Bourdon & 16 & \\
\hline & 2. Montre & 8 & \\
\hline & 3. Bourdon & 8 & \\
\hline & 4. Prestant & 4 & \\
\hline & 5. Quinte & $22 / 3$ & \\
\hline & 6. Doublette & 2 & \\
\hline & 7. Tierce & $13 / 5$ & \\
\hline & 8. Larigot & $11 / 3$ & \\
\hline & 9. Cornet 5 rangs & & \\
\hline & 10. Fourniture 4 rangs & & \\
\hline & 11. Cymbale 3 rangs & & \\
\hline & 12. Basson/Trompette & 8 & basse/dessus \\
\hline & 13. Voix humaine & 8 & \\
\hline \multirow{8}{*}{$\begin{array}{l}\text { Positif, } c-d \text { '' } \\
51 \text { notes }\end{array}$} & 1. Bourdon & 8 & \\
\hline & 2. Prestant & 4 & \\
\hline & 3. Flûte & 4 & \\
\hline & 4. Nazard & $22 / 3$ & \\
\hline & 5. Doublette & 2 & \\
\hline & 6. Tierce & $13 / 5$ & \\
\hline & 7. Fourniture 3 rangs & & \\
\hline & 8. Cromhorne & 8 & \\
\hline \multirow{6}{*}{$\begin{array}{l}\text { Pédale, } C-g \\
20 \text { notes }\end{array}$} & 1. Soubasse ouverte & 16 & \\
\hline & 2. Octavebasse & 8 & \\
\hline & 3. Prestant & 4 & \\
\hline & 4. Bombarde & 16 & \\
\hline & 5. Trompette & 8 & \\
\hline & 6. Clairon & 4 & \\
\hline \multicolumn{4}{|l|}{ Tremblant doux } \\
\hline \multicolumn{4}{|l|}{ Tremblant fort } \\
\hline 4 soufflets & & & \\
\hline
\end{tabular}


Les pleins-jeux d'André et de Jean André Silbermann.

\section{Répertoire chronologique}

Toutes les compositions de pleins-jeux citées sont des relevés personnels, à l'exception de celui de Sankt-Blasien - détruit attesté par Franz Stieffell.

Il convient de rectifier les relevés concernant Marmoutier et Ebersmunster, partiellement ou totalement erronés chez Rupp, Gessner, Bender et Fellot.

Marmoutier, 1710 .

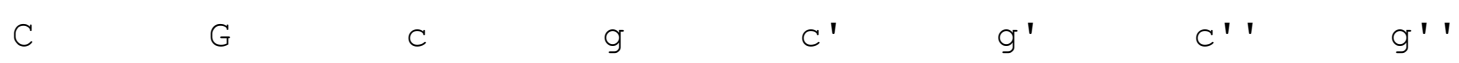

Fourniture G.O.III

$\begin{array}{lllllllll}1 & 1 / 3 & 2 & 2 & 2 / 3 & 4 & 5 & 1 / 3 & 8 \\ 1 & 1 & 1 / 3 & 2 & & 2 & 2 / 3 & 4 & 4 \\ 2 / 3 & 1 & 1 & 1 / 3 & 2 & 2 & 2 / 3 & 2 & 2 / 3\end{array}$

Cymbale G.O.III

$\begin{array}{lllllllll}1 / 2 & 2 / 3 & 1 & 1 & 1 / 3 & 2 & 2 & 2 / 3 & 4 \\ 1 / 3 & 1 / 2 & 2 / 3 & 1 & 1 & 1 / 3 & 2 & 2 & 2 / 3 \\ 1 / 4 & 1 / 3 & 1 / 2 & 2 / 3 & 1 & 1 & 1 / 3 & 2\end{array}$

Fourniture Pos.III

$\begin{array}{llllllll}2 / 3 & 1 & 1 & 1 / 3 & 2 & 2 & 2 / 3 & 4 \\ 1 / 2 & 2 / 3 & 1 & 1 & 1 / 3 & 2 & 2 & 2 / 3 \\ 1 / 3 & 1 / 2 & 2 / 3 & 1 & 1 & 1 / 3 & 2\end{array}$

Bischwiller, 1724 .

Fourniture G.0. III

C

C $C^{\prime}$

1

24

$2 / 3$

$11 / 3$

$22 / 3$

$1 / 2$

1

2 
Colmar, Dominicains, 1726 (Niedermorschwihr)

$\mathrm{C}$ $\mathrm{C}$ $\mathrm{C}^{\prime}$

$C^{\prime}{ }^{\prime}$

Fourniture G.O. III

\begin{tabular}{|c|c|c|c|}
\hline $11 / 3$ & 2 & 4 & 8 \\
\hline 1 & $11 / 3$ & $22 / 3$ & $51 / 3$ \\
\hline $2 / 3$ & 1 & 2 & 4 \\
\hline
\end{tabular}

Positif Fourniture III

$111 / 3$

$2 / 3 \quad 1$

$2 / 3$

2

4

$1 / 2$

$11 / 3$

$22 / 3$

1

2

Altorf, 1730

C C

$C^{\prime}$

$C^{\prime}{ }^{\prime}$

Fourniture G. O. III

12

$2 / 3 \quad 11 / 3$

$1 / 2$

1

4

$22 / 3$

4

2

$22 / 3$

Cymbale G.0. II

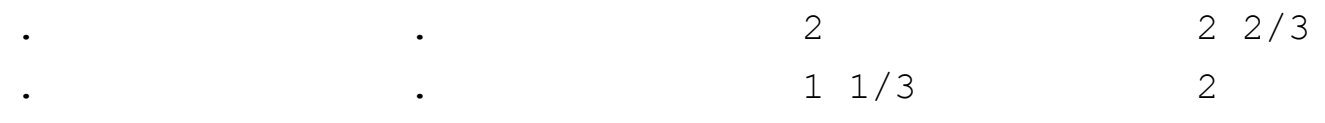

Ebersmunster, 1731.

C

C

$C^{\prime}$

$C^{\prime}$ '

Fourniture G-0. III

$2 \quad 22 / 3$

$4 \quad 8$

$11 / 3$

2

$22 / 3$

4

1

$11 / 3$

2

$22 / 3$

Cymbale G.O. III

1

$11 / 3$

2

4

$2 / 3$

1

$11 / 3$

$22 / 3$

$1 / 2$

$2 / 3$

1

2

Fourniture Pos. III

1

$11 / 3$

2

4

$2 / 3$

1

$1 / 2$

$2 / 3$

$11 / 3$

$22 / 3$

1

2 
Rosheim, 1733.

$\begin{array}{lcll}\text { C } & \text { C } & C^{\prime} & C^{\prime}{ }^{\prime} \\ \text { Fourniture G.O. III } & & \\ 1 & 2 & 4 & 51 / 3 \\ 2 / 3 & 11 / 3 & 22 / 3 & 4 \\ 1 / 2 & 1 & 2 & 22 / 3\end{array}$

Fourniture Pos. III (Lixhausen)

$\begin{array}{llll}1 & 11 / 3 & 2 & 4 \\ 2 / 3 & 1 & 11 / 3 & 22 / 3 \\ 1 / 2 & 2 / 3 & 1 & 2\end{array}$

Strasbourg, Saint-Thomas, 1740.

$\mathrm{C}$

Fourniture G. O. IV

$11 / 3 \quad 2$

1

$2 / 3$

$1 / 2$
$11 / 3$

1

$2 / 3$
$C^{\prime}$

$C^{\prime}{ }^{\prime}$

4

$22 / 3$

2

$11 / 3$
8

4

$22 / 3$

2 
Guebwiller, Dominicains, 1745 (Wasselonne)

C $\mathrm{C}$

$\mathrm{C}^{\prime}$

$C^{\prime}$ '

Fourniture G.O. III

\begin{tabular}{|c|c|c|c|}
\hline $11 / 3$ & 2 & 4 & 8 \\
\hline 1 & $11 / 3$ & $22 / 3$ & 4 \\
\hline $2 / 3$ & 1 & 2 & \\
\hline
\end{tabular}

Châtenois, 1765.

Fourniture G.O. III

$2 / 3$

$11 / 3$

$C^{\prime}$

$C^{\prime}{ }^{\prime}$

$1 / 2$

1

$1 / 3$

$2 / 3$

2

$22 / 3$

$11 / 3$

2

1

$11 / 3$

Ettenheimmunster, 1769.

$\mathrm{C}$

C

$C^{\prime}$

$C^{\prime}{ }^{\prime}$

Fourniture G.O. III

$2 / 3$

$11 / 3$

$1 / 2$

1

$1 / 3$

$2 / 3$

2

$11 / 3$

4

$22 / 3$

1

2

Sankt-Blasien, 1774

C C

$C^{\prime}$

$C^{\prime}{ }^{\prime}$

Fourniture G.O. IV

$11 / 3$

2

1

$11 / 3$

$2 / 3$

1

$1 / 2$

$2 / 3$

4

$22 / 3$

8

2

$51 / 3$

4

$11 / 3$

$22 / 3$

Cymbale G.O. III

$\begin{array}{ll}2 / 3 & 1 \\ 1 / 2 & 2 / 3 \\ 1 / 3 & 1 / 2\end{array}$

$11 / 3$

4

$22 / 3$

1

2 
Blodelsheim, 1779.

C C

Fourniture G.O. III

$2 / 3 \quad 11 / 3$

2

4

$2 / 3$

1

$11 / 3$

$22 / 3$

$1 / 2$

$2 / 3$

$11 / 3$

2

Gries, 1781.

C C

$C^{\prime}$

$C^{\prime}{ }^{\prime}$

Fourniture G.O. III

$2 / 3$

$11 / 3$

2

4

$2 / 3$

1

$1 / 2$

$2 / 3$

$11 / 3$

$22 / 3$

$11 / 3$

2

Cymbale G.O. II

$1 / 2$

1

$1 / 3$

$2 / 3$ 
Pérennité et continuité de l'oeuvre des Silbermann.

Nous n'avons pas l'intention de traiter dans leur intégralité tous les aspects de l'oeuvre des Silbermann. Nous nous permettons de renvoyer à la bibliographie quasi exhaustive, dans la dernière partie. Nous étudierons l'oeuvre des Silbermann en fonction d'une ligne directrice, en nous intéressant à la pérennité et à la continuité de l'oeuvre dans son ensemble, et à la diversité et aux divergences des différents protagonistes.

André Silbermann fut le fondateur d'une véritable dynastie de facteurs d'orgues, qui ne compta pas moins de neuf membres. Il fut le mâ̂tre de son frère Gottfried et de son fils Jean André. Lors de sa première apparition en Alsace, à savoir en 1699 à Bouxwiller il a 21 ans - il est d'emblée qualifié de facteur d'orgues. Notons que dans les archives il est mentionné à cette occasion conjointement avec un autre facteur d'orgues du nom de Johannes Ims, dont nous ignorons l'origine. (1) André Silbermann a-t-il déjà effectué un apprentissage avant cette date? Nous l'ignorons. Si nous étudions le développement de l'art d'André Silbermann, force nous est de constater que les origines de cet art restent assez indéfinies.

Certes nous savons qu'André Silbermann a travaillé un certains temps auprès de Frédéric Ring, facteur d'orgues et d'instruments à Strasbourg. Mais si nous admettons que l'art d'André Silbermann est largement tributaire de l'orgue parisien de la fin du $17^{\circ}$ siècle, nous constatons qu'en revanche l'art de Frédéric Ring tel qu'il apparâ̂t dans son projet pour l'orgue du Temple-Neuf Strasbourg était de type rhénan plutôt que parisien. Nous pensons qu'avant la construction de son premier orgue, terminé en 1703, André Silbermann s'est initié à la facture d'orgues française, c'est-à- 
dire au goût nouveau, à la manière nouvelle qui très rapidement s'imposera en Alsace. En effet l'orgue du couvent de SainteMarguerite à Strasbourg, construit avec la collaboration de Gottfried Silbermann, avait déjà une composition toute française, mis à part un seul jeu de Gambe.(3) Avant 1703 André Silbermann n'avait effectué que des réparations: Bouxwiller 1699, Dominicain de Haguenau 1701, (4) Strasbourg St-Nicolas 1702.(5) D'ailleurs si André Silbermann est allé travailler à Paris, chez François Thierry, de 1704 à 1706, c'était pour se perfectionner dans la manière française, et non pour l'apprendre de toutes pièces: "um sich im Frantzöschen Orgel=Gousto noch mehr zu perfectioniren". (3)

De retour à Strasbourg, André Silbermann construit en 1707 toujours avec la collaboration de son frère Gottfried qui porte à présent le titre de facteur d'orgues, l'orgue de l'église Saint-Nicolas à Strasbourg. Cet instrument fut également de style français, un jeu de Gambe mis à part.(6) Il est significatif de l'évolution des mentalités qu'en 1719 à la demande de l'organiste Johann Georg Thenn il remplaça ce jeu de Gambe 8 par un Bourdon 16. (7) Dès 1708, date de signature du marché pour l'orgue de Saint-Pierre-leVieux protestant, (8) les jeux de Gambe disparaissent des compositions proposées par André Silbermann, même pour l'orgue de l'abbaye de Weingarten en Souabe. (9) Par contre Gottfried, de retour en Saxe en 1710, conservera les jeux de Gambe dans ses compositions.

L'art d'André Silbermann est représentatif de l'orgue classique français à une restriction près, à savoir l'emplacement et la composition des jeux de Pédale. Placés toujours derrière le soubassement et non dans le buffet du Grand Orgue, les jeux de Pédale comportent souvent un jeu de 16 pieds ouvert, voire une 
Bombarde 16, comme à Marmoutier ou à Ebersmunster. La Pédale a une fonction de clavier de basse, et non de ténor; elle comporte généralement une Soubasse 16. Rappelons qu'à Marmoutier la Soubasse 16, ouverte, était à l'origine dépourvue de tirant de jeu, ce qui rendait impossible l'exécution d'un plain-chant en taille du type du premier Kyrie de la Messe à l'usage des Paroisses de François Couperin (1690).

De plus, les Pédales du Temple-Neuf (1708) et de Saint-Pierre-leVieux (1709) comportent des Mixtures respectivement de 6 et 4 rangs; elles sont en cela tributaires de l'orgue allemand. Dès 1710, avec l'orgue de Marmoutier (10), elles disparaissent des compositions d'André Silbermann, pour ne réapparâ̂tre qu'une seule fois chez Jean André, au Temple-Neuf en 1749.

En ce qui concerne le plein-jeu, nous constatons une légère évolution chez André Silbermann. L'orgue de Marmoutier, qui est le plus ancien orgue Silbermann conservé, possède un plein-jeu de type harmonique, comportant de nombreuses reprises, sur c et g, aussi bien pour les Fournitures que pour les Cymbales.(11) Ce type est voisin de celui de l'orgue classique français, qui n'a guère varié pendant plus d'un siècle et qui fut codifié par Dom Bedos. (12) Très tôt cependant André Silbermann modifia ce type harmonique au profit d'une progression plus polyphonique, comportant des reprises uniquement sur les c, aussi bien pour les Fournitures que pour les Cymbales. L'orgue d'Ebersmunster (1731) représente ce type. Cette formule fut appliquée également à Bischwiller (1723), aux Dominicains de Colmar (1726), maintenant à Niedermorschwihr, à Altorf (1732) et à Saints-Pierre-et-Paul de Rosheim (1733). De façon générale le plein-jeu d'André Silbermann plafonne à un niveau 
plus bas que celui de Dom Bedos: au milieu du clavier, en c', il ne dépasse jamais 1', au lieu de 2/3'.

En conclusion nous pouvons affirmer que l'orgue d'André Silbermann est l'orgue des Thierry modifié et adapté au milieu rhénan.

Jean André Silbermann apprit le métier dans l'atelier de son père at participa activement à la construction des derniers orgues de celui-ci. En 1734, à la mort de son père, il est âgé de 22 ans et prend en charge une succession difficile. Ses premiers orgues se situent dans la continuité du travail paternel. Mais dès 1736, dans un projet pour Neuf-Brisach (13), il invente un nouveau type de buffet, qui se caractérise par une tourelle centrale basse, flanquée de deux demi-plate-faces cintrées. Ce parti très élégant au demeurant, fera fortune: les orgues de Saint Thomas à Strasbourg, de Soultz et d'Arlesheim, pour n'en citer que trois, en témoignent. (14)

En 1741 eut lieu un événement important pour l'évolution du style de Jean André: son voyage d'étude en Saxe. Nous avons par ailleurs montré qu'il s'agissait bien d'un voyage d'étude et non d'un voyage de noces comme le prétendent Mathias et Wörsching (15). Pendant six semaines Jean André participe à la construction de l'orgue de Zittau, l'un des grands ouvrages de Gottfried.(16) Pendant près de quatre mois Jean André voyage, visite les orgues de son oncle en Saxe, et voit des instruments importants d'autres facteurs, en Saxe, à Berlin, Fulda, Magdeburg, Francfort, etc. De ce voyage il résultera un élargissement de la palette sonore de Jean André. Un certain nombre de jeux nouveaux, pratiqués par Gottfried, seront introduits dans les compositions proposées Jean André: le Sifflet 1', s'intégrant au plein-jeu, le Larigot 1 1/3', le Quintaton 16' ou 8', le Chalumeau 8, le Basson 8', le Cornetto 2' et le Posaune 
16'. De plus, les pleins-jeux de Jean-André se caractériseront par une acuité plus prononcée. A deux reprises Jean André construira des claviers d'Oberwerk, à l'image des grands instruments à 3 claviers de Gottfried. Il le proposera également à schuttern. Malheureusement les deux orgues comportant un tel clavier d'Oberwerk ont été anéantis: celui du Temple-Neuf à Strasbourg (1749) et celui de l'abbaye de Sankt-Blasien en Forêt-Noire (1774). Ces deux instruments avaient largement contribué à la célébrité de Jean André Silbermann, de son vivant.

Le collaborateur le plus important de Jean André fut son frère Jean Daniel, organiste et compositeur. Il eut des contacts étroits avec son oncle Gottfried, auquel il rendit visite en 1749 (2) et dont il prendra la succession.

Jean Henri, le plus jeune frère de Jean André, tout en collaborant à la facture d'orgues, se spécialisa dans la construction de clavecins, d'épinettes et de piano-forte. Ses instrumerts sont les joyaux d'un certain nombre de musées d'Europe.

Quant à Josias, fils de Jean André, il n'aura pas vécu assez longtemps pour faire oeuvre personnelle. Après avoir achevé l'orgue de Lahr, commencé par son père, il effectua un certain nombre de réparations et mourut à l'âge de 21 ans.

La manufacture des Silbermann représente le type de l'entreprise familiale. Les différents membres de la famille travaillaient en étroite collaboration, aidés par quelques ouvriers ou compagnons. Le plus important de ces derniers fut sans conteste Johann Conrad Sauer, qui après la mort de Josias Silbermann prendra la succession de l'entreprise et continuera la tradition de Jean André. Il sera 
relayé par son fils Johann Conrad junior, qui continuera lui-aussi à oeuvrer dans le même sens, jusqu'en 1828. (17)

Pendant près d'un siècle les Silbermann ont marqué la facture d'orgues en Alsace de leur empreinte. La comparaison avec le travail des concurrents, Merckel, Waltrin, Rohrer, Toussaint et d'autres, montre la supériorité des réalisations des Silbermann. Le travail des Silbermann se caractérise par une conscience professionnelle très élévée et une fierté artisanale très grande: choix des matériaux de première qualité, finition extrêmement soignée, sens aigu des proportions et de l'équilibre, efficacité maximum quant au rendement sonore des tuyaux, harmonisation dénotant une oreille très musicale où s'allient la puissance, la gravité, la finesse, la poésie.

La trame de ce travail de qualité ne s'est jamais interrompue et la renommée des Silbermann n'a pas terni jusqu'à nos jours. Notons que le mouvement de l'orgue alsacien ou la réforme alsacienne de l'orgue, animée par Albert Schweitzer et Emile Rupp, s'est cristallisée autour d'un retour à la facture des Silbermann. De nos jours encore la facture d'orgues en Alsace montre une certaine dépendance par rapport à l'art des Silbermann. 
(1) ABR. Archives du Consistoire de Bouxwiller. $\mathrm{N}^{\circ}$ 155. Comptes 1699, p. 262. "... Andres Silbermann undt Johannes Imsen beeden Orgelmachern ... den 16.ten 7 bris 1699 ..."

(2) Marc Schaefer, Les anciennes orgues Silbermann du Temple-Neuf à Strasbourg, in La Musique en Alsace, hier et aujourd'hui, Strasbourg 1970, p. 95 et suiv.

(3) Jean André Silbermann, ms. "Orgeln von Andreas Silbermann", p. 1 et suiv.

(4) Jean André Silbermann, ms. "Elsassische Orgeln", p. 41.

(5) ABR. Archives de la paroisse Saint-Nicolas de Strasbourg, Comptes 1702 .

(6) Jean André Silbermann, ms. "Orgeln von Andreas Silbermann", p. 17 et suiv.

(7) ABR. Archives de la paroisse Saint-Nicolas de Strasbourg, Protocollum der Fabric zu Sanct Niclauss in Strassburg de Annis 1703. 1704 biss 1726, séance du 10.6.1719.

(8) Jean André Silbermann, ms. "Orgeln von Andreas Silbermann", p. 25 et suiv. - AMS. Archives de la paroisse protestante Saint-Pierre-le-Vieux à Strasbourg, liasse 22, $\mathrm{N}^{\circ} 11$ : marché passé avec André Silbermann le 10. 8. 1708.

(9) Joseph Wörsching, Andreas Silbermann und Weingarten, in Der Kirchensänger, année 36, $\mathrm{N}^{\circ}$ 6/7, 1936.

(10) Jean André Silbermann, ms. "Orgeln von Andreas Silbermann", p. 81 et suiv.

(11) Marmoutier. Orgues Historiques. Harmonia Mundi. Musique de tous les temps, $N^{\circ}$ 25. Saint-Michel-de-Provence, 1963.

(12) Jean Fellot, A la recherche de l'orgue classique, Paris 1961

(13) Bernd Sulzmann, Ein Orgelbauprojekt Johann Andreas Silbermanns für die Kirche St. Louis in Neuf-Brisach (Neubreisach) 
aus dem Jahre 1736, in Ars Organi, année 31, N² 2, juin 1983, p. $90-93$.

(14) Albert Hohn, Die Orgeln Johann Andreas Silbermanns, in Acta Organologica, IV (1970), p. 11 et suiv.

(15) Marc Schaefer, in Saint-Quirin. Orgues Historiques $N^{\circ} 13$, Harmonia Mundi. Saint-Michel-de-Provence, 1965, p. 24.

(16) Ulrich Dähnert, Historische Orgeln in Sachsen, Francfort 1980, p. 281.

(17) Pie Meyer-Siat, Die Orgelbauer-Familie Sauer in Strassburg, in Acta Organologica, III (1969), p. 100-132. 
Pièces justificatives 
Pièces justificatives I.

Extraits de registres paroissiaux, actes

d'état civil.

Inventaire après décès de Jean André Silbermann.

Notice nécrologique concernant

Jean André Silbermann. 
Acte de naissance de Frédéric Ring.

$$
1.6 .6 .6 \text {. }
$$

† den 5 May morgens früh vmb zwey Vhr, ist Dominico Ringen, Pfarrherrn zu Berstett, vnnd Dorotheae seiner ehelichen Haussfrawen ein Söhnlein gebohren, den 8 huius zu Berstett inn der Kirchen, von H.-M. Barthen, Pfarrherrn zu Lamptheim getaufft, vnnd Fritricus genanndt worden. Die Pfetteren seindt gewest: Junker Georg Conrad Schaualitzky von Muckothal, dessen Stell hatt vertretten, Junker Johann Jacob von Berstett. Vnnd dann H.Gottfrid Rederer, Burger vnnd Dreyerknecht des Pfennigthurns zu Strassburg. Die Göttel aber Fraw Maria Charitas Berstetterin, gebohrene von Rathsamhausen.

Archives paroissiales de Berstett.

Kirchen Buch / Beede Pfarren Berstett vnd / Olvisheim betreffend 1664 bis 1685 .

p. 28 . 
Acte de mariage de Frédéric Ring.

Heüt den 12 Octobr. 1689 ist von mir M. J. Th. Heinrici past. et Can. Thom. nach ordentlicher Proclamation eingesegnet worden Friderich Ring der ledige Schreiner, H.-Dominici Ringen, Diaconi zu Aurelien, ehlicher Sohn, mit Jfr. Anna Catharina, weyl H.- Joh. Geörg Artopoei, gewesenen Pfarrers zu St. Willhelm, nachgelassene ehliche Tochter. Bezeügen solches,

Friderich Ring als Hochzeitter

Anna Catharina Artopösin. Als Hohzeiterin

Dominicus Ring Diaconus Aurelianus als Vatter. Sponsi

Joh. Christophorus Artopoeus pp. alss nächster Anverwanter p.

M. J. Th. Heinrici, past. et Can. Thom. mppr.

Archives Municipales de Strasbourg

M 122. fol. 13. $\mathrm{N}^{\circ} 63$. 
Acte de naissance de Frédéric Ring fils.

Eodem [22 oct. 1690] ist von mir pastori getaufft worden ein Kindt mit Nahmen Friderich, dessen Eltern Friderich Ring, der Burger undt Schreiner undt Fr.- Anna Catharina Artopoeusin, die Tauff Zeugen waren H.- Johannes Heupel, Pfarrer zum Jungen St. Peter, H.- M. Joh. Ludwig Engelhardt, Diac. Thom. undt Frl. Anna H. D. Sebast. Schmidii, prof iss. sen. undt Kirch Conv. Praesidenten Haussfr.-. Das Kindt ist gebohren 20 Octobr. 1690 morgens vor 9 Uhr.

Friderich Ring als Vatter

Johannes Heüpelius, Past: Neopetr: alss Pfetter.

M. Joh. Ludov. Engelhardt Diac. Thom. als Pfetter.

Anna Schmiden, als Göttel.

M. J. Th. Heinrici p. et C. Thom. mppr.

Archives Municipales de Strasbourg

$\mathrm{N}$ 252, fol. 70 b. $\mathrm{N}^{\circ} 287$. 
Acte de naissance de Maria Dorothea Ring.

Den 9 Martii $A^{\circ}$. 1693 ist von mier M. Joh. Ludov. Engelhardt Diac. Thom. ein Kind getaufft worden, mit Namen Maria Dorothea, dessen Eltern Fridericus Ring, Burger v. Schreiner allhier, V. Fr. Anna Catharina gebohrene Artopoeusin, seine ehl. Haussfr. Die Tauffzeugen, H.- Michael Bosch, Burger v. Barcherthändler allhier, Fr. Maria Elisabetha Engelhardtin, gebohrene Kresslerin, v. Fr. Maria Ursula Holtzbergerin, gebohrene Artopoeusin. Das Kind ist gebohren den 7 Martii $1 / 4$ nach 1 Uhr nachmittag.

Friderich Ring alss Vatter

Michael Bosch der Jüngere alss Pfätter.

Maria Elisabetha Engelhardtin, alss Göttel.

Maria Vrsula Holtzbergerin alss Göttel

M. Joh. Ludov. Engelhardt. Diac. Thom. mppr.

Archives Municipales de Strasbourg

$\mathrm{N}$ 252. fol. 119 a. $\mathrm{N}^{\circ} 497$. 
Acte de naissance d'Anna Catharina Ring.

Anno 1695, den 7 Januarii ist von mir M. Philip Strohlen Diac Thom. öfentlich bestätiget worden ein nach guter ordnung geschehene Nothtauff, da das Kind genennet worden Anna Catharina dessen Eltern sein Friederich Ring Schreiner und Burger alhie und seine Ehefraw, Anna Catharina, gebohrne Artopoeusin. Die Tauffgezeugen waren Herr Johann Georg Bemberg Ratherr und Handelsmann alhie und Fr. Martha Metzgerin gebohrne Vlmännin und Jgf.- Maria Salome Hamin. Das Kind ist gebohren worden, den 3 Januarü 1695. nachts zwischen 10 et 11. Vhr.

Friderich Ring als Vatter

Johann Geörg Bemberg als Pfetter

Martha Metzgerin als Göttele.

Maria Salome Haamin als Göttel

M. Philip Strohl. Diac Thom. mppr.

Archives Municipales de Strasbourg

$\mathrm{N}$ 252. fol. 158 a. $\mathrm{N}^{\circ} 662$. 
Acte de décès d'Anna Catharina Ring.

Den 5 Aug. $A^{\circ}$. 1697 ist umb den Mittag, ein jünges Töchterlein gestorben, mit Namen Anna Catharina, seines Alters, 2 Jahr v. 7 Monat, dessen Eltern, Joh. Fridericus Ring, Burger v. Schreiner allhier, v. Fr. Anna Catharina Artopoeusin seine ehl. Haussfr. Ist darauff den 7 Augst zu St. Helenen begraben, V. durch mich M. Joh. Ludov. Engelhardt Diac. Thom. die gewohnliche Vermahnung abgehalten worden: bezeugen solches,

Friderich Ring alss Vatter.

Dominicus Ring alss Grossvatter.

M. Joh. Ludov. Engelhardt Diac. Thom. mppr.

Archives Municipales de Strasbourg

D 201. fol. 25 a. $\mathrm{N}^{\circ} 10$. 
Acte de naissance de Johannes Ring.

Heut den 16 Xbr. 1697 ist getaufft worden Johannes, dessen Eltern Friderich Ring, der Orgelmacher undt Schreiner allhier, undt Fr. Anna Catharina geb. Artopoeusin. Die Tauffzeügen H.-Georg Christoph Lautensack, Organist in der Prediger Kirch, H.- Isaac Unselt junior Horndräher undt Burger allhier, Jfr. Anna Maria Sohnin, Christoph Sohns, Riemenschneiders seligen, nachgelassene Tochter. Das Kind ist gebohren den 14 Xbr. 1697 vormittag 10 Uhr. Friderich Ring alss Vatter

Georg Christoph Lauthensackh als Pfetter.

Isaac Vnselt der Jünger alss Pfetter

Anna Maria Sohnin alss Göttel

M. Joh. Theob. Heinrici, past. Thom. mppr.

Archives Municipales de Strasbourg

$\mathrm{N}$ 252. fol. 214 a. $\mathrm{N}^{\circ} 931$ 
Acte de naissance de Johann Daniel Ring.

Heüt den 7 April 1700 ist getaufft worden Johann Daniel dessen Eltern H.- Friderich Ring Orgelmacher undt Burger, undt Fr.- Anna Catharina Ringin gebohrne Artopoeusin. Die Tauffzeügen H.- M. Johann Georg Artopoeus, Sup. Gymnas. Praeceptor et Ecclesiastes. H.- Daniel Seüpel Goldarbeiter undt Burger. Jfr.-Maria Salome Stuberin. Das Kind ist gebohren den 5 April. Ao 1700. morgen zwischen 12 und 1 Uhr.

Friderich Ring alss Vatter.

M. Johann Georg Artopoeus, alss Pfetter.

Daniel Seupel alss Pfetter

Maria Salome Stuberin alss Göttel

M. J. Th. Heinrici, past. Thom. mppr.

Archives Municipales de Strasbourg

N 252. fol. 251 a. $\mathrm{N}^{\circ} 1104$. 


\section{Acte de décès de Frédéric Ring.}

Den 19 Mertz 1701 ist zu S. Thomae die Leichpredig gehalten worden H.- Friderich Ring, Burger Schreiner V. Orgelmacher allhier, so den 16 Mertz abends mit einem Flussüberfall noch selbige Nacht vor 11 Uhr verschieden, seines Alters 34 Jahr 10 Monat 1 Tag, ist begraben zu s. Gallen.

M. J. Th. Heinrici, Past Thom mppr.

Joh: Heüpelius, Past: Neopetr. dess Seel. Verstorbenen Mutter, Bruder.

Johann Martin Ring, Als Verwander.

M. J. Th. Heinrici, Past. Thom. mppr.

Archives Municipales de Strasbourg

D 200. fol. $91 \mathrm{a} / \mathrm{b} \cdot \mathrm{N}^{\circ} 602$. 
Acte de décès de Johann Daniel Ring.

Den 27 Septembris 1703. ist nach gehaltener Vermahnung zu S.

Thomae, zu S. Helenae begraben worden Johann Daniel, dessen Eltern weyl. H.- Friderich Ring, Burger, Schreiner undt Orgelmacher, undt Fr. - Anna Catharina Ringin gebohrne Artopoeusin, das Kind starb den 25 Sept. 1703 vormittag umb halb zehen Uhr, seines Alters 3 $1 / 2$ Jahr 2 Tag.

M. Georg. Fridericus Heupelius als Vogt dess seelig verstorbenen M. Joh. Georgius Artopoeus, als Pfetter.

M. J. Th. Heinrici, past. Thom. mppr.

Archives Municipales de Strasbourg

D 200. fol. 106 b. $\mathrm{N}^{\circ} 708$. 
1678. 17. 5 .

Acte de baptême d'André Silbermann

Den 17 May ist Andreas, Michael Silbermann, Einwohners in

Kleinbobritzsch u. Hofzimmermans, ehleibl. Sohn christlich getauft

u. den 16 huj. früh umb 3 Uhr gebohren worden. Uxor Anna Maria,

Casspar Preusslers, Einwohners zu Grosswaltersdorf, ehleibl.

Tochter.

Testes (1) Michael Wünsch, Bauer zu Kleinbobritzsch

(2) Martin Wiesener, Churfl. Voigt zu Neubau

(3) Salome, Christoph Trögers, Bauers daselbst Ehweib.

Gott gebe dem lieben Kinde Leben u. Seegen.

Pfarramt Frauenstein, Taufbeurkundung $\mathrm{N}^{\circ}$ 17/1678. 
1708. 13.6 .

Acte de mariage d'André Silbermann

Mittwochs den 13. Junii 1708 seind nach zweymahliger Aussruffung in der Pfarrkirchen zu S. Niclauss in Strassburg copulirt und eingesegnet worden: H.- Andreas Silbermann, der ledige Orgelmacher vnd Burger alhier, H.- Michael Silbermannss, dess Burgers vnd zimmerwerckmeisterss zu Grauenstein in Sachsen ehelicher Sohn, und Jfr. Anna Maria Schmidtin, H.- Caspar Schmidss, des Wagnerss und E. E. grossen Raths alhier alten Beysitzers ehel.- Tochter, welche auch neben mir und nachgesetzten zeugen unterschrieben haben. Andreas Silberman. als Hochzeyter.

Anna Maria Schmidtin als Hochzeiterin.

Michael Keck alss Beystandt

Caspar Schmidt als Vatter

M. Johannes Christianus Treitlinger. Diac. Nicol. mpria.

AMS, M 53, fol. 113 a. (St. Nicolas) 
1710. 24.11.

Acte de baptême de Johann Caspar Silbermann

Montag den 24. Nov: 1710. ist von mir, M. Johann Leonhardt Fröreissen Pfarrer zu St. Nic. H. Andrea Silbermann, dem Burger und Orgelmacher alhier, und seiner ehel. Haussfr. Anna Maria, gebohrner Schmidin, ein Söhnlein mit Nahmen Johann Caspar getauff worden. Dabey zu Gevattern waren, H. Johann Friedrich Pfeffinger, der Metzger und Burger alhier, und H. Michael Keck, der Biersieder zum Hirsch und Burger alhier, und Fr. Maria Cleophe Bühlerin, H. Stephan Bühlers, dess Handelsmanns und Burgers alhier, ehel. Haussfr. welches bezeugen,

Caspar Schmidt als Grossvatter.

Michael Keck alss Pfetter.

Johann Friderich Pfeffinger alss Pfether

Maria Cleophe Bihlerin als Göttel.

M. Johann Leonhard Fröreissen, Pf. zu St. Nicol. mpia.

AMS, N 111, fol. 217 b (St. Nicolas) 
1711. 28.7 .

Acte de décès de Johann Caspar Silbermann

Anno 1711. starb d. 28. july den Dienstag um 11. Uhren Joh: Caspar Herrn Andreas Silbermanns Burgers u. Orgelmachers allhier u. Anna Maria, gebohrner Schmittin, Söhnlein, u. wurde den Donnerstag darauff vormittag um 10. Uhren auff dem Gottes Acker zu St. Urban begraben, seines Alters 8. Monat u. 6. Tag.

Caspar Schmidt als Grossvatter

Johan Friderich Pfeffinger als Pföther

Michael Keck alss Pfetter

M. Joh: Andreas Schmuts, Diac. Nicol.

AMS, D 94, fol. 5 b (St. Nicolas) 
1712. 26.6 .

Acte de baptême de Jean André Silbermann

Sontag den 26. Jun. 1712. ist von mir M. Johann Leonhard Fröreissen Pfarrer zu St. Nicolai, H.- Andreas Silbermann, dem Orgelmacher vnd Burger alhier, und seiner ehelichen Haussfr.Anna. Maria, gebohrene Schmidin, ein Söhnlein, nahmens Johann Andreas getaufft worden. Dabey zu Gevattern waren, H.- Philipp Jacob Mader, der Notar. Publ. vnd H.- Johann Heinrich Vigera, der Handelssmann vnd Burger alhier, vnd Fr.- Anna Maria, Cladi Hanss Metzgers, dess Metzgers vnd Burgers alhier, eheliche Haussfr.welches bezeugen.

Andreas Silbermann als Vatter Johann Heinrich Vigera als Pfetter

Philipp Jacob Mader, alss Pfetter

Anna Maria Hanss Metzgerin alss Göttel

M. Johann Leonhardt Fröreissen

Pfarrer zu St. NiCOl.

AMS, N 111, fol. 252 a. (St. Nicolas) 
1714. 5.4.

Acte de baptême de Gottfried Silbermann

Donnerstag den 5. April 1714. ist von mir M. Johann Leonhardt Fröreissen Pf. zu St. Nicol: H. Andreas Silbermann, dem Orgelmacher und Burger alhier, und seiner Ehelichen Haussfr. Anna Maria, gebohrner Schmidin, ein Söhnlein mit Nahmen Gottfried getaufft worden. Dessen Pfettern und Göttel waren H. Daniel Vogt der Holtzhändler und Burger alhier, und Johann Heinrich Schall, der Carduanmacher und Burger alhier, und Fr. Susanna Zeudlerin, Gerhardt Zeudlers, dess Metzgers und Burgers alhier, eheliche Haussfr. welches

bezeugen

Andreas Silbermann als Vatter.

Daniel Vogt als Pfötter.

Johann Henrich Schall als Pfetter.

Susanna Zeudlerin als Göttel.

M. Johann Leonhardt Fröreissen

Pfarrer zu St. Nicol. mp.

AMS, N 111, fol. 297 a (St. Nicolas) 
1715. 5.2.

Acte de décès de Gottfried Silbermann

Dienstag den 5. Febr. 1715. vormittag 1/4 nach 6. Uhr frühe ist alhier in Strassb. seel. verschieden, und 4 den 7. hujus auff den Gottesacker S. Urbani begraben worden, H. Andreä Silbermans, dess Orgelmachers und Burgers alhier, und seiner Ehe-Haussfr. Annä Mariä geb: Schmidin, eheliches Söhnlein, Nahmens Gottfried. Seines Alters 9. Monat u. 2. Tag. welches bezeugen.

M. Johann Leonhard Fröreissen, Pf. zu St. Nicol. Andreas Silbermann, als Vatter. Daniel Vogt als Pfötter.

AMS, D 93, fol. 60 bo (St. Nicolas) 
1715. 5.12.

Acte de baptême de Johann Friedrich Silbermann

Donnerstag den 5. Dec: 1715. ist von mir M. Johann Leonhardt Fröreissen, Pfarrer zu St. Nicol: H. Andreas Silbermann, dem Orgelmacher und Burger alhier, und seiner ehelichen Haussfr. Anna Maria, geb: Schmidin, ein Söhnlein mit Nahmen Johann Friedrich getaufft worden. Dabey zu Gevattern waren H. Carl Bolgaw, der Zinngiesser und Burger alhier, und H. Johann Jacob Stamm, der Schiffmann und Burger alhier, und Fr. Susanna Dorothea Bühlerin, weyl. H. Stephan Bühlers, gewessenen Kauffmann und Burgers alhier, hinterlassene Wittib, welches bezeugen.

Andreas Silbermann als Vatter.

Carl Balckau alss Pfetter.

Johann Jacob Stamm als Pfätter

Susanna Dorothea Bihlerin als Göttel

M. Johann Leonhardt Fröreissen

Pfarrer zu St. Nicol. mpr.

AMS, N 111, fol. 332 b (St. Nicolas) 
1717. 31.5 .

Acte de baptême de Jean Daniel Silbermann

Eodem (Montag 31. Mai 1717) ist auch von mir M. Joh. Leonhardt Fröreissen, Pfarrer zu St. Nicol: H. Andreas Silbermann, dem Orgelmacher und Burger alhier, und seiner ehelichen Haussfr. Anna Maria, gebohrner Schmidin, ein Söhnlein mit Nahmen Johann Daniel getaufft worden. Dabey zu Gevattern waren, H. Johann Georg Thenn, Med. D. et Pract. alhier, und H. M. Joh. Jacob Dieterlin, St. Theol. H. M. Israel Dieterlins, Praec. Class. Inf. Gymnas. alhier, ehel. Sohn, und Jfr. Susanna Barbara Pfeffingerin, H. Johann Friedrich Pfeffingers, dess Metzgers u. Burgers alhier, ehel. Tochter, welches bezeugen.

Andreas Silbermann als Vatter. Joh. Georg. Thenn Med. D. alss Pfetter.

M. Joh. Jacob Dieterlin, alss Pfetter.

Susanna Barbara Pfeffingerin, alss Göttel.

M. Johann Leonhardt Pröreissen, Pf. zu St. Nic.

AMS, N 112, fol. 22 b (St. Nicolas) 
1717. 28.10 .

Acte de décès de Johann Friedrich Silbermann

Donnerstag den 28. Octobr. 1717. frühe umb 3. Uhr ist alhier in Strassburg seel. verschieden, und $\frac{1}{2}$ den 30. hujus auff den Gottesacker S. Urbani begraben worden, H. Andreas Silbermanns dess Orgelmachers u. Burgers alhier, und seiner ehe.- Haussfr. Anna Maria, geb. Schmidin, ehe.- Söhnlein, nahmens Johann Friedrich. Seines Alters 2. Jahre weniger 1. Monat u. 6. Tag. welches bezeugen.

Andreas Silberman als Vatter.

Carl Bolckau alss pfetter

M. Johann Leonhardt Fröreissen, Pf. zu St. NiC.

AMS, D 93, fol. 74 a (St. Nicolas) 
1718. 16.10 .

Acte de baptême d'Anne Marquerite Silbermann

Sontag den 16. Octobr. 1718. ist von mir M. Johann Leonhardt

Fröreissen Pf. zu St. Nic: H. Andreas Silbermann, dem Orgelmacher und Burger alhier, und seiner ehelichen Haussfr. Anna Maria, geb: Schmidin, ein Töchterlein mit Nahmen Anna Margaretha getaufft worden. Dabey zu Gevattern waren H. Johann Lauth, der Dinssen Müller und Burger alhier, und Fr-. Maria Magdalena Münchin, Johannes München, dess Kerbsbecken und Burgers alhier, ehel. Haussfr. und Fr. Maria Barbara Fändlerin, Jonas Fändels, dess Huffschmids und Burgers alhier, ehel. Haussfr. welches bezeugen

Johan Jacob Lauth alss Pfetter

Maria Magdalena Muchin als Gedel

Maria Barbara Fändlerin als Goettel

M. Johann Leonhard Fröreissen, Pf. zu St. Nicol.

AMS, N 112, fol. 55 b (St. Nicolas) 
1720. 17.2.

Acte de décès d'Anne Marquerite Silbermann

Sambstag den 17. Febr. 1720. nachmittag gegen 3. Uhr, starb alhier in Strassburg, H. Andreas Silbermans, dess Burgers und Orgelmachers alhier, und seiner ehe. Haussfr. Anna Maria, beg: Schmidin eheliches Töchterlein, nahmens Anna Margaretha, wurde \& den 19. huj. auff den Gottesacker S. Urbani begraben, seines Alters 1. Jahr, 4. Monat und 2. Tag, welches bezeugen, Andreas Silberman, als Vatter. Josephus Jeremias Titot im Nahmen des Pfetters. M. Johann Leonhard Fröreissen, Pf. zu St. Nicol.

AMS, D 93, fol. 90 a (St. Nicolas) 
1720. 18.8 .

Acte de baptême de Johann Caspar Silbermann

Sontag den 18. Aug. 1720. ist von mir M. Johann Leonhardt Fröreissen, Pf. zu St. Nicol. H. Andreas Silbermann, dem Orgelmacher und Burger alhier, und seiner ehel. Haussfr. Anna Maria geb: Schmidin, ein Söhnlein mit Nahmen Johann Caspar getaufft worden. Dabey zu Gevattern waren, H. Caspar Schmid, der Wagner, und E.E.Grossen Raths alter Beysitzer alhier, und Jacob Mähn, der Weissbeck und Burger alhier, und Fr. Martha Lampin, Johannes Lampen dess Metzgers und Burgers alhier ehel. Haussfr. welches bezeugen.

Andreas Silbermann als Vatter

Caspar Schmidt als Pfetter

Jacob Mähn alss Pfetter

Mardan Lampin als Gettel

M. Johann Leonhardt Fröreissen Pfarrer zu St. Nicol.

AMS, N 112, fol. 99 a (St. Nicolas) 
1721. 20.2.

Acte de décès de Johann Caspar Silbermann

Anno 1721. starb den 20ten Hornung Donnerstag Abends um 10. Uhren Joh: Caspar, Herrn Andreas Silbermanns, Orgelmachers u. Burgers allhier, u. Frauen Annä Mariä gebohrner Schmittin ehliches Söhnlein, u. wurde den 22ten dito vormittag um 10 Uhren, auff dem Gottesacker St. Urbani begraben. Seines Alters 25. Wochen, wenig.2. Tag. Solches bezeugen

Andreas Silberman als Vatter.

Jacob Mähn als Pfetter

M. Joh: Andreas Schmutz, Diac: zu St. Niclaus

AMS, N 94, fol. 19 b (St. Nicolas) 
1722. 15.1 .

Acte de baptême des jumeaux Johann Jacob et Gottfriedt Silbermann

Donnerstag den 15. Jan. 1722. seind von mir M. Joh: Andrea Schmutz, Diac: zu St. Niclaus, H. Andreas Silbermann dem Orgelmacher und Burger alhier, und seiner ehelichen Haussfrawen Anna Maria, geb: Schmidin, zwey Zwillings Söhnlein getaufft worden, davon das erste, Johann Jacob genennet wurde. Dabey zu Gevattern waren, H. Johann Jacob Öhlinger, notar. Publ. und Burger alhier, und Johann Jacob Biermeyer, der Werckmeister auff dem Zimmerhoff, undt Fr. Maria Salome Schmutzin, H. M. Andreas Schmutzen, dess Diaconi bey diesser Kirchen, eheliche Haussfr. Andreas Silberman als Vatter. Johann Jacob Ölinger not. publ. Johann Jacob Biermayer alss Pfeter Maria Salome Schmutzin als Göttel M. Joh: Andreas Schmutz. Diac. zu St. Niclaus.

Das andere Söhnlein empfieng den Nahmen Gottfriedt, dabey zu Gevattern waren, H. Johann Heinisch, der Kübler und Burger alhier, und H. Johann Matthäus Knauer, der ledige Handelsmann und Burger alhier, undt Fr. Elisabetha Schallin, Johann Heinrich Schallen, dess Carduanmachers und Burgers alhier, eheliche Haussfr. welches bezeugen.

Andreas Silberman als Vatter.

Johann Matthäus Knauer als Pfetter

Johannes Heinisch alss als Pfetter

Elisabetha Schallin als Götel.

M. Joh: Andreas Schmutz. Diac: zu St. Niclaus.

AMS, N 112, fol. 129 b (St. Nicolas) 
1722. 23.8 .

Acte de décès de Johann Jacob Silbermann

Anno 1722 starb Sonntag den 23t. Augusti Abends um 5. Uhren, Joh: Jacob, Herrn Andreä Silbermanns, dess Orgelmachers u. Burgers allhier, u. Fr. Annä Mariä, gebohrner Schmittin, eh.- Söhnlein, u. ward den 25sten dato darauf nachmittag um 2. Uhren auf dem Gottes: Acker St. Urbani begraben; Seines Alters 7. Monat 3 Wochen u. 2 Tage. Solches bezeugen

Andreas Silberman als Vatter. Johann Jacob Ölinger nots. publ. alss Pfetter. M. Joh: Andreas Schmutz. Diac: zu St. Niclaus.

AMS, D 94, fol. 23 a (St. Nicolas) 
1723. 14.4.

Acte de baptême de Maria Cleophe Silbermann

1723.

Mittwoch d. 14t. Aprill ist von mir, M. Joh: Andrea Schmutz, Pfarrer zu St. Niclaus, Herrn Andreä Silbermann, dem Orgelmacher u. Burger allhier, u. seiner ehl.- Hausfrauen, Fr. Anna Maria, geb: Schmittin, ein Töchterlein, namens Maria Cleophe getaufft worden: Dabey waren zu Gevattern, H. Gottfried Silbermann, Orgelmacher zu Freiberg in Sachsen, Fr. Maria Magdalena, Hn. Matthäi Edels, dess Glockengiessers, ehl. Hausfrau, u. Fr. Maria Elisabeth Rebin Samuel Schragen, dess Seilers, ehl. Hausfr. Solches bezeugen Andreas Silbermann, als Vatter.

Conrad Hüttner, in Nahmen Herrn Gottfrid Silbermanns, Orgelmachers zu Freyberg in Meissen, als Pfetters.

Maria Magdalena Edelin als Göttel

Maria Elisabetha Schragin als Göttel

M. Joh: Andreas Schmutz, Pfarrer zu St. Niclaus.

AMS, N 112, fol. 161 b (St. Nicolas) 
1723. 19.5 .

Acte de décès de Maria Cleophe Silbermann

1723.

Mittwoch den 19ten May starb vormittag um 10. Uhr, Maria Cleophe, Herrn Andreä Silbermanns, Burgers u. Orgelmachers allhier, u. seine eh.- Hausfrauen, Fr. Annä Mariä, geb: Schmittin, eh.Töchterlein u. war d. 21. dito vormittag um 10. Uhr, auff dem Gottesacker St. Urbani begraben, seines Alters 1. Monath u. 5. Tag. Solches bezeugen

Johann Heinrich Schall, im Namen des abgewesten Vatters. Conrad Hüttner als Pfetter.

M. Johann Andreas Schmutz. Pfarrer zu St. Niclaus.

AMS, D 93, fol. 110 b (St. Nicolas) 
1726. 8.3.

Acte de baptême de Jean Michel Silbermann

1726.

Freytag den 8. Mertz, ist von mir, M. Joh: Andreas Schmutz, Pfarrer zu St. Niclaus, Herrn Andreä Silbermann, dem Orgelmacher u. Burger allhier, u. seiner ehl. Hausfrau Anna Maria, geb: Schmidtin, ein Söhnlein getaufft, u. Joh: Michael, genennet worden. Die Tauffzeugen waren H. Johannes Dierbach, Schulmeister u. Choragus zu St. Aurelien, Johann Friedrich Eisenmann, der Gastgeber u. Burger allhier, u. Fr. Catherina Elisabeth, Herrn Johannes Blümels, dess Steinschneiders und Burgers allhier, ehliche Hausfrau.

Andreas Silberman als Vatter. Johannes Dierbach als Pfetter.

Johann Friederich Eisenmann als Pfetter. Catherina Elisabetha Blimlin, als Göttel. M. Johannes Andreas Schmutz, Pfarrer zu St. Niclaus.

AMS, N 112, fol. 236 a (St. Nicolas) 
1727.24 .9$.

Acte de baptême de Jean Henri Silbermann 1727.

Mittwoch den 24. Sept: ist von mir, M. Johann Andreas Schmutz, Pfarrer zu St. Niclaus, Herrn Andreas Silbermann, dem Orgelmacher und Burger allhier, u. seiner eh. Hausfrauen, Anna Mariä, geb. Schmidtin, ein Söhnlein getaufft, und Joh: Heinrich benennet worden.

Die Tauffzeugen waren H. Johannes Hamel, E. E. Kleinen Raths Beysitzer allhier, H. Joh: Christoph Frauenholtz, Capellmeister u. Burg. allhier, u. Fr. Maria Jacobe, Johannis Ehremanns, dess Macklers und Burgers allhier, ehl. Hausfrau.

Andreas Silberman als Vatter.

Johannes Hamel, als Pfetter.

Johann Christoph Frauenholtz, als Pfetter.

Maria Jacobea Ehrmännin als Göttel

M. Johannes Andreas Schmutz, Pfr. zu St. Niclaus.

AMS, N 112, fol. 276 b. 
1729. 17.4.

Acte de décès de Jean Michel Silbermann

1729.

Sonntag den 17. Apr. starb abends um 5. Uhren, Joh: Michael, Herrn Andreä Silbermanns, Orgelmachers u. Burgers allhier, u. Frau Annä Mariä, geb: Schmidtin, eh.- Töchterlein, und war darauf den 19ten ejusd. auff dem Gottesacker St. Urbani zur Erd.- Bestattet; seines Alters 3. Jahr u. 6. Woch.

Andreas Silberman als Vatter

Johannes Dirbach als Pfetter

M. Joh: Andreas Schmutz, Pfr. zu St. Niclaus.

AMS, D 93, fol. 153 a (St. Nicolas) 
1734. 16.3.

Acte de décès d'André Silbermann

Dienstag den 16. Mertz, starb vormittag zwischen 8. u. 9. Uhr, H. Andreas Silbermann, Orgelmacher und Burger allhier, und war 4 den 18. ejusdem, nach gehaltener Leich: Sermon zu St. Niclaus, nachmittag um 2. Uhr, auff dem Gottes:Acker St. Urbani zur Erden bestattet: seines Alters 55. Jahr, 9. Monat, 27. Tage.

Johann Andreas Silbermann, als Sohn.

Philipp Jacob Nacken, als Tochtermann

Johann Andreas Schmutz, Pfr. zu St. Niclaus.

AMS, D 93, fol. 187 a (St. Nicolas) 
1742. 22.8 .

Acte de mariage de Jean André Silbermann et d'Anne Salomé

Mannberger.

1742.

\& d. 22 Aug. seind nach ordentl. Proclamation ehl. copul. u. eingesegnet worden $H$. Johann Andreas Silbermann der led. Orgelmacher u. B. allhier, weyl. H. Andreae Silbermanns gewess. Orgelmachers u. B. allhier nachgelassener ehl. Sohn u. J. Anna Salome weyl. H. Johann Nicolai Mambergers gewessenen Langmesserschmidts u. $B$. allhier nachgelassene ehl. Tochter.

Johann Andreas Silbermann als Hochzeiter

Anna Salome Mambergerin als Hochzeiterin

Philipp Jacob Eberts als Zeug

Sigmund Falckenheimer als Vogt

M. Joh. Fried. Rollwagen Diac.

AMS, M 114, fol. 211 a (Temple-Neuf) 
1745. 2.10

Acte de naissance de Johann Daniel Silbermann.

1745

† den 2 Octobr. frühe morgens um $91 / 4$ Uhr ist ein Söhnlein geboren, $\odot$ den 3 seq. getaufft, u. Johann Daniel genannt worden.

Parentes: H. Johann Andreas Silbermann Orgelmacher und Burger allhier, und Fr. Anna Salome, geb. Mannbergerin, seine Ehefr. Susc. 1. H. Johann Fridrich Mannberger, Handelsmann und Burger allhier. 2. Herr Johann Daniel Silbermann, der ledige Orgelmacher, weyl. Herrn Johann Andreas Silbermann, gew. Orgelmachers u. Burgers allhie, hinterl. ehel. Sohn. 3. Fr. Anna Margaretha, H. Johann Jacob Ottmann, des Müllers und Burgers allhie Ehefr.

Johann Andreas Silbermann als Vatter

Johann Friederich Mannberger als Pfetter

Johann Daniel Silbermann als Pfetter.

Anna Margaretha Ottmänin als Göttel

M. Joh. Phil. Lobstein, Pastor tauff.

AMS, N 114, fol. 109 a (St. Nicolas) 
1748. 6.3 .

Acte de mariage de Jean Daniel Silbermann et Marie Elisabeth Bäer

1748.

\& den 6. Mart. seynd nach zweymahliger Proclamation, in der Evangelischen Kirche zu St. Nicolai, in den Stand der H. Ehe eingesegnet worden, Herr Johann Daniel Silbermann, der ledige kunsterfahrene Orgelmacher u. Burger allhir, wey. H. Andreas Silbermann, gewessten kunsterfahrnen Orgelmachers, u. Burgers allhir hinterl. ehel. Sohn, und Jungfr. Maria Elisabetha Bäerin, weyl. Johann Philipp Bäer, gewessten Handelsmanns u. Burgers allhir hinterl. ehel. Tochter.

Johann Daniel Silbermann als Hochzeither.

Maria Elisabetha Bäerin als Hochzeitherin.

Johann Nicolaus Mannberger als Beystandt

Johann Daniel Bäer als Ocle

M. Joh. Phil. Lobstein, Pfarrer copulirte.

AMS, M 54, fol. 90 a (St. Nicolas) 
1750. 13.9.

Acte de décès de Marie Elisabeth Silbermann, nee Bäer

$\odot$ den 13 Sept 1750. vor Mitternacht zwischen 11. und 12. Uhr, starb zu Sultz, oberhalb Colmar, Frau Maria Elisabetha Silbermännin, geborne Bäerin, Herrn Johann Daniel Silbermann, des kunsterfahrnen Orgelmachers und Burgers allhie gewesste Ehefr. Dero entseelter Leichnam ward $\sigma^{\top}$ den $15 \mathrm{seq}$. von gedachtem Sultz, vormittag auf den Gottesacker St. Urbani gebracht, und nachmittag um 4 Uhr vor gehaltener Leichenpredigt zur Erde bestattet. Aet 27 Jahr, 3 Wochen.

Johann Daniel Silbermann als Wittwer. Johann Georg Bruckner als Schwager M. Joh. Phil. Lobstein, Pfarrer.

AMS, D 96, fol. 41 a (St. Nicolas) 
1752. 14.12 .

Acte de naissance de Johann Andreas Silbermann.

4 den 14. Decembr. 1752. morgens um 8 3/4 Uhr, ward ein eheliches Söhnlein geboren, 우 den 15. seq. getaufft, und Johann Andreas benahmet worden.

Parent. H. Johann Andreas Silbermann Orgelmacher und Burger allhie, und Fr. Anna Salome geb. Mannbergerin.

Susc. 1. H. Johann Fridrich Lobstein, Handelsmann und Burger allhie. 2. H. Johann Heinrich Silbermann, der ledige Orgelmacher und Burger allhie. 3. Fr. Anna Maria, H. Johann Saum, des Weinhändlers und Burgers allhie, auch Fabric=Pflegers bey der Evang. Kirche zu St. Nicolai Ehefr.

Johann Andreas Silbermann als Vatter

Johann Friederich Lobstein, als Pfetter

Johann Heinrich Silbermann als Pfetter

Anna Maria Saumin als Göttel

M. Joh. Phil. Lobstein, Pfarrer, tauff.

AMS, N 114, fol. 445 a (St. Nicolas) 
1759. 9.1 .

Acte de baptême de Catherine Henriette Silbermann

Dienstag d. 9 Jan (1759) morgens gegen 6 Uhr ist H.- Joh Heinrich Silbermans Orgel und Instrumenten machers ehl.- Hausfr Catharina Margaretha g. Mossederin eines Töchterl.- genesen welches folgenden Mittwoch getaufft und Catharina Henrietta genennett worden. Patr. H.- Joh Jacob Mosseder Weinhandler und B. allh. Fr Anna Salome $g$ Mambergerin H.- Joh Andreas Silbermans Orgel und Instrumenten machers ehl. Husfr und Fr Anna Maria g Silbermannin H.- Joh Adam Saum Weinhändlers und B. allh.- ehl.- Hausfrau Johann Heinrich Silbermann als Vatter Johann Jacob Mosseder als Pfetter Anna Maria Saumin als Göttel Ann Salom Silbermannin als Gottel M. Joh. Frieder Rollwagen Diac.

AMS, N 230, fol. 421 a. (Temple-Neuf) 
1762. 2.5 .

Acte de décès de Gottfried Sibermann

1762.

Sonntag d. 2. Maj. morgens nach 10. Uhr, starb weyl: Herr Gottfried Silbermann, (lediger Herr) Kunstmahler und Burger allhier, wurde begraben Dienstag Nachmittag um 2. Uhr auff $S$. Urbani. Alt 40. Jahr, 4. Monat, 2. Wochen u. 2. Tag. Eltern waren $H$. Andreas Silbermann Orgelmacher u. Burger allhier, u. Fr. Anna Maria g. Schmidtin.

Johann Andreas Silbermann als ältster Bruder Johann Heinrich Silbermann als jüngster Bruder M. Joh. Joachim Röcklinger, Pf. zu S. Nicol.

AMS, D 96, fol. 115 b (St. Nicolas) 
1765. 17.2.

Acte de naissance de Johann Josias Silbermann.

1765.

Ein tausend, siebenhundert und fünff und sechzig Sonntags den siebenzehenden Hornung abends ein Viertel vor zehen Uhr ist ein Söhnlein geboren den zweyten Tag, als Dienstags darauff getaufft, und Johann Josias genennet worden.

Der Vatter ist $H$. Johann Andreas Silbermann, Orgelmacher und Burger alhier.

Die Mutter ist Frau Anna Salome, geborne Mambergerin, dessen Ehefrau.

Die Pfettern sind Herr Josias Weigen Medicinae Doctor und Practicus alhier, wie auch hochgräflich Leiningischer Hoffrath, und Burger alhier und Herr Johannes Pfeffinger, Medicinae Doctor und Professor bey löblicher Universitaet alhier, und Burger hieselbst. Die Göttel ist Jungfer Maria Magdalena Goullet, Herrn Johann Daniel Goullet, Banquier und Burgers alhier eheliche Tochter, wie solches bezeugen

Johann Andreas Silbermann als Vatter

Josias Weigen, Med. D.r \& Hof Rath, als Pfetter.

Dr. Jo. Pfeffinger, Prof. P. Ord. als Pfetter.

Maria Magdalena Goullet als Göttel

Johann Daniel Mülberger

Pfarrer zu St. Nicolai tauffte.

AMS, N 115, p. 463 (St. Nicolas) 
1766. 9.5 .

Acte de décès de Johann Daniel Silbermann.

Montag, den 12. Maji (begraben)

Ein Mann 48. Jahr, H. Johann Daniel Silbermann, Churfürstl.

Sächss. Hoff-Comissair und Hoff-Orgelbauer in Dressden, in der Grimmischen Gasse, st. 우

Leipzig, Stadtarchiv

Ratsleichenbuch der Stadt Leipzig, Vol. 30, fol. 308 b. 
1769. 3.5 .

Acte de décès d'Anne Salomé Silbermann, née Mannberger

Ein tausend, siebenhundert, neun und sechzig Mittwoch den dritten May Mitternacht ein Viertel vor zwölff Uhr ist gestorben weyland Frau Anna Salome, geborne Mannbergerin Herrn Johann Andreas Silbermanns, Orgelmachers und Schöffen Einer Ehrsamen Zunfft der Zimmerleuth im Leben geweste Ehefrau. Samstags darauff wurde derselben verblichener Leichnam mit gewohnlichen Cerimonien auf den Gottesacker zu St. Urbani zur Erden bestattet. Ihres Alters drey und vierzig Jahr, fünff Monath und zwanzig Tag. Ihre Eltern sind gewessen weyland H. Johann Niclaus Mannberger Eines Ehrsamen kleinen Raths gewester alter Beysitzer, und Langmesserschmid alhier, und dessen geweste Ehefrau Anna Salome, geborne Füssingerin, wie solches bezeugen.

Johann Andreas Silbermann, als Wittwer

Johann Daniel Silbermann,. als Sohn

Johann Andreas Silbermann, als Sohn

Johann Daniel Mülberger, Pfarrer zu St. Nicolai.

AMS, D 99, fol. 12 b (St. Nicolas) 
1772. 2.2 .

Acte de mariage de Jean André Silbermann et d'Anne Elisabeth Schatz

Ein Tausend, Siebenhundert, zwey und siebenzig Sonntags den zweyten Hornung sind mit gnädiger Erlaubnus des Regierenden Herrn Ammeister in der Kirch zu St. Nicolai zum ersten und andrenmal zugleich ausgeruffen und Dienstags darauff zu Hauss ehelich eingesegnet worden Herr Johann Andreas Silbermann Eines Ehrsamen Grossen Raths alter Beysitzer, vornehmer Burger, und weyland Frauen Anna Salome, geborner Mannbergerin, hinterlassner Wittwer, und Jungfer Anna Elisabetha Schatzin, weyland S.T.Herrn Johann Jacob Schatz gewesten Löblicher Universitaet alhier Gymnasiarchae und Bibliothecarii, auch vornehmen Burgers alhier mit weyland Frauen Anna Margaretha, gebohrner Arnoldin, hinterlassne ehelich erzeugte Tochter wie solches bezeugen

Johann Andreas Silbermann, als Hochzeiter.

Anna Elisabetha Schatzin, als Hochzeiterin. Johann Heinrich Silbermann als zeuge

Lt Johann Heinrich Faust alss Zeug

M. Joh. Schulmeister Pfar. Test.

Joh: Jac: Walther

Johann Daniel Mülberger, Pfarrer zu St. Nicolai.

AMS, M 55, fol. 124 b (St. Nicolas)

Im Jahr eintausend, Siebenhundert, Siebenzig und zwey, Dienstag den vierten February, sind auf obrigkeitliche Erlaubnus am vierten Sonntag nach Epiphaniae zu St. Thomae und zu St. Nicolai zum ersten und andern mahl zugleich aussgerufen und darauf zu Hauss ehelich copuliret und eingesegnet worden: Herr Johann Andreas Silbermann, der Wittwer, Orgelmacher, und E.E. grosen Raths alter Beysitzer und Burger allhier, und Jungfrau Anna Elisabetha Schatzin, weiland Herrn M. Johann Jacob Schatz, gewesenen Gymnasiarchae und Bibliothecary, auch Burgers allhier nachgelassene eheliche Tochter, wie solches bezeuget

M. Philippus Jacobus Engel

Pastor Thomanus mppria.

AM, M 123, fol. 222 b (St. Thomas) 
1783. 11.2.

Acte de décès de Jean André Silbermann

Dienstag, den eilften des Hornungs, Tausend Siebenhundert Achtzig und Drei, starb weiland Herr Johann Andreas Silbermann, gewester Orgelmacher, Bürger und E.E. grossen Raths alhier alter Beisitzer. Nächstfolgenden Freitag ward, zu St. Urbani, unter Amtsführung des unterschriebenen Diaconi, die Leiche christlich beerdiget. Zeugen sind die hier mit unterschriebenen. Er war im Jahr siebenzehenhundert und zwölffe, den vier und zwanzigsten des Brachmonates, geboren.

Joh: Andreas Silbermann als Sohn

Johann Heinrich Silbermann als Bruder

Johann Georg Stuber, Diaconus.

AMS, D 207, fol. 158 a (St. Thomas). 
1786. 3.6 .

Acte de décès de Josias Silbermann

Samstag, den dritten des Brachmonates, tausend siebenhundert achtzig und sechse, starb weiland Herrn Johann Josias Silbermann, gewester lediger Orgelmacher und Bürger allhier, der auch weiland Herrn Johann Andreas Silbermann, gewesten Orgelmachers und Bürgers, auch E. E. grossen Raths alten Beisitzers allhier, mit weiland Frau Anna Salome, geborner Mambergerin, ehelich erzeugter Sohn, den siebenzehenden des Hornungs, im Jahr sechzig und fünfe, geboren. Nächstfolgenden Montag wurde, zu St. Urbani, unter Amtsführung des unterschriebenen Diaconi, die Leiche christlich beerdiget. Zeugen sind die hier mit unterschriebenen:

Joh: Andreas Silbermann als Bruder.

Johann Heinrich Silbermann als Oheim

Johann Georg Stuber, Diaconus

AMS, D 207, fol. 203 a (St. Thomas). 
179915.1.

Acte de décès de Jean Henri Silbermann

1799.

Silbermann Jean Henri, âgé de 71 ans, 6 mois, mort le 26. Nivôse. III. arrondissement. Apoplexie.

Cejourd'hui vingt septieme Nivôse l'an sept de la République française une et indivisible, vû par moi, Alexandre Reichard, Officier municipal de la commune de Strasbourg Département du Bas-Rhin, faisant les fonctions d'Officier public en l'absence d'icelui, la copie du procès-verbal de ce jour, dressé par Jean Frédéric Wehrlen commissaire de police du troisième arrondissement de cette municipalité, qui constate que Jean Henri Silbermann, agé de vingt sept ans, homme de Lettres, et Philippe Jâcques Strohl, agé de trente deux ans, marchand pelletier, tous deux domiciliés en cette municipalité, le premier fils, le second gendre du defunt, sont comparûs par devant le dit commissaire et lui ont déclaré, que Jean Henri Silbermann, agé de soixante onze ans six mois, facteur de clavecins et forté pianos, né en cette commune, fils légitime de feû Jean André Silbermann, facteur d'orgues et de feûe Anne Marie Schmitt, veuf de Catherine Marguerite Mosseder, est mort hier vingt six Nivôse à dix heures du matin en sa demeure située Rue Finckwiller $N^{\circ}$ 64, que ledit Commissaire s'y est transporté et s'est assuré du dit décès. Moi Alexandre Reichard, après que les dits comparans m'ont eû certifié la vérité de cette déclaration, j'ai rédigé le présent acte, qu'ils ont signé avec moi. Fait en la maison commune de strasbourg, les jour, mois et an ci-dessûs.

Silbermann Strohl Jn. Alexandre Reichard
off publ pro.re

AMS, Décès an VII, acte 366. 
Inventaire après décès de Jean André Silbermann.

Etat

Der, von Herrn RathHerrn Silbermann hinterlassenen Strassburger Geld= und Denck= Münzen, Zeichnungen, Plans, auch Manuscripten vön dessen eigener Hand und fremder Personen Händen:

\section{I $\underline{\text { Münz }}=$ Cabinet.}

Tablette 1.

A. $N^{\circ} 1$. Drey silberne Bracteaten auf beiden Seiten geprägt.

2. Zwey Münzen von Metall wie das Corintische Erzt, einseit eine Kirch, anderseit ein Creuz mit ohnleslichen Schriften

3. Eine silberne Münz von Kayser otto 1.

4. Vier Stuck silberne Engels Pfenning.

5. Ein alter silberner Pfenning mit dreyfachen Bischofs stab in Gestalt einer Lilie.

B. 1. Fünf gar alte Pfenning.

2. Sieben etwas neuere Pfenning.

3. Ein Örtlein oder Quatraetlein.

4. Zwey alte Creuzer oder Zweyling.

5. Zwey alte Vierer oder Halbbatzen.

6. Vier Stuck alte Plappert.

7. Vier Stuck Schillings Groschen.

Tablette 2 .

Münzen so meistens im XVI.den Jahrhundert geschlagen worden.

A. $\quad 1$. sechs Stuck Pfenning.

2. drey Stuck Helbling oder Heller.

3. zwey Örtlein oder Quatraetlein.

4. vier alte Creuzer und zwey kleine neuere Kreuzer.

5. zwey Stuck halbe Batzen oder Sitz halb Bazen.

6. zwey Halb Bazen etwa Raths Halb Bazen genant.

7. zwey Halb Bazen.

8. Vier Stuck neue Halbbazen oder 11. Kreuzer Stuck.

9. zwey Stuck drey Kreutzner. 
B. Ein dickes Stuck mit 11. Kreuzer Gepräg.

Zwey dike Stuck Pfenning Gepräg.

Ein sechsfacher doppelter Rathsgroschen.

Ein dreifacher dito.

Ein achtfacher dito.

Tablette 3.

Acht und dreisig Stuck einfache Rathsgroschen sonsten auch drey Kreuzner oder Raths Plappert genannt, von neunzehnerley Gepräg.

1. Zwey Stuck etwas grösere und schwerere Rathsgroschen. Sechs doppelte Rathsgroschen oder neuen Schilling gros.

\section{Tablette 4.}

1. Zwey Stuck dick Pfenning von zweyerley Stempel.

2. Zwey alte drey Bätzner.

3.4.5.6. Acht Stuck viererley drey Bätzner.

7. Ein sechs Bäzner zu XXIIII. X.r

8. Ein Pfening.

9. Zwey geringere Pfenning.

10. Sechs kleine Münzen vermuthlich Kreuzer.

11.Ein drey Bäzner oder XII Kreuzer Stuck.

12.Ein sechs Bäzner oder XXIIII. Kreuzer Stuck.

13. Ein falscher drey Bäzner.

Tablette 5 .

Fünf Gulden oder LX. X.r von verschiedenem Gepräg.

Vier Stuck halbe Gulden.

Eine Hildsheimische Stadt Münz oder 24. Marien Groschen.

Tablette 6 .

A. Drey theils alte theils neuere einfache Thaler 
B. Vier Stuck halbe Thaler.

Zwey Orths Thaler.

drey Stuck doppelte Thaler.

Tablette 7.

Münzen von annis 1682. bis 1687.

zwey XXX. Sols stück.

zwey XV. " " "

zwey $X$. Sols "

vier IIII. " "

dreyzehen II." "

Sechs 1. Sol stück.

Tablette 8 .

Von der Stadt Strasburg geschlagene GoldMünzen.

$N^{\circ}$ 1. Ein gar alter GoldGulden. Urbem Virgo tuam servo.

1. Ein neuerer Goldgulden mit nemlichem Virgo Gepräg.

2. Zwey Goldgulden nach der sogenanten Reformation geschlagen mit der Umschrift Urbem Christae tuam serva.

3. zwey Stuck doppelte Goldgulden von zweierlei Stämpel.

4. drey Stuck Dukaten jede von einem besondern Gepräg.

5. Eine doppelte Ducat.

6. Eine vierfache Ducat.

7. Zwey Stuck dreifache Ducaten.

8. Eine Ducat nach dem Gepräg eines alten Halb Bazens.

9. Ein Goldgulden Rathsgroschen Gepräg.

10.Eine doppelte Ducat dreybäzner Gepräg.

11.Ein Goldstück einseit die Stadt Strasburg ein Gewölk ein Engel mit einem Kranz und Jahrzal 1627.

12. Eine Academische Jubilaeums Münz von Gold und vier Gold Gulden schwehr einseit idemoria Secularis Academiae Argentinensis neben der stadt Schild M D C L X V I I, anderseit Kayser Maximilianus II. p.

13. Ein sogenannter Goldener Helbling. 
Tablette 9.

Academische Münzen.

$N^{\circ}$. Zwey gleichlinge Jubilaeums Münzen, einseit ein Altar, anderseit Pietas Secularis Academiae Argentinensis 1667.

2. drey grösere Jubilaeums Münzen mit zweierlei Stempel und Gewicht geschlagen, in der Gröse und Gepräg wie die goldene Tabl. 8. $N^{\circ} 12$

Eine dicke Praemium Münz von 1680.

Sechszehen dünnere Praemium Münzen de annis 1681. 82. 83. 84. 85. 86. 87. 88. 89. 90.91.92. 93.94.95. et 1696. von verschiedenem Gewicht, jede mit einer besondern Überschrift und Vorstellung.

Tablette 10 .

Jubilaeums Münzen von 1617.

zwey grose viereckigte.

" runde mit nemlichem Gepräg.

" kleine viereckigte.

Eine runde vom nemlichen Gepräg.

Alle einerlei Inhalt der Schrift, einseit Pro. Religionis Centum ante annos \&C. Anno 1617. cal. Novembr. anderseit der Stadt Schild und die Schrift: [Lux post Tenebras 1517 Omnis Terra adoret Deum et Psallet ei. Unter diesen seind 2. viereckigte und zwey runde: auf der einen Seite stehet, Post Tenebras Lux 1517. anderseit] Jubilaeum Argentoratense 1617. Zwey kleine viereckigte Münzen von eben dem Inhalt. Zwey runde mit nemlicher schrift.

Zwey gar kleine $\diamond$ jedes.

Eine Jubilaeums Münze so 1630. zum Andencken der vor 100. Jahren zu Augspurg übergebenen Glaubens Bekanntnuss geschlagen worden von feinem Silber.

Tablette 11 .

A. Zum Gedächtnus des im Jahr 1576. gehaltenen Büchsen und StahI oder Armbrust Schiesens. 
1. Zwey der ersten gemünzten silbern Gulden von 1576. die eine seit mit der Umschrift Solius Virtutis flos perpetuus.

2. Zwey halbe dito so über der Lilie eine Armbrust mit zweyen Creuzweis übereinander liegenden Büchssen haben und auf der andern Seit 1576.

B. Gedächtnuss Münzen auf das im Jahr 1590. auf der Metzger Au gehaltene Schiesen mit Cartaunen.

3. Eine vergoldte 4eckigte Münz.

4. Zwey runde.

5. Eine grose viereckigte mit der Stadt Strasburg.

6. Zwey Stuck 6. b. grösse.

7. Eine Thaler grosse Münz.

8. Eine gegossene Münz von nemlicher Grösse.

9. Eine eben dergleichen Münz im Gepräg und Gröse.

10. Eine gegossene Münz in der Gröse eines Thalers aber leichter.

Alle mit verschiedenen Inschriften.

C. Drey Klippen oder viereckigte Gedächtnus=Münzen so wegen dem Anno 1592. entstandenen Bischöflichen Krieg geschlagen und nur auf einer Seite geprägt, darauf der Stadt Strasburg des Bischoff Johann Georg und des Capitels Wappen und die Jahrzahl 1592. befindlich. Der Wert ist zu 80. 40. und 20. Creuzer.

Tablette 12 .

Als die Stadt Strasburg den Bund mit den Schweizer Cantons Zürich und Bern erneuert.

$N^{\circ} 1$. Eine kleine 4eckigte Münz mit der Jahrzahl 1588.

2. 3. zwey kleine viereckigte Bunds Münzen und eine doppelte dito so im Gepräg einander gleich mit erstgemelter Jahrzahl, davon die erst 2. ein Loth, leztere aber zwey Loth wieget.

4. Zwey Stuck so wegen dem Nimwegischen Frieden 1679. zu Strassburg geschlagen worden.

5. Eine vergoldte Medaille so der Stadt 1615. dedicirt worden.

6. Zwey kleine Münzen auch auf den Nimwegischen Frieden sich beziehend mit der Jahrzahl 1679. 
7. Ein viereckigt vergoldt Stuck mit der Jahrzahl 1628.

8. Eine viereckigte Münz mit doppeltem Thaler Gepräg.

9. Eine $\diamond$ wie eines dickpfennings Gepräg.

10. Zwei Münzlein mit der Jahrzahl 1627.

11. Eine vergoldte Medaille de 1629.

12.Zwey gleichlinge Münzlein de 1628.

Vorherige $N^{\circ}$ 1. - 7. und 10. - 12. bezeichnete Münzen mit verschiedenen Emblematibus und Schriften.

Tablette 13..

$N^{\circ}$ 1. Eine kleine DenckMünz einseit der Stadt Lilie mit der Umschrift Solius Virtutis flos perpetuus.

2. Zwey gleichlinge vergoldte Münzen einseit die stadt Strassburg anderseit der Stadt Wappen 1629.

3. Zwey gleichlinge Medailles einseit die Stadt Strasburg mit der Jahrzahl 1627. anderseit der stadt Wappen mit doppelten Reihen Umschriften-

4. Zwo gleichlinge Münzen, einseit die Stadt Strasburg mit der neuen Befestigung, anderseit in der Mitte der Stadt Wappen mit den Wappen der 20. Zünfte samt Hohensteeg.

5. Eine Medaille de 1678. mit der Umschrift in 20. Zünften und Gottes Huth, Strasburg die stadt bestehen thut.

6. Eine vergoldte Medaille, einseit die stadt Strasburg mit der Jahrzahl 1627. anderseit nebst der Stadt Wappen die von den 20. Zünften.

7. Eine silberne Medaille wie die vergoldte Tabl. $12 . N^{\circ} 11$.

8. Eine Medaille mit 2. Bildern den Frieden und die Liebe vorstellend.

9. Eine Hochzeit Münze von 1629.

10. Eine grose Medaille auf dem Avers das Brustbild Ludwig XIV. mit den Umschriften Ludovicus Magnus Rex und dem Revers Argentorat. Alsat. Metrop.

11.Eine vergoldte Medaille einseit die Stadt Strasburg anderseit in der Mitte deren Wappen und um denselben die Wappen der 10. Elsassischen Reichs Städten. 
Tablette 14.

A. $N^{\circ}$ 1. 2. Zwey französische Thaler de 1695. und 1704.

3. Ein sogenantes Piece de Trente Sols de 1705.

4. 5. 6. drey zwölf Schillinge de 1709. 1714. und 1716. drey Costnizer Pfenning.

B. Alte Bischöfliche Münzen.

1. Eilf Stuck von den ältesten Bischöflichen Strasburgischen Pfenningen.

2. 3. 4. drey alte Stücklein von den Bischöffen Widerolch, Werner und Conrad.

5. Eine viereckigte Münz von dem Bischoff Johann Landgraff in Elsas.

6. Eine grösere Klippe oder viereckigte Münz von diesem Bischoff mit der Jahrzahl 1575.

7. Ein Gold Gulden vom nemlichen Jahr.

8. Zwey gleichlinge Münzen mit dem Brustbild Bischoff Carl Card. Loth.

9. Eine Münze vom Erzherzog Leopold zu Österreich de 1626. 10.Ein Bischöflicher Strasburgischer Halb Batzen.

Über dieses gesamte vorherbeschriebene Strasburgische Münz Cabinet befindet sich eine ausführliche Beschreibung nebst den Abdrücken der Münzen selbsten in einem Manuscript in folio gebunden vorhanden so von Herrn Rathherrn Silbermann verfertiget. 
II. Zeichnungen und Plans von der Stadt Strassburg und Gegenden im Elsass.

In dem Buch in Folio Lit: $A$.

Pag. 2.- 8. Sieben Plane über den Ursprung, die erste Gelegenheit der Stadt Strassburg und deren 5 malige Erweiterungen.

10.- 19. Zehen Perspectivische Grund Risse über den völligen Umfang der Stadt gegen dem Ende des XVIden Jahr Hunderts.

20. Prospect des Spitals, St. Erharts Capelle, und Speculi Astronomici samt spithal Thors im XVII. Jahr Hundert.

21. Perspectivischer Grundriss vom Metzger und dem ohnweit davon gestandenen Catharinen Thor samt dem Dauben Graben und Catharinen=Thurn.

22. Perspectivischer Grund Riss vom Armbrust Schiessrain samt dem Juden und Fischer Thor und St. Clara wörth.

23. Idem von der Gegend des Steinstraser Thors, des Veldenzischen Hoffs, Burg Thor, Thurn im Rausch und dem Rosseneck de 1577.

24. St. Elisabethen Thor de 1576.

24. St. Johannis Thor, gedeckten Bruck und gelben Thurn de 1576.

25. Weissthurn Thor samt Bollwerck und Acht Räder Mühl wie auch Cronenburger Thor de 1576.

26. - 37. Verschiedene Perspectivische Abriss verschiedener Klöster offentlicher Gebäude, Brucken \&. in der stadt.

39. -64. Grund und Profil=Riss, Bildniss, Monumenten von verschiedenen Gegenden der Stadt, Gebäuer, Häusser, Kirchen, ruinirten Gebäuden, Statuen und hin und wieder in der stadt befindlicher Monumenten, Statuen, Grabstein \&C.

65. - 69. Grundriss, Fundamenter, Zeichnungen vom Münster, dessen Thurn oberhalb der vier Schnecken, dessen obersten Theil, Pfeilern, Gewölben und Dach. 
71. - 76. Zeichnungen Riss und Perspectivische Vorstellungen von der IIl und Preusch nebst andern bey der Stadt befindlichen Wassern, des Schiess Rayns, Gegend ausserhalb des Steinstraser Thors von 1680. Gerichts Platz, Gegend um Strasburg de 1760. Vorstellung der Stadt 1680. und Rheinbrück de 1606 .

In dem Buch in Folio Lit: B.

Mehrere Grundriss von der Gegend wo das Römische Argentoratum gestanden, des neuen Marckts, wie die Stadt in ihrer Befestigung 1632. beschaffen war, des alten Herren Stalls vom deutschen Haus, des Rheins, der Breusch, der Gegend vom Dorff Kehl, der Bernhardiner Abtai Pairis, Grabstein \&C.

In dem Buch in Folio Lit: $C$.

68. Zeichnungen von einigen im Elsass liegenden Orten, Gebürgen, Schlössern und Gegenden.

In dem Buch in Folio Lit: D.

36. Zeichnungen von verschiedenen im Elsass liegenden Orten, Gebürgen, Schlössern und Gegenden.

In dem Buch in Folio Lit: E.

38. auswärtige Prospecte von Gebürgen und alten Schlössern wie auch Gegenden ausserhalb des Elsasses.

In einem Carton in gros Folio.

Eine mit Farben gezeichnete General=Postkarte durchs ganze Elsass, und eine solche Karte, aber ohne Schrift.

Und 15. gezeichnete Karten theils auf öhl=Papier und theils mit Farben, wie auch Plans verschiedener Gegenden im Elsass, Lauf des Breusch Canals und sonsten:

Nota! unter diesen seind 9. gezeichnete Karten von der Schweiz begriffen.

In einem Buch in klein Folio.

2. mit Farben und zwölf auf Oel Papier gezeichnete Karten vom Elsass solche vierzehen Karten machen zusammen das Elsass aus: 
In einem Buch in gros Folio.

Zwey Zeichnungen mit Farben, die eine von der eigentlichen Gröse des steinernen Knopfs so Anno 1751. zu Oberst auf den Münsterthurn gesetzt worden und die andere die eigentliche Gestalt und Gröse des von Kupfer getriebenen Marien=Bilds so zu Oberst auf dem Chor Thurn gestanden vorstellend. Sodann 39. gestochene Plans sowohl der äussern, als auch innern Theile des Münsters in verschiedenen Vorstellungen.

In einem langen Küftgen seind folgende von Herrn Rathherrn gezeichnete Prospectus jeder besonders aufgespannt.

1. Vue de la Tour de la Paroisse de Müllhouse du Coté des Montagnes des Voges.

2. Vue de la Tour de la Cathedrale de Selestatt du Coté des [Montagnes des Voges].

5. Vue des Environs des Chateaux des Montagnes, Landsperg Andlau et Spesbourg.

4. Vue vers le Chateau de Landsperg et de ses Environs.

5. Vue de la Tour de la Cathedrale de Colmar du Coté des Montagnes des Voges.

6. Vue du Cloitre de St. Jean des Choux vers Saverne.

7. Die Aussicht aus dem Gotteshauss St. Märgen über den Schwarzwald.

Sechs mit der Feder gezeichnete Risse hinter Glässern in vergoldenen Rahmen, von ersterer Gelegenheit und fünf maligen Erweiterung der Stadt Strassburg von Herrn Rathherr Silbermann gezeichnet.

Zeichnungen hinter Glässern in vergoldten Rahmen von Herrn Rathhern Silbermann.

1. Erstlich sechs mit der Feder gezeichnete Risse von erster Gelegenheit und fünfmaliger Erweiterung der Stadt Strasburg.

2. Klein Landau ein Dorf im Obern Elsass samt dem Rhein und die Gegend dies und jenseits desselben.

3. Die Benedictiner Abtei Ettenheim Münster und die dabey liegende St. Landelins Kirche und Bad.

4. Das Portrait Georg Haag eines Seilers und seiner Hausfrau bede von Strasburg.

5. Prospect vom Michels Berg bei Riegel gegen dem Breisgauischen 
Gebürg und ein anderer von solchem Berg gegen das Vogesische.

6. Titisee auf dem Schwarzwald.

7. Grundriss des Fürstl.- Reichsstifts St. Blasius auf dem Schwarzwald auf grau Papier mit schwarz und weiser Kreyd.

8. Das Bildnuss Martin Kiens mit seinem ausserordentlichen langen Barth, zu Sulz im obern Elsass auf grau Papier mit gedachter Kreyd.

9. Das Bildnuss Berthold Schwarzen, Erfinder des Pulvers auf solch Papier mit der nemlichen Kreyd. 
Local Geschichte der Stadt Strassburg von H.- Rathh.herausgegeben 1775.

Gedruckt in Folio.

Von dieser Local Geschichte befinden sich annoch Zwei Hundert Drey und Achtzig Exemplaria ohngebunden vorhanden nebst den dazu gehörigen Kupferstichen. Und dann seind in dem vorher bey den Manuscripten angeführten Carton in Folio sub Lit: O. annoch verschiedene Projecte des zweyten Theils dieser Local Geschichte, Vorbericht, Abtheilung der Stadt in X. Cantons, Plan und Anmerkungen vorhanden, alles in Manuscripten von H.- Rathh.- Silbermann.

IV

Eigene Manuscripten des Herrn Silbermanns in folgenden Cartons in gros Folio.

Vol: sub Lit A. Von Gebürgen, alten Bergschlössern und Merckwürdigkeiten im Elsass von A. - P. nebst den dazu verfertigten Zeichnungen.

B. Von Gebürgen, alten Bergschlössern und Merckwürdigkeiten im Elsass von R. - Z. mit den dazu gehörigen Zeichnungen: bey welchem annoch Anmerckungen einiger ausserhalb des Elsass liegender Orten und Merckwürdigkeiten, mit den dazu gehörigen Zeichnungen.

C. Historische, und andere Samlungen aus geschriebenen Chronicken und Manuscripten.

D. a.) Regiments Verfassung der Stadt Strassburg. b.) nebst von den Rittern, Knechten, Burgern, Constoflern, Haussgenossen und gleven Burgern ingleichen von Handwerckern und Zünften:

c.) das Hüttengericht der Steinmetzen in Strasburg,

d.) die Meistersänger,

e.) die Strafen und Missethäter,

f.) Lupanaria argentinensia,

g.) Monumenta von Strassburg auch Römische Gräber,

h.) Excerpta einer Cbronick der Stadt Ensisheim in Manuscript,

i.) die Begräbnisse vom XV.den Jahrhundert,

k.) Reformations Historie,

1.) Acta wegen einem ausgegrabenen Körper, 
m.) gemahlte Fenster.

E. Auszug von Jahr Zedeln, worinnen von unterschiedenen Zeiten die Anzahl der zu Strasburg geborenen, eingeseegneten und verstorbenen Menschen zu ersehen, wobey noch einige Anmerckungen sich befinden.

F. Beschreibung wolfeiler und theurer Zeiten, wobei der jeweilige Preis von Früchten und Wein nebst anderen zur Unterhaltung, nöthigen Sachen zu ersehen.

G. Vom Münster zu Strasburg.

H. die Geistliche Stifter, Kirchen und Klöster samt den Capellen, Clausen, Samlungen und Beginnen Häussern welche in und nechst der Stadt Strasburg gelegen waren.

J. Das Münzwesen der Stadt Strasburg.

K. Die in der Stadt Strasburg befindliche Gassen und Plätze und die Eintheilung derselben in $X$ Cantons b.) Auszug des Haussuchung Buchs von den Jahren 1580. bis 1605.

c.) Gassen und Häusser aus Allmend Büchern von den Jahren 1350. 1417. 1427. 1431. und 1450.

d.) Adeliche und andere Höfe ingleichem Privat Häusser mit besondern Benennungen.

L. a.) Befestigung der stadt Strasburg.

b.) die in Strassburg bei Geschöll Zeiten verordnete Lärmen Plätze wohin sich die gewafnete Burgerschaft versamlet und die an den Häussern angeordnete Schwefel Pfann die bei solchen Läuften des Nachts anzuzünden befohlen worden,

c.) der Allmend Bezirk ausserhalb der Stadt von 1350. 1427. et 1450 .

d.) Auszug des Achtherren=Buchs von 1475.

e.) die Bronnen auf der Stadt Allmend,

f.) die Wasser in und bey der stadt ingleichen die Brücken und Fähren,

g.) die Landstrassen so von der Stadt abgehen,

h.) der Burgbann der Stadt Strassburg. 
M. a.) Edificia publicia oder offentliche Gebäude in Strasburg

b.) Von Dohlen, Landfesten, Mauren und Brücken vom Jahr 1530

N. Gelehrte und Künstler welche entweder im Elsass gebohren oder sich durch ihre Geschicklichkeit bekannt gemacht.

o. a.) Vocabula antiqua.

b.) Wirths=Häusser.

Bänd in Folio.

P. Collectanea über die Antiquitates Locales der Stadt Strassburg und umliegenden Gegend.

Q. Excerpta Historica von der Stadt Strassburg und der umliegenden Gegend.

R. die Bischöffe von Strasburg.

S. a.) Von dem Regiment der Stadt Strasburg,

b.) Excerpta historica aus Johann Walters Strasburger Chronick.

T. Historische Samlungen von der Stadt Strassburg aus lauter Manuscripten zusammen getragen.

U. Kurze Beschreibung etlicher Stätte, Schlösser und Dörfer welche um Strasburg gelegen, gedruckt zu Anfang 1675. mit vielen von Herrn Rathh.- Silbermann dazu verfertigten Zeichnungen und geschriebenen Anmerckungen und Berichten.

Ein Register über vorherige Antiquitates und Excerpta Historica welches zugleich als ein Auszug dieser 2. Bücher gebraucht werden kan.

In einem Band klein Folio.

Excerpta aus D. Specklin Collectaneis

Manuscriptis.

Von Kriegen welche sich im Elsass und benachbarten Gegenden zugetragen haben, in folio

ohneingebunden.

Ausser vorherigen Manuscripten von H.- Rathh.Silbermann befinden sich annoch einige in quarto vorhanden, so die stadt und anders mehr betreffen 
und theils in Excerpten theils eigenen Entwürfen bestehen.

2) Folgende Werke von andern Autoribus sind mit H.- Rathh.Silbermann schriftlichen Anmerckungen versehen.

1. Franz Ruprecht von Ichtersheim Elsassische Topographia mit verschiedenen Zeichnungen und schriftlichen Anmerckungen von H.Rathh. - in 4 to.

In fine dieses Wercks befindet sich ein Manuscript von 100. Seiten betitult Structurae et Emendationes, cum Annotationibus variis in hanc Topographiam, von Künast.

2. Elsassische und Strasburgische Chronick von Jacob von Königshoffen 1698. mit vielen schriftlichen Anmerckungen von Herrn Rathherrn versehen. 4to.

3. Bevestigung der stadt Strasburg 1683. mit vielen schriftIichen Anmerckungen. 4to.

4. Descriptio particulae territorii Argentinensis von Bernegger, mit $H^{-n}$ Silbermanns schriftlichen Anmerckungen in folio.

3) Manuscripten von andern Personen.

1. Handschriften von Gelehrten Strasburgern zwey Theil in Folio.

2. Handschriften von fremden Gelehrten zween Theil in Folio.

3. Handschriften von Edlen 1. Vol. in Folio.

4. Strasburgische Chronick von den ältesten Zeiten bis auf das Jahr 1630. in drei dicken folianten Bänden.

5. Eine Sammlung von lateinischen Gedichten von Strassburgischen Gelehrten mit ihrer eigenen Hand geschrieben 1 Band in folio.

6. Altes Manuscript aus dem fünfzehenden Jahrhundert von Königshoven Strassburgischer Chronick ein dicker Foliant.

7. Meistersänger Lieder Anno 1540 geschrieben ein Band in folio.

8. Ein Ammeister XIIIr und XVr Buch von dem Jahr 1333 bis auf das Jahr 1680. 1. Vol. in Folio.

9. Der Stadt Strasburg Rathsbuch von 1333. bis 1609. ein dicker geschriebener Band in Folio.

10.Strasburgische Chronick von einem unbekannten von den ältesten Zeiten bis auf das Jahr 1587. 1 Band in folio. 11.Der Stadt Strasburg Rathsbuch von 1220. bis 1716. in folio 
1. Band mit einer Vorrede von Künast.

12. Chronick von den Bischöffen zu Strassburg von einem ungenannten 1 Band in folio.

13. Strasburger Chronick, von einem ungenannten auch von den ältesten zeiten bis auf das Jahr 1505. I Band in folio, scheinet eine Abschrift von einer neuern Hand zu seyn.

14.Kunastii argentoratum ex aedificiis publicis sacro-profanum oder kurze Beschreibung aller Stifts-Pfarr- und anderer Kirchen, Clöster, Capellen pp. 1 Band in quarto. Eine sehr merckwürdige Sammlung von einem fleissigen und geschickten Mann verfertiget.

15. Collectanea zur Strassburgischen Geschichte, welche sich insbesondre auf die Religion beziehen unter der Aufschrift Strassburger Chronica ein Band in 4.to

16. Musaeum geminum Kunastianum oder Beschreibung der zweien Kunastischen Kunst-Kammern durch Philipp Ludwig Künast dem ältesten Sohn. ein Band in 4 1683. MS. Enthält viele interessante Strasburgische Nachrichten.

17.Verzeichnus aller Domm- und Frey Prediger, Pfarrhh.- und Helfer der 7. Evangelischen Lutherischen Kirchen in Strasburg seit der Reformation bis 1736. ein dünner Band. in folio.

18. der Stadt Strassburg ältere Ordnungen und Artickel in Handschrift, ein dicker Band in folio.

19.Eine geschriebene Sammlung von alten Strassburgiscben Ordnungen 1. Band in 4.to

20. Argentoratensium ager sepultus, retectus das ist Collection aller theils gestandener und theils noch stehender Monumentorum Sepulcralium Inscriptionum atem Epitaphiorum, welche auf denen drei Gottsäckern um Strassburg gelegen zu befinden von Jentgraven bis auf das Jahr 1736. in Ordnung gebracht 1 vol. in 4.

21. Roll über 8. Compagn. zu Fuss und 2. Reuter Comp. zu Strassburg nebst verschiedenen schöngemahlten Wappen 1. Vol. in 4. to de 1633.

22. Kurzer Summarischer Auszug aus der Strasburger Cronica vom Anfang der Ammeister de 1333 bis auf das Jahr 1632. ein Band in fol. Dazu kommen noch verschiedene andre curiose Strassburgische sammlungen

1) Amplificatio urbis argent.

2) Chronologia Consularii bis auf das Jahr 1738 . 
3) Varia Schemmata Genealogica

4) designatio der Oberherrn und Schöffen auf den 20 zünften seit 1 1/2 seculis.

23. Ein Carton von allerhand Kupferstichen, Zeichnungen etc so auf Strassburg beziehen.

24. Ein Carton mit verschiedenen theils teutsch und theils französischen manuscripten über 1'Origine des Beguines, Cimetiere des Juif, die Fortifications hiesiger Stadt \&C.

25. Decreta Potiora Conventus Eelesiastici Argentinensis.

26. Memoire pour l'intelligence de la Carte du Cours du Rhin par $M^{r}$ de Balincourt fol.

27. Jacob Wurmbsers Reise nach Jerusalem von dem Jahr 1560. MS. 1 Band in 4.to

28. Miscellanea manuscripta von Strassburg. ein Band in folio.

Gedruckte Bücher in Canzleyschrift so sich auf Strassburg beziehen.

1. Bericht von Erfindung der Buchdruckerei in Strasburg. Strasburg 1640. ein Bändlein in 4.to

2. Kriegs Sachen Strassburger Bischtums de 1590. et 1592. ein Band in 4 .to

3. Joh. Huberi Pfarrers von St. Wilhelm Christliche Danckund DanckPredigt pp 1657. 1. Band in 4.to mit einigen Anmerckungen von H.- Rathh.-

4. Miscellanea von Strasburg 1. Band in 8. vo

5. Elsass und Breyssgau von Ursenson, Strasb. 1679. 1 Band in $12 .^{\circ}$

6. Verzeichnus der Naturalien so in Künastischer Kunst=Cammer zu Strasburg zu finden. 1. Bändlein in 4.to

7. Ein Psalterium ohne Druck Jahr von den Initiis Typographicis. in $12 .^{\circ}$

Kupferstich und Holzschnitt so Strassburg und das Elsass betreffen. in Canzleyschrift.

1. Ständ und Orden der H. Römischen Catholischen Kirchen darinnen aller Geistlichen Personen, Herren Ritter und deren Verwanten Habit und Kleidung in 95. Figuren in Holzschritt bestehend 1. Band in quarto.

2. Strasburgische Tracht und Kleidung Manns und Weibs Personen in 54. Figuren in Holzschnitt bestehend, ein 
Bändl. - in 4.to von 1573.

3. Strasburgische Tracht und Kleidung de 1706 in 60 Figuren in Holzschnitt bestehend 1 Bänd.- in 4.to

4. Strasburgisch Trachtenbüchlein von Anfang des XVII.den Saeculi bis in die Mitte desselben in 31. Figuren in Holzschnitt bestehend, ein Bänd.- in 4.to

5. Strassburger Trachten Büchlein de 1680. in 61. Kupferstichen bestehend in 4 . to

6. Strassburger Trachten Büchlein in 4.to und 32. Stichen bestehend.

7. Strasburger Kleider Trachten 1. Bändlein in 4.to mit 20. Stichen de 1731.

8. Ein Wappenbuch in 4.to dabei eine beträchtliche Sammlung von gemalten Strassburgischen Wappen von Stettmeistern Ammeistern p.

9. Portraits von Strasburger Gelehrten 2. Bänd in folio mit 170. theils Kupfer, theils Holzstichen.

10.Samlung von Helden und Gelehrten in 120. Holzstichen in 4. to

11. Sechs gestochene Karten von verschiedenen Gegenden des Elsass.

12.Ein Holzschnitt über das Armbrust Schiessen de 1576.

13. Ein groser gestochener Plan von der Stadt Strassburg.

14. Joh. Wilhelm Baurs von Strassburg wunder würdige Meer- und See-Porten nach dem Leben abgezeichnet und von Melchior Küsel in Kupfer gebracht ein Band in folio.

15.Joh. Wilhelm Bauer von Strassburg unterschiedliche Prospecten welche Er in denen Landen Italie und dan auf seiner Heimreisse in Kupfer gebracht durch Melchior Küsel zu Augspurg 1681.

$\mathrm{ABR}, \quad 6 \mathrm{E} 41,1430$. 
Nachricht / von dem Tode / des Hrn. Johann Andreas Silbermann./ Strasburg.

Den 11. Hornung starb allhier der verdienstvolle Hr. Joh. Andreas Silbermann, E. E. grossen Raths alter Beysizzer und weitberühmter Orgelbauer. Er war geboren im Jahr 1712. den 26 Brachmonath. Sein Andenken hat er durch ungefähr fünfzig Orgelwerke, welche er in dem Elsass und umliegenden Ländern verfertigt, auf das rühmlichste gestiftet. Es zeichnen sich unter denselben besonders zwey aus, deren die eine allhier zu strasburg in der Prediger $=$ Kirche, die andere in der hochfürstl. Abtey St. Blasi auf dem Schwarzwald befindlich ist.

Nebst dem zeugen von seinem unermüdeten Fleisse 1) eine unglaubliche Menge Auszüge und Bemerkungen, welche er aus Chroniken und andern auf dem Archiv löbl. Stadt und sonst verwahrten Schriften gesammelt, und aus welchen sowohl die im Jahr 1781. von ihm herausgegebene Beschreibung des st. Odilienbergs, als auch die Local = Geschichte von Strasburg entstanden, deren erster Theil im Jahr 1775. abgedruckt worden, die andere noch in Handschrift sich vorräthig befindet. 2) Die grosse Anzahl von Planen und Rissen, welche er von den Gegenden des Elsasses und des Brisgaus verfertiget, und welche eine ausnehmende zierde seines Kabinets ausmachen. 3) Die herrliche Sammlung der strasburgischen Münzen, von welchen er selbst eine Beschreibung verfertiget, die allerdings würdig wäre ans Licht zu treten. 4) Eine Sammlung von Zeichnungen und Beschreibungen, welche die Alterthümer unserer 
Stadt und Landes betreffen. Wie er denn immer beflissen gewesen, Denkmahle, die zu Zeiten aufgebracht wurden, anzukaufen und zu bewahren.

Der sel. Hr. Rathhr. Silbermann hinterlässt zwey Söhne, deren der jüngere, Hr. Johann Josias, die Orgelkunst von seinem Vater erlernt hat und fortführt.

Auch ist bemeldten Herrn Rathherrn jüngerer Herr Bruder, Johann Heinrich, annoch am Leben.

Seine Arbeiten bestehen in allerley Clavier = Instrumenten, deren einige von ihm durch eigenes Nachsinnen und lange Uebung in einem hohen Grad verbessert, andere ganz neu erfunden worden; worunter besonders drey; als nähmlich (1) ein ausserordentlicher grosser 16 Fuss Ton klingender Flügel. (2) ein forté piano en pédales. (3) ein forté piano manuel merkwürdig sind. Diese seine Instrumenten haben, wie bekannt, ein so allgemeines Lob erhalten, dass sie nicht nur nach Frankreich, der Schweiz und Teutschland, sondern auch nach Curland, Russland, Schweden,. England u.s.w. ja gar nach Ostindien versendet worden und noch zu versenden sind.

Auch dieser Hr. Joh. Heinrich Silbermann hat einen Sohn Joh. Friderich, der in die väterlichen Fussstapfen auf eine löbliche Weise tritt, und dieselbe Kunst mit gutem Erfolge treibt.

Es ist allerdings erfreulich zu sehen, dass der Ruhm, welchen der im Jahr 1734. verstorbene und von Frauenstein unweit Dresden gebürtig gewesene Hr. Andreas Silbermann, dessen Orgeln und Claviere noch immer hochgeschätzt werden, auf seine Söhne 
gebracht, bey dieser ehrwürdigen Familie sich bisher vermehrt hat, und auch inskünftige durch göttliche Hülfe sich erhalten wird. Diese unsere Nachricht wird allen ächten Kunstliebhabern willkommen seyn. Sie kann auch dienen eine Stelle in Hrn. Prof. Beckmanns Beyträgen zur Geschichte der Erfindungen zu berichtigen, da im vierten Stükke, unter dem Artikel Pantaleon, die beyden Herren Silbermänner, der verstorbene und noch lebende, nicht gehörig unterschieden waren. Dem Hrn. Joh. Heinrich gehört allerdings die Erfindung des Pantaleons; hingegen wird ihm unrichtig die Local = Geschichte der Stadt Strasburg zugeschrieben, welche ein Werk des verstorbenen ist, bey dessen Hinterbliebenen solche fernerhin für $10 \mathrm{Liv}$. wird zu haben seyn.

Archives Municipales Strasbourg A 53. 376 Ludwig Mooser, Das Brüderpaar die Orgelbaumeister Andreas und Gottfried Silbermann. Feuille collée à la dernière page de la brochure. 
Documents d'archives relatifs aux ouvrages d'André et de Jean André Silbermann.

Documents complémentaires concernant J. J. Baldner et Frédéric Ring.

Les documents sont groupés par orgue. 
Répertoire chronologique des instruments concernés:

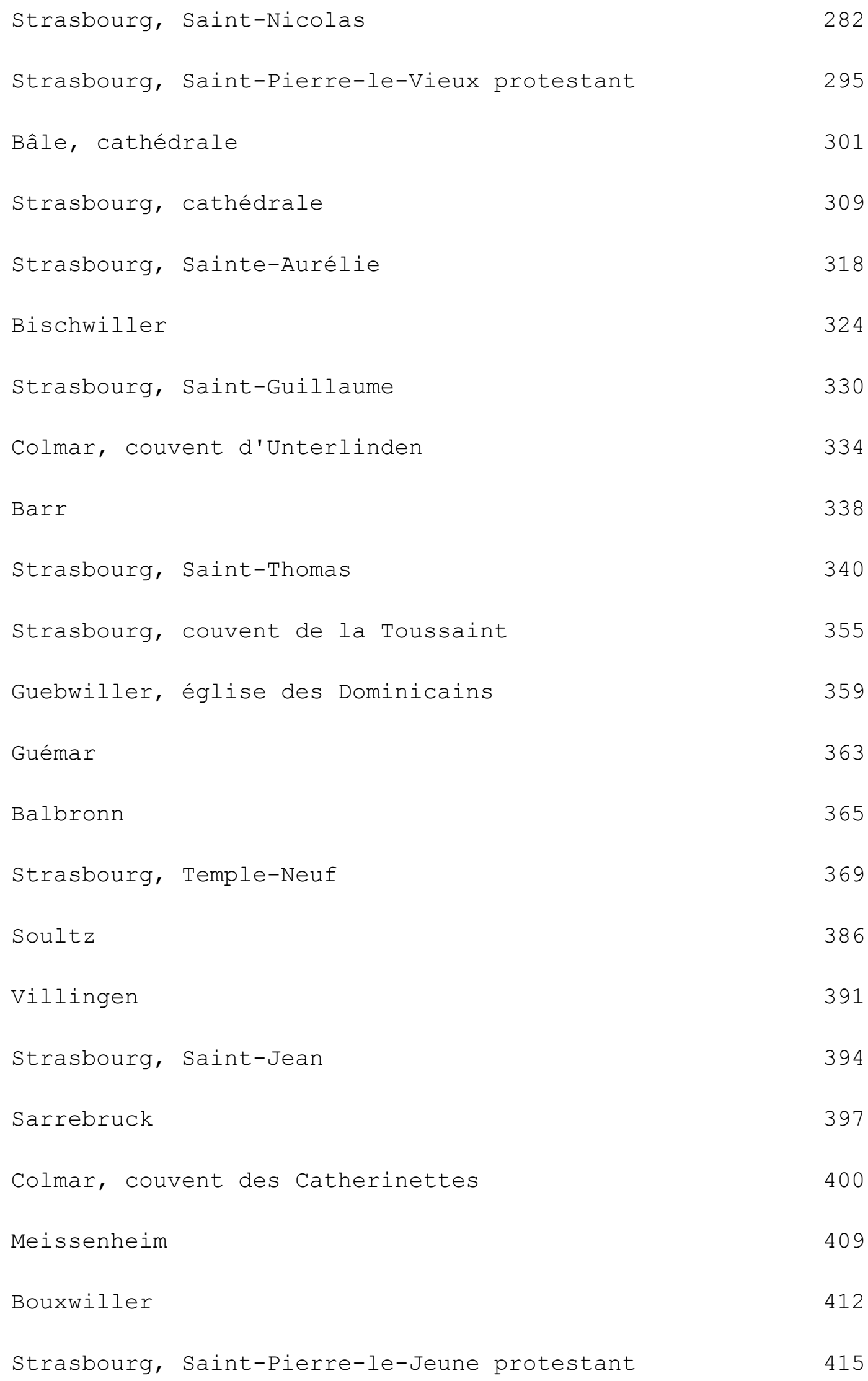


Strasbourg église protestante Saint-Nicolas.

1702 et 1706: réparations à 1'orgue Baldner. Quittances d'André Silbermann.

Description de l'orgue Baldner et projet d'orgue neuf par André Silbermann. Autographe non daté.

Marché du 18 janvier 1707 avec André et Gottfried Silbermann. Quittances d'André Silbermann. 1707. 1716.

Décision de remplacer la Viole de Gambe 8 par un Bourdon 16. 10 juin 1719.

Quittances de Jean André Silbermann. 1735. 1758. 1768. 
$(1702)$

N. 16 :

Ich unterschriebner beschein, und quitir himit dass mir der Herr Hanss Georg Wolff, als Fabric Pfleger, en 23 Octobris, von wegen der Blassbälg bey S. Nicolai fünf und sibencig Gulden zu Danck bezahlt hat.

\section{Andreas Silbermann Burger und Orgelmaoher.} N. 21

Anno 1706. den 20 Octobre.

Ich Ends benenter habe auf Begehren der Herren Kirchen Pflegern zu St: Nicolaus, die Blassbälg der Orgel bemelter Kirchen, hin und wider verleumbt mit neuem Leder, so die Ratten verfressen, wie auch etliche mahl der Orgel dass Heulen corrigiret. Fordere vor meinen verdindten Lohn, und aussgelegte Materialen ...fünf. fl.

\section{Andreas Silberman \\ Orgelmacher.}

Dass obenbemeldes so viel der Orgel Zustand zugelassen reparirt, und so viel möglich verbessert worden, bescheinet J.U.Frid, Org.

ABR. Arch. de la paroisse Saint-Nicolas. 
Verzeugnüss was an der Orgl zu St: Niculas müste gemacht werden.

Erstlich alles zihnnernne Pfeifwerck muss umbgegossen werden, weihl es von neuen nicht recht gegossen und gelödet ist worden, so auch gantz verrissen und ruinirt ist das es anderst nicht kan zurechte gemacht werden.-

Zum andern weihl dass Werck von neuen zu weydtleuffig ist angelegt worden, davon alles so dicke ineinander verstecket dass man zu nichts kommen kan, darumb müssen neue Windtlahten und Canäl, Clavir, und Regirung gemacht werdten, und dass Pedal so anitzo hinter der Orgl liget, muas hinter die Orgl aufrecht gestellet werden.-

Dryttens auss denen 8 Blassbälgen so vorhanden, können 2 davon genommen werden, und auss diesen 2 Bälgen kdnnen in die übrigen 6 so bleyben müssen, mehr Falden gemacht werden dass sie weyder aufgehen, mehr und beständigern windt fassen und von sich geben. Es müssen auch die Bälge anderst eingerichtet werden, dass man sie mit Händen zihen kan. Es muss auch ein jetweder Blassbalg besonder mit einen Kästlein eingefasset werden, damit ihn die Ratten und Meuss dass Leder nicht abfressen wie schon geschen.-

Dass Corpus sowohl am Rückpositif als Oberwerck, bleibt in äusserlichen Ansehen ohnverendert, ausgenommen die zwey aufrechten Kästen, so beyderseydts der grossen Orgl stehen worinnen die itzigen Windtlahten dess Pedals ligen werden weggethan.

Die Register so sich itzont in der alten Orgel befinden seyndt diese

als. 1. Quintathön 8 Schu gedeckt und 16 Schu Thon

2. Principal .............. 8 schu

3. Violdigamb .............. 8 .

4. Nazard. 3 Schu gedeckt ..... 6.

5. Octav ................. 4 .

6. Holflöt ................ 4 .

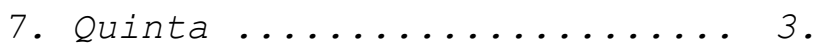

8. Superoctav ............. 2 .

9. Mixtur. 4 facht die gröste Pfeif 1 Schu

Diese 9. Register stehen in Obernwerck, und haben 2 Clavir. 
Im Rückwerck oder Positif befinden sich volgente Register.-

Als. 1. Quintathön 4 Schu gedeckt 8 Schu Thon

2. Coppel von Holtz gedeckt.. 8 .

3. Principal ............. 4.

4. Octav ................. 2 .

5. Flöt 1 Schu gedeckt...... 2 .

6. Superoctäven ............ 1 .

Im Pedal sindt 2 Register
als 1. Supbas von Holtz
16 Schu Thon
2. Gross Octavenbas
8. -

Summa 17 Register in allen.

In dass neue Werck aber müsten folgente Register gesetz werden

als. 1. Principal ............ 8. schu

2. Violdigamb ........... 8 .

3. Coppel ................ 8 .

4. Octav ................. 4 .

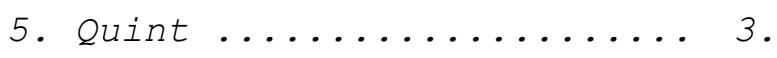

6. Superoctav ............. 2 .

7. Mixtur. 4 facht -

8. Cimbal 3 facht -

9. Cornet 5 facht -

10. Tierce.-

Diese 10 Register müssen in Obern Wercke stehen, auf einen Clavir, und diese seyndt alle nöthig zum Coral aussgenommen die letztern 2 als Cornet und Tierce, welche aber schöne Veränderungen machen in spielen.

Im Rückpositif aber müssen folgende Register stehen.

als. 1. Coppel ............. 8 Schu Thon.

2. Principal .............. 4 .

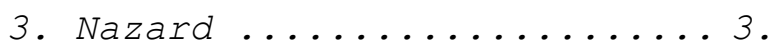

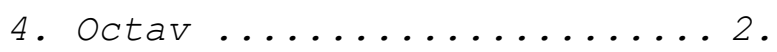

5. Mixtur 3 facht -

6. Tierce - 
Im Pedal aber können die obrigen 2 Register bleyben
als 1. Supbas
16 Schu
2. Octaven
8.

Summa 16 Register im Manual, und 2 im Pedal nebenst 2 Tremulanten, und 2 Clavir. Obgleich die Summa der Register sich ebenso hoch beläuft als in der itzigen alten Orgel, so man aber 2 Clavir haben wil, so müssen diese Register alle da seyn, sonsten kan die Orgel nicht nach heutigen Gebrauch tractiret werden.

ABR. Arch. de la paroisse Saint-Nicolas. 
18. Januar 1707.

Zuwissen seye hiemit dass ane zu End gemeltem Dato zwischen denen hochwohlverordneten respectivé Herren Kirchen vnd

Fabric=Pflegeren, wie auch denen Herren Predigeren zu St:Niclauss ane der Preusch alhier zue Strassburg, eines: vnd dann Herren Andres und Gottfrid Silbermann beeden Gebrüderen und Orgelmachern andern Theils, nachfolgender Accord, Vergleich und freundlicher Verding, wegen Auffstell: und Verfertigung einer newen Orgel cum appertinentiis in der Pfarrkirchen zu besagtem Niclaus, volgender massen, in praesentia mein vnderschriebenen Notarii beschehen und getroffen worden.

Nemblichen und zum Ersten, so obligiren und versprechen obgemelte beede Brüdere die Orgel so anietzo da stehet zu bevorstehendem newen Werckh, so gäntzlichen nach dem bereits gefertigten: vnd überlüfferten Abriss gemacht werden solle, zugebrauchen, die alte zinnene Pfeiffen, welche anfangs nicht recht gegossen und gelödet worden, dahero gantz verrissen und ruinirt sind, von newem vmbzugiessen, und was noch ahne zinn darzu erfordert würd, selbiges selbsten, von der besten prob zu fourniren, vnd beyzuschaffen, und angeregtes verdingte Orgelwerckh so wohl ins gemein was das Haubtwessen, als auch ein: vnd das andere Stuckh insonderheit betrifft, so ohnmüglich zu specificiren, dergestalten zu verfertigen, und instand zu stellen, dass es zu ihr der Herren Orgelmacher höchster Ehre und Ruhm, und zu der Kirchen und dero Herren Vorstehere, wie auch der gantzen Gemeinde Vergnügen und Satisfaction aussschlage und gereiche.

Zweittens, promittiren sie Herren Orgelmacher, ohnerachtet zwar das Corpus der newen Orgel, dem Abriss nach, weilen das alte nur zu weitläuffig angelegt gewessen, davon alles so dickh in einander verstecket, dass mann zu nichts kommen können, vmb etliche schue schmähler würd, dannoch folgende Register zu verfertigen.

AlsS.

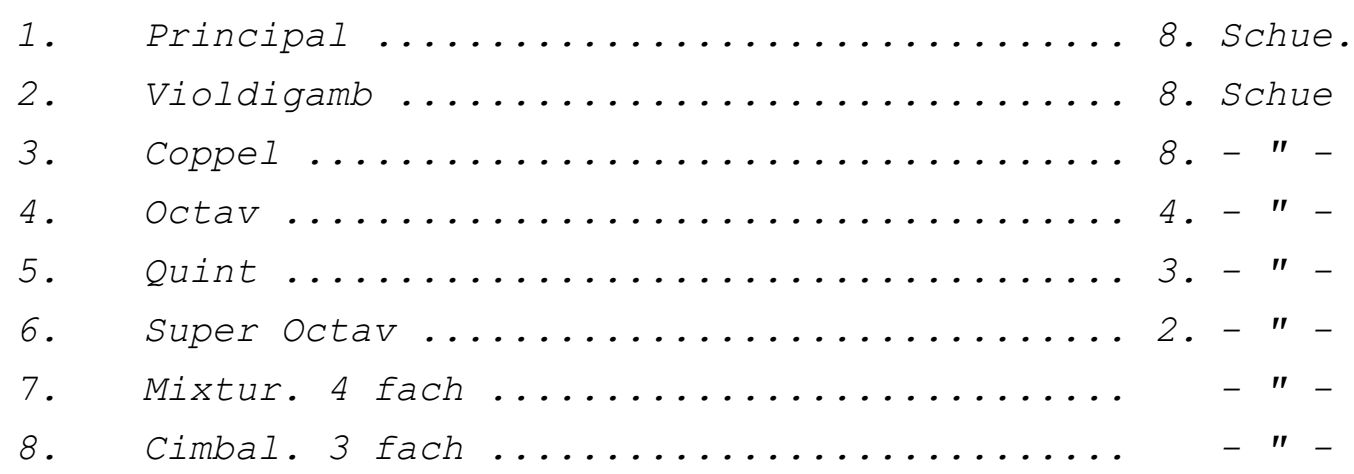


9. Cornet. 5 fach ................... - "

10. Tierce ..................... -

Welche 10. Register im Oberen Werckh stehen müssen, auff einem Clavir und alle ausser die zwey letztere zum Coral nöthig sind, diesse aber schöne Veränderungen im Spielen machen. Drittens versprechen sie auch 2. Clavir und ein Tremulanten als ein geschwinden und langsamen solchem Orgelwerckh beyzufügen. Vierdtens was das Ruckpositiv betrifft, bleibt solche in Corpore wie sie ist, ausser dass die Pfeiffen auch vmbgegossen und folgende Register darauss zustellen sind. Nemblichen. 1. Coppel ...................... 8. Schuthon.

2. Principal ...................... 4. " -

3. Nazard ......................... - " -

4. Octav ......................... - "

5. Mixtur. dreyfach ................ - " -

6. Tierce.

Vnd sollen im Pedal die oberen 2. Register bleiben, alss. 1. Sup: Bass .................... 16. Schue

2. Octaven ..................... 8. Schue

Mit der ferneren Erläuterung dass alle obgemelte Register im Cornet Thon zu verfertigen.

Fünfftens weilen das newe Werckh in seiner äusserlichen Form gegen der alten eine Veränderung bekombt, wie aus dem Abriss zu ersehen, und also die alten Zierathen und Bildwerckh verschnitten undt geändert werden müssen, alss solle ihnen den Herren Orgelmacheren erlaubt seyn, was von solchem alten Zierath noch kan gebraucht werden, darzu zu employren, das Abgehende aber sollen diesselbe auff ihren costen new zu machen schuldig sein. Sechstens sollen aus denen vorhandenen 8. Blasspälgen nur 6 . gemacht, aber besser und mit mehr Falten als die vorigen verfertigt werden, dass sie weiter auffgehen, vnd mehr wind fassen und von sich geben, auch mit den Händen gezogen werden können. Dessgleichen soll ieder Blasspalg besonders mit einem Kästlein eingefasst seyn, damit das Leder von dem Vngezieffer, wie vorhin öfters geschehen, nicht verbissen werde.

Siebendtens versprechen die Herren Orgelmacher, alle andere Mitarbeiter, die sie zu Aussführ: und Verfertigung obbesagten Orgelwerckhs quocunqmodo nöthig haben, es seyen Schreiner, zimmerleuth, Maurer, Schlosser, Mahler, Bildschnitzler oder andere dergleichen wie die Nahmen haben mögen, dessgleichen auch den 
Calcanten oder Orgeltretter den sie beständig bey ihrer Arbeit haben müssen, in ihrem Costen zu übernehmen, und die Kirch desswegen schadloss halten, angesehen solches alles in diessem Accord und hernachgemelten ihren Verdienst eingerechnet seyn solle.

Achtens solle alles das jenige was hierinnen nicht axpressé oder austrucklichen gedacht, underdessen zu vorbesagtem costbahren newen Orgelwerckh dessen Haubtzweckh Gottes Ehre ist vollkommlich und eigentlich erfordert, vnd in acht zu nehmen nöthig, würd von seithen der Kirchen und dero Herren Vorstehere, der Herren Orgelmacher Fleiss, Dexteritaet und gewissen anheim gestellt, und überlassen, alles zu observiren, was nochmahlen wie oben bereits gedacht, zu ihrer Reputation vnd der Kirchen Contentement dienlich sein mag.

Vnd haben volglich die Herren Orgelmacher sich erklärt, ane ihrem Fleiss nichts erwinden zu lassen, damit längstens innerhalb drey vierttel Jahrs Frist von dato gerechnet, bevorstehendes Orgelwerckh in seine Perfection möchte gestellet seyn, als warzu sie sich hiemit nicht allein verbinden, sondern auch zugleich promittiren, dass im Fall wieder Verhoffen, in zwischen Göttlichem Willen nach ein Todfall unter ihnen begeben solte, so doch Gott gnädig abwenden wolle, der Letstlebende alssdann vnder ihnen nichts desto weniger das versprochene Werckh vollends ausszuführen, und obgemelter Masen in stand zu stellen pflichtig seyn solle.

Vor obgemelte Arbeit dann von seithen der Kirchen und dero Grossg-. Herren Vorstehern, ihnen denen Herren Orgelmacheren /: nemblichen vor alles was sie zu bevorstehender Orgel anzuwenden, es habe sonst Nahmen wie es wolle :/ baar zu bezahlen versprochen worden Eintaussend vnd Fünfftzig Gulden Strassburger à 60 . Creutzer gerechnet, davon ihnen uff anständiges Ansuchen, weilen sie noch zur zeit keine Leuth von Capital sind 300. R. avancirt, und folgents nach und nach, nach Proportion ihrer Arbeit und erzeigenden Fleiss mit ferneren Avances continuirt werden solle.

Welchen vorstehenden Verglich mit allem seinem Innhalt, Puncten vnd Clausulen beede Theil, wahr, steth vnd vest zu halten, einander mit Mund vnd Hand /: cum renunciatione aller Exceptionen Beneficien, und anderer Ausszüg, dessen sich je einer wider den anderen zu Vmbstossung diesses bedienen könte :/ versprochen und 
zugesagt, auch zu desto mehrerer Bekräfftigung, diesen Accord so in duplo aussgefertiget, und jedem Theil ein Exemplar davon zugestellt worden, neben mir Notario eigenhändig vnderschrieben. Actum Strassburg den achtzehenden Januarii, Anno Eintaussendt Siebenhundert und Sieben.

Lucas Sebastian Ritter Pfarrer zu S. Niclauss

Caspar Schmidt als Kirchenpfleger

Johann Daniel Würtz als Fabrick Pfleger

Andreas Silberman.

Gottfried Silbermann.

Beyde Orgel Macher.

Jacob Christoph Pantrion, als hierzu in specié requirirter Notarius

Mpp.

Archives de St. Nicolas déposées aux Archives du Bas-Rhin

2 ex., portant au verso:

-Vergleich. Mit H-. Andreas: vnd Gottfrid Silbermann, denen beeden Orgelmachern, wegen reparirter Orgel zu St.Niclaus vom 18. Jan:1707.

-Vergleich Mit denen Herren Silbermännern, wegen der Orgel zu St.Nicolaus. vom 19. Januarii. 1707.

Note de M. Moeder: Invent Pantrion $n^{\circ} 24$. 
(1707)

N. 23 .

Ich Endes unterschriebner bekenne das ich von Herrn Hanss George Wolff als Fabrik Pflegern, die in dem Accord bemelte drey hundert Gulden zur Avance wegen der neuen Orgel zu St: Niclaus bestermasen empfangen habe.

Den 24 Januarii

1707.

(1707)

(1707)

N. 24 .

Den 24 Martii 1707.

N. 28 .
Ich Endes unterschriebner bescheine himit . das ich von Herrn Hanss Georg Wolff Fabric Pflegern über die vormahls empfangenne drey hundert Gulden, noch drey hundert Gulden empfangen habe, also zusammen sechs hundert Gulden. Andreas Silberman Orgelmacher.

Ich Endes unterschriebner bescheine dass ich von Herrn Johan George Wolff, Fabric Pflegern bey der Kirchen St: Niclaus, heut dato den 26 Junius zweyhundert fl. auf Abschlag der neuen Orgel empfangen habe, quitire himit. Den 26 Junii Andreas Silberman. 1707. Orgelmacher. 
$(1716)$

N. 21 .

Dass von S: T: Herren Rathherrn Manberger Fabrick-Pflegern zu St: Niccolaus vor 1 Fuder Thilen empfangen habe zehen Gulden, sage .......................... 10 I bescheine himit

Andreas Silberman.

ABR. Arch. de la paroisse St. Nicolas. P.J. des comptes

Den 10. Juny. 1719.

Ist auff Ansuchen Herrn D. Thenen des Organisten und Herrn Silbermanns Gutbefinden von Herren Kirchenpflegern und Herren Predigern, erkndt: dass bey vornehmender Stimmung der Orgel anstatt des Viol de Gamba Registers, welches heraus genommen werden solle, ein 16.schühiges Coppelregister darein gesetzet werden solle.

ABR. Arch. de la paroisse St. Nicolas. Protocollum der Fabric zu Sanct Niclauss in Strassburg de Annis 1703. 1704 biss 1726. Séance $\mathrm{du} 10.6 .1719$. 
Verzeugnus was ich Ends unterschriebener, auf erhaltenen Befehl an der Orgel zu St. Niclaus gearbeitet habe.

Alles Pfeiffenwerck der gantzen Orgel ausgehebt, den darin befindlichen Staub herausgethan.

Alle Windlathen, sambt dem gantzen Werck vom Staub und Unrath gesäubert.

die äusserlichen Scheinpfeiffen widerum hell geputzt.

Ein neues Windrohr in die Bourdon 16 pieds gemacht, weilen das alte von den Ratten zerfressen gewesen, dessgleichen noch zehen Pfeiffen theils neu gemacht, theils aber neue Füsse und Ohren angelödet.

Letzlich alles Pfeiffenwerck widerum eingesetzt, und das Manual, Positif und Pedal widerum zusamen gestimbt, dafor der Billigkeit gemäs $46 \mathrm{fl}$. Strassburg den 7 9bris 1735.

Johann Andreas Silbermann

Dieser Zettel ist mit $45 \mathrm{fl}$ zu Danck bezahlt worden Johann Andreas Silbermann

ABR. Arch. de la paroisse St. Nicolas. P.J. des comptes 
Verzeugnus was ich Ends unterschriebener auf erhaltenen Befehl an der Orgel zu St: Niclaus gearbeitet habe.

Anno 1753. den 22 et 23 Juny, die 6 Blassbälge hin und wider wo si Lufft hatten beledert, und den langsamen Tremulanten reparirt.

Aô: 1758. im Augusto. Alles Pfeiffenwerck der gantzen Orgel ausgehebt, den darin befindlichen staub daraus gethan. Alle Windladen und das gantze Werck vom Staub gesäubert, die Pedal=Stegtur reparirt. Und das gantze Werck durch alle Register widerum rein gestimbt. Vor alles $50 \mathrm{fI}$ Strassburg den 23 7bris

1758 .

Zu Danck bezahlt.
Johann Andreas

Silbermann.

Verzeuchnuss was ich Ends unterschriebener an die Orgel zu St: Niclaus, auf Begehren habe arbeiten lassen.

Aô: 1767 den 14. 15 und 16 May, habe die Blassbälge wo hin und wider verrissen waren und Luft gehabt, wider mit Leder versehen lassen. Ingleichen die Orgel durch einige Register gestimt ... 4-5Aô:'1768. den 17ten Febr: einen lossgerissenen Pedal=Clavem angehenckt. den 5ten Mertz die nemliche Arbeit verrichten lassen, und einige Pfeifen gestimt \&:

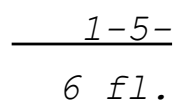

Strassburg den 11. July

1768. Johann Andreas Silbermann

Zu Danck bezahlt.

ABR. Arch. de la paroisse St. Nicolas. P.J. des comptes 
Strasbourg, église protestante Saint-Pierre-le-Vieux.

Marché du 10 août 1708 avec André Silbermann.

Contrat d'entretien du 26 août 1727 avec Andre Silbermann.

Certificat établi par André Silbermann au sujet de l'organiste Schübler, s.d. 
Kundt und Zuwissen seÿe hiemit, dass nachdem der Hoch=Edele Gestreng, Hochgelehrte hoch=und wohlweisse herr jacob Wencker alter herr Ammeister und dreyzehener alss hoch: und wohl meritirter Ober Kirchen Pfleger beÿ der Evangelischen Gemein zum Alt St. Peter in Strassburg, wie auch übrige Kirchen und fabric Pfleger und Herren Prediger daselbst befunden, dass es höchst nöthig und erforderlich ein gantz neüe Orgel zu machen, weilen die alte gantz untauglich unstimmig und nicht mehr zu repariren seÿe; Alss hat man sich mit auch genehmhalturg der E. Gemein und Kirchenkinder zum Alt - St. Peter, welche auch eine willige beÿsteur zu solchem Werck zugeben versprochen auch den 8. undt 9. Aug. bereits guthwillig den Anfang daran gemacht, dahien sich resolvirt, nachfolgenden Accord mit Hn. Andreas Silbermann, burgern und Orgelmacher allhier, zu treffen, und zur Ehre Gottes eine gantz newe Orgell machen zu lassen, welche auch bemelter $H$. Silbermann zu machen über nommen, bestanden und versprochen, dass nemlichen uff nechst kommende Pfingsten 1709 solche fertig, aussgemacht undt in der Kirchen stehen soll. und bestehet der mit Hn. Andreas Silbermann getroffene Accord in nachfolgenden Puncten, alss Erstichen verspricht $H$. Silbermann ein gantz newes Werck und Orgel, so im Gesicht Acht Schue in dem Hauptwerck aber von dem Mundtloch ahngerechnet, haben solle, mit einem ruckpositiv - sambt 
aller zugehör, mit sauberem aussschnitzel Arbeit aussgeziehret, alles von dürrem sauberem eichen Holtz, nach dem darüber uns zugestelltem und verfertigtem Abriss, uff den Cornet Thon, in die allhiessige Evangelische Kirch zum alten St. Peter, von Ein und Zwantzig Register zu machen auch alles wass von nöthen seÿn wirdt, wie es auch nahmen haben mag, darzu zu verschaffen, in seinem Kosten zu verfertigen und uffuurichten. Zum Anderen sollen folgende Register in dieses Werck kommen. (i.) princibal im schein Achtschue (2). Bourdon Sechszehen Schue (3.) Coppel Achtschue (4.) Octav Vier Schue (5) Quinte dreÿschue (6.) super octav zweÿschue (7) mixtur dreÿfach $11 / 2$ (8) Cimbel dreÿfach (9.) Cornet fünff fach (10) tierce $13 / 5$ schue. Jm Ruckwerck (1.) princibal Vierschue (2) Coppel 8 schue, (3). nazard, 3. schue (4) Octav 2 schue (5) Mixtur 3 fach (6) tierce $13 / 5$ schue. Jm pedal (1) supus offen von Holtz, Sechszehenschue (2) Octav - bas offen acht schue (3) prestent von zinn Vierschue (4) mixtur vierfach 1 1/2 schue (5) trompeten Bass Achtschue. Summa Ein: und zwantzig undt 2. tremulanten zweÿ völlige manual Clavier, Vier grosse Blässbalg, und allsso das Corpus gantz new von lauter gutem gesundtem eichenem Holtz.

Vor oben gemeltes Werck, welches von dato uff Pfingstag da man zahlen wirdt 1709 verfertiget und auffgerichtet werden solle, anbey auch H. Andreas Silbermann, uff Jahr undt tag Wehrschafft versprochen, beÿ Verpfändung aller seiner Haab und Nahrung, haben wohl Edel gemelter Herr Ober Kirchen Pfleger wie auch Hn. Kirchen 
undt fabric Pfleger undt Hn. Prediger im Nahmen der Evangelischen Gemein, versprochen Ein undt zwantzig hundert Gulden, neben der alten Orgel, jedoch dass H. Silbermann, wann die newe stehen wirdt, uff seinen Kosten solche alte abbrechen, undt den orth wiederumb mit frischen Diehlen zu belegen wolle und solle schuldig seÿn. Vndt zwar sollen die zwey tausend Einhundert gulden, in nachfolgenden terminen bezahlt werden, als erstlichen jetze gleich zum ahngeldt Vierhundert gulden, uff nechst kommendt Weÿhenachten Sechss hundert gulden, uff fassnach darauff fünffhundert gulden, und den Rest aber, wann das Werck verfertiget, auffgericht, approbirt undt vor guth und ohne mangel befunden seÿn wirdt, alles ohne gefahrdten urkundtlich seindt hierüber zwey gleichlautende Accord verfertiget und von uns underschrieben worden. Actum Strassburg, den 10.ten Augusti Anno 1708.

$\left(n^{\circ}\right.$ 44. Accord mit Herrn Andreas Silbermann wegen der Newen Orgell. Anno 1708.)

AMS. Arch. de la paroisse St-Pierre-le-Vieux, liasse 22, $\mathrm{N}^{\circ} 11$. 
Zu wissen, dass zwischen S. T. denen Herren Kirchen: und Fabric Pflegern bey der Evangelischen Pfarrkirchen zum Alten St: Peter an einem: und Herrn Andreas Silbermann dem Orgelmacher am andern Theil, wegen der in gedachter Kirchen sich befindlichen orgel volgender Accord getroffen worden.

Nemblichen, es solle er Herr Silbermann verbunden seyn, die in ermelter Orgel befindliche Pfeiffen auffuuheben, den darinnen befindlichen staub und alles anders, so die Pfeiffen hindert, ihren Dienst zu thun, auffs beste inn: und ausswendig zu accomodiren und widerumb inn Ordnung zu setzen, wann nemblichen die Orgel heulet, ein Clavis stecken bleibet oder abreisset, item die Blassbälckh und Windladen, soviel daran blessirt, auff das beste zu versehen, wie auch dann und wann bey Veränderung dess Wetters, die Zungen Register zu stimmen. Es solle auch die Orgel von vier Jahren zu vier Jahren gantz und völlig gestimmet werden. Wann dann diessem allem, massen er Herr Silbermann versprochen hat, ein völliges Genügen geschehen wird, haben obehrenernante Herren Kirchen: und Fabric Pflegere.demselben verwilliget von Herrn Fischern dem jetzigen jüngsten Fabric Pflegern desswegen sechtzig Gulden courrent Geltt zu empfangen, jedoch mit diesser Bedingung, dass damit auch diejenige fünffzehen Gulden, welche er Herr Silbermann annoch zu erfordern hätte, auffgehoben seyn sollen.

Es würde auch von obehrenberührten Herren Kirchen: und Fabric Pflegern ihme Herrn Silbermann accordirt, dass derselbe ohne Halt eines Jahr: oder Wartgelts von der Fabric Jahrs zehen Gulden zu empfangen haben, so auff Weyhenachten dieses $1727^{\mathrm{n}}$ Jahrs angehen solle, jedoch auff obaccordirte Weiss und Bedingung, damit alles in der Orgel in gutem Standt erhalten werde.

Urkundichen dessen seind vonn solchem Accord zwey gleichlautende Exemplaria von einer Hand geschrieben verfertiget von beeden Partheyen underschrieben und jeder eines davon nachrichtlichen zugestellt worden.

Actum Strassburg, Dienstags den sechs und zwantzigsten Augusti Anno ein taussendt sieben hundert zwantzig und sieben.

Johann Paul Schübler Johann Joachim Fischer Fabric Pfleger. 
(Acort mit Herren Andreas Silbermann, wegen Reparierung der Orgel zum Alten St. Peter. 1727)

AMS. Arch. de la paroisse Saint-Pierre-le-Vieux, liasse 22, $n^{\circ} 13$

Dass Herr Schübler als Organist zum altten St: Peter die Orgel so lange er Organist gewesen sehr schlecht gespielet, aber dass Clavir hart tractiret und fast dass Mannual durchgegriffen, und dass Pedal durchgetretten, bescheine mit Grundt der Wahrheit.

Andreas Silberman

Orgelmacher.

AMS. Arch. de la paroisse Saint-Pierre-le-Vieux, liasse 22, n 23 
Bâle, cathédrale.

-Marché du 7 mai 1710 avec André Silbermann.

-Répertoire des tuyaux de l'orgue, par André Silbermann.

-Quittance du 12 septembre 1711 valant contrat d'entretien. 
Bâle, cathedrale.

Zu wissen seye hiemit; dass an heut zu Ende gemeltem Dato mit Consens Unserer Gnädigen Herren der Herren Hauptern Ihr Str: Ehrs: Wh:, zwüschen Herren Emanuel Merian, alhiesigem Thumb-Probstey Schaffner an einem: Sodann Herrn Andreae Silbermann Burger und Statt Orgelmacher der Statt Strassburg am andern Theil wegen Reparir= und Verbesserung des Münsterorgelwerckhs alhier zu Basel folgender Verglich und Anred getroffen worden; Benanntlich so verspricht er Herr Andreas Silbermann bemelte Reparation dergestalt zu verrichten, damit das Orgelwerckh nach jezigem Gebrauch in guten Stand komme, auch das Pfeiffwerckh so annoch vorhanden zum Theil gebraucht werden könne, anstatt der ohnbrauchbaren aber newe zu verfertigen, dessgleichen anstatt etlichen geringen und unnötigen kleinen Registern andere nüzliche grosse und schöne Register in das Werckh zu sezen, mithin auch die Clavier zu ergenzen und die ermanglenden Claves im Bass als C. D. DS E FS Gs sodann im Discant gs. b. h c. zu ersezen, und solches nach jezigem Gebrauch der Music und Choral also einzurichten, dass das ganze Werckh auss rechtem Grund und Fundament renovirt werde, damit es sowohl in der Gütte und Wehrschafft als zum Gebrauch so gutt als new seye;

Und das auff Weiss und Maass wie underschiedlich hernach folgt: Namentlich sollen die Register im Obern Werckh oder grossen Manual Clavier also eingerichtet werden

1 Principal acht Schueh Thon, die alten Pfeiffen reparirt, die ermanglenden Claves aber im Bass von Holz und Disscant von Zinn zu verfertigen

2. Copel hat neu sein müssen.

3. Octave halb neu.

4. Quinta, an dessen Stat weihl sie ohnbrauchbar ist 1 neues Nazardsregister nach heutigen Gebrauch.

5. Superoctave hat gantz neu von Zihn sein müssen
2 Copel acht Schueh reparirt, die Bass Claves von Holz, die Discant Claves von Zinn

3 Octave vier Schueh reparirt, die ermanglenden Pfeiffen von Zinn

4 Quinte drey Schueh reparirt, die ermanglenden Pfeiffen von Zinn

5 Superoctave zum wenigsten halb neue Pfeiffen 
6 Ist in der Orgel eine offene Flöt drey Schueh weil solches ohnnöthig, soll an dessen statt eine Terce 1 3/5tI Schueh nach jezigem Gebrauch verfertiget und hinein gesezt werden.

7 Mixtur die alte so vorhanden ist von gar kleinen Pfeiffen sechs facht, welche ganz ruinirt, an dessen statt eine neüwe auff jezige Manier vierfacht von zinn muss gemacht werden

8 Cimbal die alte zweyfacht muss ganz neüe dreyfacht seyn

9 Ist noch ein kleines von 1 1/2 Schueh vorhanden, welches ohnnöthig und ohnbrauchbar an dessen statt soll eine Trompette acht Schueh von Zinn gesezet werden

10 Ist vorhanden eine kleine ohntaugliche Terce, solle an dessen statt Voix humaine gesezet werden.

Das Ruckh Positif betreffend soll selbiges wegen Mangel Plazes so viel als nöthig verendert werden, wie auch wegen ohntauglichen Windladen soll dannenhero das Corpus nach diesem eingerichtet werden und sollen folgende nuzliche und gebräuchliche Register hinein gesezt werden

Als

1 Principal vier $Z u$ diesen zweyen Schueh Thon Registern werden von

2. Copel. Dieses Register 2 Copel acht den alten Pfeiffen so ist auch gantz neue, und Schueh Thon viel tauglich noch so gross als dass gebraucht alte vorige wahre.

3 Nazard drey Schueh hanz new

4. Octave hat gantz neu 4 Octave zwey Schueh fast ganz neuw von Zihn sein müssen

5 Terce 1 3/5t I Schueh ganz neuw

6 Mixtur dreyfacht ganz neüw

Das Pedal belangend, bleibet solches in voriger Disposition also dass nicht mehr Claves darinnen vonnöthen seyn, die Register desselben sollen seyn wie folgt: 
Als

2. Octave ist halb neu.

3. Superoctave hat gantz neu sein müssen.

4. Mixtur. Diese ist so ohntauglich und so von kleinen Pfeiffen gewesen, dass sie nothwendig hat neu sein müssen. Ist jez vierfach

5. Der Posaunen oder Bomparten Bass ist gantz neue.

Die windtlathen sein bey Aushebung der Orgel so in schlechten stant gefunden wordten, dass so ich solche gebraucht hätte so wehre nichts gutes erfolget, sondern währe aufs längste eine Arbeyt auff 10 Jhar gewessen. Sindt dannenhero die Windlahten, Structur, nebsts der gantzen Regirung gantz neu, und bequem auf itzige neue Ardt gemacht wordten. Die Canäl gantz neue auch die Blassbälge also bequem, und dem Werck selbsten zum Nutz geleget auf heutiger Ardt dass man sie ub
1 Principal sechzehen Schueh so vorhanden, soll reparirt werden

2 Octave acht Schueh welches etliche newe Pfeiffen erforderet

3 Superoctave vier Schueh, welches hin und wieder newe Pfeiffen vonnöthen hat

4 Mixtur so vorhanden und sechsfacht, muss an vielen ortten mit neüwen Pfeiffen aussgebesseret werden

5 Ist vorahnden ein alter Posaunen Bass, welcher gänzlich ohntauglich, ohn allein dass mann davon etliche Corpus oder Obertheile brauchen kan, an dessen Statt soll gemacht werden eine Bompartte oder Posaunen 16 Schueh

Ferner soll die obere Windladen aussgehoben werden, so viel als die ermanglenden Claves erforderen, dazu gemacht werden, die Ventil und Structur an derselben geendert und also ganz verneüweret werden, dass sie in den Stand als wie von ganz newem zu stehen komme.

Die 2 Manual Clavier sollen ganz neüw seyn und also eingerichtet werden, dass das Oberwerckh und Ruckhpositiv kan nach Belieben zusammen geschlagen werden. Alle 4 Blassbälge sollen abgehoben werden und ganz neuw beläderet werden, wie auch die canäl meistens new seyn. Es soll auch ein new Tremulanten Register gemacht werden, und also überkombt das ganze Werckh zwey und 
mit Händten aufzihet.

2 Tremulanten Register sindt gemacht worden.

Summa dass gantze Werck ist neu, und hat 1256 Pfeiffen darunter seint nicht mehr als 129 noch von dem alten verhandten, und so ich so ein Orgel Werck, als wie dieses jtz imstandt ist, verdingen solte, so könte auf dass wönigste wöniger nicht nehmen als $3000 \mathrm{f}$. zwanzig Register den Tremulanten mit eingerechnet.

Dieses Werckh nun wie vorgemelt, verspricht Herr Silbermann in den stand zu sezen dass es der Gütte und Wehrschafft nach, so gutt als neüw seyn werde, alle Materialien wie sie auch Namen haben mögen, darzu zu verschaffen, auch die Verenderung das Ruckhpositivs mit dem Corpus über sich zu nemmen.

Für alle obbeschriebene Arbeit, wann sie dem Versprechen gemes Wehrschafft verfertiget seyn wirt, sollen ihme Herren Silbermann neben Losament und zweyen Bettern, wie auch für sein und seiner Bedienten Kost, wehrender Arbeit und so er sich deswegen alhier auffhalten möchte, desgleichen für seine Ruckhreys hiemit für alles zusammen vierhundert und fünffzig Louis blancs bezahlt, seinen Bedienten aber nach völliger Expedition ein Trinckhgeltt; auch inmittelst zu Abhebung des Werckhs nöthiges Gerüst und erforderliche Handlanger verschafft und an die Handt gegeben werden.

Alles auffrecht, ehrbarlich und ohne Gefehrde, gestalten, solchem allem getrewlich nachzukommen, zwey dergleichen contracten an Wortten gleichlautend abgeschrieben, sich auch zu Gezeugnus und wahren Urkhund dessen beyde Eingangs ernannte Partheyen selbs eygenhändig underschrieben, und ihre gewohnliche Pitschafften hiefür auffgetruckht haben; So beschehen in Basel den siebenden May Anno 1710 .

T. Emanuel Merian. Im Nahmmen als obgedacht Andreas Silberman.

Staatsarchiv Bâle, Kirchenarchiv V 29. Münster Orgel 1710-1850. 
Bâle, cathédrale.

Accord die Orglen im Münster betreffendt.

Verzeugnus alle dererjenigen Pfeiffen die sich dato in der von mir Ends unterschriebenem neuerdinge reparirten Orgel in dem Münster befinden.

Erstlich in Oberwerck

1. Principal 8 Schu Thon hat 48 Pfeiffen

2. Copel ... 8 Schu ...... 48 .

3. Octave .. 4 Schu ...... 48.

4. Nazard .. 3 Schu ...... 48.

5. Superoctave 2 Schu ..... 48.

6. Terz ...1 3/5 Schu .... 48.

7. Mixtur 4 facht die gröste Pfeif 1 1/2 Schu, hat.. 192.

8. Cimbal 3 facht hat .... 144.

9. Trompette 8 Schu ......4 48.

10. Voix humaine 8 Schu .....48. Summa in Oberwerck $\quad 720$ Pfeiffen

Im Pedal

1. Principal 16 Schu Thon hat 19.Pfeiffen

2. Octave... 8 Schu ....... 19.

3. Superoctave 4 Schu ...... 19.

4. Mixtur 4 facht die gröste Pfeif 1 1/2 Schu ....... 76.

5. Bompartte 16 Schu .......19. Summa in Pedal 152 Pfeiffen

Im Positive

1. Principal 4 Schu Thon hat 48.Pfeiffen

2. Copel ... 8 Schu ...... 48.

3. Nazard .. 3 Schu ...... 48.

4. Octave.. 2 schu ...... 48.

5. Terz ..1 3/5 Schu ..... 48.

6. Mixtur 3 facht ........144. Summa in Positive 384 Pfeiffen 
Im Oberwerck befindet sich zusamen Pfeiffen ...... 720.

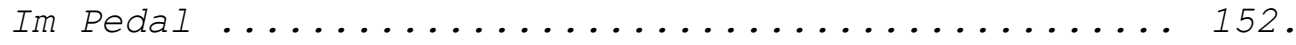

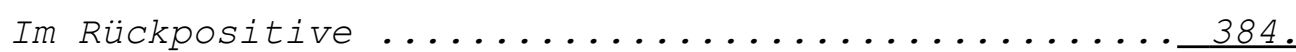

Haupt summa

1256 .

Welche Pfeiffen insgesambt in guter Ordnung stehen u: am obern Enden schön gleich und rundt abgestimbt sein.

Andreas Silberman.

Orgelmacher.

Staatsarchiv Bâle, Kirchen-Archiv V 29. 
Bâle, cathedrale.

Nachdeme die Orgel in Münster verfertiget, und vor meine Arbeit, und anders nach Vergnügen condentiret und bezahlt worvor schuldigen Danck sage, daneben verspreche, dass im Fal innerhalb Jahres Jahresfrist, sich ein Hauptfeler finden solte an der Orgel, so aus der Arbeyt selbsten endtstehen solte, selben in meinen Kosten widerumb zu repariren, und gut zu machen.

Bassel den 12 septembis 1711.

Andreas Silberman, Orgelmacher von Strassburg.

Staatsarchiv Bâle. Kirchen-Archiv V 29. 
Strasbourg, Cathédrale.

- Devis d'André Silbermann, 24 juillet 1713.

- Copie du contrat d'entretien avec Jean André Silbermann, 10 novembre 1739. 


\section{Strasbourg Cathédrale}

(24 juillet 1713 Devis estimatif de la réparation de l'orgue de la Cathédrale dressé par André Silbermann)

Auff Begehren Herrn Langhanssen wohlverordneten Schaffners, dess Frawen Hausses, habe ich ends undersehriebener das grosse Orgelwerckh im Münster zue underschiedenen mahlen, fleisig und genaw übersehen, welches dann in einem solchen schlechten stand sich befindet, dass alles stimmens und daran flickens umbsonst und vergebens ist, wie ich dann schon von etlichen Jahren her öffters Herrn Schaffner dessen berichtet, und es die Erfahrung zum öffteren genugsam bezeiget hat, dass alle daran angewante grosse Kösten vergebens gewessen, und künfftig hien auch noch alles angewendes Gellt umbsonst seyn wird, wann nicht das Orgelwerckh vorhero durch eine Haupt Reparation undt Erneuerung, in einen besseren, rechten und guten beständigen stand gesetzet wirdt, welches dann geschehen muss.

Erstens durch Abhebung aller Pfeiffen dess gantzen Werckhs

2.tens mit neuen Windlathen, Clavir, Regierung, grösseren Bälgen, Canälen, mit Reparirung der grossen und Verneuerung der klein Pfeiffen, welche theils von der Älte durch den Salpeter verfressen, dass mann sie in Händen verreiben kan, theils auch durch grosse Unwissenheit der jenigen, so sich unterstanden die orgel zu stimmen, verrissen, vertrückt und verschnitten seyndt.

3. ens durch Veränderung und Vermehrung der Register, damit die Orgel nach jetztmahliger Arth und Gebrauch in rechten Stand gebracht wärde, dann zuwissen, dass im obern grossen Werckh anjetzo nicht mehr dann 11. Register, alss

Erstl: Principal .................... 16. Schuhe.

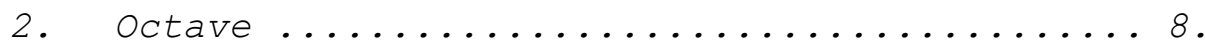

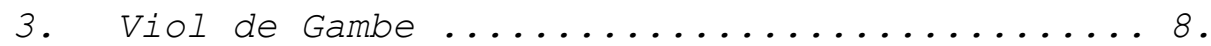

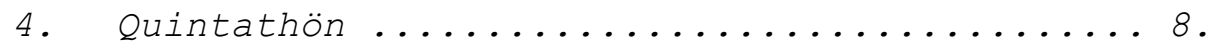

5. Quinta ........................ Schuhthon

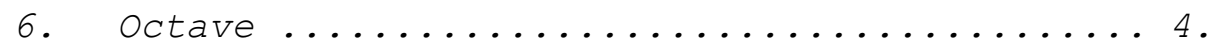

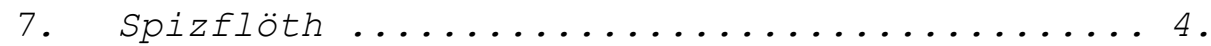

8. Mixtur. 5. fach ................. 1 1/2.

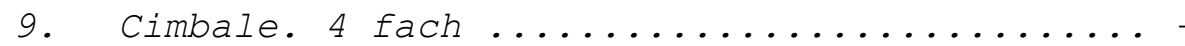

Folget im Pedal. 
10. Ein sehr altes ohnbestündiges Posaunen Register von kurtzer Mensur ................ 16. Schuhthon

11. Gross Principal Bass in Schein so in dem grossen mitleren Thurn stehet, dabey allzeit noch ein Pfeiff von einer Octaven ............. 32. Schuhe Summa im gantzen obern Werckh. 11. Register darinnen 888 Pfeiffen. Im Rückh Positive seind folgende Register

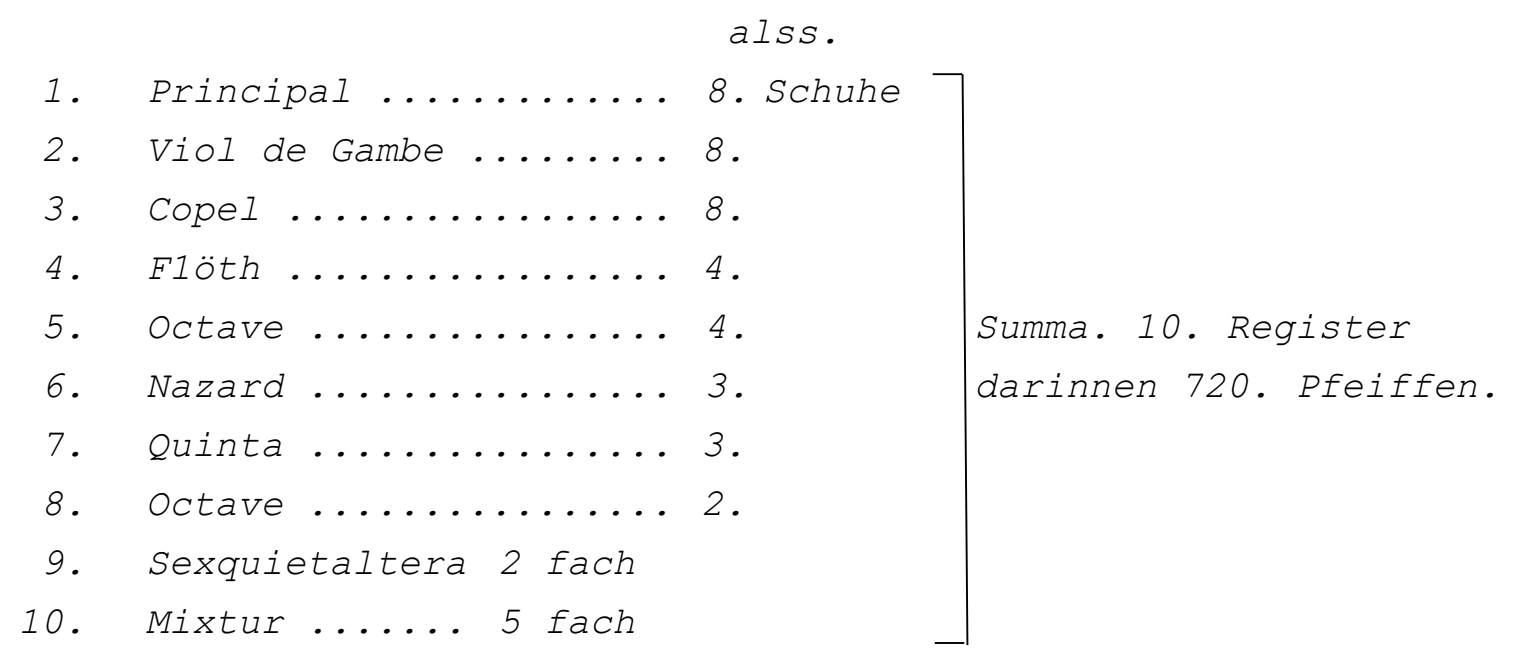

Im Brust Positive seind. 7. kleine untaugliche Register darinnen ohngefehr. 384. Pfeiffen, so niehmahlen auch von neuem nicht ist in recht gutem stand gewessen.

Ferners hat das alte Werckh. 12. Blassbälge, die zu klein undt so untauglich, dass sie gar zu nichts zu gebrauchen. Weilen daran alle obere und untere Blätter zue dinn, auch mit zue vielen kleinen Falten, und vielem Leder, dahero so lange sie gelegen ein immer wehrendes Flicken gebraucht haben. Davor müssen 6 grössere so bey nahem 12. Schuhe in die Länge, und 6. Schuhe in der Breitte haben und nur eine Falten /: an welchen lange zeit sich nichts verändert :/ gemacht werden.

Das alte Clavir ist sehr unbequem eingerichtet, und was das übelste, ist es so hart zue spielen, dessgleichen glaube weit und breitt keines mehr zue finden seyn wirdt.

Die Wind Ladthen seind so untauglich, dass mann den Wind ahn allen Enden deroselben höret durchgehen, als wie ein Wasser Rauschen, desswegen auch nothwendig neue seyn müssen, die also eingerichtet, dass, das Clavir leicht zu tractiren, und dass noch darzue, die jetzt gebräuchlichen schöne Register darauff stehen können. 
So habe ich dann mit vorhero gutem Bedacht, genawer Überlegung und Abmessung des Platzes /: besonders im obern grossen Werckh :/ befunden, dass mit Erneuerung der Windlathen diese nachfolgende Register können undt müssen hienein gesetzet werden; alss im obern Werckh zum grossen Manual Clavir muss erstens stehen.

1. Principal .......... 16. Schuhe, darzue wird das alte so in der Orgel gebraucht, die grossen Pfeiffen in Bass reparirt, die kleinen im Discant meistens neue gemacht.

2. Copel ............ 16. Schuhe, dergleichen keine in der alten Orgel dahero gantz new, die untersten 2. Octaven in Bass von Holtz wie gebräuchlich, die übrigen Discant von zinn und Bley.

3. Octave ...........8. Schuhe, darzue wird das alte verschnitten, auss den grossen so das zinn noch guth, kleinere gemacht, und vor die grossen neue gemaoht, dahero so viel als gantze neu.

4. Copel ........... 8. Schuhe, gantz neu, vor dessen Platz die Viol de Gambe aussen bleibt.

5. Octave ............ 4. Schuhe gantz neu.

6. Grosse Terz auss 4. Schuhe gantz neue, vor dessen Platz die Quint 6. Schuhe, welche in den Orgelen nicht mehr gebräuchlich aussbleibt.

7. Nazard............3. Schuhe gantz neu, vor dessen Platz die untaugliche Quintathön aussen bleibt.

8. Doublette.........2. Schuhe Vor dessen Platz Spizflöth

9. Terz auss ..........2. Schuhe 4. Schuh aussenbleibt

10. Mixtur 4 fach, gantz neu, vor die alte Mixtur 5. fach.

11. Cimbale 3 fach, gantz neu, vor die alte Cimbale 4 fach.

12. Grosser Cornet 5. fach gantz new.

13. Trompette .........8. Schuhe

14. Clairon ............ 4. Schuhe von Zinn gantz new.

15. Voin humaine ........8. Schuhe

Pedal.

Das Pedal betreffend, muss solches in seiner Disposition bleiben, also dass desselben Wind lath in Mitten der grossen Orgel repariret werde, auff welcher Wind Lath stehet. 
1. Gross Principal Bass ......

32. Schuhe 7 diese

2. Darbey stehet allezeit ein Octaven ... 16. Schuhe repariret

3. ist das Principal auss dem Obernwerck 16. Schuhe, undt

4. die Octave desselben ............ 8. Schuhe, also disponiret dass sich diese beyde Register so wohl pedaliter alss auch manualiter gebrauchen lassen.

5. kan die Trompette und Clairung auss dem obern Werckh eingerichtet werden, dass sie auch vor sich allein so wohlen pedaliter alss manualiter zu gebrauchen; vor das

6. muss von dem alten unbeständigen kleinen Posaunen Bass, eine jetzt gebräuchliche Bompartten 16. Schuh gemacht werden, dessen Corpora völlig die Länge von 16. Schuhe haben, und von zinn gemacht, konte wohl was die Bass Claves anbetrifft von Holtz seyn, weil aber diese von Holtz mehr Platz einnimbt, und jene von zinn besser klingt, so halte nicht vor nöthig hierinnen zue sparen.

Summa überkäme dass Ober Werckh mit dem Pedal 17. Register, die in sich begreiffen 1109. Pfeiffen.

Im Rückh Positive müssen seyn folgende Register

1. Principal .......... 8. Schuhe Tohn

2. Copel .............. 8 .

3. Octave ..............4.

4. Flöth .............4.

5. Nazard ...............

6. Doublette ...........2.

7. Terz auss ........... 2 .

8. Mixtur 3 fach .........1.

alles new von 10. Registern die in sich begreiffen 672. Pfeiffen.

9. Cimbale 3 fach ........ -

10. Cromhorne ..........8.

Im Brust Positive können stehen die jetzt gebräuchlichen Echo, desse dass Clavir im Tenor $f$. anfanget biss $C^{\prime \prime} '$. 
1. Copel auss .........8. Schuhe

2. Octave auss ........4.

3. Nazard auss .......... 3 .

4. Doublette auss .......2. 2 .

5. Terz. auss .........2.

Diese repontiren auff den grossen Cornet, oder grand

6. Mixtur, 3 fach ........ jeu.

7. Trompette auss ......8.8.

dieses auff beyde Plein jeu

8. Doin humaine ......... 8 . dieses auff die ober Trompette dieses auff die Voin humaine im Obernwerckh.

Haubt Summa überkäme das Orgelwerckh in allem 35 Register, ohne die Tremulanten, welche in sich begreiffen 2001. Pfeiffen und zwar anderst nicht alss wieder im cornet Thon.

Auff andere geringere Arth und Weise, wie hieoben beschrieben, kan dass Orgel Werck nicht vorgenommen werden zue repariren; dann mit wenigern Registern kan es nicht seyn, einen kunsterfahrnen Organisten damit zue contentiren, jedoch wäre auff diese Arth der Register kein Mangel, und kan sich ein künstlicher Organist darauff so wohl hören lassen, als wann er ein Werck mit mehrern Registern hätte.

Solte sich aber ein Orgelmacher finden, so sich unterstehen wolte, dass Orgel Werck auff leichtere und geringere Weisse mit wenigern Kösten zue repariren so sage dass derselbe sich entweder solches nicht getrauet zu machen, oder es nicht verstehet.

Noch besser aber und mit mehreren Registern, dem Münster auch zue grösserer zierdte, könte nach Belieben wohl ein Orgelwerckh im Münster gemacht werden, aber nicht auff diesen Platz, wo anjetzo die alte Orgel stehet, sondern darzue müsste ein besonder Gewölb, oder Bogen gemacht werden, an dem Orth wo anjetzo die Glocken Sayl hencken, unter dem grosen Rosenfenster, wie an der gleichen Orgelwerckh von 60. Registeren mein Bruder zue Freyberg in Meysen in der ThomKirchen schon drey Jahr in Arbeith ist, und in einem Jahr hoffet fertig zue werden /: davon nach Belieben einen kleinen Abriss zeigen kan :/ vor welches Werckh Ihm allein vor seinen, und seiner Arbeither Lohn 8000. RhIr: versprochen.

Das Orgelwerckh nun mit den oben benanten 35. Registern einzurichten, neue Wind Lathen, Bälge, Canäl, Clavir, Regierung, Reparirung, und neue Verfertigung der Pfeiffen, Aufstellung, und Stimmung, darzue Lüfferung der benöthigten Materialien, alss 
Holtz, Zinn, Bley, Leder, Leim, Thrath, Nägel etc:

Vor meiner, und meiner Arbeither Lohn, habe ich befunden, nachdeme ich meine Menssures vorhero fleissig genommen, auss getheilet und auffgerissen, nach diesen eines nach dem andern der Billigkeit gemess, so viel alss möglich genau überschlagen, dass sich die Summa belaufft auff Drey Tausendt, Sechs Hundert und Viertzig Gulden.

sage ......................... 3640. fl.

Inn dieser Summa der 3640. fl. aber, nicht begriffen, wass an Schlosser Arbeith, geendert oder neu seyn muss, oder so etwas am Corpus zue ändern vorfile dazue Schreiner, undt Bildhauer zu gebrauchen, welches mann noch anjetzo weil die Pfeiffen in der Orgel stehen nicht alles sehen kan, wie auch ist nicht mit eingerechnet die Handreichung der Maurer oder Zimmer Leuth bey Abhebung und Widerauffstellung der Orgel mit Rüstungen, Seilen, und Flaschenzügen an Handt zu gehen, ingleichem auch 2. Persohnen bey Stimmung und Auffstellung der Orgel die Blassbälge zu ziehen.

So aber im Fall die vorherbeschriebene summa der 3640. fl. zue viel zue seyn scheinen solte, so offerire alles noch einmahl in Beyseyn verständiger Herren oder Organisten so sich recht auff Orgelen verstehen von Stück, zu Stück zu specificiren, oder so beliebig diesen meinen Aufsatz, entweder auff Paris, oder einen Orth in Teutschlandt, an kunstverständige Organisten oder Orgelmacher zue sendten, ich gar gerne leiden will, weil versichert, dass ihm jeder kunstverständige wohl sehen darff so wohl wegen der Arbeith, alss auch wegen der Anforderung der Geld Summa weil ich versichert, dass so ich die Gnade haben solte, und mir Gott mein Leben fristet, dass ich mehr auff die Ehre sehe, diese Arbeith zu machen, als auff die daran gewinnete Geld Summ Mit diesem allem habe meinen hochgeehrten Herrn Schaffner nach Begehren gerne auffwartten wollen und sollen dessen ich Zeit lebens verbleibe Strassburg den 24t July 1713. 
Copia eines schrifftlichen Accords, von dem Stifft Frauenhauss, mit Herrn Silbermann Orgelmacher, die Aussstäubung und Stimmung der Orgel im münster betreffend.

Zu wissen seye hiermit, jedermänniglichen, insonderheit aber denen es zu wissen nöthig, dass ane heut zu End gesetztem Dato zwischen denen hoch und wohl verordneten Herren Pflegeren des löblichen Stifft Unsser Lieben Frauen Wercks alhier zu Strassburg, ane einem: und dann Herrn Johann Andreas Silbermann, dem Orgelmacher und Burgern alhier, am andern Theil wegen der im Münster befindlichen, und von ermeltem Stifft zu erhalten Orgel, auch deren nöthigen Abstaub= Stimmen und Säuberung nachfolgender Accord wissend: und bedächtlichen getroffen, und aufgerichtet worden ist. Nehmlichen es obligiert sich obernannter Herr Silberman, die in dem Münster stehende Orgel, von dato an und ins künfftige, so lang diesser Accord andauren wird, so offt es die Nothdurfft erfordert durch sich und die Seinigen ordentlich abstauben, die an solcher befindlichen Pfeiffen und Blassbälg säubern und stimmen zu lassen, mithin dass gantze Werck bestmöglichst zu besorgen und in Obacht zu nehmen dass niemahlen einige Klag entstehen und geführet, hingegen alle Zufriedenheit bezeugt werden soll. Dahingegen und vor solche Beobachtung auch hiebey verrichtender Sorgfalt und Arbeith, erbieten sich die hoch und wohlverordnete Herren Pflegere obbesagten Stiffts, ihme Herren Silbermann alle Jahr auf Weyhenachten und nechst komendes eintausend siebenhundert und viertzigsten Jahrs, zum erstenmahl fünfftzig Gulden, Strassburger courent Wehrung, zu reichen, auch wann an denen Pfeiffen oder anderem Orgelwerck so Hauptsachen genannt werden, etwas gebrochen, dass solche ohnbrauchbar würden, und neue an der abgegangenen Stück, Platz gemacht werden müssten, dieselbe auss des Stiffts Mittlen herbey und angeschafft, einfolglich dess Herren Silbermanns daran verrichtende Arbeith aparté: ausser diesem aber nichts weiters desswegen demselben bezahlt werden, sondern er alles andere es habe Nahmen wie es immer wolle, für obige ihme zugesagte fl 50 auss dem seinigen und ohne den geringsten ab dem Stifft zu thun habenden Beytrag, zu machen und das gantze Werck wieder so gut möglich, zu repariren schuldig und gehalten seyn: im übrigen aber solle diesser Accord, so lang es beederseits Parthen beliebig seyn wird, andauren. Urkundlich dessen ist diesser Accord hierüber gefertigt von beederseits Parthen eigenhändig unterschrieben, das Original desselben 
bey dem Stifft aufbehalten und gegenwärtige Copia davon zu nehmen und Herrn Silbermann zuzustellen abgeordet worden so geschehen in Strassburg den 10 ten Novembris 1739 in dem Original seind unterschrieben J. L: Wormser Denner Johann Daniel Städel und Johannes Andreas Silbermann, Orgelmacher.

AMS, Archives du Temple-Neuf. Liasse 82. 
Strasbourg, Sainte-Aurélie.

- Décompte, par André Silbermann.

- Quittance du 6 septembre 1718, par André Silbermann.

- Quittance du 4 octobre 1720, par André Silbermann.

- Quittance du 31 décembre 1767, par Jean André Silbermann. 
Die Orgel ist verdingt worden umb $1650 \mathrm{fl}$.

mehr ward mir verdingt 1. 8tave ins Pedal â $50 \mathrm{fl}$.

Käme also die gantze Orgel auf ..............1700 fl.

Hirauf habe von Herr Gefatter Notar Matern in dreymahlen empeangen

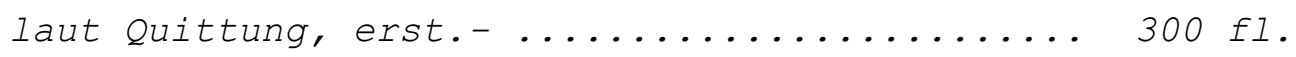

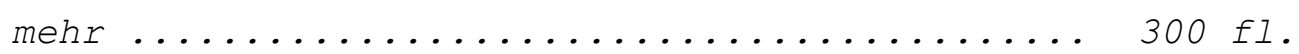

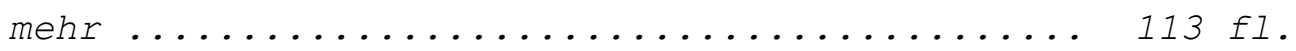

Den 5 Junii in anno 17, als am ersten Pfingst Feyertag von Herrn

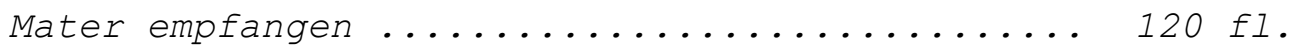

Als ich in anno 18 im Friejahr vor Ostern nach Basel reisen wollte, damahls mir die Aurelen Kirchen noch 867 fl. Schuldig war, so habe an einem Sontag morgens nach der Predig mit sämbtliche Herren Pflegern vor der Kirchenthüre geredet umb Geld zu haben. Es gabe mir aber damahls Herr XV Lemp zur Andword sie hätten anjetzo keines, ich sollte anderwertig Geld aufnehmen, sie wollten mir von dato an auch die restirente $867 \mathrm{fl}$ verzinssen. Kortz darauf aber als ich zu Bassel gewesen, und dass Geld aufgeschlagen war, ist $H$. Mater mit neuen Louis d'ors kommen, und solche meiner Fraue gegeben, ohngefehr bey $300 \mathrm{fl}$. so aber ich in meinem Buch nicht wegen Abwesenheit aufnotirt finde.

In anno 20 habe ich mit sämbtliche Herren Pfläger /: ohngefehr in Fastenzeit : / in damahls H. XV Lempen Haus abgerechnet, da sie mir auch $8 \mathrm{fl}$ wegen eines gelehnten Positives schuldig wahren, und mir auch ohne Zweiffel den verfallenen Zins von Capital werden versprochenermassen gebessert haben, damahls blieben sie mir schuldig ........... $600 \mathrm{fl}$. laut meines Buchs.

Kortz aber darauf an einem Samstag, als eben nachmittag darauf das Geld aufgeruffen wurde, kam Herr Nessman, und H. Reubel, mit Herrn Matern in mein Haus und wollten mir 300 fl in 3 s pies abzahlen, als aber ich mich damahls nicht gleich resolvieren woltte solche Geld anzunehmen, gaben sie mir 3 Tag Bedenckzeit. und kamme montags darauf diese bemelte 3 Herren sämbtlich in mein Hauss, da wir uns dan so verglichen, dass sie mir $200 \mathrm{fl}$ am

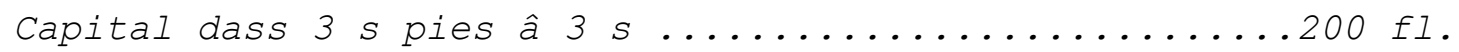
gerechnet zahlten und dan auch den Zins biss auf Pfingsten 1720 mit $24 \mathrm{fl}$. mehr den zins von $200 \mathrm{fl}$. wegen vierteljähriger

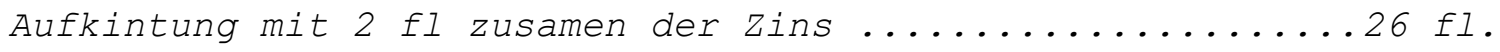
NB. ich emp[f]inge aber vor den zins dass 3 s pies â $4 s$. 
Mehr in Anno 1723 auf Pfingsten empfieng von H. Gefatter Matern 2 neue Louis d'ors â. $22 \mathrm{fl} .5 \mathrm{~s}$. thut.......... $45 \mathrm{fl}$.

mehr bracht $H$. Mater mir wieder kortz darauf 9 dergleichen Louis d'ors thut ................... 202 fl. 5 s. macht $247 \mathrm{fl} .5 \mathrm{~s}$. mit dieser sum hat er mir abgereicht - den 400 fl - am Capital und dan den zins pro 1721. 22. et 23. macht 48. fl. mehr wegen ermangelter Aufkintung vor 200 fl Capital macht 2 fl nicht zusamen $50 \mathrm{fl}$. ich habe aber an diesen zins nicht mehr empfangen, als .................... 57. s. restirte mir also noch 25 ss. NB. welches mir aber bey zahlung

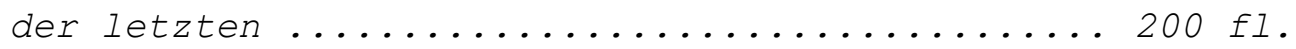
an Capital von Herren Pfarh.- Ehrlin, nebst dem verfallenen Zins von $200 \mathrm{fl}$. von anno 1723 an Pfingsten an gerechnet biss den 11 gbris bemelten Jahrs, sambt $1 / 4$ tI jähricher Aufkindung 2 fl wohl empfangen habe.

AMS. AST. $n^{\circ} 30$, carton 16,8 . 
Dass ich Entes bemeltter ferners auf Abschlag der neuen Orgel in St: Aurelien Kirchen, von S. T. Herren Phielib Jacob Matern empfangen habe dreyhundert Gulden, sage $300 \mathrm{fl}$. bescheine hiemit bestermassen.

den 6 gbris. 1718.

Andreas Silberman.

Archives de la Paroisse Ste-Aurélie. Carton 12, liasse 3 (mémoires d'artisans \& d'ouvriers 1718), quittance $\mathrm{N}^{\circ}$ 23, texte autographe. 
Dass mir Herr Nots. Philipp Jacob Mader Schaffner der Fabric Stae. Aureliae in Abschlag, wegen verdungener Orgel baar bezahlt habe, die Summe von zweyhundert Gulden, ane dem restirenden Capital der 600. fl. so dann von solchen 600. fl. verfallenen Zinss mit zwantzig und sechs Gulden, also zusammen mit zweyhundert zwantzig und sechs Gulden, welches hiemit quittirend bescheine, strassburg d. 4ten 8bris 1720 .

Andreas Silberman.

Archives de la Paroisse Ste-Aurélie. Carton 12, liasse 3 (mémoires d'artisans \& d'ouvriers 1720), quittance $\mathrm{N}^{\circ} 11$, texte non autographe. 
Dass von S. T. Herrn Notarius Stöber mir Ends unterschriebenem, vor Stimmung der Orgel zu St: Aurelien diejenigen 10. fl, welche jährlich auf Martini verfallen sind, vor dieses 1767.ste Jahr, wohl bezahlt worden, solches wird hiemit danckbarlich beschienen. Strassburg d 31. Xbris 1767. Johann Andreas Silbermann.

Archives de la Paroisse Ste-Aurélie. Bô̂te $\mathrm{N}^{\circ}$ 9, liasse 22 (memoires d'artisans \& d'ouvriers 1767), quittance $\mathrm{N}^{\circ} 22$, autographe de Jean André Silbermann. 
Bischwiller, élise protestante.

Marché du 8 décembre 1723 avec André Silbermann. 
Zuwiszen seÿe hiemit, dasz nach dem

Unsers Gudgstn Fürsten und Herren

Hoch Fürst-.e durchläucht gudgst beliebet,

ein neues Orgel Werck in allhiesiger

Pfarr Kirchen verfertiget und auffge=

stellet zu sehen, auff zu end gemeltem

dato, nahmens der Evangelisch=Lutherisch

so wohl als auch deren Teutsch= und

Frantzösisch Reformirten Gemeinden

von Uns unterschriebenen, als hierzu

special committirt= und bevollmächtigt

an Einem, so dann H-.n Andreas Silber-

mann Burgern und Orgelmacher

zu Straszburg, am andern theil

dieser ursachen, hernach folgender

auffrichtiger accord geschloszen

und zwar.

Erstlichen die Beschaffenheit dies

begehrten Orgelwercks betreffend

solle solches bestehen, in 13. Registern

alsz.

Dem Principal 8 Schuh thon, und

zwar dasz wegen ermanglender

Höhe, die ersten Grösten 5. bis 6. P...

im pasz, inwendig von Holtz, ...

übrigen aber, von gutem feinem

Zinn, auszwendig im Schein ge= stellet

seÿen . 
Der Octav 4. Schuh von der gleichen Zinn, davon die gröste, auch im Schein stehen sollen.

Der Coppel deren die gröste Octav von Holtz, die übrige von zinn und bleÿ. Dem Nazard 3. Schuh Thon von zinn und bleÿ Der super Octav 2. Schuh Thon von zinn Der Tertz ausz 2. Schuh von Zinn und Bleÿ. Der mixtur 3. fach.

Dem Cymbale 3. fach beÿde von Zinn

Der Trompet 8. Schuh von Zinn

Dem Cornet von Zinn und Bleÿ fünff fach Im Pedal

Dem Supbas gedeckt von Holtz 16 Schuh Thon Der Octav von Holtz 8. Schuh Dem Trompeten pasz, von Zinn 8. Schuh, Und sollen zweÿtens alle diese register, nach dem Frantzösischen opera Thon gestimmet, zweÿen völligen Manual Claviren und zweÿen tremulanten versehen werden.

Drittens, solle das Corpus von Eichenen Holtz, mit der benöthigten Bildschnitzler arbeit, nach dem bald einzuschickenden abrisz versehen, so viel aber die Ver= täffelung zu dem pedal hinter der 
Orgel belangt, solche von Thannen Holtz und eingefaszter arbeit gemacht seÿn .

Viertens die benöthigte Blaszbälcke betreffend, so sollen derer dreÿ, jeder 6. Schuh lang und 3. Schuh breit, welche auff die Kirchen Bühn zu liegen kommen und hinter der Orgel mit Seÿlen zu ziehen seind, verfertiget werden, Vor dieses neue Orgelwerck nun, davon Fünfftens das Corpus und die Schein... längstens zwischen Ostern und Pfingsten auff den dazu gehörigen Lettner geste... das gantze Werck aber nach und nach jedoch auff die darauff folgende Weÿhenachten gäntzlich verfertiget und auffgestellet seÿn solle, ... Er ein Jahres frist Wehrschafft zu ... hat, wird mehr gemelten $H_{-}^{-}$. Silber= mann Nahmens der auch mehr ge= dachten gemeinden zu zahlen ver= sprochen, die Summa von Tauszend Fünffhundert Gulden Straszburger Wehrung, desz... Frauliebste aber Eine Louis d'or zu 19. R. 8. b als eine discretion 
und zwar folgender gestalt, dasz die Summ so man durch eine allgemeine collect zu= sammen zu bringen suchen wird, in folgenden terminen bezahlt werden solle. Als wann das Corpus Sambt dem Schein auffgestellet seÿn wird, auff Pfingsten künfftiges Jahr .......... 500. R --Nach völliger Verfertigung des Wercks auff die darauff folgende Weÿhenachten

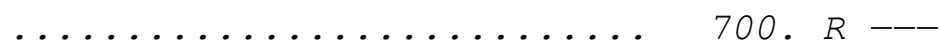

Die restirende 300 . $R$. aber ein Jahr darnach, oder auff Weÿhenachten 1725. da die geleistete Wehrschafft wird ihr end genommen haben.

Schlieszlichen und Sechstens, verspricht man hiesiger Orthen, alle arbeit fracht und zoll freÿ herausz lieffern zu laszen, und nach Verfertigung derselben, eine Fuhr nacher Straszburg zuruck zu geben, wie dann auch ferner in zeit der auffstellung dem $H^{-}$. Beständer und seinen Gesellen, freÿe Kost und Logement versprochen wird, alsz deszentwegen eingangs Höchstgedachte Hochfürst-. Durch-.t, dasz es von gndgst-. Herrschafft wegen geschehen solle, sich gndgst declarirt haben. 
Deszen nun allen zu wahrer urkund,

ist gegenwertiger Accord in duplo

verfertiget, von beÿden theilen unter=

schrieben, und jedem ein exemplar

zugestelt worden, jedoch denen hierzu

committirten oder denen Ihrigen

zu keinem praejudiz und nachtheil,

So geschehen Bischweiler den $8^{t}$. Xbris

1723.

M. Johann Daniel Germann

Pfarrer der Evangelisch=

Lutherischen Gemeinde.

J. P. Engelbach RTMstr

Phil. Gottfriedt Geisel ...

der Reformirten Teutschen ...

A. Carey Pa...

françoise Reform...

Bischweiler.

Joh: Christ: FrauenHoltz, Ca...

Andreas Silbermann, Org ...

Bischwiller. Archives communales, GG 9. 
Strasbourg, Saint-Guillaume.

- Projet non daté, non signé.

- Projet de marché du 11 novembre 1726 avec André Silbermann. 
Specification der Register, so nothwendig in die Orgel nach St: Wilhelm müsten gemacht werden.

1. Montre, im Schein von Zinn ........... Fuss Thon

2. Bourdon, von Holtz und Medal ........... 8 .

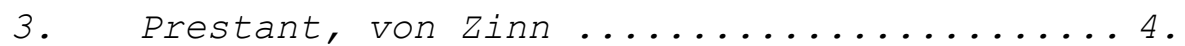

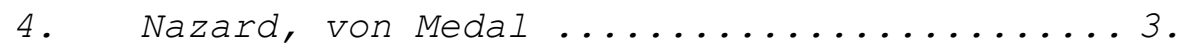

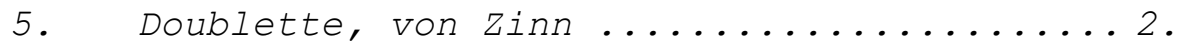

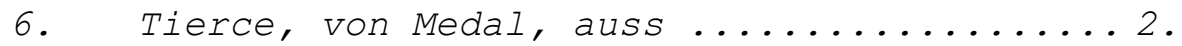

7. Fourniture, von Zinn, 3 facht, die gröste Pfeiffen ........................ $1 / 2$.

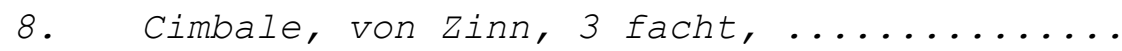

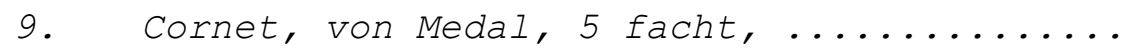

\section{Im Positif.}

1. Prestant, von Zinn, im Schein.........4 Fuss thon

2. Bourdon, von Holtz, und Medal........... 8 Fuss Thon.

3. Nazard, von Medal, die ersten 2 8taven gedeck, ......................... 3 Fuss Thon.

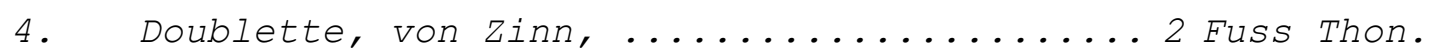

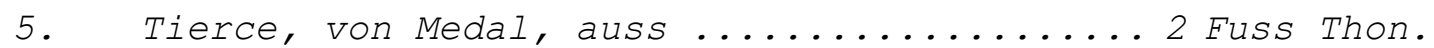

6. Fourniture 3 facht, die gröste Pfeiffen.... 1 Fuss Thon.

Pedal.

1. Supbas, von Holtz, 16 Fuss Thon offen

2. 8tave, von Holtz, 8. Fuss Thon

3. Trompette, von zinn 8. Fuss Thon.

Item 2 Tremulanten. Einen langsammen, den andern geschwind. 3 grosse Blassbälge à proportion der Orgel. Die Orgel Geheuss von saubern Eichen Holtz, mit Bildhauer Arbeit gezihrt, und mit einem von Lacvirns façon, überzogen.

Coppel 16. Sch

Archives du Bas-Rhin. Archives Paroissiales Strasbourg St. Guillaume $\mathrm{n}^{\circ} 16$. 
Specification der Register, so nothwendig in die Orgel nach St. Wilhelm müsten gemacht werden.

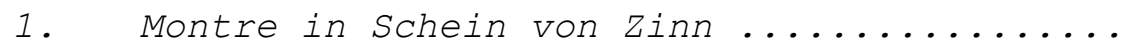

8. Fuss Thon

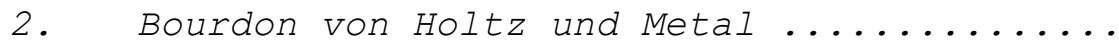

8 .

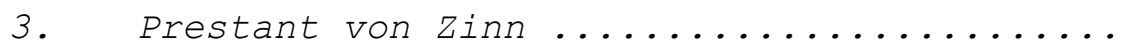

4.

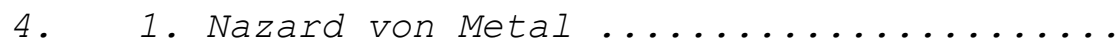

3.

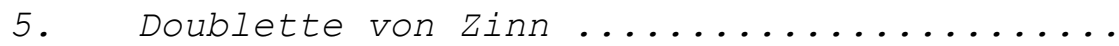

2. -

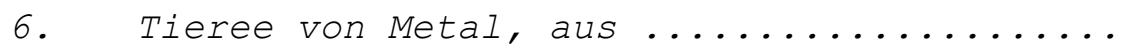

2 .

7. Forniture von Zinn 3. facht die grösste Pfeiffen 1 1/2 -

8. Cimbale von Zinn 3. facht

9. Cornet von Metal 5. facht.

Im Positif.

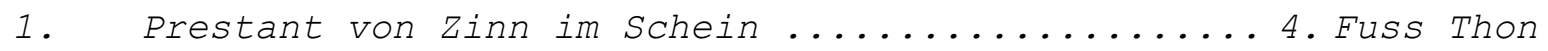

2. Bourdon von Holtz und Metal ................ -

3. Nazard von Metal die ersten 2. 8taven gedeck ....3. -

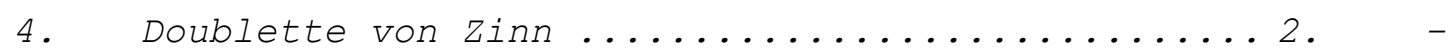

5. Tierce von Metal aus ........................ -

6. Fourniture 3. facht die grösste Pfeiffen ......... -

Pedal

1. Subpas von Holtz 16. Fussthon offen

2. Octave von Holtz 8. Fussthon

3. Trompette von Zinn 8. Fussthon

4. Coppel. 16. Schuh.

Item 2. Tremulanten einen langsamen den andern geschwind 3. grosse Blassbälg, à proportion der Orgel.

Die Orgelgehäuss von saubern Eichenholtz mit Bildhauerarbeit geziert, und mit einem façon von Lackfürniss überzogen.

Vorstehendes Werck ist zwischen denen hochedel gestreng hochedel vest und hochgelehrten Herren, Herrn Johann Reinbold Friderici hochmeritirten alten Ammeistern, Dreysehenern und Scholarchen und Herrn Johannes Dietrich, hochverdienten Dreyzehenern beeden Fabric: wie auch Herrn Daniel Richshoffer, hochverdienten Fünffzehenern als Oberkirchenpflegern zu St: Wilhelm auff vorstehenden Fuss verfertigen zu lassen beliebet, und mit Herrn Andrea Silbermann kunsterfahrenen Orgelmacher verglichen worden, dass er solches Werck zwischen hier und Michaelis eintaussend sieben- 
hundert zwantzig und sieben auf seine Kosten neu verfertigen, nach St. Wilhelm auf den gewohnlichen Platz stellen und lieffern, und alles was zum Werck selbsten gehörig, in daurhaffter Kauffmanns guter Wahr machen solle, vor solche Arbeit hingegen versprechen obwohlerwehnte Herren Fabric und Oberkirehenpflegere ihme Herrn Silbermann zu entrichten und zu bezahlen die Summam von zweytaussend siebenhundert Gulden Strassburger Current den Gulden zu 15. Batzen gerechnet und daran zwischen hier und nechst: bevorstehenden Ostern eintaussend Gulden, oder wann es seyn kan eintaussend Thaler, das übrige aber zur Helffte auff Michaelis 1727. und die letzte Helffte in anno 1728. ohne Interesse zu entrichten und zu bezahlen. In dessen Urkund sich beede contrahirende Parthen sich eigenhändig unterschrieben. So geschehen in Strassburg den eilfften Novembris anno eintaussend siebenhundert zwantzig und sechs.

Johann Reinboldt Friderici

J. Dietrich 
Colmar, couvent d'Unterlinden.

Copie du marché du 28 novembre 1736, avec Jean André Silbermann. 
Heuth zu Endt gemeltem Dato ist zwischen Ihro Hochwürdten HH. Patre Phillippo Eugenio de Surmont hochmeritirten Provinciale der Provintz S.ta Rosa in Flandteren undt Vicario Generali der Congragation des Ordtens S.ti Dominici im Elsas eines: so dann $H$. Johann Andreas Silbermann Orgelmacher zu Strasburg andteren Theils; volgenter Accord getroffen wordten, alss namblich

verbindtet sich erwähnter H. Silbermann dem Gotteshaus zu Unterlinthen ord: S.ti Dominici zu Colmar ein Orgel zu machen nach dem Abriss von ihro Hochwürdten unterschrieben so ihme H. Silbermann wider zugestellet wordten so also versehen undt componirt seyn solle.

au grand jeu.

1. Prestant ..............4 pieds.

2. Bourdon ............... 8 .

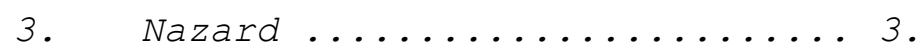

4. Flutte $\ldots \ldots \ldots \ldots \ldots \ldots \ldots$.

5. Doublette .............. 2 .

6. Tierce .............. $13 / 5$.

7. Cornet .............. 5 facht.

8. Fourn............... 3acht

9. Cromhorne separé

10. Echo ................ 5 facht, mit aparte Clavir et Windtlath

au pedal.

1. Bourdon ............... 8 pieds.

2. Trompette ............. 8 .

deux Tremblants

Die Clavir sollen anfangen von C C\# D Ds etc. bis mit C''' 4 
völlige Octaven.

Das Pedal Clavir von C C\# D Ds etc. bis mit C' 2 völlige Octaven. 2 grosse odter 3 kleine Blasbälge a proportion des Werckhes.

Also undt dergestalten dass das Werckh, inn Holtz werckh, zinnenen Pfeiffen, Bilthauerarbeith undt allem zugehörigen ohne einige Ausnahm sie mag Nahmen haben wie sie immer wolle biss Weynachten 1737. inn der Kirchen an dem Orth so ihme wirdt angewissen werdten wohl conditionirt undt verfertiget stehe, zu welchem ermelter $H$. Silbermann sich verbundten auss dessen Kosten alles Zinn, Möss, Eisen, Holtz undt Zugehöhrte ohne einige Reserve zu lüfferen undt fourniren wie auch die Schlosser, Schreiner, undt Bildthauer Arbeith auff eigenem Kosten machen zu lassen, vor welches er auch die gebührente Währschafft wie gebräuchige zu tragen versprochen.

Dahingegen verspricht ermelte ihro Hochw.- nahmens ermelten Gotteshausses, vor dieses Werckh also nach Aussag Experten auffgerichtet sambt zugehörigen Materialien unndt Arbeith berührtem $H$. Silbermann eine Summ von fünffzehen hundtert Guldten Strasburger Währung paar zu bezahlen, thut sich auch obligiren die zugehörte dess Werckhss auff dess Gotteshaus Kosten zu Strasburg abhohlen zu lassen, wie auch ihme H. Silbermann wann er selbes auffrichten wirdt sambt zwey Gesellen zu logiren undt verköstigen ohne einiges Entgeldt so alles zugangen, getreulich undt ohne Gefähdte wo nicht Kosten undt Schadten, inn Beyseyn HH. Jacob Kappler Rathss Verwandten alhier undt Frantz Ernest Cons Schaffner des Stüffts $S .{ }^{t}$ Margarethan alda so sich nebst den Contrahirenten Parten undterschrieben 
Strasburg den 28. 9 bris 1736.

Pr Phil Eug De Surmont

Pro alis, Vic. G ralis

Johann Andreas

Silbermann, Orgelmacher.

J. J. Cappler

Cons

ABR. G 4334 (11) 
Barr, église (simultaneum).

Notices concernant les travaux de Jean André Silbermann en 1737 et 1739 . 
Am 19 Juni wurde nachdem der Verding wegen einem Orgelwerck mit $H$. Johann Andreas Silbermann gemach wurden und derselbe indessen ein Positiv in die Kirch stellte wurde H. Joh. Dürrbach Arg: Stud Theol zu einem Organisten von Pastore, Diacono und schriftlich in der Amtschreiberey aufgesetzten Accord ihm das Recht gegeben privatim die Jugend zu informieren.

Dessen Besoldung bestehet in hundert und zwantzig Gulden Geld und zwey Klafter Holz.

Dabey soll demselben erlaubt seyn von einer Leich oder Hochzeit 1 fl. $2 \mathrm{~s}$. von einer Kindtaufe aber $8 \mathrm{~s}$. jedesmahl von dem Besteller abzufordern, hievon aber allezit dem orgel-zieher $2 \mathrm{~s}$. zu bezahlen gehalten seyn.

$\underline{1739}$

31 Augusti Dom: XIII Trinit. wurde das neue Orgelwerck so $H$. Johann Andreas Silbermann von Strassburg verfertigt, eingeweyet und zum erstenmal gespielet. Dafür gemelten H. Silbermann tausend dreyhundert Gulden bezahlt worden. Davon die evangel. Gemeinde A C tausend Gulden erlegt, von seiten der Röm. cath. aber dreyhundert dazu gegeben worden. Die evang. Gemeinde A C brachte durch eine freywillige Steuer 581 Gulden 1 s. 60 zusammen, der Rest aber wurde aus dem Almosen darauf gelegt, wie auch aus dem Orgelsäckel. Zu dieser Orgel wurden 2 Blass Balck Zieher bestellt deren ein jeder quartaliter einen Gulden empfängt. Wann aber die Orgel extra bestellt wird, so haben sie von dem Besteller zwei Schilling zu fordern.

Texte du pasteur Johannes Herrman, dans Verzeichnus allerhand Sachen, so sich in der Evangelischen Gemeinde und Kirchen zu Barr begeben und zugetragen, angefangen von Joh. Jacob Lauten, Pastore loci ordinario in Anno MCCXVIII d. 2. Mensis Novembris.

Archives paroissiales de Barr. 
Strasbourg, Saint-Thomas.

- Premier projet par Jean André Silbermann, en 1734.

- Deuxième projet par Jean André Silbermann, don daté.

- Troisième projet par Jean André Silbermann, le 7 mai 1736.

- Marché du 4 mai 1737 avec Jean André Silbermann.

- Marché du 12 janvier 1739 concernant le buffet d'orgue, avec Jean André Silbermann.

- Contrat d'entretien du 8 février 1744 avec Jean André Silbermann. 
Disposition eines neuen Orgelwercks, wan solches in die Kirch zu St: Thoma solte gemacht werden.

Im Oberwerck, oder Manual müssten sich nachfolgente Register nothwendig befinden.

1. Montre von feinem zinn im Schein ...........8 Schu Thon

2. Bourdon, die zwey ersten Octaven von Holtz, die übrigen von Medal 8 Schu gedeckt ..........16 -

3. Bourdon, die erste Octave von Holtz, die übrigen von Medall 4 Schu gedeckt .......... 8 -

4. Prestant von $\operatorname{Zinn} \ldots \ldots \ldots \ldots \ldots \ldots \ldots \ldots \ldots \ldots \ldots \ldots$ -

5. Quinte von Zinn, die Füss Medal ..............

6. Doublette von Zinn, die Füss Medal ............ -

7. Tierce von Medal ................................

8. Cornet von Medal, 5 facht.

9. Fourniture von Zinn, die Füss Medall, 4 facht...11/2 -

10. Cymbale von Zinn, die Füss Medall, 3 facht.

11. Trompette, die Corpora von Zinn, die Füss Medall 8 -

Im Ruckwerck, oder Positife.

1. Prestant von Zinn, im Schein ............ -

2. Bourdon, die erste Octave von Holtz, die übrigen Medall 4 Schu gedeckt................... 8 -

3. Nazard von Medall .......................

4. Doublette von Zinn, die Füss von Medal........2 -

5. Tierce von Medal ........................ 3/5 -

6. Fourniture von Zinn, die Füss Medall 3 facht.... 1 -

Im Pedal.

1. Supbass, von Holtz offen ................

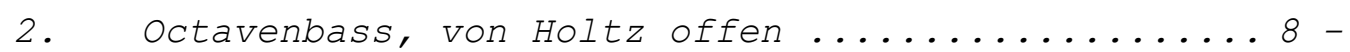

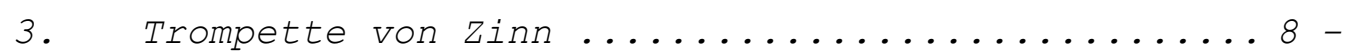

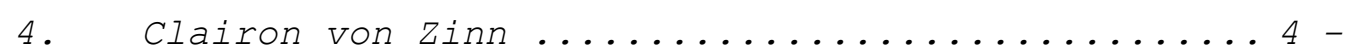

Also bekäme dieses Werck 21 Register welche nothwendig vor den Anfang müssen gemacht werden.

Dennoch aber soll das gantze Werck sambt der innern Regierung weitläuffiger und grösser gemacht werden, als zu gemelten Registern nötig wäre. Die Windlathen sollen auch alle also eingericht und gemacht werden, dass man nach Belieben folgente 5 Re- 
gister, welche theils zur Zierde und mehreren Veränderung willen, meistens aber zur Verstärckung des Wercks können hinein gesetzet werden.

Als erstlichen im Manual.

1. Clairon von Zinn ............4 Schu Thon

2. Voix humaine von Zinn .......... 8 -

Im Ruckwerck.

3. Flutte von Medal .............. 4 -

4. Cromhorne von Zinn ........... 8 -

Im Pedal.

5. Bompartte ............... 16 -

Das gantze Werck wird in den sogenanten Operathon gestimbt.

Item zwey Tremulanten, den einen langsam, und den andern geschwind durch das gantze Werck.

Die zwey Manual=Clavire von Ebenholtz, und Bein, so anfangen mit C. cis. D. dis etc. biss mit C''', vier föllige Cctaven.

Das Pedal=Clavir von Contra C. Cis. D. dis. etc. biss mit C', zwey föllige Octaven.

Vier grosse Blassbalg approportion des Wercks.

Das grosse Orgelcorpus, sambt dem Rückwerck sauber gearbeitet von Fichenholtz, der Kasten aber worin das Pedal stehet hinter dem grossen Orgelcorpus von Dannenholtz, gleichwohl alles von sauberer eingefaster Arbeit.

Was die Façon dess Werckes betrifft, und wie solches mit Ornamenten vom Bildhauer geziehret wird, kan auf Befehl, und nach dem Platz worauf das Werck stehen soll, ein Abriss gemacht werden. Auch wird das Corpus mit einem saubern Firnis überstrichen.

Vor ein solches Werck zu stellen, alle Materialien dazu zu fourniren, fortere nach genauer Überlegung dreytausend dreyhundert Gulden, sage 3000 f, vier und zwantzig Viertel Frucht, und die alte Orgel.

Zeit wärenter Stimmung der Orgel ein Bossler oder Handlanger, die Bälge zu tretten. 
In diesem Accord soll nicht begriffen seyn, was des Zimmermans, Maurers, und Schlossers Arbeit anbetrifft. Solches soll von einem hochlöblichen Capitel aparte bezahlt werden.

Johann Andreas Silbermann

(Prod. in Capit. Ord.

d. 18. Xbr. 1734.) 
Specification derer Register, vor eine neue Orgel nach St. Thoma.

Im Oberwerck.

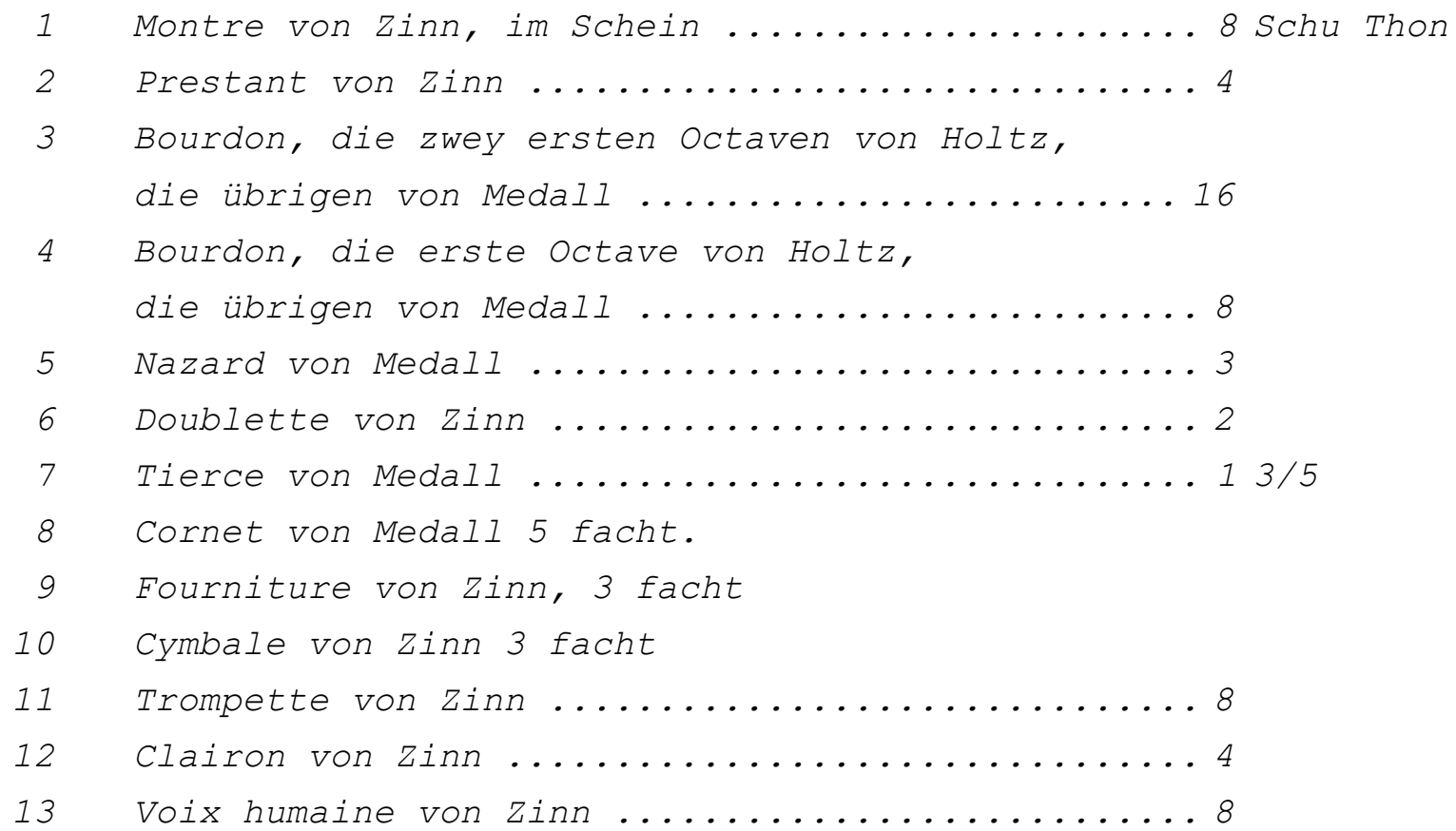

Im Ruckwerck

$1 \quad$ Prestant von Zinn, im Schein .............4

2 Bourdon, die erste Octave von Holtz, die übrigen von Medall ................. 8

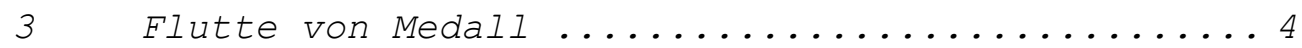

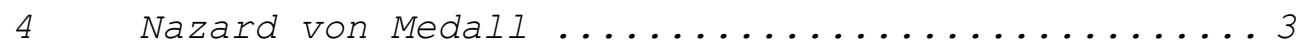

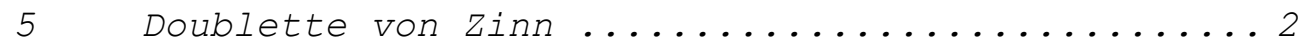

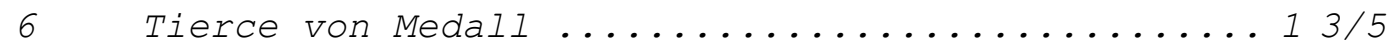

7 Fourniture von Zinn 3 facht

8 Cromhorne von $\operatorname{zinn} \ldots \ldots \ldots \ldots \ldots \ldots$

Im Pedal

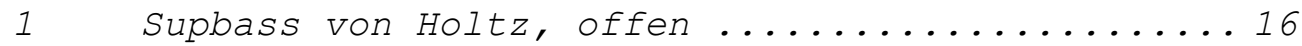

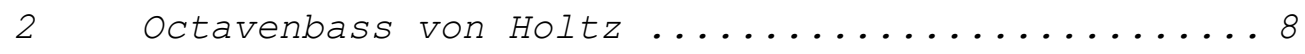

3 Quinte von $\operatorname{zinn} \ldots \ldots \ldots \ldots \ldots \ldots \ldots \ldots \ldots \ldots \ldots \ldots \ldots \ldots$

4 Frestant von $\operatorname{Zinn} \ldots \ldots \ldots \ldots \ldots \ldots \ldots \ldots$

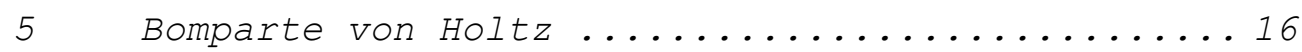

6 Trompette von $\operatorname{zinn} \ldots \ldots \ldots \ldots \ldots \ldots \ldots \ldots$

$7 \quad$ Clairon von $\operatorname{zinn} \ldots \ldots \ldots \ldots \ldots \ldots \ldots \ldots \ldots \ldots \ldots$ 
Noch einen Echo mit apartem Clavir und Windlad, von 125 Pfeiffen. Zwey Tremulanten, den einen langsam, und den andern geschwind. 4 Blassbälg aproportion des Wercks.

Drey Manual Clavire übereinander, von Ebenholtz und Bein, welche anfangen von C. C\#. D. D\# etc. biss mit C''', 4 völlige Octaven. Dass Pedal Clavir von Contra C. C\#. D. D\# etc. biss mit c', zwey octaven.

Das gantze Werck soll in den Operathon gestimbt werden.

Das grosse Orgelcorpus sambt den Ornamenten von Eichenholtz. Der Pedalkasten hinter dem Werck von Danenholtz, gleichwohl von eingefaster Arbeit.

J A Silbermann 
Specification

derjenigen Register, so in die Orgel nach diessem Abriss sollen gemacht werden.

Im Oberwerck oder Manual.

1. Montre von feinem zinn im Schein pollirt.....8. Schuh Thon.

2. Bourdon, die zwey ersten Octaven von Holtz, die übrigen von Medall 8. Schuh gedeckt ......16.

3. Bourdon, die erste Octave von Holtz, die übrigen 3. von Medall 4. Schuh gedeckt.......8.

4. Prestant von $\operatorname{Zinn} \ldots \ldots \ldots \ldots \ldots \ldots \ldots \ldots$.

5. Quinte, die Corpora von Zinn, die Füss von

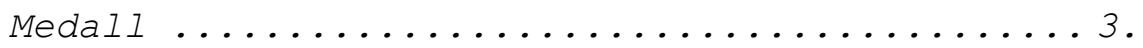

6. Doublette, die Corpora von Zinn, die Füss

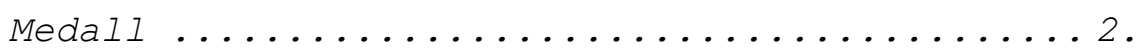

7. Tierce von Medal ................... 3/5.

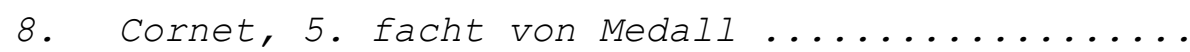

9. Fourniture 4. facht, die Corpora von Zinn,

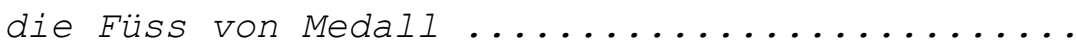

10. Cymbale 3. facht, die Corpora von Zinn, die Füss Medall ......................

11. Trompette, die Corpora von Zinn, die Füss

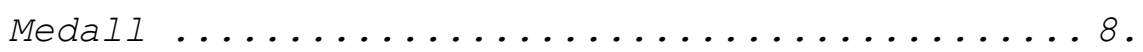

12. Clairon, die Corpora von Zinn, die Füss Medall ...........................

13. Voix humaine, die Corpora Zinn, die Füss

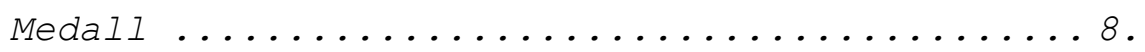
Die Trompette und Clairon bekommt edes Register zwey Züge, einen vor den Discant, den andrern vor den Bass, dass man also mehrere Veränderung machen kan.

Im Ruckwerck oder Positive.

1. Prestant von Zinn, im Schein pollirt.......4. Schuh Thon.

2. Bourdon, die erste Octave von Holtz, die Übrigen

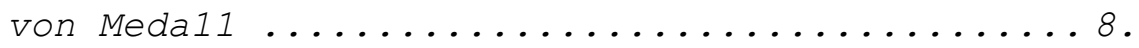

3. Flutte von Medall, 2. Schuh gedeckt.........4.

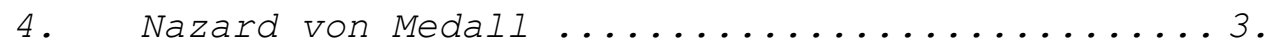

5. Doublette, die Corpora von Zinn, die Füss Medall .....................

6. Tierce von Medall .................... 3/5. 
7. Fourniture 3. facht, die obern Theil von Zinn,

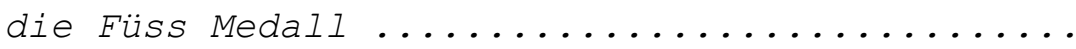

8. Cromhorne, von Zinn, die Füss Medal ........8. Im Pedal.

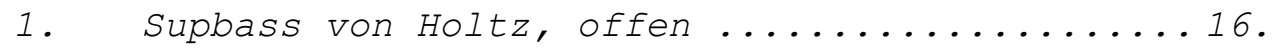

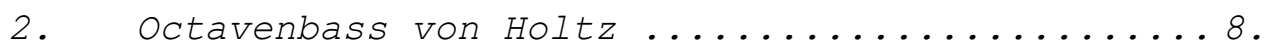

3. Quinte die Corpora von Zinn, die Füss Medall ..6.

4. Prestant von Zinn, die Füss Medall .........4.

5. Bomparte, die Corpora von Holtz,

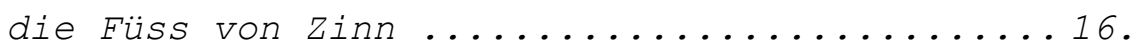

6. Trompette, die Corpora von Zinn, die Füss

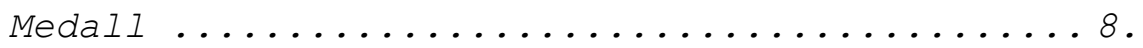

7. Clairon, die Corpora von Zinn, die Füss

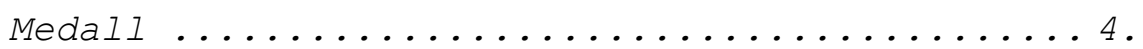

Item ein Echo mit besonderem Clavir von 125. Pfeiffen.

Also bekäme dieses Werck 29. Register, welche in sich begreiffen 1748. Pfeiffen.

Noch 2. Tremulanten, den einen langsam, und den andern geschwind, durch das gantze Werck.

Drey Manual=Clavire übereinander von Ebenholtz und Bein, welche anfangen von C. C\# D. D\# etc. biss mit C''', also 4 völlige octaven.

Das Pedal=Clavir von Contra C. C\# D. D\# etc. biss mit $C^{\prime}$, zwey völlige Octaven.

Vier grosse Blassbälge, jeden mit einer Falte.

Das gantze Werck wird in den Opera Thon gestimmt.

Das grosse Orgel=Corpus, sambt dem Rückwerck sauber gearbeitet von Eychenholtz, die Bilder, Blumen-Krüge und die andern Ornamenten alles nach dem Abriss.

Der Pedal=Kasten hinter der Orgel von Dannenholtz, gleichwohl alles von eingefaster Arbeith. Auch soll das Orgel=Corpus mit einem sauberen Firnis überstrichen werden. 
Vor ein solches Werck in meinem Kosten zu stellen, alle Materialien, alss Zinn, Bley, Messing, Leder und Holtz darzu zu fourniren, fordere auffs genaueste 4500. f. sage viertaussend fünffhundert Gulden, 20. Fiertel Frucht, und die alte Orgel.

Zeitwehrender Stimmung der Orgel einen Bossler oder Taglöhner zum Bälge tretten; was des Zimmermanns, Maurers und Schlossers Arbeith anbetrifft, soll von einem hochlöblichen capitul à parte bezalt werden.

Strassburg den 7.ten May 1736.

Johann Andreas Silbermann Orgelmacher. 
Kundt, zu wissen und offenbahr seye hiemit männiglichen, denen es zu wissen nöthig, dass ahne heut zu End gesetztem Dato zwischen E: wohlehrwürdigen Capitulo s.ti Thomae alhier ahne einem, sodann Herrn Johann Andreae Silbermann hiessigem Burger und Orgelmacher ahne andern Theil wegen der in die S.ti Thomas Kirch zu verfertigender neuen Orgel hienachstehender Accord auffrecht und redlichen getroffen und geschlossen worden: Es verspricht nemblichen erstgedachter Herr Silbermann von dato in dreyen Jahren ein neues Orgel Werck in Conformitaet des darüber gemachten und übergebenen Risses mit neunundzwantzig Registern, nemblichen im Oberwerck:

1. Montre von Zinn, im Schein .............. Schu Thon.

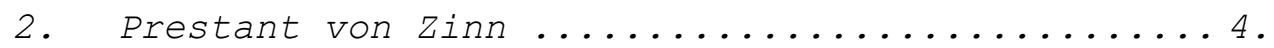

3. Bourdon, die zwey ersten Octaven von Holtz, die übrigen von Metall ................. 16. Schu Thon.

4. Bourdon, die erste Octave von Holtz, die übrigen von Metall ...................

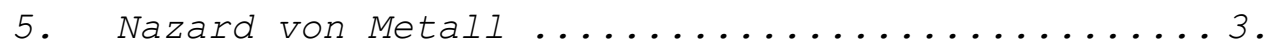

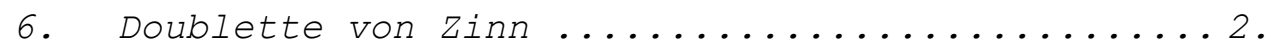

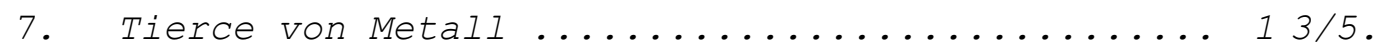

8. Cornet von Metall 5. facht.

9. Fourniture von Metall 3. facht.

10. Cymbale von Zinn 3. facht.

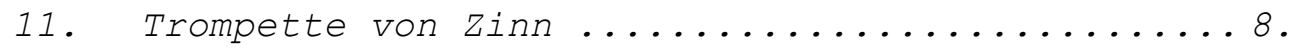

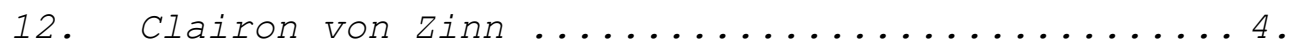

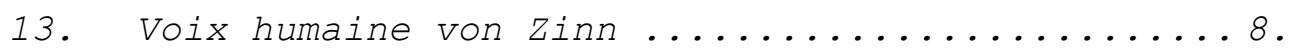

Im Ruckwerck.

1. Prestant von Zinn, im Schein .............

2. Bourdon, die erste Octave von Holtz, die übrigen von Metall ................

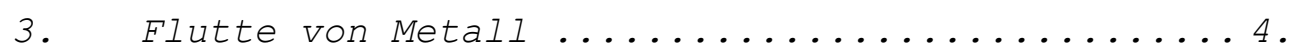

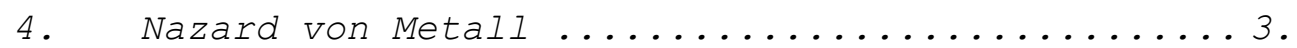

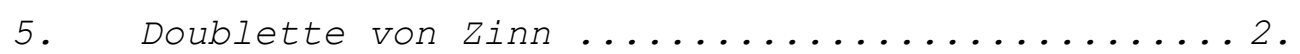

6. Tierce von Metall .................... 3/5.

7. Fourniture von Zinn 3. facht.

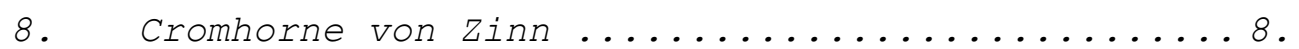


Im Pedal.

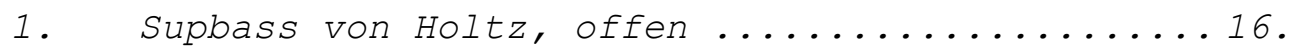

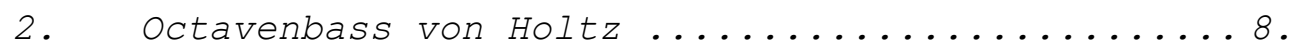

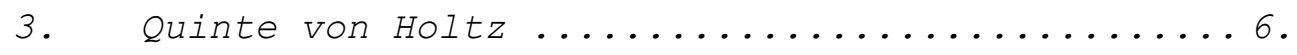

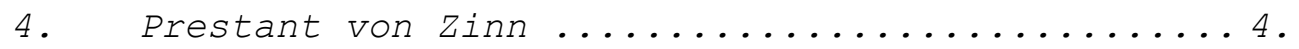

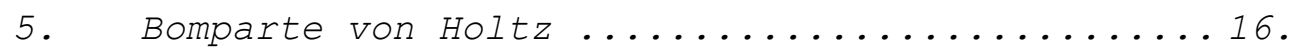

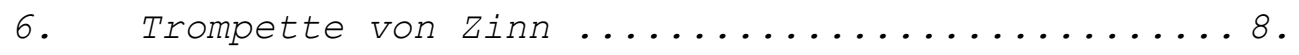

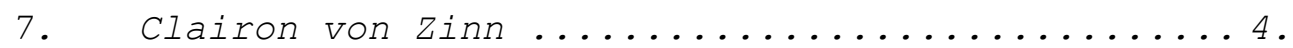

Noch einen Echo mit apartem Clavir und Windlad, von 125. Pfeiffen.

Zwey Tremulanten, den einen langsam und den andern geschwind.

Vier Blassbälg aproportion des Wercks.

Drey Manual Clavire übereinander, von Ebenholtz und Bein, welche anfangen von C. C\#. B. B\#. etc. biss mit C'''. 4. völlige Octaven.

Das Pedal Clavir von contra C. C\#. B. B\#. etc. biss mit C'. zwey Octaven.

Das gantze Werck soll in den Operathon gestimbt werden.

Das grosse Orgelcorpus sambt den Ornamenten von Eichenholtz. Der Pedalkasten hinter dem Werck von Dannenholtz, gleichwohl von eingefaster Arbeith.

Auff denjenigen neuen Lettner, welcher ihm in erwehnter S.ti Thomas Kirch angewiessen werden wird, gantz neu auffurichten und mit allen was hie vorgeschriebenermassen specifice auffgezeichnet steht, in völligen stand zu stellen. Inzwischen aber und ohngefehr in einem Jahr, solche mit einem Positiv, so zu dem Choral wird gebraucht werden können, ohne Entgeld zu versehen. Dahingegen verspricht anfangs gemeltes wohlehrwürdiges Capitulum s.ti Thomae ihme Herrn Silbermann sowohl vor seine als auch vor alle zu dem Werck selbsten und dessen völlige Auffstellung benöthigte Schmid, Schlosser, Zimmerleuth und Schreiner Arbeith wie nicht weniger vor die Handtlanger und Blassbalgtretter, im geringsten nichts ausgenommen, in baarem Geld die Summ von dreytaussendfünffhundert Gulden, hiessig Courrent und in Früchten sechs Fiertel Weitzen und sechs Fiertel Roggen in drey Terminen nemblich bei Anfang der Arbeith zu dem Werck 1166. fl. 6. s. 8. d. und in Früchten 2. Frtl. Weitzen und 2. Frtl. Roggen, sodann wann das werck halb 
fertig eine gleiche Summ von 1166. fl. 6. s. 8. d. sambt 2. Frtl. Weitzen und 2. Frtl. Roggen, und wann solches geendigt und an seinem Platz stehen wird, den Rest der 1166. fl. 6. s. 8. d. und 2. Frtl. Weitzen und 2. Frtl. Roggen nebst Überlassung der alten Orgel, welche er in seinen Kosten abzubrechen und in seine Behaussung führen zu lassen schuldig seyn solle, mit dem Anhang baar zu bezahlen und zu entrichten, dass er die Orgel zwey Jahrlang von dem Tag an, da sie an ihrem Ort in der Kirchen stehen wird, gerechnet, ohne einige Belohnung zu stimmen, ahnebey vor das gantze Werck genugsame Caution zu leisten verbunden seyn solle.

Welchen Accord beede Theil mit wohlbedächtlicher Verzich= und Begebung aller darwiederdienender rechtlicher Exceptionen und Aussflüchten, sie mögen Nahmen haben wie sie immer wollen, steth, vest und unverbrüchlich zu halten, einander nicht nur mit Mund und Hand versprochen, sondern auch in Urkundt und zu mehrerer Versicherung dessen eigenhändig unterschrieben und ist jedem Theil ein gleichlautend Exemplar davon zugestellet worden.

Alles getreulich sonder Gefährten, Actum Strassburg den 27.ten Aprilis Anno: 1737.

Joh. Georg Scherz d.

Cap. Thom. Praepositus Johann Heinrich Lederlin, P.P. Capituli Thomani Decanus. Johann Leonhard Fröreissen D. h.t. Capituli Actuarius. Johann Andreas Silbermann.

Hiernechst wurde auch noch wohlbedächtlich abgeredet, und verglichen, dass die Zahlung in obbestimmbten Terminen, jedesmahl auf den Fuss, wie das Gelst heuthzutag stehet, nehmlichen das drey Gulden stück zu drey Gulden gerechnet, bezahlet werden solle, wann es auch gleich zur zahlungszeit mehr oder weniger allhier gelten würde.

Actu Strassburg den 4.ten May 1737. Johann Leonhard Fröreissen D.

Joh. Georg Scherz D. Cap. Thome. Praepositus. Johann Heinrich Lederlin, P.P. Capituli Thomani Decanus. Johann Andreas Silbermann. 
Zu wissen seye, dass heuth dato zwischen ST. Herrn Dr. und Prof. Lorentz, S.T. Herrn Diacono Sen. Schweigheuser, als hochverordneten Herren Deputatis eines wohlehrwürdigen Capituli Sti. Thomae allhier in Strassburg ahne einem, sodann Herrn Johann Andrea Silbermann von hier am andren Theyl wegen denen zu dem neuen orgelwerck nöthigen Ornamentis nachstehender Accord auffrecht und redlich getroffen und geschlossen worden. Es obligirt sich nehmlichen gedachter Herr Silbermann ermeldtes Orgelwerck nach dem ersteren vorgelegten Riss, so mit Lit. A. bezeichnet, und von denen contrahirenden Parten unterschrieben worden, zu verfertigen, ahnebey die darinnen gezeichnete ornamenta, nichts davon aussgenommen, daran zu schaffen. Dagegen versprechen obgedachte Herren Deputati Nne Vener: Capituli, salva tamen ratificatione, ihme Herrn Silbermann, über die in dem erstern Accord stipulirte Summamm, annoch vierhundert Gulden in denen gesetzten Terminen, baar zu bezahlen. In Urkundt dessen sich beederseits contrahirende Partheyen, nebst mir dess ernandten Ven: Capituli Schaffner, hie eigenhändig unterschrieben.

Actu. Strassburg den 12ten Jan. 1739.

Johann Michael Lorentz. d. M. Joh: Georg Schweigheüser Diac. Sen:

Johann Andreas Silbermann Orgelmacher

Ben: Henr: Marbach Schaffner. 
Zu wissen seye hiemit jedermänniglichen, insonderheit aber denen es zu wissen nöthig, dass ane heut zu End gesetztem Dato zwischen einem wohlehrwürdigen Capitulo des löblichen Stiffts Sancti Thomae alhier zu Strasburg ane einem, sodann Herrn Johann Andreas Silbermann, dem Orgelmacher und Burgern dahier am andern Theil, wegen der in der Kirch zu St: Thomä befindlichen und von ermeltem Stifft zu erhalten habenden Orgel, auch deren nöthigen Abstäub= Stimm= und Säuberung, nachfolgender Accord wissend= und wohlbedächtlichen getroffen und aufgerichtet worden ist:

Nemlichen es obligiret sich obernanter Herr Silbermann, die in der Kirch zu St: Thomä stehende Orgel von dato und inskünfftige, solang dieser Accord dauren wird, so offt es die Nothdurfft erfordert, durch sich und die seinigen ordentlich abstäuben, die an solcher befindliche Pfeiffen und Blassbälg säubern und stimmen zu lassen, mithien das gantze Werck bestmöglichst zu besorgen und in obacht zu nehmen, dass niemahlen darüber einige Klag entstehen und geführet, hingegen alle zufriedenheit bezeugt werden solle.

Dahingegen und vor solche Beobachtung, auch hiebey verrichtender Sorgfalt und Arbeith, erbiethet sich ein wohlehrwürdiges Capitul obbesagten stiffts ihme Herrn Silbermann alle Jahr auf Weyhenachten undt in anno eintaussendsiebenhundertvierzigundvier zum ersten Mahl dreyssig Gulden, Strasburger Current zu reichen, auch wann an denen Pfeiffen oder anderm Orgelwerck, so Haubtsachen genannt werden, etwas gebrochen, dass solche ohnbrauchbar würden, und neue an der abgegangenen Stück Platz werden müssten, dieselbe aus des Stiffts Mittlen herbey= und angeschafft, einfolglich des Herrn Silbermanns daran verrichtende Arbeit à parté, ausser diessem aber nichts weiters desswegen demselben bezahlt werden, sondern er alles andere, es habe Nahmen wie es immer wolle, für obige ihme zugesagte dreyssig Gulden aus dem seinigen und ohne den geringsten von dem Stifft zu thun habenden Beytrag, zu machen, und das gantze Werck wieder, so gut möglich zu repariren, schuldig undt gehalten seyn, im übrigen aber solle diesser Accord, solang es beederseits Parthen beliebig seyn wird, dauren. 
Urkundlich dessen ist diesser Accord hierüber gefertigt von bederseits Parthen eigenhändig unterschrieben, das Original desselben bey dem Stifft aufbehalten und ihme Herrn Silbermann eine Copiam davon zuzustellen abgeredet worden.

So geschehen in Strasburg den achten Februarii Anno eintaussendsiebenhundertviertzigundvier.

Joh: Friedrich Böckler, Dr. Johann Andreas Silbermann h. t. Actuarius.

AMS . AST $\cdot \mathrm{N}^{\circ} 30$. 
Strasbourg, couvent de la Toussaint.

- Copie du marché avec Jean Andre Silbermann, non daté (1743) 
Demnach vor gut und nöthig erachtet worden, die Orgel in der Stiffts-Kirche zu Allerheiligen in Strassburg zu translociren, indeme die Feuchtigkeit der Mauer, alss woran sie würcklich stehet, Ursach ist, dass das Pfeiffenwerck durch den Salpeter, wovon es allbereit ziemlich angestecket ist, gäntzlich ruiniret würde, so ist nöthig, dass solche in die Lufft und zwar mitten in die Kirch gerad gegen dem hohen Altar hinüber, als wo eigentlich der rechte Platz ist, wohin die Orglen gehören, muss gesetzet werden.

Dahero ist erstlichen erachtet worden, dass die Orgel gleich vornen an das Brust-Geländer angesetzt werden solle, also zwar, dass solche von hintenwärts muss gespielet werden, dazu soll ein neu Corpus von sauberem eychenem Holtz neben denen dazugehörigen Ornamenten gemacht werden, nach Art und Grösse des Massstabs, wie es der unterschriebene Riss weiset, und dann vornen hinein soll ein Schein von zinnern Pfeifen gemachet werden.

Zweytens. Ein Echo 5. fach mit 125 Pfeifen neben einem Clavier und Windlade, welcher in diese Orgel sehr nöthig.

Drittens soll auch ein Pedal 8. Fuss, von einer völligen Octave darzu gesetzet werden.

Viertens. In das neue Corpus die Registratur völlig einzurichten, wie auch die übrige Einrichtung der Leisten und Backenwerck, Registerwellen, Canael und Blassbälg, und das alte Pfeiffenwerck wieder in guten Standt zu stellen und zu stimmen und an gehörigem Orth in die neue Orgel einzusetzen, das alte Getäffel, womit die 
alte Orgel eingeschlossen ist, um die Einschliessung des neuen Pedals einzuschliessen, offerire alles vorbeschriebene nebst Fornirung aller darzu gehörigen Materialien, als das gedachtes hochlöbliches Stifft Allerheiligen ferner keine Unkösten hat, vor vierhundert Gulden, sage 400. fl. in Stand zu stellen.

Hier ist zu mercken, dass die zinnerne Pfeiffen im Schein zu nichts anders also nur um der Zierath willen in das neue Corpus gesetzet würden, und also stumm wären, dahero habe ich unterschriebener zum Besten der Orgel und des Stifts Allerheiligen Nutzens willen auff mich genommen die alte Orgel, welche bestehet in 6. Registern, alss

1. Bourdon ........................ 8ussthon

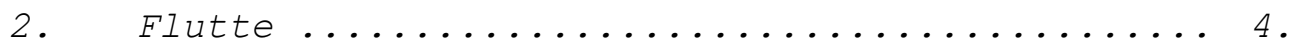

3. Doublette .....................

4. Fourniture 2. facht die gröste Pfeiff...... 1/2. -

5. Cornet 4. facht

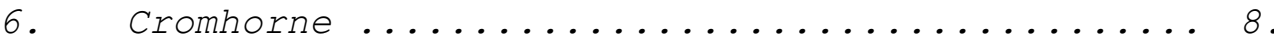
völlig in den nemlichen stand, wie sie sich dato befindet an mich zu nehmen, und dem Stifft Allerheiligen eine gantz neue Orgel zu machen in folgenden Registern bestehend

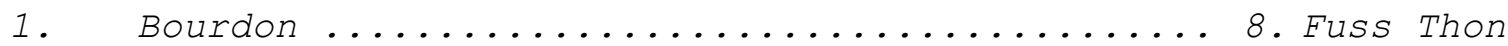

2. Flutte .................... -

3. Doublette ..................... -

4. Fourniture 2. fach die gröste Pfeiff........1/2. -

5. Cornet 4. facht.

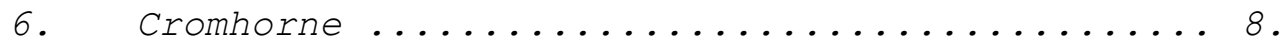
mit Zusetzung /: neben einem Echo und Pedal, wie Eingangs dieses 
schon gemeldet :/ annoch 2. andere Register als Nazard 3. Fuss und Tierce $13 / 5$. Fuss.

Dazu soll nun ein gantz neue Manual-Windlade gemacht werden von 9. Registern, worauf dann der Schein der Orgel zu einem Register nahmentlich Prestant 4. Schue gebraucht werden solle.

Ferner sollen auch 2. neue Blassbälcke, welche grösser weder die alten seynd, gemacht werden, davon jeder 1. Schue länger und 7 1/2. Zoll breyter seyn sollen dann die alten.

Weiters soll auch ein Tremblent doux hineingemacht werden.

Vor diese gantze neue Orgel mit allen Zugehörungen in völligen Stand zu setzen /: wie hierunten angemercket worden :/ dergestalt, dass gedachtes Stifft Allerheiligen weiter keine Unkösten damit zu leiden hat, ist mir Johann Andreas Silbermann dem Burger und Orgelmacher allhier durch Herrn Hermann Weinemer mehrgedachten Stiffts Allerheiligen Praebendarium accordiert und versprochen worden überhaubt eine Summa von fünffhundert und sechzig Gulden sage 560. fl. in heutigem Valor zu zahlen, nebst Überlassung der völligen alten Orgel. Betreffend die neue Orgel, so verspreche ich hiemit solche von Dato an innerhalb 5. Monathen fertig und in gutem Stand zu lieffern.

Urkund dessen auf beyden Seithen alles stets und vest zu halten, haben wir uns beyde eigenhändig zu mehrerer Versicherung in zwey gleichlauthenden Accorden unterschrieben. 
Guebwiller, église des Dominicains.

- Marché du 27 juin 1744 avec Jean André et Jean Daniel Silbermann.

- Quittances par Jean André et Jean Daniel Silbermann. 
Zu wissen seye hiemit vnd in Krafft diesses dass zwischen dem Ehrwürdigen P. Joannes Evangelista Eggs Prior des Convents Prediger Ordens zu Gebwyler vnd denen RR. PP. à Consilijs gedachten Convents ahn einen, so dan Herren Johan Andreas, vnd Daniel Silbermann kunsterfahrenen Orgelmacheren zu Strassburg andern Theils, folgender Accord /: zu Herstellung eines in Opera Thon neüwen Orgelwerckhs in die so genante Prediger Kirch der Statt Gebwyler, bestehend in einem Hauptwerckh vnd ruckh positive mit zwey Clavier von Eben Holtz gemacht :/ bedächtlichen mit nachgesetzten vnd aussfierlichen conditionen getroffen vnd geschlossen worden.

Erstlichen versprechen Sie Herren Silbermänner die Kästen zu gedachtem Hauptwerckh vnd Ruckh Positive von Eichenem Holtz vnd zierlicher Bildschnitzler Arbeit nach gegebenem Riss zu verfertigen.

So dan solle dass Hauptwerckh folgende Register haben.

1. Montre von feinem zinn poliert ...........8 Schuthon

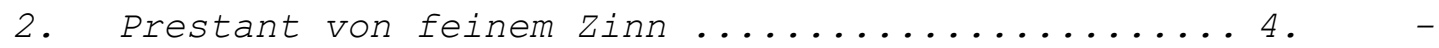

3. Bourdon gedeckht die 2 erste Octaven von Holtz die übrige von Metall .................. 16 .

4. Bourdon die erste Octave von Holtz, die übrige von Metall ..................

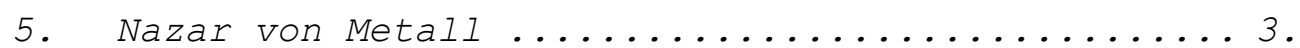

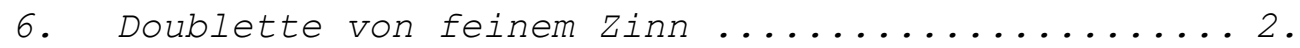

7. Tierce von Metall .................... 3/5.-

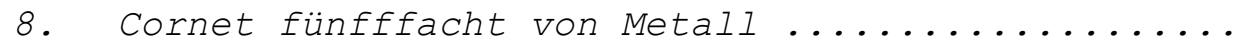

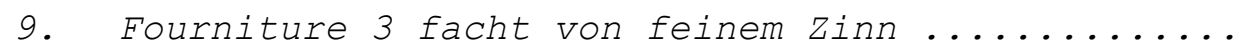

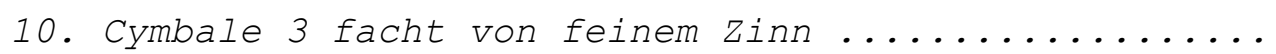

Positive.

1. Prestant im Schein .................. Schu -

2. Bourdon die erste Octave von Holtz, die übrige von Metall ................ -

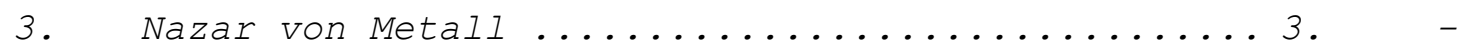

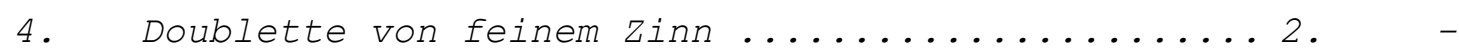

5. Tierce von Metall .................. 3/5.-

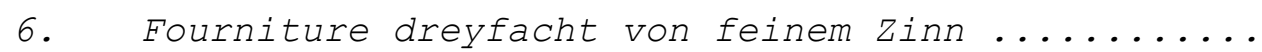


Pedal.

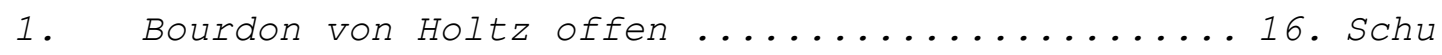

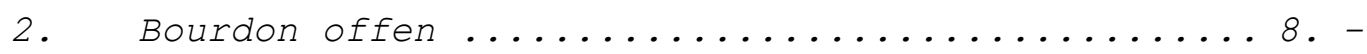

Dass Pedal Clavier soll anfangen von C. biss g. zweiter Octave, also 20 Claves.

Ferners 2 Tremulanten ein geschwinden, vnd ein langsammen.

3 Blassbälckh nach Proportion der Orgel oder des Werckhs.

Welches vorstehende Orgel = Werckh Sie Herren Silbermänner auff Ihrige Kösten nebst ein jähriger Wehrschafft auff Joannis Baptista - 1745. in vorbemeltes Gottes = Hause Prediger Ordens zu Gebwyler auff dero Lettner zu stellen sich verobligieren.

Da hingegen verspricht genanter P. Prior vnd PP. à Consilijs /: mit Gutheissung des Hochwürdigen Patris Pÿ Schmid General Prediger Vicarŷ vnd Commissarij Generalis der Clöstern Prediger Ordens im Elsass :/ Im Nammen des Convents Ihnen Herren Silbermänneren Vier Tausent zwey Hundert Livres tournois, in Silber oder Golt, oder zwey Tausent ein hundert Gulden Strassburger Wehrung, die Lovisd'or à zwelff Gulden in folgendem Termin zu bezahlen. Nemblichen so gleich bey Anfang darauff zu geben Sechs Hundert Gulden, vnd nach Verfertigung des Werckhs den Überrest in parem Gelt zu bezahlen.

NB. ESS verspricht auch dass Convent die gemachte Orgel /: welche von Herren Silbermännern in ihrem Kosten von Strassburg auff Colmar auff den Latthoff soll geliffert werden :/ in seinem Kosten allhier zu fiehren. Ahn bey verspricht auch dass Convent bey Stellung vnd Verfertigung des Orgel = Werckhs ihnen Herren Silbermännern vnd ihren Gesellen die nöthige Kost zu geben. Zur Vrkhund dessen seyn zwey gleich laudente Contracten verfertiget, vnd von denen Contrahenten /: mit Aufftruckhung des gewöhnlichen Convents Sigill :/ eigenhändig vnterschriben worden. 
Gegeben im Prediger Closter zu Gebwyler den 27 ten Juny 1744.

F: Joannes Evangelista Eggs p. T. prior mppr.

F: Bruno Gerger Magtr Thliae mppria

$F$ : Jacobus Nodler $p: t$ : Supprior mppria

Fr: Dominicus Schandalat p. T. Procurator mppria

Fr: Jordanus Hunold - - - - - - - - mppria

Fr: Augustinus Waeckerle mppria.

Authoritate offidii approbire, ratificir undt confirmire obgesetzten Contract den Tag undt Jahr wie oben $P$.

Fr. Pius Schmidt vic: gralis Congreg.

Alsaticae ord: prd... mppr.

Johan Daniel Silbermann

Auff diessen Accord habe ich von einem hochlöblichen Convent empfangen 600 R. Sage Sechs Hundert Gulden.

bescheine

Gebweyler den 27 Juny 1744.

Johann Daniel Silbermann.

Nach Verfertigung der neuen Orgel seyn wir von Einem Hochwürdtigen Convent in Gebwyler bezahlt worden biss auf $500 \mathrm{R}$ oder Tausend Livers welches wir bescheinen

Gebwyler den 14 7bris

1745

Johann Andreas

Silbermann

Johann Daniel Silbermann.

AHR. Série H, couvent des Dominicains de Guebwiller, cart. 3, chem. 7. 
Guémar, église catholique.

Notice du 16 juillet 1843 concernant le transfert de l'orgue de Guémar à Griesheim-sur-Souffel. 
1843. Transfert de I'orque de Guémar à Griesheim-sur-Souffel.

L'an 1842 les habitans de la commune de Griesheim, ayant terminé leur église, construite de leurs propres fonds, ont pris la résolution de se procurer un jeu d'orgues, pour la trés grande gloire

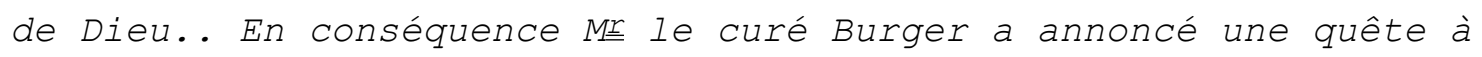
cet effet. Les souscriptions se sont élevées à la somme de 1500

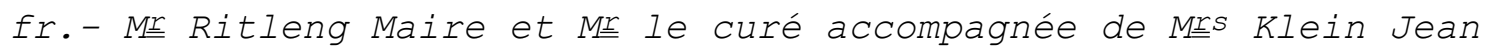
et Klein Jacques se sont transportés à Guémar et ont fait l'acquisition au nom de la commune d'un jeu d'orgues qui servait à cette église. L'accord a été conclu pour la somme de 1000 fr. Ces orgues sont l'oeuvre du célébre facteur d'orgues nommé silbermann. Mㅌ Wetzel facteur d'orgues à Strasbourg les a reparées et placées dans notre église. Cette réparation a couté 550 fr. Afin que les fils voient ce qu'ont fait leurs pères pour l'honneur de la religion, le conseil de fabrique a dressé le présent procèsverbal, qui a été signé par tous les membres du conseil. Griesheim le 16 Juillet 1843.

Burger curé

Ritleng Wurm

Klein Ehrman Klein

S. B. Hügel

Archives paroissiales de Griesheim-sur-Souffel. Protocole de Fabrique d'Eglise Griesheim a/s. 1831-1898, p. 15. 
Balbronn, église simultanée.

Marché du 15 avril 1747 avec Jean André Silbermann. 
Kundt und zu wissen seye hiermit, dass auff heut dato den 15ten Abril 1747 auff Seiten der Herren Vorsteher der Gemeind zu Ballbronn als nemlichen Herren Pfarrer, Herren Schuldheiss, Herren Burgermeister und Herren Mitregierende folgender Accord zwischen Herren Silbermann ist auffgerichtet worden, und darinen bewillget und geschlossen worden.

EsS verspricht nemlich Herr Silbermann ein gantzes neues Orgelwerck, für die obige gemelte Herren Vorsteher der Gemeint Ballbronn, dass Corpora oder Casten bestehend in saufferen eichenen Holtz auff die Fason wie gegenwertiger Riss zu machen, welches neues Werck soll in sich begreiffen folgende Register

$1 \quad$ Prestant von sauber gebalierten Zinn im Schein

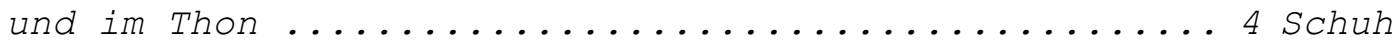

2 Bourdon 4 Schuch die zwey erste Octaven von eichenen Holtz 4 Schuh gedeckt die andere zwey Octaven von Medal.................... 8 Schuh

3 Quinte, die Corpra von Zinn, die Füss Medal ...... à 3 Schuh 4 Doublette die Corpora von Zinn die Füss von Medal ... 2 Schuh

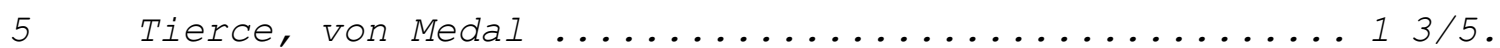

6 Cornet von Medal fünff fach zu hundert fünff und zwantzig Pfeiffen

7 Fourniture, die Corpora von Zinn die Füss von Medal.. 3 fach 8 Cimpal, die Corpora von Zinn die Füss von Medal .... 3 fach $9 \quad$ Tremblant doux

Dass Clavier von Ebenholtz und Bein sauber gearbeitet solches soll anfangen mit $C$ C\# $D$ D\# \& und soll in Discand gehen biss mit $C^{\prime \prime}{ }^{\prime}$ also mit vier volligen Octaven. 
Es soll alles nach dem Opera oder dem frantzöschen Thon gestimet seyn;

Zwey Blassbälge sollen gemacht werden, jeden derselben soll lang seyn 5 1/2 Schuh und soll breit seyn 3 Schuhe.

Dass Corpus oder Orgelcasten würde nach der Grösse und Fason gemacht wie auff den Abriss zu sehen, und von sauberen eichenen Holtz verfertiget samt den Zieraten vom Bildhauer.

Dieses obemedte Werck verspricht gedachter Silbermann von dato an biss künfftigen Herbst verferdiget und in guten Stand gestellet zu haben;

Dahingegen versprechen die Herren Vorsteher der Gemeinde Ballbronn ihme Silbermann in barren Geld zu zahlen die Suma von 800 fl sage acht hundert Gulden, und zwar in jetzigem Falor, und zehen Wagen Eychen und Buchen Holtz, jeeder solcher zehen wägen mit vier stuck Viehe besponnen, frey nach Strassburg vor mein Hauss zu lüfferen und soll die Zahlung in folgenten Terminen beschehen, nemlich gleich nach Verfertigung der Orgel 300 fl und den Rest von 500 fl auf Michaelis des 1748sten Jahrs und zwarr ohne Interssen, dass Holtz anbelanget so sollen 5 Wägen voll auff Michaelis 1747 gelüfert werden und die restiernde 5 Wagen auff Michaelis des 1748sten Jahrs.

Bey Auffsetzung des Wercks sollen ihme Silbermann nebst seinen Gesellen Kosten und Losament ohne Entgeld gereichet werden, wie auch die freyen $A b$ Zu und Abfuhr.

Urkundtlich desen ist dieser Accord in Duplo ausgefertiget von beeden Theillen eigenhändig unterschrieben, und jeeden ein gleichlautentes Exempelar zugestellet worden, so geschehen zu 
Balbronn auf Tag und Jahr wie vorgemelte.

Jörg Bucholz Schultheiss

Frantz Zörringer Burgermeister

Conrad Hammel dess Gerichts

Görg Monhart des Gerichts

Michel Hoffmann dess Gerichts

Hanss Georg Glass dess Gerichts

Hanss Jörg Motzig dess Gerichts

J. Reeber

Johann Andreas Silbermann

Balbronn. Archives communales. 
Strasbourg, Temple-Neuf.

- Marché d'octobre 1700 avec Frédéric Ring.

- Acte de vente de l'orgue du Temple-Neuf à la commune de Ribeauvillé, 16 mai 1748 .

- Marché du 27 juin 1747 avec Jean André et Jean Daniel Silbermann.

- Quittances par Jean André et Jean Daniel Silbermann.

- Compte-rendu de la construction du nouvel orgue, rédigé en 1750 .

- Compte-rendu de la séance du 2 juillet 1793 au sujet du diapason de l'orgue. 
I. N. D.

Kundt undt zuwissen seye hiemit männiglich, dem daran gelegen: Demnach die Hoch- vnd Wohlverordnete Herren Kirchen Pflegern auch wohl meritirte Herren Prediger der Newen Kirchen zu den Predigern genant, bey den vornehmst- vnd meisten Gliedern daselbst verpfarrter christlichen Gemeind, die Gedancken verspührt, denen sie selbst auch bey zu stimmen durch den Nachdruck guter Gründe sich gemässiget gefunden, dass nicht allein zur zierde eines so grossen Gebawes, sondern auch zur Nothwendigkeit, vmb genugsamer Vnterhaltung willen dess Gesanges einer so volkreichen Gemeinde, auch ein grösseres vnnd mehr proportionirtes Orgelwerck erfordert werde; Da dann auch E: löbl.-: Gemeinde zu Darreichung der nöthigen Vnkosten sich gantz willig erklärt vnnd durch Einlegung einer freyen Steür von mehr alss Sechshundert Gulden, daran würcklich einen guten Anfang gemacht hat: Dass Wohlgedachte Herren Kirchenpflegere vnndt Geistliche, dass Werck desto ehender vnd besser in Stand zu bringen, mit H.- Fridrich Rincken Burgern und Orgelmachern allhier, folgenden Accord getroffen haben.

1.) Erstlichen und zu aller vordrist verspricht der Orgelmacher, vor seine Arbeit zustehen vnd erbare Wehrschafft zu leisten, dergestalt, dass dafern dass Werck in einigem Stuck mangelhafft, oder sonst übel versehen befunden werden solte, er übernimmt, alles abgängige in seinen Kosten zu ersetzen und zu verbesseren, oder da dass Werck über bessers Verhoffen vntauglich befunden werden solte, Er der Kirch allen anwendenden Kosten auch leidenden Schaden getrew- vnd vollkomlich gut zu thun und wider zu erstatten übernimt, alles bey Verpfändung seiner Haab und Nahrung, jedoch mehr nicht, als so viel hierzu von nöthen seyn wird.

2.) Wird er sich angelegen seyn lassen, dass Werck nach dem von ihm selbst abgezeichneten Modell auff zu richten, vnd nach demselben ein neü Corpus zu verfertigen.

3.) Seind die bey jetzigem Werck befindliche Register so viel thunlich zu behalten, jedoch wo sie etwan schadhafft seyn solten, zu verbessern, zu säubern, zu stimmen, vnd sonsten in gehörigen Zuestand zu bringen.

4.) Zu solchen Registern, so in der Anzahl vierzehen sind, soll er wass sonst lieblich vnd anständig befunden wird, weiters hin zu thun: dergestalt, dass nach gemachter Abtheilung, dass 
gantze Werck folgende Register in sich begreiffe.

Ober Clavier. $\left[\begin{array}{ll}\text { 1. Coppel. } & \text { 4. Schuh gedeckt } \\ \text { 2. Quint. } & \text { 3. Schuh. } \\ \text { 3. Octav. } & \text { 2. Schuh. } \\ \text { 4. Cimbeln. } & \\ 5 . \text { Cornet 5. fach } & \end{array}\right.$

Mittler Clavier $\left[\begin{array}{ll}\text { 1. Principal. } & \text { 8. Schuh } \\ \text { 2. Viol da Gamba. } & \text { 8. Schuh } \\ \text { 3. Coppel. } & \text { 8. Schuh } \\ \text { 4. Quint. } & \text { 3. Schuh } \\ \text { 6. Octav. } & \\ \text { 7. Mixtur } & \text { 4. Schuh } \\ \text { 8. Voix humaine } & \text { 6. fach } \\ \text { 9. Trompette } & \end{array}\right.$

Unter Clavier. $\left[\begin{array}{ll}\text { 1. Principal. } & \text { 4. Schuh } \\ \text { 2. Quintaden. } & \text { 8. Schuh. } \\ \text { 3. Spitzflöten. } & \text { 4. Schuh } \\ \text { 4. Schweiglen. } & \text { 2. Schuh } \\ \text { 5. Crumhorn. } & \\ \text { 6. Echo. }\end{array}\right.$

Pedal. $\quad\left[\begin{array}{ll}\text { 1. Bordunen. } & \text { 32. Schuh. } \\ \text { 2. Principal. } & \text { 16. Schuh. } \\ \text { 3. Trompettenbass. } & \text { 16. Schuh. } \\ \text { 4. Principal. } & \text { 8. Schuh. } \\ \text { 5. Quint. } & \text { 3. Schuh. }\end{array}\right.$

Welches zusammen 25. Register aussmachet; vnd soll in dass Werck auch der Tremulant angerichtet werden.

5.) Die 3. Clavier sollen alle gantz und völlig seyn, mit 50. Claves. Dessgleichen soll auch dass Clavier zu dem Transponiren auff ein gantzen und halben Thon, ein gerichtet werden.

6.) Der Orgelmacher wird auch Blassbälg vnd Windladen nach Erforderung der Register machen: Dazu aber von dem vorhandenen anwenden und gebrauchen was nur düchtig sein wird.

7.) übernimbt man an Seiten der Kirchen alle nöthige Materialien zu bezahlen, doch dass der Orgelmacher solche zu erkundigen 
und nach Möglichkeit dass beste auss zu suchen sich angelegen lassen sein soll.

8.) Er wird auch genugsame Leüt anstellen damit wo möglich, dass neüe Werck biss auf zu künfttig Ostern nach Gottes Willen in brauchbarem stand seyn möge.

9.) Hingegen verspricht man ihm H.- Rincken, vor jeden gantzen Tag, den er arbeiten wird, vor Kosten und Lohn Einen Gulden zu zahlen vor ein halben Tag Ein halben Gulden: vnd dan auf jeden Gesellen dess Tags gleichfalls vor Kosten und Lohn, Fünff Schilling daneben man ihnen jedes Tags zum Morgen und Abendbrodt, ein Mass Wein wird zu kommen lassen.

Dess zu Vrkund und steter Vesthaltung ist gegenwertiger Verding an Seiten der Kirchen, von Hochverordneten Herrn OberKirchenpfleger, vnd anderseiten von dem Orgelmacher eigenhändig vnderschrieben worden

Actum den Octobr: 1700.

Johann Reinboldt Friderici

Friderich Ring

Orgelmacher.

Archives Municipales de Strasbourg. Archives du Temple-Neuf. fasc. 82 . 
(Verkauff der alten Orgel.)

Zu Anfang des Maji ward H.- M. Brücken Cantori die Commission gegeben den Kauff wegen der alten Orgel nach Rappoltsweyher mit dasigem H.- Amtmann Msr. Larcher zu schliessen, der nach seinem Original folgendermassen lautet.

Je soussigné reconnois avoir fait marché avec Mr. Bruck Chanteur de l'Eglise-neufve de Strasbourg, chargé de Procuration de Monsieur Hammerer Ammeistre de la d.e Ville de Strasbourg et en qualité de Chef de la Fabrique de lad.e Eglise neufve pour les Orgues qui se. trouvent actuellement dans lad.e Eglise et ce dans 1 etat actuel ou elles se trouvent avec tous les ornements, tuyaux, soufflets et touttes choses qui doivent y appartenir, \& ce pour les pris et somme de deux mil cinq cent Livres, me soumettant de payer lad.e somme au temps que l'on enlevera lesd.es Orgues, me reservant neantmoins que ce soit au plus tard pour le mois d'Avril de I'annee prochaine 1749. \& que I'on ne commence a demonter lesd.es Orgues que dans le courant de mois de Mars d.e année, et ce a peine de tous depens dommages et intérets, en foy de quoy nous avons fait le present marché double: fait à Ribeauville le seize May mil sept cent quarant huit.

Larcher.

Brück.

Archives du Temple-Neuf. Vol. 7. p. 181. 
Aufsatz von einem gantz neuen Orgelwerek vor die Prediger Kirche, wie solches in allen stücken nach gegenwärtigem unterzeigneten Abriss in einen gantz neuen Orgelkasten soll erbauet werden.

Vors Erste. Muss ein gantz neuer Orgelkasten so wohl zu dem grossen Werck als auch dem Ruckpositive in allen stücken nach dem Abriss gemacht werden. Derselbe soll vom Bildhauer mit saubern Ornamenten geziehret seyn, und soll alles vom Mahler sauber gefasst, und mit feinem Gold vergoldet werden. Wie auch müsste derselbe mit gehöriger Schlosser Arbeit versehen seyn.

2 tens. Müssen alle Windladen zum gantzen Werck sambt denen Claviren, und dem gantzen Regierwerck gantz neu gemacht werden, die Windladen von beständigem, ohnwandelbahrem beindürrem eychenen Holtz, die 3 Manual=Clavire von Ebenholtz und Bein, jedes derselben von 4 völligen Octaven, oder 49 Clavibus. Das Regierwerck aber von gehörigen thauerhafften Matherialien.

3 tens. Müssen sechs grosse Blassbälge gemacht werden, jeden derselben 9 Schue 1 Zoll lang, und 4 Schue 8 Zoll breyt, und soll jeder nur aus einer Falte bestehen. Die Blätter sollen von Dreyling Diehlen gemacht werden, woraus die Hertz geschnitten seyn, und mit starcken Hirnleisten von Eychenholtz angefasst, und meistens wegen beständiger Tauer mit Kalbleder und Pergament versehen seyn. Das Regierwerck dazu wird so eingerichtet dass sie können aufgetretten werden. Sie müssen vor die Kirche hinaus auf ein aparte Bühne geleget werden, damit man auch bey Auftrettung derselben nicht wie bey denen jetzigen Bälgen ein Gerassel oder Gepolter höhren kan.

Die sämbtliche Canaele und Windführungen sollen gleichfals von reinem Holtz verfertiget werden.

4 tens. Die Manual=Clavire sollen so eingerichtet werden, dass man nicht allein wie sonst gewöhnlich das Haupt=Manual=Clavir mit dem Ruckpositive=Clavir kopplen oder zusamen spielen kan, sondern mit dem Manual Clavir das Oberwercks= oder dritte Clavir soll sich gleichfals mit einander spielen lassen, und auch dem Werck die völlige stärcke zu geben sollen alle drey Manual Clavire sich auf einmahl können spielen lassen. 
5 tens. Soll alles in den Opera oder frantzöschen Thon gestimmet werden.

Die Register aber sollen folgente seyn.

Im Haupt= Manual.

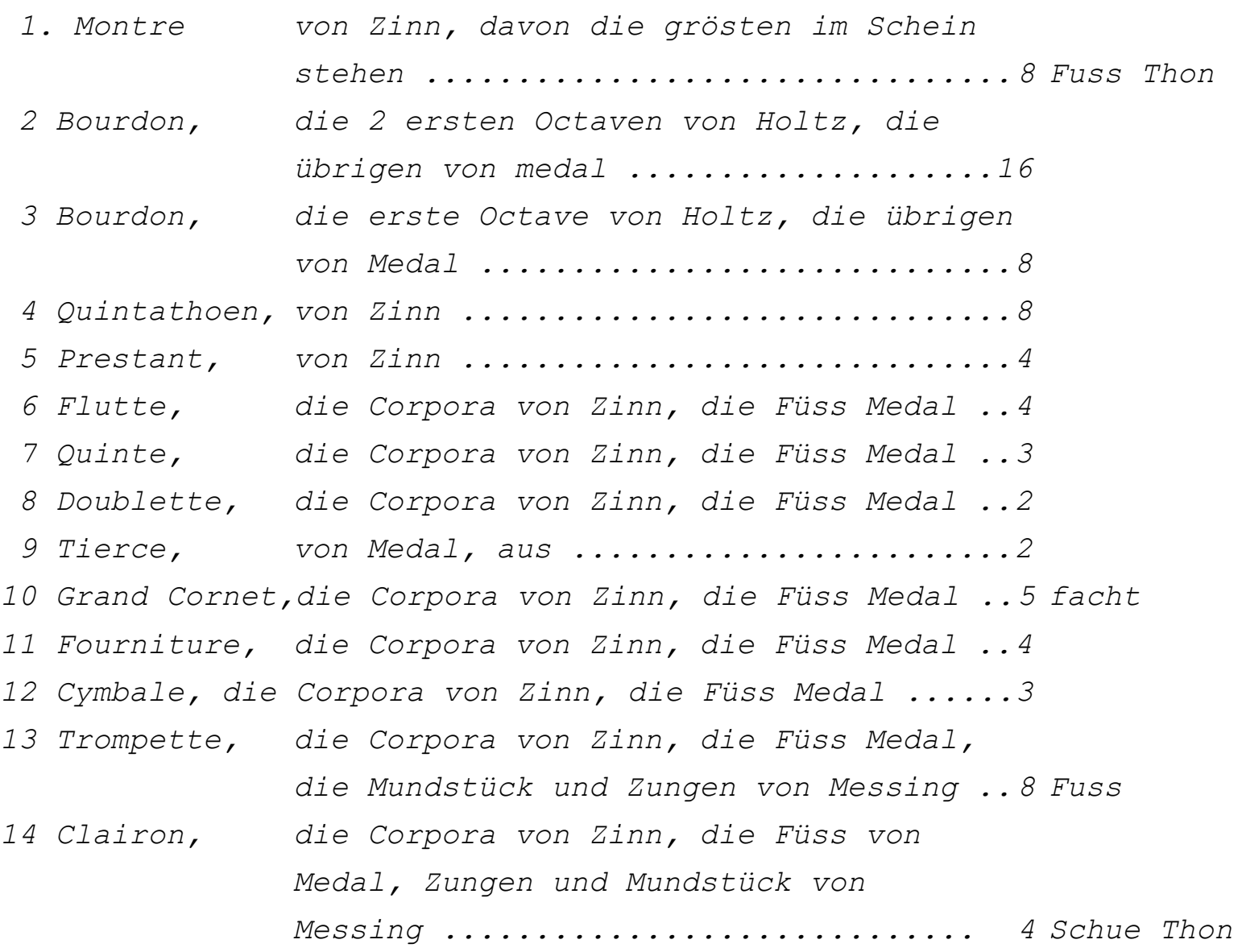

Summa .................... 14 Register

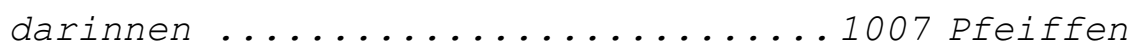


Register welche ins Oberwerck kommen sollen.

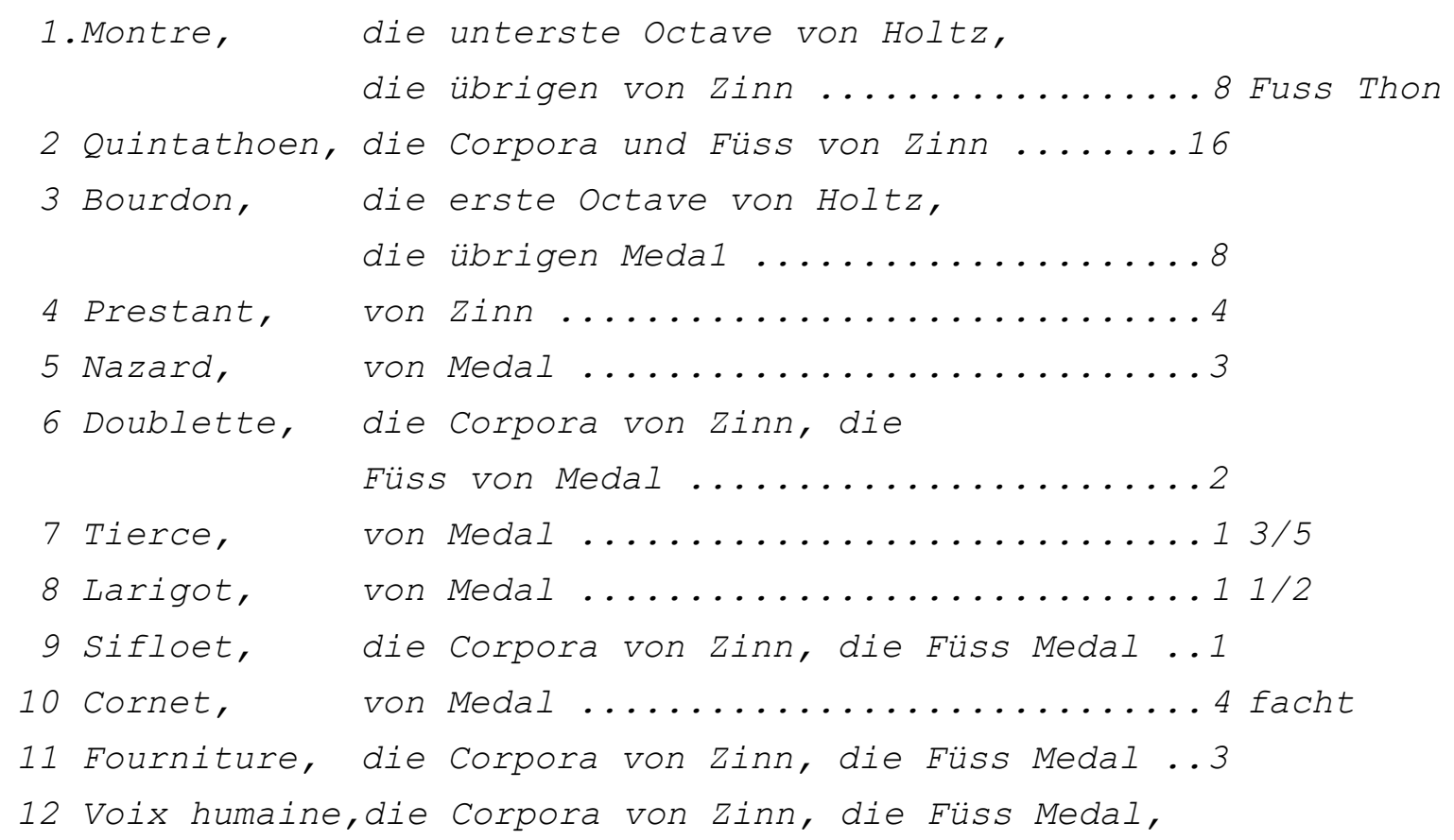


Im Pedal sollen sich folgente Register befinden.

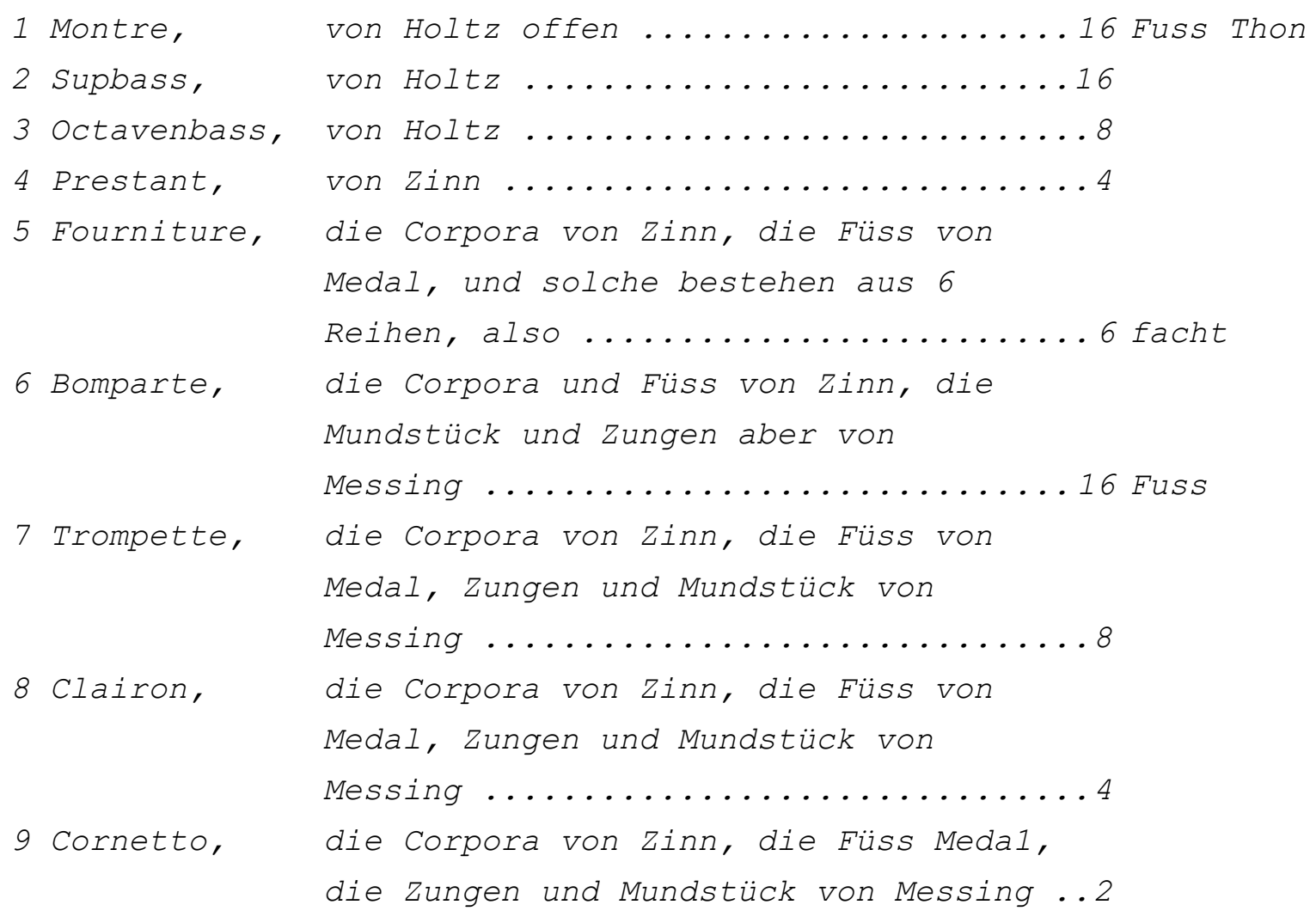

Summa .................. Register

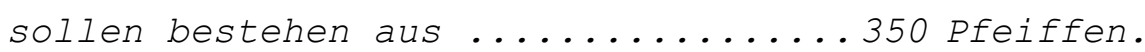

Demnach bestünde das Werck aus 44 Registern

Welche in sich begreiffen 2682 klingente Pfeiffen.

Ferner sollen zwey Tremulanten gemacht werden, davon der eine

langsam, der andere aber geschwind gehen soll. 
Vorstehentes neue Orgelwerck ist zwischen dem hoch und wohlverordneten Herren ober= Kirchen Pfleger, denen übrigen wohlverordneten Herren Pflegern, und denen wohlehrwürdtigen Herren Geistlichen der Prediger Kirche, und denen zweyen Gebrüdern Silbermänner denen Orgelmachern alhier, folgentermassen verdungen und verglichen worden. Dass gedachte Silbermänner selbiges Werck zwischen hier und Michaelis 1749 neu verfertigen, und nach dem vorbeschriebenen Aufsatz eintheilen, auf ihre Kösten auf den Platz wo die jetzige Orgel stehet, stellen, auch alle dazu erforterliche Matherialien als Zinn, Bley, Messing, Holtz, Leder, Leim, Nägel und Thratwerck, in Summa wie sie auch Nahmen haben mögen dazu geben, wie nicht weniger die Schreiner, Thräher und Schlosser Arbeit so wohl zum Orgelwesen als auch zu einem neuen Kasten, sambt der Fassung und Vergoldung und aller Bildhauer Arbeit zu besorgen und machen zu lassen über sich nehmen, so dass die Kirche weiter nichts als die zimmer Arbeit zu der Orgel in dero Kosten soll machen lassen.

Hingegen versprechen die hoch und wohlverordneten Herren Kirchen Pfleger und wohlehrwürdtigen Herren Geistlichen denen Silbermännern vor Ihre obengemelte Arbeit zu entrichten und zu bezahlen, die Summa von 9150 fl, sage neun tausend einhundert fünfftig Gulden. Welche gedachte Summa dieselbe zu empfangen haben, wie die Gelder von denen Steuren, aus dem alten Orgelwerck, und anderm eingehen, so dan was das stifft Frauenhauss dazu würcklich zu geben erkant hat, und noch ferners geben möchte, nach denen von gedachtem Frauenhauss gesetzten und noch zu setzen habenten Terminen, doch so dass biss zu End des tausend siebenhundert und fünftzigsten jahrs die völlige summa ausser was noch von dem Frauenhauss möchte zu erheben seyn, von seithen der Kirch sollte volkommen bezahlt werden. Und zwar soll alles nach heuthigem Valor die Louis d'or zu 12 fl, oder das drey Gulden Stück zu 3 fl geschehen.

Urkundlich dessen, ist dieser Accord von einer Hand in 2 gleichlautente Exemplaria verfertiget, und von beyden Theilen unterschrieben worden.

So geschehen in Strassburg den 19 May 1747. 
Obiger Accord ist in allen Stucken aprobirt und angenommen worden, nur mit dieser Reservation, dass die Zahlungen in correntem Geld wie solches gangbar seyn mag, beschehen sollen: Hingegen verspricht man alles was von seithen der Kirch zu erlegen ist, bis zu End des ein taussent sieben hundert und neun und viertzigsten Jahrs zu bezahlen, Strassburg den 27 Juny 1747.

J F Hammerer

Johann Philipp Richshoffer

Georg Friderich Bemberg

G.V.Holtzberger, Diac. im Namen

der Geistlichen.

Johann Andreas Silbermann

Johann Daniel Silbermann. 
Dass von Herrn Georg Friedrich Bemberg wohlverordneten Herrn Kirchenpfleger der Prediger Kirch nach dem mit den hoch wohlverordneten Herrn Pflegern gedachter Kirch getroffenen Orgelaccord von der aus der Gemeind gesamleten Steuer in zweyen Mahlen auf Abrechnung empfangen habe die Summam von 2542 fl, sage zwey tausend fünff hundert viertzig zwey Gulden, solches thue hiemit quittirent bescheinen. Strassburg den 19 Octobris 1747. Johann Andres Silbermann

Dass ich ferner von deme zur Orgel gesamleten Geld von Herrn Bemberg empfangen habe $160 \mathrm{fl}$, sage hundert sechtzig Gulden, beecheine Strassburg den 26 8bris

Ferner habe die zwey ersten vom hochlöblichen Stifft Frauen Hause zu zahlen versprochenen Termine so auf Michaelis des 1747sten \& 1748sten Jahrs mit 1000 fl, sage tausent Gulden verfallen sind, wohl empfangen, bescheine Strassburg den 9 Xbris 1748 Johann Andreas Silbermann

Dass ich ferners empfangen habe 625. fl. sage sechs=hundert fünff und zwantzig Gulden so vor die Helffte der verkaufften Orgel eingegangen Orgel, bescheine Strassburg den 10. Aprill

1749 . Johann Daniel Silbermann.

Dass ich Ends unterschriebener von Herren Georg Friederich Bemberg, als Pfleger der Neuen=Kirch, in zwey Mahlen zusamen achthundert Gulden, sodan Vom Frauen Hause vor den tritten Termin fünff hundert Gulden empfangen habe, thue hiemit quittierent bescheine.

Strassburg den 16. 8bris

Johann Daniel Silbermann.

Ferner habe von Herrn Bemberg hochmeritirten Herrn Pfleger der Neuen Kirch in baarem Geld empfangen 2398 fl, sage zwey tausend drey hundert neuntzig acht Gulden, solches bescheine Strassburg den 19 9bris 
$A^{\circ} 1750$.

\section{Nachricht}

was bey Auffrichtung und Inauguration der neuen Orgel, und bey der vorgenommenen Reparation der Kirch notables vorgegangen.

ad annum praecedentem gehörig.

Nachdem die 1747 d.- 27 Jun: mit H.- Joh: Andr: u. H.- Joh: Danj: Silbermann, Gebrüdern, veraccordirte neue Orgel, fertig ist gemacht worden, so wurde gleichbalden nach Rappolsweyer, lals wohin die alte Orgel in die cathol.-: Kirch daselbst vor $1250 \mathrm{fl}$ verkaufft war,) berichtet, dass man laut Accord, diesselbe auff dero Kosten sollte abbrechen, u. abhohlen lassen: woran auch gleich d.- \& nach ostern als d.- 9 Apr: 1749, der Anfang durch ein von der Gemeind Rappolsweyer bestellten Orgelmacher, tit: Rohrer, ist gemacht worden. Nachdem nun das

Der neue Platz vor die Blassbälg der Orgel. alte Orgelwerck, völlig hinweg war, so wurde der bisshero offene Platz über dem Creutzgang, wo dermalen die Blassbälg liegen, auff Uncosten dess Frauenhausses mit Balcken belegt, und in den Stand gesetzt, dass die Blassbälck an diessen Ort konnten logiret werden. Darauff dann das Orgelwerck selbsten an den Platz wo die vorige Orgel gestanden, auffgeschlagen worden.

Indem man aber wahrnahm, dass diesses so schöne, u. kostbare Werck, gleichsam geschändet würde, wan man nicht auch eine Reparation mit Aussweissung u. Aussziehrung der gantzen Kirch würde vornehmen, (als worauff man biss dato gar keine Gedancken gemacht hatte, weil man sich vorstellete, die Uncosten würden allzugross seyn, als dass man solche auffbringen könnte,) / so liess man dissfalls einen Überschlag wegen Aussweissung der Kirch machen, welcher dann sammt den Gerüsten auff $900 \mathrm{fl}$ ist

H. - Andreas abgeschätzet worden. Ein genereuses Mitglied aber Stahl, weisst die Kirch von diesser Gemeinde, nemlich H.-Andreas Stahl, gratis auss. Burger und Maurer Meister allhie, welcher keine Kinder hatte, und bey guten Mittlen stund, ent- 
schloss sich sehr grossmüthig die gantze Kirch auff eygene Kosten ausszuweissen, u. an gehörige Orten mit Farben einzufassen. Da er dann seine eygene aussgelegte Uncosten, auff vier biss fünff halb hundert Gulden schätzte, so er für Materialien, u. Arbeitslohn musste anwenden. Nun der Entschluss wurde würcklich von Ihme gefasst, es koste was es wolle, diesses Werck mit Aussweissung der Kirch, in Gottes Nahmen vorzunehmen. H.- Stahl so diesse Reparation unternahm, stellte auch alsobald Leuth genug an; wie dann bey 20 Personen zugleich arbeiteten. Das Holtz zu den Gerüsten, (welche alle schwebend in seyle gehenckt wurden, u. H.- Stahl auff $100 \mathrm{fl}$ zu kauffen, kosteten,) wurde von Seiten der Stadt, so lang man es nöthig hatte, darzu fourniret. Das allererwünschteste Wetter beförderte das Werck ungemein, so dass in Zeit von 5 Wochen die gantze Kirch ihre neue, schöne, weisse Gestallt hatte. Während solcher Reparation, u. Aussweissung der Kirch, wurde das schon auffgeschlagene neue Orgelwerck sorgfälltigst mit Tüchern umhängt; welche, H.- Bemberg, Kirchen und Fabric-Pfleger gütigst fournirte.

200. Die Diesse Helle und Schönheit verdunckelte den Glantz Cantzel u. der der Cantzel u. dess Altars; man glaubte sich dahero Altar nebst genöthiget zu seyn, auch diesse Stück, nebst den anderem werden Lednern, Tafflen, Leuchtern, u. in Summa allem was neu verguldt einer Reparation nöthig hatte, anjetzo auff einmal u. renovirt. vorzunehmen, und alles auff einmal in einen schönen und aussgezierten stand zu.stellen. Es geschah auch würcklich. Man accordirte mit H.-Huber dem ältern, L. t. Stadt-Mahlern, vor 1200 fl. die Cantzel, den Altar, samt den eissernen Gegittern so an den Lednern herum sich befinden, die Consolen, oder erhabene Bilder so zwischen diessen Tafflen sind, die eissernen Stangen, woran die Leuchter hangen, und alles andere von Eissen oder Holtzwerck, wie es diesse Reparation erforderen würde, theils zu vergulden, theils anzustreichen.

Neues Orgel- Zu gleicher Zeit, wurde das Stübel auff der Orgel, 
Stübel.

201. Neue

Orgel-Steeg.

Notification wegen der Inauguration der Neuen Orgel.

dem unteren Kirchen-Stübel gleich gemacht, und folglich erweitert. Dann das alte stübel stund um etliche Schuh von den Fenstern weg: damit der Tag an dem Ort, wo seit 1747 das Kirchenstübel ist erbaut worden, ehmals konnte herab fallen. Dann es war damals noch kein Fenster unten in der Mauer. Die Baucösten bey diesser Reparation dess OrgelStübels geschahen von Seiten dess Frauenhausses. Da auch die alte steeg auff die Orgel, eine sehr enge, und fünstere Schneckensteeg war, so wurde auff Uncosten dess Frauenhausses eine neue Steeg verfertiget, und die Thüre zum Eingang in die Orgel erweitert.

Als nun alles biss auff etliche Kleinigkeiten in vollkommenem stand gesetzt war, so wurde beschlossen, auff den 16 Nov: als Dom: XXIV Trinit: so der Sonntag vor der Herbst-Predigt 1749 war, die Inauguration oder Einweyhung der Orgel in Specie, vorzunehmen; dann alles übrige bestund nur in einer

Notificat: der Renovation. Den zuvor als den 23 Trinit: wurde Steuer an dem solches nach der Predigt der Gemeinde notificirt, Tag der Orgel mit Vermelden, dass über 8 Tag, als auff den Tag Einweyhung. der Inauguration der Orgel, an allen Kirch-Thüren, Verspruch hiebey, das verdrüssl. Herumgehen Schüsslen, zu einer freywilligen Steuer, (weil man noch eines sehr starcken Beytrags nöthig habe,) würden nach der Morgen Predigt, gehallten werden. Bey Verkündigung diesser Steuer, versicherte man derer Musicanauch die Gemeinde, dass wo diesse Steuer ansehnlich ten einzustel- und erwünscht würde aussfallen, man das verdrüsslen.

202 liche / Herumgehen vor die Häusser am Weyhnachten bey den Herren Musicanten sowohl der Vocalisten als Instrumentisten wollte auffheben. So auch würcklich geschah.

Inauguration

Dom: XXIV Trinit: oder der 16. Nov: war dann der der Orgel Tag der Orgel-Einweyhung selbsten. Da dann in der selbsten. Morgenpredigt eine von dem H.- Frauenholtz als Capell-Meister oder Chorago diesser Kirch; u. in der Abendpredigt eine von H.- M. Brück, Cantore diesser Kirch, besonders gestellte schöne Music unter Trompeten und Paucken Schall zu beedenmalen 
feyerlichst auffgeführet wurde. Freytags d.- 7 Nov: wurde solche Music zuvor probirt. Der Musictext des H.- Choragi Frauenholtzes wurde auch getruckt. Die Predigt selbsten, verrichtete H.- D. Froereisen, als Pastor diesser Kirch, und erwählte zum Text den 150 Psalm. Die Abendpredigt hatte, H.- D. Fridr: Jac: Reuchlin, welchen diesse Predigt in circulo traff. Er tractirte die ordentl.- Epistel, richtete aber im Eingang $u$. in den Usibus vortrefflich seine Predigt nach den Umständen und Gelegenheit diesser Solennitaet ein.

Nach der Morgenpredigt wurden wie man es 8 Tage vorher verkündiget, an den Kirchen Thüren, von lauter Rathh.- die Schüsslen gehallten, und eingelegt $2250 \mathrm{fl} .1 \mathrm{~s} .41 / 2$ d. Da man auch die Erlaubnus erhielt, dassjenige was diessen gantzen Tag in der Amtpredigt, Mittags und Abendpredigt in die Säcklein eingeleget werden sollte, zu diesser Orgelsteuer dörffen einzusammlen, so befand sich in dem Säckel bey der Mittags und Abendpredigt $243 \mathrm{fl}$ 3 s $31 / 2$ d. Das Säckel in der Amtpredigt schätzte man ohngefehr gegen $50 \mathrm{fl}$. Es wurde aber solches ohn auffgezehlt in den Opfferstock, der vor diesse Kirch in specie gewidmet ist, gethan; weil man sich noch nicht wegen diessem Säckel in der Morgenkirch, unterredet gehabt, auch nicht verkündiget worden war, so wurde auch nichts als Kupffer eingelegt, wie zu anderen Zeiten auch zu geschehen pflegt.

AMS. Archives du Temple-Neuf. Vol. 7 (Délibérations 1695-1761) p. 198-203. 
(Séance du 2. 7. 1793.)

Es erschiene Bürger Hepp, Organist dieser Kirche und batte, dass man die ehemalige stimmung der Orgel bey der Muaic, so wie sie vorher üblich war, wieder verordnen möge, indem das Transponiren der Orgel nicht nur sehr mühsam seye, sondern auch die erwünschte Würckung unmöglich machen könne, im Gegentheil der Music nachtheilig seyn müsse. Er führte hauptsächlich an, dass man nirgend um einen halben Ton transponire, weilen kein halber dem andern gleich, sondern bey jedem Ton immer einer grösser oder höher als der andere ist, folglich immerwährendes Dissoniren verursacht, ferner: dass man durch solches Transponiren fast immer auf solche Tonarten kommt, die bei keiner gutgestimmten orgel rein sind; weiter: dass die Hautbois niemalen der Orgel in der stimmung gleich kommen, indem diese immer etwas höher ist als jene. Des immerwährenden Wiederspruchs in Ansehung der Würckung und Eigenschaft jeder Tonart nicht zu gedencken. Ausser dem seye dieses Transponiren sehr mühesam, ingrat und nicht eines jeeden Sache, gebe auch vielen Anlass zu Feindschaft und streitigkeiten zwischen dem Music = Director und Organisten; weil jede von diesen fremden Tonarten auf zweyerley Art bezeichnet werden kann und doch immer nur eine Art davon brauchbar ist. So seye auch dis Transponiren für die Vocal Music sehr nachtheilig, weil durch die übertriebene Höhe der Gesang Noth leiden muss, wodurch allerdings die deutliche Pronunciation gehindert wird, welche doch bey dem Gesang ein wesentliches stück ist p.p.

Worauf beschlossen wurde, dass Bürger Hepp, von dessen Kunst und Geschicklichkeit in der Music, besonders was die Orgel, betrift, man überzeugt ist, zu ersuchen seye die Music in dem transponirten Orgelton fernerhin zu accompagniren, so wie er solches seit einiger zeit mit allem Beyfall gethan hat.

AMS. Archives du Temple-Neuf. Vol. 93 (Délibérations 1793-1822) p. $1-2$. 
Soultz, église catholique.

Copie du marché du 23 octobre 1747 avec Jean André et Jean Daniel Silbermann. 
Heut dato den drey und zwantzigsten Octobris ein tausent siben hundtert viertzig und siben, ist nachvolgenter Accord zwischen alhiessiger Obrigkeit, Rath, Zunfftmeister, und überigen Deputierten Nahmens alhiessiger Gemeindt ahn einem; so dann H. Daniel Silbermann Orgelmacher löblicher Statt Strassburg, dermahlen sich alhier befündtent, sowohl für sich als nahmens seines E. Bruedters Johann Andres Silbermann andteren theils, gemacht wordten, undt zwahr über diejenige Orgel so vermög Plan definiert undt von denen Partheyen undterem heütigen Tag ahngenohmen undt unterschriben wordten, welche Orgel bestehen solle in volgenten Registern als

1. Montre von Zinn in Schein ............. 8. Schuhe Thon

2. Prestant von $\operatorname{Zinn} \ldots \ldots \ldots \ldots \ldots \ldots \ldots \ldots \ldots \ldots \ldots$

3. Bourdon die zwo undterste Octaven von Holtz die überige Metaille ............... 16 .

4. Bourdon die erste Octave von Holtz die überige

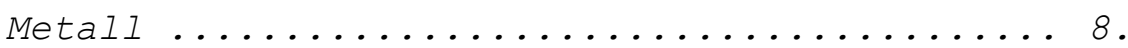

5. Cornette fünff fach von Metall ins halbe Clavier

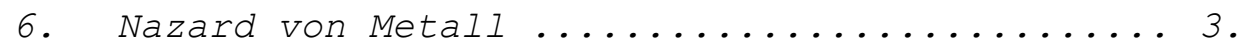

7. Doublette die Corpora von Zinn, die Fuess von

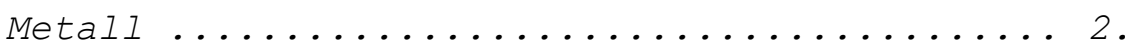

8. Tierce von Metall ................... 1 3/5 -

9. Fourniture drey fach die Corpora von zinn die Fuess aber von Metall ............... 1 1/2 -

10. Cymbal drey fach von Zinn undt die Füess von Metall

11. Trompette von $\operatorname{Zinn} \ldots \ldots \ldots \ldots \ldots \ldots \ldots \ldots . \ldots$. .

12. Flutte von Metall ................ -

13. Voix humaine von $\operatorname{Zinn} \ldots \ldots \ldots \ldots \ldots \ldots \ldots \ldots$. - 
14. Echo mit besondterem Clavier undt Windtladt soll anfangen von dem Tenor a. biss ahn dass oberste von C'''. Bestehet also der gantze Echo auss 140. Pfeiffen von Metall, hat also drey Claves mehr als die Ensisheimber. In welchem Echo ein Register von Menschenstimm auch stehen solle.

\section{Positive}

1. Prestant im Schein von Zinn ........... -

2. Bourdon die erste Octave von Holtz, die überige

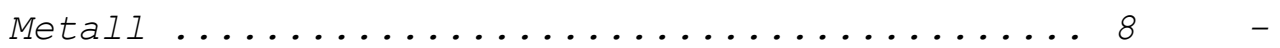

3. Flutte von Metall ............... -

4. Nazard von Metall ................... 3 -

5. Doublette von $\operatorname{zinn} \ldots \ldots \ldots \ldots \ldots \ldots \ldots \ldots \ldots \ldots \ldots \ldots \ldots$ -

6. Tierce von Metall .................. 2 -

7. Larigot von Metall .................. $11 / 2$ -

8. Carillon.

9. Fourniture drey fach von Zinn ............ -

10. Cromhorn von $\operatorname{zinn} \ldots \ldots \ldots \ldots \ldots \ldots \ldots \ldots \ldots$ -

Pedal.

1. Bourdon von Holtz offen ............... 16 -

2. Bourdon von Holtz ................ 8 -

3. Trompette von $\operatorname{Zinn} \ldots \ldots \ldots \ldots \ldots \ldots \ldots \ldots \ldots \ldots$ -

4. Clairon von Zinn ................

Zwey Tremelant einer langsam, und den andern geschwindt.

Die Trompette undt Clairon in dem Manual soll jedtes Register zwey Zug haben damit mann nach Belieben abwechslen kann. 
Die drey Manual = Clavier sollen sein von Ebenholtz undt Bein welche anfangen von C. C.\# etc. biss C''' also vier völlige Octaven.

Dass Pedal Clavier soll bestehen auss zwo völligen Octaven von dem grossen C. biss $C^{\prime}$.

Vier Blassbälg nach Proportion dess Werckhs, welche sollen hinter die Orgel auff ein klein Bihnlein gelegt werdten, undt mit Rollen, als wie zue Ensisheimb, sollen auffgezogen werdten.

Dass gantze Werckh soll in den Opera odter frantzösischen Thon gestimet werdten.

Dass Corpus sambt dem Ruckhpositive soll von eingefastem eichenem Holtz dessgleichen auch die ornamenten von obgemeltem Holtz gemacht werdten. Der Kasten aber worin dass Pedal stehet, soll von Thannenholtz wie gebräuchlich gemacht werdten, die gantze Orgel soll bestehen in ein taussent acht hundtert dreysig acht klingenten Pfeiffen.

Alles obgemeltes nach obangezogenem kostbahren Riss sambt denen zwey Engelen wie alles der angezogenen Riss weisset, zue machen, undt zue verferdtigen, wie auch alle Materialien als Holtz, Zinn, Pley, Messing, Ledter, Leim, Dratwerckh undt Schlosserarbeit, in Summa alles wass zue Verferdtigung der Orgel gehört, wie auch die Ornamenten in des H. Silbermanns Kosten anzueschaffen undt verferdtigen zue lassen, undt geschahe der Preyss undt Handtel für undt umb die Summa von siben tausent zwey hundtert Livres Tournois in heütigem Valor, die Species möchten auff odter abgerueffen 
werdten, neben sechs Ohmen Wein, zue bezahlen nach gefüfferter undtt gestelter Arbeith, welches geschehen solle von Dato über drey Jahr, anbey annoch überkommen wordten, dass die Orgel gleich solle propieret undt von dem Ladhoff zue Colmar, durch hiessige Gemeindt ohne Endgelt H.. Silbermanns abgeführt werdten. Doch solle der Gemeindt freystehen die Bälg auff Stangen odter Rollen zue stellen, undt verspricht ermelter H. Silbermann Jahr undt Tag Währschafft zue leisten für ebgemelte Orgel und Arbeith, Urkundt dessen sich die Partheyen im Original eigenhändtig undterschriben haben.

Actum Sultz ut supra,

Undt ist H. Silberman dass Logement auff der Tiergarthen Zunfft frey verwilliget wordten.

Stehet im Original undterschriben Immelin ..

Collationniert

Remy

Not: und Stattschreiber

Archives communales de Soultz, liasse GG 5(1683-1753) 
Villingen.

Description de l'orgue de Villingen par Jean André Silbermann. 
Disposition der Orgel welche sich in dem hochlöblichen Gotteshauss zu St: Georgen in Villingen befindet.

Register im Manual vors mittlere Clavir.

1 Montre, oder Principal, von feinem polirtem Zinn im Schein ................8 Schu Thon

2 Bourdon, oder Coppel, die 2 unterste Octaben von Holtz, die übrigen Medal /: nemblich von einer Composition von zinn und Bley:/ ................... 16

3 Bourdon, die erste Octav von Holtz, die übrigen von Medal .................. -

4 Prestant, oder Octava, von.Zinn ............ -

5 Nazard, von Medal ...................... -

6 Doublette, oder Superoctav, die Corpora von Zinn, die Füss Medal ................ -

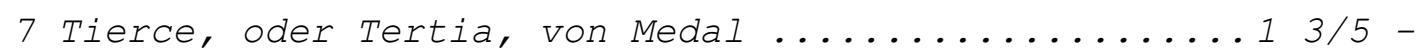

8 Siflet, die Corpora von Zinn, die Füss Medal .. 1 -

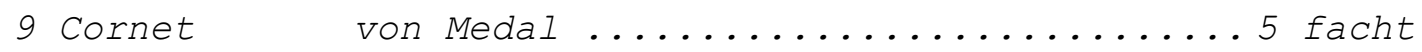

10 Fourniture oder Mixtur, die Corpora von Zinn, die Füss Medal .................. 3 facht

11 Cymbal die Corpora von Zinn, die Füss Medal . . 3 facht

12 Trompet die Corpora von Zinn, die Füss Medall, Mundstück und Zungen von Messing .....8 Schu Thon

13 Vox humana, die Corpora von Zinn, die Füss Medal, Mundstück und Zungen von Messing .....8 8

Register im Positif, oder dem untersten Clavir.

1 Prestant oder Principal von feinem polirtem Zinn

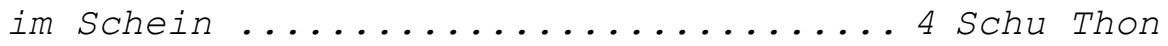

2 Bourdon, die grösten von Holtz, die übrigen von Medal ......................

3 Flutte oder Flöte, von Medal ............... -

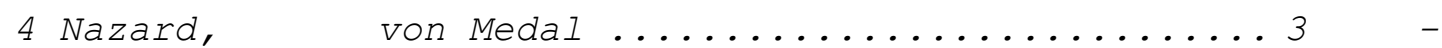

5 Doublette, die Corpora von Zinn, die Füss Medal .. 2 -

6 Tierce von Medal ..................... $3 / 5$ -

7 Fourniture, die Corpora von Zinn, die Füss Medal .. 3 facht

8 Cromhorn, die Corpora von Zinn, die Füss Medall, Mundstück und Zungen von Messing .....8 Schu Thon 
Register im dritten Clavir.

1 Bourdon, die 2 untersten Octaven von Holtz, die übrigen Medal ................ 8

2 Fagot Bass oder Basson, die Corpora und Füss von Zinn, die Mundstück Medal, die Zungen von Messing. Durch die 2 unterste Octaven, von $C$ biss mit $h \ldots \ldots \ldots .8$

3 Nazard d'Echo

4 Doublette d'Echo

5 Tierce d'Echo

6 Prestant d'Echo

durch die 2 obern Octaven.

Register vors Pedal

1 Supbass von Holtz, 8 Schu gedeckt.............. Schu Thon

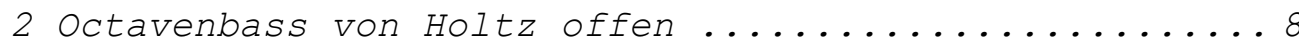

3 Trompettenbass, die Corpora von Zinn, die Füss Medall, Mundstück und Zungen von Messing .................. 8

2 Tremulanten, davon einer langsam der ander geschwind.

3 Blassbälge, jeder 6 Schu lang, 3 Schu 4 Zoll breyt, jeder mit 7 Falten.

Jedes der 3 Manual Clavire bestehet aus 4 völligen Octaven.

Das Pedal Clavir aber aus 2 völligen Octaven.

Und ist die Orgel im Cammerthon gestimbt.

Badisches Generallandesarchiv Karlsruhe. Akten St. Georgen, Fasc.47 
Strasbourg, Saint-Jean.

Notice descriptive, au moment de la vente de l'orgue à la Konkordienkirche de Mannheim en 1800. 
Composition de l'orgue de J.A.Silbermann à l'église St. Jean de Strasbourg, vendu à Mannheim en 1800.

\begin{tabular}{|c|c|c|}
\hline \multicolumn{3}{|c|}{ Manual } \\
\hline 1. & Montre & 8 \\
\hline 2. & Prestant & 4 \\
\hline 3. & Cornet 5 fach & \\
\hline 4. & Bourdon & 16 \\
\hline 5. & Bourdon & 8 \\
\hline 6. & Nazard & $22 / 3$ \\
\hline 7. & Doublette & 2 \\
\hline 8 . & Tierce & $13 / 5$ \\
\hline 9. & Siflet & 1 \\
\hline 10. & Fourniture & \\
\hline 11. & Cymbale & \\
\hline 12 . & Trompette & 8 \\
\hline 13. & Voix humaine & 8 \\
\hline
\end{tabular}

Echo

$\begin{array}{lllll}\text { 1. } & \text { Prestant } & \text { Bass } 4 & \text { Prestant } & \text { DisC. } 4 \\ \text { 2. Bourdon } & \text { Bass } 8 & \text { Bourdon } & \text { Disc. } 8 \\ \text { 3. Fagot } & \text { Bass } 8 & \text { Nazard } & \text { DisC. } 2 / 3 \\ \text { 4. } & & & \text { Doublette } & \text { DisC. } 2 \\ \text { 5. } & & & \text { Tierce } & \text { DisC. } 13 / 5\end{array}$

Positif

1. Prestant 4

2. Bourdon 8

3. Flöte 4

4. Nazard $22 / 3$

5. Doublette 2

6. Tierce $13 / 5$

7. Larigot $11 / 3$

8. Fourniture

9. Cromhorne 8 
Pedal durch 2 Octaven

1. Subbass 16

2. Octavbass 8

3. Trompettenbass 8

2 Tremulanten

Manualschiebekoppel.

Konkordienkirche Mannheim, Pfarrarchiv. 
Saarbrücken, Ludwigskirche.

Projet non exécuté, du 18 février 1762. 
Disposition einer neuen Orgel vor eine evangelische Kirche

Register ins Manual

1 Montre

2 Bourdon

3 Bourdon

4 Prestant

5 Quinte

6 Doublette

7 Tertz

8 Siflet

9 Cornet

10 Fourniture

11 Cymbal

12 Trompet

13 Vox humana

1 Prestant

2 Bourdon

3 Flöte

4 Nazard

5 Doublette

6 Tertz

7 Larigot

8 Fourniture

9 Cromhorne oder Principal von feinem polirten zinn im Schein ................. 8 Schu Thon oder Coppel, die 2 untersten Octaven von Holtz, die übrigen von Medal, welches eine Composition von Zinn und Bley ist 16 " " die unterste Octav von Holtz, die übrigen Medal ................ 8 " " oder Octav von Zinn ............. 4 " " die Corpora von Zinn, die Füss Medal . . 3 " " oder Superoctav, die Corpora von Zinn, die Füss Medal .................. 2 " "

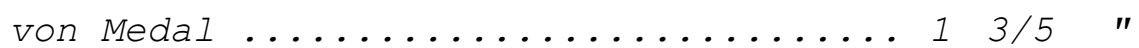
die Corpora von Zinn, die Füss Medal .. 1 " " von Medal .................. 5 facht oder Mixtur, die Corpora von Zinn, die Füss Medal ................. 4 facht die Corpora von Zinn, die Füss Medal .. 3 facht die Corpora von Zinn, die Füss Medal, Mundstück und Zungen von Messing ..... 8 SchuThon disponirt von 4 völligen Octaven, welche inclusive in sich begreifen, Discand, Alt, Tenor und Bass, zusamen 49 Pfeifen davon die gröste klingen soll .... 8 " "

\section{Register im Positif}

von feinem polirten Zinn im Schein.... 4 Schu Thon die grösten Pfeifen von Holtz, die übrigen von Medal ............. 8 " von Medal ..................... $4 "$ " von Medal .................... $3 "$ die Corpora von Zinn, die Füss Medal . 2 " " von Medal ....................... 1 3/5" von Medal ......................... $11 / 2$ " die Corpora von Zinn, die Füss Medal .. 3 facht die Corpora von Zinn, die Füss Medal, Mundstück und Zungen von Messing ..... 8 SchuThon 
Register vors Pedal

1 Supbass

2 octavenbass

3 Prestant

4 Bomparte von Holtz offen ................ 16 Schu Thon

von Holtz offen ............... 8 "

von Medal .................... $4 "$

die Corpora von Holtz, die Füss von

Zinn, die Mundstück und Zungen Messing 16 " "

5 Trompettenbass von Zinn, die Füss Medal ......... 8 " "

6 Claironbass von Zinn, die Füss Medal .......... " " forte, Tremblant doux.

Die 3 Manualclavire sollen jedes aus 4 völligen Octaven oder 49 Clavibus bestehen, welche im Bass mit C Cis D Dis und sich im Discand mit c''' endigen, von Ebenholtz und fein sauber gearbeitet und soll sich nach Erfortern das Manual- und Positifclavir miteinander kopplen oder zusammen ziehen lassen.

Das Pedalclavir aber soll aus 2 fölligen Octaven bestehen. Das Trompettenregister im Manual soll 2 züge haben, einen vor den Bass, den andren vor den Discand.

4 Blassbälge sollen gemacht werden, davon jeder 6 Schu lang, und 3 Schu 4 Zoll breyt seyn soll und aus 6 Falten bestehen.

Die zu diesem Werck erforderliche Windladen sollen von beindürrem eychenem Holtz und apart dazu bereitetem Leder verfertigt werden und also angelegt seyn, damit man bequem dazu kommen kan. Wie nicht weniger sollen die Blasebälge, Canaele und die gantze Structur und Mechanic des gantzen Werks mit allem möglichen Fleiss so gearbeitet seyn, damit alles von einer beständigen Dauer seyn möge.

Vor ein solches Werk in meinen Kosten zu verfertigen und alle zu dem Orgelwesen benötigte Matherialien als Zinn, Bley, Holtz, Leder, Messing und andere dazu gehörige Sachen, ausser dem Orgelkasten, dazu anzuschaffen, offeriren über mich zu nehmen um $5350 \mathrm{fl}$. , sage fünftausend dreyhundert fünffzig Gulden, wobey nichts anderes ausgedungen ist, als dass der Orgelkasten föllig fertig auf den Platz gestelt werden soll und wan das Orgelwesen in Strassburg wird verfertigt seyn, dass solches von da nacher Saarbrücken soll abgehohlt werden. Strassburg, den 18 Fabruarij 1762.

Johann Andreas Silbermann

Stadtbücherei Saarbrücken, Archivaliensammlung des Hist. Vereins f. d. Saargegend, Abteilung A, Nr. 382, fol. 1 a - 2 a. 
Colmar, couvent des Catherinettes.

- Projet, non exécuté, par Jean André Silbermann.

- Marché du 25 septembre 1771 avec Jean André Silbermann, avec quittance du 4 octobre 1772 . 
Disposition eines 4. schühigen Orgelwercks mit Ruck=Positive, welches in die Kirche der wohlehrwürdtigen Closterfrauen zu St: Catharinen in Colmar gemacht werden könte.

Register im Manual Werck.

1. Prestant, von feinem pollirtem Englischem Zinn im Schein ............... 4 Schu Thon.

2. Bourdon, die 15. grösten von Holtz von 8. Schu offen, die übrigen von Metall ...... 8 -

3. Nazard, von Metall ..................

4. Doublette, die Corpora von Zinn, die Füss Metall .................. 2 -

5. Tierce, von Metal ....................... 3/5 -

6. Siflet, die Corpora von Zinn, die Füss

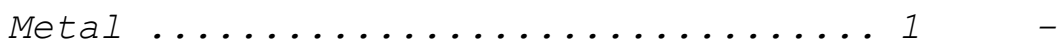

7. Fourniture, die Corpora von Zinn, die Füss Metal .................. facht

8. Cornet, von Metal ................. facht.

9. Fagot=Bass, oder Basson, vom grossen C biss mit d', also 27. Claves, die Corpora und Füss von Zinn, die Mundstück Metal, die Zungen von Messing ............. 8.Schu Thon.

10. Trompette de Reçit, vom mittelsten ds' biss mit d''', die Corpora von Zinn, die Füss Metal, Mundstück und Zungen von Messing 
Das Siflet=Register soll zwey züge haben, einen vor den Discand, den andern vor den Bass.

\section{Register im Ruck Positiv.}

1. Bourdon, die grösten von Holtz, die übrigen von Metal ............... 8.Schu Thon

2. Quint, von feinem polirtem Englischem Zinn, wovon die grösten im Schein stehen ... 3 -

3. Flutte, die 2 grösten von Holtz, die übrigen von Metal .................

4. Doublette, die Corpora von Zinn, die Füss Metal 2 -

5. Tierce, von Metal .................... 3/5 -

6. Pourniture, die Corpora von Zinn, die Füss $\operatorname{Metal} \ldots \ldots \ldots \ldots \ldots \ldots \ldots \ldots \ldots \ldots$ facht

Register im Echo.

1. Bourdon

2. Prestant

3. Nazard

4. Doublette

5. Tierce

6. Flagolet

von 2. Octaven, nemlich von dem mittlern $c^{\prime}$ biss d'''

Tremblant forte

Tremblant doux.

Die 3. Clavire sollen von Ebenholtz und Bein verfertigt seyn, und jedes aus 51. Clavibus bestehen, nemblich von C im Bass biss mit d''' im Discand. 
Das Pedal Clavir soll aus einer Octave oder 13. Clavibus bestehen. Damit sollen sich vermittelst doppelter Ventilen in der Manual= Windlade, von den oben gemelten Manual=Registern die unterste Octave gebrauchen lassen, nemlich vom

Bourdon

Prestant

Fagot=Bass.

Zu diesem Werck sollen zwey Blassbälge gemacht werden, jeder 5 1/2 Schu lang, und 3. Schue breyt, und soll jeder aus 7. Falten bestehen.

Diese zwey Bälge müssen mit Rollen aufgezogen werden, und werden in den Orgel=Kasten gelegt, und hinter demselben aufgezogen.

Den Orgelkasten betreffent, so soll derselbe nach dem darüber vorgezeigtem Plan von sauberm Eychenem Holtz mit eingefastem Getäffel und benötigten Thüren, und geschnittenen Zierathen vom Bildhauer verfertigt werden. Ingleichem soll der Kasten welcher hinter der Orgel die Blassbälge einschliesset, ebenfals von sauberer Schreiner=Arbeit von Eychenholtz gemacht werden.

Diese Arbeit in meinen Kösten in Strassburg zu verfertigen, alle dazy gehörige Matherialien als Zinn, Bley, Messing, Holtz, Leder und Leim, und wie sie sonsten Namen haben mögen, anzuschaffen. Ingleichem die Schreiner= Bildhauer und Schlosser= Arbeit in meinen Kösten zu besorgen, so dass von Seiten der wohlehrwürdtigen Closterfrauen, weiter nichts als der Transport, und bey Aufsetzung und Stimmung der Orgel, längstens $31 / 2$ Woche der Kosten und Losament gegeben würde, offerire über mich zu nehmen um 5000 Id 
sage fünftausend Livers.

Johann Andreas Silbermann

AHR, Série H (Couvent des Catherinettes), Dossier 12. 
Disposition eines Orgelwercks, welches in die Kirche der wohlehrwürdtigen Closterfrauen zu St: Catharinen gemacht werden soll.

$$
\text { Register im Manual. }
$$

1. Montre, davon sollen die 7. grösten Pfeiffen von Holtz inwendig gestellet werden, die übrigen von feinem pollirten Englischen Zinn im Schein .........8 Fuss Thon

2. Prestant, die Corpora von Zinn, die Fuss Metal ..4

3. Bourdon, die 15. grösten von Holtz, die übrigen von Metal ............ -

4. Nazard, von Metal ................... -

5. Doublette, die Corpora von Zinn, die Füss Metal ..2 -

6. Tierce, von Metal .......................... $3 / 5$ -

7. Larigot, von Metal ........................ $1 / 2$ -

8. Siflet, die Corpora von Zinn, die Füss Metal .. 1 -

9. Cornet, von Metal .................. 5 facht.

10. Fourniture, die Corpora von zinn, die Füss Metal .. 3. facht.

11. Fagot=Bass oder Basson, vom grossen C. biss mit d.' also 27. Claves, die Corpora und Füss von Zinn, die Mundstück Metal, die Zungen von Messing 8.Fuss Thon

12. Trompette de Reçit, vom mittelsten ds' biss mit d.''' die Corpora von Zinn, die Füss Metal, Mundstück und Zungen von Messing. 
Das Siflet = Register soll zwey züge haben, damit um mehrerer Veränderung willen der Bass und Discant jedes besonders kan gezogen werden.

Register im Ruck Positive.

1. Bourdon, die grösten von Holtz, die übrigen Metal ................. 8 Schu Thon

2. Flutte, einige der grösten von Holtz, die andern Metal ................. 4

3. Quinte, die grösten von feinem polirtem Zinn im Schein ................... 3

4. Doublette, die Corpora von Zinn, die Füss von

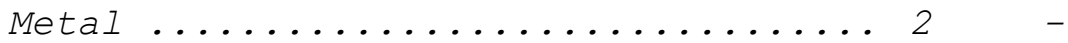

5. Tierce, von Metal ...................... 3/5 -

6. Fourniture, die Corpora von Zinn, die Füss $\operatorname{Metal} \ldots \ldots \ldots \ldots \ldots \ldots \ldots \ldots \ldots \ldots \ldots$ facht Register im Echo.

1. Bourdon

2. Prestant

3. Nazard

von zwey Octaven, nemlich vom $c^{\prime}$

4. Doublette biss mit d.' ''

5. Tierce

6. Flagolet

Tremblant forte Tremblant doux. 
Die 3. Clavire sollen von Ebenholtz und Bein verfertigt seyn, und jedes aus 51. Clavibus bestehen, welche anfangen mit C. im Bass, und sich im Discant mit d.''' endigen.

Das Pedal Clavir soll aus einer Octave oder 13. Clavibus bestehen. Damit sollen sich vermittelst doppelter Ventilen in der Manual = Windlade, von den obgemelten Manual = Registern die unterste Octave gebrauchen lassen, vom

Montre

Bourdon

Prestant

Fagot $=$ Bass .

Was den Thon betrifft worein die Orgel soll gestimmet werden, so ist dazu der Frantzösische gewählet worden.

Zu diesem Werck sollen zwey Blassbälge gemacht werden, jeder 5 1/2. Schue lang, und 3. Schue breyt, und soll jeder aus 7. Falten bestehen. Diese zwey Bälge müssen mit Rollen aufgezogen werden, und werden in den Orgel = Kasten gelegt, und hinter demselben aufgezogen.

Den Orgelkasten betreffend, so soll derselbe nach dem darüber vorgezeigtem Plan von sauberm Eychenem Holtz mit eingefastem Getäffel und benötigten Thüren, und geschnittenen Zierathen vom Bildhauer verfertigt werden. Ingleichem soll der Kasten welcher hinter der Orgel die Blassbälge einschliesset, ebenfals von sauberer Schreiner = Arbeit von Eychenholtz gemacht werden. Diese Arbeit in meinen Kösten in Strassburg zu verfertigen, alle 
dazu gehörigen Matherialien, als Zinn, Bley, Messing, Holtz, Leder und Leim, und wie sie sonsten Namen haben mögen, anzuschaffen. Ingleichem die Schreiner = Bildhauer $=$ und Schlosser $=$ Arbeit in meinen Kösten zu besorgen, so dass von seiten der wohlehrwürdtigen Closterfrauen, weiter nichts als der Transport, und bey Aufsetzung und stimmung der Orgel $31 / 2$ Wochen lang vor mich und zwey Gesellen der Kosten und Losament gegeben würde.

Vor diese vorbeschriebene Arbeit ist von denen wohlehrwürdtigen Closterfrauen zu zahlen versprochen worden die Summa von 5300. Livers, sage fünftausend dreyhundert Livers. Und zwar soll die Helffte von dato an in einem halben Jahr bezahlet werden, und denen 2 Gesellen 1 Louisd'or zu einem Trickgeld gereichet werden. Colmar den 25. September 1771.

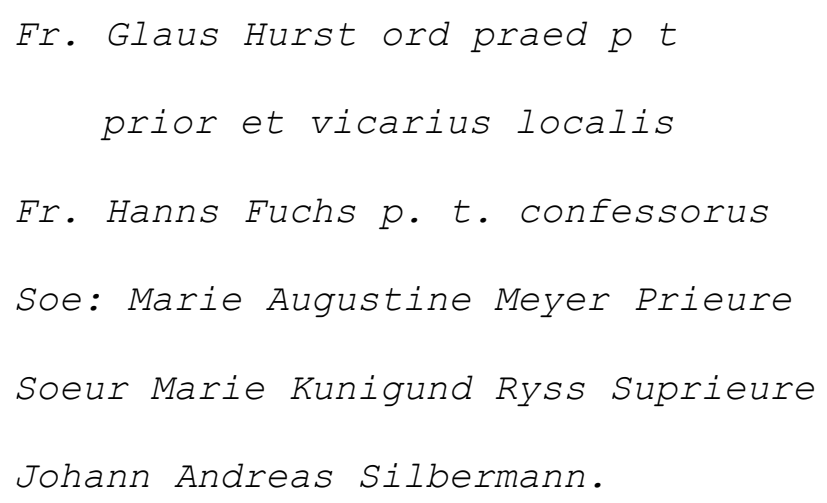

Dass nach Verfertigung der neuen Orgel von denen wohlehrwürdtigen Closterfrauen vollkommen laut dieses Accords bezahlt worden, solches wird hiemit nebst schuldigster Dancksagung beschienen Colmar den 4. October Johann Andreas 1772. Silbermann 
Meissenheim, église protestante.

- Devis du 27 février 1772 par Jean André Silbermann, valant marché.

- Quittances par Jean André Silbermann. 
Disposition einer neuen Orgel, welche [in] die Kirche zu Meissenheim gemacht werden könte.

Register im Manual

1 Montre, wovon die 6. grösten inwendig von Holtz, die übrigen aber auswendig in den Schein von feinem Englischen polirtem zinn gesetzt würden ........ 8 Schu Thon

2 Bourdon. wie gewöhnlich die 15. grösten von Holtz, die übrigen von Metal, dass ist eine Composition von Zinn und Bley ... 8 -

3 Prestant. von Zinn ................. -

4 Flutte. von Metal .................. 4 -

5 Nazard.

6 Doublette. von Metal ..................... 3 -

7 Tierce. die Corpora von Zinn, die Füss Metal .. 2 von Metal ...................... $13 / 5$

8 Cornet.

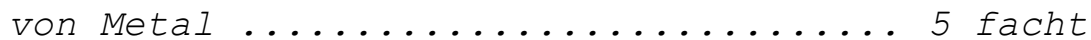

9 Fourniture die Corpora von Zinn, die Füss Metal . . 3 facht 10 Cimbale. die Corpora von Zinn, die Füss Metal .. 2 facht

\section{Register im Pedal}

1 Supbass von Holtz 8 Schu gedeckt...........16Schu Thon

2 Octavenbass von Holtz ................. schu

3 Prestant

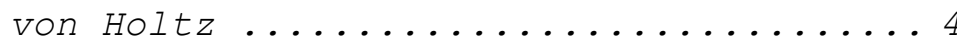

Tremblant forte Tremblant doux.

Das Manual = Clavier soll von Ebenholtz und Bein verfertigt seyn, und aus 4. völligen Octaven bestehen, oder 49. Clavibus. Das Pedal = Clavier aber aus einer Octav, oder 13. Clavibus. Den Thon betreffend, so sollen die Register in den Kammerthon gestimmet werden.

Die zwey Blassbälge sollen jeder aus 7. Falten bestehen, und lang seyn 6. Frantzösische Schue, und 3. Schu 4. Zoll breyt. Die zu diesem Werck erforterende drey Windladen, sollen von beindürrem Eychenem Holtz, und apart dazu bereitetem Leder verfertigt werden. Wie nicht weniger sollen die Blassbälge, Canaele und Mechanick von dauerhaften Matherialien verfertigt seyn. Was die äusserliche Façon des Orgelkasten betrifft, so wird derselbe nach schon vorgewiesenem Abriss verfertiget, jedoch dass dersel- 
be beynahe um 2. Schu breyter, und auch so viel höher gemacht wird. Demnach werden 39. hellpolirte zinnene Pfeiffen ins Gesicht zu stehen kommen.

Der Orgelkasten soll vornen und auf den Seiten von sauberm Eychenem Holtz mit geschnittenen Zierathen vom Bildhauer sich zeigen. Die Ruckwand desselben, nebst dem Kasten worein das Pedal gestellet wird, soll von Dannenholtz, gleichwohl von eingefaster Arbeit verfertigt seyn. Ein solches Werck in meinen Kösten zu verfertigen, alle dazu erforterliche Matherialien als Zinn, Bley, Messing, Holtz, Leder, Leim, und wie sie sonst Namen haben mögen dazu zu forniren; ingleichen die Schreiner $=$ Bildhauer $=$ und $=$ Schlosser $=$ Arbeit $z u$ besorgen, so dass von Seiten einer Gemeinde nur die freye $\mathrm{Zu}=$ und Abfuhr zu besorgen, und mir nebst zweyen Gesellen gegen 3. Wochen das Losament und die Verköstigung zu verschaffen, offerire über mich zu nehmen vor $1800 \mathrm{fl}$. sage eintausend acht hundert Gulden Französisch Geld, die Louisd'or zu 12. fl. oder den doppelten Thaler zu 3. fl. gerechnet Strassburg den 27. February 1772 .

Johann Andreas Silbermann.

Als ein Angeld auf diesen Accord habe ich Ends unterschriebener den 26. Mertz 1774. empfangen ...........300 fl Ferner den 28. November 1775. wider.... $300 \mathrm{fl}$ Und dann nach Verfertigung der Orgel den 21. May 1776. den Rest von ....... 1200 fl

Summa $1800 \mathrm{fl}$

Für welches hiemit nebst schuldigster Dancksagung quittire. In Meissenheim den 21. May

$$
1775 .
$$

benebst 18 fl Trinkgeld Johann Andreas Silbermann
Johann Andreas

Silbermann.

Meissenheim, Gemeindearchiv 
Bouxwiller, église protestante.

Copie du marché du 6 février 1777 avec Jean André Silbermann. 
Akkord über die Orgel, welche in die evangelisch lutherische Kirche zu Buchsweiler gemacht werden soll.

I. Register im Manualwerk.

1. Montre. Die grossen Pfeifen von feinem englischen Zinn

8 Schuh

2. Bourdon. Die 2 ersten Oktaven wie gewöhnlich von Holz, die übrigen von Metall

16 Sch.

3. Bourdon. Die erste Oktave von Holz, die übrigen Metall.

8 Sch.

4. Prestant. Von Zinn.

4 SCh.

5. Nazard. Von Zinn.

3 Sch.

6. Doublette. Die Corpora von Zinn, die Füss Metall 2 Sch.

7. Tierce von Metall

$13 / 5 \mathrm{Sch}$.

8. Cornet von Metall

5 facht

9. Fourniture. Die Corpora von Zinn, die Füss Metall

3 facht

10. Cimbale, die Corpora von Zinn, die Füss Metall

2 facht

11. Siflet Corpora Zinn, Füss Metall

1 Sch.

12. Trompette. Die Corpora von Zinn, Fuss Metall, Mundstück und Zungen Messing

8 Sch.

13. Voix humaine. Corpora von Zinn, Füss Metall, Mundstück und Zungen Messing

8 Sch.

II. Register im Positiv.

1. Prestant. Von feinem englischen Zinn 4 Sch.

2. Bourdon. Die grössten von Holz, die übrigen Metall 8 Sch.

3. Nazard, von Metall 3 Sch.

4. Doublette, die Corpora von Zinn, die Füss Metall 2 sch.

5. Tierce, von Metall $13 / 5$ Sch.

6. Flutte, von Metall 4 Sch.

7. Fourniture, Corpora von Zinn, Füss Metall 3 facht

8. Cromhorne, die Corpora von Zinn, Füss Metall, Mundstück und Zungen Messing

8 Sch.

III. Register im Pedal.

1. Supbass von Holz, 8 Schuh gedeckt 16 Sch.

2. Oktavbass von Holz, offen $8 \mathrm{Sch}$.

3. Bompartte, die Corpora von Holz, die Füss von Zinn, Mundstück und Zungen Messing 16 Sch.

4. Trompette, die Corpora von Zinn, Füss von Metall, Mundstück und Zungen Messing

8 Sch. 
Die Orgel soll im Kammerton gestimmt werden. Zwei Tremulanten, den einen langsam, den andern geschwind. Das Trompetteregister im Manual soll 2 züge haben, damit man den Bass und Discand jeden besonders spielen kann. Die 2 Manualclaviere sollen von Ebenholz und Bein verfertigt sein. Das Pedalclavier soll in $C$ anfangen und sich im $g$ der zweiten Octave endigen. Zu diesem Werk sollen 3 Blasbälge gemacht werden, davon jeder 6 französische Schuh lang und 3 Schuh 4 zoll breit sein soll, und jeder aus 7 Falten bestehen. Die 5 zu diesem Werk gehörigen Windladen sollen von beindürrem, gespaltenem eichenem Holz und apart dazu bereitetem Leder verfertigt werden, wie nicht weniger die ganze Mechanik des Werks von dauerhaften Materialien verfertigt werden. Die beiden Kästen des grossen Werks und des Positivs sollen von sauberem eichenem Holz mit sauber geschnittenem Zierat und auf der Höhe in der Mitte mit dem herrschaftlichen Wappen von 2 Löwen gehalten und dem Fürstenhut versehen sein. Der Kasten aber des Pedals nebst der Rückwand der Orgel von Tannenholz, gleichwohl aber von eingefasster Arbeit.

Für dieses Werk, welches ich bis zu End des Sommers 1778 aufzustellen verspreche in meinen Kosten zu verfertigen, alle dazu erforderliche Materialien als Zinn, Blei, Leder, Holz, Messing und andere Sachen mehr dazu fournieren, wie nicht weniger die Schreiner-, Schlosser- und Bildhauerarbeit über mich zu nehmen, fordere ich 4400 Gulden Elsasser Valor, wie er zu Anfang dieses 1777 Jahres als dem dato dieses geschlossenen Accords gilt. Welche Summe von seiten eines evang. Iuth. Consistorii auch dafür versprochen worden. Wobei von seiten wohlbesagten Consistoriums weiter nichts als der Transport und bei der Aufstellung des Werkes das Losament für mich und meine Leute zusammen in 4 Personen bestehend, besorgt werden darf.

Gegenwärtiger Accord ist auf oben beschriebene Art zwischen dem evang. Iuth. Consistorio zu Buchsweiler und Johann Andreas Silbermann geschlossen und von beiden Teilen unterschrieben worden.

Buchsweiler, den 6. Februarii 1777.

F. Kauffmann, Die Silbermann-Orgel zu Buchsweiler, Elsassland. Lothringer Heimat, 7, 1927, p. 245-247. 
Strasbourg, église protestante Saint-Pierre-le-Jeune.

- Description de l'orgue après les travaux de J.J.Baldner en 1642.

- Marché du 31 juillet 1779 avec Jean André Silbermann, avec quittances de 1779 et 1780 . 
Description de l'orque de l'église Saint-Pierre-le-Jeune à Strasbourg en 1642 .

Gantze und vollkommliche Beschreibung dess Orgelwercks zum Jungen St: Peter, wie dieselbige /: nachdem sie von Herrn Hanss Jacob Baldneren Burger und Orgelmacher allhie zu Strassburg in Anno 1642. renovirt und verfertiget./ befunden worden, dessgleichen was in specie daran gemacht und verbessert worden ist.

Erstlichen so bestehet das gantze Werck in nachfolgenden Registeren

Manual Register.

Principal.

Copel.

octav.

Superoctav.

Quint.

Flöten.

Mixtur.

Schweiglein.

zincken.

zimbel.

Manual Register, alss Prineipal, Copel, Octav, Superoctav, Quint, Flöten, Mixtur, Schweiglein, Zincken und Zimblen, so all von zinnen Pfeiffen, stehen auf einer absonderlichen Windladen in der Mitten.

Pedal Register, alss Principal, Octav, Mixtur und Posaunen, so auch all von zinnen Pfeiffen, biss auff drey so der Orgelmacher von Holtz gemacht, stehen auf zwo absonderlichen Windladen, nemblichen die eine auf der rechten, und die andere auf der lincken Seiten.

Archives de la paroisse protestante Saint-Pierre-le-Jeune à Strasbourg. Saal=Buch 1724. p. 229-230.
Pedal Register.

Subbass.

Principal.

octav.

Mixtur.

Posaunen. 
31.7.1779. Marché pour la construction de 1'orgue de l'église protestante St. Pierre-le-Jeune à Strasbourg.

Disposition einer neuen Orgel, welche in die Evangelische Kirche zum Jungen St: Peter hier in Strassburg soll gemacht werden.

Register im Manual.

1. Montre, die grösten Pfeiffen von feinem Englischen Zinn, polirt..........8 Schu Thon

2. Bourdon, die zwey ersten Octaven von Holtz, die übrigen Metal .................16---

3. Bourdon, die erste Octave von Holtz, die übrigen Metal ................. 8 ---

4. Prestant, die Corpora Zinn, die Füss Metal ..... 4---

5. Nazard, die Corpora von Zinn, die füss Metal .. 3---

6. Doublette, ebenso .....................

7. Tierce, von Metal ................... $3 / 5$ -

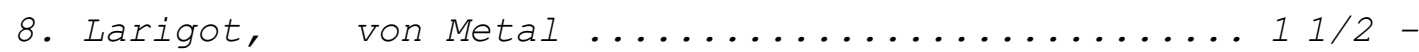

9. Cornet, von Metal..................... 5 facht

10. Fourniture, die Corpora von Zinn, die Füss Metal .. 3 facht

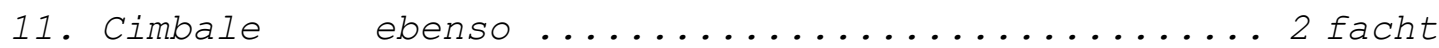

12. Trompette, die Corpora von Zinn, die Füss Metal, Mundstück und Zungen von Mössing ..... 8 Schu.

Register im Pedal.

13. Supbass von Holtz, 8 Schu, gedeckt.........16 Schue Thon

14. Octavenbass, von Holtz offen ...............--

15. Bompartte, die Corpora von Holtz, die Füss von Zinn, Mundstück und Zungen Mössing ... 16---

16. Trompette, ebenso ................. 8 ---

Zwey Tremulanten, der eine langsam der andere geschwind.

Die Orgel soll in dem so gewöhnlichen Kammerthon gestimmet werden.

Das Trompetten=Register im Manual soll zwey Züge haben, den einen für den Discand, den andern für den Bass.

Das Manual Clavier soll von Ebenholtz und Elffenbein verfertigt werden, und aus 51. Clavibus bestehen, nemblich im Bass von C C\# D D\# E F \&: und im Discand biss ins d''' gehen. 
Das Pedal=Clavier aber soll im C anfangen, und sich im g. der zweyten Octave endigen.

Drey Blassbälge, wovon jeder 6 Schu lang, und in der Breyte 3 Schu 4 Zoll Haben soll. Jeder soll aus 7. Falten bestehen.

Die 4. zu diesem Werck erfordernte Windladen, sollen von beindürrem gespaltenen eychenem Holtz, und apart dazu bereitetem Leder verfertigt seyn, wie nicht weniger die gantze Mechanic des Werks von dauerhafften Matherialien verfertigt werden.

Das Gehäuse oder Orgelkasten soll vornen und auf der Seite von eychenem, die Ruckwand desselben nebst dem Pedalkasten aber von thannenem Holtz verfertigt werden.

Für ein solches Werk in meinen Kösten zu verfertigen und auf den Platz zu stellen, und alle dazu erforterliche Matherialien, als Zinn, Bley, Leder, Holtz, Mössing und andre Sachen mehr dazu zu forniren, wie nicht weniger die Schreiner= Schlosser= und die höchstnöthige Bildhauer= Arbeit über mich zu nehmen, ist von Seiten Eines HochwohlEhrwürdtigen Capitels, nebst Überlassung der alten Orgel, die Summa von 4600 Livers, sage viertausend sechshundert Livers, die Louisd'or zu 24 Livers oder das drey Gulden Stück zu 6. Livers, bewilliget worden.

Vû et approuvé en Chapitre, en consequence nous sommes convenus avec Monsieur Silbermann que les susdits quatre mille six cens livres seront payes en trois termes, dont le premier luy a été payé en signant les presentes, le second le sera lorsque son ouvrage sera fait en moitié, et le dernier lorsqu'il sera entierement fini et approuvé: ce faisant luy avons aussitot payé ledit premier terme par la somme de quinze cents livres, et signé avec luy !es presentes faites doubles à Strasbourg ce 31. juillet 1779.

Lantz

Doyen de St. Pierre le Jeune.

Dass ich Ends unterschriebener auf diesen Accord empfangen habe 750 f. oder 1500 Livers, solches bescheine hiemit Strassburg den 31. Julius

Johann Andreas

1779. Silbermann 
Dass von E. Hochwürdtigen Kapitel den zweyten Zahlungs Termin mit 750 f. oder 1500 Livers empfangen habe, bescheine

Strassburg den 26. February 1780 .
Johann Andreas

Silbermann

Dass von E. Hochwürdtigen Kapitel den letzten Zahlungs Termin mit $800 \mathrm{f}$. wohl empfangen, da also für die völlige Zahlung dieses Accords nebst schuldigster Danksagung quittire Strassburg den 15. Julius Johann Andreas 1780. Silbermann

ABR. G 5151. 
Bibliographie 


\section{Plan de la bibliographie.}

I. Histoire d'Alsace. Généralités.

II. Périodiques.

III. Dictionnaires, catalogues.

IV. Facture d'orgues, histoire de l'orgue en général.

V. Buffets d'orgues.

VI. L'orgue en France.

VII. L'orgue en Alsace.

VIII. La musique en Alsace. Les organistes.

IX. Monographies de localités et d'églises.

X. Les Silbermann de Strasbourg et leurs oeuvres.

XI. Les orgues de la cathédrale de Strasbourg.

III. Les Silbermann facteurs d'instruments à clavier.

XIII. Le tempérament des orgues des Silbermann.

XIV. Jean André Silbermann collectionneur et historiographe.

XV. Gottfried Silbermann et ses oeuvres en Saxe.

XVI. Les élèves de Gottfried Silbermann.

XVII. Les Silbermann et l'orgue de Jean-Sébastien Bach.

XVIII. Mozart et l'orgue.

IXX. L'orgue en Allemagne et en Autriche.

XX. L'orgue en Suisse.

XXI. L'orgue aux Pays-Bas. 
422

I. Histoire d'Alsace. Généralités. 
Affiches de Strasbourg. Strasburgisches Wochenblatt.

Strasbourg, 1793 .

Albrecht, P. Dionysius

History von Hohenburg oder St. Odilienberg, 1751.

Annuaire du Bas-Rhin, 1782.

Baquol, J.

Dictionnaire géographique, historique et statistique du Haut et du Bas-Rhin. 2/Strasbourg, 1851.

Boislisle, Arthur de

Le grand hiver et la disette de 1709. Revue des

questions historiques 73 (avril 1903) p. 442-509. 74

(octobre 1903) p. 486-542.

Bopp, M.-J.

Die evangelischen Geistlichen und Theologen in Elsass

und Lothringen. Neustadt a.d. Aisch, 1959, 709 p.

Chailley, Jacques

Précis de Musicologie. Paris,1958, 431 p.

Courrier du Bas-Rhin. Strasbourg, 1817.

Gerber, E.L.

Historisch-Biographisches Lexikon der Tonkünstler. 2 vol. 1790/92. Leipzig.

Gerbert, Martin

Korrespondenz des Fürstabtes Martin II. Gerbert von St. Blasien, éd. Georg Pfeilschifter 2 vol. 684 et 670 p. Karlsruhe, 1931. 1934.

Haug, Hans

L'artisanat d'art à Strasbourg. Strasbourg,1952. 19 p. ill.

Haug, Hans

L'Art en Alsace. Paris, 1962. 301 p., 318 ill., 6 pl. couleurs. 
Haug, Hans

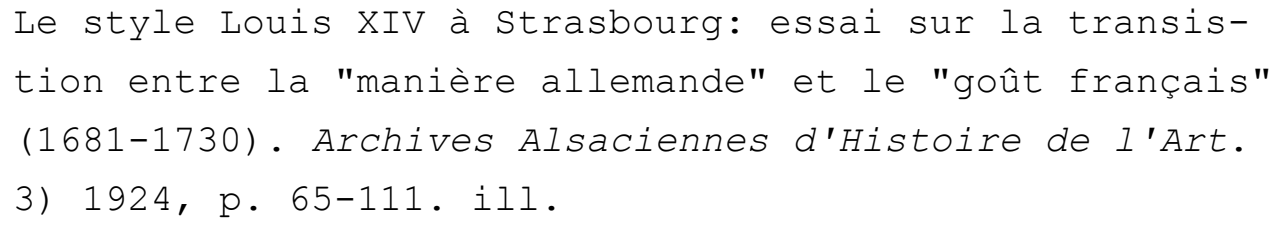

Heitz, F.-C.

Das Zunftwesen in Strassburg. Geschichtliche Darstellung begleitet von Urkunden und Aktenstücken. Strasbourg 1856 .

Hermann, Jean-Fréd.

Notices historiques, statistiques et littéraires sur la ville de Strasbourg. vol 2. Strasbourg, 1819.

Hiller

Wöchentliche Nachrichten und Anmerkungen die Musik betreffend. 1766 .

Himly, François-J.

Glossaire Allemand-Français des institutions de I'ancienne Alsace. Strasbourg, 1965. 75 p. (Publications de la Fédération des Sociétés d'Histoire et d'Archéologie d'Alsace Tome IV).

Hoffmann, Charles

L'Alsace au dix-huitième siècle au point de vue historique, judiciaire, administratif, économique, intellectuel, social et religieux. publié par A.-M.-P. Ingold. Colmar, 4 t., 1906-1907.

Horrer, Ph.X.

Dictionnaire géographique, historique et politique de I'Alsace. Tome 1. Strasbourg, 1787.

Juillard, Etienne Les péages en Alsace au XVIII siècle, Revue d'Alsace, t.84 (1937), p. 16-34. 
König, Josef Hermann

Die katholischen Körperschaften des Unterelsasses vor und während der grossen Revolution, Strasbourg, 1915, $186 \mathrm{p}$.

Livet, Georges

L'Intendance d'Alsace sous Louis XIV, 1648-1715, Strasbourg, 1956, $1084 \mathrm{p}$.

Livet, Georges

Le XVIIIe siècle et l'esprit des lumières, in Histoire de l'Alsace (éd. Ph. Dollinger), Toulouse, 1970, p.305 355 .

Livet, Georges

La Guerre de Trente Ans et les traités de Westphalie. La formation de la province d'Alsace (1618-1715), in Histoire de l'Alsace (éd. Ph. Dollinger), Toulouse, 1970, p. $259-303$.

Murr, Christoph Gottlieb von

Journal zur Kunstgeschichte und zur allgemeinen Litteratur, Achter Theil, Nürnberg, 1780.

Schmidt, Charles

Wörterbuch der Strassburger Mundart, Strasbourg, 1896 , $123 \mathrm{p}$.

Seyboth, Adolphe

Das Alte Strassburg, vom 13. Jahrhundert bis zum Jahre 1870, Geschichtliche Topographie, Strasbourg,1890, ill.

Seyboth, Adolphe

Strasbourg historique et pittoresque, depuis son origine jusqu'en 1870, Strasbourg, 1898.

Sitzmann, E.

Dictionnaire de biographie des hommes célèbres de I'Alsace, Rixheim, 1909-1910, 2 vol.

Wochentliche Strassburger Frag= und Anzeigungs= Nachrichten, Strasbourg. 
426

Wolff, Christian

Guide des recherches généalogiques en Alsace, Strasbourg, 1975, 257 p. 
II. Périodiques. 
Acta Organologica. Berlin,1967 et suiv.

Ars Organi. Berlin,1952/1953 et suiv.

Caecilia. Organ des Elsässischen Vereins für Kirchenmusik. Organe de la Société alsacienne de musique religieuse. Colmar, Strasbourg, 1884 et suiv.

Connaissance de I'Orgue. Paris, 1971 et suiv.

Echos des sanctuaires de Sainte-Odile. Publications de l'Institut Saint-Léon IX, Strasbourg, 1920-1921, 1930-1932.

L'Est musical. Revue trimestrielle. Strasbourg, 1, 1939, $\mathrm{N}^{\circ} 1-2$. Instrumentenbau-Zeitschrift. Constance, 1946/47 - 1956. Siegburg, 1957 et suiv.

ISO-Information. Lauffen, 1969 et suiv.

Musik und Kirche. Kassel, 1929 et suiv.

The Organ. Londres, 1921 et suiv.

The Organ Yearbook. Amsterdam, 1970 et suiv.

L'Organo. Brescia, 1960 et suiv.

Het Orgel. Rotterdam, 1904 et suiv.

L'Orgue. Paris, 1930 et suiv.

Orgues Historiques. Saint-Michel-de-Provence, 1962 et suiv.

Renaissance de I'orgue. Paris, 1968 - 1970. 8 Nos.

La Tribune de 1'Orgue. Lausanne, 1948 et suiv. 
III. Dictionnaires, catalogues. 
Alberti, H.J. von

Mass und Gewicht. Berlin, 1957.

Baquol, Jacques

L'Alsace ancienne et moderne, ou Dictionnaire géographique, historique et statistique du Haut et du BasRhin. Strasbourg 2/1851. 556 p.

Dictionnaire de la Musique publié sous la direction de Marc Honegger. I-II. Les hommes et leurs oeuvres. Paris, 1970, 1200 p., ill.

Eitner, R.

Biographisch-bibliographisches Quellen-Lexikon der Musiker und Musikgelehrten christlicher Zeitrechnung bis Mitte des 19. Jahrhunderts. Graz, 2/1959-1960, 11 t. en 6 vol.

Fétis, F. -T.

Biographie universelle des musiciens et bibliographie générale de la musique. Paris, 2/1870-1875, 8 t, en 4 vol.

Forkel, Johann Nikolaus Musikalischer Almanach für Deutschland auf das Jahr 1782. Leipzig, 1782 .

Gerber, E.L.

Historisch-Biographisches Lexikon der Tonkünstler. 2 vol. Leipzig, 1790/1792.

Graaf, G.A.C, de

Literatuur over het orgel. Amsterdam, 1957, 71 p.

Himly, F.-J. (éd. )

Arts et techniques du document écrit. Strasbourg.

Archives Départementales 14 octobre - 16 novembre 1963. Strasbourg, 1963, $120 \mathrm{p}$.

Die Musik in Geschichte und Gegenwart. Allgemeine Enzyklopädie der Musik. Kasse1, 1949/51 - 1968, 14 vol., ill., mus. 
Refardt, Edgar

$$
\begin{aligned}
& \text { Historisch-biographisches Musikerlexikon der Schweiz. } \\
& \text { Leipzig, 1928, XV - } 355 \text { p. }
\end{aligned}
$$

Das Reichsland Elsass-Lothringen. 3 vol. Strasbourg,1898-1903.

Reuter, Rudolf

Bibliographie der Orgel. Literatur zur Geschichte der Orge1 bis 1968. Kasse1, 1973, 267 p.

Riemann

Musiklexikon, Personenteil 1-2, Sachteil. Mayence, 12/1959-1967, 3 vol., mus.

Schoepflin, J.-D.

Alsatia illustrata, celtica, romana, francica. 2 vol. Colmar, 1751 et 1761 .

Sitzmann, E.

Dictionnaire de biographie des hommes célèbres de l'Alsace. Rixheim, 1909-1910, 2 vol.

Thieme, U. - Becker, F.

Allgemeines Lexikon der Bildenden Künstler. 37 vol. Leipzig 1907-1950.

Walther, J. G.

Musikalisches Lexicon oder musikalische Bibliothec. Leipzig 1732. Réédition Kassel, 1953 (Documenta musicologica, série I).

Wickersheimer, E.

Catalogue général des manuscrits des bibliothèques publique de France. Départements. Tome 47. Strasbourg. Paris 1923. XXII-591 p.

Zedler, Johann Heinrich

Grosses Vollständiges Universal Lexicon aller Wissenschafte und Künste. Halle et Leipzig, 64 vo1., 17321770. Supplément 4 vol., 1751-1754. 
IV. Facture d'orques, histoire de l'orque en général. 
Adelung, Wolfgang

Einführung in den Orgelbau. Leipzig, 1955, 186 p., ill.

Andersen, Paul-Gerhard

Organ Building and Design. Londres, 1969, 359 p., ill.

Burney, Charles

The Present State of Music in Germany, The Netherlands and United Provinces. Londres, 1773, 2/1775. trad. all. de J.J.C. Bode, Carl-Burney's Tagebuch seiner MusikaIischen Reisen. II. Durch Flandern, die Niederlande und am Rhein bis Wien. Hambourg 1773. III. Durch Böhmen, Sachsen, Brandenburg, Hamburg und Holland. Hambourg, 1773. Facsimile Kasse1, 1959.

Burney, Charles

The Present State of Music in France and Italy. Londres 1771, 2/1773. Trad. all. de C.D. Ebeling, Carl Burney's ... Tagebuch einer Musikalischen Reise durch Frankreich und Italien, Hambourg, 1772. Facsimile Kasse1, 1959.

Dufourcq, Norbert

L'Orgue. Paris, 1948, 126 p.

Ellerhorst, Winfred

Handbuch der Orgelkunde. Einsiedeln, 1936, 850 p., ill.

Erici, Einar

Inventarium över bevarade äldre kyrkorglar i Sverige. Stockholm, 1965, 315 p., ill.

Frotscher, Gotthold

Geschichte des Orgelspiels und der Orgelkomposition. 2 vol. Berlin, 1935. 2/1959.

Haake, Walter

Orgeln. Königstein-im-Taunus, 1954, 48 p., ill.

Haake, Walter

Orgeln in aller Welt. Königstein-im-Taunus, 1965, 112 p., ill. 
Hopkins, E.J. et Rimbault, E.F.

The Organ, its History and Construction. Londres, 1855. $3 / 1877,636 \mathrm{p}$.

Internationales Regulativ für Orgelbau (Wien 25. - 29. Mai 1909). Leipzig et Vienne, 1909.

Jakob, Friedrich

L'Orgue. Lausanne, 1970, 96 p., ill.

Klotz, Hans

Das Buch von der Orgel. Kasse1, 1937. 5/1955, 161 p., ill.

Klotz, Hans

Uber die Orgelkunst der Gotik, der Renaissance und des Barock. Kasse1, 1975, 427 p.

Krams, Peter

Wechselwirkungen zwischen Orgelkomposition und Pedalspieltechnik. Wiesbaden, 1974, 199 p., mus.

Mahrenholz, Christhard

Die Orgelregister. Ihre Geschichte und ihr Bau. Kassel, 2/1942, $329 \mathrm{p} ., 8 \mathrm{pl}$.

Lange, Martin

Kleine Orgelkunde. Kasse1, 1955, 71 p., ill.

Lottermoser, $\mathbf{W}$.

Fehler bei der Umrechnung von Graden der Foernerschen Windwaage in Millimeter-Wassersäule, Das Musikinstrument, XVII, cahier 10, p. 1146 (1968) et XVIII, cahier 8, p. 943 (1969).

Merklin, A.

Geschichte und Entwicklung des Schwellwerkes der Orgel, Zeitschrift für Instrumentenbau. 45. 1925. p. 131.

Quoika, Rudolf

Vom Blockwerk zur Registerorgel. Kasse1, 1966, 88 p., 7 $\mathrm{pl}$. 
Quoika, Rudolf

Das Positiv in Geschichte und Gegenwart. Kasse1, 1957, 119 p., ill.

Raugel, Félix

Les Organistes. Paris, 1923. 2/1962, 103 p.

Ritter, A.G.

Zur Geschichte des Orgelspiels im 14. bis zum Anfange des 18. Jahrhunderts. Leipzig, 1884.

Rössler, Ernst Karl

Klangfunktion und Registrierung. Kasse1, 1952, 68 p.

Rokseth, Yvonne

La musique d'orgue au $X V^{\circ}$ siècle et au début du XVI․ Paris, 1930. 418 p., 8 pl.

Rupp, Emile

Die Entwicklungsgeschichte der Orgelbaukunst. Einsiedeln, 1929. 467 p., ill.

Töpfer, Johann Gottlob

Lehrbuch der Orgelbaukunst nach den besten Methoden

älterer und neuerer ... Orgelbaumeister... 4 vol.

Weimar 1855. revu par P. Smets, Mayence, 3/1936.

Wangemann, $\mathrm{O}$.

Geschichte der Orgel und der Orgelbaukunst von den ersten Anfängen bis zur Gegenwart. Demmin, 1880.

Wangemann, 0 .

Die Orgel, ihre Geschichte und ihr Bau. Leipzig, 1891.

Williams, Peter

The European Organ 1450-1850. Londres, 1966. 336 p., ill. 
436

V. Buffets d'orgues. 
Blanton, Joseph Edwin

The revival of the organ case. Albany, 1965. $127 \mathrm{p} .$, ill.

Blanton, Joseph Edwin

The organ in church design. Albany, 1957, 492 p., ill.

Brommer, Hermann

Colmarer Bildhauer des XVIII. Jahrhunderts, Annuaire de Colmar XXII. 1972. p. 33-65.

Brunzema, Daniel

Die Gestaltung des Orgelprospektes im friesischen und angrenzenden Nordseeküstengebiet bis 1670 und ihre Bedeutung für die Gegenwart. (Abhandlungen und Vorträge zur Geschichte Ostfrieslands, Heft XXXV) Aurich 1958; 72 p., ill.

Hill, A.G.

The organ-cases and organs of the Middle Ages and Renaissance. Londres, 1883. réimpression Hilversum, 1966.

d'Ixnard, Pierre-Michel

Recueil d'architecture. Strasbourg, 1791.

Jacobsson, Johann Karl

Technologisches Wörterbuch oder alphabetische Erklärung aller nützlichen mechanischen Künste, Manufacturen, Fabriken und Handwerken. Berlin, 1781-1794.

Lehni, Roger

Aspects de l'art baroque. Peter Thumb et les maîtres du Vorarlberg. Saisons d'Alsace, $\mathrm{N}^{\circ}$ 32, p. 435-482.

Lévy-Coblentz, Françoise

L'art du meuble en Alsace. Tome 1: Du gothique au baroque 1480-1698. Strasbourg, 1975, 531 p., ill. 
VI. L'orgue en France. 
Arbus, Marie-Réginald A.

Une merveille d'art provençal. Le grand orgue de la

basilique de Saint-Maximin-la-Sainte-Baume et l'histoire générale de l'orgue, Aix-en-Provence, 1955, 132 p., ill.

Bedos de Celles, Dom François

L'Art du facteur d'orgues, Paris, 1766 - 78, 3 vol, infolio, XXXII, 676 p., 137 pl. Réédition en facsimile, Kassel, $1963-66$.

Bouvet, Charles

Les Couperin, Paris, 1919, 304 p., 16 pl.

Brossard, Pierre

La facture d'orgue dans le nord-est de la France au début du l8e siècle. The Organ Yearbook, vol. V, 1974, p. $57-65$.

Brunold, Paul

Le grand orgue de St. Gervais à Paris, Paris, 1934.

Cavaillé-Coll, Cécile et Emmanuel

Aristide Cavaillé-Coll, ses origines, sa vie, ses oeuvres. Paris, 1929, 183 p., ill.

Clicquot, François Henri

Théorie-pratique de la Facture de l'Orgue d'après l'expérience de M. Clicquot, Facteur d'Orgue, dessinée et mis en ordre sur ses modèles en l'année 1789. Edition facsimile Kassel, 1969, 90 p., 87 p1.

Clutton, Cecil

Two early French organs (La Flèche, Poitiers). The Organ, 40, 1961, p. 169.

Diederich, Susanne

Originale Registrieranweisungen in der französischen Orgelmusik des 17. und 18. Jahrhunderts. Kassel, 1975.

Douchain, Olivier

Quinze années d'histoire de l'orgue à la cathédrale de Toul (1740-1755). Documents inédits pour servir à l'histoire de l'orgue en Lorraine. Annales de l'Est, 5e série, 23e année, $\mathrm{n}^{\circ} 2$ (1971) p.157 - 205. 
Douchain, Olivier

Les orgues anciennes de Lorraine. A 31, Mensuel de la Lorraine et du Luxembourg, décembre, 1973, p. 59 - 64, ill.

Douglass, Fenner

The language of the classical French Organ. A musical tradition before 1800. (Yale Studies in the History of Music, (5) Yale University, 1969, 235 p., ill.

Dufourcq, Norbert

Les orgues et organistes de Saint-Germain-des-Prés du XVIIe siècle à nos jours. Bulletin paroissial de saintGermain-des-Prés, mars 1927.

Dufourcq, Norbert

Documents inédits relatifs à l'orgue français (XIVXVIIIe s.). Paris, 1934, 486 p.

Dufourcq, Norbert

Esquisse d'une histoire de l'orgue en France du XIIIe au XVIIIe siècle. Etude technique et archéologique de l'instrument. Paris, 1935, 508 p., ill.

Dufourcq, Norbert

Le livre de l'orgue français. Tome I; Les sources, Paris, 1973, 700 p., XLV pl. Tome II: Le buffet, Paris, 1969, 309 p., CCLXXII pl. Tome III:/1: La facture, de la première à la seconde Renaissance, Paris, 1975, 198 p., XXIV pl. Tome IV: La musique, Paris, 1972, 202 p., XXIV pl.

Dufourcq, Norbert et Krug-Basse, Jean

A propos de l'orgue dit "des Couperin", Mélanges François Couperin, publiés à l'occasion du Tricentenaire de sa naissance 1668-1968. Paris, 1968, p. 72-104, ill.

Engramelle, Joseph

La tonotechnie ou l'art de noter les cylindres. Paris, 1775, 236 p., 5 pl. Réédition Genève, 1971. 
Fellot, Jean

L'orgue classique français. Musique de tous les temps. Numéro spécial. Saint-Michel-de-Provence, 1962. 63 p., ill., avec disque $17 \mathrm{~cm}$.

Fleury, Paul de

Dictionnaire biographique des facteurs d'orgues nés ou ayant travaillé en France, Paris, s.d. (1921), 197 p.

Fromageot, Paul

Orgues et organistes de Saint-Germain-des-Prés. Bulletin de la société historique du VIe arrondissement de Paris, t.IX, 1906, ill.

Gardien, Jacques

L'orgue et les organistes en Bourgogne et en FrancheComté au dix-huitième siècle. Paris, 1943. 577 p., 26 $\mathrm{Pl}$.

Gay, Dom Claude

Notes pour servir à la registration de la musique d'orgue française des XVIIe et XVIIIe siècles. L'Organo 2 (1961), p. 169-199.

Gravet, Nicole

L'orgue et l'art de la registration en France du XVIe siècle au début du XIXe siècle. L'Orgue, n 100 (1961), p. 202-257.

Hamel, Marie-Pierre

Nouveau manuel complet du facteur d'orgues, ou traité théorique et pratique de l'art de construire les orgues, contenant l'orgue de Dom Bedos et tous les progrès et perfectionnements de la facture jusqu'à ce jour; précédé d'une notice historique sur l'orgue, et suivi d'une biographie des principaux facteurs d'orgues français et étrangers. Paris, 1849, 3 t. et un atlas. 2/Paris 1903, éd. Joseph Guédon, Nouveau manuel du facteur d'orgues, 1 vol.

Hardouin, Pierre

Le grand orgue de Saint-Gervais à Paris. Paris, s.d. (1955), 32 p., ill. 


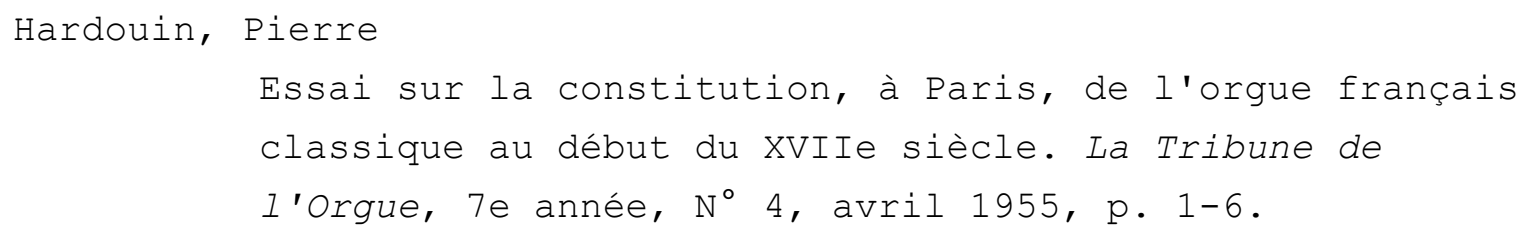

La Musique en Lorraine. Etude rétrospective d'après les archives locales, Paris, 1882, 197 p., ill.

Jacquot, Albert

Essai de répertoire des artistes lorrains. Les facteurs d'orgues et de clavecins lorrains, Paris, 1910, 16 p., ill.

Lhôte, Georges

Remarques sur l'orgue français, in ISO-Information, $\mathrm{N}^{\circ}$ I, 1969, P. 57-82, ill.

Mehl, Maurice et Chanoine Martin

L'orgue de l'église paroissiale de Vézelise, in L'Orgue, $\mathrm{N}^{\circ} 99$ (1961), p. 65-69 et $\mathrm{N}^{\circ} 101$ (1962), p. 8-13, ill.

Meignien, Jean-Marie

L'architecture des jubés, tribunes et buffets d'orgues du département de I'Aube, extrait de l'Almanach 1974 de Libération-Champagne, p. 161 - 192, ill. 
Mersenne, Marin

Harmonie Universelle contenant la théorie et la pratique de la musique, Paris, 1636.

Meyer, Hermann

Karl Joseph Riepp, der Orgelbauer von Ottobeuren, Kassel, 1938.

Moucherel, Christophe

Mémoire instructif pour faire les Devis, Desseins, Plans, Marchez et Réception des Orgues, Rodez, 1734.

Raugel, Félix

Le grand orgue de Saint-Germain-des-Prés de Paris. The Organ, juin 1923.

Raugel, Félix

Les orgues et les organistes de Saint-Germain-des-Prés depuis 1653. Musique et instruments, juillet 1923.

Raugel, Félix

Recherches sur quelques mâtres de l'ancienne facture d'orgues française. Les L'Epine, Jean-Pierre Cavaillé, Dom François Bedos de Celles. Paris, 1925, 26 p., ill.

Raugel, Félix

Les Grandes Orgues des Eglises de Paris et du Département de la Seine. Paris, 1927, 278 p., ill.

Riemann Musik-Lexikon

article "Thierry" 12e éd., vol. 2 (1961), p. 788-789.

Sauveur, Joseph

Application des sons harmoniques à la composition des Jeux ' d'Orgues. Collection des Mémoires de l'Académie royale des Sciences. Paris, 1702. 
Schmitt, Georges

Nouveau Manuel complet de l'organiste. Première partie conténant l'histoire de l'orgue, sa description et la manière de le toucher, ouvrage renfermant un apercu sur la facture ancienne et moderne, la comparaison des factures française, allemande et anglaise, la description des orgues les plus remarquables, la biographie des organistes les plus célèbres depuis le XIVe siècle. Paris, 1855.

Schweitzer, Albert

Deutsche und französische Orgelbaukunst und Orgelkunst. Leipzig 1906, 51 p., ill. réédition facsimile Wiesbaden, 1968 .

Servières, Georges

La décoration artistique des buffets d'orgues. Paris et Bruxelles, 1928, 228 p., 48 pl.

Souberbielle, Léon

La Théorie Louisquatorzienne du Plein-Jeu de l'orgue français. Une source de son histoire, à Paris, en 1702. Sèvres, 1971, $14 \mathrm{p}$.

Villard, Jean-Albert

L'oeuvre de François-Henri Clicquot, Facteur d'Orgues du Roy (1732-1790). Poitiers, 1973, 235 p., ill.

Weigert, M.A.

Plans et vues de St. Germain des Prés au Cabinet des Estampe de la Bibliothèque Nationale. Paris et Ile de France, Mémoires publiés par la Fédération des Sociétés Historiques et Archéologiques de Paris et de l'Ile de France. Paris, 1958.

Wörsching, Joseph

Riepp und Dom Bedos. Musica Sacra, 63, p. 163 ss.

Wörsching, Joseph

Der Orgelbauer Karl Riepp. Mayence, 1940. 327 p., 24 pl. 
VII. L'orque en Alsace. 
Bachmeyer, L.

Die Orgel und die Organisten der Zaberner Pfarrkirche. Journal de Saverne, 1922 (21, 23, 25, 28, 30 novembre) . $6 \mathrm{p}$

Barth, Médard

Elsass "das Land der Orgeln" im 19. Jahrhundert. Haguenau 1966, 452 p., ill. 37 pl. (Archives de l'Eglise d'Alsace, 1965-66).

Gessner, A.

Zur elsässisch-neudeutschen Orgelreform. Ein Wort der Kritik und Abwehr. Strasbourg s.d. (1913).

Haeberlé., Martin

Les orgues de la vallée de Munster, Annuaire de la Société d'histoire du Val et de la Ville de Munster, 14, 1959, p. 48-54.

Klaus, P.G.

Elsässisch-süddeutsche Beziehungen im Orgelbau. Caecilia, $68^{\circ}$ année, $\mathrm{N}^{\circ} 11 / 12$ 1960, p. 185 - 193.

Klein, E.

Kurze Geschichte der Orgel in der katholischen Kirche von Sarre-Union, Sarre-Union, 1927, 8 p., ill.

Kwasnik, Walter

Emile Rupp als Orgelreformer, Kirchenmusiker und Mensch. Francfort/M., s.d. (1967), 42 P.

Lang, M.

Bibliographie de l'histoire de la musique en Alsace, La musique en Alsace, hier et aujourd'hui. Strasbourg, 1970. p. 373-459. (Publications de la Société Savante d'Alsace et des régions de l'Est. Tome X.).

Lévy, J . Le sort des orgues dans la Haute Alsace pendant la grande Révolution, (1793-1801), Revue catholique d'Alsace, 1913. 
Lévy, J .

Nouvelles notes sur le sort des orgues dans le Haut-Rhin du temps de la grande Révolution. Revue catholique d'Alsace 1929.

Mathias, F.X.

Compte-rendu du Congrès d'orgue tenu à l'Université de Strasbourg du 5 au 8 mai 1932, Strasbourg, Sostralib, 1934 .

Meyer-Siat, Pie

La facture d'orgues en Alsace au XIX ${ }^{\circ}$ siècle, Artisans et ouvriers d'Alsace, Strasbourg, 1965, p. 237-251.

Meyer-Siat, Pie

La réforme alsacienne de l'orgue, Bulletin des

professeurs du Lycée d'Etat de Garçons de Mulhouse. $1965 / 6 \mathrm{~N}^{\circ} 3 \mathrm{p} \cdot 13-20$.

Meyer-Siat, Pie

Les Callinet facteurs d'orgues à Rouffach et leur oeuvre en Alsace. Strasbourg, Istra, 1965, $457 \mathrm{p} ., 48 \mathrm{pl}$. (Publications de la Société Savante d'Alsace et des régions de l'Est).

Meyer-Siat, Pie

Le séjour en Alsace du facteur d'orgues Antoine Lété, Société d'Histoire et d'Archéologie de Saverne et environs, cahier 60 (IV 1967) p. 25-28.

Meyer-Siat, Pie

Le projet d'orgue Callinet pour la cathédrale de Metz, Annuaire de la Société d'Histoire et d'Archéologie de la Lorraine, Tome LXVII-LXVIII, 1967-68, p. 189-229.

Meyer-Siat, Pie

Les travaux du facteur d'orgues Pierre Rivinach en Alsace, Société d'Histoire et d'Archéologie de Saverne et environs, cahier 63-64 (III-IV 1968) p. 35-38.

Meyer-Siat, Pie

Die Orgelbauerfamilie Callinet, Acta Organologica Vol. 2 Berlin, 1968, p. 82-100. 


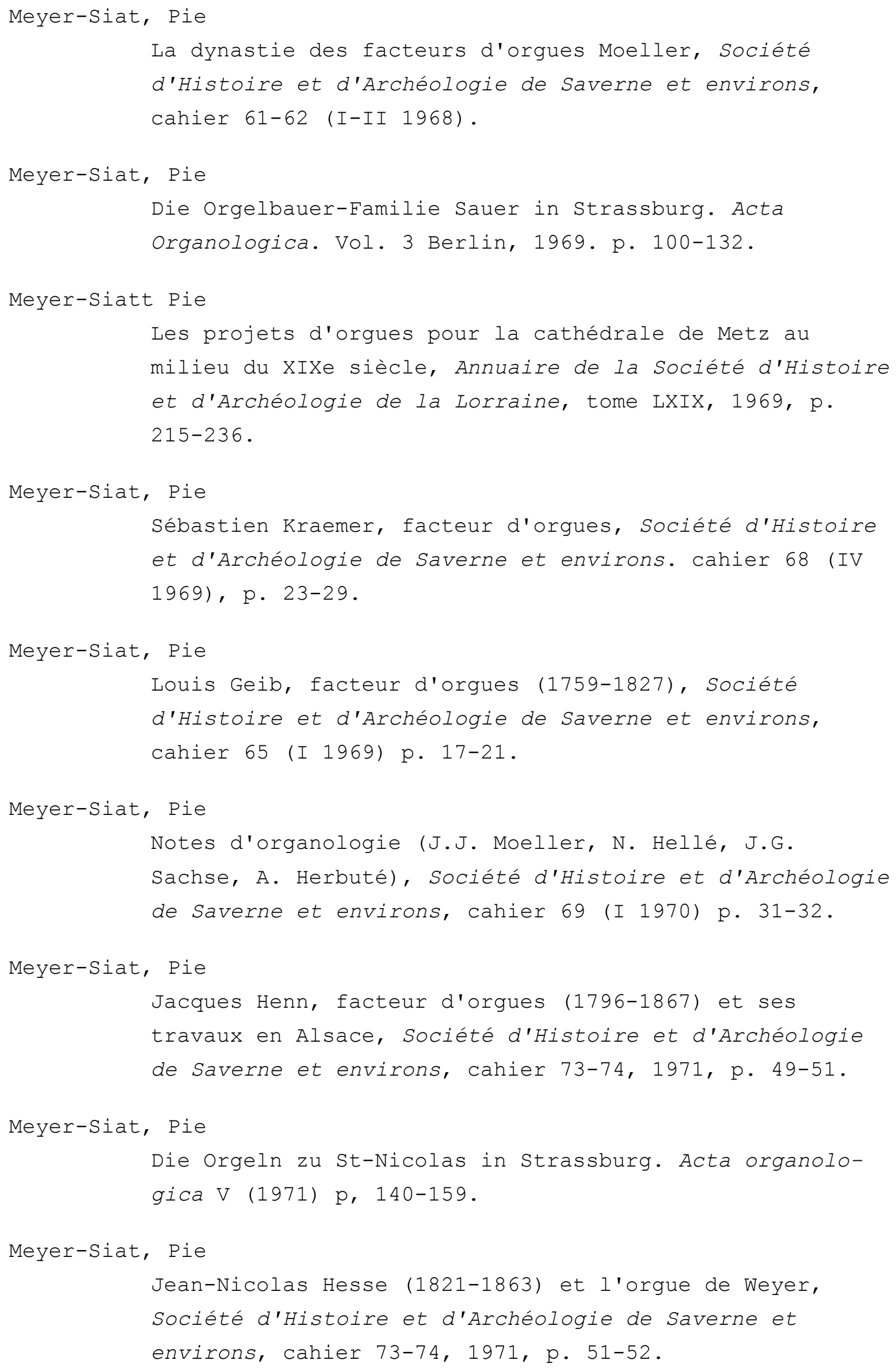




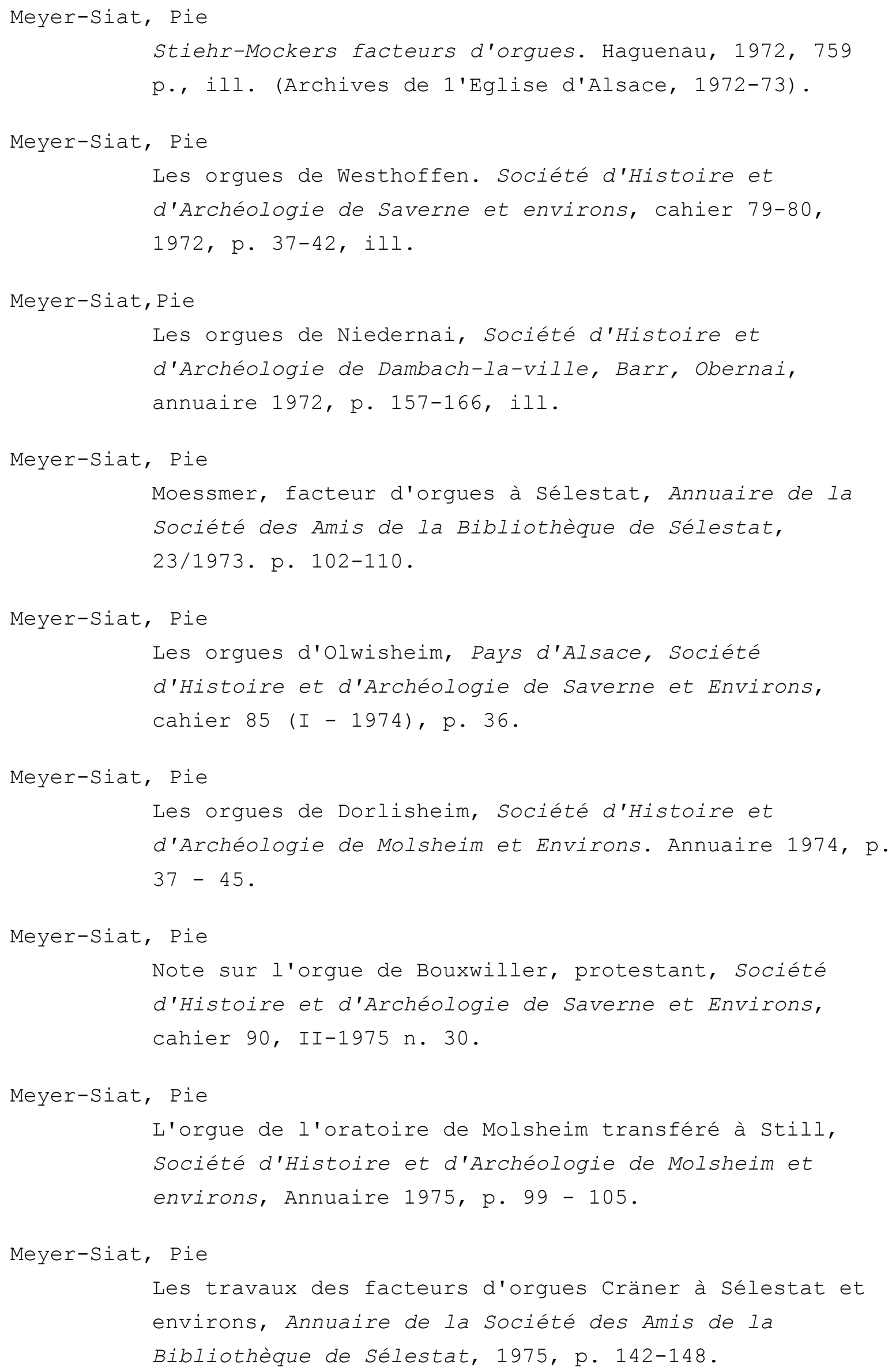


Meyer-Siat, Pie

George Wegmann, facteur d'orgues, Archives de l'Eglise d'Alsace, 1975, p. 273-322.

Meyer-Siat, Pie

Les orgues de l'église St-Pierre-le-Vieux catholique du XVIII ${ }^{\circ}$ siècle à nos jours, Annuaire de la Société des Amis du Vieux-Strasbourg, Strasbourg, 1975, p. 118-128.

Meyer-Siat, Pie

L'orgue Wegmann de Schalkendorf, Société d'Histoire et d'Archéologie de Saverne et environs, cahier 94, 1976, p. 27-28, ill.

Meyer-Siat, Pie

L'orgue Wegmann de Mackwiller, Société d'Histoire et d'Archéologie de Saverne et environs, cahier 94, 1976, p. $23-24$

Meyer-Siat, Pie

Notes sur le facteur d'orgues Tollay et son orgue de Wingersheim, Société d'Histoire et d'Archéologie de Saverne et environs, cahier 94, 1976, p. 15.

Meyer-Siat, Pie

Les orgues des temples protestants de Saverne, Société d'Histoire et d'Archéologie de Saverne et environs, cahier 94, 1976, p. 13-15, ill.

Pirro, André

Orgues et organistes de Haguenau, de 1491 à 1525

environ, Revue de Musicologie, 1926, p. 11 - 17.

Pugh, R.

Nos orgues régionales. Le grand orgue de la collégiale de Wissembourg. Revue d'Alsace, 1926, p. 228-238.

Ringue, J.

Le plus vieil orgue d'Alsace (Eglise catholique de Bouxwiller, Bas-Rhin), L'Orgue, 1961, p. 51-52.

Rupp, Emile

Der elsässische Orgelbau in alter und neuer Zeit, Der Elsässer, $\mathrm{N}^{\circ} 138(3.3 .1923), \mathrm{N}^{\circ} 162$ (14.3.1923). 
Rupp, Emile

Die elsässisch-neudeutsche Orgelreform, Kirchenmusikalisches Archiv, $\mathrm{N}^{\circ} 8$ (1910).

Schaefer, Marc

Les orgues Stiehr-Mockers de Riquewihr, Strasbourg, 1961, 58 p., ill. (Orgues d'Alsace, 3).

Schaefer, Marc

Les Orgues de l'église Sainte Marguerite, Riquewihr, Colmar, Ingersheim, 1969, 23 p., ill.

Schaefer, Marc

Notes sur l'orgue Dupont de Neuwiller-lès-Saverne, Société d'Histoire et d'Archéologie de Saverne et environs, cahier 73-74, I-II 1971, p. 35-37.

Schaefer, Marc

Louis Dubois et l'orgue des Récollets de Saverne, Société d'Histoire et d'Archéologie de Saverne et environs, cahier 85, I - 1974, p. 7 - 11.

Schneider, E.

Der Orgelbauer Josef Bergöntzle, Das Musikinstrument, XIV, cahier 9 (1965), Bodensee-Hefte, cahier 2 (1965).

Schweitzer, Albert

Die Strassburger Sängerhaus=Orgel, Strasbourg,1909, 20 p., ill.

Wiltberger, H.

Orgelbauerei M. u. J. Rinkenbach in Ammerschweier, Strasbourg et Colmar, 1909.

Wollbrett, A.

Weiterswiller, Les origines de l'orgue de Saint-Michel. Société d'Histoire et d'Archéologie de Saverne et environs, cahier 47-48, (1964 III-IV).

Wollbrett, A.

L'orgue de Bouquenom et son temps, Société d'Histoire et d'Archéologie de Saverne et Environs, tiré à part 1962. 
(Wollbrett, A.)

L'Eglise paroissiale de Saverne, Société d'Histoire et d'Archéologie de Saverne et environs, cahier 55-56 (IIIIV 1966). p. 50-54: Wollbrett, A., Les Orgues, p. 45-49: Will, R., Les tombeaux des évèques.

(Wollbrett, A.)

Les Eglises de Neuwiller-lès-Saverne, Société d'Histoire et d'Archéologie de Saverne et environs, cahier II-III 1959. p. 17-23: Wollbrett, A., Les orgues (Dans l'église Saint-Pierre et Saint-Paul, Souvenirs d'ancien régime).

Wollbrett, A.

Notes sur le facteur d'orgues Nicolas Dupont (1714-1781) de Domnom-les-Dieuze, Les Cahiers Lorrains, $16^{\circ}$ année, $\mathrm{N}^{\circ}$ 2. Avril 1964. P. 35-42.

Wollbrett, A.

Nouveaux propos biographiques sur Nicolas Dupont, constructeur des orgues de S. Pierre et S. Paul de Neuwiller et son neveu Nicolas Tholé, Société d'Histoire et d'Archéologie de Saverne et environs, cahier 57 (I 1967) p. 18. 
VIII. La musique en Alsace. Les organistes. 
Brück, Johann Friedrich

Cantates inédites. Strasbourg, Bibliothèque du Séminaire Protestant.

Frauenholtz, Johann Christoph

Cantates inédites. Strasbourg, Bibliothèque du Séminaire Protestant.

Gérold, Théodore

Les plus anciennes mélodies de l'Eglise protestante de Strasbourg et leurs auteurs. Paris, 1928, $80 \mathrm{p}$.

Goehlinger, F.A.

La musique à la cathédrale de Strasbourg après le premier retour de l'Alsace à la mère-patrie sous Louis XIV. Die Musikpflege am Strassburger Münster nach der ersten Rückgabe des Elsass an Frankreich. Strasbourg, 1920 (Studia Leontina) 216 p.

Kopff, René

Johann Georg Rauch (1658-1710), organiste de la cathédrale de Strasbourg. Sa vie et son oeuvre. Thèse de doctorat de $3^{\circ}$ cycle. Strasbourg, Institut de Musicologie, (dact.) 1971.

Lobstein, J.F.

Beiträge zur Geschichte der Musik im Elsass und besonders in Strassburg von den ältesten bis auf die neueste Zeit, Strasbourg, 1840, $147 \mathrm{p}$.

Merkling, G.

Die Organisten an der St. Thomaskirche in Strassburg vom Jahre 1517 an, Elsass-lothringische Gesang und Musikzeitung (1911/12), p. 82-83.

La Musique en Alsace, hier et aujourd'hui. Strasbourg, 1970, 459 p., ill. (Publications de la Société Savante d'Alsace et des Régions de l'Est, tome X).

Pirro, A.

Notes sur un claveciniste alsacien (Célestin Harst). Revue de Musicologie 9, 1925, février, 1 - 5, mus. 


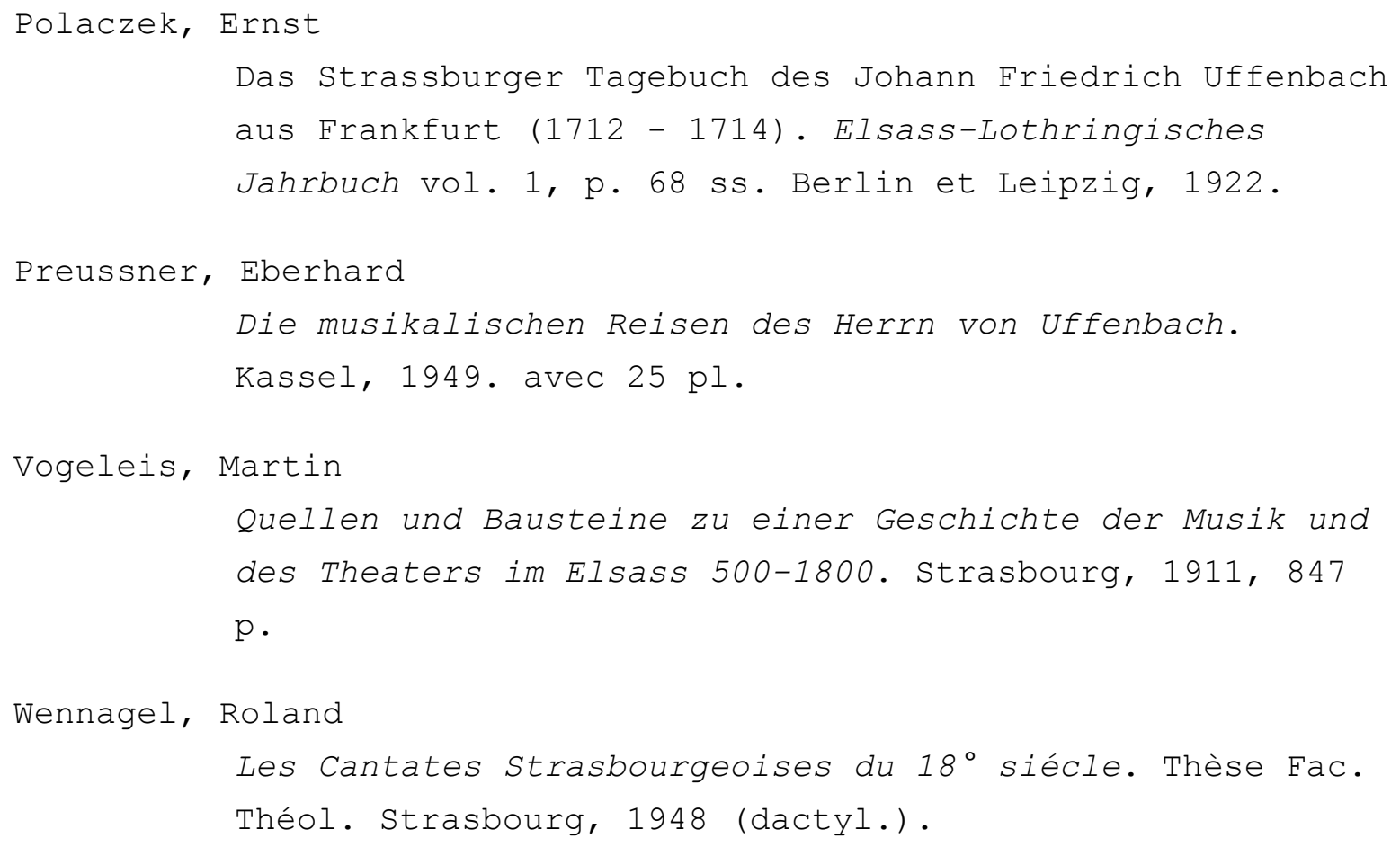


456

IX. Monographies de localités et d'églises. 
Barth, Médard

L'église paroissiale de Molsheim, Strasbourg, 1964, 25 p., $54 \mathrm{pl}$.

Chèvre, André

Lucelle, histoire d'une ancienne abbaye cistercienne, Delémont, 1973, $351 \mathrm{p}$.

Dammron, E.

Geschichtliche Notizen über die evang.= luth. Pfarrgemeinde Bischheim=Hönheim, Strasbourg, 1907.

Dehio, G.

Handbuch der Deutschen Kunstdenkmäler, Berlin, 1937.

Eggenberger-Billerbeck, Dorothee

Die barocke Klosterkirche von Ebersmünster und ihre

Stellung im Werke Peter Thumbs, Diss., Bâle, 1970.

Ehrhard, E.

Historische Skizze der Pfarrei Sulzbad, Molsheim, 1908.

Erichson, Alfred

Das theologische Studienstift Collegium Wilhelmitanum 1544 - 1894, Strasbourg, 1894.

Festschrift zum Goldenen Jubiläum der Einweihung der Pfarrkirche von Eckbolsheim 1886 - 1936, Strasbourg, 1936.

Friedel, René

Geschichte des Fleckens Erstein, Erstein, 1927, 702 p.

Ginter, Hermann

Die Kirche von Ebersmünster, Oberrheinische Kunst, X, 1942, p. $151-170$.

Goehlinger, François Auguste

Histoire de I'Abbaye de Marbach, Colmar, 1954, 351 p., ill.

Goehlinger, François Auguste

Histoire du Chapitre de l'Eglise Collégiale St-Martin de Colmar. Colmar, 1951, 359 p., ill. 
Gubler, Hans-Martin

Der Vorarlberger Barockbaumeister Peter Thumb (1681 -

1766). Ein Beitrag zur Geschichte der süddeutschen

Barockarchitektur, Sigmaringen 1972.

Guggenbühl, Willy

Gries. Chronik einer unterelsässischen Landgemeinde. Saverne 1957, 264 p., ill.

Guggenbühl, Willy

Sessenheim. Chronik einer elsässischen Landgemeinde. Saverne 1961, 241 p., ill.

Hecker, F.

Die Stadt und das Tal zu Münster im Gregorienthal. Munster, 1890 .

Heinemann, J. G.

Die Kirche Sanct-Aurelien in Strassburg. Strasbourg, 1865 .

Heitz, Friedrich Carl

Die St. Thomas-Kirche in Strassburg. Strasbourg, 1841, $140 \mathrm{p}$.

Jung, Erik

Le Choeur de St-Guillaume de Strasbourg. Strasbourg, 1947, 272 p., ill.

Klein, C.

Pfarrerbuch und Kirchenchronik der älteren evangelischen Gemeinde zu Buchsweiler. Strasbourg, 1914, 156 p. (Beiträge zur Geschichte der ehemaligen Grafschaft HanauLichtenberg und ihrer Residenz-Stadt Buchsweiler, vol. 2) .

Kuntz, Charles

Die romanische Kirche St. Peter und St. Paul zu Rosheim. Saverne, s.d. (1923), 62 p., ill.

Lambs, Johann Philipp

Die Jung St. Peter=Kirche in Strassburg. Eine geschichtliche Darstellung. Strasbourg 1854. 108 p. 
Merklen, M.

Ensisheim, jadis ville libre-impériale et ancien siège de la régence archiducale des pays antérieurs d'Autriche ou Histoire de la ville d'Ensisheim. 2 t. en 1 vol. 362 et 402 p. Colmar 1840, 1841.

Ohleyer

Die Kirche von St-Peter und Paul zu Weissenburg. Wissembourg, 1863.

Ohresser, Xavier

Eglise et Abbaye d'Ebersmunster. Sélestat, 1957.

Ohresser, Xavier

L'ancienne église d'Obernai. Archives de l'Eglise d'Alsace, 8 (1957), p. 81-83.

Reinhard, Aimé

Le Temple-Neuf à Strasbourg. Notice commémorative. Strasbourg, 1888 .

Schmitt, J.-M.

Le Prieuré de Saint-Quirin. Sarrebourg, 1970.

Schneegans, Louis

L'église de Saint-Thomas à Strasbourg, et ses monuments. Strasbourg, 1842 .

Stoeber, August

Auswahl aus den hinterlassenen Gedichten von Johann Friedrich Wenning, Officier d'Académie, ehemaligem Oberlehrer und Organisten in Barr, im Unter=Elsass. Mulhouse, 1879, 188 p. ill.

Stöber, E.

Sämmtliche Gedichte und kleine prosaische Schriften, III Strasbourg 1836.

Will, Robert et Rieger, Théodore

Eglises et Sanctuaires d'Alsace. Strasbourg 1969, 210

p., $124 \mathrm{pl}$. 
Wolffer, G.

Geschichte der Gemeinde und Kirche zum alten Sankt Peter zu Strasbourg, zusammengestellt nach Originalen Copien, Berichten und Buchauszügen. Strasbourg 1974 (dactyl.). 
461

$\underline{X}$. Les Silbermann de Strasbourg et leurs oeuvres. 
Anonyme

Zur Geschichte des Orgelbaues im Elsass, Landes-Zeitung für Elsass-Lothringen, 28.12.1885.

Barth, Th.

Eine alte Orgel (Bâle, St-Théodore), Monatsschrift für Gottesdienst und kirchliche Kunst, XIX (1914) cahier 6, p. $173-177$.

Baser, Friedrich

Weshalb ging Silbermann nach Strassburg ?, Musik und Kirche, 12.1940/5. p. 110-114.

Baser Friedrich

Klingende Denkmäler einer deutschen Künstlerfamilie.

Silbermann-0rgeln in ganz Elsass, Baden und der Schweiz, Die Musik. 32. 194T, p. 337.

Baur, E.

Das Orgelbauergeschlecht Silbermann in Basel, Basler Jahrbuch, 1927. p. 192-206.

Bender, Antoine

Les orgues Silbermann d'Altorf, Caecilia, 1960. p. 115118 .

Bender, Antoine

Rectificatif (Positif de Sessenheim), L'Orgue, $\mathrm{N}^{\circ} 91$

(1959) p. 85 .

Bender, Antoine

Les Orgues Silbermann de Soultz (Haut-Rhin), Strasbourg, 1960,28 p., ill. (Orgues d'Alsace, 1).

Bender, Antoine

Les orgues Silbermann de St. Pierre et Paul à Rosheim, caecilia. 1960 .

Bender, Antoine

Les Orgues Silbermann de Marmoutier et Ebersmunster (avec la collaboration de Marcel Thomann) Strasbourg, 1960, 46 p ill; (Orgues d'Alsace, 2). 
Bernard, J.J.

Les orgues Silbermann de Wasselonne. Mémoire présenté à l'Ecole normale d'instituteurs de Strasbourg. S. 1., 1966, 71 p., dactyl., ill.

Bovet, Guy

Essai d'un parallèle entre le Silbermann d'Arlesheim et deux d'Alsace. La Tribune de I'Orgue, 14, 1962, $\mathrm{N}^{\circ} 4 \mathrm{p}$. $1-3$.

Braun, Luc

Orgues historiques: Marmoutier, St-Michel-de-Provence, 1963, 32 p., ill., 1 disque (Harmonia Mundi).

Bury, J. P.

L'orgue de St. Thomas à Strasbourg. Strasbourg, s.d., dactyle, ill.

Dufourcq, Norbert

L'orgue Silbermann de Marmoutier en Alsace, Société

d'Histoire et d'Archéologie de Saverne, 1956, $\mathrm{N}^{\circ} 1, \mathrm{p}$. $1-2$.

Dufourcq, Norbert

L'orgue Silbermann de Marmoutier en Alsace, L'Orgue. 1955, p.105-108, ill., 1956, p. 63-64.

Edel, A.

Les orgues de l'église paroissiale de Turckheim, $75^{\circ}$ Anniversaire de la Chorale Ste-Cécile Turckheim, Turckheim s.d. (1953), p. 10-15, ill.

Edel, A.

L'orgue de l'église Ste-Anne de Turckheim, Turckheim 1976, 28 p., ill.

Frossard, Claude

Quelques orgues modernes d'Alsace, La Tribune de I'Orgue, 8. 1956, $\mathrm{N}^{\circ}$ 5, p. $1-6$, ill.

Frossard, Claude

Anciennes orgues d'Alsace, La Tribune de l'Orgue, 7. 1955. octobre. 
Ginter, L.

Die Silbermannsorgel von Bischofsheim, Elsassland. Lothringer Heimat, 19, I939, p. 19 - 23.

Goehlinger, F.A.

Des Orgelbauers J.-A. Silbermann Briefwechsel mit dem Abt Preiss von Marbach (1736-1740), Etudes Alsaciennes, Tome 1, Strasbourg 1947, p. 103-129. (Publications de la Société Savante d'Alsace et des régions de l'Est).

Gramm, J.J .

Collégiale d'Arlesheim. L'unique orgue Silbermann encore en Suisse est restauré. La Tribune de I'Orgue, 14, 1962, $\mathrm{N}^{\circ} 3, \mathrm{p} \cdot 4-6$.

Guggenbühl, Willy

Geschichte der Grieser Silbermann-Orgel, Almanach de, l'Eglise évang. Iuth. 1969, p. 49-52., ill.

Hardouin, Pierre

André Silbermann à Paris. La Tribune de l'Orgue, 8, $1956, \mathrm{~N}^{\circ} 4, \mathrm{p} \cdot 1-5$.

Herrenschwand, F.

Silbermann organs in Switzerland, Organ Institute

Quarterly Andover (Mass.), 5, 1955, 15 et suiv.

Hesselbacher, Martin

Die Silbermann-Orgel in der evangelischen Kirche Meissenheim, Geroldsecker Land. Jahrbuch für den Landkreis Lahr, cahier 6. 1963/64.

Hesselbacher, Martin

Die Silbermann-Orgel in Meissenheim. Nachrichtenblatt der Denkmalpflege in Baden-Württemberg, $9^{\circ}$ année, cahier $3 / 4,1966$.

Hohn, Albert

Die Orgeln Johann Andreas Silbermanns. Acta organologica, IV (1970) p. 11 - 58. compléments et corrections dans Acta organologica VI (1972), p. 178-183. 
Hohn, Albert

Die Silbermannorgel in St. Märgen, 850 Jahre St. Märgen, St. Märgen, 1968, p. 131-143.

Hohn, Albert

Klosterkirche ... Offenburg. Geschichte der Orgel. offenburg, s.d. (1968) 10 p., ill.

Hohn, Albert

Die Orgel des Johann Andreas Silbermann in der Pfarrund Wallfahrtskirche St. Landolin zu Ettenheimmünster, Instrumentenbau 2eitschrift, XVII année, septembre 1963. p. 392-407.

Huck, Jos. L.

Les orgues de Marmoutier, témoins d'une époque. Société d'Histoire et d'Archéologie de Saverne, $1954 \mathrm{n}^{\circ}$ 3-4, p. $21-23$.

Kauffmann, Friedrich

Die Silbermann-Orgel zu Buchsweiler, Elsassland. Lothringer Heimat, 7, 1927, p. 245-248 ill.

Klein, CH. D.

Les orgues de l'église protestante de Colmar. Annuaire de Colmar, IV. 1938. p 135-151.

Klotz, Hans

article "Silbermann", Die Musik in Geschichte und Gegenwart vol. 12 (1965), col. 694-701.

Kobel, Heinz

Die Orgel im Dom zu Arlesheim BL von Johann Andreas

Silbermann 1761. Bâle s.d. (1960), 101 p., dactyl ill.

Kobel, Heinz

Die Orgel des Johann Andreas Silbermann im Dom zu Arlesheim, Caecilia, 1961, p. 118-122.

Kobel, Heinz

Die Orgel des Johann Andreas Silbermann von 1761 im Dom zu Arlesheim und ihre Restauration 1959-1962. Katholische Kirchenmusik 87, 1962, p. 75-84, ill. 
Kobel, Heinz

Silbermann und Callinet. Ars Organi, cahier 30, 1967, p. 1045-1053.

Kuhn, Th. AG

Die Silbermannorgel in der Kirche St. Leonhard in Basel. Männedorf, s.d., 110 p., ill.

Laffont, J.-E. Emile (Dr.)

A propos d'un orgue à Sessenheim. Cannes 1950, 19 p., ill.

Lanz, H.

Die Silbermann-Orgel aus St. Theodor, Historisches Museum, 1951 Jahresberichte und Rechnungen. Bâle, s.d. (1952) p. 29-35., ill.

Lapresté, J .

Lettre ouverte de $\mathrm{M}$. Jean Lapresté, président du Groupement national des facteurs d'orgue de France à M. l'abbé Jean Ringue, en réponse à son article relatif à l'orgue de Soultz. Caecilia 1950, p. 25-26.

Lottermoser, $\mathbf{W}$.

Akustische Untersuchungen an der Andreas SilbermannOrgel von Ebersmünster, Archiv für Musikwissenschaft, 11 (1954) p. 300-314.

Lottermoser, $W$, et Jenker, E. Klanganalytische Untersuchungen im Anschluss an die Restauration der J.A. Silbermann-Orgel von Arlesheim/Basel, Das Musikinstrument, XV, cahier 2 (1966).

M. Ch.

Notabilités alsaciennes nées en juin. Revue alsacienne, Paris-Nancy X (1886-1887) p. 368.

Mathias, F.X.

Die über Silbermann zirkulierenden Irrtümer, Der Elsässer, 4 et 7 février 1928.

Mathias, F.X.

Andreas und Johann Andreas Silbermann im Kampf mit "Orgelwölfen", Der Elsässer, N. 54, 3 mars 1928. 
Mathias, F.X.

L'histoire des Silbermann, la plus éloquente apologie de l'art français, Revue catholique d'Alsace, 43. 1928, p, $667-681$.

Mathias, F.X.

A. Silbermann en route pour Paris, Revue catholique d'Alsace, 1929, p. 420-426.

Mathias, F.X.

André Silbermann d'après les notes biographiques que lui a consacrées son fils aîné: Jean André Silbermann, I'Est Musical, avril-juin 1939.

Mathias, F.X.

André Silbermann en route pour l'Alsace. André Silbermann s'installe à Strasbourg, Echos des Sanctuaires de Ste-Odile, 1930, p. 57-60.

Mathias, F.X.

Die Pragmatik der Orgelbaugeschichte des 18. Jhs. im Lichte des Pariser Silbermann Archivs, Société Internationale de Musicologie. I. Congrès de Liège. Compterendu. 1. - 6. Sept. 1930. I. p. 182.

Mathias, F.X.

Jean André Silbermann de Strasbourg (suivant les Archives Silbermann à Paris) et l'histoire de l'orgue au $18^{\circ}$ siècle, Echos des Sanctuaires de Ste-Odile, 5. 1933, p. $244-262$.

Mathias, F.R.

Das liturgische Musikwesen an der Strassburger St. Magdalenenkirche, Speich, E., St. Magdalena in Strassburg. Geschichte des Klosters und der Pfarrei, Strasbourg 1937, p. 281-313.

Mathias, F.X. et Wörsching, J.

Die Orgelbauer=Familie Silbermann in Strassburg i/E. aus dem Nachlass der Verfasser herausgegeben von Paul smets I. et II. livraison, Mayence, s.d. (1960), 160 p. 
Meckel, E.

Die Orgelbauer Silbermann am Oberrhein, Welt am Oberrhein, 1961, cahier 6.

Meyer-Siat, P.

L'orgue Silbermann de Marmoutier menacé en 1805, Société d'Histoire et d'Archéologie de Saverne, cahier 77 (I1972), p. 23-24.

Meyer-Siat, P. et Raber, A.

L'orgue Silbermann de l'église St. Georges de Sélestat et de l'église des Dominicains de Colmar. Annuaire de Colmar, 1968. p. $96-125$.

Miramon Fitz-James, B. de

André Silbermann d'après les notes biographiques que lui a consacré son fils aîné et son successeur dans la manufacture d'orgues à Strasbourg: J.A. Silbermann, et d'après les travaux récents du chanoine F.X. Mathias, I'Est Musical, Strasbourg 1939, $\mathrm{N}^{\circ} 1-2$.

Miramon Fitz-James, B.de

André Silbermann (1678-1734) et l'art français de l'orgue, Bulletin trimestriel des Amis de l'orgue, $\mathrm{N}^{\circ}$ 18, juin 1934, p. 3.

Mooser, Ludwig

Das Brüderpaar die Orgelbaumeister Andreas und Gottfried Silbermann. Historische Skizze nach kirchlichen und amtlichen Urkunden entworfen. Strasbourg, Gustave Silbermann, 1861. $31 \mathrm{p}$.

Nardin, Paul

Les nouvelles orgues de Saint-Pierre-le-Jeune à

Strasbourg. L'Orgue, $\mathrm{N}^{\circ}$ 58-59 (1951) p. 14-19.

Nardin, Paul

Quelques précisions sur les orgues Silbermann de l'Eglise Abbatiale de Marmoutier, La Tribune de I'Orgue, 8. 1956, $\mathrm{N}^{\circ} 3, \mathrm{p} .2-4$. 
Pugh, R.

Glanes sur Silbermann, I. L'orgue d'Ebersmunster, Revue d'Alsace, Tome 72, $\mathrm{N}^{\circ}$ 475, mars-avril 1925, p. 159-164.

Pugh, R.

Glanes sur Silbermann, II. L'orgue de Soultz (HautRhin), Revue d'Alsace, Tome 72, $\mathrm{N}^{\circ} 478$, septembreoctobre 1925, p. 454-461.

Pugh, R.

Ancient Alsatian Organs, The Organ. 6. 1926/1927. p.237.

Rahner, H.E.

Der Neubau der Stiftsorgel st. Blasien unter Abt Martin Gerbert durch Johann Andreas Silbermann, Archiv für Musikforschung, 2, 1937, p. 433-454.

Raugel, Félix

Les Silbermann facteurs d'orgues alsaciens et les organistes et organiers français de leur temps, Liber Amicorum Charles Van den Borren, Anvers 1964, p. 192195 .

Raugel, Félix

Les Silbermann et la France musicale de leur temps, Alsace Française 19, 1939, p. 65-66 et Fédér. des soc. de mus. d'Alsace, 1953, $\mathrm{N}^{\circ} 4, \mathrm{p} .13-15$.

Reinbolt, Claus

Die Silbermannorgel von Marmoutier, Le Nouvel Alsacien 17 Nov. 1954.

Ringue, Jean

Un orgue qu'il faut préserver: celui d'Altorf, Caecilia, 1950 , p. $73-75$.

Ringue, Jean

L'orgue de Saint-Pierre, près d'Eichhoffen (Bas-Rhin), L'Orgue, $\mathrm{N}^{\circ} 104$ (1962) p. 97-99.

Ringue, Jean

Jean-André Silbermann, Petite Revue. Supplément littéraire du Nouvel Alsacien, 4. 10. 1950. 
Ringue, Jean

Les facteurs d'orgues Silbermann en Alsace, Caecilia, 1952, p. 59-63, 91-93, 135-138.

Ringue, Jean

Un cas terriblement typique: l'orgue de Soultz. Caecilia, 1949, p. 161-165.

Schaefer, Marc

Découverte d'un orgue Silbermann à Gries (Bas-Rhin), Revue d'Alsace, tome 101, 1962, p. 130-133.

Schaefer, Marc

L'orgue de Saint-Ouirin, Jean-André Silbermann,

Documentation technique. Orgues Historiques, SaintOuirin, St-Michel-de-Provence, 1966 (Harmonia Mundi).

Schaefer, Marc

Les trois orgues Silbermann de l'église Saint-Pierre-leJeune, Eglise luthérienne St-Pierre-le-Jeune, bulletin trimestriel, 1966, $\mathrm{N}^{\circ} 4, \mathrm{p} .5-6$.

Schaefer, Marc

Les anciennes orgues de l'église Saint-Etienne de Mulhouse en souvenir du $2 e$ centenaire de l'orgue de Jean-André Silbermann. Bulletin du Musée Historique de Mulhouse, Tome LXXIV, Année 1966, p. 135-161., ill.

Schaefer, Marc

Les anciennes orgues du château de Saverne. Le Château de Saverne (A. Wollbrett éd.), 1969. p. 60.

Schaefer, Marc

Les anciennes orgues Silbermann du Temple-Neuf à Strasbourg. La Musique en Alsace, hier et aujourd'hui. Strasbourg 1970, p. 95-122., ill. (Publications de la Société Savante d'Alsace et des régions de l'Est Tome $\mathrm{X)}$.

Schaefer, Marc

L'orgue Silbermann de l'église Sts-Simon-et-Jude d'ottrott, Annuaire de la Société d'Histoire et d'Archéologie de Dambach-la-Ville, Barr, Obernai, $N^{\circ}$ 4/1970. p. 5156., ill. 
Schaefer, Marc

article "Silbermann". Dictionnaire de la Musique (Marc

Honegger éd.) Paris, 1970, Tome II, p. 1026-1028.

Schaefer, Marc

L'orgue Silbermann de l'église paroissiale St-Georges de Molsheim, ancienne église des Jésuites, Société

d'Histoire et d'Archéologie de Molsheim et environs, Annuaire 1971, p. 47-55.

Schaefer, Marc

L'orgue Silbermann d'Altorf. Société d'Histoire et

d'Archéologie de Molsheim et environs, Annuaire 1973, p. 29-31, i11.

Schaefer, Marc

Notre orgue (Bischwiller). Bulletin interparoissial

protestant de Bischwiller, 1973, $\mathrm{N}^{\circ}$ 8, p. 12-13.

Schilling, $\mathrm{H} .-\mathrm{L}$.

Eine Silbermann-Orgel in Meissenheim-Baden, Musik und Kirche, 34, cahier 2 (1964)

Sulzmann, Bernd

Silbermannorgeln in der Ortenau, Badische Heimat. Mein Heimatland 48. cahier 1/2 1968. p. 196-208, ill.

Sumner, W.L.

The Silbermanns and their organs, The Organ. 18. 1938/39. p. 129, 221.

Thomann, Marcel

Albert Schweitzer et les orgues Silbermann de Marmoutier, Société d'Histoire et d'Archéologie de Saverne, cahier 89 (I-1975) p. 15-16, ill.

Thomann, Marcel

Les orgues Silbermann de l'église abbatiale de Marmoutier, Vie musicale et théatrale, 1959, décembre, p. 140-141. 
Thomann, Marcel

Les Orgues Silbermann (Marmoutier), I'Eglise Abbatiale de Marmoutier, Complément I-II, 1958 du Bull. de la Société d'Histoire et d'Archéologie de Saverne. Saverne, 1958, p, 6-7, ill.

Thomann, Marcel

Rund um Silbermann, Petite Revue, Supplément littéraire du Nouvel Alsacien, 7.9.1960, p. 1 et 4.

Thomann, Marcel

Une inscription de 1709 découverte dans les orgues Silbermann de Marmoutier, Société d'Histoire et d'Archéologie de Saverne, 1957, $\mathrm{N}^{\circ} 1 / 2, \mathrm{p}, 18-19$.

Walter, Rudolf

Der Orgelbau für die Fürstabtei St. Blasien 1772/75, Musicae Sacrae Ministerium. Festgabe für Karl Gustav Fellerer, 1962, p. 259-319.

Walter, Rudolf
Johann Andreas Silbermann e i suoi criteri costruttivi. Carteggio inediti con l'abbazia di St. Märgen (Selva Nera), L'Organo, IX, $N^{\circ}$ I(1971) p. 3-34. IX, $N^{\circ} 2$ (1971) p. 153-199., ill.

Weber, Jean-Daniel

Die umgebaute Orgel der Evangelischen Aurelienkirche zu Strassburg im Elsass, Musik und Kirche. Kassel. 23. 1953. p. 173-175.

Weber, Jean-Daniel

Les orgues restaurées de l'église protestante SainteAurélie, à Strasbourg. L'Orgue, $N^{\circ} 66$ (1953) p. 20-26.

Wild, Georges

Zur Restaurierung der Silbermann-Orgel in der SanktThomas-Kirche zu Strassburg, Die Auslese, Zeitschrift für Offerten auserlesener Firmen des kirchlichen Bedarfs, 1972, 10 mrars. p. 1, 2, 4. 


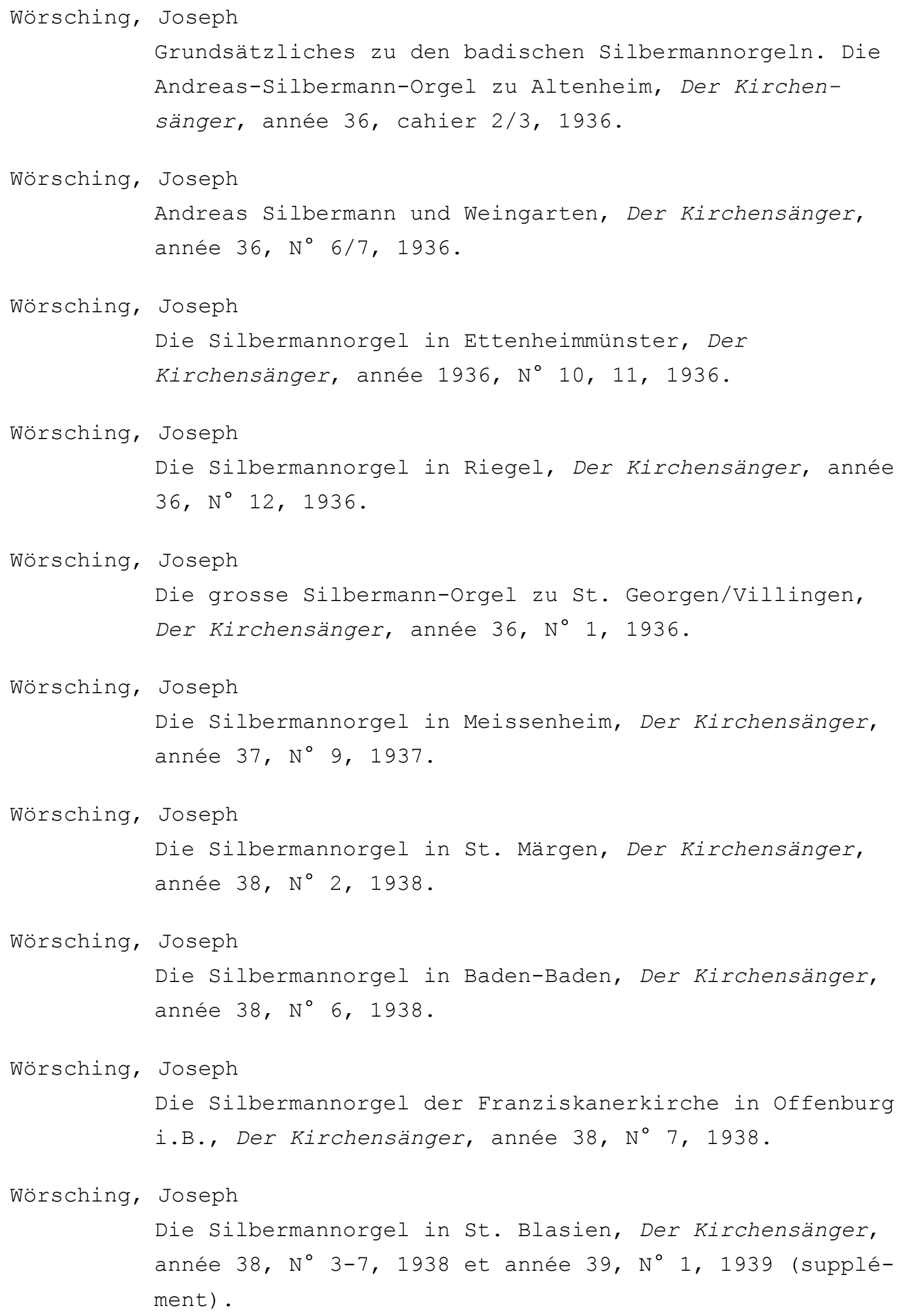




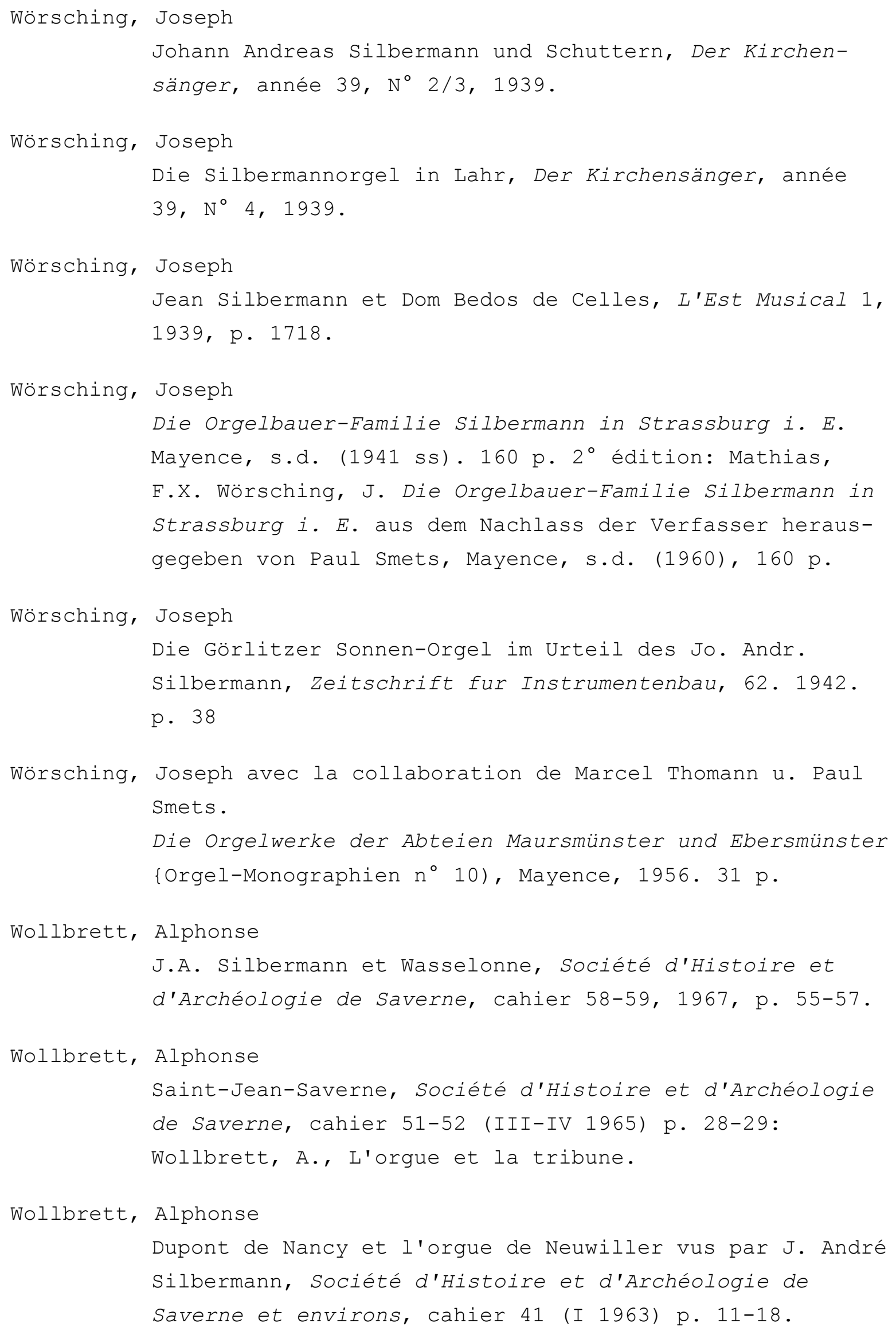


$\underline{\text { XI. Les orques de la cathédrale de Strasbourg. }}$ 
Allihn, Max

Die Orgel des Strassburger Münsters. Zeitschrift für Instrumentenbau, 13, 1893, $\mathrm{N}^{\circ} 27$

Bender, Antoine

Restauration des orgues de la cathédrale de Strasbourg. L'Orgue, 1960, p. 51-52.

Beyer, Victor

Les grandes orgues de la cathédrale. La sculpture strasbourgeoise au XIVe siècle, Paris et Strasbourg, 1955, p. $44-48$.

Clauss, E.

Zur Frage der Restaurierung der Münsterorgel, Caecilia, 1929, p. 51; 1931, p. $159-160 ; 1932$, p. 50, $156-157$.

Clauss, E.

Der Wiederaufbau der Silbermann'schen Münsterorgel, Caecilia, 1931, p. 50 - 51; 1933, p. 53, 149 - 151.

Clauss, E.

Einweihung und Uebergabe der neuen Münsterorgel,

Caecilia, 1935, p. 116 - 117, 134 - 136, ill.

Clauss, E.

Die neue Strassburger Münsterorgel, Caecilia, 1935, p. 158 - 161; 1936, p. 136 - 137.

Daguillon, J.

Le frère prêcheur Ulrich Engelbrecht a-t-il construit

les premières orgues de la cathédrale de Strasbourg?

Revue Catholique d'Alsace, 42, 1927, p. 583-598.

Dehio, G.

Das Strassburger Münster, Munich, 1922.

Disposition der grossen Münsterorgel zu Strassburg, Caecilia, 1897, p. 62 .

Fornaçon, S.

Die Krebs-Orgel in Strassburg. Der Kirchenmusiker. Darmstadt. 5. 1954. p. 186-187. 
Fröhlich, F.

Die Strassburger Münsterorgel und ihr Baumeister

Friedrich Krebs aus Onoldsbach. 1489-1491. Bergfried.

Rothenburg O.T. 10. 1958. $\mathrm{N}^{\circ}$ 5. p. 38-39.

Gallagher, E.W.

The Organ at Strasbourg Cathedral. The Organ. 31.

1951/52. p. 1-7.

Gass, J.

Les orgues de la Cathédrale de Strasbourg à travers les siècles. Strasbourg, 1935. 35 p.

Gessner, A.

Gutachten über die von Hrn. Orgelbaumeister H. Koulen in Oppenau umgebaute Orgel im Münster zu Strassburg i. E. Caecilia, 1899, p. 19-20, 59-60.

Geyer, J.

Les orgues que réclame la cathédrale de Strasbourg.

Echos des Sanctuaires de Sainte-Odile, 1930, p. 4-11.

Grandidier, Philippe-André

Essais historiques et topographiques sur l'église

cathédrale de Strasbourg, Strasbourg 1782.

Heinrich, L. \& Stark, W.

L'orgue Silbermann de la cathédrale de Strasbourg, le parfait emblème de la mission Silbermann dans l'histoire de l'orgue. L'Est Musical, I.1. 1939, p. 12-16.

Mathias, F.X.

La réfection des orgues de la cathédrale de Strasbourg. Echos des Sanctuaires de Sainte-Odile 1931, p. 34-42.

Mathias, F. X.

Die Silbermann-Orgel im Strassburger Münster nach den Aufzeichnungen von Johann Andreas Silbermann, Jahrbuch der Elsass-Lothringischen Wissenschaftlichen Gesellschaft zu Strassburg, vol. 1, 1928, p. 113-133. 


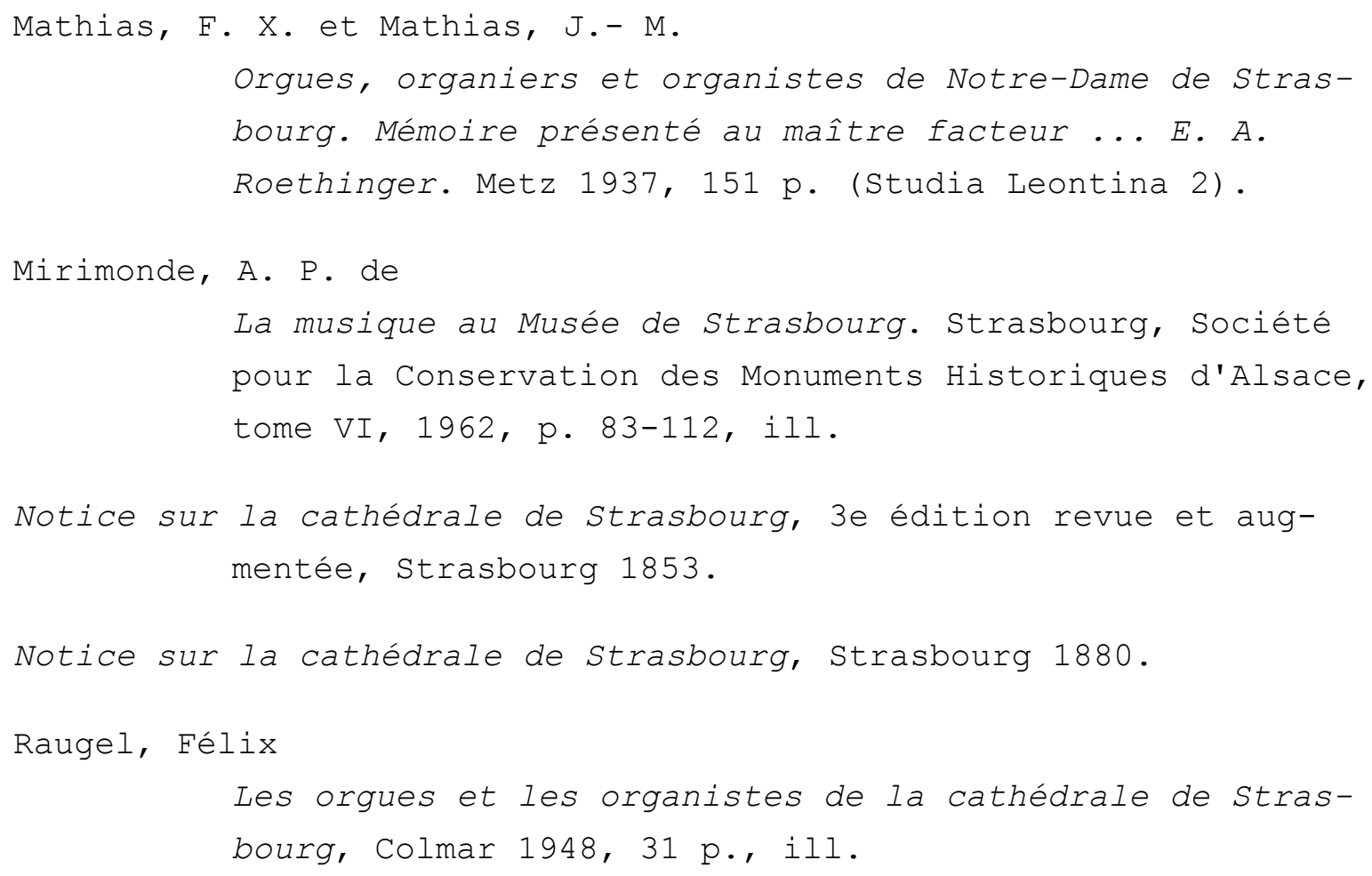

Projet d'Orgue de Jubé pour la Cathédrale de Strasbourg, L'Orgue, $\mathrm{N}^{\circ}$ 83, p. 23-24, ill.

Reinbolt, Claus

Zum Problem und Umbau der Strassburger Münsterorgel, Strasbourg 1935, extrait de Jahrbuch der ElsassLothringischen Wissenschaftlichen Gesellschaft zu Strassburg, vol. VIII, 1935, p. 11-61.

Reinbolt, Claus

Die neue Orgel im Münster zu Strassburg, Strasbourg 1936, extrait de Jahrbuch der Elsass-Lothringischen Wissenschaftlichen Gesellschaft zu Strassburg, vol. IX, 1936, p. 159-207.

Reinhardt, Hans

La Cathédrale de Strasbourg. Paris, 1972, 266 p., ill.

Reinhardt, Hans

Petit historique des orgues de la cathédrale, 1260-1714. Bulletin de la Société des Amis de la Cathédrale, XI1974, p. 57-68, ill. 
Rupp, Emile

Die grosse Orgel des Strassburger Münsters.

Geschichtlicher Rückblick und Aktuelles. Caecilia. 1927. p. 44-47, 65-69, 97-102.

Roethinger, E.A.

Zum Wiederaufbau der Strassburger Münsterorgel.

Caecilia, 1934, p. 125-126.

Roethinger, E.A.

Stand des Wiederaufbaues der Strassburger Münsterorgel. Caecilia, 1935, p. 92-93.

Rupp, Emile

Die grosse Orgel des Strassburger Münsters.

Geschichtlicher Rückblick und Aktuelles. Caecilia. 1927. p. 44-47, 65-69, 97-102.

Schadaeus, 0 .

Summum Argentoratensium Templum. Strasbourg, 1617.

Schmidlin, A.

Geschichte der Strassburger Münsterorgel. Caecilia, 1935, p. 136-140.

Schmitt, 0 .

Johann Jakob Arhardt und das Strassburger Münster.

Elsass-Lothringisches Jahrbuch VII. 1928. p. 126-137.

Schuler, Th.

Das Strassburger Münster. Strasbourg, 1817. $6 \mathrm{pl}$.

Strasburger Münster= und Thurn=Büchlein; oder kurtzer Begriff der merkwürdigsten Sachen, so im Münster und dasigem Thurn zu finden. Strasbourg, 1732, in-12.

Vix-Beulay, A.M.

Essai sur les mannequins articulés du buffet des orgues de la cathédrale de Strasbourg. L'énigme du Rohraffe. L'Alsace française. Strasbourg, 15. 1939/1940, p. 205220 . 
Wagner, P.

Les nouvelles orgues de la cathédrale de Strasbourg.

Bulletin trimestriel des Amis de l'orgue, sept. 1935.

Wörsching, Joseph

Die Strassburger Münsterorgel. Musica Sacra, 67, 1937, p. $82-85$.

Wörsching, Joseph

Die Strassburger Münsterorgel. Die Kirchenmusik, 5, 1942, p. 8-9.

Wörsching, Joseph

Die Orgelwerke des Münsters zu Strassburg i.E. (OrgelMonographien $\mathrm{n}^{\circ}$ 21), Mayence, 1947, 23 p., ill. 
Boalch, Donald H.

Makers of the harpsichord and clavichord 1440 to 1840, Londres, 1956. XXV, 169 p. + 32 planches.

Boltz, Th.

Die Musikerfamilien Stein und Streicher. Vienne, 1917.

(Buhle, E, et Sachs, C.)

Verzeichnis der Sammlung alter Musikinstrumente im Bachhause zu Eisenach. Leipzig, 2/1919. 52 p.

Goehlinger, F.A.

Geschichte des Klavichords, Bâle, 1910.

Herrmann, Heinrich

Die Regensburger Klavierbauer Späth und Schmahl und ihre Tangentenflügel. Diss. Erlangen, 1928.

Hertz, Eva

Johann Andreas Stein (1728-1792). Ein Beitrag zur

Geschichte des Klavierbaues. (Diss. Fribourg i.B. 1937)

Wolfenbüttel et Berlin 1937. 93 p.

Hirt, F.J.

Meisterwerke des Klavierbaus, Olten, 1955.

Hubbard, Frank

Three centuries of harpsichord making. Cambridge, Mass., 1965. 4/1972. 373 p. $41 \mathrm{pl}$.

James, Philip

Early keyboard instruments from their beginnings to the year 1820, Londres, 1930, reprint 1960. 153 p.

Kinsky, Georg

Musikhistorisches Museum von Wilhelm Heyer in Cöln.

Katalog, Vol. 1. Tasteninstrumente, Cologne, 1910, 478

p.

Marpurg, Friedrich Wilhelm

Raccolta delle più nuove compositioni di Clavicembalo, 2 vol. Leipzig 1756-57. 
Nef, Walter

Alte Musikinstrumente in Basel, Bâle, 1974. Bd. 2, Schriften des Historischen Museums Basel.

Rindlisbacher, Otto

Das Klavier in der Schweiz, Berne - Munich, 1972, 268 p., ill.

Rühlmann, J .

Gottfried Silbermann und sein Cimbal d'Amour; das Cimbal royal von J.E. Hänel und die Erfindung des Pianoforte, Monatshefte für Musikgeschichte, 1870. p. 129-140, 149162 .

Russell, Raymond

Victoria and Albert Museum Early keyboard instruments, Londres, 1959. $28 \mathrm{pl}$.

Russell, R.

The harpsichord and clavichord, Londres, 1959, 2/1973.

Samoyault-Verlet, Colombe

Les facteurs de clavecins parisiens. Notices biographiques et documents (1550-1793). Paris, 1966, 189 p.

Victoria \& Albert Museum

A picture book of keyboard musical instruments. Londres, 1929. $20 \mathrm{pl}$.

Vienne

Kunsthistorisches Museum, Wien. Katalog der Sammlung alter Musikinstrumente. I. Teil, Saitenklaviere. Vienne, $1966,95 \mathrm{p} .+32 \mathrm{pl}$.

Winternitz, Emanuel

Keyboard Instruments in the Metropolitan Museum of Art. A Picture Book. New York, 1961, 43 p. 
Billeter, Bernhard

Die Silbermann-Stimmungen, Archiv für Musikwissenschaft, année 27, cahier 1/avril 1970. p. 73-85.

Bormann, Karl

Orgel- und Spieluhrenbau. Kommentierte Aufzeichnungen des Orgel- und Musikwerkmachers Ignaz Bruder (1829) und die Entwicklung der Walzenorgeln. Zurich, 1968, 332 p,, ill.

Dupont, Wilhelm

Geschichte der musikalischen Temperatur, Kassel, 1935. $138 \mathrm{p}$.

Eger, Arthur

Stimmungshöhe und Stimmungsart und ihre Probleme bei der Wiederherstellung alter wertvoller orgeln mit einer Untersuchung der Temperatur Gottfried Silbermanns. 1956.

Kelletat, Herbert

Zur musikalischen Temperatur insbesondere bei Johann Sebastian Bach. Kassel, 1960, 78 p.

Lange, Helmut K.H.

Die Silbermann-Stimmung. Instrumentenbau-Zeitschrift, 20e année, (1966), n², p. 116-122.

Lange, Helmut K.H.

Die Orgelstimmung Gottfried Silbermanns. Ein Beitrag zur Ausführungspraxis alter Musik. ISO-Information, $\mathrm{N}^{\circ} 8$ (Septembre 1972) p. 543-556. N 9 (Février 1973) p. 647658. $\mathrm{N}^{\circ} 10$ (Novembre 1973) p. 721-730. Acta Organologica 7, 1973, p. 154-188.

Legros, Henri

Le tempérament dans la musique d'orgue française classique. La Tribune de I'Orgue. 27e année, $\mathrm{N}^{\circ} 3$, sept. 1975, p. $1-9$.

Lottermoser, Werner

Die Temperierung nach G. Silbermann. Das Musikinstrument, XIV, cahier 11 (1965). p. 999-1001. 
Sorge, Georg Andreas

Gespräch zwischen einem Musico theoretico und einem

Studioso musices von der ... Silbermännischen Temperatur. Lobenstein, 1748 . 
XIV. Jean André Silbermann collectionneur et historiographe. 
Benoit, A.

Collections et collectionneurs alsaciens 1600 - 1820. Strasbourg 1875 .

Friese, J.

I. Neue Vaterländische Geschichte der Stadt Strassburg. Ein Lesebuch für die Jugend. Strasbourg, 1791.

II. Neue Vaterländische Geschichte der Stadt Strassburg, von den ältesten Zeiten bis auf das Jahr 1791. Ein Lesebuch für die Jugend. Strasbourg, 1791 .

III. Neue Vaterländische Geschichte der Stadt Strassburg und des ehemaligen Elsasses. Von den ältesten Zeiten bis auf das Jahr 1791. Strasbourg, 1792.

IV. Id. Strasbourg, 1793.

V. Neue Vaterländische Geschichte der Stadt Strassburg, und des ehemaligen Elsasses. Von den ältesten bis auf unsere Zeiten. Strasbourg, 1801.

Friese, J.

Historische Merkwürdigkeiten des ehemaligen Elsasses, aus den Silbermännischen Schriften gezogen, Strasbourg, 1804 .

Reiber, F.

Iconographie alsatique. Catalogue des estampes et des livres de la collection de Ferdinand Reiber. Préface de Rodolphe Reuss. Strasbourg, 1896.

Reuss, Rodolphe

Catalogue des livres, manuscrits, dessins, gravures, cartes, autographes, etc. de feu M.F.C. Heitz. Strasbourg, 1868, $333 \mathrm{p}$.

Schneegans, Louis

Note de Silbermann (Ensisheim), Bulletin de la Société pour la conservation des monuments historiques d'Alsace, XVIII (1897), appendice p. 22. 
Silbermann, Jean André

Local-Geschichte der Stadt Strassburg, avec vignettes et 16 planches gravées par Weis. Strasbourg, 1775. 232 p., in-folio.

Silbermann, Jean André

Beschreibung von Hohenburg oder dem St. Odilienberg, samt umliegender Gegend, avec 20 gravures de Weiss. Strasbourg, 1781. $134 \mathrm{p}$. in-12.

Trachsler, Beat

Der Basler Zeichner Emanuel Büchel, Bâle, 1973, 88 p., ill.

Wolff, Felix

Verzeichnis der Zeichnungen und Abbildungen der geschichtlichen Denkmäler in Elsass-Lothringen. Strasbourg, 1905 . 
XV. Gottfried Silbermann et ses oeuvres en Saxe. 
Amtskalender für evangelisch-lutherische Geistliche im Königreich Sachsen.

1883.

Anonyme

Die Silbermann-Orgel in der Dorfkirche zu Ponitz

(Thür.), Glaube und Heimat. Ev. Sonntagsblatt für

Thüringen, 4. 1949. $\mathrm{N}^{\circ} 37, \mathrm{p} .3$.

Anonyme

Die Orgel in der katholischen Hofkirche in Dresden, G. Silbermann's letztes und grösstes Werk, Zeitschrift für Instrumentenbau, 26. 1906, $\mathrm{N}^{\circ} 16$.

Anonyme

Kurzgefasste Uebersicht der Bauweisen des Königl. Polnisch. und Churfürstl. Sächs. Land- und Hoforgelbauers Silbermann, Sächsische Schulzeitung, 50. 1883, p. 278 .

Anonyme

Vaterländische Erinnerungen. Aus dem Leben Gottfried

Silbermanns, Sonntags-Beilage zur Norddeutschen

Allgemeinen Zeitung, $\mathrm{N}^{\circ} 42,16$ oct. 1881.

Anonyme

200 Jahre der Silbermann-Orgel in der St.-Georgen-Kirche zu Rötha, Der Kirchenchor, 1922, $\mathrm{N}^{\circ} 2$.

Bewilogua, A. L.

Geschichte der Parochie Helbigsdorf. Grosshartmannsdorf, 1909.

Böhm, H.

Orgeltage und Silbermann-Gedenken. Dresden und Freiberg, Musica, Kassel, 18. 1964. 5, p. 258.

Böhmke, Jörg H.

Die Silbermann-Orgeln von Grosshartmannsdorf und Helbigsdorf, Ars Organi, $\mathrm{N}^{\circ}$ 28. 1966, p. 963-966. 
Bonitz, Eberhard

Die Silbermann-Orgel der Dresdener Hofkirche.

Auslagerung - Wiederaufbau - Erstes Konzert, Ars Organi, $\mathrm{N}^{\circ} 39,1971, \mathrm{p} .1638-1640$.

Culca, Zdenek

Silbermanns Reise nach Prag. Die Quellen über die Prager Reise Gottfried Silbermanns im Jahre 1723, Ars Organi, 44. 1974, p. 1940-1945.

Dähnert, Ulrich

Die mitteldeutschen Orgeln Gottfried Silbermanns, Natur und Heimat, 1. 1953, p. 10-13.

Dähnert, Ulrich

Gottfried Silbermann als Erbauer von Orgeln und Klavierinstrumenten, Musik und Gesellschaft, 8. 1953, p. 13-17.

Dähnert, Ulrich

Freiberg - die Stadt der Silbermannorgeln, Bach-Fest-

Buch, 43. Deutsches Bach-Fest der Neuen Bach-

Gesellschaft 6. - 9. Dez. 1968 in Dresden, p. 37-39.

Dähnert, Ulrich

Die Silbermannorgel in Pfaffroda im Erzgebirge, Der

Kirchenmusiker, 19/4. 1968, p. 167-169.

Dähnert, Ulrich

Orgel in der Wehrkirche zu Mittelsaida, Der Kirchenmusiker, 17/6. 1966, p. 250.

Dähnert, Ulrich

Die Orgellandschaften Sachsen und Thüringen, Acta

Organologica, 1. 1967, p. 45-62. ill.

Dähnert, Ulrich

Ist das Hilbersdorfer Brüstungspositiv im Leipziger

Instrumentenbau-Museum ein Werk Gottfried Silbermanns?

Instrumentenbau-Zeitschrift, 18. 1934, cahier 12.

Dähnert, Ulrich

art. Silbermann, Riemann Musik-Lexikon, vol. 2, Mayence, 1961, p. 684. 
Dähnert, Ulrich

Nicht ausgeführte Orgelbaupläne Gottfried Silbermanns,

Walcker Hausmitteilung, $\mathrm{N}^{\circ} 27,1961$.

Dähnert, Ulrich

Die Orgeln Gottfried Silbermanns in Mitteldeutschland, Leipzig 1953 (Forschungen zur Sächsischen Kunstgeschichte, vol. 2.) $222 \mathrm{p}$. ill.

$2^{\circ}$ éd. augm., Amsterdam, 1971 (Bibliotheca Organologica; vol. 34) 222 p. ill.

David, Werner

Silbermann-Orgel auf Reisen, Musica, Kassel, 6. 1952, p. $214-215$.

Flade, Ernst

Die Silbermann-Orgel des Freiberger Doms und die bei ihrer Erbauung beschäftigten Künstler, Handwerker, Kaufleute und Händler, Zeitschrift für Kirchenmusiker, 14. 1932 .

Flade, Ernst

Gottfried Silbermann als Orgelbauer, Bericht über die Freiburger Tagung für deutsche Orgelkunst vom 27. bis 30. Juli 1926 (éd. Wilibald Gurlitt), Augsbourg, 1926, p. 59-67.

Flade, Ernst

Der Orgelbauer Gottfried Silbermann. Ein Beitrag zur Geschichte des deutschen Orgelbaues im Zeitalter Bachs, Leipzig, Kistner \& Siege1, 1926, 162 p. ill. $2^{\circ}$ éd. augm., Gottfried Silbermann. Ein Beitrag zur Geschichte des deutschen Orgel- und Klavierbaus im Zeitalter Bachs, Leipzig, Breitkopf \& Härte1, 1953, 291 p. ill.

Flade, Ernst

Der Zukunftswert der Silbermannorgel, Bericht über die dritte Tagung für deutsche Orgelkunst in Freiberg i. Sa. vom 2. bis 7. Oktober 1927 (éd. Christhard Mahrenholz), Kassel, 1928. 
Flade, Ernst

Verschiedene Strömungen im Orgelbau unserer Zeit in ihrem Verhältnis zu Gottfried Silbermann, Zeitschrift für Kirchenmusiker, Dresden, 15. 1933, p. 57-60.

Flade, Ernst

Silbermanniana, Musik und Kirche, 10. 1938, p. 79-81.

Flade, Ernst

Gottfried Silbermann. Leben und Werk. Gedenkschrift der Stadt Freiberg zur 200. Wiederkehr seines Todestages, Leipzig, 1953. 26 p.

Flade, P.

Dresdens Orgeln, Dresdener Anzeiger, 8 mai 1900.

Frotscher, Gotthold

Dispositionsgrundsätze Gottfried Silbermanns, Zeitschrift für Kirchenmusiker, 17.

Gärtner, Rudolf

Gottfried Silbermann, der Orgelbauer (Schriftenreihe Grosse Sachsen, Diener des Reiches, 7.), Dresden, 1938.

Gautsch

Der berühmte Orzelbauer Gottfried Silbermann, Saxonia, 2. 1877 .

Grabow, Hans W.

Die Orgeln von Gottfried Silbermann, Ars Organi, 44. 1974, p. 1946-1951.

Haase, 0 .

Ueber die älteren Orgeln Sachsens und deren Erhaltungszustand, Dresden, v. 1944.

Haase, 0 .

Die Kleinorgel G. Silbermanns, Musik und Kirche, 9. 1937, p. 148 .

Habermann, H. et D.

Der Orgelbauer Gottfried Silbermann, Berlin, 1953. 
Haufe, Armin

Unser Sachsen, das Land klingender Kleinode Gottfried Silbermanns, Der Kirchenchor, 1940. $\mathrm{N}^{\circ} 7 / 8$, p. 43.

Hentschel, Walter

Der Orgelbauer Gottfried Silbermann, Heimatschutz. Mitteilungen, 21. 1932.

Hoffmann, F.

Die Silbermann-Orgel in Grosskmehlen, Glaube und Gewissen, Halle/Saale, 6. 1960, p. 149-151.

Hüllemann, Herbert

Die Tätigkeit des Orgelbauers Gottfried Silbermann im Reussenland, Leipzig, 1937. 68 p.

Kemper, K.

Die Freiberger Domorgel und ihre Veränderungen seit

ihrer Erbauung durch Silbermann, Zeitschrift für

Instrumentenbau, Leipzig, 48. 1928, p. 879.

Monatschrift für Gottesdienst und kirchliche Kunst, 33.

1928, p. 340

Kirchenmusik, Berlin, 9. 1934, p. 317-319.

Kemper, K.

Nochmal: Die Domorgel zu Freiberg, Zeitschrift für Instrumentenbau, Leipzig, 49. 1929, p. 596.

Kirsten, Alfred

Die Lebensgeschichte der Silbermannorgel in der Georgenkirche zu Rötha, Rötha, s.d. (1935)

Kirsten, Alfred

Das klingende Wunder von Rötha, Die Nationalkirche, Weimar, 8. 1940, p. 208.

Kirsten, Alfred

An einem Markstein in der Geschichte der Röthaer Silbermann-Orgeln (Marienorgel), Rötha 1935, et in Kirchenchor, 46. 1935, p. 59-61.

Liesche, R.

Das Silbermann-Positiv (im Bremer Dom), Bremen, 1939. 
Löhn-Siegel, Anna

Gottfried Silbermanns Lebensgang, Wissenschaftliche

Beilage der Leipziger Zeitung, 1906. 44 a.

Lottermoser, Werner et Jenkner, E.

Klanganalytische Untersuchungen an der G. SilbermannOrgel in Reinhardtsgrimma, Das Musikinstrument, 17. 2, p. 172., 1968 .

Lottermoser, Werner

Die Akustik des Raumes und der Orgel in der Frauenkirche zu Dresden, Lottermoser, Werner et Meyer, Jürgen, Orgelakustik in Einzeldarstellungen, Francfort/M., 1966. Das Musikinstrument, vol. 16. 1966, p. 149-154.

Märker, M. J. F.

Chronik von Grosshartmannsdorf, Marienberg, 1840.

Magnus, E.

Der Klang der Freiberger Silbermann-Orgel, Zeitschrift

für Instrumentenbau, Leipzig, 48. 1928, p. 984.

Mahrenholz, Christhard

Grundsätze der Dispositionsgestaltung des Orgelbauers Gottfried Silbermann, Archiv für Musikwissenschaft, 16 . 1959, p. 174-186.

Mahrenholz, Christhard

Musicologica et Liturgica, Kassel, 1960.

Matthews, John

Gottfried Silbermann and his times, The Organ, 4. $1924 / 25$, p. 25, 128 .

Mengert, Maximilian

Gottfried Silbermann, der Erbauer der Orgel der St. Georgenkirche zu Glauchau, Glauchauer Zeitung und Neueste Nachrichten, 4 mai 1913.

Mooser, Ludwig

Gottfried Silbermann der Orgelbauer. Ein historisches Lebensbild, Langensalza, 1857, 158 p. 
Müller, Fritz

Die Besetzung des Pedals der Orgeln von Gottfried

Silbermann, Instrumentenbau-Zeitschrift, Constance, 11. 1956/57, p. 7-8.

Müller, Werner

"Weil Frauenstein mein Heimatland..." (Aus der Heimat des Orgelbauers Gottfried Silbermann), Walcker Hausmitteilung, $\mathrm{N}^{\circ}$ 34. 1965.

Müller, Werner

Auf den Spuren Gottfried Silbermanns, Brand-Erbisdorfer Zeitung, 1965.

Müller, Werner

Die Werktage des Gottfried Silbermann, InstrumentenbauZeitschrift, 20. $\mathrm{N}^{\circ}$ 8. 1966, p. 389-392.

Müller, Werner

In der Werkstatt Gottfried Silbermanns, Walcker Hausmitteilung, $\mathrm{N}^{\circ}$ 36. 1966.

Müller, Werner

Gottfried Silbermanns Nachlass, Instrumentenbau-Zeitschrift, 21, 1967, p. 176.

Müller, Werner

Das Geburtshaus des Orgelbauers Gottfried Silbermann, Sächsische Heimatblätter, 1967, N 1 .

Müller, Werner

Auf den Spuren von Gottfried Silbermann. Ein Lebensbild des berühmten Orgelbauers nach urkundlichen Quellen, Berlin, Evangelische Verlagsanstalt, et Kassel-Bâle, Bärenreiter, 1968, 252 p. ill.

$4^{\circ}$ éd. augm., Berlin 1975, 336 p. ill.

Oehme, Fritz

Handbuch über ältere und neuere berühmte Orgelwerke im Königreich Sachsen von 1710 an bis zur Gegenwart, Dresden, 3 vol. 1889-1897. 
Oswald, E.

Drei Orgeln im Freiberger Dom. Eine wertvolle Silbermann-Orgel gerettet, Zeitschrift für Kirchenmusiker, Dresden, 21. 1939, p. 33-35.

Pape, Uwe

Die Silbermann-Orgel im Bremer Dom, Ars Organi, $\mathrm{N}^{\circ} 24$, 1964, p. 739-741.

Paulik, G.

Registrierung auf Silbermann Orgeln, Zeitschrift für Kirchenmusiker, 10. 1928, p. 93, 101.

Paulik, G.

Zur 200-Jahrfeier der Einweihung der Silbermannorgel in der Frauenkirche zu Dresden, Nov. 1736, Zeitschrift für Kirchenmusiker, 18. 1936, p. 87.

Quoika, Rudolf

Ueber die Orgellandschaft J.G.Silbermanns, Musik und Kirche, 23. 1953/3, p. 91.

Ritter, J.L.

Etwas zur Feyer des ersten Jubiläums der beyden Silbermannschen Orgeln in Rötha, Leipzig, 1821.

Rubardt, Paul

Die Silbermannorgeln in Rötha, Leipzig, Breitkopf \& Härtel, 1953, 32 p. ill.

Rubardt, Paul

Die Silbermannorgeln in Rötha, Bach-Fest-Buch zum 27. Deutschen Bachfest in Leipzig 1950.

Ruger, Gerhard

Die Silbermann-Orgel zu Glauchau, St. Georgen, s.l.n.d.

Schäfer, Oskar

Bisher unbekannte Orgel Gottfried Silbermanns, Zeitschrift für Kirchenmusiker, Dresden, 13. 1931, p. 101. 
Schäfer, Oskar

Eine Silbermann-Orgel in einem Schradendorf, Zeitschrift für Kirchenmusiker, Dresden, 1. oct. 1931, et Zeitschrift für Musikwissenschaft.

Schäfer, Oskar

Noch ein Kleinod in der "Schraden". Eine Silbermann-

Orgel in der Kirche zu Kmehlen, Mitteilungen des

Landesv. für sächs. Heimatschutz, 20. 1931, p. 379.

Schlecht, Gottfried

Die Orgel der Dresdener Frauenkirche und ihr Erweiterungsplan vom Jahre 1912, Einführungsheft zur 3. Tagung für deutsche Orgelkunst in Freiberg/Sa., Kassel, 1927.

Schlecht, Gottfried

Originale Registermischungen auf Silbermannschen Orgeln, Einführungsheft zur 3. Tagung für deutsche Orgelkunst in Freiberg/Sa., Kassel, 1927.

Schlitterlau, Rudolf

Gottfried Silbermann, Der Kirchenchor, 1918. $\mathrm{N}^{\circ} 3$.

Schnorr von Carolsfeld, E.

Gottfried Silbermann und wir, Zeitschrift für Instrumentenbau, Leipzig, 52. 1932. $\mathrm{N}^{\circ} 17$.

Schütz, F.

Die Silbermann-Orgel im Dome zu Freiberg, Zeitschrift

für Kirchenmusiker, Dresden, 9. 1928. p. 185.

Schwarzenberg, Christoph

Die Silbermannorgel Crostau, The Organ Yearbook, 5. 1974, p. 40-56, ill.

Stöbe, Paul

Geschichte des Orgelbaues in Sachsen, Der Kirchenchor, 1900-1902, 1904 .

Stöbe, Paul

Die Silbermann-Orgeln in Frauenstein, Zeitschrift für Instrumentenbau, Leipzig, 50. 1930, p. 716, 748; et Der Kirchenchor, 41. 1930, p. 73-76. 
Sumner, William Leslie

Silbermann and his work, Hinrichsen, Max, 8th Music Book, Londres, 1955.

Türcke, M.

Gottfried Silbermann, Sächsische Schulzeitung, 1891.

Türcke, $O$.

Gottfried Silbermann, der Altmeister der sächsischen Orgelbauer, Sächsische Schulzeitung, 1891, p. 291-303.

Ungelenk, Manfred

Geschichte der Silbermannorgel von Schloss Burgk, Schleiz, 1958, $31 \mathrm{p}$.

Walde, P.

Die Silbermann-Orgel der kath. Hof- und Propsteikirche zu Dresden, Musica Sacra, 58. 1929, p. 96; et Organon, Munich, 6. 1931, p. 24.

Walde, P.

Die Orgel der kath. Hof- und Propsteikirche zu Dresden (G. Silbermanns letztes Werk), Monatshefte für kath. Kirchenmusik, 11. 1929, p. 73-77.

Walde, P.

175 jähr. Jubiläum der Orgel in der kath. Hofkirche zu Dresden, Zeitschrift für Instrumentenbau, Constance, 49. 1929, p. 258.

Walther, Kurt

Der Orgelbauer G. Silbermann und sein Werk in der Kirche zu Ponitz, Altenburger Heimatblätter, 1937, $\mathrm{N}^{\circ} 3$.

Williams, Peter

Organs in Freiberg, Saxony, The Organ Yearbook, 2. 1971, p. 13-22, ill.

Wörsching, Joseph

Gottfried Silbermanns erstes und letztes Meisterwerk, Musica Sacra, 58. Regensburg, 1928. 
Wörsching, Joseph

Die Silbermann=Orgel im Dome zu Freiberg (Sachsen), Mayence, s.d., ill. 16 p. (Orgel=Monographien $\mathrm{N}^{\circ}$ 58).

Ziebel, Fr.

Geschichtliches über die Silbermann Orgel in

Grosskmehlen, Die evangelische Kirchenmusik der Provinz Sachsen, 5, 1939, p. 76-78.

Zschaler, G.

Gottfried Silbermann, Hof- und Landorgelbauer zu Sachsen, Dresden, 1858, 70 p. 
XVI. Les élèves de Gottfried Silbermann. 
Armstrong, William H.

Organs for America. The life and work of David

Tannenberg. Philadelphia, 1967, 154 p., ill.

Dähnert, Ulrich

Der Orgel- und Instrumentenbauer Zacharias Hildebrandt, Leipzig/Wiasbaden, 1962, 266 p., ill.

Dähnert, Ulrich

Der Orgelbauer Zacharias Hildebrandt, Musik und Kirche. 27. 1957. p. 285-286.

Dähnert, Ulrich

Zacharias Hildebrandt zu seinem 200. Todestag am 11. Oktober 1957, Ars Organi, 11. 1957, p. 189-191.

Hofner, Hans

Die Silbermannschule im Markgrafentum Bayreuth, Zeitschrift für Instrumentenbau, 53. 1933. p. 315, 331, $348-351$.

Hofner, Hans

Die Orgelbauer Graichen und Ritter als SilbermannSchüler, Ars Organi, 22. 1963. p. 589-610.

Nichterlein, W.

Die Hildebrandorgel in der St. Wenzelskirche zu Naumburg a.S. und ihr Erbauer, s.1.n.d. (1933)

Poppen, H.

Der Orgelbauer Joachim Wagner - ein Schnitger-Schüler, Archiv für Musikforschung, V (1940) p. 230.

Rubardt, Paul

Zacharias Hildebrandt und seine Orgel in Störmthal, 1934 .

Steves, Heinz Herbert

Der Orgelbauer Joachim Wagner (1690-1749). Archiv für Musikforschung, IV (1939), p. 321; V (1940), p. 17. 
Allihn, Max

Bach-Silbermann-Orgel, Zeitschrift für Instrumentenbau, Leipzig, 1909, p. 971, 1017.

Bach-Dokumente

vol. 1: Schriftstücke von der Hand Johann Sebastian

Bachs, éd. Werner Neumann et Hans-Joachim

Schulze, Leipzig et Kassel, 1963, 288 pp. ill.

vol. 2: Fremdschriftliche und gedruckte Dokumente zur

Lebensgeschichte Johann Sebastian Bachs 1685-

1750, éd. Werner Neumann et Hans-Joachim

Schulze, Leipzig et Kasse1, 1969, 573 pp. ill.

vol. 3: Dokumente zum Nachwirken Johann Sebastian Bachs 1750-1800, éd. Hans-Joachim Schulze, Leipzig et Kasse1, 1972, 750 pp. ill.

Dähnert, Ulrich

Johann Sebastian Bach's ideal organ, The Organ Yearbook, 1. 1970, p. 21-37. ill.

David, Werner

Johann Sebastian Bach's Orgeln, Berlin, 1951, 107 pp., ill.

Ernst, Friedrich

Bach und das Pianoforte, Bach-Jahrbuch 1961, p. 61. Réimpression: Das Musikinstrument $\mathrm{N}^{\circ}$ 6, Francfort/M., s.d. $23 \mathrm{pp}$.

Flade, Ernst

Bachs Stellung zum Orgel- und Klavierbau seiner Zeit, Bericht über die wissenschaftliche Bachtagung der Gesellschaft für Musikforschung, Leipzig, 1950.

Kloppers, Jacobus

Die Interpretation und Wiedergabe der Orgelwerke Bachs, Francfort/M., 1966, 398 pp.

Paumgartner, Bernhard

Johann Sebastian Bach. vol. I: Bis zur Berufung nach Leipzig, Zurich, 1950, 543 pp. ill. 
Quoika, Rudolf

Albert Schweitzers Begegnung mit der Orgel, Berlin, 1954 .

Rougier, Adrien

Les orgues de Jean-Sébastien Bach, Lyon, 1962, 55 p.

Rubardt, Paul

Zwei originale Orgeldispositionen J.S.Bachs, Festschrift Heinrich Besseler, Leipzig, 1962. p. 495.

Rubardt, Paul

Bachorgeln in Leipzigs Umgebung, J.S.Bach. Das Schaffen eines Meisters im Spiegel einer Stadt, Leipzig, 1950.

Schrammek, Winfried

Johann Sebastian Bach, Gottfried Silbermann und die französische Orgelkunst, Bach-Studien, 5/1975, p. 93107, éd. Rudolf Eller et Hans-Joachim Schulze, Leipzig, 1975 .

Schweitzer, Albert

J.S.Bach, le musicien-poète, Leipzig, 1905, 455 p.

Schweitzer, Albert

J.S.Bach, Leipzig, 1908. 791 p. ill.

Sommer, William Leslie

The organ of Bach and Gottfried Silbermann, Organ

builder, Londres, 1956, 432 p.

Williams, Peter

The Organs in St. Wenzel, Naumburg, with some thoughts on G. Silbermann and J.S.Bach, The Organ, 46. 1966/67, p. 31 . 
XVIII. Mozart et l'orgue. 
Bauer, W.A. et Deutsch, O.E.

Mozart, Briefe und Aufzeichnungen. vol. 2. Kasse1, 1962.

Hartmann, C.

Die Orgel von St. Bavo; Mozart's Reise nach Haarlem. Iserlohn, 1947.

Lievense, W.

De familie Mozart op bezoek in Nederland. Eeen reisverslag. Hilversum, 1965, 48 p., ill.

Pröger, Johannes

Mozarts Verhältnis zur Orgel und zur Orgelkomposition. Berlin, 1965, $27 \mathrm{p}$.

Wörsching, J .

Wolfgang Amadeus Mozart und die Orgel. Zeitschrift des Historischen Vereins für Schwaben, Vol. 55-56 (1942-

1943) Augsburger Mozartbuch. p. 452-486, ill. 
XIX. L'orgue en Allemagne et en Autriche. 
Adlung, Jacob

Musica Mechanica Organoedi. Berlin, 1768. Facsimile par

Chr. Mahrenholz, Documenta Musicologica, Kassel, 1961.

Bärnwick, F.

Die grosse Orgel im Münster zu Weingarten in

Württemberg. Weingarten 1922. 4/1948 Kassel.

Bendeler, Joh. Philipp

Organopoeia, oder Unterweisung wie eine Orgel nach ihren Hauptstücken ans wahren mathem. Gründen zu erbauen sei. Francfort et Leipzig, 1690.

Biba, 0 .

Zur Lebensgeschichte des Wiener Orgelbauers Johann

Hencke, Geseker Heimatblätter, 26, N 140 (1968)

Biehle, Johannes

Die Tagung für Orgelbau in Berlin 1928. Kasse1, 1929,

$111 \mathrm{p}$, ill.

Biermann, J.H.

Organographia Hildesiensis specialis, Hildesheim, 1738.

Bösken, Franz

Die Orgelbauerfamilie Stumm aus Rhaunen-Sulzbach und ihr Werk. Ein Beitrag zur Geschichte des Orgelbaus am Mittelrhein. Mayence 1960, 108 p., 16 pl.

Bösken, Franz

Quellen und Forschungen zur Orgelgeschichte des Mittelrheins Vol. 1: Mainz und Vororte - Rheinhessen Worms und Vororte. Mayence, 1967, 541 p., ill.

Bormann, Karl

Orgel- und Spieluhrenbau. Kommentierte Aufzeichnungen des Orgel- und Musikwerkmachers Ignaz Bruder (1829) und die Entwicklung der Walzenorgeln. Zurich, 1968, 332 p., ill.

Bormann, Karl

Die gotische Orgel zu Halberstadt. Berlin, 1966, 192 p., ill. 
Boxberg, Christian Ludwig

Ausführliche Beschreibung der Grossen Neuen Orgel in der Kirchen zu St. Petri und Pauli allhie zu Görlitz. Görlitz, 1704.

Brommer, Hermann

Die Orgel der ehemaligen Gengenbacher Abteikirche im Augustinermuseum zu Freiburg, tiré-à-part de la revue Schau-ins-Land, 86. Jahresheft des BreisgauGeschichtsvereins, Fribourg-en-Brisgau, 1968, p. 77-102.

Bruggaier, E.

Studien zur Geschichte des Orgelpedalspiels in Deutschland bis zu Zeit Johann Sebastian Bachs. Francfort/M., 1959.

Bullmann, F.G.

Die rheinischen Orgelbauer Kleine - Roetzel - Nohl. Leben und Werk einer Orgelbanerfamilie des 18. und 19. Jahrhunderts im rheinischen und südwestfälischen Raum. Giebing über Prien am Chiemsee, 1969. (Schriften zur Musik, Vol. 6)

Burgemeister, Ludwig

Der Orgelbau in Schlesien. Strasbourg, 1925. 2/Francfort 1973, $376 \mathrm{p} ., 70 \mathrm{pl}$.

Dähnert, Ulrich

Tobias Trost, Die Musik in Geschichte und Gegenwart. Vol. 13. Kasse1, 1966. col. 828-829.

David, Werner

Die Orgel von St. Marien zu Berlin und andere berühmte Berliner Orgeln. Mayence, 1949 (Orgelmonographien $\mathrm{N}^{\circ}$ $60)$.

Eisenmann, P.

Zur Wiederherstellung der alten Orgel aus Kleinkems in der alten evangelischen Kirche in Schopfheim. Nachrichtenblatt der Denkmalpflege in Baden-Württemberg, $4^{\circ}$ année, cahier 3, 1961. 
Fischer, Hermann et Wohnhaas, Theodor

Süddeutsche Orgeln aus der Zeit vor 1900. Eine Bestandsaufnahme auf Grund der Aufzeichnungen der Orgelbauer Strebel in Nürnberg. Francfort/M., 1973, 319 p., ill.

Fock, Gustav

Arp Schnitger und seine Schule. Kasse1, 1974, 310 p., 46 $\mathrm{pl}$.

Förner, Christian

Vollkommener Bericht, wie eine Orgel aus wahrem Grunde der Natur in allen ihren Stücken nach Anweisung der mathematischen Wissenschaft solle gemacht, probirt und gebraucht werden. Berlin, 1684.

Frotscher, Gotthold

Deutsche Orgeldispositionen aus fünf Jahrhunderten. Wolfenbüttel et Berlin, 1939, 68 p.

Frotscher, Gotthold

Geschichte des Orgelspiels und der Orgelkomposition. 2 vol. Berlin, 1935. 2/ 1959.

Gernhardt, K. et Schrammek, W.

Fehler bei der Umrechnung von Graden der Foernerschen Windwaage in Millimeter-Wassersäule, Das Musikinstrument, XVIII cahier 2, p. 191 (1969).

Graf, A.

Die Pfalz als Orgellandschaft, Der Turmhahn, 12, cahier $5 / 6$ (1968).

Gurlitt Wilibald (éd.)

Bericht über die Freiburger Tagung für deutsche Orgelkunst vom 27. bis 30. Juli 1926. Augsburg, 1926, 175 p., ill.

Haake, W.

Caspariniana, Ars Organi, cahier 28, juillet 1966, p. $966-970$.

Hackel, W.

Uber die Orgelbauer Ulisch und Kayser, Ars Organi, $\mathrm{N}^{\circ}$ 40, juin 1972, p. 1701-1702. 
Halle, Johann Samuel

Die Kunst des Orgelbaues, theoretisch und praktisch beschrieben. Brandenburg, 1779.

Halle, Johann Samuel

Theoretische und praktische Kunst des Orgelbaues. Brandenburg, 1779 .

Harrassowitz, Hermann

Geschichte der Kirchenmusik an St. Lorenz in Nürnberg, extrait de Mitteilungen des Vereins für Geschichte der Stadt Nürnberg. Vol. 60, 1973.

Haupt, L.

Die Geschichte der berühmten Orgel in der Haupt-und

Pfarrkirche zu St. Peter und Paul in Görlitz, Neues Lausitz. 36

Haupt, R.

Die Orgel von St. Sixtus zu Northeim (Hannover). Orgelmonographien, $\mathrm{N}^{\circ}$ 18, Mayence, s.d.

Heiling, Hans

Johann Ignaz Egedacher und seine Orgel in der Stiftskirche zu Zwettl in Niederösterreich, Mayence, 1959. (Orgelmonographien, 49) .

Hofner, Hans

Matthias Tretzscher, ein Kulmbacher Orgelbauer der Barockzeit, Ars Organi, cahier 23, février 1964.

Hofner, Hans

Das Orgelbauergeschlecht der Wiegleb in Mittel- und Oberfranken, Kulturwarte (Hof) 11, 1965.

Hohn, A.

Die Orgeldispositionen (Jesuitenkirche Mannheim), Jesuitenkirche Mannheim. 30 édition. Mannheim, 1965. n. 16-19.

Hohn, A.

Die Orgel der Gebrüder Stieffell in der Stadtkirche zu Rastatt. Rastatt, 1964. 
Kaufmann, Walter

Die Orgeln des alten Herzogtums Oldenburg. Oldenburg, 1962, $200 \mathrm{p} ., 32 \mathrm{pl}$.

Kaufmann, Walter

Die Orgeln Ostfrieslands. Aurich, 1968, 387 p., 33 pl.

Klaus, Gregor

Die grosse Orgel zu Weingarten, s.l., 1959.

Kleemann, Gotthilf

Die Orgelmacher und ihr Schaffen im ehemaligen Herzogtum Württemberg. Stuttgart, 1969, 266 p., ill.

Leucht, K.F.

Die Orgel in der Karlsruher Schlosskapelle aus dem Jahre 1786. Tübingen, 1938 .

Lindner, H.

Unsere alte Orgel und ihr Schicksal (Jesuitenkirche

Mannheim), Jesuitenkirche Mannheim. $3^{\circ}$ édition,

Mannheim, 1965. p. 7-13.

Mahrenholz, Chr. (éd.)

Bericht über die $3 e$ Tagung für deutsche Orgelkunst in

Freiberg i. Sa. vom 2. bis 7. Oktober 1927, Kasse1, 1928 .

Marks, Günther

Eine 200 jährige Joachim Wagner=0rgel wurde wiederhergestellt, Musik und Kirche, 12, 1940, p. 43-47.

Mattheson, J.

Critica Musica. Hambourg, 1722-25.

Merian, M.

Topographia Sveviae. Francfort, 1643.

Nicolai, D.T.

Kurze doch zuverlässige Beschreibung der grossen Orgel

in der Hauptkirche zu Görlitz, 1797. 
Niedt, Friedrich E.

Musikalische Handleitung mit einem Anhang von mehr als 60 Orgel-Wercken versehen durch J. Mattheson. Hambourg, 1721.

Pape, Uwe

Arp Schnitger, ISO-Information. $\mathrm{N}^{\circ} 5$ (1971) p. 357-376.

Peine, Theodor

Der Orgelbau in Frankfurt am Main und Umgebung von den

Anfängen bis zur Gegenwart. Francfort, 1956, 288 p.

Praetorius, Michael

De Organographia (Syntagma Musicum II). Wolfenbüttel,

1619. facsimile W. Gurlitt, Documenta Musicologica,

Kassel, 1958.

Quoika, R.

Joh. Georg Freundt, Die Musik in Geschichte und

Gegenwart. Vol. 4. Kassel, 1955, col. 931-933.

Quoika, R.

Egedacher, Die Musik in Geschichte und Gegenwart vol. 3. Kassel, 1954. col. 1150-1156.

Reuter, Rudolf

Orgeln in Westfalen. Inventar historischer Orgeln in

Westfalen und Lippe. Kassel, 1965, 375 p., 259 pl.

Reuter, Rudolf

Die Orgel in der Denkmalpflege Westfalens. Kasse1, 1971, 96 p., ill.

Ritter, A.G.

Zur Geschichte des Orgelspiels, vornehmlich des deutschen, im 14. bis zum Anfange des 18. Jahrhunderts. Leipzig, 1884. vol. I, texte, 225 p. vol. II, exemples musicaux, $230 \mathrm{p}$.

Rücker, Ingeborg

Die deutsche Orgel am Oberrhein um 1500. Fribourg-enBrisgau, 1940, $168 \mathrm{p}$. 
Schefold, Max

Die Ulmer Orgel- und Klavierbauerfamilie Schmahl. Zeitschrift für Musikwissenschaft, $\mathrm{N}^{\circ}$ 12, Leipzig, 1930, p. $531 \mathrm{ff}$.

Schlick, Arnold

Spiegel der Orgelmacher und Organisten. Spire, 1511. Réédition par Paul Smets, Mayence, 1959, 134 p.

Schnitger, Arp

De nagelaten geschriften van de orgelmaker Arp Schnitger (1648-1719). éd. C.H. Edskes. Sneek, 1968.

Schubart, R.H.

Die Orgel der Ludwigskirche in Saarbrücken, Zeitschrift für die Geschichte der Saargegend, XVII/XVIII (1969/70) p. $345-363$.

Seifert, Hans et Supper, Walter

Die Hauptorgel des Ulmer Münsters von 1969. Ulm, 1969, 31 p., ill.

Smets, Paul

Die grosse Gabler-Orgel der Abtei Weingarten, Mayence, 1940. (Orgelmonographien, 9).

Smets, Paul (éd.)

Orgeldispositionen (Dresdener Handschrift). Kasse1, 1931.

Strube, Wilhelm

Johann Christian Köhler, ein unbekannter mitteldeutscher Orgelbauer in Hessen-Darmstadt. Ars Organi, 26. 1965, p. 885-886.

Stüven, W.

Orgel und Orgelbauer im Halleschen Land vor 1800. Wiesbaden, 1964. 
Sulzmann, Bernd

Die Orgeln der Gebrüder Stieffell in Köndringen und

Ichenheim. Nachrichtenblatt der Denkmalpflege in BadenWürttemberg, année 11, 1968, cahier 1, p. 19-26.

Sulzmann, Bernd

Der Orgelbau am Oberrhein im 18.. und 19. Jahrhundert. Acta organologica, vol. 2, Berlin, 1968, p. 54 - 81.

Sulzmann, Bernd

Die Orgelbauerfamilie Martin in Waldkirch im Breisgau. Wiesbaden, 1975. 235 p., ill.

Sulzmann, Bernd

Die Orgel der Katholischen Stadtpfarrkirche St. Bartholomäus zu Ettenheim. Ettenheim, 1973. 47 p., ill.

Walter, R.

Die Orgel der ehemaligen Abteikirche Schwarzach und ihr Erbauer Joh. Georg Rohrer, Die Ortenau, Veröffentlichungen des Historischen Vereins für Mittelbaden, $47^{\circ}$ année, Offenburg, 1967. p. 216-229.

Walter, R.

Der Würzburger Hoforgelmacher J. Ph. Seuffert, Die Mainlande, Würzburg. 12. 1961. Nº 18.

Walter, R.

Beziehungen zwischen süddeutscher und italienischer Orgelkunst vom Tridentinischen Konzil bis zum Ausgang des Barock, Acta organologica, V (1971) p. 160-205.

Walther, J.F.

Die in der Königl. Garnisonskirche zu Berlin befindliche neue Orgel. Berlin, 1727.

Walther, J.F.

Die Gute Hand Gottes über der Garnisonskirche und SchulAnstalten. Berlin, c. 1729. 
Werckmeister, Andreas

Erweiterte und verbesserte Orgel=Probe. Quedlinburg, 1698. (Réédition Kassel, 1970).

Werckmeister, Andreas

Organum Gruningense redivivum. Oder kurtze Beschreibung des in der Grüningischen Schlos-Kirchen berühmten OrgelWercks. Wie dasselbe anfangs erbauet und beschaffen gewesen: Und wie es anitzo auf allergnädigsten Befehl Sr. Kön. Preuss. Majestät ist renoviret und mercklich verbessert worden. Quedlinburg et Aschersleben 1705. (Réédition Mayence, 1932).

Winter, Helmut

Das Winddruckproblem bei den norddeutschen orgeln im 17. und 18. Jahrhundert, Acta Organologica Vol. 3 Berlin, 1969. p. 176-182.

Wörsching, J.

Die Baugeschichte der Ottobeurer Riepporgeln 1754 bis

1766, Zeitschrift für Instrumentenbau. Leipzig, 60. 1940. p. 182184, 206-208, 61. 1941.p. 2.

Wörsching, J.

Alte Orgelpläne für Ottobeuren, Christliche Kunst, à partir de 1938: Die neue Saat. 1938. p. 209-213.

Wörsching, J.

Schwäbische Orgelbauer des 18. Jahrhunderts in Franken, Musica sacra. Cologne. 68. 1937. p. 151-158.

Wörsching, J.

Die Silbermannorgel in Schwarzach, Der Kirchensänger, année 37, $\mathrm{N}^{\circ}$ 6, 1937.

Wörsching, J.

Die berühmten Orgelwerke der Abtei Ottobeuren, erbaut 1754 - 1766. Mayence, 1941.

Wörsching, J.

Die Orgeln der Abteikirche Ottobeuren. 1959. 
Wunderlich, H.

Die Schnitger-Orgel der Hauptkirche St. Jacobi zu Hamburg und ihre Bedeutung für die Orgelbewegung, Die Arp-SchnitgerOrgel der Hauptkirche St. Jacobi Hamburg, Hambourg, 1961. 
XX. L'orgue en Suisse. 
Boerlin, P. - H.

Die Orgelflügel-Entwürfe von Hans Holbein d. Ae. Zum Problem der Künstlerischen Gestaltung des Orgelgehäuses. Oeffentliche Kunstsammlung Basel. Jahresberichte 195960. p. 141-164.

Fluri, A.

Orgel und Organisten in Bern vor der Reformation. Berne, 1905.

Fluri, A.

Zur Geschichte der Münsterorgel, Der Münsterausbau in Bern, Jahresbericht 1909.

Gramm, J.J.

Esquisse historique des anciennes orgues de la cathédrale de Lausanne (1411-1954). La Tribune de I'Orgue, $8^{\circ}$ année, $\mathrm{N}^{\circ} 3$ (février 1956) p. 8-10; $\mathrm{N}^{\circ} 4$ (avril 1956) p. 6-10; $\mathrm{N}^{\circ} 5$ (juin 1956) p. 7-9.

Jakob, F.

Der Orgelbau im Kanton Zürich. (Publikationen der Schweizerischen Musikforschenden Gesellschaft, série 2, vol. 18) 1971 .

I. Textband. $296 \mathrm{p} ., 45 \mathrm{pl}$.

II. Quellenband, $470 \mathrm{p}$.

Majon, Luc

Das Berner Münster. (Die Kunstdenkmäler des Kantons Bern, vol 4) Bâle, 1960.

Mooser, R.A.

Aloys Mooser, facteur d'orgues à Fribourg. Tirage à part des Etrennes Fribourgeoises, 1935.

Münger, Fritz

Schweizer Orgeln von der Gotik bis zur Gegenwart. Berne, $1961,20 \mathrm{p} ., 39 \mathrm{pl}$.

Refardt, Edgar

Historisch-Biographisches Musikerlexikon der Schweiz. Leipzig, Zurich, 1928. 
Walter, Rudolf

Zur Orgelgeschichte des Domstiftes Arlesheim. Katholische Kirchenmusik, cahier 5/1966. 
523

XXI. L'orgue aux Pays-Bas. 
Bouman, A.

Nederland ... Orgelland. Leiden 1964, 165 p., ill.

Edskes, C.H.

De nagelaten geschriften van de orgelmaker Arp Schnitger (1648-1719). Sneek, s.d. (1968), 80 p., ill.

Edskes, C.H.

Repertorium op de archivalia betreffende het orgel in de Martinikerk te Groningen. Groningue, 1971.

Edskes, C.H.

Rapport betreffende het orgel in de Martinikerk te Groningen. Groningue, 1972 .

Edskes, C.H.

The Organs of the Oude Kerk in Amsterdam at the Time of Sweelinck. Alan Curtis, Sweelinck's Keyboard Music, Leyden, 2/1972, p. 163-200.

Hess, Joachim

Dispositien der merkwaardigste Kerk-orgelen, welke in de zeven Vereenigde Provincien alsmede in Duytsland en Elders aangetroffen worden. Gouda, 1774. Reprint Buren, 1976.

Jongepier, Jan

Frieslands Orgelpracht. Deel I 1500-1800. Sneek, 1970, ill.

Knock, N.A.

Dispositien der merkwaardigste Kerk-Orgelen. Groningue 1788. Réédition Sneek, 1968, 64 p., ill.

Moortgat, Gaby

Oude Orgels in Vlaanderen. II. Anvers, 1965, 156 p., ill.

Peeters, F. et Vente, M.A.

L'Orgue et la Musique d'Orgue dans les Pays-Bas et la Principauté de Liège du $16 e$ au $18 e$ siècle. Anvers, 1973 341 p., ill. 
Radeker, Joh.

Korte beschrijving van het beroemde en prachtige orgel in de Groote of St. Bavooskerk te Haarlem. Haarlem, 1775 .

Arp Schnitger (1648-1719) en zijn werk in het Groningerland. Groningue, 1969, 104 p., ill.

Vente, M.A.

L'influence des Flamands sur les Français en matière de construction d'orgues. L'Orgue, 48, p. 78; 49, p. 110.

Vente, M.A.

Die Brabanter Orgel. Amsterdam, 1963, 275 p. 45 pl.

Vente, M.A.

Vijf eeuwen Zwolse orgels 1447-1971. Amsterdam, 1971, 82 p., $18 \mathrm{pl}$. 


\section{Supplément à la bibliographie}

Livet, Georges et Rapp, Francis

Histoire de Strasbourg des origines à nos jours, 4 vol., Strasbourg, 1980 .

Dähnert, Ulrich

Historische Orgeln in Sachsen, Francfort, 1980.

Sulzmann, Bernd

Ein Orgelbauprojekt Johann Andreas Silbermanns für

die Kirche St. Louis in Neuf-Brisach (Neubreisach) aus

dem Jahr 1736, in: Ars Organi, année 31, $\mathrm{N}^{\circ} 2$ (juin

1983), p. 90-93.

Sulzmann, Bernd

200 Jahre Orgelbaugeschichte in St.Blasien, in: St.Bla-

sien. Festschrift aus Anlass des 200jährigen Bestehens

der Kloster- und Pfarrkirche, Munich, 1983, p. 232-275.

Lange, Helmut K.H.

Uber die Bedeutung des Syntonischen Kommas und seine Verwendung in der Orgelstimmung Gottfried Silbermanns, in: Acta Organologica, XVI (1982), p. 235-248.

Kühl, Jürgen

Die Orgelstimmungen von Ignaz Bruder und Andreas Silbermann, in: Acta Organologica, XVI (1982), p.249-264.

Müller, Werner

Gottfried Silbermann. Persönlichkeit und Werk. Eine Dokumentation, Francfort, 1982.

Die Orgeln des Doms zu Arlesheim, mit Beiträgen von Rudolf Walter und Marc Schaefer, Arlesheim, 1983, 119 p.

Hummel, Jörg-Dieter

Friderich Ring. Der vergessene Instrumentenbauer aus dem Elsass und sein Clavicymbal von 1700, mit Beiträgen von Marc Schaefer, Strassburg, Martin Scholz, Basel, und einem Geleitwort von Wilhelm Kempff, Augsburg, 1976, 77 P., ill. 
Bopp, Marie-Joseph

Die evangelischen Gemeinden und Hohen Schulen in Elsass und Lothringen, Neustadt a. d. Aisch, 1963.

Meyer-Siat, Pie

Die Silbermann-Genealogie nach den Strassburger Akten, in Acta Organologica, 11, 1977, p. 137-145.

Sulzmann, Bernd

Historische Orgeln in Baden, München-Zürich, 1980, 296

p., ill.

Meyer-Siat, Pie

Historische Orgeln im Elsass, München-Zürich, 1983, 304

P., ill.

Wettstein, Hermann

Die Orgelbauerfamilie Silbermann. Bibliographischer

Beitrag zu ihrem Leben und Werk (1683. 1783. 1983),

Fribourg/Br., 1983, 89 p. Schriften der Universitäts-

bibliothek Freiburg im Breisgau, 6 .

Weber, Edith

La recherche musicologique. Objet, méthodologie, normes de présentation, Paris, 1980, 171 p. Collection Guides Musicologiques, 1.

Schaefer, Marc

L'orgue du jubé de la cathédrale de Strasbourg, in

Visitatio Organorum. Feestbundel voor Maarten Albert

Vente, (éd. Albert Dunning), Buren, Frits Knuf, 1980,

tome 2, p. 537 - 550 .

Schaefer, Marc

Le grand orgue de la cathédrale de Strasbourg au XVIIe siècle, in Bulletin des Amis de la Cathédrale de Strasbourg, XIV, Strasbourg, 1980, p. 41 - 54.

Pfrimmer, Robert

Les nouvelles grandes orgues de la cathédrale de Strasbourg, in Bulletin des Amis de la Cathédrale de Strasbourg, XV, Strasbourg, 1982, p. 77 - 98. 
Gerard, Bernadette et Machard, Roberte

Le grand orgue et les organistes de Notre-Dame de Paris, Béziers, 1980, 54 p., ill.

Soinne, Paavo

Urkujen vastaanotto-tarkastuksesta 1700-luvulla- eräs dokumentti (1766) ja sen historiallista taustaa, in Pro Organo Pleno. Juhlakirja Enzio Forsblomill 14. 3. 1980 (éd. Seppo Murto et Markku Heikinheimo), Helsinki, 1980, p. $321-331$.

Gugger, Hans

Die Bernischen Orgeln, Berne, 1978, 713 p., ill. 


\section{Archives consultées}

Altenheim, Pfarrarchiv

Bâle, staatsarchiv

Barr, arch. communales

" arch. de la paroisse protestante

Berstett, arch. de la paroisse protestante

Bischoffsheim, arch. communales

Bischwiller, arch. communales

" arch. de la paroisse réformée

Boersch, arch. de la paroisse catholique

Bouxwiller, arch. communales

" arch. du Consistoire

Châtenois, arch. communales

" arch. de la paroisse catholique

Colmar, Archives du Haut-Rhin (AHR)

" Archives Municipales de Colmar (AMC)

" archives de la paroisse protestante

Goxwiller, arch. de la paroisse protestante

Gries, arch. communales

Griesheim-sur-Souffel, arch. de la paroisse catholique

Haguenau, Archives Municipales de Haguenau (AMH)

Heiligenstein, arch. communales

" arch. de la paroisse protestante

Hessenheim, arch. de la paroisse catholique

Karlsruhe, Badisches Generallandesarchiv (GLA)

Kaysersberg, arch. communales

Kolbsheim, arch. de la paroisse protestante

Lahr, Gemeindearchiv

Meissenheim, Gemeindearchiv

Metz, Archives de la Région de Lorraine et du département de la Moselle

" Archives Municipales de Metz

Mittelberghaim, arch. de la paroisse protestante

Molsheim, arch. communales

" arch. de la paroisse catholique

Mulhouse, Archives Municipales de Mulhouse (AMM)

" arch. de la paroisse réformée

Munich, Hauptstaatsarchiv

Nancy, Archives de Meurthe-et-Moselle et des anciens Duchés de Lorraine et de Bar 
Neuwiller-lès-Saverne, arch. communales

Niedernai, arch. de la paroisse catholique

Nuremberg, Landeskirchliches Archiv Nürnberg

Obernai, archives communales

Paris, Archives Nationales

" Bibliothèque Nationale

Riquewihr, arch. communales

" arch. de la paroisse protestante

Rosheim, arch. communales

Sarrebruck, Stadtbücherei Saarbrücken

Saverne, arch. communales

Sélestat, Archives et bibliothèque de Sélestat (AMSél.)

Soultz, arch. communales

" arch. de la paroisse catholique

Strasbourg, Archives du Bas-Rhin (ABR)

" Archives Municipales de Strasbourg (AMS)

" Bibliothèque Municipale

" Archives Administratives

" Archives de l'Evêché

" Archives des Monuments Historiques du Bas-Rhin

" Musées de la Ville de Strasbourg

" Bibliothèque Nationale et Universitaire de Strasbourg (BNU)

" Archives de la Fondation Saint-Thomas

" Archives du Consistoire de Saint-Thomas

" Archives du Chapitre de St-Thomas (déposées aux AMS)

" Archives du Temple-Neuf (déposées aux AMS)

" Archives de la paroisse protestante St-Pierre-le-Jeune

" Archives de la paroisse Sainte-Madeleine

" Archives de la paroisse Saint-Guillaume

" Archives de la paroisse Saint-Nicolas

" Archives de la paroisse Sainte-Aurélie

" Archives de la paroisse protestante Saint-Pierre-le-Vieux (déposées aux AMS)

" Bibliothèque du Grand Séminaire

Stuttgart, Württembergisches Hauptstaatsarchiv

Waldolwisheim, arch. de la paroisse catholique

Wissembourg, arch. communales

" arch. de la paroisse protestante

" arch. de la paroisse catholique

Wurtzbourg, Universitätsbibliothek Würzburg 
Archives privées:

Barr, famille Stoltz

Boulay, famille Haerpfer

Molsheim, famille Kriess

Paris, famille Koehler

Paris et Versailles, famille Mallet-Rosetti

Strasbourg, famille Schwenkedel

Stuttgart-Sillenbuch, famille Hummel-Klinckerfuss 


\section{Table des matières}

1. Introduction.

a) Aperçu général sur l'orgue en France

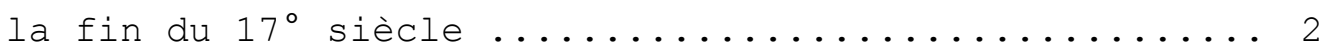

b) La facture d'orgues en Allemagne

la fin du $17^{\circ}$ siècle .......................

c) La facture d'orgues en Alsace

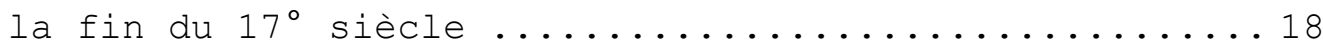

2. Introduction aux "Archives Silbermann de Paris" ....... 38

3. Le prétendu apprentissage d'André Silbermann

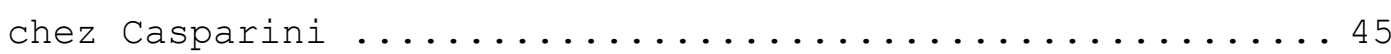

4. Le travail d'André Silbermann auprès de

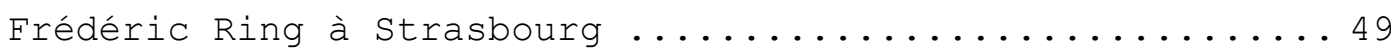

5. La généalogie de la famille Silbermann ............. 57

a) Généalogie $N^{\circ} 1$. Les ancêtres des facteurs

d'orgues saxons et strasbourgeois ...............59

b) Généalogie $\mathrm{N}^{\circ}$ 2. Les ancêtres du facteur

d'orgues Xavier Silbermann à Thonon .............. 72

c) Généalogie $\mathrm{N}^{\circ}$ 3. La lignée haut-rhinoise

originaire de Burnhaupt-le-Bas .............76

6. Documents iconographiques concernant la famille

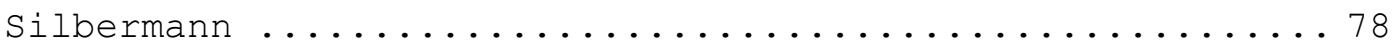

7. Répertoire chronologique des orgues construits

par André Silbermann ...................... 82

8. Répertoire chronologique des orgues construits

par Jean André Silbermann ....................... 84 
9. Répartition confessionnelle des orgues construits par André et Jean André Silbermann .............. 86

10. Les orgues d'André Silbermann.

Compositions, selon l'ordre chronologique .......... 89

11. Les orgues de Jean André Silbermann.

Compositions, selon l'ordre chronologique ............ 129

12. Les pleins-jeux d'André et de Jean André

Silbermann. Répertoire chronologique ............. 198

13. Pérennité et continuité de l'oeuvre des

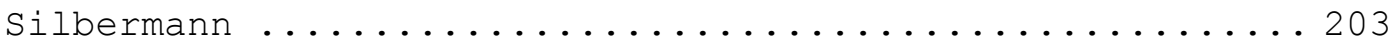

14. Pièces justificatives I.

Extraits de registres paroissiaux, actes

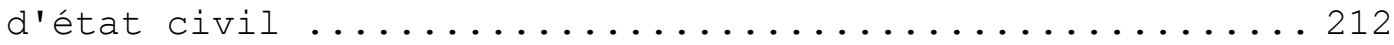

Inventaire après décès de Jean André Silbermann ....... 259

Notice nécrologique concernant Jean André Silbermann . . . 277

15. Pièces justificatives II.

Documents d'archives relatifs aux ouvrages

d'André et de Jean André Silbermann. Documents

complémentaires concernant Baldner et Ring .........280

16. Bibliographie.

I. Histoire d'Alsace. Généralités ............... 423

II. Périodiques ...................... 428

III. Dictionnaires, catalogues ................ 430

IV. Facture d'orgues, histoire de l'orgue en général .. 433

V. Buffets d'orgues ................... 437

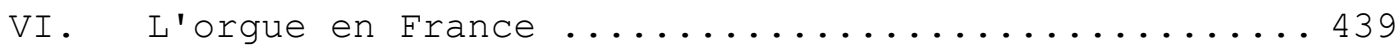

VII. L'orgue en Alsace .......................446 
VIII. La musique en Alsace. Les organistes ...........4454

IX. Monographies de localités et d'églises ........4457

X. Les Silbermann de Strasbourg et leurs oeuvres ....4 462

XI. Les orgues de la cathédrale de Strasbourg .......4 475

XII. Les Silbermann facteurs d'instruments à clavier ... 481

XIII. Le tempérament des orgues des Silbermann ........484

XIV. Jean André Silbermann collectionneur

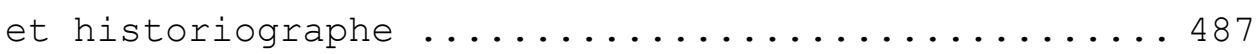

XV. Gottfried Silbermann et ses oeuvres en Saxe ......490

XVI. Les élèves de Gottfried Silbermann ........... 502

XVII. Les Silbermann et l'orgue de Jean-Sébastien Bach .. 504

XVIII.Mozart et l'orgue ................... 507

XIX. L'orgue en Allemagne et en Autriche ............509

XX. L'orgue en Suisse ..................... 520

XXI. L'orgue aux Pays-Bas .................. 523

Supplément à la bibliographie ............... 526

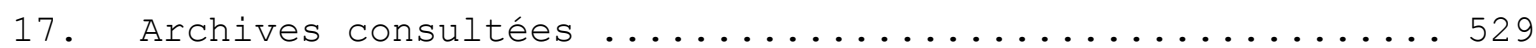

Illustrations:

Page specimen des "Archives Silbermann de Paris" ....... 44

Signatures de Friderich Ring, Andreas Silbermann

et Johann Andreas Silbermann .............. 56

Cartes géographiques (en fin de volume):

a) Localisation initiale des orgues Silbermann.

b) Localisation actuelle des orgues Silbermann.

c) Répartition confessionnelle des orgues Silbermann. 
REPARTITION CONFESSIONNELLE DES ORGUES SILBERMANN

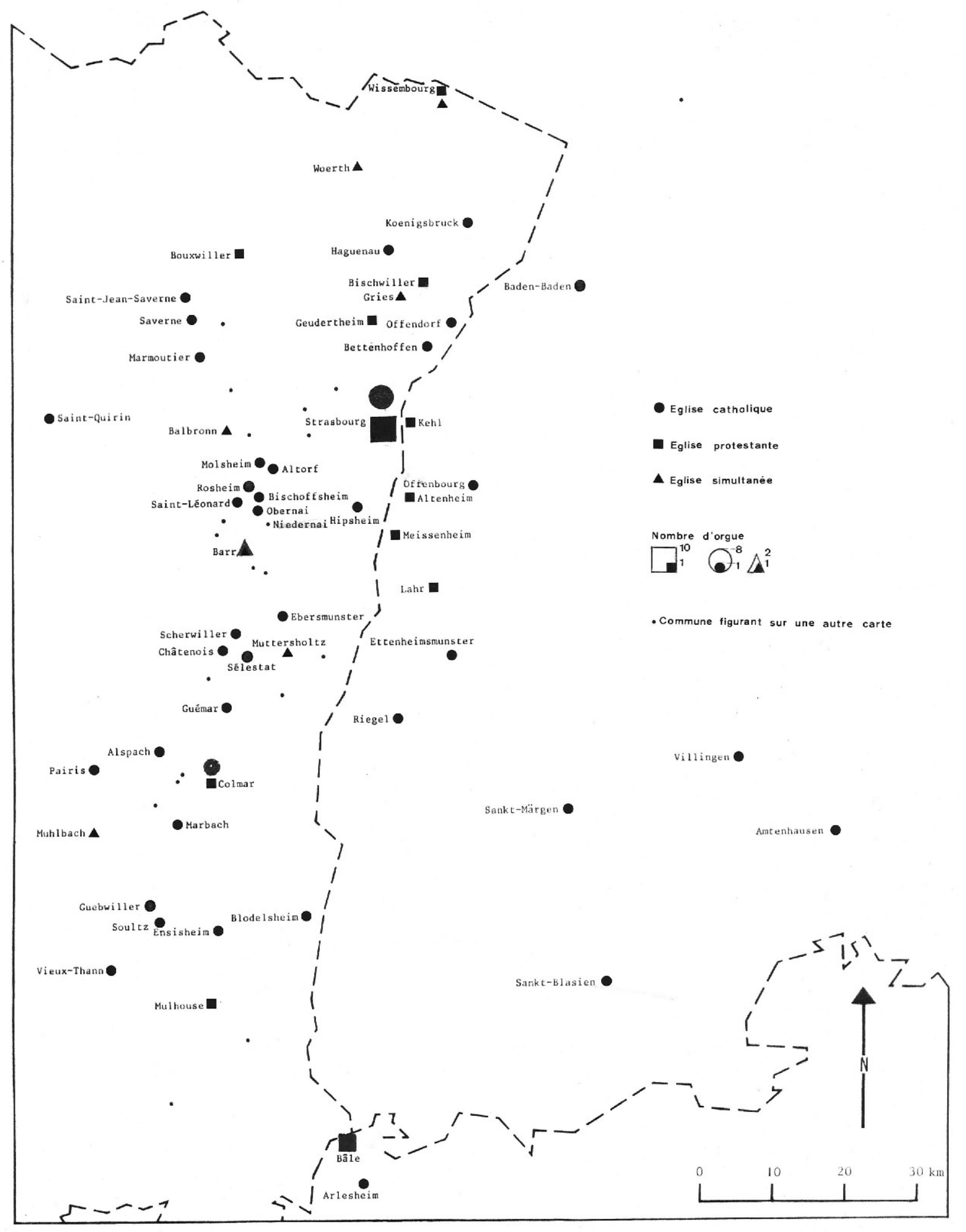




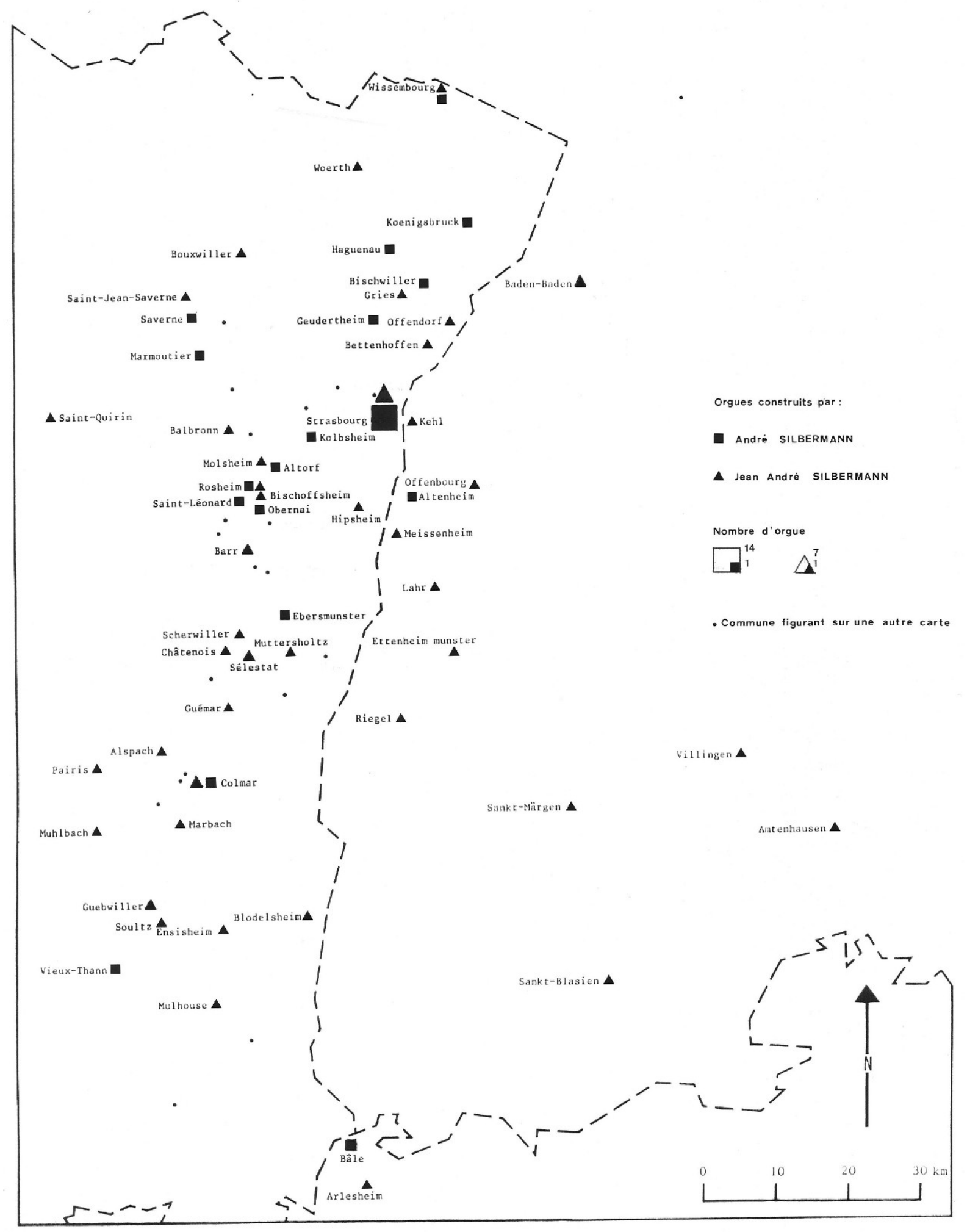




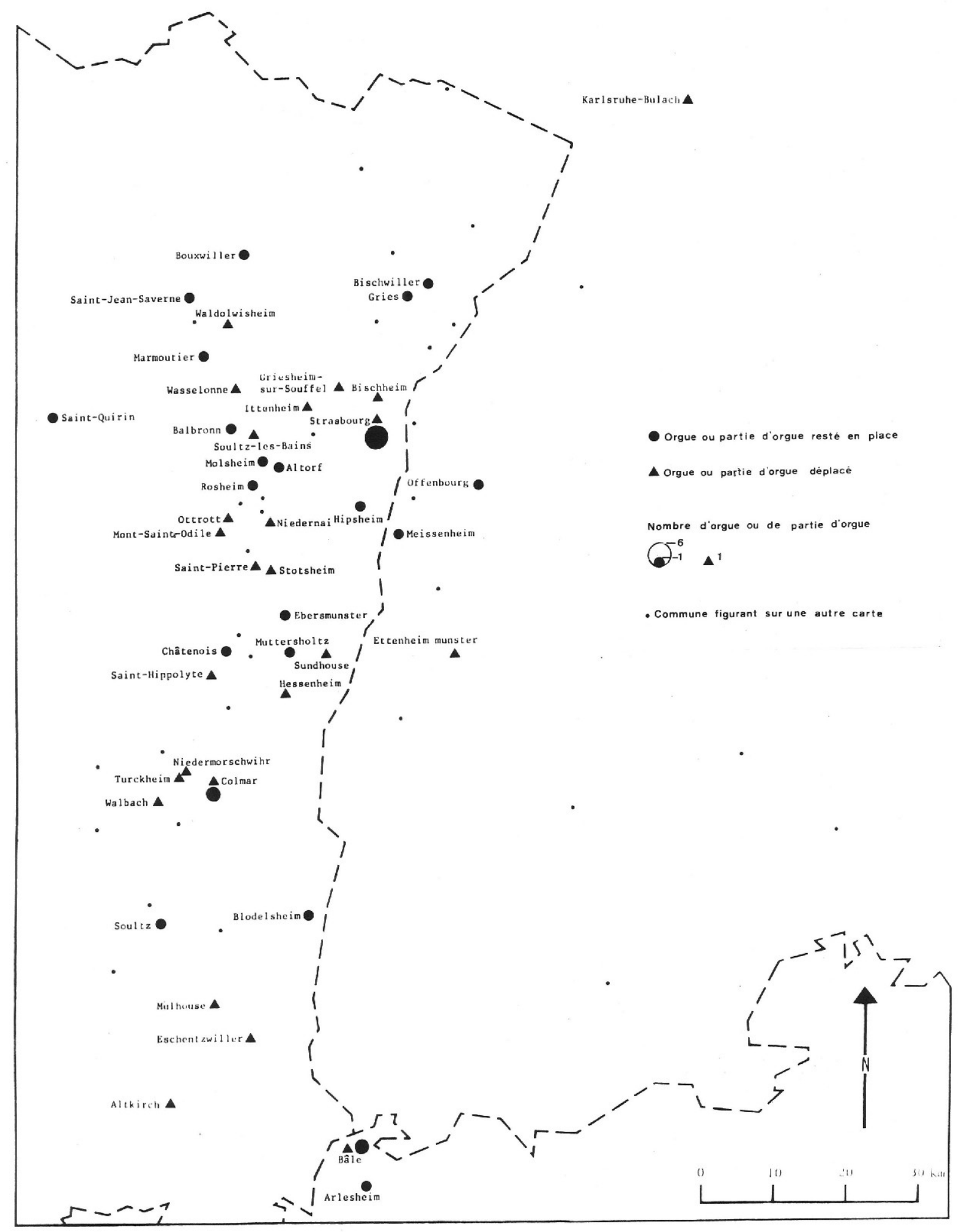

